Travaux et documents du CIRAC

\title{
RELATIONS SOCIALES
}

DANS LES SERVICES D'INTÉRÊT GÉNÉRAL

Une comparaison France-Allemagne

Sous la direction de

Solène HAZOUARD, René LASSERRE, Henrik UTERWEDDE

\section{CIRAC}




\section{Relations sociales dans les services d'intérêt général \\ Une comparaison France-Allemagne}

\section{Solène Hazouard, René Lasserre et Henrik Uterwedde (dir.)}

DOI : 10.4000/books.cirac. 425

Éditeur : IFAEE

Année d'édition : 2011

Date de mise en ligne : 13 décembre 2017

Collection : Travaux et documents du CIRAC

ISBN électronique : 9782905518644

\section{ononatition \\ Qbooks}

http://books.openedition.org

\section{Édition imprimée}

ISBN : 9782905518408

Nombre de pages : 308

\section{Référence électronique}

HAZOUARD, Solène (dir.) ; LASSERRE, René (dir.) ; et UTERWEDDE, Henrik (dir.). Relations sociales dans les services d'intérêt général : Une comparaison France-Allemagne. Nouvelle édition [en ligne]. CergyPontoise : IFAEE, 2011 (généré le 02 octobre 2020). Disponible sur Internet : <http:// books.openedition.org/cirac/425>. ISBN : 9782905518644 . DOl : https://doi.org/10.4000/books.cirac. 425

(c) IFAEE, 2011

Conditions d'utilisation:

http://www.openedition.org/6540 
Travals et documents du CIRAC

\title{
RELATIONS SOCIALES
}

\section{DANS LES SERVICES D'INTERET T GENERAL}

\author{
Une comparaison France-Allemagne
}

Sous la direction de

Solene HAZ OUARD, Rene LASSERRE, Henrik UTERUEEDE 




\section{RELATIONS SOCIALES}

DANS LES SERVICES D'INTÉRÊT GÉNÉRAL

Une comparaison France-Allemagne

Sous la direction de

Solène HAZOUARD, René LASSERRE, Henrik UTERWEDDE

\section{CIRAC}

TRAVAUX ET DOCUMENTS DU CIRAC 


\section{Travaux et documents du CIRAC}

Collection dirigée par René Lasserre

Ouvrage publié avec le concours du Centre Interdisciplinaire d'Etudes et de Recherches sur l'Allemagne (CIERA) et de l'Université de Cergy-Pontoise (Civilisations et identités culturelles comparées des sociétés européennes et occidentales, CICC)
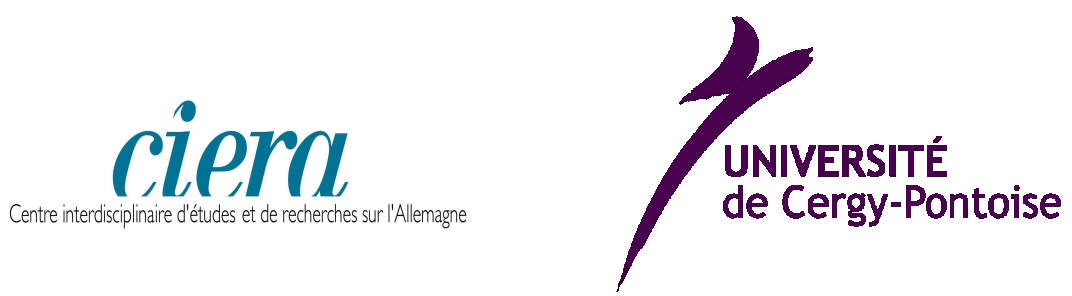

Les contributions publiées dans cet ouvrage engagent uniquement la responsabilité de leurs auteurs.

(C) CIRAC, 2011

www.cirac.u-cergy.fr

CIRAC, c/o Université de Cergy-Pontoise

33 Boulevard du Port - 95011 CERGY-PONTOISE CEDEX

ISBN 978-2-905518-40-8 


\section{RELATIONS SOCIALES \\ DANS LES SERVICES D'INTÉRÊT GÉNÉRAL}

UNE COMPARAISON FRANCE-ALLEMAGNE

TABLE DES MATIÈRES

REMERCIEMENTS

\section{INTRODUCTION}

Solène Hazouard, René Lasserre, Henrik Uterwedde

Le bilan socio-économique contrasté de la dérégulation

des services publics en France et en Allemagne.

I - LA LIBÉRALISATION DES SIG DANS L'UNION EUROPÉENNE ................... 15

Jean-Claude Boual

Le processus d'européanisation des services d'intérêt général

dans le contexte de la construction européenne.

Richard Pond

Syndicats et libéralisation en Europe

Pierre Bauby

Les enjeux sociaux de la dérégulation des services publics marchands

à l'échelle communautaire. Quels défis pour les représentants patronaux?

Jean Lapeyre

Les enjeux sociaux de la dérégulation en France et en Allemagne

dans le contexte européen.

II - Mutations du dialogue social en France et en Allemagne

Jean-Pierre Basilien

L'évolution de la représentation et des stratégies syndicales

dans les services publics en France

Wolfgang Schroeder, Samuel Greef

Nouveaux acteurs de la représentation des intérêts des salariés en Allemagne.

\section{Samuel Greef, Viktoria Kalass}

Les relations sociales à la Deutsche Bahn : nouveaux acteurs et

évolution des rapports de force sur fond de privatisation et de libéralisation

Corinne Dequecker, Pierre-Eric Tixier

Les enjeux de la régulation des entreprises de service public en France

face au processus de leur libéralisation.

Torsten Brandt, Thorsten Schulten

Le rôle de la libéralisation et de la privatisation des services publics

dans l'érosion de la convention collective de branche en Allemagne 
III - VERS DE NOUVELLES FORMES D'EMPLOI ET DE MANAGEMENT ?

Jean-François Amadieu

Développement des ressources humaines

dans les entreprises françaises de service public

Dieter Wagner, avec la collaboration rédactionnelle de Magdalena Chmielewska

Les entreprises publiques en mutation.

Organisation et gestion interne dans les régies municipales

Achim Meerkamp

Nouvelles conditions de travail dans les services publics en Allemagne.

Marnix Dressen

Ouverture du marché du fret ferroviaire. Du statut du cheminot à son refoulement?

Claudia Panke

Gestion des conséquences de la privatisation

dans le domaine des ressources humaines : l'exemple de la Deutsche Bahn AG

IV - MISES EN REGARD SECTORIELLES FRANCO-ALLEMANDES

Dominique Bailly

La régulation sociale dans les services postaux

Bernard Caron

Historique et évolution du statut. Libéralisation du service public de l'électricité

Heinz-J. Bontrup

Régulation sociale et conditions de travail dans le secteur électrique allemand

Bernard Parmantier

Le secteur des industries électriques et gazières en France.

Evolution des relations sociales de la nationalisation à la privatisation

Christophe Gardavaud

Relations sociales et gestion des ressources humaines à la Deutsche Bahn AG

dans le contexte de l'ouverture à la concurrence.

Dominique Aubry

Les évolutions de la régulation sociale

dans le contexte de la libéralisation des transports ferroviaires en France

V - ÉCLAIRAGES D'AUTRES PAYS MEMBRES DE L'UE

Richard Pond

De la nationalisation à la privatisation : l'exemple de la Grande-Bretagne.

Christoph Hermann, Dominik Lindner

Libéralisation des services publics, stratégies d'entreprises

et conséquences pour l'emploi : le secteur postal et électrique en Autriche

Monika Andersson Bäck, Jesper Hamark, Christer Thörnqvist

Conséquences sociales des processus de « marchéisation »

dans le secteur électrique et les services postaux suédois

LES AUTEURS 


\section{REMERCIEMENTS}

Cet ouvrage collectif constitue la synthèse des travaux de deux journées d'étude et d'un colloque organisés en 2009-2010, dans le cadre du projet Relations professionnelles et régulation sociale dans les services d'intérêt général. Comparaison France-Allemagne. Mené par le Centre d'Information et de Recherche sur l'Allemagne contemporaine (CIRAC), en partenariat avec le Deutsch-Französisches Institut de Ludwigsburg (DFI), ce projet s'inscrit dans le cadre des Programmes de formation-recherche soutenus par le Centre Interdisciplinaire d'Etudes et de Recherches sur l'Allemagne (CIERA). Il avait pour ambition de comparer les transformations de l'organisation des relations professionnelles, des modalités et des enjeux de la régulation sociale dans le secteur des services d'intérêt général en France et en Allemagne depuis la fin des années 1990, dans le contexte de la déréglementation et de la libéralisation des anciens services publics intervenues à la suite des directives de l'Union européenne.

Pour mener à bien ce projet d'assez grande ampleur, les organisateurs ont pu bénéficier, en majeure partie, du concours financier apporté par le CIERA, auquel s'est associé le Centre de recherche «Civilisations et Identités culturelles comparées des Sociétés européennes et occidentales» (CICC) de l'Université de Cergy-Pontoise. Nous remercions vivement les auteurs venus de divers pays européens et de différents horizons scientifiques ou professionnels qui ont apporté leur contribution à cet ouvrage, ainsi que Marie Gravey qui a assuré la traduction de leurs travaux.

Nous tenons également à remercier l'équipe du CIRAC de son concours, en particulier Werner Zettelmeier, chargé de recherches, pour ses relectures attentives, ainsi que Cécile Santoul, documentaliste, pour sa coopération active aux travaux d'édition.

Solène Hazouard

Ingénieur d'études au CIRAC
René Lasserre

Directeur du CIRAC
Henrik Uterwedde

Directeur adjoint du DFI 



\title{
Le bilan socio-économique contrasté de la dérégulation des services publics en France et en Allemagne
}

\author{
Solène HAZOUARD, René LASSERRE, Henrik UTERWEDDE
}

Dans le contexte de la promotion et de l'intégration du marché intérieur européen initiées par l'Acte unique, un processus de dérégulation dans les services publics d'intérêt général a été engagé à l'aube des années 1990 au niveau communautaire en vue de favoriser l'émergence d'un grand marché des services à l'échelle européenne. Ce processus de dérégulation avait pour objet d'ouvrir à la concurrence les services d'intérêt général des pays membres qui, jusqu'alors, étaient généralement organisés en monopoles publics et constituaient pour ainsi dire des enclaves nationales échappant à la fois aux règles communes de la concurrence et, de surcroît, au droit commun du travail dans les pays membres. De ce fait même, les directives successives adoptées au niveau communautaire, puis transposées en droit national, ont non seulement modifié les modes de régulation économique, mais plus profondément encore les systèmes de régulation socioprofessionnelle et de gestion des ressources humaines de ce secteur, remettant ainsi en question les particularités sociales du service public qui s'étaient affirmées dans la plupart des Etats membres depuis la fin de la Seconde Guerre mondiale.

Tel est l'objet du présent ouvrage qui étudie et compare, principalement à travers le cas de la France et de l'Allemagne qui sont au centre de l'analyse, mais aussi à partir d'autres exemples européens, tels que celui de l'Autriche, de la Grande-Bretagne et de la Suède, les incidences de cette politique de dérégulation sur les relations collectives de travail et le management social des entreprises dans le secteur, désigné désormais sous le terme de services d'intérêt économique général (SIEG). Au-delà des singularités des expériences nationales qui ont marqué la structuration initiale, puis la transformation des anciens services publics, il ressort de l'analyse des effets que la dérégulation a induits dans le champ social un tableau d'ensemble très différencié, dans lequel coexistent des évolutions régressives en termes d'acquis sociaux statutaires, mais également de nouvelles formes d'organisation des rapports de travail. Les unes et les autres concourent à la modernisation économique et sociale de ce vaste ensemble qui, incluant des secteurs aussi essentiels que ceux de l'énergie, de la communication et des transports, sont d'une importance vitale pour la compétitivité des économies européennes.

\section{Enjeux et bilan de la libéralisation des SIEG en Europe}

L'analyse rétrospective de la libéralisation des SIEG au sein de l'Union européenne, qui est menée dans la première partie de l'ouvrage, en retrace les grandes étapes et les principales modalités, notamment à travers la privatisation d'une très large part des opérateurs historiques nationaux et la politique d'ouverture 
du marché à de nouveaux opérateurs de droit privé. Elle dresse également un bilan plutôt critique de ses effets économiques et sociaux. Sur le plan économique, les résultats sont globalement mitigés au regard des objectifs poursuivis, que ce soit en matière de coûts, d'efficacité, de potentiel d'emplois ou de compétitivité, ou que ce soit en termes mêmes de concurrence avec, souvent, la création de nouveaux oligopoles ou de cartels de fait, à l'instar de ce que l'on a pu observer dans le secteur des télécommunications. Sur le plan de la régulation sociale, les conséquences de la libéralisation varient d'un pays à l'autre et conduisent également à des évolutions contrastées, notamment dans le réaménagement des garanties statutaires ainsi que des conditions d'emploi, de rémunération et de travail des salariés. Mais ce sont peut-être plus encore les modalités du dialogue social qui ont été structurellement modifiées sous l'effet des nouvelles conditions dans lesquelles s'exercent la concurrence, mais aussi des nouveaux défis techniques et qualitatifs auxquels les différents opérateurs se trouvent confrontés dans la prestation des services.

\section{Les mutations du dialogue social en France et Allemagne}

C'est en effet ce qui ressort très nettement de l'analyse comparée des mutations du dialogue social dans les SIEG en France et en Allemagne qui fait l'objet de la seconde partie. En France on assiste globalement, en dépit de la forte résistance syndicale opposée à ce que l'on considère généralement comme un démantèlement du service public, à un recul marqué de la syndicalisation, à une perte de cohésion interne des organisations et à une concurrence accentuée entre elles pour conserver un niveau de représentativité suffisant sur le terrain. Ceci incite les organisations à pratiquer une contractualisation progressive, et à bien des égards constructive, des rapports sociaux au sein des grands opérateurs publics, lesquels pratiquent des modes de management rénovés, tandis que prévaut une régulation très inégale dans le champ des nouveaux opérateurs privés, dont le développement est par ailleurs disparate. L'une et l'autre conduisent à une convergence, voire à une normalisation des rapports sociaux au sein des SIEG sur le modèle du secteur concurrentiel classique où prévaut une dichotomie de plus en plus marquée du dialogue social entre d'un côté le monde de la grande entreprise où il progresse dans une logique «maison » de type «néo-paternaliste » et celui de la PME où il devient sporadique pour ne pas dire quasi inexistant. A noter que dans ce nouveau paysage social polarisé, où l'ancienne dichotomie entre secteur public «nationalisé » et secteur privé s'estompe, l'Etat sort progressivement du jeu social et tend à s'effacer en tant qu'acteur patronal direct et de tout premier plan dans un secteur d'intérêt économique vital.

Mais alors qu'en France, mises à part quelques poches de résistance dans le secteur des grands services logistiques des services portuaires, aéroportuaires et ferroviaires, où la conflictualité reste forte, le contraste public/privé tend pro- 
gressivement à se réduire, il semblerait au contraire qu'en Allemagne le paysage des relations sociales, autrefois plus unifié, se polarise et se différencie entre le secteur industriel et commercial concurrentiel classique, et celui des SIEG. Alors qu'auparavant le secteur public globalement homogène, organisé autour d'acteurs et de procédures stables, s'alignait assez largement sur les pratiques de la régulation contractuelle autonome de branche du secteur privé, sa dérégulation et son ouverture à la concurrence ont conduit à des rapports sociaux pour le moins contrastés, pour ne pas dire instables et conflictuels, qui tendent au contraire à le singulariser. Sous l'effet de la concurrence, les services allemands d'intérêt général semblent en effet s'orienter à la fois vers une fragmentation et une sur-régulation du dialogue social, lesquelles s'incarnent sous des formes diverses, que ce soit par l'émergence d'organisations syndicales et de régulations de type catégoriel, à l'instar de celles développées par les pilotes de ligne ou les conducteurs de locomotives, ou encore par la mise en place d'une régulation sectorielle d'ordre public avec l'introduction en 2008 d'un salaire minimum légal dans le secteur postal. Autant de signes qui témoigneraient en Allemagne d'une évolution des services d'intérêt général vers un corporatisme accentué et conflictuel, articulant des intérêts catégoriels, et finalement assez éloigné d'une régulation homogène que laissait normalement présager l'extension d'une logique de concurrence dans de larges secteurs d'activité dérégulés.

Cette évolution apparemment paradoxale s'explique essentiellement par le fait que sous l'effet de la concurrence introduite par l'ouverture des différents services à de nouveaux compétiteurs, l'ancienne régulation par grandes branches de service public entre employeurs publics (fédéraux, régionaux et communaux) et syndicats unitaires des services publics a volé en éclats. L'opposition d'intérêts entre opérateurs historiques et nouveaux compétiteurs s'est dès le départ cristallisée autour des écarts de conditions de rémunération et de travail, hypothéquant d'emblée la constitution d'un front patronal uni. La négociation sectorielle s'est ipso facto recentrée vers le niveau de l'entreprise, en se reconstituant entre l'opérateur historique en cours de privatisation et l'organisation syndicale qui y était fortement implantée et s'y montra avant tout préoccupée de sauvegarder les acquis sociaux. Et ce d'autant plus qu'il s'agissait de faire pièce à la pression à la baisse que ne manquèrent pas d'instaurer les opérateurs privés à travers les contrats négociés avec leurs personnels. Cette déconcentration sectorielle de la négociation et son déplacement vers l'entreprise à laquelle on a assisté en Allemagne n'est certes pas propre aux SIEG, puisqu'elle s'est également fortement développée au cours des vingt dernières années dans les principales branches de l'industrie et du commerce du secteur concurrentiel. A ceci près, toutefois, que dans ce vaste socle du partenariat social allemand, la négociation d'entreprise a été développée conjointement par les partenaires sociaux et a été précisément intégrée et articulée avec la négociation de branche, ceci afin de dégager des marges de flexibilité négociées jugées nécessaires dans la 
gestion des conditions d'emploi et de travail des entreprises du secteur considéré. La dérégulation contractuelle dans les SIEG allemands a procédé tout au contraire d'une logique de désintégration partielle non contrôlée, provoquée par l'intrusion du marché dans un secteur antérieurement protégé, et conduisant à une polarisation entre une régulation de type néo-corporatiste et protectionniste autour des opérateurs historiques, et une régulation sociale a minima dans la sphère concurrentielle. Cette fragmentation initiale ne semble cependant pas devoir perdurer au-delà d'une phase d'adaptation transitoire dans le contexte d'une dérégulation qui apparaît très largement effective à la fois dans la privatisation des entreprises publiques et dans l'ouverture tangible des marchés à de nouveaux compétiteurs, la première conduisant à des innovations managériales fortes au sein des anciens opérateurs, la seconde à une contractualisation sectorielle intégrant progressivement l'ensemble des acteurs comme c'est déjà le cas dans le secteur ferroviaire.

\section{Nouvelles conditions d'emploi et nouvelles formes de management social}

Si l'on se concentre, comme le font la troisième et la quatrième partie de l'ouvrage, sur l'étude des conditions d'emploi et l'évolution des formes de management, ainsi que sur la mise en regard des processus de transformation intervenus en France et en Allemagne dans les principaux secteurs, on relève toutefois d'assez nombreuses convergences. Notamment dans la gestion de la variable emploi avec une tendance générale et parallèle à la compression des effectifs, laquelle se décline de façon relativement comparable selon les activités, et un recours accentué à des formes de travail temporaire constaté dans la plupart des secteurs. A quoi vient s'ajouter un alignement progressif des standards sociaux sur ceux du secteur concurrentiel de droit commun.

Dans l'évolution des statuts des personnels, la situation est plus tranchée dans la mesure où en France les agents du secteur public relèvent généralement de statuts particuliers leur assurant la garantie de l'emploi, des avantages et des régimes de protection spéciaux, tandis qu'en Allemagne prévaut, en dehors des Chemins de fer, de la Poste ou des Télécoms - où une part non négligeable du personnel bénéficiait du statut de fonctionnaire - un statut d'employé contractuel modulable. La régression de la part du personnel sous statut au bénéfice d'un nombre croissant d'employés contractuels à laquelle on assiste en France n'a cependant pas un impact comparable, en termes de gestion des ressources humaines, à la mutation des statuts qu'a entraînée la privatisation des grands opérateurs publics allemands, laquelle a été accompagnée de la mise en place de structures de gestion déléguée des personnels fonctionnaires, qui ont permis de les réaffecter à des fonctions soumises aux obligations de droit commun. En Allemagne, la privatisation couplée à la dérégulation a conduit à une transformation plus radicale des modes de 
management sur le modèle du management privé chez les grands logisticiens que sont aujourd'hui, dans leur secteur respectif, Deutsche Bahn et Deutsche Post ou encore les grands opérateurs internationaux que sont devenus les quatre majors de l'énergie (E.ON, RWE, EnBW et Vattenfall Europe) ou Deutsche Telekom. Dans ces entreprises qui ont adopté une logique d'opérateurs internationaux à l'intérieur desquels les diverses activités ont été filialisées et soumises à l'impératif de rentabilité, le changement de management a été profond sur tous les plans. Dans le secteur de la gestion des personnels, il a été complètement réaligné sur le principe de flexibilité de l'emploi, à la fois dans sa dimension géographique, dans les modes de rémunération et le déroulement de carrière en fonction de la qualification et des compétences.

En France, où au contraire ont continué de prévaloir des systèmes de management relativement spécifiques hérités des entreprises à statut, se profile actuellement une normalisation de la régulation sociale vers un système contractualisé qui certes se rapproche du secteur privé, mais dans laquelle se maintiennent des formes de management hybride où coexistent des garanties héritées du statut avec de nouvelles pratiques de gestion des ressources humaines relevant du secteur privé, en particulier dans la gestion des compétences et du développement du personnel. Cette évolution permet aux grands opérateurs de pouvoir faire face, sans pour autant renoncer aux positions privilégiées et aux acquis sociaux de l'opérateur historique, aux enjeux de mobilité, de flexibilité et de qualité que requièrent des services de plus en plus concurrentiels, tant au plan du marché domestique que du marché transfrontières. Il n'est cependant pas sûr que cette modernisation adaptative suffise à les hisser avec succès au rang de majors européens.

La stratégie de modernisation incrémentale qui prévaut ainsi en France dans la plupart des grands services restés dans l'orbite publique s'apparente en fait, dans sa philosophie, au modèle qui a été mis en œuvre en Allemagne au niveau régional et local, principalement dans les anciennes régies autonomes de transports, d'électricité et de gaz, ou des services de l'eau. Ces entreprises de structuration oligopolistique, qui demeurent encore peu concurrentielles plus de dix ans après leur ouverture au marché, affichent néanmoins, elles aussi, de nouveaux modes de gestion qui relèvent des normes du secteur privé. Ceci à la fois en termes de rentabilité économique et de développement local, de qualité environnementale et de gestion des ressources humaines, et aux fins de conserver leurs positions territoriales, mettant ainsi en œuvre un modèle de management d'intérêt général axé à la fois sur la responsabilité sociale interne de l'entreprise, sa rentabilité et la qualité du service rendu. Le modèle que défendent les grands opérateurs français restés dans le giron public est du même ordre à l'échelle du territoire et du marché national. Ce modèle économique et 
social n'est pas pour autant complètement en phase avec les ambitions qu'ils affichent par ailleurs au plan européen et international.

LA COMPARAISON FRANCO-ALLEMANDE FONDÉE SUR UN ASSEZ LARGE ÉVENTAIL D'ÉTUDES DE CAS apporte ainsi des analyses riches d'enseignements sur l'évolution des SIEG en Europe. La focalisation sur deux modèles nationaux dont les parentés historiques, au moins en termes de philosophie de l'Etat, demeurent néanmoins assez proches, risquerait de donner une vision quelque peu réductrice de la problématique générale des SIEG en Europe. C'est pourquoi l'ouvrage ouvre, dans sa dernière partie, sur quelques éclairages issus d'autres pays membres de l'UE. Nous ne pouvions naturellement en aucune manière faire l'impasse sur le cas de la Grande-Bretagne qui a été indiscutablement le pays précurseur en matière de libéralisation et de rénovation des services publics et dont l'expérience, audacieuse dans ses méthodes et ambivalente dans ses résultats, a servi de référence et bénéficié à l'ensemble de l'Union. Le modèle social suédois, dont on connaît la capacité novatrice permanente, nous invitait à analyser son expérience de modernisation des services publics qui nous apporte des éléments originaux quant à la mise en œuvre déjà ancienne et récurrente d'une logique de marché dans les services d'intérêt public. Enfin le modèle autrichien, dont on peut dire qu'il a fait preuve d'exemplarité dans la mise en œuvre des orientations de l'UE en matière de dérégulation, contribue à en illustrer la praticabilité et l'efficacité économiques mais n'en montre pas moins les limites en termes de progrès social. Bref, un cas d'école en tant que tel, lui aussi tout à fait instructif et qui montre que, loin d'être une atteinte aux identités sociales ou une panacée économique, la dérégulation n'aura été que ce que les Etats membres auront bien voulu en faire. 
$-1-$

LA LIBÉRALISATION DES SIG

DANS L'UNION EUROPÉENNE 



\title{
Le processus d'européanisation des services d'intérêt général dans le contexte de la construction européenne
}

\author{
Jean-Claude Boual
}

\begin{abstract}
Après un rappel des missions réalisées par le Comité européen de liaison sur les services d'intérêt général (Celsig) dont il est Animateur, Jean-Claude Boual retrace les grandes étapes de l'européanisation des services d'intérêt général pour se pencher ensuite sur les trois types de démarches liées à leur élaboration à l'échelle de l'UE (législative, relative aux Traités et jurisprudentielle). Pour conclure, il ouvre le débat sur deux questions : celle de la subsidiarité dans le cadre de la construction du marché unique et celle de la définition d'une économie sociale de marché au niveau communautaire.
\end{abstract}

Au sein de l'Union européenne, un processus juridique est en cours en vue d'élaborer une conception européenne des services d'intérêt général (SIG), tout comme le Conseil d'Etat a établi une conception juridique du service public en France à la fin du $\mathrm{XIX}^{\mathrm{e}}$ et au début du $\mathrm{XX}^{\mathrm{e}}$ siècle.

\section{Création et missions du Celsig}

Le Comité européen de liaison sur les services d'intérêt général (Celsig) a été créé à l'issue d'un séminaire organisé au titre d'une association française à Bruxelles, intitulé «le premier forum des services d'intérêt général ». C'est d'ailleurs suite à ce forum que l'utilisation du terme «services d'intérêt général » s'est imposée au niveau communautaire.

Ce comité européen a été fondé à la demande des participants. Il s'agit d'un lieu de rencontre, de travail en commun et d'un réseau de réseaux de la société civile, auquel participent de façon volontaire, en conservant leur liberté d'expression (le Celsig ayant lui-même une expression qui est le résultat d'un travail commun), un grand nombre d'organisations : la Confédération Européenne des Syndicats (CES), la Confédération Européenne des Cadres (CEC) et leurs fédérations membres, le Centre européen des entreprises à participation publique et des entreprises d'intérêt économique général (CEEP) ou encore les organisations de la plate-forme des ONG sociales travaillant au niveau communautaire, parmi lesquelles ATD Quart Monde, le Secours populaire et l'UNIOPSS à l'échelle de la France et, au niveau européen, les associations de consommateurs et les associations de protection de l'environnement. Le Celsig travaille également avec les mutuelles, des réseaux d'universités et d'universitaires, notamment le CIRIEC, ainsi qu'avec le CECODHAS, en charge du logement social. 
A la demande de la Fédération des services publics de la CES, le Celsig organise une journée de réflexion stratégique sur la stratégie globale des syndicats et des ONG par rapport à la politique menée actuellement sur les SIG au niveau communautaire.

Sur son site Internet, ce think tank publie un bulletin bimensuel, bilingue français-anglais. Ses animateurs réalisent des contributions régulières en fonction des consultations de la Commission et des problèmes posés. Les colloques organisés par le Celsig se tiennent en général au Parlement, au Comité économique et social européen ou au Comité des régions.

Le think tank travaille par conséquent avec les institutions, au regard desquelles il est considéré comme le point fédérateur par rapport à la problématique des SIG au niveau communautaire.

Le Celsig se réunit toutes les six semaines à Bruxelles, généralement chez l'un de ses partenaires, en raison du fait que le peu de moyens dont il dispose lui permet uniquement de financer les manifestations organisées. Les organisations membres du Celsig reconnaissent l'utilité de son travail, notamment la CES, qui a pu surmonter ainsi les contradictions internes des grandes Confédérations nationales sur la problématique des SIG dans le cadre communautaire. A partir du Congrès d'Helsinki en 1999, l'apport extérieur du Celsig a permis à la CES de faire des services publics un des axes majeurs de ses revendications.

\section{La politique de libéralisation des SIG à l'échelle communautaire}

Depuis le début des années 1990, un processus de libéralisation s'est développé au niveau communautaire, sous l'impulsion notamment de la Commission. Cette politique de libéralisation a commencé par les services publics de réseau, c'est-à-dire les transports, les télécommunications, la poste et l'énergie (essentiellement pour ce qui est de l'électricité et du gaz). Puis de proche en proche, tous les services publics locaux et les services publics sociaux ont été intégrés au processus. Ce n'est d'ailleurs pas un hasard si à partir de 2004, la Commission a introduit la notion de services sociaux d'intérêt général (SSIG) dans le débat. Dès lors, la problématique recouvre l'ensemble des SIG au niveau européen. Ce processus a entraîné des modifications dans l'organisation des services publics de chacun des Etats membres.

Si, d'une part, cette politique de libéralisation est progressive, de l'autre, elle n'est pas unilatérale. Elle a été impulsée notamment par la société civile (syndicats, ONG, CEEP), par un certain nombre d'Etats membres, en tenant compte d'une réalité sociale : les services d'intérêt général constituent en effet un élément fondateur du modèle social européen qui, au-delà de sa diversité, regroupe un certain nombre de grandes caractéristiques.

Ce processus de libéralisation s'est accompagné d'un processus de maintien, voire dans certains cas de développement d'obligations de service public. 


\section{Les étapes de l'européanisation des SIG}

Dans le contexte de la construction européenne, le processus d'européanisation des services d'intérêt général s'est fait selon trois étapes.

1957-1987 : ignorance de la problématique

Cette phase débute avec la signature du Traité de Rome, pour s'achever avec la mise en place de l'Acte unique. Certes, l'ancien Article 90 du Traité de Rome soustrayait à la concurrence les entreprises chargées d'un monopole fiscal ou d'une mission de service public quand la concurrence faisait échec, en fait ou en droit, à la bonne conduite de ces missions. Et l'ancien Article 77, relatif aux transports, reconnaissait la possibilité de missions de service public dans ce domaine (c'est d'ailleurs le seul endroit dans le Traité de Rome où le terme de service public est utilisé). Malgré tout durant cette période, peu d'évolutions se sont produites. Le règlement de 1969 sur les obligations de service public dans les transports par route, chemin de fer et voies navigables se contentait finalement de prendre en compte la situation existante, sans bouleverser toutefois les conditions d'exercice des missions ou de fourniture du service public dans l'ensemble des Etats membres. Il faut tenir compte dans ce contexte du fait que la CEE était composée en 1969 de seulement six Etats membres.

1987-1997/98 : une période conflictuelle

Durant cette seconde phase, le débat fait rage entre d'un côté, ceux qui souhaitent libéraliser les secteurs concernés via une approche très dogmatique de la libéralisation et du marché, incluant la problématique de l'accès des tiers au réseau (ATR), et ceux qui s'y opposent, notamment en raison de la spécificité de leur secteur. Peut-on considérer par exemple que l'électricité est une marchandise comme une autre, bien qu'on ne puisse pas la stocker? La discussion portait sur les grands services de réseau gérés par des monopoles naturels, induisant parfois des effets de club (télécoms).

Avec la mise en œuvre de l'Acte unique, l'année 1987 a créé une véritable rupture pour plusieurs raisons. Au milieu des années 1980 se sont développées de grandes avancées technologiques, avec l'essor des technologies de l'information et de la communication, l'exemple le plus caractéristique étant celui des télécommunications et notamment du téléphone. En 1987, il existait encore un monopole d'Etat dans ce domaine, y compris sur les terminaux. Les évolutions technologiques ont touché tous les secteurs (distribution d'électricité, transports, chemins de fer...).

En deuxième lieu, ces grands services publics, construits pour la plupart après la Seconde Guerre mondiale, entraient dans une phase de dysfonctionnements importants. Les premières libéralisations ont été réalisées au Royaume- 
Uni sous un gouvernement travailliste, au prétexte de lutter contre le corporatisme (notamment des syndicats). Sont venus se greffer ensuite des problèmes politiques, notamment à travers la grande grève des mineurs de 1984-1985 sous le gouvernement de Margaret Thatcher. Mais les dysfonctionnements étaient réels : beaucoup d'évolutions se sont faites au nom de l'intérêt des consommateurs, compte tenu de la capture corporatiste des services publics par le management et les organisations syndicales. Car le corporatisme n'est pas uniquement le fait des organisations syndicales. C'est aussi en grande partie, et peut-être en premier lieu, le fait du management des entreprises.

Troisième facteur d'évolution : l'application des théories néolibérales au niveau mondial, avec l'arrivée au pouvoir de Margaret Thatcher au Royaume-Uni et de Ronald Reagan aux Etats-Unis. L'école de Chicago a commencé à rayonner sur le plan idéologique dans toutes les universités ainsi qu'à travers les débats communautaires.

L'Acte unique constitue le quatrième et dernier facteur d'évolution. Ce bond qualitatif a permis de relancer la construction européenne sous l'impulsion de Jacques Delors. L'idée était de passer d'une conception d'un marché commun organisé alors en 12 marchés nationaux (certes sans barrières douanières pour la circulation des marchandises et des capitaux) à celle d'un marché unique doté d'une réglementation commune sur l'ensemble du territoire européen. Induisant près de 400 directives, cette démarche impliquait notamment de déconstruire les monopoles nationaux des services publics de réseau. Comme mentionné dans les textes communautaires, cela passait par la politique de concurrence qui a pris un poids particulier, devenant un véritable dogme.

\section{7/98-aujourd'hui : l'articulation entre le marché et les missions des SIG}

De l'article 16 du Traité d'Amsterdam a découlé une inflexion dans le processus de libéralisation. L'article reconnaissait l'importance des services d'intérêt économique général (SIEG) dans la cohésion sociale et territoriale de la construction européenne; il renvoyait la Commission et les Etats membres à leurs propres responsabilités afin de faire fonctionner ces services correctement. Il s'agit dès lors non pas d'une période d'apaisement, mais d'une période (certes conflictuelle) de recherche d'articulation entre le marché (la politique de concurrence) et les missions que doivent fournir les SIG. Il n'existe pas de consensus au sein du Parlement ni au sein du Conseil, bien que la position de la société civile soit relativement homogène, le Celsig ayant joué un rôle important de ce point de vue.

Cette recherche d'articulation des grands réseaux de service public est loin d'être évidente : les approches des Etats membres diffèrent. On assiste par conséquent à des situations assez paradoxales : certains pays qui, comme la Suède, ont de nombreuses missions de service public se retrouvent du côté des pays ultralibéraux comme le Royaume-Uni dans les débats au Conseil. Cela se pro- 
duit du fait d'une incompréhension liée à des problèmes de vocabulaire, des différences culturelles, ou des ambiguités concernant la subsidiarité.

De nombreuses évolutions se sont produites durant cette période. Désormais commence à émerger l'idée de construire des services publics au niveau européen, et pas seulement à travers les demandes de la société civile. Dans un contexte de crise financière, économique, sociale et écologique, les acteurs appellent à des avancées fortes. Par conséquent, il est peut être en train de s'ouvrir une quatrième période plus positive par rapport à la construction des SIG au niveau européen.

\section{Les démarches adoptées dans la construction des SIG au niveau communautaire}

Dans ce processus, les démarches législative, relative aux Traités et jurisprudentielle sont entremêlées.

\section{La démarche législative}

Il s'agit essentiellement des directives de libéralisation de l'énergie, du secteur postal, des télécoms, et des règlements dans le domaine des transports. Dans tous ces secteurs, la libéralisation s'est faite de manière progressive, sur une période de 10 à 15 ans, voire davantage. Elle se situe aujourd'hui dans une phase d'achèvement du point de vue de la réglementation communautaire. Pour ce qui est de la transposition et de l'application effective des dispositions dans les Etats membres en revanche, la problématique est différente. Dans le secteur de l'électricité notamment, une bonne vingtaine d'Etats membres font l'objet d'une procédure de la part de la Commission pour non application des obligations de service public.

Les différents secteurs sont d'ores et déjà libéralisés : les transports aériens (1998), les télécoms (2000), le secteur postal (2009), l'électricité et le gaz (2007) et le fret ferroviaire (2006, à l'exception du transport régional des voyageurs). Dans tous les secteurs, les directives et les règlements de libéralisation ont été adoptés en codécision avec Parlement et le Conseil, c'est-à-dire que tous les Etats membres les ont approuvés.

Sous la pression de certains Etats membres, mais aussi plus particulièrement de la société civile, dans l'ensemble des directives et règlements ont été introduites des obligations de service public: soit de service universel (secteur postal, télécoms, électricité), soit de service public (transports). Selon les secteurs, ces obligations figurent dans la directive de libéralisation, dans des règlements pour les transports ou dans une directive particulière, comme dans le cas des télécoms (secteur dans lequel le débat sur le contenu du service universel est toujours ouvert, notamment au sujet de l'accès à Internet). Certains Etats membres et la société civile souhaitent qu'il s'agisse d'un service universel. 
Mais la majorité des Etats membres s'y oppose. Pour les transports, un règlement spécifique détermine les obligations de service public. Le débat autour de ce règlement a duré plus de dix ans : à travers un processus de libéralisation économique, la Commission a tenté de mettre en concurrence les régies avec les autres entreprises, remettant en cause la libre administration des collectivités locales et le libre choix de telle ou telle collectivité qui est le résultat du suffrage universel. Le blocage s'est fait au Parlement.

Le processus de libéralisation n'est pas un processus strictement économique. Il est également social et politique. L'argumentation portait sur des questions fondamentales telles que le poids des services publics, leur rôle dans nos sociétés, la garantie d'exercice des droits fondamentaux, la garantie de la citoyenneté, l'accès à des services essentiels. Il ne s'agissait pas seulement de libéraliser parce que c'était dans l'air du temps.

La question de la souveraineté se pose également. Outre les obligations de service public, ou des définitions de service universel au niveau communautaire, sont mis en place des régulateurs nationaux dans chacun des secteurs, $\mathrm{y}$ compris actuellement dans les chemins de fer. Ces régulateurs se sont plus ou moins rassemblés en club européen. Malgré les propositions de la Commission dans un certain nombre de secteurs, les Etats membres ont refusé jusqu'à présent l'existence de régulateurs européens. Il s'agit là de l'une des grandes interrogations pour les années à venir : comment peut-on avoir une réglementation communautaire commune sur l'ensemble du territoire européen avec une régulation parcellisée en 27 Etats membres ? Cette problématique n'est pas encore parfaitement réglée.

Il ne faut pas assimiler privatisation et libéralisation. Certes, l'idéologie néolibérale et la libéralisation poussée de façon dogmatique conduisent souvent à la privatisation. Mais celle-ci n'est pas obligatoire. Elle est de la responsabilité des Etats membres et non des instances communautaires. Dans les différents secteurs, les statuts sont différents selon les opérateurs. C'est moins vrai dans les télécoms où la privatisation est allée beaucoup plus loin.

La libéralisation s'est faite par vagues successives, la Commission passant à l'étape suivante sans réelle évaluation de celle en cours. Parfois, les directives de libéralisation ont été adoptées au niveau communautaire sans que les transpositions des directives précédentes aient été faites dans tous les Etats membres. Cette fuite en avant constitue un problème patent. Aujourd'hui encore, les conséquences de la libéralisation n'ont pas fait l'objet d'une réelle évaluation dans le domaine économique, le domaine social, ou pour ce qui est des prix et de la qualité de service fourni aux utilisateurs. Cela ne veut pas dire qu'il n'existe aucun rapport sur le sujet: la Fédération des services publics de la CES a réalisé des études sur la qualité et l'emploi dans ces secteurs, avec des résultats contradictoires. L'étude « Mapping of the public services » du CEEP est la première publication portant sur la place de l'ensemble des SIEG dans l'économie communautaire. Bien qu'elle ait été menée à la demande de la Commission, il est 
regrettable de constater que ces questionnements apparaissent vingt ans après le début du processus de libéralisation.

A l'issue d'un rapide tour d'horizon, on s'aperçoit que les effets concrets de la libéralisation sont contrastés selon les secteurs et selon le point de vue adopté (emploi, prix, qualité du service). Dans quelques cas, cela s'est traduit par une amélioration, dans la majorité des cas, les résultats sont beaucoup plus discutables.

\section{Les concepts et traités}

Concurremment à cette démarche législative de libéralisation, souvent sous la pression de la société civile et de quelques Etats membres, la Commission alimentait le débat sur les concepts à travers des textes dans lesquels elle développait une approche générale de la problématique. Plusieurs communications ont été publiées, la première (septembre 1996) portant sur les services d'intérêt économique général (SIEG) et la construction européenne. Sur proposition de la Belgique et de la France, cela a abouti à l'adoption de l'article 16 du Traité d'Amsterdam, reconnaissant l'importance des SIEG sur la cohésion territoriale et la cohésion sociale, dans le contexte de la construction européenne. Ont suivi une deuxième communication de la Commission (janvier 2001), un rapport au Conseil (fin 2001), un livre vert (mars 2003), un livre blanc (avril 2004) et une communication (2007) dans le cadre plus large du marché intérieur. La réflexion a été alimentée avec des définitions plus ou moins précises. Malgré tout, notamment dans le livre blanc d'avril 2004, la Commission a reconnu qu'en cas de contradiction entre l'intérêt général et la concurrence, l'intérêt général devait l'emporter. Cela ne signifie pas néanmoins qu'elle applique ce principe dans chacune de ses propositions.

De son côté, le Parlement a adopté plusieurs résolutions (en 2002, 2004 et 2006). Répondant à une demande du Celsig et de l'ensemble de la société civile, il a créé un intergroupe « services publics » en janvier 2010, extrêmement suivi par toute une série de parties prenantes, d'ONG et de syndicats : plus de cent personnes participent aux réunions de l'intergroupe, qui se tiennent une fois par mois lors de chaque session parlementaire.

De même, le Comité des régions ainsi que le Comité Economique et Social ont mené de véritables travaux sur ces questions. L'idée d'une directive cadre a été émise par la société civile dans les années 1998-1999. En 2005-2006, cinq projets ont été proposés. Ils étaient initiés par un groupe politique au Parlement européen (le PSE), le Comité des régions, la CES, le CEEP et le Celsig. Un séminaire du Celsig a porté sur la convergence de ces projets de façon à peser sur les instances communautaires et la Commission. Ces travaux n'ont pas abouti au niveau législatif, mais ils ont amené les acteurs à aborder la problématique des Traités : l'ancien Article 90 du Traité de Rome (l'article 86 du Traité de Maastricht) sur l'exception des services publics dans la politique de concurrence et l'article 16 introduit dans le Traité d'Amsterdam. Ce dernier a repré- 
senté une évolution significative : il évoque la cohésion sociale et territoriale. Les services d'intérêt économique général doivent pouvoir fonctionner sur la base de principes et dans des conditions leur permettant d'accomplir leurs missions. Dès que cet article a été adopté, s'est engagée une polémique avec la Commission pour savoir s'il était susceptible de générer du droit dérivé. Cette question a incité les acteurs à poursuivre le débat par rapport au contenu du traité.

Les évolutions menées portent d'une part sur la Convention $\mathrm{n}^{\circ} 1$ (celle qui a adopté la Charte des droits fondamentaux européenne) dont l'article le plus débattu (l'article 36) reconnaissait le droit d'accès aux SIEG. De nombreux articles de la Charte des droits fondamentaux traitent des SIG, qu'il s'agisse de l'aide au logement, des aides sociales, de la perte d'emploi, de la vieillesse, de la dépendance, des accidents du travail, des services sociaux, de la sécurité sociale ou du droit à l'éducation. Par conséquent, la Charte a apporté des éléments importants, bien qu'initialement, elle ait été seulement déclaratoire et qu'elle n'ait pris de puissance politique et normative que depuis l'entrée en vigueur du Traité de Lisbonne, au $1^{\text {er }}$ décembre 2009 , de sorte qu'elle n'a pas produit tous ses effets.

La Convention $\mathrm{n}^{\circ} 2$ (celle qui a élaboré le projet de Traité établissant une Constitution pour l'Europe) s'est également emparée de ce sujet sous l'impulsion de la société civile. A chaque séance, la problématique des SIG a été débattue ou évoquée. Le projet de Traité établissant une Constitution établissait une hiérarchie des normes en proposant des lois à la place des directives et des règlements. De ce fait, la Commission européenne qui, au titre de l'article $86 \$ 3$ du Traité, peut adopter de sa propre initiative sans passer par le législateur (le Conseil et le Parlement) des directives dans le domaine de la concurrence pour les SIEG, perdait cette capacité car les directives perdaient leur caractère législatif pour entrer dans le domaine règlementaire au sens français du terme. Hélas, avec le Traité de Lisbonne cette disposition a disparu puisque ce traité se contentant d'amender les traités précédents a repris des termes de directive et de règlement pour les actes législatifs communautaires et que la hiérarchie des normes a disparu.

Cependant dans le Traité de Lisbonne, nous observons deux avancées importantes: d'une part une modification de l'article 16 (qui devient l'article 14) qui, sans préjudice des articles 106 et suivants sur les problèmes de concurrence, permet de faire sous la forme de la codécision c'est-à-dire de la procédure législative normale (Conseil/Parlement), des règlements sectoriels et transversaux sur la problématique des services publics. Le deuxième apport essentiel (qui est le résultat d'un débat relatif au logement social dans les Pays-Bas) réside dans le protocole $\mathrm{n}^{\circ} 26$. Celui-ci reconnaît un large pouvoir discrétionnaire aux Etats membres, définit les secteurs de SIG ainsi que les grands principes sur lesquels doivent se fonder les SIG au niveau communautaire: un niveau élevé de qualité, de sécurité, d'accessibilité, l'égalité de traitement, la 
promotion du service universel et les droits des utilisateurs. Ce protocole a la même valeur juridique que le Traité, mais il ne peut donner lieu à du droit dérivé. Par conséquent, le droit dérivé se fera à partir de l'article 14.

\section{La démarche jurisprudentielle}

Plusieurs centaines d'arrêts de la Cour de Justice de l'Union européenne (CJUE) portent sur les questions de SIEG. Dans un certain nombre de cas, les textes législatifs (directives et règlements) ne font que reprendre la jurisprudence de la Cour.

Cette jurisprudence suit trois grands axes : les conditions spécifiques d'application des règles de la concurrence, la distinction entre services économiques et services non-économiques (au cas par cas) et les conditions de financement des SIEG afin d'éviter les distorsions de concurrence. Les derniers jugements abordent en outre les relations entre les collectivités locales et les conditions permettant le «in-house », c'est-à-dire la régie ou la quasi-régie. Le débat s'est noué à partir de trois grands arrêts au début des années 1990 :

- l'arrêt Port de Gênes de 1991: les entreprises publiques ou privées pouvant assurer indifféremment des SIEG peuvent bénéficier de dérogations aux règles de la concurrence, à la condition expresse que ces règles fassent obstacle à l'accomplissement de la mission. C'est la confirmation juridique de l'article $86, \S 2$;

- l'arrêt Corbeau, qui admet la possibilité d'un droit exclusif à travers l'exemple de la poste ;

- l'arrêt Almelo, qui autorise le monopole d'importation et d'exportation à partir de l'exemple de l'électricité.

A partir de là, compte tenu du fait que des problèmes concrets se posaient dans la vie de tous les jours, de nombreuses affaires sont arrivées devant la Cour. Celles-ci l'ont amenée à préciser un certain nombre d'éléments, notamment la distinction entre services économiques et non économiques. Dans l'arrêt Poucet et Pistre, la CJUE précise que la sécurité sociale est un service exclusivement social, c'est-à-dire non économique. Elle indique par ailleurs que l'Organisation européenne pour la sécurité de la navigation aérienne (arrêt Eurocontrol) poursuit une mission d'intérêt général. C'est également la CJUE qui a défini les conditions de financement des SIEG à travers les quatre conditions de l'arrêt Altmark. Malgré tout, le débat autour des aides d'Etat n'est toujours pas stabilisé : une affaire a été portée devant la Cour en 2010 pour savoir si le financement des parcs naturels constituait une aide d'Etat. L'affaire oppose la Commission à l'Allemagne, cette dernière ayant demandé à la France de se joindre à ses côtés.

La Cour a défini les conditions de prestation du « in-house » à travers l'arrêt Teckal et toute une série d'autres arrêts, portant notamment sur les entreprises 
locales de service public, les Stadtwerke, en Allemagne, en Autriche ou en Italie du Nord. Ils précisent les conditions dans lesquelles une collectivité locale peut attribuer un SIEG sans passer par la concurrence : celle-ci doit contrôler cette entreprise comme s'il s'agissait de son propre service, l'entreprise devant fournir l'essentiel de ses services sur le territoire de la collectivité en question. A travers l'arrêt Tragsa (qui porte sur une affaire espagnole) et surtout un arrêt de 2009 portant sur les collectivités locales en Allemagne, la CJUE définit également les relations entre les collectivités locales elles-mêmes et les conditions dans lesquelles elles peuvent utiliser les services les unes des autres.

Les arrêts de la CJUE ont incité la Commission à préciser la situation en 2005 à travers trois textes dits «le paquet Monti-Kroes ». Il définit les conditions dans lesquelles une entreprise peut échapper à la mise en concurrence quand elle est soumise à des obligations de service public, à condition qu'il n'existe pas de surcompensation par rapport au coût des missions de service public.

\section{Perspectives}

Via l'intergroupe «services publics », le Parlement européen pourra jouer un rôle plus offensif, notamment en ce qui concerne l'application du Traité de Lisbonne. Aux yeux de la Commission, l'article 14, s'il oblige à une réglementation, ne précise pas les délais : il n'y a donc pas urgence car du point de vue de la Commission européenne, la réglementation en matière de services publics est suffisante. Or nous nous situons dans une situation relativement nouvelle de crise économique et sociale. Dans son rapport de 2008, l'OCDE indique que les services publics en général ont été l'élément le plus efficace pour amortir les effets de la crise, leur effet ayant été plus important pour cela que celui des prestations sociales. Dès lors, à travers les problématiques et les crises que traverse l'UE, l'idée de service public européen commence à prendre davantage corps. Le Comité Economique et Social élabore un rapport sur les moyens de redynamiser la méthode communautaire par rapport au processus décisionnel de l'UE, mettant en avant l'idée de service public européen sur l'exemple des chemins de fer. Le paquet Monti-Kroes va être révisé : il s'agira d'élargir l'éventail des secteurs susceptibles de se soustraire à la politique de concurrence (qui comprend aujourd'hui le secteur hospitalier et le logement social).

Il y a également nécessité de créer des obligations de service public (ou des SIEG ou SIG) européennes dans des secteurs aujourd'hui stratégiques tels les domaines financiers ou bancaires. Par ailleurs, la question écologique et la nécessité de promouvoir des biens communs de l'humanité appellent également des dispositions particulières pour le financement et la gestion de ces biens communs qui s'apparentent à des services publics ou services d'intérêt général ou services d'intérêt économique général.

Les trois démarches législative, relative aux Traités et jurisprudentielle permettent de dessiner une conception européenne des SIG. Certaines cultures na- 
tionales se rapprochent : l'Allemagne, l'Autriche et l'Italie du Nord partagent la conception des entreprises locales de service public (les Stadtwerke) tandis que la France discute d'une loi de création d'entreprises locales.

DANS LE CADRE DES DiAlogueS FRANCO-ALLEMANDS, deux grandes questions mériteraient d'être débattues :

- la question de la subsidiarité dans le contexte de la construction du marché unique. La subsidiarité est-elle possible dans un marché ouvert, dans la mesure où un concurrent peut toujours ester en justice pour contester une décision d'une collectivité quelle qu'elle soit ? Comment articuler la réglementation commune communautaire avec le Protocole sur les SIG qui affirme que les Etats membres, les régions et les collectivités locales ont un large pouvoir discrétionnaire pour définir les missions de service public ? Il reste un travail à faire pour préciser le contrôle de l'erreur manifeste par la Commission (et en dernier ressort par la Cour) et l'articulation de la subsidiarité. D'autant plus que les différences culturelles sont marquées entre Allemands et Français en matière de subsidiarité. Les Français ne sachant pas réellement ce que cela recouvre, ils ont une conception jacobine de la décentralisation descendante. En Allemagne, le processus décisionnel part davantage du terrain, selon une approche de bottom-up. Les Etats membres utilisent la subsidiarité pour conserver des domaines de compétences face à la Commission, qui leur répond que ce processus est valable dans les deux sens ;

- la question de la définition d'une économie sociale de marché au niveau communautaire. L'économie sociale de marché, née en Allemagne, a été introduite à l'échelle européenne dans le Traité de Lisbonne. Quelques éclaircissements sur sa signification seraient nécessaires dans le cadre communautaire, regroupant 27 pays, de traditions et niveaux de développement différents, notamment entre les «15 anciens » et les 12 «nouveaux». Cette question est d'autant plus pertinente en temps de crise, à l'heure où chacun a pris conscience du fait que les ressources naturelles sont limitées. Un nouveau débat apparaît sur la gestion des biens communs européens. Par conséquent, la problématique sur les SIG à l'échelle européenne a encore de beaux jours devant elle. 



\section{Syndicats et libéralisation en Europe}

\section{Richard POND}

Du point de vue de la Fédération Syndicale Européenne des Services Publics (FSESP), la Commission européenne aborde la libéralisation des services publics sous l'angle du marché unique. Préoccupée par cet état de fait et malgré quelques avancées sur le sujet (protocole annexé au Traité de Lisbonne, création d'un intergroupe au Parlement européen), la FSESP rappelle le rôle primordial de services publics de qualité, véritable " pierre angulaire de la société européenne ».

\section{Impact social de la libéralisation des services publics}

La libéralisation a eu un impact lourd et significatif sur les salariés de toute l'Europe, en particulier sur ceux des services publics et ceux qui travaillaient dans le secteur public mais se retrouvent maintenant en situation de fournir des services publics en tant que salariés d'opérateurs du privé. La Fédération Syndicale Européenne des Services Publics (FSESP ${ }^{1}$ ) représente environ huit millions de travailleurs, dont beaucoup ont vécu la libéralisation personnellement et, dans le secteur de l'énergie en particulier, les salariés ont subi les politiques de libéralisation menées surtout par la Commission européenne.

Cela ne veut pas dire que les syndicats européens sont irrévocablement opposés à la concurrence ou au corollaire probable d'une libéralisation accrue : la privatisation. La préoccupation de la FSESP réside dans le fait que ces politiques sont initiées par la Commission européenne et approuvées avec plus ou moins d'enthousiasme par les gouvernements nationaux, sans que ceux-ci n'en reconnaissent véritablement l'impact social. A ce jour, les témoignages suggèrent que non seulement des centaines de milliers de travailleurs ont été perdants dans cette libéralisation mais on se demande aussi véritablement si le processus a porté les fruits promis par ses défenseurs.

\section{Une politique inspirée du marché unique}

L'approche de la Commission européenne vis-à-vis de la question des services publics a largement consisté à envisager les choses du point de vue du marché unique. Il n'y a pas eu de véritables programmes concernant les services publics, mais un programme dans le cadre duquel les politiques sont principale-

\footnotetext{
${ }^{1}$ La FSESP est une fédération syndicale européenne affiliée à la Confédération Européenne des Syndicats (CES). Elle comprend plus de 250 organisations membres dans 49 pays et couvre les secteurs de la santé et des services sociaux, les gouvernements centraux, régionaux et locaux ainsi que les services publics d'énergie, d'eau et de gestion des déchets.
} 
ment définies en fonction de leur contribution à l'extension du marché unique à pratiquement tous les secteurs.

Même lorsque la Commission a débattu d'une politique affectant directement le secteur public, comme son initiative sur la qualité des finances publiques, elle s'est concentrée sur une gestion «a minima» et sur de faibles niveaux de dépenses publiques. Plutôt que de chercher des manières de développer réellement une politique des services publics, la Commission européenne a créé son propre langage qui fragmente la discussion. Nous n'avons pas de services publics, mais nous avons des services d'intérêt général, des services d'intérêt économique général, des services sociaux d'intérêt général et des services sociaux non économiques d'intérêt général!

La vision de la Commission en ce qui concerne de nombreux services publics, en particulier ceux que fournissent les industries de réseau, est focalisée sur la libéralisation. Dans les secteurs fédérés par la FSESP, l'impact de la libéralisation a été particulièrement net dans l'énergie : électricité et gaz. Depuis le début des années 1990, la FSESP observe les effets de la libéralisation à mesure que la législation européenne contraint les Etats membres d'ouvrir leurs marchés à la concurrence. En 2007, une étude commandée par la Commission européenne a confirmé l'estimation de la FSESP selon laquelle le secteur énergétique a perdu environ 300000 emplois depuis le début du processus de libéralisation. Il ne s'agit pas nécessairement de destructions d'emplois car nombre d'entre eux auraient été externalisés mais, selon l'expérience de la FSESP, ces externalisations impliquent le transfert d'emplois vers des entreprises ou des secteurs appliquant des conditions d'emploi et de rémunération moins favorables aux salariés.

La Commission semble considérer les services publics et la coopération comme l' "exception » à la règle de la concurrence et de la recherche de profit, ce qui apparaît aussi dans son programme de dérégulation. Dans ce cadre, la Commission promeut la directive relative aux services, une meilleure régulation et une politique commerciale européenne plus libérale.

\section{Campagne de la FSESP en faveur des services publics}

La FSESP a fait valoir que, globalement, les organisations du secteur public sont les meilleurs fournisseurs de services publics mais que, en tout état de cause, il faudrait laisser au moins les modalités de leur fourniture à la discrétion des pouvoirs publics. Des services publics de qualité dépendent d'un ensemble d'éléments importants. Il y va de l'efficacité de leur fourniture et de leur organisation, basée sur des finances saines. La transparence est vitale, de même que des mécanismes permettant la participation efficace des usagers et des partenaires sociaux. La fourniture de ces services doit être évaluée en termes d'égalité, de cohésion et d'inclusion sociale, et la FSESP est convaincue qu'il faut, pour avoir des services de qualité, préserver une main-d'œuvre bien formée, 
dont l'engagement s'appuie sur un emploi et des conditions de travail de bonne qualité.

Il est essentiel d'assurer l'accessibilité universelle des services, à un prix abordable, selon un principe d'équité. Il n'est pas impossible que ces conditions soient compatibles avec celles du marché, mais elles ne peuvent être laissées au seul marché. Il est impératif de mettre en place certaines formes de régulation et, de préférence, d'une régulation qui, en substance, prendrait la forme d'un contrôle démocratique.

Préoccupée par la focalisation de la Commission européenne sur le marché unique, la FSESP a mené une campagne, en collaboration avec un large éventail de syndicats, organisations politiques et sociales, pour tenter d'obtenir une directive cadre sur les services publics. Nous avions beaucoup d'objectifs majeurs. Nous voulions faire clairement passer le message selon lequel des services publics de qualité sont la pierre angulaire de la société européenne et qu'ils devraient à ce titre être au cœur de l'élaboration des politiques européennes et non pas considérés simplement comme une pierre d'achoppement sur la voie de la création d'un marché unique.

La campagne visait aussi à identifier qui était responsable de la gestion des services publics avec un rôle et une responsabilité clairs des pouvoirs publics locaux, régionaux et nationaux élus qui prennent en compte les besoins des usagers. La FSESP a voulu souligner le risque que les grands opérateurs puissent entrer sur le marché afin d' « écrémer » les parties rentables des services publics, créant un déséquilibre qui laisserait au secteur public la responsabilité uniquement d'un ensemble de services non économiques. La campagne consistait en outre à souligner combien il est important de rendre les services accessibles aussi largement que possible, de les fournir gratuitement ou à des prix abordables à ceux qui en ont besoin.

\section{Le protocole annexé au Traité de Lisbonne}

L'approche de la Commission européenne nous posait de nombreux problèmes. Premièrement, la Commission était réticente même à reconnaître les services publics, puisqu'elle utilise le terme de « services d'intérêt général » et n'a toujours pas de direction chargée de gérer spécifiquement les services publics. Deuxièmement, elle s'est focalisée très largement sur le marché unique, qui s'est vu accorder la priorité sur pratiquement toutes les autres politiques.

Le Traité de Lisbonne constitue néanmoins une évolution relativement positive en termes de reconnaissance de l'importance des services publics, et le protocole annexé à ce Traité donne quelques perspectives de protection et de promotion des services publics. Le Président de la Commission, Jose Manuel Barroso, a fait la remarque suivante : "Dans le Traité de Lisbonne, il y a déjà des dispositions très claires sur la défense et la garantie des services publics. Je suis convaincu que les services publics remplissent une fonction essentielle 
dans notre modèle de société européenne. Je suis prêt à organiser un débat avec le Parlement européen sur la meilleure manière de garantir cette protection et la spécificité des services publics».

Le protocole comporte trois sections principales à l'article 1, concernant respectivement :

- la subsidiarité - c'est l'idée principale, selon laquelle les services doivent être fournis «d'une manière qui réponde autant que possible aux besoins des utilisateurs »;

- le besoin d'une diversité de services ; et

- surtout, les principes communs inhérents à tous les services publics reconnus dans cette formule : « un niveau élevé de qualité, de sécurité et quant au caractère abordable, l'égalité de traitement et la promotion de l'accès universel et des droits des utilisateurs ».

La FSESP estime que cette dernière phrase l'oblige clairement à poursuivre son action pour que le respect de ces principes puisse être évalué concrètement. Le défi consiste dorénavant à essayer d'appliquer ces éléments clés du protocole, et la FSESP souhaite se concentrer sur un certain nombre de questions spécifiques.

\section{Propositions de la FSESP}

La FSESP attend maintenant de la Commission européenne qu'elle utilise le protocole relatif aux services publics comme base pour établir une liste de contrôle à partir de laquelle les changements apportés aux services publics peuvent être évalués en termes de qualité, de sécurité et de prix, d'égalité de traitement et de promotion de l'accès universel et des droits des usagers. Evaluer la législation à la lumière de cette liste de critères serait la première étape franchie par la Commission avant d'engager un processus d'évaluation d'impact plus vaste. Ce serait reconnaître la primauté des obligations de service public sur la conformité au marché, comme le prévoit le protocole.

La FSESP estime aussi que pour s'assurer que cette liste est utilisée dans la pratique, la Commission européenne devrait dédier une unité (de préférence au bureau du Président) pour la faire valoir face à toute proposition susceptible de modifier la nature d'un service public.

Une autre initiative qui mériterait, selon la FSESP, d'être débattue à la Commission, est un projet de Statut des services européens d'intérêt général. Celui-ci suivrait les mêmes lignes que le statut de société européenne et servirait de référence aux fournisseurs de services publics à tous les niveaux requis.

Une autre étape serait la publication par la Commission d'une communication sur l'accès universel. Ce document devrait souligner que la garantie d'accès universel comporte, par définition, une dimension collective et mettre en avant les mécanismes assurant l'accès universel par la solidarité. 
DEPUIS LES DERNIÈRES ÉLECTIONS EUROPÉENNES, il y a eu une évolution importante avec la création au Parlement européen d'un intergroupe sur les services publics. Il rassemble des députés d'horizons politiques divers, ainsi que d'autres acteurs comme les syndicats, qui se retrouvent maintenant régulièrement pour discuter de sujets de préoccupation, en particulier lorsque la législation proposée risque d'avoir un impact sur les services publics. L'existence de cet intergroupe va contribuer à sensibiliser davantage le Parlement européen aux services publics et devrait permettre de former plus facilement des coalitions lorsqu'un lobbying coordonné est nécessaire sur des initiatives susceptibles d'avoir des répercussions, positives ou négatives, sur les services publics.

Alors que le protocole relatif aux services publics donne des perspectives d'action en ce domaine et semble marquer des changements dans l'approche de la Commission, il reste des défis importants. La FSESP a essuyé une grande déception en constatant que la stratégie Europe 2020, comme la précédente définie à Lisbonne, n'arrive pas à reconnaître le rôle que jouent les services publics pour répondre à certains de ses principaux objectifs. Les pouvoirs publics sont des acteurs majeurs sur les questions du développement économique, de la hausse des taux d'emploi et de la réalisation des objectifs d'inclusion sociale. Ils peuvent en outre, en tant qu'employeurs, avoir une ou deux longueurs d'avance sur le secteur privé dans des initiatives destinées à donner de meilleures chances aux femmes, aux jeunes, aux personnes en situation de handicap et de chômage de longue durée.

Il faut aussi citer les projets de la Commission en matière de gouvernance économique et de surveillance plus attentive des projets de dépenses publiques dans toute l'UE. Pour la FSESP, le problème est que cette approche se concentre bien trop étroitement sur la consolidation fiscale. Elle ne répond pas aux besoins d'action coordonnée afin d'assurer que l'Europe prend résolument la voie de la reprise en accordant un rôle central aux services publics et à l'investissement public.

Traduction de Marie GRAVEY 



\section{Les enjeux sociaux de la dérégulation des services publics marchands à l'échelle communautaire. Quels défis pour les représentants patronaux?}

\section{Pierre BAUBY}

Après avoir explicité le rôle du Centre européen des entreprises à participation publique et des entreprises d'intérêt économique général (CEEP), l'auteur revient sur la politique communautaire de libéralisation des services d'intérêt général et les formes du dialogue social européen, pour clore son propos sur les enjeux actuels en la matière, soulignant au passage la difficile construction d'une conception commune des services d'intérêt économique général.

J'introduirai cet exposé par trois remarques relatives au titre de la contribution : « les enjeux sociaux de la dérégulation des services publics marchands à l'échelle communautaire ». Ces précisions ne sont pas que sémantiques car elles abordent des questions de fond:

- je n'emploie pas pour ma part le terme de «dérégulation », parce qu'il laisse entendre implicitement qu'on fait disparaitre les anciennes règles pour ramener ces services au droit commun de la concurrence et aux règles communes du marché. En réalité, il y a non seulement dérégulation (c'est-à-dire qu'on change les anciennes règles), mais aussi « rerégulation », au sens où on met en place de nouvelles règles, souvent plus nombreuses et complexes que les précédentes. Je préfère par conséquent parler de politique de libéralisation progressive ;

- comment définir les « services publics » ? Il n'existe pas actuellement au plan européen d'équivalent identique à la conception française dans toutes les langues, dans toutes les traditions et les cultures nationales. Le terme allemand de Daseinsvorsorge n'est pas complètement équivalent à ce que nous appelons services publics en France. Les Anglais utilisent pour leur part trois concepts : «public service » au singulier, «public services » au pluriel et «public utilities ». J'emploierai plutôt le terme européen de «services d'intérêt général » (SIG), qui a l'avantage de mettre l'accent sur l'intérêt général, c'est-à-dire sur les objectifs, et non pas sur la forme publique ou privée des opérateurs. De ce point de vue, le terme « services d'intérêt général » permet d'aborder les deux grandes conceptions qui coexistent derrière le terme « service public » :

- la conception organique, selon laquelle est service public un service dont la propriété est publique, 
- la conception fonctionnelle, qui met l'accent sur les objectifs, les finalités, le droit à la santé, à l'éducation ou encore l'accès aux transports. Je préfère pour ma part mettre l'accent sur la conception fonctionnelle,

- les règles et les textes européens ne mentionnent pas de « services marchands ». Ils évoquent des services d'intérêt économique général (SIEG) et, depuis le Traité de Lisbonne, de services non économiques d'intérêt général (SNEIG).

\section{Le CEEP et ses évolutions}

L'acronyme du CEEP est resté identique depuis sa création en 1961. Toutefois, les mots qu'il désigne ont évolué. A l'origine, le CEEP était le Centre Européen de l'Entreprise Publique. A l'époque, dans les six Etats membres de la CEE, il existait de grandes entreprises publiques suite à la vague de nationalisations d'après-guerre. Elles sont toutefois organisées différemment selon les pays. A l'initiative des Français notamment se crée une sorte d'amicale des entreprises publiques au plan européen. Il s'agit des prémices d'un lobbying des entreprises publiques, d'un lieu de discussion des grandes entreprises publiques nationales. Il n'est alors pas question d'entreprises publiques locales.

Le changement se fera par étapes : d'abord avec la modification de statut des entreprises et les ouvertures de capital, puis les élargissements progressifs de la Communauté européenne (de six à 27 Etats membres), le CEEP deviendra le Centre européen des entreprises à participation publique. Ensuite, avec la politique communautaire de libéralisation des services d'intérêt général, le CEEP sera le Centre européen des entreprises à participation publique et des entreprises d'intérêt économique général. Ainsi l'organisme s'ouvre aux entreprises privées dont l'objectif est de fournir des SIEG, ainsi qu'aux entreprises locales, qui peuvent être soit $100 \%$ publiques, soit des sociétés d'économie mixte. Depuis 2008, l'acronyme correspond à un titre en anglais : European Center of Employers and Enterprises Providing Public Services. Il met l'accent sur les employeurs (publics ou privés) qui fournissent des services au public. Cela concerne tous les employeurs, y compris potentiellement les employeurs administratifs. Le slogan du CEEP est « Serving the public ».

L'organisme fonctionne sur deux piliers :

- il est le représentant des entreprises de service d'intérêt général ;

- il est le partenaire officiel du dialogue social européen, avec du côté syndical la Confédération Européenne des Syndicats (CES), dont fait partie la Fédération Syndicale Européenne des Services Publics (FSESP) et, du côté patronal privé, l'ancienne UNICE, devenue BUSINESSEUROPE, à laquelle est intégrée l'Union Européenne de l'Artisanat et des Petites et Moyennes Entreprises (UEAPME). 


\section{La politique communautaire}

La politique communautaire s'est développée de manière progressive depuis 1957. A cette date, le Traité de Rome prévoit d'éliminer les obstacles aux échanges en vue de créer un marché commun. De 1957 à 1986, un consensus règne entre les Etats membres et entre les institutions européennes, en vertu duquel chaque Etat continue à définir, organiser, gérer et financer ses services publics selon ses traditions nationales. Par conséquent, il n'existe pas de politique communautaire à l'égard des SIEG pendant les trente premières années du Traité de Rome. L'article 86 du Traité prévoit seulement une dérogation aux règles de la concurrence pour les « services d'intérêt économique général ».

Cela va changer avec l'Acte unique de 1986. Dans le cadre des quatre grandes libertés de circulation (circulation des hommes, des marchandises, des services et des capitaux), le texte fixe explicitement l'objectif d'européaniser les SIEG mentionnés dans le Traité de Rome. Il s'agit de faire passer le niveau où sont définies les grandes règles du plan de chacun des Etats au plan communautaire. Il règne à l'époque un consensus sur le fait que les SIEG sont les services de réseau nécessaires aux libertés de circulation (communications, transports, énergie). Mais l'Acte unique fixe l'objectif de libre circulation sans, et c'est là son gros défaut, définir les prémisses d'une conception européenne des SIEG.

Dès lors, comment européaniser les SIEG? Il s'agira d'utiliser les «armes » du Traité (un droit communautaire de la concurrence extrêmement précis et les principes du libre-échange) pour casser les frontières, tout en cherchant à améliorer l'efficacité de ces services. Car partout existaient des monopoles nationaux, régionaux ou locaux selon les histoires, les secteurs, les pays. Un monopole peut être efficace comme il peut ne pas l'être. Par conséquent, l'idée sous-jacente est d'introduire un peu de concurrence, qui de manière progressive aidera à moderniser, à rendre plus efficace les anciens monopoles. Il s'établit une convergence entre les objectifs de libre circulation et de modernisation dans des politiques de libéralisation progressive avec des directives sectorielles destinées à constituer le marché intérieur.

Toutefois, la libéralisation va mener à des polarisations :

- économique : concentrations et concurrence oligopolistique au plan européen ;

- sociale : clientèle solvable et gros consommateurs ;

- territoriale : zones denses ;

- temporelle : survalorisation du court terme ;

- financière : conséquences de l'externalisation en termes d'environnement ou en matière d'emploi.

Ces évolutions ont poussé les responsables politiques, depuis les années 1990, à se poser la question d'une libéralisation certes, mais maîtrisée, régulée, contrôlée. Il ne s'agissait pas de situer les SIEG hors du marché intérieur mais de 
prendre en considération leurs missions particulières. De ce point de vue, depuis cette période, on a assisté à un processus par étapes afin de construire une conception commune, allant du traité d'Amsterdam en 1997 et de la Charte des droits fondamentaux en 2000 au Traité de Lisbonne, qui comporte en annexe le Protocole 26 définissant au plan communautaire des principes extrêmement importants pour l'avenir.

Cela m'amène à évoquer l'étude menée par le CEEP à la demande de la Commission européenne, sur financements de la direction Emploi et affaires sociales, afin de mieux connaitre la réalité des services d'intérêt général au plan européen et au plan des 27 Etats membres. Intitulée « Mapping of the public services », cette cartographie des services publics est publiée et disponible sur Internet ${ }^{1}$.

\section{Le dialogue social européen}

Dès le traité de Rome, la dimension sociale est évoquée bien qu'il ne se passe pas grand-chose dans les premières années. L'élément clé réside dans une initiative de Jacques Delors en novembre 1985, consistant à demander aux représentants de l'UNICE (patronat traditionnel), du CEEP et de la Confédération Européenne des Syndicats de se réunir. L'idée de Jacques Delors est de faire intervenir un troisième partenaire social, le CEEP, représentant les entreprises de service public, comme «facilitateur » du dialogue social européen. La réunion, connue sous le nom de processus de Val Duchesse, va déboucher sur un accord le 31 octobre 1991, intégré dans le protocole social annexé au Traité de Maastricht. Il s'agit d'une sorte de révolution en la matière : les trois partenaires sociaux ont le pouvoir d'élaborer la norme sociale communautaire, alors qu'auparavant la Commission européenne avait, dans ce domaine comme de manière générale, le monopole de proposition des normes communautaires. Le Conseil des ministres peut formaliser un accord cadre signé entre les acteurs du dialogue social européen. Toutefois, cela donnera peu de résultats immédiats.

Dans ce contexte, trois accords ont été ratifiés par le Conseil des ministres, faisant dorénavant partie de la législation :

- l'accord sur le congé parental de 1996, révisé en 2009 ;

- l'accord sur le travail à temps partiel (1997) ;

- l'accord sur les contrats à durée déterminée (1999).

Des accords autonomes ont été conclus sur :

- le marché du travail inclusif (2010), devant intégrer les personnes jusqu'à présent exclues du marché du travail ;

- le harcèlement et la violence au travail (2007);

- le stress lié au travail (2004) ;

- le télétravail (2002).

${ }^{1}$ www.ceep.eu (version complète en anglais, deux premières parties en français) [consulté le 12/05/11]. 
Enfin, des cadres d'action ont été signés sur :

- l'égalité hommes-femmes (2005) ;

- la formation tout au long de la vie (2002).

Il est compréhensible que le dialogue social ait eu des difficultés à émerger puisque traditionnellement, les trois partenaires sociaux assuraient davantage au plan communautaire une mission de coordination qu'une véritable mission d'initiative et d'action, qu'il s'agisse du patronat, organisé au niveau de chacun des pays, de la Confédération Européenne des Syndicats ou du CEEP. Il a donc fallu constituer chacun de ces trois acteurs comme acteur européen, en tenant compte du fait qu'ils sont en lien avec 27 Etats membres, cultures et traditions nationales, ce qui vient compliquer l'établissement de positions communautaires.

\section{Les formes du dialogue social européen}

Le dialogue interprofessionnel/intersectoriel

Le dialogue intersectoriel est mené au niveau de l'économie dans son ensemble. Il réunit les organisations syndicales et patronales de tous les secteurs d'activité confondus. Six types d'organisations participent au dialogue social interprofessionnel :

- les travailleurs sont représentés par la Confédération Européenne des Syndicats (CES) créée en 1973. Au dialogue social participent aussi EUROCADRES (Conseil des cadres européens) et la CEC (Confédération Européenne des Cadres), qui sont membres de la délégation de la CES ;

- les employeurs européens sont représentés par trois organisations différentes :

○ le CEEP,

- BUSINESSEUROPE, qui remplace l'Union des Confédérations de l'Industrie et des Employeurs d'Europe (UNICE), fondée en 1958,

- à la suite d'un accord de coopération signé en 1995, l'Union Européenne de l'Artisanat et des Petites et Moyennes Entreprises (UEAPME) participe aussi au dialogue social en tant que membre de la délégation de BUSINESSEUROPE.

Les partenaires sociaux élaborent des groupes de travail et de discussion pour essayer de forger des positions communes. En mai 2009, ils ont adopté leur troisième programme de travail multi-annuel, qui s'est achevé en 2010. Ces discussions se déroulent pour l'essentiel au niveau interprofessionnel.

\section{Le dialogue sectoriel}

L'autre niveau du dialogue social, le dialogue social sectoriel, réunit les organisations patronales et syndicales par secteur d'activité. Depuis 1985, les comités 
du dialogue social sectoriel ont été mis en place dans 36 secteurs industriels différents (19 en 1999) :

- $\quad$ secteurs primaires : agriculture, industrie extractive, pêche ;

- secteurs secondaires : industrie chimique, construction, électricité, gaz, sucre, bois, chaussure, ameublement, acier, chantiers navals, tannerie et cuir, textile et habillement ;

- secteurs tertiaires : audiovisuel, aviation civile, banques, restauration collective, commerce, hôpitaux, nettoyage industriel, navigation intérieure, assurances, spectacle vivant, gouvernement local et régional, transport maritime, services aux personnes, postes, sécurité privée, football professionnel, chemins de fer, transports routiers, télécommunications, travail intérimaire.

On remarque que les autorités publiques et administrations ne font pas partie de la liste ci-dessus. Pourtant, il existe une demande, notamment du côté syndical, en vue d'établir un dialogue social sectoriel dans ce domaine. Cela démontre les réticences et les résistances des Etats membres en la matière. Le CEEP hésite à se lancer dans cette voie, bien qu'aujourd'hui, des organisations des autorités publiques, en particuliers locales, soient membres à part entière du CEEP.

\section{Trois types d'activités}

- La concertation tripartite désigne les échanges réguliers entre partenaires sociaux et autorités publiques européennes (Conseil, Commission européenne). Selon l'article 152 de l'actuel Traité sur le Fonctionnement de l'Union européenne (TFUE), « le sommet social tripartite pour la croissance et l'emploi contribue au dialogue social ». A l'échelle des Etats membres, le dialogue social est souvent tripartite, c'est-à-dire qu'il implique les gouvernements ; cela tient à l'histoire des pays, à leur culture, leur tradition. Dans d'autres pays, il est uniquement bipartite ;

- la concertation des partenaires sociaux couvre les activités des comités consultatifs et les consultations officielles dans l'esprit de l'article 153 TFUE ;

- le dialogue social européen désigne les travaux bipartites des partenaires sociaux, découlant ou non des consultations officielles de la Commission, en vertu des articles 154 et 155 TFUE.

\section{Les enjeux}

\section{Evolutions socio-économiques}

En ce qui concerne les SIG, on est passé en l'espace de trente ans du paradigme dominant du monopole au paradigme dominant de la concurrence, le monopole 
et les droits exclusifs afférents ne pouvant être que résiduels. Ce changement de paradigme va avoir des effets considérables sur la structuration des entreprises, la structuration syndicale et le dialogue social.

On assiste par ailleurs à un phénomène de concentration rapide des entreprises, non plus à l'échelle nationale, mais à l'échelle communautaire. Dès lors, comment mesurer et autoriser ou non ces concentrations, qui par définition sont à l'opposé de la notion de concurrence ?

Les règles européennes sont sectorielles (télécoms, transports, électricité, gaz...) afin d'intégrer les spécificités macroéconomiques de chaque secteur. On ne peut pas organiser de la même manière les télécoms et l'eau, et plus encore l'éducation et la santé. Mais dans ce contexte réglementaire sectoriel, on voit apparaître un nombre croissant de groupes multiservices. Dès lors, comment maîtriser, réguler ce fonctionnement?

Dans le même temps, des entreprises locales subsistent, voire reprennent de l'importance dans de nombreux Etats membres. Le principe de proximité, de service sur-mesure adapté aux besoins de chacun, fait que les entreprises locales conservent toute leur place dans l'avenir de la structuration des SIG. On voit là des tensions extrêmement fortes dans ce domaine des évolutions socio-économiques, qu'il va falloir maîtriser.

\section{Transformations des entreprises}

Entre les anciens monopoles nationaux traditionnels et les grands groupes européens dans chacun des secteurs, il se produit des évolutions considérables. On assiste à une concentration des stratégies de production de valeur dans chaque groupe, doublée d'une décentralisation du management au niveau local, afin que les centres de profits locaux participent à la création de valeur. Une nouvelle problématique se développe par conséquent autour de l'enjeu de la centralisation/décentralisation.

En outre, quelle place accorder au dialogue social ? Du côté des entreprises (y compris des entreprises fournisseurs de services publics), il existe des tensions très importantes. La solution de demain réside-t-elle dans le dumping social ? Cette potentialité existe. Par conséquent, est-ce la course au moins-disant social pour faire plus de profits, voire éventuellement baisser les prix, ou s'agit-il d'établir des règles communes, y compris dans le domaine social?

Les directives communautaires Marché intérieur par secteur incluent toutes désormais des obligations de service public ou des obligations de service universel. Pourquoi n'intégreraient-elles pas des Obligations de Régulation Sociale $(\mathrm{ORS})$, c'est-à-dire un minimum de règles sociales pour faire en sorte que le jeu de la concurrence ne se résolve pas dans le dumping social, mais qu'il existe des bases communes y compris dans les directives communautaires? 
Ces règles ne peuvent être que le fruit d'un dialogue social européen.

En France, l'ancien statut du personnel des industries électriques et gazières est étendu maintenant à tous les acteurs en concurrence, qu'ils soient publics ou privés.

De même, l'introduction en 2008 d'un salaire minimum dans le secteur postal allemand, annulée début 2010 pour vice de procédure, visait à préserver les conditions sociales dans le cadre de l'ouverture à la concurrence. Il est clair que ce salaire minimum freine l'ouverture à la concurrence, dans une industrie de main-d'œuvre (pas moins de $75 \%$ des coûts postaux sont des coûts salariaux).

\section{Les mutations sociales}

Les mutations sociales, évoquées dans le rapport «Mapping of the public services » mentionné plus haut, concernent dans un premier temps une tendance progressive au rapprochement des statuts de l'emploi public du système général d'emploi.

Par ailleurs, le droit de négociation collective est progressivement étendu aux groupes d'employés publics, alors que jusque là, les droits de négociation collective n'existaient pas nécessairement dans les secteurs d'emplois publics.

\section{Graphique 1 : Taux de syndicalisation (en \%)}

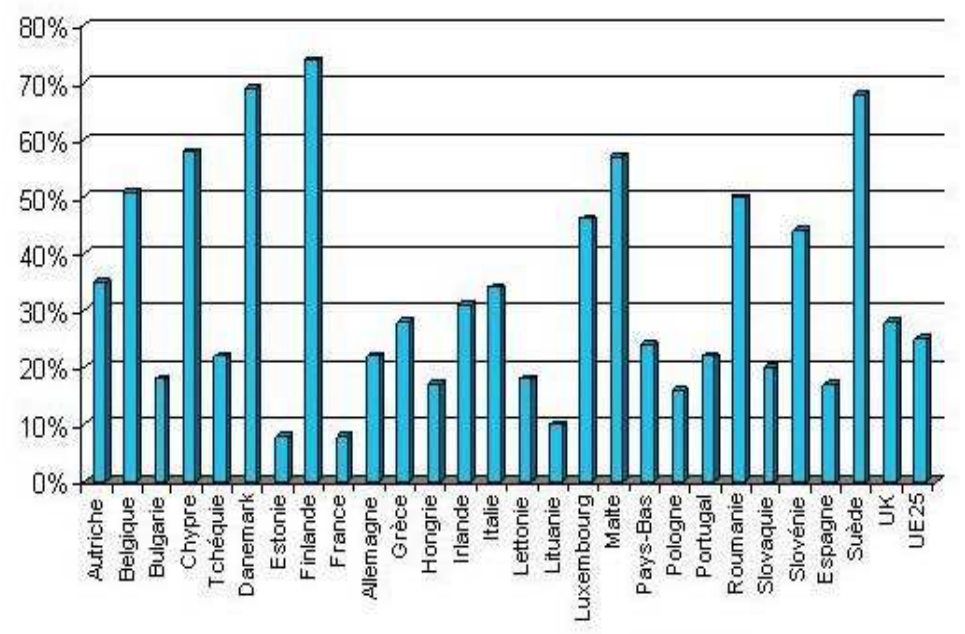

Source : Observatoire européen des relations industrielles (EIRO).

Une autre transformation réside dans la tendance générale, dans l'Union européenne, au déclin de la syndicalisation avec des écarts importants entre les pays (les taux de syndicalisation varient de $5 \%$ à $75 \%$ ). Il existe une disparité 
énorme, bien qu'il s'agisse de chiffres globaux, couvrant l'ensemble des secteurs et pas seulement ceux des SIG. Dans beaucoup de pays, en France en particulier, la syndicalisation est plus forte dans les services publics que dans la moyenne générale. Or pour exister, le dialogue social implique la présence de représentants du patronat et des syndicats.

Des différences persistent entre les Etats membres quant aux exigences attachées à l'emploi dans les SIG. Les caractéristiques et tendances sectorielles perdurent par ailleurs. L'étude « Mapping of the public services » a pour intérêt de considérer aujourd'hui que les évolutions des SIG au plan européen sont le fruit de trois tendances lourdes : une politique générale de libéralisation progressive mais régulée au plan communautaire d'un côté, et de l'autre, de très fortes traditions nationales et des logiques sectorielles qui continuent à marquer l'organisation, la structuration et le fonctionnement des services publics dans chacun des Etats membres. Ces trois tendances se conjuguent, de sorte qu'aujourd'hui, il n'existe pas d'uniformisation au niveau communautaire.

\section{Diversité des relations sociales}

A la lumière des études publiées en la matière, on distingue cinq «modèles » ou « régimes » de relations sociales au sein de l'Union européenne :

- au nord : le « corporatisme organisé » (Danemark, Finlande, Suède) ;

- au centre-ouest : le «partenariat social» (Autriche, Allemagne, Belgique, Luxembourg, Pays-Bas, Slovénie) ;

- au sud : l'approche «centrée sur l'Etat» (France, Grèce, Italie, Portugal, Espagne);

- à l'ouest : le «pluralisme libéral »(Chypre, Malte, Irlande, Royaume-Uni);

- au centre-est : une approche «mixte » avec un régime polarisé ou centré sur l'Etat (Bulgarie, République Tchèque, Estonie, Lettonie, Lituanie, Hongrie, Pologne, Roumanie, Slovaquie), qui tient au fait que les pays d'Europe centrale et orientale sortent d'un système étatique et sont confrontés à des évolutions très importantes.

\section{Quelles relations sociales?}

Le dialogue social est plus ou moins développé dans les différents Etats membres. Les variations tiennent aux systèmes de relations industrielles. Dans la plupart des secteurs et des pays coexistent des régimes de droit public et de droit privé, y compris souvent au sein d'une même entreprise. Cela concerne les statuts et, par conséquent, les modes de négociation sociale.

On note également une différence en termes de représentativité et de relations industrielles entre le secteur public, en particulier dans les activités ou les entreprises de SIG contrôlées par l'Etat, et le secteur privé de SIG. A cause de leur particularité, les SIG contrastent significativement par rapport aux autres 
secteurs en ce qui concerne leur impact sur le dialogue social et les relations industrielles. Les obligations de service public peuvent impliquer des normes particulières de travail, de relations industrielles et de dialogue social. Enfin, le rôle de l'Etat au sens large (autorités publiques) dans le dialogue social varie considérablement en Europe alors que les autorités publiques ont un rôle clé, d'une part, dans la définition, l'organisation et la régulation des SIG et, d'autre part, dans le dialogue social et la négociation.

POUR RÉSUMER, LE JEU N'EST PAS AUSSI FERMÉ qu'il paraît au regard d'un certain nombre d'éléments de libéralisation. Dans ce cadre ouvert, chacun des acteurs doit agir, se constituer comme acteur pour essayer de construire une Europe de l'avenir qui soit aussi celle des services d'intérêt général. 


\title{
Les enjeux sociaux de la dérégulation en France et en Allemagne dans le contexte européen
}

\author{
Jean LAPEYRE
}

Les services publics s'inscrivent dans le modèle social européen, dont les valeurs fondatrices sont ici explicitées. L'auteur, anciennement Chargé de mission auprès du Secrétaire Général du Comité Economique et Social Européen (CESE), évoque tour à tour le Traité de Lisbonne, le problème des ententes illicites et les conséquences de la libéralisation sur l'emploi. A ce sujet, il prône une nouvelle organisation des relations sociales qui tiendrait compte de la diversité des situations d'emploi.

Ce sujet si important des services publics est en pleine actualité car ce qui est extraordinaire avec la crise, c'est que nous sommes en train de réhabiliter par l'absurde le rôle de l'Etat, de l'intérêt général et de la fiscalité. L'avenir des services publics se joue au travers du choix de société que nous voulons et la crise est le moment le plus opportun de se reposer cette question (le pire moment est le meilleur moment comme le disait Mark Twain).

Au-delà de l'espace de paix et de démocratie que représente l'Europe, il y a ce que nous appelons le modèle social européen, non pas un modèle fondé sur l'uniformité mais un modèle basé sur des valeurs communes dont voici les trois principales :

- la cohésion économique et sociale qui, au travers des Fonds structurels, doit permettre un développement de toutes les régions d'Europe en particulier, en compensant les retards structurels existants et par le rôle des services publics, et plus largement des Services d'Intérêt Général, des Services d'Intérêt Economique ou Non Economique Général, des Services Sociaux d'Intérêt Général (SIG, SIEG, SINEG, SSIG... termes barbares typiquement bruxellois) favorisant l'accès de tous les citoyens à des services de qualité, en particulier d'éducation ou de santé. La faiblesse des moyens budgétaires de l'Union et la crise risquent de peser lourdement sur la réussite de l'intégration des nouveaux pays ;

- la solidarité qui, au travers d'un socle de droits sociaux, doit permettre de faire converger les situations sociales et d'éviter le risque de dumping social. C'est pourquoi il est d'ailleurs nécessaire d'ancrer les droits fondamentaux des citoyens dans le Traité de Lisbonne. Solidarité aussi dans la préservation, la modernisation et le développement d'une protection sociale qualitative et solidaire et non pas individualisée, même si des systèmes complémentaires peuvent se développer ; 
- la qualité des relations sociales comme moyen le plus efficace économiquement et socialement pour anticiper et gérer par la négociation collective, les mutations industrielles et technologiques et le progrès social.

Le Traité de Lisbonne donne de nouvelles bases mais qui devraient être consolidées par une ou des directives communautaires pour que cela ne reste pas déclaratif. Le jargon communautaire sur les SIG, les SIEG les SSIG et les SINEG ne facilite pas les choses et la Commission conduite par le Président Barroso enlise toute initiative permettant de garantir juridiquement ces Services aux citoyens.

D'ailleurs que veut dire Service Non Economique d'Intérêt Général quand on sait que les services publics sont de la compétence de la DG Marché Intérieur qui est celle chargée d'éliminer les obstacles à la concurrence ? L'expression «non économique » n'a pas de sens en dehors du contexte légal européen de la concurrence et c'est la CEJ qui statue dans chaque cas particulier sur la question de savoir si un service est ou non économique...

La prééminence du droit à la concurrence a créé nombre de conflits entre les règles du marché intérieur et les services publics qui ont eu une incidence considérable sur la structure des services publics dans les Etats membres, par la libéralisation, les procédures intentées contre le financement des services publics, les procédures intentées contre l'offre directe de services par les pouvoirs publics ou contre les restrictions imposées à l'offre de services dans un pays (dans les soins de santé par exemple), par le risque de libéralisation du commerce des services (AGCS) à l'OMC.

N'oublions pas que si nous avons gagné une bataille contre la Directive Bolkestein, nous n'avons pas gagné la guerre et la CEJ au travers d'un certain nombre d'arrêts (Viking, Laval, Ruffert, Luxembourg...) est en train de mettre en cause les droits sociaux conventionnels, qui font force de lois dans les pays nordiques par exemple, mais aussi le droit de grève.

Pourtant, le Traité de Lisbonne offre une possibilité importante de consolider la place des services publics. L'article 6.1 sur la Charte des Droits Fondamentaux ouvre sur l'article 36 de cette Charte qui garantit un droit d'accès aux SIEG «afin de promouvoir la cohésion sociale et territoriale de l'Union». D'autres articles offrent des bases de références telles que l'article 14 sur l'éducation, l'article 24.1 sur les droits des enfants à la protection sociale et à la garde, l'article 34.4 sur le droit à l'aide sociale et au logement, l'article 35 sur le droit aux soins de santé... Dans le Traité lui-même également, l'article 14 donne une base juridique possible mais relative, due à l'obligation de l'unanimité pour établir un Règlement communautaire.

La CES demande une clause sociale pour clarifier la relation entre marché intérieur et droits sociaux fondamentaux et spécifiant que la législation communautaire sur la concurrence ne peut interférer avec le droit du travail et les règles de sécurité sociale.

En ce qui concerne les entreprises publiques et à participation publique, je 
dois dire que le mouvement syndical européen a toujours eu une coopération exemplaire avec le CEEP (une des trois organisations reconnues comme partenaires sociaux au niveau du dialogue social européen). Nous avons même signé le premier accord européen, le 6 septembre 1990, pour les secteurs de l'énergie et du transport ferroviaire. Il préfigurait l'établissement de relations industrielles dans le cadre européen obtenu dans l'accord du 31 octobre 1991 intégré dans le Protocole Social du Traité de Maastricht.

Le Comité Economique et Social Européen et le Comité des Régions accueillent régulièrement le Collectif européen SSIG et ont soutenu l'étude de janvier 2008 sur les «Services sociaux d'intérêt général dans le marché intérieur du $\mathrm{XXI}^{\mathrm{e}}$ siècle : la nouvelle donne du Traité réformateur ».

Nous avons longtemps traîné des incompréhensions sur le concept de service public. Il y a une quinzaine d'années, la France défendait avec une certaine arrogance le service public «à la française », le seul qui semblait digne d'intérêt, avant de découvrir que dans les pays à forte organisation décentralisée, les services publics fonctionnaient aussi bien, voire mieux, avec une plus grande proximité et rapidité de réaction vis-à-vis des demandes. Heureusement, ce débat est dépassé.

Même si je pense que la définition des missions et un cadre strict pour les faire respecter sont plus importants que la nature de la propriété, qu'elle soit publique, mixte ou privée, force est de constater que les privatisations et les partenariats public/privé n'ont pas forcément répondu positivement aux affirmations des libéraux qui les ont mis en œuvre et à la Commission européenne qui les a encouragés. Ils ont même parfois coûté fort cher aux citoyens.

Bien sûr la Grande Bretagne est un exemple caricatural de cette politique, mais une étude faite par l'Université de Greenwich pour la Fédération Syndicale Européenne des Services Publics montre que les arguments développés sur le fait que le secteur privé est intrinsèquement supérieur pour délivrer des services et des biens, que cela représente des économies pour l'Etat, que les risques sont transférés et que finalement le privé est plus efficace, se sont révélés surévalués voire faux ; l'Etat assurant le plus souvent les coûts d'infrastructures et leur entretien, celui-ci paye cher le transfert de risque. Nous avons même pu constater la reconstitution de monopoles privés là où on mettait en cause le monopole public. Ce monopole au détriment des citoyens ne se joue pas forcément uniquement par une entreprise, mais parfois par les ententes illicites de quelques entreprises importantes comme on l'a vu dernièrement dans les télécommunications.

Mais les ententes illicites dans le secteur privé sont plus courantes qu'on ne le croit, condamnées au niveau national comme Carrefour/Hasbro France/Maxi Toys et Jouet Club condamnés en 2007 pour entente sur les prix des jouets de Noël ou Henkel/Sara Lee/Colgate Palmolive/Unilever, condamnés en Allemagne en 2008 pour entente sur les prix des dentifrice, gel douche et liquide vaisselle... et au niveau européen la Commission a pris huit décisions infligeant des amendes à 41 entreprises pour une somme de 3,334 milliards €. Vous pourriez vous dire 
que s'il y a condamnation, c'est que le système fonctionne, pour ma part j'ai tendance à penser que les entreprises tentées par ces ententes illicites seront plus habiles à frauder mais pas forcément plus honnêtes.

$\mathrm{Si}$ on prend le domaine de l'emploi, la création attendue dans le secteur privé, suite à la privatisation de la Deutsche Post, de Deutsche Telekom ou d'une partie des services communaux et hospitaliers, est loin d'avoir compensé les suppressions de postes dans le secteur public. L'institut Hans Böckler des syndicats allemands arrive ainsi à un solde négatif de 600000 emplois supprimés en 20 ans. Entre 1991 et 2006, le service public allemand a donc supprimé près de 2,1 millions d'emplois, soit presque un tiers de tous les emplois publics. La moitié de ces 2,1 millions d'emplois a réellement disparu pendant que l'autre moitié a été privatisée. Les auteurs de l'étude, qui relèvent aussi un fort recul de l'emploi au niveau communal dans les secteurs du traitement des ordures, du sport, de l'éducation et de la culture, précisent que dans tous les secteurs fortement touchés par les privatisations, ce n'est pas seulement l'emploi qui a reculé mais aussi le niveau des salaires, les accords collectifs perdant de plus en plus leur fonction de référence.

Un rapport récent de l'OCDE a montré que «malgré une relative stabilité des rémunérations des agents publics en pourcentage du PIB en Grande-Bretagne, en Italie et au Danemark, les coûts de production des biens et services publics ont fortement augmenté en raison de la hausse des coûts de ces biens et services fournis par le secteur privé » et qu'au Pays-Bas et en France, « une diminution relative de la part des rémunérations s'est accompagnée d'une hausse encore plus marquée des biens et services financés par les administrations publiques, mais fournis par le secteur privé ».

Une enquête de la direction générale de la santé et de la protection des consommateurs, la «DG Sanco» de la Commission européenne, dans un bilan de deux décennies de dérégulation, montre des résultats décevants pour les consommateurs, notamment les plus démunis. Nous sommes donc loin de l'intérêt général...

Les réformes menées depuis 20 ans se sont focalisées sur l'efficience, c'està-dire la taille de l'administration et son coût, mais pas sur l'efficacité, c'est-àdire le contenu des politiques et les procédures. On s'est occupé du tuyau mais pas de ce qui passait dans le tuyau...

Le lancement en France de la Révision Générale des Politiques Publiques en juillet 2007 n'échappe pas à cet objectif de rationalisation par des mesures de réorganisation, par des regroupements et fusions quelquefois rationnels, mais dont le but «secondaire» est la réduction du nombre d'emplois (36 000 en 2008 dont la moitié dans l'éducation nationale) et des économies budgétaires (objectif de 9 milliards $€$ entre 2009 et 2012).

Alors on audite à grands frais, avec l'aide de grands cabinets externes, dans toutes les administrations (vous connaissez la blague qui court dans les administrations : « un auditeur c'est celui qui te pique ta montre et qui après te dit l'heure 
qu'il est »). La plupart du temps les cadres intermédiaires sont court-circuités, ce qui pose après le problème de l'opérationnalité des réformes.

\section{Une nouvelle gouvernance des services publics}

Il faut avancer sur une nouvelle organisation des relations sociales qui, à partir d'un tronc commun, peut permettre de développer des espaces de dialogue social plus diversifiés, prenant en compte la spécificité des situations de travail et leur diversité de statuts.

Au travers de ce problème se pose aussi bien sûr celui du statut des fonctions publiques qui peut apparaitre, au risque de choquer, trop rigide et finalement ne pas tenir compte suffisamment de l'évolution de l'emploi dans les administrations et collectivités territoriales, avec un système à deux vitesses dû à un nombre de salariés non statutaires avec un statut de contractuel de plus en plus important: $12 \%$ dans l'ensemble des ministères et $34 \%$ dans la fonction publique territoriale (avec les assistantes maternelles). La fonction hospitalière française est la moins concernée par les non titulaires. Cela est vrai dans l'ensemble de l'Union européenne. Une étude de la Fondation de Dublin sur les relations industrielles dans le secteur public montrait cette évolution croissante des salariés non statutaires : plus de $35 \%$ en Allemagne, plus de $25 \%$ en Belgique jusqu'à $45 \%$ en Bulgarie mais aussi $50 \%$ en Espagne.

Bien sûr, la situation du nombre de statutaires et de non statutaires est très diverse aux différents niveaux de décentralisation.

Dans 12 pays de l'UE, il n'y a pas de statut particulier pour les salariés des collectivités territoriales. Seule la négociation collective permet de régler les conditions d'emploi et de salaire. Mais dans les autres pays où il y a statut, la proportion du personnel concernée peut être très variable. En Allemagne, on passe de $61 \%$ de statutaires au niveau des régions à $14 \%$ au niveau municipal alors qu'en Espagne, où $89 \%$ des salariés employés par les régions ont un statut, ils ne sont plus que $49 \%$ au niveau provincial et $39 \%$ au niveau municipal.

IL EST DONC INDISPENSABLE QUE L'UNION aide à développer un dialogue social voire un nouveau système de négociation collective d'autant plus que dans certains pays, le droit de négociation n'existe pas pour les fonctionnaires comme en Allemagne, en Bulgarie, en Hongrie et dans les pays Baltes. Les salaires et conditions d'emploi des agents statutaires sont déterminés unilatéralement par l'Etat et le droit existant dans d'autres pays est tout relatif comme en France, les décisions étant souvent unilatérales, prises par les autorités politiques, même si un accord signé en 2008 par certaines organisations syndicales ouvre la voie à une concertation renforcée possible mais non certaine.

Il faudrait trouver une voie de décentralisation du dialogue social avec des mécanismes centraux de coordination. L'Italie est un bon exemple de négociations articulées. 
Au niveau européen, la Fédération Syndicale Européenne des Services Publics a réussi à ouvrir, après beaucoup d'efforts, deux espaces de dialogue social avec des organisations européennes d'employeurs au niveau des collectivités territoriales et des hôpitaux. Malheureusement, au niveau de la fonction publique d'Etat, ce dialogue reste inexistant malgré quelques concertations.

Enfin, je voudrais souligner le rôle de la Responsabilité Sociale des Entreprises (RSE) dans les services publics. Il est indispensable que les fonctions publiques développent cette responsabilité dans le cadre d'une politique de développement durable, en particulier au niveau des collectivités territoriales en prise directe avec les citoyens et au travers les appels d'offres de marchés publics. 
- II -

Mutations du dialogue SOCIAL

EN France et en ALLEMAGNe 



\title{
L'évolution de la représentation et des stratégies syndicales dans les services publics en France
}

\author{
Jean-Pierre BASILIEN
}

L'article suivant met en évidence la difficulté de décrire l'évolution de la représentation et des stratégies syndicales dans les services d'intérêt général. Car les situations sont particulièrement contrastées d'une entreprise à l'autre, qu'il s'agisse de leur degré d'ouverture à la concurrence ou de leur histoire syndicale. Néanmoins, le système des relations professionnelles tendrait à se banaliser, s'alignant progressivement sur le droit commun.

Entreprise\&Personnel est une association d'entreprises qui regroupe aujourd'hui près de 130 membres. Elle a été créée en 1969 par un groupe d'entreprises non seulement issues du privé mais aussi du public. Entreprise\&Personnel a été conçue pour être un lieu d'échange, de partage des pratiques entre des acteurs qui, dans l'entreprise, souhaitent partager leurs visions, leurs analyses sur l'évolution des systèmes sociaux, des politiques de gestion de ressources humaines, du monde syndical ou encore des politiques de management. Dans cette période de crise, les entreprises demandent à l'association d'organiser de nombreux échanges car elles souhaitent confronter leur vécu propre avec celui d'autres entreprises, y compris de secteurs d'activité très différents. A partir de ces échanges, Entreprise\&Personnel produit chaque année une quinzaine de documents de synthèse. Certains sont très orientés vers les pratiques, tandis que d'autres ont plutôt pour ambition de faire le lien entre les travaux de recherche et le monde de l'entreprise. On retrouve parmi les adhérents d'Entreprise\&Personnel un certain nombre d'entreprises issues du secteur public (La Poste, France Télécom, la RATP, EDF, GDF SUEZ) et des grandes entreprises du secteur privé.

\section{De quoi parle-t-on?}

Lorsque l'on veut parler de l'évolution du paysage syndical et des stratégies, deux difficultés apparaissent. La première vient de la diversité des situations qui existent dans les différentes entreprises de service d'intérêt général et de l'hétérogénéité des périmètres de référence. On relève des situations très contrastées, tant du point de vue de l'évolution des secteurs concernés que du paysage syndical d'origine et de son évolution récente. Ainsi, il s'avère difficile de parler de l'évolution de la représentation et des stratégies syndicales comme si on décrivait un ensemble homogène pour en dégager quelques traits communs. France Télécom qui, de même que La Poste, est issu de la transformation en 1990 du ministère des PTT, la SNCF, EDF ou encore GDF SUEZ, recouvrent des réalités totalement différentes. On peut certes évoquer l'énergie, des télécommunica- 
tions et des transports publics, mais leur exposition concurrentielle, leur degré d'ouverture au marché sont différents ; chaque entreprise a sa propre histoire. Les composants du corps social, les évolutions managériales et les histoires syndicales varient d'une entreprise à l'autre. Il faut par conséquent veiller à ne pas globaliser trop rapidement les analyses.

La seconde difficulté réside dans la nécessaire distinction des acteurs syndicaux et de leurs niveaux d'action. Il existe deux niveaux de lecture, selon que l'on se place au niveau des confédérations, de leurs prises de position sur les dossiers clés, ou que l'on parle des stratégies concrètes d'une fédération ou d'une organisation syndicale dans le contexte propre de son entreprise. Cette différence d'enjeux entre le niveau confédéral et le niveau fédéral permet d'expliquer les stratégies concrètes menées sur le terrain dans les négociations sociales. Par ailleurs les acteurs syndicaux ne sont plus, si tant est qu'ils l'aient jamais été, des entités homogènes. La « marque » syndicale n'est plus la garantie d'un contenu homogène comme cela a pu être le cas dans le passé. On trouve donc une diversité des positions prises dans les entreprises d'un même secteur au nom d'une même organisation syndicale, et à l'intérieur même d'une entreprise selon les différents métiers, les différents départements, les différents corps de métiers...

Au niveau confédéral, un des enjeux stratégiques de la CGT, sur un dossier comme celui de la réforme des régimes spéciaux par exemple n'avait pas de lien exclusif avec le dossier lui-même mais était aussi en relation avec l'image que la CGT voulait donner à l'ensemble du salariat non directement concerné par ces réformes. L'enjeu premier au niveau confédéral était certes de faire avancer le dossier dans un sens qui était le plus favorable possible à ses adhérents, mais aussi d'éviter la cristallisation d'une représentation négative dans l'opinion publique de son action. Si elle avait été perçue comme uniquement circonscrite à la seule défense des intérêts des salariés des entreprises publiques, elle aurait accru la coupure entre Privé et Public. Dans un dossier comme GDF SUEZ, l'enjeu de la Caisse Centrale d'Activités Sociales du personnel des industries électrique et gazière (CCAS) a été dissocié dès 2007 des autres enjeux de la fusion car il n'avait rien à voir avec la nature de la réforme proposée et rentrait dans une stratégie pilotée à l'échelle confédérale.

Au niveau des fédérations professionnelles, les actions sont largement déterminées par le système de contraintes constituées par la concurrence syndicale. La stratégie de la CGT ne peut être comprise, par exemple, sans l'intégration, dans la grille d'analyse, de la stratégie propre de SUD, dont un des objectifs est de peser sur celle de la CGT. On est ici dans un système d'interactions, au sein duquel une stratégie ne peut être élaborée indépendamment de celle développée par d'autres acteurs. La loi du 20 août 2008 a renforcé le contexte de concurrence entre organisations. Selon ce texte, pour pouvoir exister dans la négociation collective, un syndicat doit rassembler au moins $10 \%$ des voix et suffrages exprimés aux élections professionnelles. En outre, deux conditions 
doivent être remplies en vue de la validation d'un accord : celui-ci doit être signé par une organisation représentant $30 \%$ des voix, l'opposition ne devant pas représenter plus de $50 \%$ des voix des salariés. L'atteinte de ces seuils devient un enjeu en soi. Enfin, les fédérations ont à gérer le poids croissant des retraités parmi les syndiqués. Les salariés qui ont fait ces entreprises il y a 20 ans qui ne sont pas forcément ceux qui construisent actuellement le nouveau modèle social des entreprises de services publics. Une fédération, comme n'importe quelle organisation, ne peut ignorer l'existence de cette force en son sein puisqu'elle aussi va participer à la constitution de majorités dans les fonctionnements internes de ces organisations.

Il existe enfin une interaction très forte entre l'adaptation des stratégies des organisations syndicales et ce que leur proposent les dirigeants d'entreprise en termes d'espace de dialogue social, de négociation collective, de marge de négociation, de style de management et de logique d'action. La façon dont on peut décoder les stratégies syndicales, entreprise par entreprise, est aussi à mettre en relation avec les politiques de management et de dialogue social conduites dans ces entreprises. A partir de situations identiques il y a dix ans, on voit bien, lorsque l'on accompagne certaines d'entre elles et qu'on échange avec les partenaires sociaux, combien les conditions et l'ambiance du dialogue social ont pu évoluer différemment. On pourrait citer l'exemple de la RATP qui mène depuis longtemps une politique cohérente dans la conduite du dialogue social conduisant à des accords collectifs importants accompagnant l'évolution de l'entreprise.

\section{Des difficultés communes}

Dans l'analyse de l'évolution du syndicalisme des entreprises du secteur public, quelques points peuvent être dégagés. Le premier concerne l'ensemble des organisations syndicales : le mouvement syndical dans ces secteurs n'échappe pas aux difficultés de l'ensemble du mouvement syndical en France. Si la syndicalisation reste plus forte que dans les entreprises du privé, elle est cependant en recul sensible d'après les données disponibles, bien que celles-ci demeurent difficiles à traiter. Le taux de syndicalisation des actifs s'y établit aujourd'hui autour de $20 \%$. Bien qu'il reste nettement plus élevé que la moyenne nationale (7\% à 8\%), il a chuté de moitié par rapport au début des années 1990. A ce sujet, on peut citer les travaux de Dominique Andolfatto et Dominique Labbé sur les syndiqués en France, qui a fait l'objet d'une publication en mai 2007. La même tendance à la baisse se retrouve dans la fonction publique à proprement parler, y compris dans des ministères historiquement fortement syndicalisés comme le ministère de l'Education nationale. Par conséquent, malgré les difficultés des salariés et leurs inquiétudes dans le cadre de transitions rapides, les entreprises du secteur public n'échappent pas aux problèmes de la syndicalisation en France. 
Le second point concerne un fractionnement du mouvement syndical beaucoup plus marqué qu'au début des années 1990, avec l'apparition dans certaines de ces entreprises de SUD ou encore de l'UNSA. Cependant, au fur et à mesure des élections qui se déroulent depuis la loi du 20 août 2008, le nombre d'organisations représentatives se réduit.

\section{L'évolution des rapports de force}

Au sein des entreprises de secteur public, l'organisation la plus importante reste la CGT (cf. tableau 1) mais elle perd des voix au fil des élections (avec des reculs supérieurs à $3 \%$ en 2010).

Tableau 1 : Paysage syndical en fonction des dernières élections professionnelles (au 30 avril 2011)

\begin{tabular}{l|l|l|l|l|l|l|l|} 
& CGT & CFDT & FO & CGC & CFTC SUD & UNSA \\
\hline SNCF & $37,30 \%$ & $13,7 \%$ & $8,5 \%$ & $0,4 \%$ & $1 \%$ & $17,3 \%$ & $21,4 \%$ \\
\hline RATP & $33,9 \%$ & $10,1 \%$ & $10,06 \%$ & $5,2 \%$ & $0,9 \%$ & $14,09 \%$ & $25,7 \%$ \\
\hline LA POSTE & $32,8 \%$ & $18,1 \%$ & $16,5 \%$ & $\begin{array}{l}6 \% \\
\text { UNSA) }\end{array}$ & $4,7 \%$ & $22 \%$ & \\
\hline $\begin{array}{l}\text { France } \\
\text { Télécom } \\
\begin{array}{l}\text { Orange } \\
\text { EDF }\end{array}\end{array}$ & $26,43 \%$ & $22,01 \%$ & $12,25 \%$ & $9,79 \%$ & $6,77 \%$ & $22,48 \%$ & \\
\hline
\end{tabular}

Source : Entreprise\&Personnel.

Dans le même périmètre, la CFDT se positionne suivant les cas en deuxième ou troisième place. Bien que la situation se stabilise, voire se redresse, la CFDT a été durement sanctionnée pour les positions qu'elle a été amenée à prendre au sein de ces entreprises ou sur des dossiers nationaux, qui n'ont pas toujours été comprises de la part des salariés, en particulier dans une logique qui jusque récemment privilégiait la négociation de l'adaptation des entreprises publiques en vue de concilier l'idée du développement économique et industriel de l'entreprise et la notion de service public. Aujourd'hui, l'entreprise où la CFDT a les positions les plus fortes est France Télécom, où l'organisation a réuni $22 \%$ des voix aux élections professionnelles de 2009. On retrouve ensuite une dispersion entre la RATP, où son dernier résultat a dépassé le seuil des $10 \%$ d'à peine $0,1 \%$ et EDF, où l'organisation rassemble plus de $21 \%$ des voix.

Un autre élément important est l'émergence de SUD, qui occupe la deuxième position chez France Télécom et à La Poste avec respectivement 22,48\% et 22\% 
des voix, et la troisième position à la SNCF avec $17,3 \%$ des voix. Il faut remarquer que SUD n'a pas «profité » des positions radicales prises pendant le conflit des retraites de 2010. L'organisation est également présente au sein de la RATP, mais peu dans les entreprises EDF et GDF. Cependant, SUD Energie est une organisation syndicale en phase de structuration dont on peut penser qu'elle va se développer au fil des élections professionnelles.

Force Ouvrière, qui conserve des positions solides dans la fonction publique, est surtout présente à La Poste avec $16 \%$ des voix aux élections professionnelles. Le syndicat obtient entre $12 \%$ et $13 \%$ chez France Télécom, EDF et GDF SUEZ. A la SNCF l'organisation se situe en revanche en-dessous du seuil désormais fatidique des $10 \%$.

La CGC est très peu représentée dans l'univers des entreprises publiques, en dehors d'EDF et de GDF, où elle a recueilli plus de $10 \%$ des voix aux dernières élections professionnelles. Quant au syndicat CFTC, sa présence est marginale dans ces entreprises : en dehors de France Télécom, il ne dépasse pas les 5\%. Au fil des élections professionnelles, l'ensemble de cette organisation va (du moins dans un premier temps) progressivement disparaître du paysage syndical.

Enfin l'UNSA, dont les succès ont été largement relayés dans la presse, a gagné quelques points au cours des dernières élections professionnelles. Par exemple au sein de la SNCF où elle s'appuie sur une implantation historique, l'UNSA se situe désormais en deuxième place au sein du panorama syndical de l'entreprise. Lors des dernières élections, l'organisation a profité à la fois des fruits de son action mais probablement aussi des stratégies développées par d'autres organisations au sein de la SNCF, et notamment du report des voix d'un certain nombre de sympathisants CFDT, sceptiques face à l'alliance opportuniste de leur organisation avec la FGAAC, le syndicat très catégoriel des conducteurs de trains.

$\mathrm{Au}$ sein de ce paysage syndical relativement éclaté, on retient d'une part la forte présence de la CGT et de l'autre l'émergence du pôle radical incarné par SUD. Bien que la CGT conserve une position majoritaire, ses résultats électoraux s'érodent au fil des élections, sa base militante chute en valeur absolue et son influence diminue en proportion. Son poids relatif dans le jeu social interne, bien que conforté par la réforme de la représentativité et la validation des accords collectifs, a changé de nature au cours du temps.

\section{La CGT comme analyseur des difficultés du monde syndical}

Comprendre la stratégie de la CGT aujourd'hui, c'est d'abord comprendre les enjeux de la confédération tels que sa direction confédérale autour de Bernard Thibault peut les voir. L'enjeu majeur de la CGT, qui s'exprime dans le cadre de ses derniers congrès, est celui de son poids à terme, avec l'enjeu de la relève des militants qui vont partir en retraite massivement dans les prochaines années. La question qui est posée à la CGT est celle de la faiblesse relative de son implantation dans le secteur privé, de sa capacité à pouvoir exister dans le salariat 
tel qu'il est aujourd'hui et tel qu'il est en train de se transformer. A ce titre, la syndicalisation dans les secteurs publics n'est plus aussi essentielle qu'elle l'a été. Bernard Thibault révélait dans Le Peuple de février 2009: "Notre taux de syndicalisation est le plus élevé là où les effectifs des salariés sont en déclin». Cela vise à la fois les industries traditionnelles, mais aussi et surtout ces entreprises de service public où la CGT continue à avoir une présence importante. Au sein de l'organisation, la perte d'adhérents est estimée depuis 1991 à $-35 \%$ dans le secteur mines-énergie et à $-21 \%$ au niveau des cheminots.

La syndicalisation dans le secteur privé est actuellement la première préoccupation de la CGT car c'est ce qui garantira son avenir à terme, et non la défense crispée des statuts des salariés des entreprises publiques. Selon les données de la CGT, lors du Congrès de Tours, la moyenne d'âge des syndiqués était de 48 ans, tandis qu'à l'heure actuelle, $48 \%$ des syndiqués dans neuf fédérations couvrent seulement $16 \%$ du salariat. On retrouverait par ailleurs seulement $0,5 \%$ de syndiqués à la CGT sur les sept millions de salariés que comptent les secteurs du commerce et des services. Or, l'avenir du syndicalisme est là, ainsi que dans les entreprises de moins de 100 personnes, qui rassemblent $65 \%$ des salariés et dans celles de moins de 200 personnes, regroupant plus de $70 \%$ des salariés. La coupure entre salariés du privé et salariés du public est l'une des grandes inquiétudes des dirigeants syndicaux actuels. Leur développement dans l'ensemble du salariat est lié à la capacité de démontrer qu'ils ne sont pas que les gardiens des statuts et des «avantages » des salariés des secteurs publics. Cette posture est forcément en tension avec les positions prises par les fédérations dans leur champ de syndicalisation, car il ne s'agit pas de perdre les positions acquises jusqu'alors.

Dès lors, le dilemme du syndicat est de pouvoir donner une image de son action qui ne donne pas le sentiment de s'enfermer dans la seule défense d'intérêts statutaires tout en étant cependant suffisamment fort à l'intérieur des entreprises publiques où il est implanté pour ne pas laisser d'espace sur le terrain à une organisation comme SUD, prête à récupérer tous les déçus de la CGT, ou tous les salariés qui ne se reconnaîtraient plus aujourd'hui dans l'évolution de la CGT sur un certain nombre de dossiers.

L'évolution de la CGT se traduit d'un côté par la prise de conscience de la rapidité des changements à l'échelle d'une économie globalisée, de l'autre, par sa nouvelle approche de la négociation collective. Au niveau confédéral aujourd'hui, la CGT s'engage dans un syndicalisme de négociation qui se répercute sur les postures prises dans les entreprises publiques. Cette évolution très progressive a été engagée il y a de nombreuses années. Au $48^{\mathrm{e}}$ Congrès de la CGT, le mot «compromis », associé au mot «négociation », n'a pas pu être pour autant retenu car une majorité aurait mis en difficulté la direction confédérale sur ce simple terme. La CGT a sorti il y a quelques mois un guide pratique de la négociation dans le secteur privé, dont la préface est signée par Maryse Dumas, qui exprime de façon tout à fait claire la position de l'organisation : «La négo- 
ciation sociale apparaît aujourd'hui pour une grande partie de l'opinion publique comme un véritable contre-pouvoir aux décisions unilatérales d'où qu'elles viennent : patronat, gouvernement voire Président de la République. Elle va de pair avec une forte crédibilité accordée au syndicalisme, surtout lorsqu'il est uni sur des questions fondamentales ». A la CGT, on associe donc aujourd'hui clairement la négociation à l'image du syndicat dans l'opinion publique et dans le salariat. Maryse Dumas ajoute par ailleurs : «Qui dit négociation dit aussi compromis à partir de deux options contradictoires, voire opposées ». Le terme " compromis » est acté. Pour rappel, c'est en 1978, lors d'un congrès de la CFDT, que Jacques Moreau a introduit la notion de compromis. A travers cette évolution, on se rend compte que la réalité n'est pas tout à fait celle que l'on peut retenir à travers des positions quelque peu spectaculaires pouvant être prises sur des événements à chaud.

\section{L'évolution du système des relations professionnelles}

La décentralisation et la réorganisation des entreprises de services publics ont été accompagnées par une décentralisation et un développement fort du dialogue social. A la RATP par exemple, plus de 300 accords ont été signés depuis 2000 tandis que chaque année, un observatoire paritaire du dialogue social, qui s'intéresse à l'innovation dans le dialogue social et aux modalités de régulation, établit une synthèse de la façon dont le dialogue social fonctionne. Le dialogue social se situe au niveau de l'entreprise, des départements ou des unités élémentaires de management, c'est-à-dire les lignes de bus ou de métro. Des accords sont négociés et signés à ces différents niveaux. La CGT, présente dans ces négociations, signe aussi un certain nombre d'accords de plus en plus en cohérence avec sa position au niveau national, même si le cheminement n'est pas linéaire et que le double objectif de garder la cohérence a minima de l'organisation et d'éviter de trop s'exposer aux critiques de la part de SUD n'apparaît pas aussi clairement.

La professionnalisation de la fonction RH est un autre élément d'amélioration du dialogue social dans les entreprises de services publics, de sorte que l'on trouve aujourd'hui dans la fonction RH d'excellents experts des questions du dialogue social et des managers tout à fait en mesure de développer une négociation sociale positive, le rôle du management de proximité étant décisif. La volonté de gagner la «bataille des idées » se retrouve en première ligne dans l'évolution des entreprises du secteur public. Dans un document issu du congrès des cheminots de 2007, la CGT des cheminots explique comment déployer un syndicalisme de proximité, insistant sur le fait que c'est à ce niveau que se gagnent les batailles revendicatives. "C'est sans conteste et sans complexe que la direction [de la $\mathrm{SNCF}$ ] fait du management de proximité une priorité pour gagner ni plus ni moins la bataille des idées. Elle ambitionne de contester en permanence le terrain de la CGT et de peser ainsi sur la capacité de mobilisation 
des cheminots. Elle s'est dotée en ce sens de dirigeants de proximité qui doivent faire l'objet de formations ». Dans l'évolution des entreprises publiques et dans la régulation sociale, on voit apparaître un niveau de régulation sociale de proximité, entre le manager et ses équipes, qui n'existait pas, ou à des degrés extrêmement limités, dans les années qui suivirent la création de ces entreprises. Il s'est construit progressivement, au point de devenir un lieu de régulation important qui déplace le jeu des acteurs dans la régulation sociale d'ensemble. Par une capacité d'écoute et de négociation au plus près du terrain, le management de proximité permet de régler un plus grand nombre de sujets.

POUR CONCLURE, NOUS ASSISTONS dans les entreprises de services publics à une évolution progressive du système de relations sociales pour se rapprocher de celui des entreprises du secteur privé. Historiquement, le système de relations professionnelles dans les entreprises publiques s'articulait dans un jeu complexe entre l'Etat, les organisations syndicales, le management et les salariés. On assiste aujourd'hui à une banalisation progressive du système de relations professionnelles dans ces secteurs. Les adaptations et les changements de statuts sont négociés par étapes. Ces négociations conduisent à des compromis de fait ou actés dans des accords. On voit apparaître des conventions collectives de branche regroupant des acteurs historiques de ces secteurs et les nouveaux entrants. Il se produit un alignement sur le droit syndical commun des dispositifs qui étaient jusqu'alors spécifiques à ces secteurs d'activité, que ce soit au titre des IRP (les Instances Représentatives du Personnel) ou au titre de la négociation collective. Dans ce processus, la CGT aura certainement un rôle majeur à jouer dans les années à venir. Elle va devoir se déterminer en matière de négociation collective tout en sachant qu'un pôle de radicalité devrait se maintenir durablement dans ces entreprises pour un certain nombre de raisons, parmi lesquelles les inquiétudes continues d'une partie des personnels face aux évolutions engagées. 


\title{
Nouveaux acteurs de la représentation des intérêts des salariés en Allemagne
}

\author{
Wolfgang SCHROEDER, Samuel GREEF
}

\begin{abstract}
Dans cette contribution, les auteurs exposent l'évolution des relations sociales et des syndicats en Allemagne par le biais de l'association des pilotes Cockpit et du syndicat de médecins Marburger Bund. Ils révèlent ainsi comment, à la faveur du recul des syndicats unitaires de branche et de la décentralisation du dialogue social, ces organisations se sont progressivement hissées au rang d'acteurs autonomes de la négociation collective.
\end{abstract}

Dans le modèle syndical allemand, la représentation des salariés part du principe que c'est de manière collective, pour une même branche de l'économie et par le biais d'un syndicat généraliste que la défense des intérêts communs est la plus efficace. Au-delà de ce modèle de représentation unitaire et sectorielle dominant, la République fédérale compte cependant depuis toujours une multitude d'organisations, qui œuvrent toutefois très majoritairement en complémentarité : soit elles coopèrent avec les organisations membres de la confédération allemande des syndicats (DGB) pour leurs négociations, soit elles défendent, en tant qu'associations professionnelles, des intérêts spécifiques, sans s'engager parallèlement dans les négociations collectives.

Mais, depuis quelques années, on constate que quelques associations professionnelles assument des missions syndicales ou revendiquent même, de manière offensive, une position d'acteur à part entière, au centre de l'arène des négociations. Les succès remportés par l'organisation Cockpit en 2001, par le syndicat des conducteurs de locomotives en 2008 ou par le syndicat de médecins Marburger Bund en 2007 sont le signe d'un profond changement dans les relations de travail en Allemagne. Certains observateurs mettent même en garde face à l'érosion du partenariat social et pronostiquent une radicalisation durable des relations industrielles suivant le modèle américain ou anglo-saxon, autrement dit une évolution du corporatisme vers le pluralisme.

C'est pourquoi cette contribution se concentre sur les conditions dans lesquelles certaines associations professionnelles sont parvenues à élargir les ressources à leur disposition et les atouts de leurs organisations face aux gros syndicats de branche au point d'avoir pu les mobiliser pour se positionner de manière autonome dans les négociations collectives. Les associations professionnelles ont longtemps été considérées comme des organisations de métier et non comme des représentants actifs des intérêts économiques et sociaux de leurs membres (Heyde, 1956, p. 623). Nous nous demanderons ici dans quelle mesure certaines évolutions de la configuration, dans le contexte des relations sociales, ont initié un changement de fonction et un renforcement du rôle des associations professionnelles. Répondre à cette question requiert une approche ciblée 
des caractéristiques et des ressources des organisations (Olsen, 1965; Offe, 1974 ; Traxler, 1999) mettant en relation la force primaire liée au marché de l'emploi (Jürgens, 1983; Kädtler, 2006) et les structures d'opportunité possibles. Nous appuyant sur le concept de «political opportunity structure » (conditions, opportunités et limites de l'environnement/des conditions structurelles de naissance de mouvements sociaux) (cf. Kriesi, 1991), nous entendons par structure d'opportunité les facteurs qui mettent une association professionnelle en situation de réaliser concrètement l'option (de décision) théoriquement possible de négociations autonomes. Selon Kingdon (2003, p. 20), l'élément déterminant est une "fenêtre d'opportunité » dans laquelle des évolutions ou processus indépendants les uns des autres se retrouvent dans une configuration telle qu'une possibilité d'action s'ouvre pour l'acteur (ici : l'association).

Nous examinons dans une perspective comparative les cas de l'organisation Cockpit et du Marburger Bund, dans un contexte de mutation des structures syndicales et sectorielles, ainsi que de défis posés aux négociations collectives, en nous interrogeant sur l'efficacité des structures d'opportunité. Les associations choisies ici sont, d'un point de vue quantitatif et qualitatif, les acteurs les plus importants issus d'une structure professionnelle, qui ont opté ces dernières années pour une stratégie de négociation autonome ${ }^{1}$. Tout en s'étant toujours préoccupées de questions liées aux conventions collectives, ces organisations se sont longtemps considérées principalement comme des associations professionnelles, acceptant la position de leader des syndicats de la DGB en matière de négociation. Ces deux cas ont en commun un environnement à la configuration semblable qui se caractérise, d'une part, par l'organisation dérégulée et basée sur la performance du secteur public et parapublic sous le signe de l'Europe et, de l'autre, par d'importants changements ou bien une baisse du nombre d'adhérents chez les acteurs syndicaux établis. Nous renvoyons en particulier, avec ce dernier aspect, à la création de ver.di et aux problèmes d'intégration dans les négociations collectives. Par ailleurs, ces deux associations existent depuis longtemps, elles ont un taux d'adhésion exceptionnellement élevé, une base d'adhérents homogène, détiennent une force primaire importante ${ }^{2}$ dans les processus de travail des entreprises ainsi qu'une forte position de blocage basée sur le niveau de qualification moyen à élevé de leurs membres. L'hypothèse déjà formulée par Olson (1965), selon laquelle de petits groupes homogènes sont plus faciles à fédérer, se voit confirmée par l'évolution favorable du nombre d'adhérents et la capacité de mobilisation de ces associations.

Cette contribution fournit à la fois un bilan structuré de la concurrence nouvelle à laquelle se livrent les syndicats professionnels et une analyse compara-

\footnotetext{
${ }^{1}$ Selon Lesch (2008b, p.145), on recense six exemples de ce type. Aux acteurs ici analysés viennent s'ajouter l'organisation indépendante des agents de bord (UFO), le syndicat des contrôleurs aériens (GdF), la fédération des assistants médicaux (VMF) et le syndicat des conducteurs de locomotives (GDL) (au sujet de GDL, voir aussi Greef/Kalass dans le présent ouvrage).

${ }^{2}$ La force primaire des membres de ces associations réside dans leur pouvoir de faire directement pression sur l'entreprise en refusant de collaborer ou en menaçant de le faire (cf. par ex. Kädtler, 2006, p. 19-20).
} 
tive des conditions et conséquences qui ont conduit à ces changements dans les secteurs du rail, des transports aériens et de l'hôpital. Les questions suivantes seront au centre de nos réflexions :

- quelles structures d'opportunité peuvent expliquer cette évolution d'associations professionnelles, qui entrent en concurrence avec les syndicats établis en poursuivant une politique autonome de négociation?

- comment réagissent les syndicats et employeurs concernés face à ces nouveaux acteurs, quels impacts cette concurrence nouvelle a-t-elle pour les relations sociales au sein du secteur et qu'implique cette évolution, d'une manière générale, pour le modèle syndical allemand ?

- les configurations examinées constituent-elles des processus réversibles ou irréversibles?

En partant du modèle de Wolfgang Streeck (1993), qui établit une classification des types et dimensions de syndicats, nous commencerons par analyser les différents modèles organisationnels d'associations de salariés en République fédérale. Nous présenterons dans ce cadre l'évolution des syndicats et des associations professionnelles et de travailleurs. Nous nous intéresserons ensuite aux deux études de cas choisies et montrerons comment l'organisation Cockpit et le Marburger Bund sont devenus des acteurs importants dans les négociations collectives. C'est l'identification de structures d'opportunité qui sera au centre de nos préoccupations. Troisièmement, nous présenterons la réaction des partenaires sociaux établis face aux nouveaux acteurs. Quatrièmement, nous apporterons une réponse à la question des facteurs qui ont permis aux associations professionnelles de se transformer en acteurs de la négociation. En partant des caractéristiques structurelles traditionnelles des deux associations professionnelles retenues, nous chercherons les facteurs qui ont contribué à cette évolution. Sur cette base, nous présenterons les caractéristiques essentielles des structures d'opportunité et évoquerons les perspectives d'évolution ultérieure possible dans le champ du dialogue social en Allemagne.

\section{Syndicats et associations de salariés en Allemagne}

La nouvelle situation de concurrence en matière de négociation collective entre syndicats membres de la DGB et associations professionnelles bénéficie d'un intérêt majeur (synthèse par exemple dans Bispinck/Dribbusch, 2008 ; Keller, 2008a ; Lesch, 2008a ; Schroeder, 2008a). On compte ainsi quelques études approfondies sur d'anciens acteurs professionnels, notamment les travaux de Müller/Wilke (2006) sur la concurrence entre les syndicats dans le secteur ferroviaire et de Hoffmann/Schmidt (2008) sur la grève des conducteurs de locomotives en 2008. Pour le secteur hospitalier et le changement de rôle du Marburger Bund, ce sont Bandelow (2005) et Martens (2008) qui ont posé les premiers jalons. Le débat sur les implications juridiques de cette concurrence nouvelle dans 
ses rapports à la convention collective unique, à l'avenir de la convention de branche, au droit de signer des conventions et au droit de grève (Weisemann, 2007 ; Bayreuther, 2006 ; Greiner, 2007) est un peu plus approfondi.

Nos réflexions partent des idéaux-types possibles : modèles organisationnels de classe, de métier et d'entreprise (cf. Streeck, 1993 ; tableau 1). Le modèle allemand a été marqué, depuis la fin de la Seconde Guerre mondiale, par le modèle de classe, inscrit dans le contexte national de branche. Ont coexisté et coexistent toutefois encore dans le pays des organisations de représentation des salariés structurées suivant le modèle de métier et celui de l'entreprise. La situation actuelle est donc diverse et nuancée. Une première étude réalisée par nos soins $^{3}$ avait identifié 531 organisations représentant les intérêts des salariés, dont 94 sont reconnues au titre de syndicats ou se considèrent comme tels. 64 de ces syndicats font partie de l'une des trois confédérations (DGB, dbb, CGB), les 30 autres étant des syndicats indépendants.

Tableau 1 : Types et dimensions des groupes d'intérêts

\begin{tabular}{|c|c|c|c|}
\hline & Modèle de classe & Modèle de métier & Modèle de l'entreprise \\
\hline Identité & $\begin{array}{l}\text { Position sur le } \\
\text { marché } \\
\text { Nation }\end{array}$ & $\begin{array}{l}\text { Capital humain, } \\
\text { qualification } \\
\text { Profession } \\
\end{array}$ & $\begin{array}{l}\text { Relation de travail, } \\
\text { adhésion à l'entreprise } \\
\text { Organisation }\end{array}$ \\
\hline Valeurs & $\begin{array}{l}\text { «Solidarité » } \\
\text { Egalité des revenus } \\
\text { et des statuts } \\
\text { Universalisme social }\end{array}$ & $\begin{array}{l}\text { «Méritocratie »: } \\
\text { Différenciation des } \\
\text { revenus et des statuts } \\
\text { Particularisme de groupe }\end{array}$ & $\begin{array}{l}\text { «Séniorité »: } \\
\text { Droit à l'emploi, } \\
\text { promotion } \\
\text { Particularisme d'entreprise }\end{array}$ \\
\hline Organisation & $\begin{array}{l}\text { Syndicat politique } \\
\text { d'industrie : global- } \\
\text { inclusif }\end{array}$ & $\begin{array}{l}\text { «organisation de mé- } \\
\text { tier », craft union: } \\
\text { spécialisé-exclusif }\end{array}$ & $\begin{array}{l}\text { Syndicats d'entreprise, } \\
\text { «jaunes »: } \\
\text { englobant l'entreprise }\end{array}$ \\
\hline Institution & $\begin{array}{l}\text { Corporatisme: } \\
\text { Statut public } \\
\text { d'intérêts organisés }\end{array}$ & $\begin{array}{l}\text { Pluralisme : } \\
\text { Libéralisme, } \\
\text { volontarisme }\end{array}$ & $\begin{array}{l}\text { Paternalisme : } \\
\text { Coopération et } \\
\text { participation volontaires }\end{array}$ \\
\hline Politique & $\begin{array}{l}\text { Productivisme } \\
\text { Politique de } \\
\text { répartition } \\
\text { «responsable» } \\
\text { Politique sociale } \\
\text { universaliste }\end{array}$ & $\begin{array}{l}\text { Fermeture au marché } \\
\text { «Militant sectionalism » } \\
\text { Politique de métier } \\
\text { différenciée }\end{array}$ & $\begin{array}{l}\text { Coopération pour assurer } \\
\text { la compétivité de } \\
\text { l'entreprise } \\
\text { Politique du personnel à } \\
\text { l'échelle de l'entreprise }\end{array}$ \\
\hline
\end{tabular}

Source : Streeck, 1993, p. 43.

\footnotetext{
${ }^{3}$ Faute de liste exhaustive détaillant l'ensemble des organisations de représentation des salariés, leur nombre ne peut être déterminé que de manière approximative. Sources : «Liste officielle des associations enregistrées auprès du Bundestag et de leurs représentants » (http://www.bundestag.de/wissen/archiv/sachgeb/lobbyliste/index.html) [consulté le 01/06/11], «Oeckl» (Deutschland 2007. Taschenbuch des öffentlichen Lebens) et «Hoppenstedt » (Schroeder/Kalass/Greef, 2008, p. 34-38).
} 
On compte en outre 429 unions de professions libérales et autres associations et fédérations professionnelles. Jusqu'à présent, ces organisations ne se sont pas comportées en acteurs de la négociation. Faut-il s'attendre à l'avenir à une multiplicité de nouveaux syndicats professionnels et de métier qui créeraient des « relations à l'anglaise » dans le dialogue social allemand ? Même si l'on peut clairement répondre par la négative aujourd'hui, cela n'indique pas pour autant quelles conséquences auront les cas existants. Il est évident que le statut de syndicat et la possibilité de transformation d'associations professionnelles en acteurs autonomes du dialogue social supposent des conditions préalables d'une extrême complexité. Les associations doivent pouvoir être «efficaces» et «puissantes » (Lesch, 2008b, p. 147). Dans quelles conditions les associations professionnelles parviennent-elles à étendre leur domaine de responsabilité au champ, réservé aux syndicats, de la négociation collective ? Quelles conséquences peut-on en déduire pour le système allemand de dialogue social ? Cette contribution montre, à l'aide de deux études de cas, le changement de positionnement des organisations professionnelles.

\section{Etudes de cas}

Les deux cas retenus - organisation Cockpit et Marburger Bund - sont soumis ci-dessous à une analyse comparative. Cette comparaison porte sur la structure des associations telle qu'elle s'est constituée historiquement, le contexte sectoriel et les stratégies respectives de négociation collective. Il est particulièrement important d'identifier les structures d'opportunité qui, selon notre thèse, ont contribué à ce que ces associations puissent s'établir comme acteurs autonomes. En font partie les répercussions structurelles du changement de perception subjective des acteurs et les modifications des structures de dialogue social et de l'environnement organisationnel.

\section{Organisation Cockpit}

L'association des pilotes de ligne et des ingénieurs de l'air en Allemagne ${ }^{4}$ (Vereinigung Cockpit - VC), qui défend les intérêts de l'ensemble de l'équipage technique, a une place particulière quand on considère la nouvelle concurrence entre les syndicats. En réussissant à obtenir en 2001, à l'issue d'une grève, une convention collective séparée pour les pilotes, ce syndicat a endossé le rôle de précurseur pour d'autres organisations professionnelles (comme celle des médecins ou des conducteurs de locomotives). Ce succès s'est appuyé sur le haut niveau de qualification et l'importante force de blocage des membres de l'organisation et sur l'homogénéité de ses adhérents.

\footnotetext{
${ }^{4}$ Désormais, cette association ne rassemble plus que des pilotes, les ingénieurs d'aviation ayant été mis à la retraite ou formés pour devenir pilotes. L'Office fédéral des statistiques (Jahreshefttabellen 2006, Tableau 10.1) recensait encore 88 ingénieurs d'aviation en 2006.
} 


\section{Organisation : histoire et évolution}

La VC fut fondée en 1969, pour représenter les pilotes de la Lufthansa. Depuis 2001, elle défend les intérêts de 8200 pilotes $^{5}$ dans les négociations, tout en continuant de se définir comme une association professionnelle. La majorité des pilotes réunis dans cette association (environ $70 \%$ ) sont salariés chez Lufthansa. Les principales missions de $\mathrm{VC}$ sont la négociation des rémunérations et le lobbying, en particulier dans les domaines de la formation, des conditions de travail et de la sécurité des vols. Mais Cockpit tente aussi de se positionner aussi fortement que possible dans le champ de la cogestion d'entreprise. Cette organisation dispose uniquement d'instances fédérales, du fait de son petit nombre de membres et de la structuration des compagnies aériennes plus petites (ayant une représentation VC directe via le conseil d'entreprise). L'organe suprême de décision est l'assemblée générale annuelle. Les négociations collectives sont menées et préparées par la commission de négociation VC.

Le nombre d'adhérents de l'association connaît une évolution positive. De 2000 à 2007 seulement, les effectifs sont passés de 6000 à 8 200. La croissance la plus importante $(+52 \%)$ a coïncidé avec le début de la politique de négociation autonome (1999-2001). En 2006, VC arrive à un taux de syndicalisation de 80 à $90 \%$, ce chiffre atteignant même $95 \%$ chez Lufthansa Cargo (cf. Würfel, 2001). Du fait de ce taux de syndicalisation, VC revendique le droit de représentation exclusive des pilotes, refusé par ver.di, même si ce syndicat ne compte pratiquement pas de personnels de l'équipage technique parmi ses membres (cf. Lesch, 2008b, p. 151) ${ }^{6}$.

\section{Evolution du contexte sectoriel}

Le secteur des transports aériens a beaucoup changé au cours des deux dernières décennies. Lorsque la libéralisation a commencé, dès le milieu des années 1980, la Lufthansa a réagi avec une grande frilosité. Du fait de sa situation de monopole dans un secteur très régulé par l'Etat, l'entreprise espérait sans doute pouvoir ignorer certaines évolutions ou pouvoir s'en tenir à une politique des «petits pas». En se concentrant sur l'excellence technique et non sur l'innovation commerciale, elle s'est retrouvée à la traîne face à ses nouveaux concurrents européens, très fortement axés sur le marché. La privatisation progressive a été amorcée en $1992^{7}$, le gouvernement fédéral se retirant peu à peu comme opérateur des aéroports et des lignes aériennes. La conjugaison de réac-

\footnotetext{
${ }^{5}$ En 2006, on comptait 9359 pilotes, parmi lesquels $8054(86 \%)$ travaillaient pour les 17 principales compagnies aériennes. Une seule entreprise, la Lufthansa, rassemble 55\% d'entre eux, soit 4400 pilotes (Off. fédéral des statistiques, Jahreshefttabellen 2006, Tableau 10.2).

${ }^{6}$ La situation de concurrence diffère ainsi totalement de celle qui règne parmi le personnel de cabine, où il existe une quasi-parité entre ver.di et l'organisation indépendante des agents de bord (UFO).

${ }^{7}$ Sur les causes et les répercussions d'un management d'entreprise de plus en plus tourné vers les actionnaires, selon l'approche adoptée à partir des années 1990, voir Höpner, 2003.
} 
tions réservées aux nouvelles conditions de marché, à la privatisation et au début de récession des années 1990 aux Etats-Unis a considérablement aggravé la situation économique de la Lufthansa (cf. Lehrer, 2001, p. 361-362, 372 sq.). La réaction cruciale à cette situation a consisté en une politique de redressement qui, dans le cadre de conventions collectives garantissant l'emploi, imposa des efforts financiers considérables, en particulier aux catégories de salariés les plus qualifiés. Seifert (2001, p. 204 sq., p. 424) cite des pertes de revenus de l'ordre de $30 \%$ à la suite de baisses de salaires. L'externalisation de certaines activités de l'entreprise, la privatisation complète et la transformation de la Lufthansa en société par actions ont permis à l'entreprise d'enregistrer des bénéfices dans les années suivantes ${ }^{8}$. Mais la concurrence s'intensifie dans le secteur aérien ${ }^{9}$, si bien qu'il faut s'attendre à l'avenir à d'autres restructurations, avec leurs conséquences pour le personnel.

Ces évolutions ont surtout eu des répercussions sur le statut des pilotes, qui se sont vus de plus en rétrogradés au rang de «conducteurs de bus des airs ». Cette expression ne renvoie pas seulement au fait que les pilotes ne sont plus payés à la hauteur de leurs responsabilités ${ }^{10}$ et de leurs performances (cf. Ramge, 2003, p. 90). Dans les années 1990, ils se sont aussi associés aux concessions négociées pendant la période d'assainissement financier de la Lufthansa, sans que l'augmentation de compétitivité de l'entreprise et l'amélioration de la conjoncture du secteur ${ }^{11}$ ne donnent lieu ultérieurement à des contreparties. Les salaires des pilotes de la Lufthansa étaient inférieurs d'environ $27 \%$ en moyenne à ceux de leurs homologues internationaux (Kolf, 2001, p. 19). En 2001, VC revendiqua par conséquent une augmentation moyenne de salaire de plus de $30 \%$.

\section{Politique de négociation collective}

De 1973 à 1999, VC a coopéré avec le syndicat des employés (DAG), qui défendait alors les intérêts des pilotes dans les négociations collectives. Lorsqu'il est apparu que le DAG allait être absorbé par le nouveau syndicat ver.di, Cockpit a décidé de revoir son positionnement et sa stratégie de négociation. Cette organisation n'a donc pas souhaité d'accord de coopération avec ver.di, mais

\footnotetext{
${ }^{8}$ En 2006, le chiffre d'affaires de la Lufthansa atteignait 19,8 milliards $€$ (pour 803 millions $€$ de bénéfices), ce qui représente une hausse de $77,3 \%$ par rapport à l'année précédente (Lufthansa, 2008).

${ }^{9}$ Entre 2001 et 2006 , le nombre de grandes entreprises dont le chiffre d'affaires est supérieur à 50 millions $€$ (qui constituent ensemble $97 \%$ du chiffre d'affaires total et emploient $86 \%$ des pilotes) est passé de 13 à 17 . Au total, le nombre d'entreprises aéronautiques a grimpé de 133 à 331 (Off. fédéral des statistiques).

${ }^{10}$ Cette responsabilité se reflète dans la grande confiance accordée aux pilotes. Dans les sondages, $93 \%$ des personnes interrogées leur accordent une confiance très élevée à plutôt élevée, ce qui les place juste derrière les pompiers (94\%) mais devant les médecins (86\%) (Reader's Digest, 2007).

${ }^{11}$ Si le chiffre d'affaires de la branche aéronautique augmentait de $8 \%$ à $11 \%$ dans les années 1980, la hausse s'est réduite à 3-5\% dans les années 1990 pour tomber à moins de $1 \%$ en 2001-2002. Depuis 2003, la croissance a repris, enregistrant un taux de 9-10\% (Off. fédéral des statistiques, statistiques sur les entreprises aéronautiques).
} 
s'est efforcée, lors du round de négociations de 2001, d'avoir une position autonome. Au cours de ces négociations, le syndicat des services publics et des transports (ÖTV) et le DAG ont obtenu une augmentation de salaire de 3,5\% pour les personnels au sol et de cabine. Ce résultat étant très inférieur aux attentes des pilotes, $\mathrm{VC}$ a refusé son extension aux équipages techniques, négociant alors pour la première fois séparément avec l'entreprise. Les premiers rounds de négociations se soldèrent cependant par un échec, les pilotes demandant une revalorisation de plus de $30 \%$ alors que la Lufthsansa proposait 8,7\%. Après plusieurs grèves (d'avertissement), une procédure d'arbitrage a commencé sous Hans-Dietrich Genscher, alors ministre des Affaires étrangères. Les négociations se sont conclues par une augmentation de base de $20 \%$ du salaire associée à une participation variable aux résultats de l'entreprise, soit une augmentation totale de revenu d'environ $28 \%$ pour 2001 (cf. Handelsblatt, 2001, p. 5).

Les syndicats membres de la DGB critiquèrent cet accord, en raison principalement de la désolidarisation qu'il impliquait (cf. Bispinck, 2001, p. 424) ${ }^{12}$. S'ajouta à cet aspect le fait que les pilotes du secteur du fret aérien sont nettement moins bien payés que ceux des transports de voyageurs, une disparité sur laquelle se penche également la commission VC depuis 2007, notamment en raison de la pression exercée par ver.di (Tarp, 2007).

\section{Structure d'opportunité utilisée}

Les facteurs internes comme le taux de syndicalisation très élevé et le pouvoir de blocage dû à la qualification des adhérents ne sont pas les seules clés du succès de VC dans les négociations. Le moment où l'organisation s'est positionnée comme acteur du dialogue social (1999-2001) correspond aussi à une structure d'opportunité spécifique, qui a joué un rôle essentiel dans cette victoire. La création de ver.di - le plus important changement structurel pour les syndicats allemands depuis 1945 - a eu de profondes répercussions sur d'autres acteurs. Le DAG, qui menait jusqu'alors les négociations pour les pilotes regroupés au sein de VC, fut complètement absorbé par le nouveau syndicat multisectoriel. L'organisation Cockpit elle-même insista sur le fait que les syndicats étaient, avec la création de ver.di, «trop occupés par leurs propres problèmes », si bien que la séparation d'avec le DAG fut relativement simple, faute de réactions de la part des autres syndicats (cf. Sturm, 2000). Parallèlement, compte tenu de la fusion imminente, la crainte grandit au sein de VC que les besoins de ses adhérents ne soient plus pris en considération au sein du nouveau grand syndicat.

La stratégie managériale de la Lufthansa favorisa également la scission des pilotes. Car les accords tarifaires peu favorables, établis pendant la période de redressement des années 1990, devaient être prolongés alors que la situation fi-

\footnotetext{
${ }^{12}$ Le « Darwinisme social », le «cavalier seul au détriment des collègues » ou « l'atteinte à l'équité salariale entre les collaborateurs » constituent certains des reproches soulignés par la DGB et ver.di.
} 
nancière de la Lufthansa s'était rétablie. Les pilotes ne voyaient quant à eux pas la nécessité de poursuivre leur contribution à l'assainissement financier (cf. Bispinck, 2001, p. 423-424). La séparation de leur association d'avec ver.di créa les conditions préalables nécessaires à une politique de négociation autonome. VC exprima en même temps un refus de la politique solidaire et corporatiste de ver.di. Mais, malgré l'issue favorable de ses propres négociations, Cockpit se considère encore comme une association professionnelle et, à titre secondaire seulement, comme un syndicat (Schewe, 2007).

\section{Marburger Bund}

Le Marburger Bund (MB) défend les intérêts professionnels des médecins salariés et fonctionnaires en Allemagne, et est reconnu depuis 2006 comme leur représentation syndicale. Comme le secteur aérien décrit plus haut, le secteur sanitaire et hospitalier est touché par d'importants changements affectant son organisation, son cadre économique et d'emploi, ainsi que par des mesures de privatisation impliquant aussi une évolution de l'image de la profession et une dégradation du statut des médecins. Le positionnement du MB comme acteur de la négociation s'est fait dans le sillage de celui de l'organisation Cockpit.

\section{Organisation : histoire et évolution}

Le Marburger Bund fut créé en 1947 sous le nom de «Marburger Gemeinschaft ", lié à l'ordre des médecins (Ärztekammer), à Marburg/Lahn. Cette association avait pour objectif premier l'amélioration des conditions de travail, et en particulier des temps de travail. Un an après sa création, l'organisation se sépare de l'ordre des médecins et constitue l'union syndicale Marburger Bund (cf. Marburger Bund, 2008). Le MB est structuré en 14 associations régionales. Les petite et grande commissions décident des mesures d'action syndicale, de la stratégie de négociation et de la signature ou de la dénonciation d'accords collectifs. Comparé aux grands syndicats, le Marburger Bund n'a pratiquement pas de personnel salarié. Font exception le secrétaire général et le président de l'association fédérale, les secrétaires généraux des associations régionales ainsi que quelques juristes et personnels de secrétariat. Les associations de district sont constituées de bénévoles.

$\mathrm{Au}$ cours des quarante dernières années, le $\mathrm{MB}$ a vu son nombre d'adhérents passer de 17000 (1967) à $108000(2007)^{13}$. Ceux-ci travaillent à $90 \%$ dans des hôpitaux ; s'ajoutent à eux quelques médecins issus du secteur ambulatoire, des administrations et d'entreprises privées. Ver.di voit d'un œil critique la politique de recrutement du Marburger Bund. Premièrement, les médecins peuvent rester membres de l'association après s'être installés en cabinet

\footnotetext{
${ }^{13}$ Une croissance de près de 10000 adhérents par rapport à l'année précédente, malgré l'accroissement de la concurrence avec les associations de médecins spécialistes (cf. Bandelow, 2007 p. 275).
} 
(et donc avoir quitté leurs fonctions en tant que salariés). Deuxièmement, les médecins-chefs salariés ont des responsabilités budgétaires qui font d'eux «[...] des médecins dirigeants dans des fonctions d'employeur [...]», en conséquence de quoi la condition syndicale de l'indépendance n'est plus remplie et l'association n'est pas habilitée à négocier (Dielmann, 2006, p. 14). Avec au total 148000 médecins dans les hôpitaux allemands, le MB atteint un taux de syndicalisation de plus de $60 \%$ chez les médecins hospitaliers (cf. Bundesärztekammer, 2007, graphique 1).

Le Marburger Bund dispose d'une importante capacité d'organisation et de gestion des conflits, reposant notamment sur la forte volonté de regroupement du corps médical (cf. Schmitt, 1966, p. 17-84) et sur la grande homogénéité de ses membres, qui ne tient pas uniquement à la durée de la formation universitaire. L'emploi presque exclusif dans des hôpitaux implique des situations professionnelles, organisationnelles et des problèmes semblables. Le corps médical allemand est en outre soudé par une «[...] cohérence idéologique relativement forte sur fond de valeurs libérales, conservatrices, et de situations d'intérêts similaires [...] $\gg($ Bandelow, 2007, p. 284) et par l'obligation partagée que représente le serment d'Hippocrate. Il résulte de ces facteurs un important potentiel d'action et de pouvoir mobilisable dans les relations avec des tiers. Mais il existe également entre les membres des différences considérables de revenus et de statuts, qui reflètent les structures hiérarchiques de l'hôpital. Car le MB regroupe toutes les catégories de personnel, depuis les internes jusqu'aux médecins-chefs, en passant par les spécialistes et les chefs de service. La principale ligne de partage sépare les médecins-chefs des autres catégories de médecins, les médecins-chefs se trouvant structurellement dans une situation de conflit d'intérêts entre solidarité avec le corps médical et responsabilité entrepreneuriale du budget de la structure.

\section{Evolution du contexte sectoriel}

Depuis le milieu des années 1990, les hôpitaux sont eux aussi de plus en plus confrontés à des processus qui ont transformé les conditions d'affrontement et d'organisation des personnels et ont conduit le Marburger Bund à gagner de l'importance. Il faut citer en premier lieu la privatisation de nombreux hôpitaux ${ }^{14}$. Ce processus s'est accompagné d'une décentralisation de la négociation collective au niveau des établissements, les hôpitaux privés ayant quitté les conventions collectives du service public au profit d'accords d'établissement ou de groupe (cf. Böhlke/Schulten, 2008, p. 25-26). Deuxièmement, la loi de réforme du système de santé (GSG) adoptée en janvier 1993 a entraîné le plafonnement du budget des hôpitaux et l'introduction de forfaits (remboursement d'un acte médical au forfait et non en fonction des coûts réellement survenus) (cf. ibid., p. 25 ; Simon, 1997, p. 3-

\footnotetext{
${ }^{14}$ La part des établissements publics est passée de $46 \%$ en 1991 à $34 \%$ en 2006 tandis que, sur la même période, celle des hôpitaux privés grimpait de $15 \%$ à $27 \%$.
} 
4, p. 13-14). La conséquence de ces deux évolutions est, d'une part, une pression financière accrue sur les salariés, les mesures de rationalisation se focalisant le plus souvent sur les dépenses de personnel, qui représentent $60 \%$ des dépenses ${ }^{15}$. D'autre part, l'image de la profession a changé, la direction des hôpitaux ayant été reconsidérée pour donner de plus en plus la priorité aux modèles de pensée et d'action économiques face à l' " état de la science et à ce qui est médicalement nécessaire ». Les missions médicales ne cessent d'augmenter, de même que les tâches administratives comme le calcul des coûts et des performances ou les obligations documentaires destinées aux caisses-maladie. Les médecins-chefs ont eux aussi été intégrés à cette nouvelle forme de gestion administrative et ont une responsabilité budgétaire (cf. Simon, 1997, p. 25, p. 45 ; Tödtmann, 2005, p. 1).

La coïncidence entre baisses de salaires et pression administrative à l'efficacité a favorisé le sentiment d'une perte de statut au sein du corps médical, dégradé du rang de "demi-dieux en blanc » à celui d' "ouvriers qualifiés en gestion de la santé » (Martens, 2008, p. 20 sq.). La limitation des coûts dans le secteur de la santé publique a en outre pré-programmé des conflits de répartition entre les salariés dès lors qu'une catégorie donnée souhaite élargir son rayon d'action financier. S'ajoute l'augmentation du nombre de médecins travaillant en ambulatoire (dont le nombre est passé de 92000 en 1990 à 136000 en 2006) (cf. Bundesärztekammer, 2007, tableau 1). Ce contexte a donc fortement limité non seulement les possibilités d'intégrer la hiérarchie hospitalière, mais également les perspectives d'évolution. De plus, l'alternative d'une installation en cabinet privé s'est trouvée réduite par les restrictions budgétaires qui affectent également la médecine libérale (cf. Busch/Stamm, 2006, p. 17-18).

Les conséquences financières de ces évolutions touchent surtout les jeunes médecins. Selon une étude réalisée par l'Institut de recherche économique DIW Berlin ${ }^{16}$ (Spengler, 2004), le revenu mensuel net moyen des jeunes médecins employés à plein temps dans le service public est tombé de $2170 €$ (1993) à $2009 €$ (2002) ; parallèlement, le temps de travail hebdomadaire est passé de 45 à 46,3 heures : cette évolution correspond à un recul du salaire horaire de 12,10 à 10,80€. Si l'on considère l'ensemble des médecins du service public, on observe il est vrai une hausse du revenu net, mais celle-ci tient non pas à une hausse du salaire horaire, mais à une augmentation de la durée hebdomadaire du travail. Selon une étude de la société NERA, les médecins hospitaliers allemands se trouvent, comparés à leurs homologues européens $^{17}$, au bas de l'échelle des salaires (NERA 2004, p. 6) ${ }^{18}$.

\footnotetext{
${ }^{15}$ Ainsi, il a été fait largement machine arrière sur les dispositions relatives aux heures supplémentaires, qui seules donnent droit à rémunération. Toutefois, le nombre d'heures supplémentaires réellement effectuées n'a pas varié, en raison du fait que celles-ci étaient considérées comme nécessaires d'un point de vue médical ou adaptées à la formation.

${ }^{16}$ Cette étude repose sur les données du microrecensement officiel de 1993 et 2002.

${ }^{17}$ La fourchette des salaires donnée pour l'année 2003 se situait entre 35000 et $56000 €$ en Allemagne. A titre de comparaison en France, elle s'échelonnait entre 93000 et $107000 €$ en 2001 (cf. NERA, 2005, p. 5).

${ }^{18}$ Ver.di critique le fait que les données allemandes de l'étude proviennent du MB. Les cas sur lesquels repose

l'enquête seraient peu réalistes, tandis que l'ensemble des prestations ne serait pas pris en compte dans
} 


\section{Politique de négociation collective}

La politique de négociation collective relève, depuis la création du MB, du champ d'activités de l'association. Mais, comme le syndicat ÖTV et les employeurs lui ont dénié le droit de signer des conventions et n'ont donc pas négocié avec le $\mathrm{MB}$, ce dernier a dû chercher un partenaire de coopération pour défendre ses intérêts à sa place; pendant de nombreuses décennies, ce fut le DAG. Le contrat de coopération avec ce syndicat (1950) permit au Marburger Bund de peser sur les négociations collectives et de participer efficacement, de 1959 à 1961, à la convention des employés relevant de la fonction publique fédérale (BAT). En 1971, la question durablement conflictuelle du temps de travail des médecins entraîna une première grève autonome. Refusant la réduction progressive de la durée du travail, acceptée par l'ÖTV et le DAG, le MB déclara l'échec des négociations collectives. Du fait de cette pression, les employeurs intégrèrent le MB au cours du round de négociations suivant. En 1976, le Marburger Bund, le DAG et l' " union de syndicats et associations du service public» constituèrent une union syndicale séparée de l'ÖTV. Cet accord de coopération dura jusqu'à la dissolution du DAG, absorbé dans ver.di (cf. Marburger Bund 2008). Mais, contrairement à ce qui se passa chez les pilotes, il n'y eut pas de négociation séparée dès 2001. La rupture entre le MB et ver.di intervint au moment des négociations préliminaires à la convention du service public (TVöD). Le Marburger Bund voyait dans ce nouvel accord une amélioration financière unilatérale des autres catégories professionnelles au détriment des médecins, les « intérêts propres au corps médical n'étant qu'insuffisamment pris en compte [par ver.di] dans les négociations collectives du service public » (Marburger Bund 2006, p. 9). Les changements d'échelon ne devaient plus dépendre de l'âge et de la situation de famille (principe de séniorité) mais d'une évaluation, basée sur la performance, de l'expérience professionnelle et de l'ancienneté de service. Les primes exceptionnelles de Noël et de congés devaient être diminuées. Sur ces questions, ver.di était globalement disposé à coopérer avec les employeurs, ce qui du point de vue du Marburger Bund rendait impossible toute collaboration ultérieure (cf. Lindhorst, 2007, p. 1). En juin 2006, 14000 médecins se mirent en grève pour demander une convention collective séparée ${ }^{19}$. La fédération des Länder (Tarifgemeinschaft deutscher Länder) fut le premier acteur à faire des concessions. Suivit en août la convention signée par les employeurs communaux, après une grève de plus de 17000 médecins.

l'estimation des rémunérations. Selon ses calculs, les médecins allemands devraient se situer dans la moyenne haute (cf. Dielmann, 2005, p. 16).

${ }^{19}$ Il existe un contentieux sur la possibilité alors donnée au MB de signer une convention collective distincte (c'est-à-dire une nouvelle convention). Diverses comparaisons reconnaissent certes au texte des «améliorations », mais évoquent également un faible degré de divergence par rapport à la convention d'origine (p. ex. Schwarzkopf-Steinhauser, 2006, p. 11). 
Dans cette convention collective, ver.di critiqua le fait que les prétentions salariales excessives des médecins pénalisaient les autres catégories professionnelles et, en entraînant une désolidarisation des salariés, étaient un facteur de division. Ver.di reproche en outre au MB d'avoir obtenu ces augmentations des salaires supérieures à la moyenne principalement en échange de temps de travail prolongés et, ainsi, de ne pas avoir amélioré mais aggravé les conditions de travail (cf. Dielmann, 2006, p. 14).

\section{Structure d'opportunité utilisée}

A la différence de l'organisation Cockpit, le MB a d'abord accepté que les négociations soient menées par ver.di, pour ne s'en affranchir qu'à l'automne 2005. Cette évolution eut plusieurs causes : d'une part, les changements survenus au cours des dix dernières années dans le secteur accrurent les restrictions budgétaires ainsi que les exigences de performance à l'égard des médecins, entraînèrent une augmentation des tâches administratives et eurent des répercussions négatives sur l'évolution des salaires. D'autre part, ces processus, associés à une augmentation du nombre de médecins, firent craindre à ces professionnels une dégradation croissante de leur statut. S'ajoutait en outre le mécontentement relatif aux mauvaises conditions de travail comme la durée importante du travail et les heures supplémentaires, ainsi que la perspective, à partir de 2005, de pertes financières supplémentaires avec le changement de convention (passage de la BAT à la TVöD). Ce fut sans doute ce réajustement de la structure des salaires et rémunérations qui motiva réellement le retrait de ce groupement de négociation. En septembre 2005, l'assemblée générale du MB décida de rejeter la TVöD et retira à ver.di le pouvoir de représenter les médecins afin de demander une convention collective séparée pour ces derniers, sous le mot d'ordre «BAT $+30 \%$ \%. Cette démarche avait été précédée d'une grève des internes, soumis à du travail supplémentaire sans compensation de salaire, en particulier dans les hôpitaux universitaires, après la dénonciation pure et simple par certains Länder de la convention BAT encore en vigueur. Le MB s'empara de ce mécontentement et prit la tête du mouvement de grève (cf. Busch/Stamm, 2006, p. 17-18 ; Martens, 2008, p. 33) ${ }^{20}$.

Avec une première victoire séparée dans les négociations en 2006, le MB a nettement renforcé son positionnement de syndicat $^{21}$. La transformation en syndicat n'est néanmoins absolument pas achevée. Les rapports d'opposition avec les autres catégories de salariés hospitaliers et l'organisation hiérarchique

\footnotetext{
${ }^{20}$ Vient s'ajouter à cela le très fort crédit accordé aux médecins par l'opinion publique. Sur l'échelle du prestige professionnel, les médecins occupent régulièrement, et de loin, la première place. Alors que le degré de considération a légèrement reculé entre 1991 et 2005, il est remonté de $6 \%$ en 2006 , après le succès de la grève des médecins (Institut für Demoskopie Allensbach).

${ }_{21}$ Lors de la $111^{\mathrm{e}}$ assemblée générale, la petite commission de négociation a renforcé ses effectifs, passant de 11 à 15 membres. Les médecins fonctionnaires bénévoles se sont intéressés davantage à la politique concrète de négociation collective, évinçant ainsi les juristes au sein de la commission.
} 
du corps médical laissent supposer une orientation de l'association encore fortement liée au métier. Cette opiniâtreté corporatiste se trouve renforcée par la volonté de préserver son propre statut face aux autres associations de médecins et par la longue tradition d'organisations puissantes au sein de la profession ${ }^{22}$. Dans son étude sur la grève des médecins, Martens (2008, p. 47) parvient lui aussi à la conclusion suivante : «Le profil à venir de l'association reste flou [...]»; mais, selon lui, le débat sur la manière dont les choses peuvent et doivent évoluer bat son plein.

Comme l'a montré l'analyse des configurations dans l'un et l'autre cas, le chemin qui a conduit les organisations à mener des négociations autonomes était depuis longtemps un objectif prioritaire, auquel ont été subordonnés les objectifs concurrents. Il a en même temps fallu écarter toute une série d'obstacles qui barraient la voie. Pour ce faire, il fallait des structures d'opportunité significatives qui ont offert un cadre permettant aux deux associations de s'établir en acteurs autonomes de la négociation collective.

\section{Réactions des partenaires sociaux établis}

Les hypothèses selon lesquelles l'évolution présentée dans ces deux études de cas pourrait représenter une concurrence si vivifiante en matière de politique salariale (cf. Hensche, 2007) qu'elle pourrait même soulager les syndicats d'industrie, ne sont pas réalistes. Le renforcement des associations professionnelles est au contraire critiqué par les partenaires sociaux traditionnels et a suscité une résistance farouche chez les syndicats membres de la DGB et chez les employeurs (cf. Lech, 2008b ; Keller, 2008a ; Bispinck/Dribbusch, 2008). Les partenaires sociaux établis considèrent cette évolution comme une menace pour le rôle pacificateur et pour les forces du système allemand.

Le renforcement accru du rôle des associations professionnelles dans le système de dialogue social va encore exacerber la pression exercée jusque-là sur les syndicats membres de la DGB. La profonde mutation structurelle de l'économie et de la société et les crises qui en résultent chez les adhérents (Ebbinghaus, 2003), les opposants ${ }^{23}$ (Schroeder/Silvia, 2003), ainsi que les difficultés à s'imposer et à s'implanter ${ }^{24}$ (Hassel, 2006 ; Schroeder, 2008b), ont déjà dans le passé imposé des changements structurels majeurs du côté des syndicats.

C'est dans ce contexte qu'il faut voir le renforcement des associations professionnelles et l'apparition de nouveaux acteurs du dialogue social car des or-

\footnotetext{
${ }^{22}$ Par ex. : le taux de syndicalisation de l'union des associations de médecins (Ärztevereinsbund) dépassait $60 \%$ à la fin du XIX ${ }^{\mathrm{e}}$ siècle ; il atteignait même 95\% dans les années 1920 (Schmitt, 1966, p. 38).

${ }^{23}$ On entend par là les difficultés liées aux adhérents et les problèmes d'intégration et de gouvernance au sein des fédérations d'employeurs ainsi que les organisations exemptes de conventions collectives (OT-Verbände) constituées par les fédérations en vue de résoudre ces problèmes.

${ }^{24}$ On entend par là l'affaiblissement du lien entre les syndicats et la démocratie sociale ainsi que les difficultés à s'imposer, illustrées par l'échec de la grève de 2003 d'IG Metall en Allemagne de l'est.
} 
ganisations professionnelles plus puissantes menacent d'affaiblir encore davantage les syndicats DGB établis. Il est caractéristique que cette concurrence nouvelle se limite aux secteurs dans lesquels ces syndicats sont depuis longtemps confrontés à un faible taux de syndicalisation ${ }^{25}$. Cette observation vaut pour les pilotes comme pour les médecins hospitaliers. Le modèle privilégié et jusqu'à présent dominant pour la DGB de l'organisation syndicale unitaire tend vers une politique salariale solidaire, intégrant dans un même syndicat des intérêts plus ou moins forts, cette dimension étant considérée comme essentielle. C'est dans ce contexte que s'inscrivent les réactions parfois virulentes des syndicats membres de la DGB à cette concurrence de surenchère (cf. Hassel, 1999, p. 89 ; Hassel, 2007, p. 180 ; Streeck, 2003, p. 99 ; Schroeder, 2005, p. 121 ; Biebeler/Lesch, 2007, p. 140 sq.). En dénonçant des accords réservés aux personnels les plus qualifiés, ces syndicats critiquent surtout le fait que ces accords auront selon eux des répercussions négatives pour les catégories de salariés plus faibles, d'où le qualificatif de «non solidaires ${ }^{26}$. Comme les catégories professionnelles concernées ne mobilisent pas leur potentiel pour s'imposer au profit de l'ensemble des salariés, les syndicats membres de la DGB voient non seulement un risque que l'alliance ne se trouve affaiblie et que les employeurs puissent finalement opposer les uns aux autres les différents groupes dans les négociations ; mais ils craignent surtout que les catégories de personnels les plus faibles ne se retrouvent complètement exclus de cette alliance et qu'une concurrence de surenchère ne s'exerce au détriment de ces catégories (Bispinck/Dribbusch, 2008, p. 159-160). Enfin, ce phénomène peut mener à l'affaiblissement global des syndicats de la DGB. La question étant de savoir qui regroupe les salariés hautement qualifiés et comment ils peuvent être défendus, elle se résume principalement à une concurrence pour le recrutement. Dans cette situation, la stabilité organisationnelle des syndicats DGB est effectivement menacée, ce qui exacerbe la concurrence latente entre les syndicats auprès des adhérents potentiels (cf. Keller, 2008a, p. 167). C'est pourquoi ver.di mais aussi IG Metall tentent par exemple, à travers de nouvelles campagnes ciblées, de séduire les salariés qu'ils ont eu des difficultés à toucher jusqu'à présent (p. ex les ingénieurs hautement qualifiés) - mais le risque de voir éventuellement d'autres catégories professionnelles suivre l'exemple des pilotes ou des médecins joue sans doute également un rôle. Outre des initiatives nouvelles à destination de groupes ciblés, les syndicats de la DGB essaient de revoir la

\footnotetext{
${ }^{25}$ Cela vaut aussi bien pour la concurrence de surenchère que pour celle du moins-disant social. La concurrence de surenchère va de pair avec une faible syndicalisation des salariés hautement qualifiés dans les organisations membres de la DGB, la concurrence du moins-disant social avec une faible syndicalisation des employés peu qualifiés (Allemagne de l'est, artisanat, travail intérimaire) (cf. Schroeder, 2008, p. 14 ; Bispinck/Dribbusch, 2008, p. 159 sq.).

${ }^{26}$ Wolfgang Rose (responsable ver.di du district de Hambourg) a commenté comme suit l'approche de l'union Cockpit: «Nous n'approuvons pas le fait que les hauts salaires s'enrichissent au détriment des plus faibles revenus » (Hamburger Abendblatt, 2001). La politique de négociation de GDL a été également critiquée par M. Sommer, le chef de la DGB : "Il n'est pas concevable qu'un syndicat et ses membres prennent leurs distances à l'égard de la solidarité qui unit tous les salariés »(Der Spiegel, 2007, p. 20).
} 
communication et la légitimation de leur politique de répartition dans les négociations imminentes ${ }^{27}$. Les syndicats membres de la DGB n'ont en définitive le choix qu'entre deux options réalistes (cf. Keller 2008a, p. 167-168) : a) empêcher que de puissantes associations professionnelles ne continuent de se développer en coopérant, de manière pragmatique, à la politique de négociation ou b) axer leur propre stratégie de recrutement, leur politique de négociation et leurs offres de services sur les intérêts particuliers de certaines catégories de salariés.

Dans une situation classique, la différenciation des standards salariaux au sein de l'entreprise est aussi dans l'intérêt des employeurs. Mais, dans le cas de la concurrence de surenchère, employeurs et syndicats de la DGB demandent, d'une même voix, le maintien du principe de convention collective unique (au niveau de l'entreprise). Ils craignent au fond une augmentation des dépenses de personnel, et redoutent que des négociations à venir avec différentes organisations de salariés ne soient plus difficiles et plus conflictuelles (cf. Bispnick/Dribbusch, 2008 ; Lesch, 2008a). Avec des durées de validité potentiellement distinctes, la convention de branche perd de son rôle pacificateur, ce qui crée un risque de multiplication des actions de lutte sociale. Les différences de salaires, qui influent sensiblement sur les motivations des salariés, sont de moins en moins liées à la performance des différentes catégories et davantage à leur pouvoir respectif de faire grève (cf. Lesch, 2008b, p. 149).

Dans cette situation, Keller (2008a, p. 168-169) voit dans la négociation avec des organisations de travailleurs concurrentes une stratégie possible pour les employeurs, ce qui impliquerait une reconnaissance de facto de plus petits syndicats comme partenaires sociaux dans le cadre d'accords collectifs (d'établissement). Mais les employeurs pourraient aussi, dans le même temps, poursuivre le dialogue social existant et tenter de coopérer avec les syndicats d'industrie. Keller juge irréaliste le scénario du lock-out en réaction aux grèves que le nombre de conflits du travail pourrait renforcer.

\section{Syndicats catégoriels : similitudes et différentes}

La naissance de syndicats catégoriels n'est ni un hasard ni une nécessité. Les configurations concrètes dans lesquelles les associations professionnelles étudiées ici sont parvenues à s'établir comme acteurs autonomes de la négociation montrent que ce processus requiert des conditions préalables (cf. également Keller, 2008a, p. 164 ; Müller-Jentsch 2008, p. 62 ; Lesch, 2008b, p. 147). Parmi ces données fondamentales complexes, un facteur essentiel est une organisation solide, en mesure de s'appuyer sur des ressources matérielles assurées et des valeurs communes. De petits groupes homogènes de salariés sont en outre plus enclins à s'organiser et à se fédérer au sein d'une association. On peut donc

\footnotetext{
${ }^{27}$ Voir la déclaration d'Helga Schwitzer (membre de la direction d'IG Metall). Cette dernière recommande de «prendre davantage en considération les intérêts distincts » (Steiger, 2008, p. 4).
} 
postuler une coïncidence d'intérêts plus importante que dans des groupes plus hétérogènes (Olson, 1965, p. 29, 34). Les deux associations analysées présentent ainsi l'une comme l'autre un taux de syndicalisation comparativement élevé. Elles sont en situation de mettre à la disposition de leurs membres des biens individuels constituant une incitation directe à l'adhésion (ibid., p. 130133). Dans certaines conditions, ce taux de syndicalisation important permet aux associations de convertir la position centrale de leurs membres dans le processus de travail en forte capacité d'action. Le nombre d'associations et groupes de travailleurs disposant d'un potentiel comparablement élevé est faible, ce qui limite le nombre d'émules possibles. Les deux associations s'appuient en outre sur une longue histoire incluant aussi un rôle passif (au sens d'organisations associées) dans les négociations. Ces organisations disposaient déjà, avant même de s'établir comme syndicats, d'un savoir approfondi en matière de procédures et de négociations qui leur a permis de lancer plus facilement leurs propres activités en tant que partenaire social.

Ces conditions ne suffisent toutefois pas à elles seules pour expliquer le processus de transformation d'une association professionnelle en acteur de la négociation collective. Les structures décrites existent en effet, dans une configuration semblable, depuis quelques décennies. En même temps, la comparaison des deux syndicats professionnels a montré que des circonstances similaires étaient certes déterminantes pour leurs activités d'acteurs autonomes ; les particularités de chaque cas impliquent toutefois une dynamique de conflit propre à chacun d'entre eux. Cela rend plausibles les points de vue selon lesquels il n'y a pas lieu de «s'attendre à court terme à un grand nombre d'émules » (Bispinck/Dribbusch, 2008, p. 162) car «des obstacles juridiques et organisationnels s'opposent à une tendance plus large à la création de syndicats professionnels » (Lesch, 2008b, p. 147). C'est pourquoi nous nous concentrons ci-dessous sur les similitudes et différences qui peuvent être vues comme des structures d'opportunité. A partir des études de cas présentées, on peut retenir quelques observations, identifiables comme des conditions similaires dans le processus de transformation analysé :

- la concurrence entre syndicats et entre acteurs de la négociation s'installe surtout dans les catégories de salariés à haut niveau de qualification, ceux-ci étant moins sensibles à la logique de représentation unitaire - en particulier lorsqu'ils ont l'impression que leurs marges de manœuvre en matière de salaire ne sont pas exploitées pendant des périodes assez longues. Ces personnels, ainsi que les salariés détenant des postes clés, peuvent dans certaines conditions avoir une position de blocage sur le marché de l'emploi, ce qui leur permet d'obliger leurs employeurs à aller à l'encontre de leurs propres intérêts en les menaçant de changer d'entreprise ou de faire grève. Ils sont généralement très soudés à l'échelle de leur profession ou de leur entreprise. Alors que les syndicats unitaires aspirent à des écarts de salaires aussi faibles que possible 
entre les différentes catégories d'employés, les personnels hautement qualifiés, souhaitant être le mieux rémunérés possible, privilégient des salaires différenciés ;

- les organisations professionnelles et les syndicats catégoriels gagnent du terrain lorsque les profils traditionnels d'activités de ces catégories de salariés sont dévalorisés à mesure des changements qui affectent un secteur ou une entreprise ou bien au fil des évolutions technologiques. Ce phénomène peut intervenir dès lors que le nombre d'élites dites de fonction augmente sensiblement comme dans le cas des médecins et des pilotes par exemple. Mais il peut aussi avoir d'autres causes : les évolutions économiques ou technologiques peuvent également mener à utiliser des machines pour des activités autrefois mécaniques. Au fil de ces évolutions, certaines qualifications deviennent superflues. Ce changement peut s'accompagner d'une dévalorisation du profil d'activité de certaines catégories de salariés et, par conséquent, de pertes de salaire. Les personnes concernées se voient contraintes d'agir à cause de cette dévalorisation, et tentent de pérenniser les acquis liés à leurs qualifications. Si ce groupe n'est pas remplacé et qu'il continue de jouer un rôle important dans la chaîne de création de valeur ou de responsabilité du fait de la division du travail, certains facteurs spécifiques d'une lutte axée sur le statut peuvent se trouver réunis ;

- avec la modération salariale acceptée par les syndicats et des pactes pour l'emploi dans les entreprises, la sécurité de l'emploi a été troquée contre des concessions en matière de rémunération, de temps de travail et de primes. Après l'amélioration de la conjoncture, les syndicats membres de la DGB n'ont pas réussi à obtenir des compensations pour les concessions faites dans le passé2 ${ }^{28}$ (cf. Bispinck/Schulten, 2001). Pour les emplois à haut niveau de qualification, la transformation des entreprises analysées ici s'est accompagnée non seulement de concessions mais également d'une dégradation effective (ou simplement ressentie) de l'image de la profession et du statut. L'offensive menée par ces organisations professionnelles peut donc également être considérée comme une tentative de s'opposer à cette double peine : pertes de salaire et perte de statut ;

- la concurrence entre syndicats s'installe en particulier dans les secteurs publics et parapublics. On trouve plus précisément deux grands domaines d'activités : le secteur hospitalier et celui des transports. La transformation d'organisations professionnelles jusqu'alors passives dans les négociations en syndicats actifs dans le dialogue social n'est en

\footnotetext{
${ }^{28}$ Lesch (2008b, p. 148) compare la dynamique de 12 secteurs en matière de négociation salariale. Il en conclut qu'avec une croissance de $16,5 \%$ entre 1995 et 2006 , la modération salariale est très forte dans les services publics, les secteurs étudiés faisant montre d'une croissance moyenne de $25,5 \%$ pour culminer à $35,7 \%$. Lesch n'observe par ailleurs pas de différenciation au sein même des services publics.
} 
aucun cas une tendance uniforme en Allemagne. Quand on compare les succès de Cockpit, du Marburger Bund ou encore du syndicat des conducteurs de locomotives (voir Greef/Kalass dans le présent ouvrage), on remarque que ces exemples de concurrence réussie sont liés à des entreprises actuellement ou autrefois gérées par la puissance publique. De nombreux salariés ayant traditionnellement bénéficié dans ces entreprises d'un statut de fonctionnaire et des avantages associés (par exemple la garantie d'une sécurité d'emploi à vie), nous supposons que la suppression croissante de postes de fonctionnaires a créé des facteurs d'opportunité qui ont favorisé soit un renforcement des organisations existantes soit la création de syndicats catégoriels. Se trouvent ainsi réunis les éléments déterminants qui ont fait émerger les structures d'opportunité constituant le cadre des négociations autonomes de ces organisations.

Tableau 2 : Eléments structurels des associations professionnelles étudiées

\begin{tabular}{|c|c|c|}
\hline & Organisation Cockpit & Marburger Bund \\
\hline Secteur & Transports aériens & Hôpital \\
\hline Création & $\begin{array}{l}1969 \text { (en tant } \\
\text { qu'association } \\
\text { professionnelle) }\end{array}$ & $\begin{array}{l}1947 \text { (en tant qu'association } \\
\text { professionnelle) }\end{array}$ \\
\hline $\begin{array}{l}\text { Négociations menées } \\
\text { auparavant par }\end{array}$ & $\begin{array}{l}\text { 1973-1999 : coopération } \\
\text { avec le syndicat des } \\
\text { employés (DAG) }\end{array}$ & $\begin{array}{l}\text { 2001-2006: ver.di } \\
1976-2001: \text { union de } \\
\text { négociation DAG, GGVöD } \\
1950: \text { coopération avec le } \\
\text { DAG }\end{array}$ \\
\hline $\begin{array}{l}\text { Qualification des } \\
\text { adhérents }\end{array}$ & Elevée & Très élevée \\
\hline $\begin{array}{l}\text { Homogénéité / pouvoir } \\
\text { de blocage }\end{array}$ & Très élevée & Elevée \\
\hline Nombre d'adhérents & 8200 & 110000 \\
\hline $\begin{array}{l}\text { Taux de } \\
\text { syndicalisation }\end{array}$ & Très élevé & Elevé \\
\hline $\begin{array}{l}\text { Structure } \\
\text { d'opportunité }\end{array}$ & $\begin{array}{l}\text { Privatisation de la } \\
\text { Lufthansa, création de } \\
\text { ver.di }\end{array}$ & $\begin{array}{l}\text { Privatisation du secteur } \\
\text { hospitalier, introduction de } \\
\text { la convention du service } \\
\text { public (TVöD) }\end{array}$ \\
\hline $\begin{array}{l}\text { Convention collective } \\
\text { distincte depuis }\end{array}$ & 2001 & 2006 \\
\hline
\end{tabular}

Source : présentation des auteurs. 


\section{Perspectives}

Quelle va être l'évolution ultérieure des relations sociales et des syndicats allemands ? La mise en place d'une concurrence nouvelle dans les négociations estelle un processus réversible ou irréversible ? Pour répondre à ces questions, il faut avoir à l'esprit que ces formes de concurrence sont récentes et que les acteurs en présence sont encore trop occupés à gérer leurs victoires. Il est donc impossible à l'heure actuelle de prévoir de manière claire et définitive la tendance à venir. Il ne pourra sans doute pas y avoir de retour à la situation antérieure à la constitution des nouveaux syndicats. Les deux cas présentés montrent un phénomène lisible, qui ne se résume pas à un facteur comme la professionnalité. La nouvelle concurrence entre syndicats concerne quelques domaines précis (cf. Keller, 2008b, p. 63-64). Le fait que cette évolution intervienne pour l'instant essentiellement dans le secteur autrefois public ou parapublic ne signifie cependant pas nécessairement qu'elle y restera circonscrite. D'autres catégories professionnelles et organisations, qui n'ont jusqu'à présent pas attiré l'attention dans le dialogue social, détiennent le potentiel requis, au sens des configurations présentées plus haut, pour s'établir en syndicats (par exemple dans le secteur de l'éducation/enseignement supérieur). Seule une analyse complète des organisations existantes est susceptible d'apporter un éclairage sur le nombre de concurrents possibles en matière de négociation collective.

Les conséquences juridiques et sociales de la pluralisation de la représentation syndicale des salariés dépendent de l'évolution ultérieure de cette concurrence. Mais on peut déjà constater un changement quant à la pression exercée sur le modèle allemand. La convention collective de branche - pilier essentiel de ce modèle - se trouve soumise, depuis les années 1990, à un double processus d'érosion. La couverture conventionnelle se réduit, tandis que les accords d'entreprise et d'établissement ainsi que les dérogations aux dispositions prévues dans les conventions gagnent du terrain avec la multiplication des « clauses d'ouverture ». Cette décentralisation du paysage conventionnel exerce une pression permanente sur le pouvoir de légitimation et d'influence des partenaires sociaux intervenant à un échelon plus vaste que celui de l'entreprise. La concurrence des syndicats et des conventions, étudiée ici, est un facteur supplémentaire susceptible de remettre en cause la convention collective unique et, ainsi, de participer d'une nouvelle fragmentation du paysage conventionnel (cf. Lesch, 2008b, p. 147-148 ; Bispinck, 2008, p. 7-10).

Le nombre de catégories professionnelles disposant, grâce à leur position dans le processus de travail, d'une force primaire suffisante est néanmoins limité. Convertir efficacement cette force primaire en un potentiel de blocage suffisant requiert des bases structurelles non négligeables - bases qui, à elles seules, ne suffisent toutefois pas s'il manque les opportunités permettant de se séparer des syndicats membres de la DGB. Keller (2008a, p. 171) parvient également à cette conclusion : «La force primaire ou de marché est également dis- 
ponible dans quelques autres groupes, mais la force secondaire ou organisationnelle également nécessaire n'est pas là et ne peut être établie, en tout cas à court et moyen terme ». La pluralisation limitée de la représentation syndicale des intérêts semble amenée à subsister dans quelques rares secteurs (cf. Schroeder, 2008a, p. 15). Mais le modèle du syndicat de classe (suivant le modèle posé par Streeck) restera le cœur du modèle allemand. La question suivante est plus déterminante pour les tendances à long terme : dans quelle mesure les syndicats traditionnels parviendront-ils à s'accomoder de la concurrence nouvelle ? Sera$\mathrm{t}$-il possible d'instaurer de nouvelles formes de coopération et de collaboration? Dans cette configuration, « un nouveau type de coopération en matière de dialogue social et de politique syndicale $[. .]$.$» semble «bien moins exclu que dans$ le cas de la concurrence du moins-disant social » (Bispinck/ Dribbusch, 2008, p. 162).

IL N'Y AURA PAS DE CHANGEMENT BRUTAL DE MODÈLE, du corporatisme au pluralisme. Les études de cas réalisées plaident plutôt en faveur d'un rapport nouveau, mixte, entre les idéaux-types des syndicats de classe, de métier et d'entreprise, le déplacement se faisant au détriment des syndicats de branche, unitaires. La décentralisation des conventions et les clauses d'ouverture valorisent l'échelon de l'entreprise, tandis que les nouveaux concurrents, qui pratiquent la surenchère dans les négociations, renforcent celui du syndicat professionnel.

Traduction de Marie GRAVEY

\section{Indications bibliographiques}

[S.A.], « Die Zerreißprobe », Hamburger Abendblatt, 17/05/2001, p. [n.c.]

[S.A.], « Lufthansa erzielt neuen Passagierrekord 2007 », Lufthansa Financial News, 10/01/2008, p. [n.c.]

[S.A.], « Tarifabschluss der Piloten weckt Begehrlichkeiten », Handelsblatt, 11/06/2001, p. 5

ASTHEIMER S., « Neues Arbeitnehmerselbstbewusstsein », Frankfurter Allgemeine Zeitung, 14/08/2007, p. [n.c.]

BANDELOW N. C., « Ärzteverbände - Niedergang eines Erfolgsmodells? », in WinTER T. vON, WiLLEMS U. (eds), Interessenverbände in Deutschland, VS Verlag, Wiesbaden, 2007, p. 271-293

BAYREUTHER F., « Der Arbeitskampf des Marburger Bundes - Ein Lehrstück zur Tarifeinheit im Betrieb », Neue Zeitschrift für Arbeitsrecht, vol. 23, n¹2, 2006, p. 642-646

BIEBELER H., LESCH H., «Zwischen Mitgliedererosion und Ansehensverlust - Die deutschen Gewerkschaften im Umbruch », Industrielle Beziehungen, vol. 14, n 2, 2007, p. 133-153

BISPINCK R., « Tarifpolitischer Halbjahresbericht - Eine Zwischenbilanz der Lohn- und Gehaltsrunde 2001 », WSI Mitteilungen, vol. 54, $\mathrm{n}^{\circ}$ 7, 2001, p. 419-425

BISPINCK R., « Das deutsche Tarifmodell im Umbruch », Wirtschaftsdienst, vol. 88, n 1, 2008, p. 7-11 
BISPINCK R., SCHUlten T., «Zur Kritik der wettbewerbsorientierten Tarifpolitik », in WAGNER H. (ed), Interventionen wider den Zeitgeist. Für eine emanzipatorische Gewerkschaftspolitik im 21. Jahrhundert. Helmut Schauer zum Übergang in den Un-Ruhestand, VSA, Hambourg, 2001, p. 209-225

BISPNICK R., DRIBbUSCH H., « Tarifkonkurrenz der Gewerkschaften zwischen Über- und Unterbietung. Zu aktuellen Veränderungen in der Tarif- und Gewerkschaftslandschaft », Sozialer Fortschritt, vol. 57, $\mathrm{n}^{\circ} 6$, 2008, p. 53-163

BöHLKe N., SchUlten T., « Unter Privatisierungsdruck », Mitbestimmung, vol. 54, n 6, 2008, p. 24-27

BUNDESÄRZTEKAMMER (ed), Ergebnisse der Ärztestatistik zum 31. Dezember 2006. Diagramme und Tabellen [en ligne], Bundesärztekammer, Berlin, 2007. Disponible sur : http://www.bundesaerztekammer.de/down loads/Aerztestatistik2006.pdf [consulté le 20/05/09]

Busch G., Sтамм S., «Renaissance der Standesorganisation? », Infodienst Krankenhäuser, n 34, 2006, p. $16-21$

Darnstädt T., Hammerstein K. von, Schmitt J., Tietz J., « Aufstand der Zwerge », Der Spiegel, n³3, 2007 , p. 20

DielmanN G., «Zwangsjacke Solidarität? », Infodienst Krankenhäuser, n³4, 2006, p. 13-15

Doll N., « Man will uns eliminieren », Die Welt, 13/10/2007, p. [n.c.]

EbBinghaus B., «Die Mitgliederentwicklung deutscher Gewerkschaften im historischen und internationalen Vergleich », in SChroeder W., WeßELS B. (eds), Die Gewerkschaften in Politik und Gesellschaft der Bundesrepublik Deutschland. Ein Handbuch, Westdeutscher Verlag, Wiesbaden, 2003, p. 174-203

GreINER S., «Der Arbeitskampf der GDL - Überlegungen zur Parität im Sparten- und Spezialistenarbeitskampf », Neue Zeitschrift für Arbeitsrecht, vol. 24, n 18, 2007, p. 1023-1029

HASSEL A., Gewerkschaften und sozialer Wandel. Mitgliederrekrutierung und Arbeitsbeziehungen in Deutschland und Großbritannien, Nomos, Baden-Baden, 1999

HASSEl A., « Die Erosion der gewerkschaftlichen Lobbymacht », in LEIF T., SPETH R. (eds), Die fünfte Gewalt. Lobbyismus in Deutschland, VS Verlag, Wiesbaden, 2006, p. 188-198

HASSEl A., « Gewerkschaften », in WinTER T. vON, WiLlems U. (eds), Interessenverbände in Deutschland, VS Verlag, Wiesbaden, 2007, p. 173-196

HEYDE L., «Stichwort: Verbände, wirtschafts- und sozialberufliche », in Handwörterbuch der Sozialwissenschaften, tome 10, Vandenhoeck \& Ruprecht, Göttingen, 1959, p. 623-625

Hoffmann J., SсHмidT R., « Der Streik der Lokführer-Gewerkschaft GDL. Anfang vom Ende des deutschen Systems der industriellen Beziehungen? », in Prokla, vol. 38, ${ }^{\circ}$ 151, 2008, p. 323-342

HöPNER M., Wer beherrscht die Unternehmen? Shareholder Value, Managerherrschaft und Mitbestimmung in Deutschland, Campus, Francfort s/ Main, 2003

JÜRGENS U., « Die Entwicklung von Macht, Herrschaft und Kontrolle im Betrieb als politischer Prozeß - Eine Problemskizze zur Arbeitspolitik », Leviathan, Sonderheft 5, 1983, p. 58-91

KÄDTLER J., Sozialpartnerschaft im Umbruch. Industrielle Beziehungen unter den Bedingungen von Globalisierung und Finanzkapitalismus, VSA-Verlag, Hambourg, 2006

KAZIM H., « Auch ein falsches Urteil ist immerhin ein Urteil », Spiegel online [en ligne], 08/08/2007, p. [n.c.]. Disponible sur : http://www.spiegel.de/wirtschaft/0,1518,498847,00.html [consulté le 06/07/2008]

Keller B., « Der aufhaltsame Aufstieg von Berufsverbänden. Bedingungen, Ziele, Folgen », Sozialer Fortschritt, vol. 57, $\mathrm{n}^{\circ} 6,2008 \mathrm{a}$, p. 163-173

Keller B., «Renaissance von Berufsverbänden? Bedingungen, Ziele und Folgen », in BLANK T., MÜNCH T., Schanne S., StaffHorst C. (eds), Integrierte Soziologie - Perspektiven zwischen Ökonomie und Soziologie, Praxis und Wissenschaft. Festschrift zum 70. Geburtstag von Hansjörg Weitbrecht, Hampp, Munich/Mering, 2008b, p. 51-66 
KIngDON J. W., Agendas, alternatives, and public policies, $2^{\mathrm{e}}$ édition, Pearson Education, New York, 2003

KOLF F., « LH-Piloten fordern 35 Prozent mehr », Handelsblatt, 07/02/2001, p. 19

KRIESI H., « The Political Opportunity Structure of New Social Movements - Its Impact on Their Mobilization », WZB Discussion Paper, FS III 91-103, 1991, p. [n.c.]

KRUMMHEUER E., «Unbestimmt verspätet », Handelsblatt, 02/11/2007, p. 10

LEHRER M., « Macro-varieties of Capitalism and Micro-varieties of Strategic Management in European Airlines », in Hall P. A., Soskice D. (eds), Varieties of Capitalism - The Institutional Foundation of Comparative Advantage, Oxford University Press, New York, 2001, p. 361-386

LESCH H., « Das deutsche Tarifsystem zwischen Regulierung und Fragmentierung », Wirtschaftsdienst, vol. $88, \mathrm{n}^{\circ} 1,2008 \mathrm{a}$, p. 11-15

LESCH H., « Spartengewerkschaften - Droht eine Destabilisierung des Flächentarifvertrags? », Sozialer Fortschritt, vol. 57, $\mathrm{n}^{\circ} 6,2008 \mathrm{~b}$, p. 144-153

LINDHORST E., Entwicklung durch Ärztestreiks - neue Perspektiven durch neue Tarifverträge für Ärzte? BAT, TVöD, Ärztetarif(e) und ...? [en ligne], Deutsche Gesellschaft für Unfallchirurgie, Berlin, 2007, p. [n.c.]. Disponible sur: http://www.dgu-online.de/pdf/unfallchirurgie/ausderpolitik/tarifvertragsartikel19_04_2007 .pdf [consulté le 20/05/2009]

MARBURGER BUND (ed), Unterm Strich. Informationen zu den arztspezifischen Tarifverträgen des Marburger Bundes, Marburger Bund, Heiligenstadt, 2006

MARbURGer BUND (ed), 60 Jahre Marburger Bund [en ligne], Marburger Bund, Heiligenstadt, 2008, p. [n.c.]. Disponible sur: http://www.marburger-bund.de/marburgerbund/bundesverband/verband/geschichte/ 60_jahre_mb.php [consulté le 20/05/2009]

MARTENS H., « Primäre Arbeitspolitik und Gewerkschaften im Gesundheitswesen. Der Ärztestreik 2006 als Beispiel primärer Arbeitspolitik in Zeiten tiefgreifender gesellschaftlicher Umbrüche », HBS Arbeitspapier, $\mathrm{n}^{\circ}$ 143, 2008, p. [n.c.]

MÜLlER H.-P., WILKE M., „Gestaltend Einfluss nehmen“ - Bahngewerkschaft und Bahnreform 1993-2005, Sigma, Berlin, 2006

MÜLLER-JENTSCH W., « Rückkehr der Berufsgewerkschaften? », WSI-Mitteilungen, vol.61, n 2, 2008, p. 62

NERA (ed), Comparing Physician's Earnings - Current Knowledge and Challenges. A Final Report for the Department of Health, NERA, Londres, 2004, p. [n.c.]

OFFE C., «Politische Herrschaft und Klassenstrukturen », in WIDMAIER, H.-P. (ed), Politische Ökonomie des Wohlfahrtsstaates, Athenäum-Fischer-Taschenbuch-Verlag, Francfort s. Main, 1974, p. 264-293

OLSON M., The Logic of Collective Action. Public Goods and the Theory of Groups, Harvard University Press, Cambridge/Londres, 1965

RAMGE T., «Halbgötter in Blau », Brand Eins, vol. 5, n 8, 2003, p. 88-93

SCHEWE C., « Keine Berührungsängste - Berufspolitische Gesamttagung der Vereinigung Cockpit », VC Info, $\mathrm{n}^{\circ} 11+12,2007$, p. [n.c.]

SCHмIтT H., Entstehung und Wandlungen der Zielsetzungen, der Struktur und der Wirkungen der Berufsverbände, Untersuchungen über Gruppen und Verbände, tome 6, Duncker \& Humblot, Berlin, 1966

SCHROEDER W., « Gewerkschaften auf der Suche nach Stabilität im Wandel. Eine Bestandsaufnahme », Vorgänge, vol. $44, \mathrm{n}^{\circ} 3 / 4,2005$, p. 116-127

SCHROEDER W., «Die Konkurrenten der Einheitsgewerkschaft », Mitbestimmung, vol. 54, n 4, 2008a, p. 11 15

SCHROEDER W., «SPD und Gewerkschaften: Vom Wandel einer privilegierten Partnerschaft », WSI-Mitteilungen, vol. $61, \mathrm{n}^{\circ} 5,2008 \mathrm{~b}$, p. 231-237 
SChroeder W., Silvia S. J., « Gewerkschaften und Arbeitgeberverbände », in SCHROEDER W., WeSSELS B. (eds), Die Gewerkschaften in Politik und Gesellschaft der Bundesrepublik Deutschland. Ein Handbuch, Westdeutscher Verlag, Wiesbaden, 2003, p. 244-270

SChroeder W., KalaSs V., Greef S., Kleine Gewerkschaften und Berufsverbände im Wandel, Fondation Hans Böckler, Düsseldorf, 2008 (Böckler Forschungsmonitoring, 3)

SCHWARZKOPF-STEINHAUSER G., «Vdää zum Tarifabschluss zwischen VKA und MB », Infodienst Krankenhäuser, $\mathrm{n}^{\circ} 34,2006$, p. 11

SEIFERT K.-D., Der deutsche Luftverkehr 1955-2000 - Weltverkehr, Liberalisierung, Globalisierung, Die deutsche Luftfahrt, Bernard \& Graefe, Bonn, 2001

SIMON M., Das Krankenhaus im Umbruch. Neuere Entwicklungen in der stationären Krankenversorgung im Gefolge von sektoraler Budgetierung und neuem Entgeltsystem, Wissenschaftszentrum Berlin für Sozialforschung, Berlin, 1997

SPENGLER H., «Einkommen und Arbeitszeiten junger Klinikärzte in Deutschland », Wochenbericht des DIW Berlin, vol. 72, ${ }^{\circ} 34,2005$, p. 489-494

STEIGER H., « 'Wir wollen Ingenieuren nichts vorschreiben' », VDI-Nachrichten, nº, 2008, p. 4

STREECK W., «Klasse, Beruf, Unternehmen, Distrikt - Organisationsgrundlagen industrieller Beziehungen im europäischen Binnenmarkt », in STRüMPEL B., DIERKES M. (eds), Innovation und Beharrung in der Arbeitspolitik, Schäffer-Poeschel, Stuttgart, 1993, p. 39-68

STREECK W., «Gewerkschaften in Westeuropa », in SChroeder W., WeßEls B. (eds), Die Gewerkschaften in Politik und Gesellschaft der Bundesrepublik Deutschland. Ein Handbuch, Westdeutscher Verlag, Wiesbaden, 2003, p. 86-100

Streeck W., Seglow P., Wallace P., «Competition and Monopoly in Interest Representation - A Comparative Analysis of Trade Union Structure in the Railway Industrie of Great Britain and West Germany », Organization Studies, vol. 2, ${ }^{\circ}$ 4, 1981, p. 307-330

STURM T. vON, «Vereinigung Cockpit - 1 Jahr eigenständige Tarifpartei », VC-Info, ${ }^{\circ}$ 9+10, 2000, p. [n.c.]

TARP M., « Tarifpolitik der VC am Scheideweg? », VC Info, n¹1+12, 2007, p. [n.c.]

TöDTMann C., «Die Vertreibung der Ärzte ins Paradies », Handelsblatt, 18/03/2005, p. 1

TraXLER F., « Gewerkschaften und Arbeitgeberverbände. Probleme der Verbandsbildung und Interessenvereinheitlichung », in MüLLER-JENTSCH W. (ed), Konfliktpartnerschaft: Akteure und Institutionen der industriellen Beziehungen, Hampp, Munich, 1999, p. 57-77

WEISEMANN U., «Auflösung der Flächentarifverträge und des Industrieverbandsprinzips? - Streikrecht im Umbruch », Deutsche Zeitschrift für Wirtschafts- und Insolvenzrecht, vol. 17, ${ }^{\circ}$ 11, 2007, p. 441-445

WÜRFEL T., « Der Organisationsgrad als Pulsmesser einer Gewerkschaft », VC-Info, n 5+6, 2001, p. [n.c.]. 


\title{
Les relations sociales à la Deutsche Bahn : nouveaux acteurs et évolution des rapports de force sur fond de privatisation et de libéralisation
}

\author{
Samuel GREEF, Viktoria KaLASS
}

Après un exposé précis du contexte et du contenu de la réforme ferroviaire allemande de 1994, les auteurs mettent en évidence les effets de l'ouverture à la concurrence du secteur sur la structure de l'emploi. Ils abordent également les défis que les syndicats ont dû relever par la suite, dans le cadre desquels l'organisation des conducteurs de locomotives (GDL) est parvenue à devenir un partenaire social à part entière.

«Chez les syndicats de la DGB [confédération allemande, S.G./V.K.], la tendance est à la discrétion. (...) Il n'en va pas différemment à la Deutsche Bahn. Depuis la réforme ferroviaire de 1994, c'est Transnet [le plus important syndicat, unitaire, du secteur, S.G./V.K.] qui a géré l'entreprise conjointement avec la direction, et s'est penché ces dernières années en particulier sur le projet d'entrée en bourse. Le rejet de ce projet à la fois par ses propres adhérents et par la DGB n'intéressait pas Transnet.

Nous, GDL [syndicat des conducteurs de locomotives, S.G./V.K.], avons rompu avec cette logique de défensive. En 2003, pour la première fois puis, de manière très fructueuse, en 2007. Le fait qui nous est reproché par Transnet, d'être contrairement à eux un syndicat catégoriel, a été notre force. »

(Schell, 2009, p. 212-213)

C'est sur cette affirmation que Manfred Schell, membre du syndicat des conducteurs de locomotives GDL pendant près de 40 ans et président fédéral de ce même syndicat pendant 19 ans, conclut son autobiographie, parue en février 2009. Ces mots de conclusion font référence à des changements fondamentaux dans le secteur ferroviaire allemand, et à des dissensions entre les syndicats du secteur sur la manière de faire face à cette évolution. Des désaccords existent sur la manière de mener une politique syndicale efficace et sur ce qui peut être considéré comme socialement juste. A l'automne 2007, le syndicat de Schell a réussi, après plusieurs années de conflits à la fois au sein du camp syndical et avec l'employeur, la Deutsche Bahn (DB), à s'établir comme acteur autonome dans le secteur ferroviaire. Ce fut ainsi la fin du monopole du gros syndicat unitaire, Transnet, fort d'un grand nombre d'adhérents, en matière de négociations. Mais les évolutions survenues dans les rangs des syndicats du rail soulevaient aussi la question, pour l'employeur, de la manière de gérer cette concurrence nouvelle et des moyens d'empêcher que la situation ne s'envenime davantage avec une concurrence tarifaire au sein de l'entreprise. 
Cette situation nouvelle, qui requérait de la part des acteurs en présence de s'écarter des actions de routine bien rôdées, intervient - et les propos de Schell le font apparaître - sur fond de privatisation de la DB et de libéralisation du marché ferroviaire allemand. C'est pourquoi nous allons présenter plus en détail la réforme introduite en 1994 et les conséquences qui en découlent pour les relations sociales dans le groupe ferroviaire. Nous commencerons par résumer les étapes de la réforme déjà réalisées, avant d'étudier les répercussions de cette réforme en termes de conditions d'emploi à la Deutsche Bahn. Puis nous expliquerons les grands changements survenus dans le camp des syndicats du rail pour répondre, avec des stratégies diverses, à l'annonce de la privatisation et de la libéralisation des transports ferroviaires. Nous souhaitons en particulier nous demander pourquoi le syndicat des conducteurs de locomotives a réussi, dans cette configuration d'évènements, d'évolutions et de stratégies de 2007-2008 précisément, à imposer une convention collective autonome pour les conducteurs. Le GDL est à ce jour la dernière organisation à avoir réussi à s'établir, avec une convention séparée, en syndicat professionnel autonome ${ }^{1}$. Le secteur ferroviaire est ainsi l'un des rares domaines à avoir été confrontés à une réorganisation du paysage syndical à la suite de la dérégulation. Les anciens acteurs, qui mettaient autrefois l'accent sur le métier, font valoir une exigence de représentation autonome de leur clientèle dans les négociations, faisant ainsi concurrence aux grands syndicats établis et réunis au sein de la DGB.

\section{La DB avant la privatisation}

L'histoire de la Deutsche Bahn est essentielle si l'on s'intéresse à l'évolution des relations sociales au cours des dernières années. Il faut souligner deux aspects. D'une part, la Deutsche Bundesbahn, entreprise d'Etat, était très fortement tournée vers le marché intérieur allemand. Mais elle n'était pas une simple entreprise de transports : si la Lufthansa avait le caractère d' "ambassadeur politique, économique et culturel » à l'étranger (Ramge, 2003, p. 90), la Bundesbahn était un symbole de statut en Allemagne même. Après la Seconde Guerre mondiale, l'entreprise est devenue un élément majeur de la reconstruction allemande. La Bundesbahn fut utilisée comme moyen de transport du charbon et de l'acier, lesquels constituaient la base de l'économie de la RFA d'après-guerre. C'est elle qui évacua les décombres des villes détruites; elle contribua aussi à organiser le retour des prisonniers de guerre allemands (mobil 2009, p. 50). Puis, la montée des transports motorisés individuels dans les années 1960 initia une période de déclin continu du rail par rapport à la route, si bien que la Bundesbahn accumula peu à peu des dettes (van Riesen, 2007, p. 43). L'entreprise

\footnotetext{
${ }^{1}$ Les précurseurs ont été l'association des pilotes Cockpit (VC) en 2001, l'organisation indépendante des agents de bord (UFO) en 2002, le syndicat des contrôleurs aériens (GdF) en 2003, le syndicat des médecins Marburger Bund (MB) en 2006 et la fédération des assistants médicaux (VMF) en 2007 (cf. Schroeder dans ce volume ; Schroeder/Greef, 2008 ; Lesch, 2008, p. 307 ; Keller, 2008a ; Müller/Wilke, 2008b).
} 
étant un établissement public dépourvu de personnalité morale, ses déficits ne se répercutèrent pas directement sur le budget de la République fédérale, mais s'ajoutèrent, en une sorte de budget annexe, aux finances fédérales (Müller/Wilke, 2006, p. 23). Les acteurs politiques savaient qu'une réforme de la Bundesbahn était urgente avant même le bon mot du chancelier Helmut Schmidt, selon lequel la République fédérale ne pouvait se permettre qu'une chose : soit une Bundeswehr, soit une Bundesbahn. Mais cette conscience générale de la nécessité d'une réforme ne déboucha pas automatiquement sur des avancées concrètes. La Bundesbahn devint le jouet d'intérêts politiques opposés, à la fois entre les partis et entre les différents échelons du système politique. Les élus régionaux et communaux se battirent avec véhémence, par crainte de perdre des électeurs, pour préserver « leur » réseau ferré. Chaque village devait si possible être raccordé au réseau, et il fallait, dans le souci de la mission d'intérêt général de l'Etat, maintenir un trafic régulier y compris sur les lignes non rentables. Les dettes colossales de la Bundesbahn s'accrurent par conséquent. Dans les années 1960, les déficits annuels, d'un milliard à un milliard et demi de DM, furent comblés encore et encore par les finances fédérales. En 1977, l'aide issue du budget fédéral se chiffrait à 10 milliards de DM. La dette totale de la Bundesbahn continuait cependant de s'accroître : à la fin des années 1970, elle dépassait la barre des 30 milliards de DM, et monta à 44 milliards jusqu'en 1989 (Müller/Wilke, 2006, p. 23-24 ; mobil 2009, p. 54). La stratégie rigoureuse de «dégraissage »- suppression de lignes et retrait territorial de la Bundesbahn - n'eut pas les effets escomptés, notamment en raison de la victoire de plus en plus nette de la route sur le rail : entre 1949 et 1989, la part du rail dans le fret de marchandises tomba de 56 à $22 \%$. Dans le même temps, le réseau de lignes de la Bundesbahn fut réduit de $10 \%$ (de $30400 \mathrm{~km}$ à $27000 \mathrm{~km}$ ). A cette réduction du réseau ferré, associée aux progrès techniques, correspondirent les premières suppressions de postes, plus de la moitié des emplois ayant été détruits entre 1979 et 1990 (1949: 540000 emplois ; 1960 : 498000 ; 1990 : 249 000) (Müller/Wilke, 2006, p. 23-24 ; van Riesen, 2007, p. 45 ; voir également graphique 2).

La culture de l'emploi au sein du groupe ferroviaire est un deuxième aspect important. On constate d'abord chez les personnels un haut niveau d'identification à l'entreprise : ils se voient comme des cheminots. Cette identification collective crée d'une part la base d'un fort sentiment d'appartenance parmi les salariés, et a permis à Transnet (sous le nom de GdED, syndicat des cheminots d'Allemagne, avant 2000) de regrouper les personnels du rail, ouvriers, employés et fonctionnaires, dans un syndicat d'entreprise unitaire, intercatégoriel (Müller/Wilke, 2006, p. 13). L'attachement des personnels à leur statut est un autre aspect qui caractérise la culture de l'emploi de la Bundesbahn. La Bundesbahn, en tant qu'administration fédérale, tenant à la loyauté de ses collaborateurs, leur accorda le statut de fonctionnaires. Ainsi, même les activités les plus rudimentaires étaient effectuées par des fonctionnaires (ibid., p. 24). Ce 
qui assurait à la Bundesbahn la loyauté de ses salariés, impliquait en retour des dépenses de personnel élevées, qui devaient être compensées par l'Etat. Il s'établit entre la Bundesbahn et ses personnels une relation combinant loyauté des serviteurs de l'Etat d'une part et obligation d'assistance de l'employeur d'autre part. Le haut niveau de discipline et de fiabilité qui en résultait dans le travail des salariés étayait par ailleurs leur fort sentiment d'appartenance. Ces deux points de départ, le potentiel de politisation de la Bundesbahn et la charge financière représentée pour le budget de l'Etat, ainsi que l'incapacité des acteurs politiques à s'entendre pendant des années sur le cap à donner ensemble aux réformes d'une part et la conscience particulière des «cheminots » comme catégorie de salariés d'autre part sont importants pour l'évolution ultérieure.

\section{La césure de l'Unité allemande}

La réunification allemande constitue une césure importante dans l'histoire des chemins de fer allemands. L'unité politique des deux Etats fut suivie de la fusion de la Deutsche Bundesbahn et de la Deutsche Reichsbahn, en charge jusque-là du trafic ferroviaire en RDA. Ce projet de fusion rendait une réforme du secteur encore plus urgente. Au moment de la réunification, les infrastructures de la Reichsbahn, en particulier le réseau et les trains, étaient pour une grande partie dans un état de désolation important (Müller/Wilke, 2006, p. 25). La RDA n'avait ni arrêté les lignes non rentables ni assuré le maintien et le développement du réseau en réalisant les investissements nécessaires. En 1975, le train restait le moyen de transport numéro un de l'Etat est-allemand, alors qu'en RFA l'automobile et les transports routiers avaient depuis longtemps supplanté le rail. Mais la place remarquable du rail en RDA était le résultat d'orientations de politique économique. Au-delà de 50 kilomètres en effet, le fret devait obligatoirement être transporté par rail, jusqu'en 1989. De plus, l'offre de véhicules privés était toujours en retard sur la demande (mobil 2009, p. 51). Au moment de l'unification, 253000 employés de la Reichsbahn - contre 236000 salariés de la Bundesbahn - géraient un réseau exactement deux fois plus petit que celui de la République fédérale (Müller/Wilke, 2006 , p. 25). Les suppressions de postes et l'arrêt de certaines lignes étaient inévitables pour porter la Reichsbahn au niveau de la Bundesbahn, y compris d'un point de vue technique. Les deux compagnies réunissant en 1993 plus de 15 milliards de marks de dettes, la voie de la réforme était ouverte (mobil 2009, p. 53) $)^{2}$.

\section{Directives européennes d'harmonisation}

En décembre 1993, la réforme fut lancée, avec les voix du Bundesrat et du Bundestag. Mais une modification de la constitution était nécessaire, la Loi

\footnotetext{
${ }^{2}$ Endettement nouveau de la Deutsche Bundesbahn en 1993 : 9,4 milliards DM ; endettement nouveau de la Deutsche Reichsbahn en $1993: 6,1$ milliards DM.
} 
fondamentale définissant à l'art. 87 alinéa 1 l'exploitation des chemins de fer fédéraux comme institution publique, donc comme administration fédérale. Sur la base de cet article, combiné à l'article 33 alinéas 4 et 5 , la Bundesbahn relevait ainsi du champ d'application du droit public, ce qui obligeait à garantir que son exploitation fût assurée par des fonctionnaires de métier (Müller/Wilke, 2006, p. 37). Mais, du fait des obligations européennes en matière de concurrence, la gestion économique des chemins de fer ne pouvait rester aux mains de fonctionnaires. Dans le sillage de la création d'un marché intérieur commun, le législateur européen visait au début des années 1990 une harmonisation des marchés nationaux de transports afin d'instaurer - suivant les principes de l'économie de marché - une concurrence entre les opérateurs du secteur (ibid., p. 31-32). La directive européenne comportait essentiellement quatre volets (ibid., p. 33-34) ${ }^{3}$ :

- l'indépendance de la direction des entreprises ferroviaires vis-à-vis de l'influence des Etats ;

- le financement autonome, indépendant du budget de l'Etat ;

- la séparation, au moins d'un point de vue organisationnel, du réseau et de l'exploitation, permettant une comptabilité séparée des deux activités afin d'empêcher tout transfert de subventions ;

- la création d'un accès non discriminatoire au réseau, devant être assuré à l'avenir aux compagnies ferroviaires étrangères aussi.

En 1991 également, le Conseil européen actualisa le droit relatif aux services d'intérêt général (SIG) dans le secteur des transports ${ }^{4}$. Les entreprises se voyaient ainsi retirer la responsabilité des SIG, désormais déléguée à l'Etat, donneur d'ordre public. Les missions d'intérêt général devaient à l'avenir être confiées à des opérateurs de transports, et ce, dans le cadre de procédures de mise en concurrence, afin que les aides publiques puissent désormais être attribuées selon les principes de l'économie de marché et que ces missions ne soient qu'exceptionnellement imposées. La directive 91/440/CEE et le règlement 1893/91 ont été les premières étapes importantes sur la voie de la création d'un marché commun européen des transports ferroviaires. Mais ce projet n'est pas encore achevé (Müller/Wilke, 2006, p. 34 sq. ; van Riesen, 2007, p. 78-79).

\section{La privatisation des chemins de fer allemands}

Dans un premier temps, la compagnie nationale a été transformée en établissement de droit public. Elle se voyait ainsi conférer une première autonomie d'un point de vue économique et comptable, et fut soumise à une privatisation formelle (Müller/Wilke, 2006, p. 38 ; van Riesen, 2007, p. 84-85), au plan légal

\footnotetext{
${ }^{3}$ La base juridique est la directive 91/440/CEE.

${ }^{4}$ Règlement (CEE) $n^{\circ} 1893 / 91$.
} 
et financier. La Deutsche Bahn, structure de droit privé, reste toutefois détenue à $100 \%$ par la puissance publique, son financement émanant désormais à la fois de moyens publics et d'investisseurs privés (Engartner, 2008, p. 90-91). Le réseau et l'exploitation restent conjoints, notamment car l'opposition virulente des syndicats et du SPD a rendu impossible une séparation institutionnelle complète (van Riesen, 2007, p. 96). Le concept essentiel ici est celui de groupe intégré, consistant à laisser officiellement la responsabilité de l'exploitation du réseau à l'Etat fédéral, alors qu'en pratique c'est la Deutsche Bahn qui l'assure. Même dans le cas d'une privatisation matérielle complète, initialement prévue pour 2008, puis repoussée pour une durée indéterminée en raison des conséquences de la crise économique mondiale et du changement de climat politique $^{5}, l^{\prime}$ Etat fédéral resterait actionnaire majoritaire, seuls $49,9 \%$ au maximum de l'entreprise pouvant être cédés en bourse.

Deux ans après la création en janvier 1994 de la Deutsche Bahn Aktiengesellschaft (DB AG), qui réunissait les deux anciennes entreprises d'Etat Bundesbahn et Reichsbahn, la grande étape suivante fut la réforme des chemins de fer : la régionalisation des transports de proximité. Depuis 1996, les Länder sont en charge de la gestion des transports de voyageurs de proximité et reçoivent pour assumer cette mission des aides financières de l'Etat fédéral, calculées et attribuées suivant une formule de répartition fixe (Müller/Wilke, 2006, p. 59-60). Avant l'adoption de la loi de régionalisation, cette question a suscité d'importants conflits politiques. Les Länder, dont l'accord de principe sur cette réforme était indispensable pour obtenir la modification de la Loi fondamentale, sont parvenus à augmenter l'enveloppe initialement prévue pour la régionalisation et à inscrire les transports de voyageurs grandes lignes et l'infrastructure à la charge de l'Etat fédéral (Van Riesen, 2007, p. 97 ; Lehmkuhl, 1996, p. 81 sq.).

Trois ans plus tard suivit une réorganisation de plus grande ampleur, la Deutsche Bahn AG étant divisée en cinq filiales indépendantes pour les transports régionaux de voyageurs, les transports de voyageurs grandes lignes, le fret, le réseau et les gares, tous ces domaines d'activités demeurant sous la responsabilité de la holding DB.

Alors que les entreprises opéraient au départ avec une large indépendance et que la holding était une structure de regroupement peu contraignante, l'arrivée de Hartmut Mehdorn au poste de président du conseil d'administration - et la mention de synergies perdues en cas de séparation trop forte des entreprises entraîna un retour à la centralisation et un renforcement de la holding, qui n'alla toutefois pas jusqu'à la remise en cause de la séparation de la DB AG en filiales.

\footnotetext{
${ }^{5}$ Tandis qu'en 2009, le ministre fédéral des Transports Tiefensee excluait officiellement une entrée en bourse avant 2013, en raison de la crise économique mondiale et de la perte de valeur consécutive pour l'entreprise (Tagesschau, 14/03/2009), les experts supposaient que la discussion autour de la privatisation reprendrait son cours après les élections au Bundestag de 2009.
} 
D'un point de vue économique, on peut qualifier cette transformation du groupe de réussite, même si le budget de l'Etat ne put être significativement déchargé à cause des moyens consacrés à la régionalisation et des aides versées par l'Etat fédéral pour le maintien et le développement des infrastructures ; ce changement permit au moins de stabiliser les dépenses et d'empêcher l'explosion des coûts qui menaçait à la veille de la réforme (van Riesen, 2007, p. 147). L'entreprise Deutsche Bahn AG enregistre toutefois des bénéfices depuis 2003, avec en 2008 un bénéfice avant intérêts et impôts de 2,5 milliards $€$ environ. Dans le même temps, le chiffre d'affaires de la Deutsche Bahn a plus que doublé, passant de 15 à 33 milliards $€$ (voir grahique 1$)^{6}$.

\section{Graphique 1 : Evolution du chiffre d'affaires et des bénéfices du groupe DB}

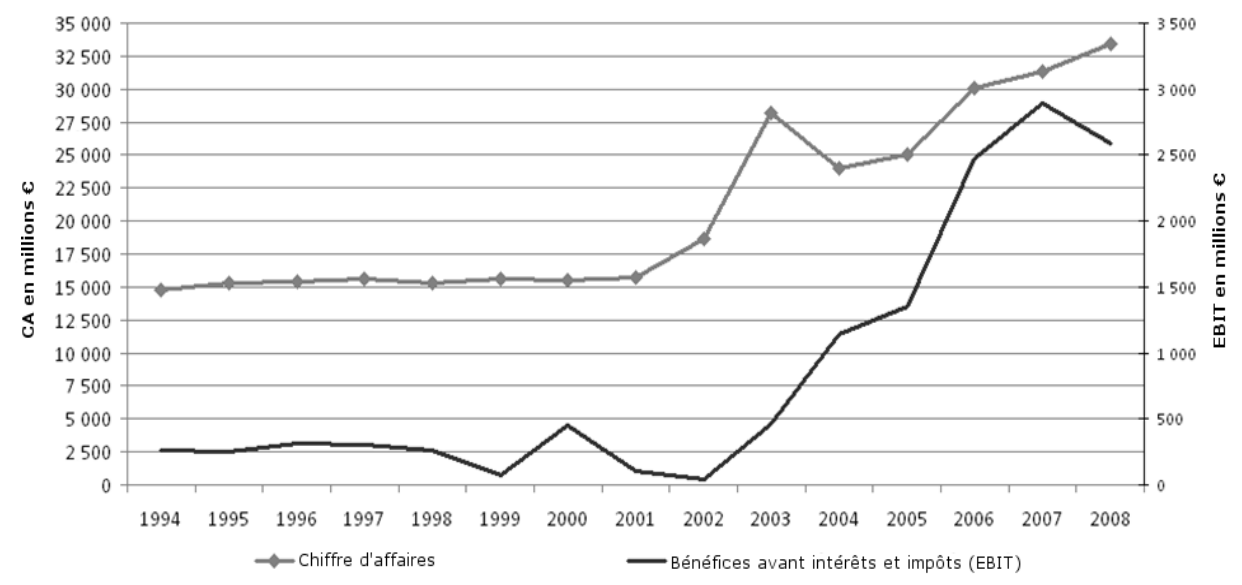

Source : rapports d'activité de la DB, 2005-2008.

\section{Modification de la structure d'emploi à la suite de la réforme}

L'évolution économique positive du groupe entraîna des changements considérables pour le personnel de l'entreprise. La fusion de la Reichsbahn et de la Bundesbahn, doublée du passage de la compagnie publique de chemins de fer à une forme juridique de droit privé, mit un terme aux recrutements sous statut de

\footnotetext{
${ }^{6}$ Le léger recul des bénéfices en 2008 révèle que Mehdorn a quitté la DB au sommet de sa progression. Outre la forte augmentation des coûts matériels (qui ont triplé depuis 2001, tandis que les coûts de personnel n'ont été multipliés que par 1,5), la crise économique mondiale aura des conséquences négatives pour l'entreprise, en raison notamment de la stratégie exportatrice de Mehdorn. Dernièrement, $58 \%$ du chiffre d'affaires revenait au transport de fret, qui sera le segment le plus durement affecté par la crise. Par ailleurs, la concurrence va continuer de s'accroître également dans les transports de proximité. Durant l'exercice 2008, la part des prestations de transport ferroviaire de proximité réalisées par des opérateurs n'appartenant pas à la DB est grimpée à 10,1\%. Jusqu'à présent, il s'agit ici notamment d'opérateurs communaux, qui constituent $53 \%$ de la concurrence. Avec $17 \%$ de parts de marché, les grands groupes internationaux se classent en revanche derrière les entreprises allemandes du Mittelstand, qui totalisent 21\% (Rapport d'activité de la DB, Geschäftsbericht 2008, p. 53).
} 
fonctionnaire. Les fonctionnaires de la Bundesbahn furent repris, mais les nouveaux personnels ainsi que ceux de la Reichsbahn se voyaient désormais appliquer le statut d'employé. Du point de vue de l'entreprise, ce changement mettait fin aux coûts exorbitants de gestion des ressources humaines du passé ; pour les personnels, les garanties d'emploi qui jadis allaient pratiquement de soi étaient supprimées. Du point de vue des syndicats, ce changement impliquait une capacité de faire grève renforcée : alors que le droit de grève est refusé aux fonctionnaires en raison de l'obligation de loyauté à l'égard de l'Etat, les employés peuvent imposer leurs intérêts dans les conflits avec celui-ci, si besoin en ayant recours à la grève et à des actions de lutte sociale.

En outre, la restructuration des chemins de fer à la suite de la réforme s'accompagnait de suppressions d'emplois massives. Dans l'Est de l'Allemagne en particulier, de nombreux emplois furent supprimés au cours de la première moitié des années 1990. De 1990 à 1994, le nombre de personnels passa, en quatre années seulement, de 460000 à 340 000, soit une réduction de $25 \%$. De même, dans les années qui suivirent, le nombre de postes ne cessa de diminuer (voir graphique 2).

Graphique 2 : Emplois dans les chemins de fer (jusqu'en 1994, Bundesbahn + Reichsbahn, état : fin d'année ; à partir de 1995, DB AG, état : moyenne annuelle)

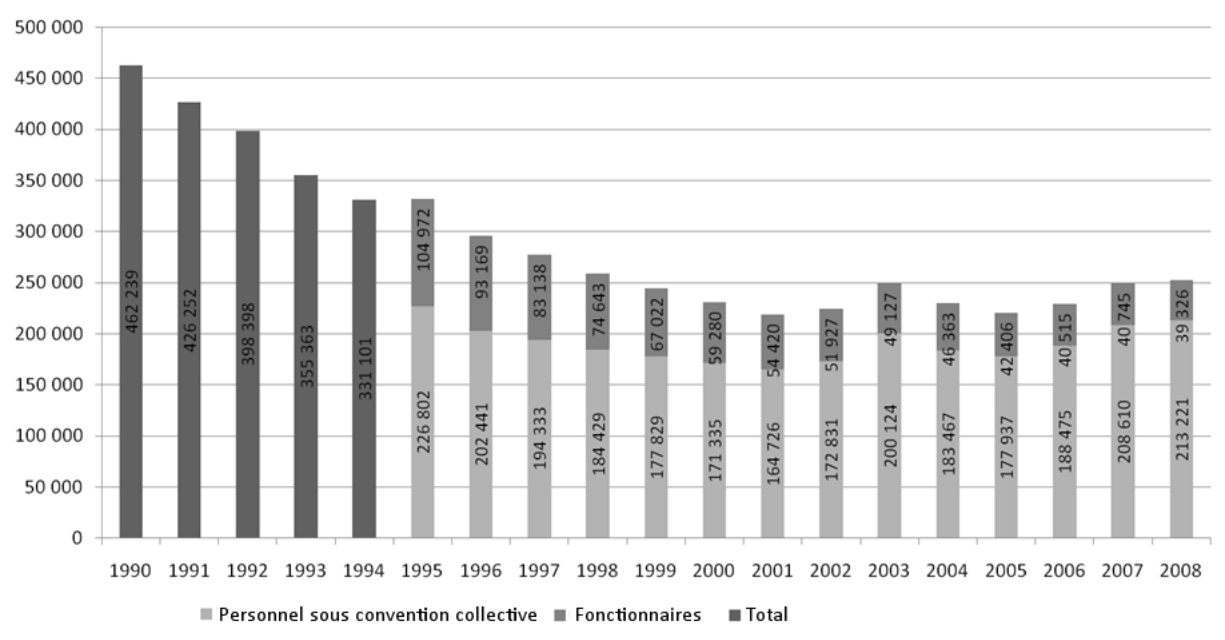

Sources : Müller/Wilke, 2006, p. 157 ; rapports d'activité de la DB, 1996-2008.

Alors que l'évolution des effectifs en 2003 et 2007 donne au premier abord l'impression d'une augmentation du personnel, ces hausses s'expliquent par les acquisitions d'autres entreprises de transports et de logistique en Allemagne et à l'étranger. Il faut signaler notamment l'intégration de Stinnes AG (2003) au groupe DB ou l'acquisition de l'entreprise américaine BAX Global par DB AG (2007). Mais si l'on se base exclusivement sur les effectifs de DB AG dans le 
secteur ferroviaire, l'ampleur de la politique de réduction de postes apparaitt clairement (voir graphique 3).

\section{Graphique 3 : Evolution des effectifs de DB AG dans le secteur ferroviaire (personnels des compagnies d'autocars DB inclus)}

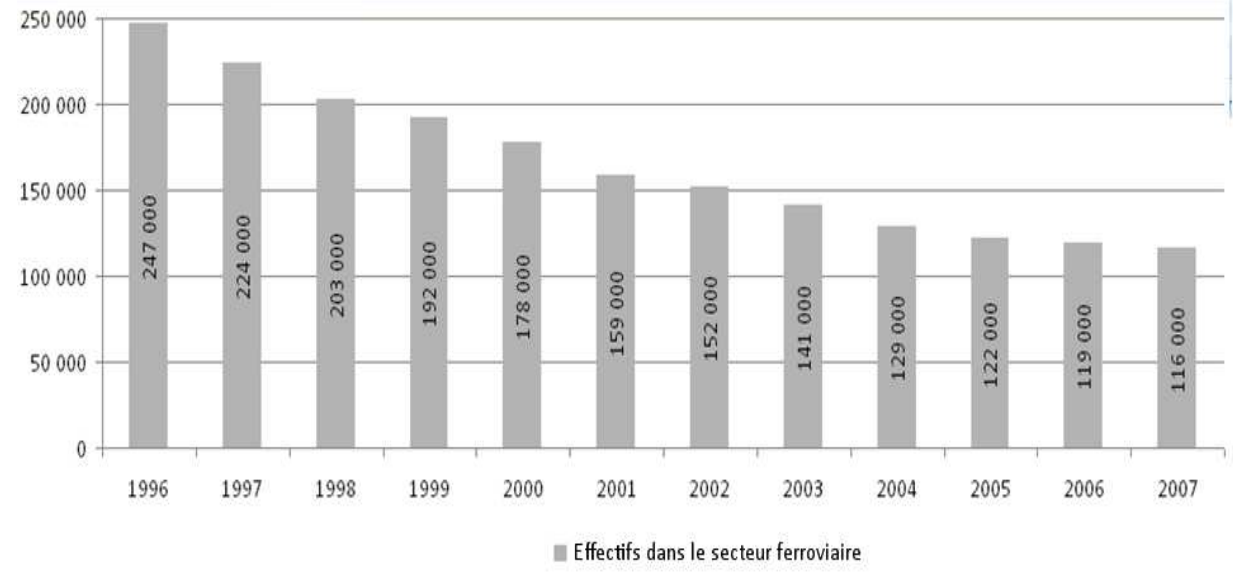

Source : DB AG; données mises à disposition par l'Agence fédérale des réseaux (BNetzA).

Malgré cette réduction nette des effectifs, les suppressions de postes ont été réalisées sans licenciements. Le gros syndicat unitaire du secteur, Transnet, et la direction de l'entreprise pesèrent ensemble pour éviter de telles mesures. En contrepartie de cette politique et de la préservation du marché de l'emploi interne au groupe dans le cadre de l'entreprise intégrée, Transnet renonça à s'opposer fondamentalement à la privatisation et choisit au contraire de coopérer au processus de réforme (Müller/Wilke, 2006, p. 11 sq.).

\section{Relations sociales après la réforme : les syndicats face à de nouveaux défis}

L'évolution des relations sociales au sein de la DB est fortement marquée par les acteurs qui y sont représentés. On trouve, d'une part, un employeur fort, dont les intérêts sont représentés depuis 2002 par $\mathrm{Agv} \mathrm{MoVe}^{7}$ (fédération d'employeurs des prestataires de services de mobilité et de transports). Du côté des personnels, on trouve les trois syndicats Transnet (anciennement GdED), GBDA (syndicat des fonctionnaires allemands du rail et affiliés) et le syndicat

\footnotetext{
${ }^{7} \mathrm{Au}$ moment de sa création, Agv MoVe rassemblait 21 entreprises membres, qui comptaient parmi les groupes d'entreprises de la Deutsche Bahn AG. Bien que leur nombre ait considérablement augmenté entre 2002 et 2009, le lien avec la holding DB est resté fort : sur les 70 entreprises membres, 67 sont des filiales de la Deutsche Bahn AG. Quand elles sont organisées au sein d'une fédération, les entreprises de transport ferroviaire non détenues par l'Etat fédéral relèvent pour leur part de la fédération d'employeurs de la concurrence AGVDE (fédération d'employeurs des chemins de fer allemands). Ces deux organisations appartiennent à la Fédération des employeurs allemands BdA.
} 
des conducteurs allemands de locomotives (GDL). La juxtaposition de ces trois organisations de salariés n'est pas une nouveauté dans le secteur ferroviaire, mais c'est plutôt une exception dans les relations industrielles allemandes en général. Alors que la pluralité syndicale dans le secteur s'est installée avec le temps, la concurrence entre les trois syndicats, longtemps latente, est devenue manifeste ces dernières années. Nous allons maintenant présenter ces trois organisations, leurs objectifs et convictions fondamentales ainsi que les stratégies qui en résultent.

\section{Transnet}

Transnet est le plus gros syndicat dans le secteur ferroviaire. Cette organisation a succédé à l'ancien GdED (syndicat des cheminots d'Allemagne) et est, comme lui, structurée en syndicat unitaire du secteur ferroviaire. Tous les personnels de la Bundesbahn pouvaient adhérer au GdED, indépendamment de leur statut professionnel. Ce syndicat faisait partie, comme Transnet aujourd'hui, de la confédération DGB. Pour la majorité des salariés de la Deutsche Bundesbahn, l'adhésion au GdED allait pratiquement de soi : au début des années 1970, le taux de syndicalisation était de plus de $70 \%$. Les personnels de la Bundesbahn étaient soudés par une forte identification à l'entreprise et une grande solidarité de groupe. Les personnels se considéraient comme membres de la Bundesbahn. Ce sens de la cohésion et de la tradition était le résultat non seulement de l'appartenance à l'entreprise mais aussi de l'adhésion au syndicat des cheminots GdED (Müller/Wilke, 2006, p. 122).

Le champ d'action de ce syndicat étant à l'origine limité à la Bundesbahn, qui n'avait de concurrents que sur quelques rares lignes régionales, le GdED a toujours présenté les caractéristiques d'un syndicat d'entreprise. Son organisation ressemblait aux structures de la Deutsche Bundesbahn. L'un et l'autre avaient leur centrale à Francfort; les hauts fonctionnaires du syndicat faisaient depuis toujours partie de la direction de l'entreprise et siégeaient en permanence au conseil d'administration de la Bundesbahn. Comme la majorité des personnels de l'entreprise et donc une grande partie des adhérents du GdED étaient fonctionnaires, le syndicat n'avait qu'un pouvoir de grève très limité et privilégiait les moyens pacifiques pour parvenir à ses fins (ibid., p. 123). L'identification des cheminots à leur entreprise à travers leur emploi allait dans le sens de la politique du GdED, qui tendait largement à la loyauté envers la DB. En raison du lien organisationnel avec l'entreprise et de la présence de ses membres dans les organes de décision de la Bundesbahn, le syndicat était en outre en situation de peser sur les décisions.

Sur la question de la réforme des chemins de fer allemands, le GdED rejeta le principe d'une stratégie de blocage. Il espérait au contraire, en participant de manière constructive au processus de réforme, pouvoir limiter les conséquences sociales de la privatisation de l'entreprise. La décision de ne pas s'opposer fon- 
damentalement au processus de réforme n'obéissait pas seulement à des traditions historiques et à des convictions spécifiques, elle résultait aussi des expériences faites par le syndicat allemand des postes (DPG). Celui-ci s'était opposé aux exigences de privatisation de l'Etat fédéral, espérant pouvoir empêcher, par une pression parlementaire et extraparlementaire, le transfert de la Deutsche Post au secteur privé.

Or, ce n'est pas ce qui se produisit, et le DPG non seulement dut finalement accepter la privatisation de l'entreprise, mais il se retrouva devant le fait accompli. Par son rejet de principe de la privatisation, le DPG avait ainsi compromis toute possibilité de faire valoir l'intérêt des personnels dans les décisions de fond de la réforme de la Poste (Müller/Wilke, 2006, p. 11). Le GdED, ne voulant pas répéter les erreurs du syndicat des postes, donna au contraire son accord de principe à la privatisation et tenta d'obtenir, en contrepartie de son attitude coopérative, que l'Etat fédéral, propriétaire des chemins de fer, s'engage à prendre en compte les aspects sociaux de la réforme. Malgré les suppressions d'emplois massives qui suivirent la privatisation, le GdED obtint, grâce à son attitude participative, des réductions d'effectifs sans licenciements. L'exigence fondamentale du syndicat concernait la non-séparation du réseau et de l'exploitation des chemins de fer, le maintien d'un groupe intégré et, partant, la préservation d'un marché de l'emploi à l'intérieur du groupe, ce qui devait offrir la possibilité, en cas de suppression de postes dans un domaine d'activité, de rester salarié dans une autre partie du groupe.

De même que l'entreprise se restructurait pour passer, dans le sillage de la réforme, d'une orientation sur le marché allemand à un positionnement d'acteur global axé sur les transports internationaux en général, le GdED revit lui aussi sa politique de recrutement. Afin de pouvoir continuer à regrouper tous les personnels de l'entreprise Deutsche Bahn AG dans un même syndicat, le champ d'action fut étendu au domaine des transports.

Cette ouverture et, par conséquent, l'évolution vers un syndicat du secteur des transports, apparaissent symboliquement dans le nouveau nom du syndicat des cheminots, rebaptisé syndicat des transports, services et réseaux (Transnet) (ibid., p. 137 sq.). En matière de négociation collective, Transnet coopère depuis 2002 avec le GDBA, qui regroupait des fonctionnaires de la Bundesbahn et affiliés et était jadis intégré aux structures de la dbb (fédération des fonctionnaires allemands), passant ainsi outre aux subdivisions qui structuraient traditionnellement le paysage syndical dans le secteur ferroviaire. Cette orientation nouvelle ne fait pas l'unanimité au sein de la DGB.

Comme les syndicats membres de la DGB en général, Transnet doit lutter depuis des années contre une perte d'adhérents (voir graphique 4). La relève syndicale manque tout particulièrement, alors que le groupe des retraités augmente. Transnet est aujourd'hui l'un des syndicats présentant la moyenne d'âge la plus élevée en Allemagne (IW, 2007). 


\section{Graphique 4 : Evolution du nombre d'adhérents de Transnet et de la DGB}

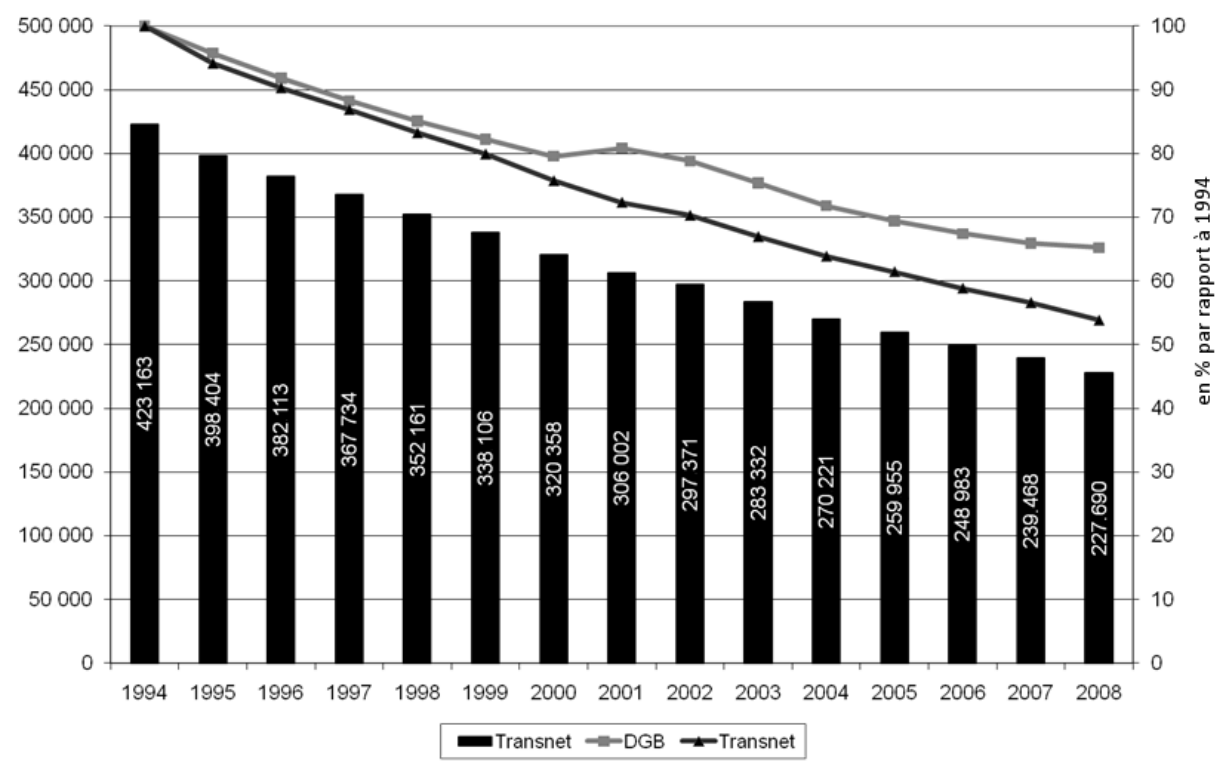

Source : DGB [en ligne]. Disponible sur : http://www.dgb.de [consulté le 30/05/2009].

\section{Le GDBA}

Le GDBA (depuis 1994, syndicat des transports GDBA) est une organisation syndicale membre de la fédération des fonctionnaires allemands, présente dans le secteur ferroviaire. L'adhésion était traditionnellement réservée aux fonctionnaires et agents non titulaires mais, comme la majorité des personnels de la Bundesbahn étaient des fonctionnaires, on peut considérer que le GDBA représente les mêmes personnels que le syndicat unitaire GdED. Le GDBA a été fondé en 1946 afin d'accueillir les fonctionnaires de la Bundesbahn, les personnels roulants et ceux des postes d'aiguillage, au-delà des syndicats d'industrie et syndicats unitaires à prédominance social-démocrate (Müller/Wilke, 2006, p. 117). Malgré la part élevée de fonctionaires parmi les personnels de la Bundesbahn $^{8}$, le GDBA n'a jamais réussi à avoir des effectifs comparables à ceux du GdED. A la fin des années 1990, alors que le projet d'entrée en bourse de la Deutsche Bahn prenait une tournure concrète et que le GdED révisait lui aussi sa politique de recrutement, le nombre de membres du GDBA était d'environ 70 000. Le GDBA coopérait traditionnellement dans les négociations avec le syndicat des conducteurs de locomotives GDL, également membre de la

\footnotetext{
${ }^{8}$ Au début des années 1950, la part des fonctionnaires était de $45 \%$. Au fil du temps, les chiffres à la base de ce calcul ont augmenté de sorte qu'en 1970, la part des fonctionnaires était passée à plus de $50 \%$ (Müller/Wilke, 2006, p. 121).
} 
dbb (ibid., p. 248). Dans le sillage de la nouvelle politique syndicale de Transnet, dont le président Norbert Hansen envisageait de fusionner les trois syndicats traditionnels du secteur (Transnet, GDBA et GDL), un rapprochement entre Transnet et GDBA s'opéra cependant. Avec la fin du recrutement de fonctionnaires à la $\mathrm{DB}$, dans le cadre de la réforme, la base d'adhérents du GDBA diminuait à vue d'œil, de sorte que la collaboration avec Transnet et les liens étroits avec ce syndicat donnaient au GDBA une perspective de survie, au-delà des menaces qui pesaient sur sa propre raison d'être. En juin 2002, l'ancienne coopération entre le GDBA et le GDL fut dissoute dans le cadre d'une union syndicale et à sa place fut instaurée une coopération avec Transnet, au départ déclarée union de négociation. Depuis 2005, GBDA et Transnet constituent officiellement une union syndicale à laquelle a été transféré le droit de représenter les intérêts des deux syndicats dans les négociations collectives (ibid., p. $252-253)^{9}$.

\section{Le GDL}

Le GDL représente traditionnellement les intérêts des conducteurs de trains dans le secteur ferroviaire allemand et, au plan organisationnel, fait partie, comme le GDBA, de la fédération des fonctionnaires $(\mathrm{dbb})$. La référence à la tradition, à laquelle le syndicat renvoie sans cesse - notamment afin d'asseoir ses propres revendications et de justifier sa raison d'être en tant qu'organisation syndicale - joue un rôle important dans l'image que le GDL a de lui-même. Le GDL fut fondé en 1867 sous le nom d'association des conducteurs de locomotives allemands (VDL) et se considère comme le plus vieux syndicat du pays. Alors qu'il mettait au début l'accent sur une représentation corporatiste des intérêts, depuis le soutien aux conducteurs ou à leurs familles qui se trouvaient dans le besoin jusqu'à l'amélioration des conditions de travail, sa politique se réorienta ensuite vers le travail syndical. Cette évolution se traduisit par un changement de nom ${ }^{10}$. Le GDL, type même du syndicat de métier qui s'affirmait à côté du syndicat unitaire GdED, constitue une exception dans le modèle syndical allemand, par ailleurs dominé par des syndicats de branche, d'industrie ou interprofessionnels (cf. Streeck/ Seglow/ Wallace, 1981, p. 315). Avec 34000 membres environ, le GDL est le plus petit des trois syndicats du secteur ferroviaire $^{11}$, mais il regroupe selon ses propres chiffres environ $80 \%$, soit une majorité remarquable, des conducteurs de trains de la DB $A^{12}$. Selon ses statuts, le GDL poursuit une stratégie semblable à celles des autres syndicats

\footnotetext{
9 Cf. http://www.transnet.org/Gewerkschaftsarbeit/Tarifpolitik/Tarifgemeinschaft/index_html/ [consulté le 30/05/2009].

${ }^{10}$ De VDL (association des conducteurs de trains allemands) à GDL (syndicat des conducteurs de trains allemands).

${ }^{11}$ Transnet compte actuellement 240000 membres (statistiques d'affiliation du DGB, 2007), parmi lesquels près de 100000 sont employés par la Deutsche Bahn AG. Le GDBA rassemble environ 50000 membres.

2 Cf. http://www.gdl.de/ [consulté le 29/05/2009].
} 
ferroviaires, et s'est, de même, ouvert à tous les personnels du secteur des transports ${ }^{13}$. Depuis 2002, le GDL mène une politique active de recrutement auprès de l'ensemble des personnels roulants, la convergence d'intérêts y étant importante de son point de vue (Schell, 2009, p. 154). Cet élargissement du champ initial a été assorti de l'exigence d'une convention collective autonome pour les personnels roulants au printemps 2007, mais cette revendication ne put être imposée. Le GDL ne pouvait en effet, s'agissant du personnel roulant en général, s'appuyer sur son succès auprès des conducteurs. Alors que le syndicat détient une grande légimité pour ces derniers, il ne représente qu'un tiers des personnels roulants (GDL, 2007) ${ }^{14}$.

A la suite de la réunification allemande, le GDL eut un succès considérable dans l'Est de l'Allemagne. Alors que le GdED perdait environ la moitié de ses adhérents est-allemands entre 1991 et 1998, le GDL parvint non seulement à réunir en son sein la majorité des conducteurs de trains de la Reichsbahn, mais également à maintenir ses effectifs à un niveau élevé (Müller/Wilke, 2006, p. 246 ${ }^{15}$. Les conducteurs d'ex-RDA ne pouvant bénéficier du statut de fonctionnaires dans la DB AG lors de la fusion entre la Bundesbahn et la Reichsbahn, ils furent recrutés sur des emplois de droit privé, ce qui changea radicalement la nature et la force de frappe du syndicat. Avec ces personnels employés et donc en droit de faire grève, le GDL accédait à une nouvelle ressource en matière de lutte sociale, qui allait devenir en 2007 la base de son cheminement vers l'autonomie tarifaire. Dès 1990-1991, le GDL Est avait organisé deux grèves d'avertissement fructueuses en Allemagne de l'Est, qui lui permirent d'obtenir une révision des salaires dans un rapport d'un pour un et la prise en compte des années passées dans le service public lors du transfert des personnels de la Reichsbahn vers un emploi dans l'Allemagne fédérale (Schell, 2009, p. 104 sq.).

\section{Le conflit social et l'établissement du GDL comme acteur de la négociation}

La privatisation de la Deutsche Bahn, lancée en 1994, est cruciale pour comprendre le conflit social. Lors de la réforme des chemins de fer, transférés d'une grande entreprise publique, orientée vers l'intérêt général national, vers une société par actions opérant à l'international, orientée vers le profit, le syndicat GdED/Transnet opta pour une stratégie de coopération. Comme il a été dit plus

\footnotetext{
${ }^{13}$ Statuts du GDL (état : 2008), à l'adresse : http://www.gdl.de/redaktionssystem/downloads/gdl-satzung.pdf, [consulté le 30/05/2009].

${ }^{14}$ Le GDL explique que parmi les 19611 conducteurs de trains à la Deutsche Bahn AG, 15500 sont affiliés au GDL (79\%). Parmi les 11844 personnels roulants de la DB AG, 3900 sont membres du GDL (33\%) (GDL, 2007).

${ }^{15}$ Contrairement à d'autres syndicats, le GDL a commencé par constituer de nouveaux groupes locaux sur le terrain et a fondé un syndicat GDL est-allemand (GDL Ost). Le 24 janvier 1990 fut ainsi créé le premier syndicat libre de RDA (GDL, 1992, p. 451). Un an plus tard, les syndicats GDL de l'est et de l'ouest fusionnèrent pour couvrir l'ensemble du territoire (GDL, 1992, p. 451 sq. ; Schroeder/Kalass/Greef, 2008, p. 60 ; Müller/Wilke, 2006, p. 247).
} 
haut, Transnet avait, dans une logique constructive, accepté le projet de privatisation et tenta de donner à la restructuration un visage social - au sens d'une sécurisation globale des emplois : Transnet ne souhaitait pas bloquer la réforme, mais choisit de donner à sa politique une orientation corporatiste, de partenariat social, ce qui se traduisit aussi par un rapport « symbiotique » entre la direction de l'entreprise et le comité directeur du syndicat. Le GDL vit cette coopération, qui transparaissait aussi dans les bons rapports personnels entre le dirigeant de la compagnie et Hansen, président de Transnet, comme une «manigance » avec l'employeur (cf. Müller/Wilke, 2008a, p. 29).

Cette stratégie participative de Transnet s'exprima en particulier dans les accords collectifs de sécurisation des emplois des années 1990. En échange d'emplois garantis pour plusieurs années, des concessions furent faites en matière de salaires et de temps de travail, notamment afin de prémunir les membres du syndicat contre des suppressions d'emplois. Pour les groupes à haut niveau de qualification - comme les conducteurs, traditionnellement protégés par un système de primes complexe - cette politique de concessions signifiait des préjudices matériels sensibles. Pour cette catégorie, les pertes de revenus allaient jusqu'à $17 \%$ (voir graphique 5).

\section{Graphique 5 : Pertes de revenus des conducteurs de trains liées à la réforme de 1994}

\begin{tabular}{|c|c|c|c|}
\hline & 1993 & 1994 & Différence en $\%$ \\
\hline $\begin{array}{l}\text { Catégorie de salaire Iz**) } \\
\text { Echelon } 1 \\
\text { Echelon } 8\end{array}$ & $\begin{array}{l}1861 \\
2079\end{array}$ & $\begin{array}{l}1611 \\
1751\end{array}$ & $\begin{array}{l}-13,4 \\
-15,8\end{array}$ \\
\hline $\begin{array}{l}\text { Catégorie de salaire Is*) } \\
\text { Echelon } 1 \\
\text { Echelon } 8\end{array}$ & $\begin{array}{l}1773 \\
1950 \\
\end{array}$ & $\begin{array}{l}1611 \\
1751 \\
\end{array}$ & $\begin{array}{c}-9,1 \\
-10,2 \\
\end{array}$ \\
\hline $\begin{array}{l}\text { Catégorie de traitement VIb*) } \\
\text { Echelon } 1 \text { ( } 21 \text { ans) } \\
\text { Echelon } 11 \text { ( } 43 \text { ans) }\end{array}$ & $\begin{array}{l}1287 \\
1575\end{array}$ & $\begin{array}{l}1289 \\
1401\end{array}$ & $\begin{array}{r}+0,2 \\
-11,0\end{array}$ \\
\hline $\begin{array}{l}\text { Catégorie de traitement Vc**) } \\
\text { Echelon } 1 \text { ( } 21 \text { ans) } \\
\text { Echelon } 10 \text { ( } 41 \text { ans) }\end{array}$ & $\begin{array}{l}1334 \\
1692\end{array}$ & $\begin{array}{l}1289 \\
1401\end{array}$ & $\begin{array}{r}-3,4 \\
-17,2\end{array}$ \\
\hline
\end{tabular}

Rémunération mensuelle de base en $€$, hors primes et compléments. Catégories de salaire : conducteurs de trains de la Deutsche Bundesbahn ; catégories de traitement : conducteurs de trains de la Deutsche Reichsbahn

Source et présentation : Lesch, 2008, p. 320.*) Conducteurs de trains ; **) conducteurs de trains en chef (Oberlokführer).

Mais les pertes de revenus ne constituaient pas le problème essentiel car, même si les conducteurs déploraient une perte disproportionnée, tous les personnels étaient touchés et donc associés à l'assainissement financier du groupe. Ce qui pesa en revanche davantage, c'est que, dans les années suivantes, alors que le chiffre d'affaires et les bénéfices connaissaient une évolution positive, Transnet ne fut pas en mesure de compenser ces salaires modérés par des progressions plus importantes et des accords sociaux plus favorables, en récompense pour les concessions acceptées auparavant par les salariés. L'augmentation de salaires 
des conducteurs de trains ouest-allemands fut de $25 \%$ de 1994 à 2007 - alors qu'elle fut comparativement de près de $56 \%$ dans l'ensemble de l'économie (Lesch, 2008, p. 318). C'est l'une des raisons qui ont ensuite contribué à renforcer la volonté d'autonomie du GDL dans les négociations. Lorsque l'employeur tenta en outre de réajuster le système de primes traditionnel, ce qui aurait signifié des coupes et des détériorations des conditions de travail, en particulier pour les personnels roulants, de nombreux salariés n'étaient plus prêts à aucune concession.

Le GDL parvint à tirer profit de cette insatisfaction générale. Il prit la tête du mouvement de protestation naissant et fit un premier pas sur le chemin de l'autonomie, avec pour objectif la pérennité du syndicat et sa reconnaissance comme partenaire à part entière par l'employeur. Le motif concret d'insatisfaction des personnels surgit lors des négociations sur l' " accord collectif complémentaire Deutsche Bahn Regio» (DB Regio ErgTV) d'octobre 2002, dont les conditions (emplois garantis contre travail supplémentaire non rémunéré) furent jugées inacceptables par le GDL. L'opposition ouverte toucha aussi les membres de Transnet et du GDBA. Les comités directeurs des deux syndicats avaient déjà donné leur bénédiction au résultat des négociations et accepté les coupes demandées par l'employeur. Les membres se sentirent exclus du processus de décision en vue de cet accord. Sur Internet, des adhérents de Transnet firent circuler ouvertement des menaces de grèves sauvages et réunirent des signatures contre cet accord collectif. Transnet perdit alors un nombre sensible de membres (Schroeder/ Kalass/ Greef, 2008, p. 61). Les démissionnaires ne furent pas rares à rejoindre le GDL qui avait, dans l'intervalle, officiellement rejeté le DB Regio ErgTV. Finalement, même les syndicats de l'union de négociation se virent contraints de se retirer de l'accord déjà signé pour stopper l'hémorragie d'adhérents (Müller/Wilke, 2006, p. 258, 268).

Le GDL tenta dans la foulée d'obtenir un accord séparé pour le personnel roulant et la représentativité pour cette catégorie de salariés, mais il échoua dans un premier temps. Il parvint tout de même, dans le cadre d'une conciliation visant à régler ce conflit social, à imposer un droit de négociation pour les intérêts des conducteurs. Mais, la définition exacte de ce droit laissant beaucoup de marge d'interprétation, le différend entre Transnet et le GDL sur leurs compétences respectives n'était pas écarté pour autant. Transnet et l'employeur ne souhaitaient accorder au GDL qu'un droit de négociation complémentaire pour les questions propres à ses adhérents, une fois closes les négociations avec Transnet, alors que le GDL comprenait la décision du conciliateur comme une reconnaissance de son droit à mener des négociations autonomes pour les conducteurs de trains (Müller/Wilke, 2006, p. 288).

Peu après, le conflit de légimité entre Transnet et le GDL se raviva. En 2007, le GDL s'adressa directement, en acteur social autonome, à la Deutsche Bahn AG. L'année précédente, le syndicat avait déjà transmis à l'employeur 
une proposition d'accord collectif spécifique au personnel roulant, autonome et indépendant des revendications de l'union syndicale Transnet/GDBA. La revalorisation de salaire demandée (+30\%) et la profonde situation de conflit, d'ailleurs incarnée personnellement par le dirigeant de la DB Mehdorn (entrée en bourse sans ménagement) d'une part et le président du GDL Schell (imposition d'un accord séparé quelles que soient les circonstances) d'autre part, débouchèrent sur plusieurs mois d'affrontement. Le GDL eut à nouveau recours à des actions de lutte sociale et la direction de l'entreprise fit appel à plusieurs reprises aux tribunaux du travail sur la question du droit de grève, avant qu'un accord ne soit trouvé fin 2007 / début 2008 (cf. par exemple Kazim, 2007).

La revendication du GDL (autonomie dans les négociations) et sa volonté d'être reconnu d'égal à égal eurent cette fois-ci plus de succès. Le syndicat parvint à imposer une convention collective séparée pour les conducteurs de trains (LokfTV). L'augmentation de salaire de $11 \%$ en deux étapes, remportée par le GDL, fut appliquée à l'ensemble des personnels ferroviaires, alors que Transnet et le GBDA n'avaient exigé que 4,5\% de revalorisation : mais, ces deux syndicats ayant fixé à titre préventif un droit de négociation complémentaire en cas d'accord ultérieur, plus favorable, tous les salariés profitèrent des succès du GDL. En contrepartie de son droit de représenter les conducteurs, le GDL dut cependant renoncer à son exigence initiale de représenter les intérêts de l'ensemble des personnels roulants. Les contrôleurs membres du GDL étant peu nombreux, le syndicat ne parvint pas à se faire accorder la représentativité pour l'ensemble des roulants. Les conducteurs de manœuvre n'entrent à ce jour pas non plus dans le champ d'application de la convention LokfTV et donc dans le champ de représentativité du GDL.

\section{La structure d'opportunité : comment expliquer que le GDL ait réussi à se faire reconnaître comme acteur social autonome ?}

La structure d'opportunité ${ }^{16}$ qui a préparé le terrain permettant au GDL, après un conflit de près d'un an avec la Deutsche Bahn AG et Transnet, d'être reconnu comme organisation autonome, est le résultat de trois éléments d'explication. Premièrement, l'orientation prise par Transnet en matière de négociation et de politique syndicale fut décisive. Du point de vue de ses membres, la politique de Transnet était trop liée aux intérêts de la direction de l'entreprise. Transnet ne s'était en outre pas engagé assez clairement en faveur d'une modernisation des conventions collectives qui permettrait de prendre davantage en compte les intérêts propres à une catégorie ou une situation particulière. Deuxièmement, le GDL profita, dans l'élaboration de sa stratégie, de la

\footnotetext{
${ }^{16}$ Le concept de structure d'opportunité émane de l'approche de la «political opportunity structure », utilisée par exemple pour expliquer la formation de mouvements sociaux (cf. Kriesi, 1991). En ce qui concerne le GDL, on entend par structure d'opportunité les facteurs grâce auxquels l'organisation est parvenue à imposer une convention collective autonome pour les conducteurs de trains dans le contexte de 2007/2008.
} 
méfiance très répandue dans l'opinion à l'égard des conséquences négatives de la privatisation ou de l'entrée en bourse, et de l'avis général selon lequel les salaires des personnels allemands n'avaient pas augmenté suffisamment par rapport à l'accroissement des bénéfices et des rendements demandés (débat sur l'équité par rapport aux salaires des dirigeants) ${ }^{17}$.

Ce contexte permit à la fois au syndicat de conquérir de nouveaux membres et de défendre dans l'espace public des revendications perçues comme justes. Pendant toute la durée du conflit avec la direction de la Deutsche Bahn, le GDL put compter, du côté des voyageurs touchés par les grèves, sur une vague de sympathie et de compréhension pour ses revendications ${ }^{18}$. Le fait que les conducteurs de trains ne fassent pas partie, contrairement aux médecins ou aux pilotes, des catégories professionnelles les mieux rémunérées, a également contribué à assurer la sympathie de l'opinion publique pour les exigences du $\mathrm{GDL}^{19}$.

Les conducteurs étaient certes traditionnellement privilégiés par rapport à d'autres catégories de salariés, du fait d'un vaste système de primes et de la garantie de l'emploi due à leur statut de fonctionnaire. Mais, après l'arrêt de la politique de «fonctionnarisation» (Verbeamtung) dans le secteur ferroviaire, dont les conducteurs est-allemands ne bénéficièrent pas du tout après la réunification, et l'offensive de la direction de l'entreprise en 2002 pour rogner sur le système de primes, les conducteurs de trains voyaient leur statut professionnel menacé. Les conventions collectives visant à assurer la pérennité des sites, négociées sous la responsabilité de Transnet, non seulement engendraient des pertes financières, mais alimentaient aussi la peur de la perte de statut. La crainte de se voir dégrader au rang de «camionneurs du rail ${ }^{20} »$ explique la véhémence et l'implication avec lesquelles les conducteurs de trains défendirent leurs revendications. L'endurance du GDL résultait toutefois non seulement de la lutte pour de meilleures conditions de travail et de rémunération, mais aussi de l'objectif d'assurer la survie et l'autonomie de sa propre organisation (pérennité) et de consolider celle-ci (positionnement et reconnaissance comme partenaire social à part entière) (cf. Keller, 2008b, p. 56).

On peut en somme parler d'une structure d'opportunité à trois dimensions (Greef, 2009, p. 122), que l'on retrouve aussi dans les autres exemples de positionnement d'associations professionnelles en syndicats (cf. Schroeder/ Kalass/ Greef, 2008 ; Schroeder/ Greef, 2008) :

\footnotetext{
${ }^{17}$ La part des Allemands qui affirment profiter personnellement de la reprise économique est tombée de $30 \%$ en juillet 2007 à $18 \%$ en décembre de la même année (Infratest dimap, 2007, 2008).

${ }^{18}$ Début octobre 2007, $57 \%$ des personnes témoignaient une certaine compréhension pour la grève des conducteurs. En novembre, ce chiffre se montait déjà à $66 \%$ (parmi les voyageurs touchés par la grève, l'approbation était encore plus forte, avec $71 \%$ ) (Infratest dimap, DeutschlandTREND, 2007).

${ }^{19}$ Avec un salaire brut horaire (2006) compris entre 19,60 et 21,77€, les conducteurs de trains se placent juste au-dessus du salaire horaire moyen, situé à $19,70 €$, tandis que la moyenne dans le secteur manufacturier est plus élevée, atteignant par exemple 25,15€ de l'heure (Brenke et al., 2007, p. 622).

${ }^{20}$ Günther Kinscher, alors président adjoint du GDL (GDL, 2004).
} 
- dimension : organisation/ adhérents : capacité de fédération et de mobilisation élevée ;

- dimension : évolution sectorielle : (peur de la) perte de statut ;

- dimension : paysage syndical : insatisfaction à l'égard de la représentation.

AVEC LA PRIVATISATION DES CHEMINS DE FER ALLEMANDS, qui s'appuyait non seulement sur des considérations de politique fiscale visant à stopper le déficit du budget de la compagnie ferroviaire, en progression constante et financé sur des moyens fédéraux, mais aussi sur des obligations européennes de libéralisation, la politique de l'entreprise fut soumise à de profonds changements. La Bundesbahn et la Reichsbahn furent fusionnées et transformées en une entreprise de droit privée, constituée en société par actions. Avec la réforme ferroviaire, on est passé d'une compagnie qui se limitait au marché national à un groupe de transports et de logistique opérant dans le monde entier et dont les activités ne s'arrêtent pas aux frontières nationales.

Cette réorientation de l'entreprise s'est accompagnée d'une restructuration des relations sociales, et les syndicats se sont trouvés confrontés à de nouveaux défis. Ils durent d'abord décider sur le principe s'ils souhaitaient participer au processus de réforme ou s'ils le rejetaient catégoriquement. En optant pour une stratégie de coopération qui à la fois était l'héritière de traditions établies dans l'histoire du GdED (politique syndicale tournée vers l'entreprise) et se basait sur les expériences négatives du DPG, avec son rejet de principe de la réforme postale, le syndicat unitaire comptait accompagner les réformes de mesures sociales afin d'en atténuer les conséquences négatives pour les personnels. En contrepartie de garanties d'emploi à long terme, des concessions furent faites en matière de conditions de travail et de rémunération. Cette modération salariale provoqua les dernières années la résistance farouche des personnels roulants, qui se virent comme les perdants de la politique de GdED/Transnet. S'agissant des conducteurs de trains, pour lesquels le GDL, qui avait d'emblée rejeté l'ensemble du processus de réforme, avait la représentativité, le plus petit des trois syndicats ferroviaires, jadis insignifiant, parvint à stopper, au moins pour un temps, la politique de nivellement, et à s'imposer comme représentant en mesure de négocier d'égal à égal sur les questions concernant les conducteurs. Ce passage à l'autonomie s'est appuyé sur une structure d'opportunité spécifique.

Concernant l'évolution à venir des relations sociales dans le secteur ferroviaire, il est particulièrement intéressant de noter que l'accord obtenu par le GDL (augmentation de salaire de $11 \%$ ) fut étendu à tous les personnels de la DB. L'employeur tente ainsi d'endiguer la pluralité syndicale et d'empêcher la concurrence en matière de négociation. L'argumentation de Transnet, selon laquelle le GDL est un syndicat catégoriel remportant une victoire pour le compte de sa propre clientèle mais sur le dos de groupes plus faibles, et mettant par 
conséquent en danger la paix dans l'entreprise en divisant les salariés en catégories plus ou moins privilégiées, est pour le moment démentie par la décision de l'employeur de faire bénéficier tous les salariés de l'acquis du GDL. Mais il est aujourd'hui impossible de dire comment les relations sociales évolueront à l'avenir dans le secteur et quel syndicat sera à long terme le gagnant des réformes. C'est pour l'instant le GDL, que le processus de réforme a propulsé, d'une niche insignifiante, sur le devant de la scène de la politique sociale et syndicale du secteur et qui, du point de vue des salariés, a pu faire valoir des victoires dans les négociations.

Traduction de Marie GRAVEY

\section{Indications bibliographiques}

[S.A.], « Nachkriegsgeschichte. Aufbruch in die neue Zeit », mobil, $\mathrm{n}^{\circ}$ 5, 2009, p. 46-53

[S.A.], Tiefensee gegen Privatisierungspläne. Bahn-Börsengang vor 2013 kein Thema mehr [en ligne], Tagesschau.de, Hambourg, 14/03/2009. Disponible sur: http://www.tagesschau.de/wirtschaft/bahn918.html [consulté le 25/05/2009]

Brenke K., Gataullina L., Handrich L., Proske S., «Zu den Löhnen der Lokomotivführer der Deutschen Bahn AG », Wochenbericht des DIW Berlin, vol. 74, n 43, 2007, p. 621-629

DEUTSCHE BAHN, [Geschäftsbericht 2008] [en ligne], Deutsche Bahn AG, Berlin, [2008]. Disponible sur : http://www.deutschebahn.com/site/shared/de/dateianhaenge/berichte/geschaeftsbericht_2008_konzern.pdf [consulté le 30/05/2009]

Engartner T., Die Privatisierung der Deutschen Bahn. Über die Implementierung marktorientierter Verkehrspolitik, VS Verlag, Wiesbaden, 2008

GDL, Erfolg und Zukunft. 125 Jahre GDL. Geschichte der Gewerkschaft Deutscher Lokomotivführer und Anwärter 1867-1992, Hauptvorstand der Gewerkschaft Deutscher Lokomotivführer und Anwärter, Francfort s/ M., 1992

GDL, « Neuer stellvertretender GDL-Bundesvorsitzender », GDL-Informationsdienst, 01/04/2004, p. [n.c.]

GDL, Der Fahrpersonaltarifvertrag: Zahlen, Fakten, Hintergründe [en ligne], Gewerkschaft Deutscher Lokomotivführer (GDL), Francfort s/ Main, 2007. Disponible sur: http://www.gdl-kassel.de/index.php/aus haenge/aushang-ne-bahnen/attachments/index.php?option=com_docman\&task=doc_details\&gid=10\&Itemid= 67 [consulté le 17/05/2011]

GREEF S., Berufsgewerkschaften. Kleine Arbeitnehmerverbände als Herausforderung für das deutsche Gewerkschaftsmodell, AVM, Munich, 2009

IW [INSTITUT DER DEUTSCHEN WIRTSCHAFT], «Gewerkschaften in Deutschland », iW Dossier, $\mathrm{n}^{\circ} 31$, 2007, p. [n.c.]

KAZIM H., « Auch ein falsches Urteil ist immerhin ein Urteil » [en ligne], Spiegel online, 08/08/2007, p. [n.c.]. Disponible sur : http://www.spiegel.de/wirtschaft/0,1518,498847,00.html [consulté le 30/05/2009]

KELlER B., «Der aufhaltsame Aufstieg von Berufsverbänden. Bedingungen, Ziele und Folgen », Sozialer Fortschritt, vol. 57, n 6,2008 a, p. 163-173

KelLeR B., «Renaissance von Berufsverbänden? Bedingungen, Ziele und Folgen », in BLANK T., MüNCH T., SChanne S., STAFFHORST C. (eds), Integrierte Soziologie - Perspektiven zwischen Ökonomie und Soziologie, Praxis und Wissenschaft. Festschrift zum 70. Geburtstag von Hansjörg Weitbrecht, Hampp, Munich/Mering, 2008b, p. 51-66 
KRIESI H., « The Political Opportunity Structure of New Social Movements - Its Impact on Their Mobilization », WZB Discussion Paper, FS III 91-103, 1991, p. [n.c.]

LEHMKUhl D., «Privatizing to Keep it Public? The Reorganisation of the German Railways », in BENZ A., GoETZ K. H. (eds), A New German Public Sector? Reform, Adaptation and Stability, Dartmouth, Aldershot, 1996, p. $71-92$

LESCH H., « Spartengewerkschaften - Entstehungsmotive und ökonomische Wirkung », Industrielle Beziehungen, vol. $15, \mathrm{n}^{\circ} 4,2008$, p. 303-328

MüLler H.-P., Wilke M., „Gestaltend Einfluss nehmen“ Bahngewerkschafts und Bahnreform 1993-2005, Sigma, Berlin, 2006

Müller H.-P.,WiLke M., « Chronik eines Dauerkonflikts », Mitbestimmung, vol. 54, n 4, 2008a, p. 28-32

MüLler H.-P., WILKE M., « Verdrängte Beruflichkeit - Renaissance des Berufsprinzips? », Industrielle Beziehungen, vol. $15, \mathrm{n}^{\circ} 4,2008 \mathrm{~b}$, p. 376-401

RAMGE T., « Halbgötter in Blau », Brand Eins, vol. 5, n 8, p. 88-93

SCHELl M., Die Lok zieht die Bahn. Autobiographie, Rotbuch Verlag, Berlin, 2009

SCHROEDER W., GREEF S., « Industrie- und Spartengewerkschaften im Konflikt. Organisatorische Voraussetzungen und realisierte Gelegenheitsstrukturen », Industrielle Beziehungen, vol. 15, n 4, 2008, p. 329-355

Schroeder W., Kalass V., GreeF S., Kleine Gewerkschaften und Berufsverbände im Wandel, Fondation Hans Böckler, Düsseldorf, 2008, (Böckler Forschungsmonitoring, 3 )

Streeck W., Seglow P., Wallace P., « Competition and Monopoly in Interest Representation: A Comparative Analysis of Trade Union Structures in the Railway Industries of Great Britain and West Germany », Organization Studies, vol. 2, n 4, 1981, p. 307-330

VAN RIESEN O., Zur Leistungsfähigkeit des Regulierungsstaates im Bahnsektor. Eisenbahnregulierung in Europa im Spannungsfeld zwischen institutionellem Design und der politischen Ökonomie des Sektors. Eine Analyse von Eisenbahnregulierungsregimen in Deutschland und Großbritannien, LIT Verlag, Berlin, 2007. 



\title{
Les enjeux de la régulation des entreprises de service public en France face au processus de leur libéralisation
}

\author{
Corinne DEQUECKER, Pierre-Eric TIXIER
}

Dans la présente contribution, les auteurs reviennent, étape par étape, sur l'impact social de la libéralisation des services d'intérêt général, au sein des entreprises concernées en France. A la lumière des transformations déjà réalisées, il s'agit de s'interroger sur le futur modèle de ces ex-entreprises publiques, qui évoluent désormais sur des marchés plus ou moins soumis à concurrence.

Afin d'aborder cette thématique, nous nous sommes livrés à une réinterprétation d'un ensemble d'études qualitatives réalisées dans des entreprises publiques comme la RATP, EDF, France Télécom, la SNCF. Nous avons relu ces enquêtes dans une perspective comparative pour formuler un diagnostic global sur leurs problèmes de régulation. Les entreprises publiques sont passées d'une période historique du statut public à une période parfois qualifiée par les entreprises de « dé-intégration » dans la période d'ouverture de leur marché. Nos travaux ont porté particulièrement sur cette période qui se caractérise par une forte interrogation sur leurs finalités, ainsi que sur la période la plus actuelle faisant émerger les questions suivantes : existe-t-il un nouveau modèle de régulation ? Et si non, quel nouveau modèle de régulation pourrait-on inventer, sachant que le risque lorsqu'on aborde un tel débat est la sur-idéologie, car chacun de nous est interrogé sur sa vision de la société ?

\section{Un modèle historique de double régulation par le statut et l'arrangement}

Le modèle des entreprises de service public était construit sur un monopole (bien que l'on retrouve le marché en périphérie) et sur un statut spécifique de leur personnel : des fonctionnaires pour la Poste avant sa séparation de France Télécom, des statuts spécifiques comme à EDF, la SNCF ou la RATP. Les fonctionnements sociaux de ces entreprises ont été construits autour du prescriptif avec une importance particulière accordée au collectif et au métier et leurs modes de régulation étaient construits autour de la technique, de l'échange politique et structurés par les rapports de force entre directions et syndicats. En matière de ressources humaines, le recrutement et les carrières s'effectuaient par la voie de concours ou via des écoles professionnelles. Enfin, les corps de règles étaient élaborés du sommet de ces entreprises, avec comme conséquence un nombre considérable d'arrangements au niveau des établissements locaux, appelés dans certains cas « le coutumier ». 
Une des caractéristiques spécifiques de ces entreprises était leurs structures de dialogue social fondées sur le compromis suivant: la technique aux ingénieurs et le social aux syndicats. Dans ce modèle dominant de la fin de la Seconde Guerre mondiale aux années 1980, les syndicats se vivaient comme co-garants de l'intérêt général. Dans certains cas, ils ont été même cofondateurs des statuts de ces entreprises comme à EDF. Les structures de dialogue social des entreprises publiques dites statutaires avaient une caractéristique que l'on retrouvait dans l'ensemble de la fonction publique avec les Commissions Administratives Paritaires (C.A.P) et les Comités Techniques Paritaires (C.T.P) qui servent à structurer les débats entre directions et syndicats, sans qu'il existe de véritable négociation collective au sens formel du terme jusqu'aux années 1990. Chacune des parties prenantes pouvant à la sortie des instances de dialogue prendre des positions différentes de celles qu'elle a tenues dans l'instance, dimension qui a participé à une sorte d'irresponsabilité collective à la fois des directions et des syndicats. Il n'y a pas ainsi d'engagement des parties juridiquement sanctionné.

Le modèle historique des entreprises publiques était construit aussi sur l'hypothèse de l'entreprise intégrée, des modes de gouvernance très centralisés et des formes de compromis implicites qui reposent à la fois sur des règles statutaires, mais aussi de nombreux arrangements qui fonctionnent au jour le jour au niveau des établissements. Dans de tels systèmes de régulation, ces ajustements sont pour une large part masqués et implicites. Mais, ils ont une place centrale dans les formes de régulation parce qu'ils structurent les conditions d'utilisation de la maind'œuvre et les rémunérations réelles. L'ensemble de ces mécanismes de régulation produisait une identité fière des agents avec le sentiment de rendre un service efficace aux citoyens français qui y étaient attachés, en contrepartie de leur statut. Or, la libéralisation des marchés va avoir comme effet induit une profonde remise en cause des rapports existant entre ces salariés, les entreprises et leurs clients.

\section{Une phase de « désoptimisation » et de bricolage institutionnel}

Il existait un certain accord dans la société française sur l'hypothèse de la modernisation du service public à travers la version Rocardienne dans les années 1980 avec la recherche d'efficience : une conception du service public qui voulait associer une hypothèse de performance avec le maintien d'une structure publique. Cette stratégie de changement consensualiste est remise en cause dans les années 1990 par l'hypothèse du marché et de la concurrence. Ce changement se fait sous l'influence de l'idéologie libérale des expériences Thatchérienne et Reaganienne. Celles-ci inspirent très fortement la Commission européenne qui veut introduire une libre concurrence sur l'ensemble des services. Deux textes fondateurs (l'Acte unique et le Traité de l'Union Européenne), suivis d'un ensemble de directives sectorielles, vont désormais organiser les conditions de la concurrence et impliquer la suppression des monopoles et un changement de statut des entreprises publiques. 
Cette option du marché a donné lieu en France à un débat social intense et à un jeu complexe à quatre partenaires entre l'Union européenne, l'Etat français, les entreprises publiques et les mouvements sociaux, dont ceux de 1995 qui ont engendré des modifications et des retards dans le processus de libéralisation initialement prévu.

La volonté d'introduire la concurrence amenait pour les entreprises publiques un ensemble de redéfinitions fondamentales sur différentes dimensions qui touchaient directement l'organisation, l'activité et le travail d'une partie des agents du service public, les ressources humaines et les relations sociales :

- en termes de fonctionnement organisationnel, la séparation des activités, nommée «dé-intégration », a été ou est un phénomène mal vécu par les salariés en interne. Elle s'est traduite par une sorte de phase dépressive collective d'une partie des agents qui valorisaient les fonctionnements antérieurs jusqu'à en faire une sorte d'âge d'or pour certains salariés qui avaient le sentiment d'un changement inéluctable qui dégradait la qualité du service et l'optimisation de l'organisation. En fait, les transformations remettaient en cause, en plus de la transformation organisationnelle, les formes d'échanges implicites antérieurs qui existaient entre ces entreprises et leur personnel, notamment les arrangements locaux finalisés ou non par des accords locaux (une adaptation des règles aux différentes activités dans un contexte donné qui «fluidifiait» les relations) et qui structuraient les rémunérations réelles et les conditions d'utilisation de la main-d'œuvre. Cette situation de changement provoque une période de l'entre-deux, où les règles du jeu antérieures ne sont plus claires et les nouvelles règles du jeu ne sont pas fixées, et entraîne une perte de confiance des agents envers la hiérarchie et les directions. Dans un certain nombre de cas, le management a parfois eu tendance à décontextualiser les impacts du changement par rapport à la situation vécue des salariés. Du point de vue formel des managers, il peut être assez facile de considérer que les changements de structures n'impactent pas le travail des agents, mais lorsque l'on interviewe ces derniers, ces petits déplacements dans l'activité peuvent s'avérer pour eux extrêmement significatifs ;

- les réformes engagées ont été par ailleurs fortement contestées par les syndicats, notamment les organisations majoritaires, souvent la CGT qui résistait doublement : d'une part parce qu'elle contestait les orientations vers le marché, l'internationalisation des entreprises, mais aussi le développement de la négociation collective qui remettait en cause le principe de représentativité prouvée sur lequel fonctionnaient les instances statutaires de dialogue social. Le développement de la négociation collective a permis notamment aux directions de passer des accords avec les organisations syndicales minoritaires. Par ailleurs, ces organisations syndicales, et particulièrement la CFDT, étaient plus favorables 
à l'enjeu de la modernisation et à l'enjeu politique européen au nom duquel la transformation des entreprises publiques s'effectuait ;

- ces transformations organisationnelles se sont accompagnées en même temps d'un renforcement de la distance entre les organisations syndicales (quelles que soient leurs étiquettes) et les salariés, qui ont continué à voter aux élections professionnelles, alors que les syndicats perdaient une part de leur capacité d'influence sur les orientations des entreprises et les changements de périmètres des établissements, excepté en matière de résolution des problèmes individuels : "la sécurité sociale syndicale » a continué à fonctionner.

Cette situation d'incertitude a entraîné une phase d'adaptation (plutôt difficile) : une conflictualité récurrente encore actuelle, de nombreux procès sur les règles applicables et une fragilisation du lien de représentation entre syndicats et salariés, ainsi que la montée de franges radicales minoritaires qui refusent les changements engagés. Ces diverses résistances ont été souvent menées par la CGT et SUD qui mettent en avant les nombreuses dysfonctions et les incertitudes pour les personnels pour engager la contestation et refuser les transformations en cours. Le changement de règle du jeu pose en fait une question difficile à tous les syndicats : qu'est-ce que défendre l'intérêt des salariés dans une telle période de changements de règles du jeu : faut-il défendre les acquis antérieurs ou inventer de nouvelles règles, mais à quelles conditions, défendre les intérêts des personnels qui sont en poste ou les nouveaux recrutés ?

\section{Quelles stratégies de changement?}

Une des difficultés majeures qu'ont rencontrées les directions des entreprises publiques est que le changement de modèle s'impose sans qu'il existe de véritable compromis politique dans la société française ou en interne entre directions et syndicats sur la nature des changements à mener. Comment conduire le changement dans de telles conditions ? Un des points majeurs de focalisation des enjeux entre les différents acteurs est centré sur l'articulation entre modernisation, marché et nouvelles règles du jeu.

Dans un certain nombre de cas, les directions ont tendance à demander à l'échelon local, par défaut d'accord au niveau central, de résoudre un certain nombre de problèmes de transformation ce qui demande une sorte de sur-compétence managériale au management local qu'il n'a pas toujours, parce que cette compétence n'était pas nécessaire dans le modèle antérieur. Une partie des salariés a alors le sentiment d'une dégradation de la qualité du travail, ce qui remet en cause le rapport identitaire antérieur au travail et à l'entreprise. Les salariés défendent ce qu'ils appellent le «travail bien fait » qui renvoie aux formes antérieures de travail, avec le sentiment qu'ils ne pourront le produire tel quel dans le nouveau modèle d'entreprise qui se dessine. Mais, cela ne veut pas dire pour autant que les salariés sont contre les transformations : lorsqu'on interviewe les 
agents, il en ressort que si pour leur majorité, ils ne souhaitent pas les changements en cours, ceux-ci peuvent être acceptables si l'entreprise met en place des politiques pertinentes, notamment de rémunération et de carrière, ce qui supposerait d'inclure les conditions réelles d'utilisation de la main-d'œuvre et les systèmes d'arrangements qui fonctionnaient antérieurement pour construire les bases d'un nouveau compromis.

Une seconde question majeure est de savoir comment vont être construits les nouveaux parcours professionnels. Auparavant, les systèmes des carrières étaient fondés sur d'un côté des concours et examens professionnels et, de l'autre, une place forte était donnée à l'ancienneté. Il était possible par ailleurs sous certaines conditions de changer d'activité, ce qui était perçu comme un élément très positif par le personnel. Dans la nouvelle configuration, quelles doivent être les règles de carrières qui doivent s'appliquer avec la séparation des activités et la création des filiales ? Quelles sont les nouvelles règles qui conditionnent la mobilité professionnelle et géographique?

Une troisième question porte sur les compromis internes. Que substituer au double compromis antérieur reposant sur les règles statutaires et la dimension implicite des formes de régulation locales ? Les changements rendent nécessaire d'expliciter ces règles du jeu parce qu'elles structurent le fonctionnement réel, ce qui supposerait à la fois de les reconnaître et de les intégrer en termes de politique. Cela représente un enjeu de confiance entre personnel, direction et syndicats, confiance qui n'est pas présente dans la majorité des cas. Autant de questions qui ne sont qu'en partie résolues et qui sont à l'origine de nombreuses insatisfactions des personnels des entreprises publiques.

\section{Demain, quel modèle pour les ex-entreprises publiques ?}

Quelles sont désormais les finalités de l'ex-entreprise publique ? Quelle doit être la fonction de ces entreprises dans la société ? Les réponses à ces questions sont fondamentales parce qu'elles donnent sens à l'activité, car les salariés en ont besoin pour donner sens à leurs tâches au quotidien. Et comme la définition de la place de l'entreprise entre l'intérêt général et le marché n'est pas complètement claire à leurs yeux, les salariés s'interrogent sur ce que doit être leur travail. Si la transformation de l'entreprise est considérée comme inéluctable, beaucoup de salariés souhaitent qu'elle ne soit "pas tout à fait comme les autres » et qu'elle conserve un certain nombre des référents du service public antérieur. Et derrière ces interrogations, se pose la question centrale des éléments du contrat social qui lie les salariés à l'entreprise, l'entreprise à la société et l'entreprise aux salariés. Un débat qui se joue autant sur l'activité quotidienne de chacun que sur un plan plus sociétal et politique. Trois grandes questions se posent donc pour les directions en termes de pilotage et de régulation :

- une première dimension est liée à la clarté et aux caractéristiques de la vision des changements. On peut observer que la façon dont les diri- 
geants présentent les transformations de l'entreprise peut être très différente suivant les cas. Ce qui est en jeu à travers la vision des orientations proposée par les directions pose la question de leur capacité à générer de la confiance, à incarner les projets et à les faire partager. Mais ces orientations ne peuvent pas découler uniquement du sommet et descendre dans le papier glacé des supports de communication interne, comme cela a été quelquefois le cas. Dans une période de bouleversements où les orientations ne sont pas complètement définies, les salariés attendent des dirigeants qu'ils échangent avec eux, qu'ils manifestent leur proximité et discutent des différentes options. Pour être écoutés, les dirigeants doivent «mouiller leur chemise » et être présents avec les femmes et les hommes qui conduisent le changement sur le terrain. C'est un élément qui nous a beaucoup frappés lors des enquêtes de terrain que d'observer les attentes des salariés en termes de contact direct avec les dirigeants pour comprendre la nature des changements qui affectent ces entreprises et pouvoir discuter avec eux de ces changements. Si on est incertain sur les objectifs, la question du leadership des dirigeants devient un élément central de la conduite du changement, seul à même de donner un sens aux transformations en cours ;

- le second point clé est lié à la capacité managériale de créer de la confiance malgré l'incertitude. Lorsque les managers locaux se comportent en patrons capables de traduire les orientations au plan local en résolvant les problèmes qui se posent pour les salariés en termes de travail, de ressources humaines, de dialogue avec les syndicats..., ils arrivent à faire passer les réformes et créent de l'efficacité. Ce travail de liaison entre les orientations stratégiques et le travail sur le terrain est essentiel, car il construit l'acceptabilité du changement au quotidien et à cet égard on observe de grandes différences au plan local ;

- le dernier point qui est au cœur de la définition du nouveau modèle est celui de l'invention de nouveaux compromis sociaux. On observe à l'heure actuelle une faiblesse de ces compromis sociaux au niveau central pour la majorité des ex-entreprises publiques. Par conséquent, la régulation ad hoc localisée dans les établissements est essentielle à construire puisqu'elle doit suppléer en partie ce qui n'est pas construit au niveau du sommet des organisations. Comment passer de formes de compromis qui intégraient à la fois des règles statutaires rigides et des compromis locaux masqués à des formes plus explicites de régulation?

L'ENSEMBLE DE CES QUESTIONS qui sont en débat dans les ex-entreprises publiques demande un ensemble d'innovations et d'investissements managériaux, ce qui pose une question centrale en termes de gouvernance. Quelle refondation du modèle entreprise/société et d'une performance pourrait intégrer à la fois des dimensions économiques, techniques, sociales internes mais aussi sociétales? 


\title{
Le rôle de la libéralisation et de la privatisation des services publics dans l'érosion de la convention collective de branche en Allemagne ${ }^{1}$
}

\author{
Torsten BRANDT, Thorsten SCHULTEN
}

Avec la décentralisation de la politique de négociation collective dans les secteurs industriels et le manque de couverture conventionnelle dans le tertiaire privé, la libéralisation et la privatisation des services publics constituent le troisième facteur, jusqu'à présent peu pris en compte, d'érosion du système allemand de conventions collectives.

La privatisation et la création de nouveaux marchés dans le domaine des services d'intérêt général (SIG) se sont accompagnées, dans de nombreux secteurs du tertiaire, d'une rupture par rapport au système conventionnel appliqué au service public. Cette situation n'a pas conduit à la signature de nouveaux accords de branche, mais à un éclatement des conventions collectives, avec une concurrence entre les systèmes des entreprises publiques et des nouvelles entreprises privées, et à des conditions de travail très hétérogènes au sein même de certaines entreprises.

Cette contribution discute pour conclure de la manière dont des standards (minimaux) de branche homogènes pourraient être imposés dans les nouveaux marchés libéralisés afin de limiter la concurrence sur les charges et les coûts du travail.

Depuis la fin des années 1990, un grand nombre d'études ont analysé, sous les intitulés de «crise » ou d' « érosion » de la convention collective de branche (Flächentarifvertrag), la mutation fondamentale du système allemand de convention collective ${ }^{2}$.

Ces études se concentrent surtout sur le recul continu de la couverture conventionnelle (Ellgut/Kohaut, 2008) et sur la tendance croissante à la décentralisation de la négociation, de plus en plus pratiquée au niveau de l'entreprise, avec une multiplication des accords d'entreprise, et des clauses d'ouverture de plus en plus fréquentes.

Ces clauses permettent aux entreprises de déroger dans certaines conditions aux normes prévues par la convention collective de branche (Bispinck, 2006a ; Kohaut/Schnabel, 2007).

\footnotetext{
${ }^{1}$ Cet article constitue la version modifiée et traduite d'une contribution parue dans le numéro 10/2008 de la revue WSI-Mitteilungen, intitulée «Liberalisierung und Privatisierung öffentlicher Dienstleistungen und die Erosion des Flächentarifvertrages ». Cette réédition est réalisée en accord avec la rédaction de WSIMitteilungen. L'article s'appuie sur des résultats de recherche obtenus dans le cadre du projet international «Privatisation of Public Services and the Impact on Quality, Employment and Productivity » (PIQUE), projet $\mathrm{n}^{\circ}$ : CIT5-2006-028478, mené avec le soutien de la Commission européenne. L'Institut des sciences économiques et sociales de la fondation Hans Böckler (WSI) s'est associé au projet pour les travaux portant sur l'Allemagne. Pour plus d'informations consulter : www.pique.at [consulté le 17/05/11].

${ }^{2}$ Cf. notamment Artus (2001); Bispinck (2006a, 2007); Bispinck/Schulten (2003) ; Oppolzer/Zachert (2000); Kohaut/Schnabel (2003, 2007).
} 
Les débats sur les causes de cette évolution ont longtemps été dominés par la thèse de la "mondialisation », selon laquelle la convention de branche, inscrite dans le cadre national, ne serait plus en mesure, compte tenu de l'internationalisation croissante de l'économie, de remplir sa fonction corporatiste classique et de définir des conditions de concurrence homogènes pour un secteur donné. Sont évoqués, à l'appui de cette thèse, les nombreux «pactes pour l'emploi », qui ont fixé des conditions de travail dérogeant à la convention collective de branche au motif de la concurrence internationale (Rehder, 2003).

La «thèse de la mondialisation» situe essentiellement la crise de la convention collective de branche dans l'industrie. C'est d'autant plus étonnant que, malgré tous les changements survenus dans ces secteurs, la couverture conventionnelle y reste relativement élevée et que les structures conventionnelles y sont relativement stables. En revanche, c'est surtout dans les branches du tertiaire, plutôt axées sur le marché intérieur, que l'érosion des conventions collectives a comparativement beaucoup progressé (Ellgut/Kohaut, 2008). Il a fallu attendre les débats sur le développement des bas salaires en Allemagne pour que ce phénomène attire davantage l'attention (Bispinck, 2007 ; Bispinck/Schulten, 2008). C'est de toute évidence moins la mondialisation que l'aptitude insuffisante des syndicats à faire bloc et à s'imposer, ainsi qu'un marché du travail favorisant des conditions d'emploi précaires, qui ont contribué à l'érosion des systèmes de conventions collectives.

Enfin, les discussions sur la crise de la convention de branche omettent les changements qui ont eu lieu au sein même du secteur public. La couverture conventionnelle y étant restée importante, le service public ${ }^{3}$ est perçu comme un champ encore largement stable de ce point de vue (Schnabel, 2007). Or, c'est ignorer les changements et disparités internes dans les relations de travail du secteur (Dribbusch/Schulten, 2007 ; Keller, 2007). Et c'est surtout oublier que, dans de nombreuses branches, la libéralisation et la privatisation des services publics sont allées de pair avec une sortie du système de conventions collectives et avec l'apparition de nouvelles structures, souvent extrêmement fragmentées. Or, ces changements jouent un rôle au moins aussi important dans l'évolution du système conventionnel allemand que la décentralisation dans le secteur industriel ou l'érosion des structures conventionnelles dans le tertiaire. L'éclatement des structures et la menace de voir une concurrence des coûts du travail remplacer celle de la qualité en raison de la libéralisation sont également démontrables, sous des formes diverses, dans d'autres pays d'Europe, dans les secteurs de l'électricité, des transports en commun, des postes et des hôpitaux (Brandt/Schulten, 2009, 2008d).

\footnotetext{
${ }^{3}$ Par le terme de « service public », on entend l'activité des fonctionnaires, employés et ouvriers. L'employeur peut être la commune, le Land, le Bund ou une autre personne morale de droit public, un établissement public ou une fondation de droit public. Tandis que les salaires des ouvriers et employés sont définis par le biais de conventions collectives, le traitement des fonctionnaires est établi par voie légale.
} 


\section{Le système de conventions collectives du service public en mutation}

Le service public constituait traditionnellement l'un des principaux piliers du système allemand de protection des personnels. Jusqu'à il y a quelques années, ce secteur était doté, avec la convention collective des employés de la fonction publique (Bundesangestelltentarifvertrag, BAT) et les conventions correspondantes pour les ouvriers (MTArb et BMT), d'un dispositif centralisé dont la portée n'a jamais été dépassée par aucune autre convention collective (Keller, 1993). Il s'agissait de facto de conventions interprofessionnelles regroupant, outre les administrations fédérales, régionales et communales, de vastes domaines des SIG. S'y ajoutaient aussi d'autres secteurs pour lesquels les accords du service public étaient le principal repère et qui s'en inspiraient largement. En faisaient partie les transports ferroviaires et les postes ainsi que l'ensemble du secteur sanitaire et social, grandes associations caritatives incluses. Enfin, les accords collectifs fixaient aussi les grilles de rémunération des fonctionnaires, régulées par la loi.

Comme les conventions collectives pour l'ensemble des services publics étaient négociées de manière centralisée, à l'échelle nationale, des conditions de rémunération et de travail très homogènes étaient définies partout. Le régime de travail du service public se distinguait surtout de celui du privé par une sécurité d'emploi nettement plus grande et par une dispersion bien moindre des salaires entre les différentes catégories de personnels (Atzmüller/Hermann, 2004a). Alors que les salariés hautement qualifiés gagnaient un peu moins dans le public que dans le privé, c'était le plus souvent l'inverse pour les personnels moins qualifiés.

Mais, depuis le début des années 1990, le secteur public est engagé dans un profond processus de restructuration, qui n'est pas resté sans conséquences sur le système de conventions collectives (Dribbusch/Schulten, 2007). Sous la pression d'une politique de restriction budgétaire, l'introduction de concepts issus du «New Public Management» s'est accompagnée de méthodes de gestion de plus en plus proches de celles du privé, visant à moderniser une administration publique critiquée pour son «inefficacité » (Bordogna, 2007). Se sont ajoutées l'externalisation et la privatisation de services publics ainsi que la concurrence des entreprises publiques avec les nouveaux opérateurs privés, renforcée par la libéralisation.

Cette vaste phase de restructuration a entraîné de massives suppressions d'emplois. Ainsi, le nombre de postes dans la fonction publique a diminué de $32 \%$ entre 1991 et 2006 (soit 2,1 millions d'emplois supprimés) pour tomber à 4,6 millions. Environ la moitié de ces suppressions d'emplois sont imputables aux privatisations, l'autre moitié résultant de mesures de rationalisation dans ce qui reste du service public (Brandt/Schulten, 2008a, p. 70-71). La pression croissante des prix et de la rentabilité a en outre constitué la toile de fond sur laquelle a été négociée une réforme en profondeur des conventions collectives du 
service public, débouchant finalement en 2005 sur l'adoption de la nouvelle « convention collective du service public » (TV-ÖD). Ce texte comporte un certain nombre de clauses d'ouverture permettant de poursuivre la décentralisation et les négociations séparées, une disposition utilisée au cours des négociations ultérieures, en particulier sur les temps de travail (Bispinck, 2006b). Les négociations à l'origine de cette nouvelle convention ont débouché dès 2003 sur une scission des employeurs entre les niveaux fédéral, régional et communal, si bien que, depuis 2006, les Länder mènent des négociations autonomes, sur la base de la «convention collective pour le service public des Länder » (TV-L).

Les modifications survenues à l'intérieur du système conventionnel du service public ont entraîné une grande dispersion des standards sociaux, laquelle limite les fonctions régulatrices de la convention de branche. Or il faut y ajouter les changements externes induits par la libéralisation et la privatisation, qui ont mené à soustraire (partiellement) de nombreuses branches du public au système de conventions collectives du service public.

\section{Répercussions de la libéralisation et de la privatisation sur la politique de régulation sociale}

La libéralisation et la privatisation des services publics entraînent une pénétration des logiques du capitalisme dans des secteurs de la société qui jusque-là n'étaient pas touchés par les possibilités de valorisation du capital à des fins de profit. La libéralisation entraîne l'ouverture à la concurrence des anciens monopoles d'Etat et la création de nouveaux marchés. Les différentes formes de privatisation constituent des réponses diverses à la pression de la concurrence dans le cadre de marchés libéralisés (Killian et al., 2006) : la première forme est la privatisation formelle ou organisationnelle, dans le cadre de laquelle une entité autrefois publique est convertie en entreprise de droit privé, toutefois encore détenue majoritairement par les pouvoirs publics.

Ce type de privatisation vise principalement l'autonomie économique, qui laisse une importante liberté d'action en termes de gestion et soustrait la nouvelle entreprise à l'influence des institutions politiques. La privatisation des missions constitue une deuxième option consistant, pour des entreprises publiques, à externaliser certaines activités à des entreprises privées. Enfin, la troisième forme dite de privatisation matérielle ou privatisation des biens va jusqu'à la vente réelle de l'entreprise publique à des investisseurs privés.

Quelles que soient la forme juridique et la forme de propriété, la libéralisation déclenche un processus de marchandisation qui contraint le régime de travail traditionnel du secteur public à s'adapter à la concurrence et a des conséquences lourdes sur les conditions de travail et d'emploi et sur les structures traditionnelles de la régulation sociale. Ainsi, les branches concernées par la libéralisation sortent généralement largement du système de protection 
sociale du service public. De nouvelles structures, très fragmentées, s'y substituent; elles se caractérisent par une double dualité (Brandt/Schulten, 2007, 2009): d'une part, se forme au niveau sectoriel un système de protection des personnels en deux volets, avec des structures conventionnelles relativement stables dans les anciens monopoles d'Etat et dans les entreprises restées publiques d'un côté, et des structures plutôt décentralisées, avec une couverture conventionnelle nettement moins favorable, du côté des nouveaux opérateurs. D'autre part, une division croissante s'opère au niveau des entreprises entre différentes catégories de salariés, avec des dispositions distinctes pour les anciens et les nouveaux, et pour les personnels permanents et temporaires.

S'ajoute à ce phénomène l'externalisation de vastes domaines d'activités, souvent accompagnée du passage à une convention collective moins protectrice ou même de la fin de toute couverture conventionnelle.

Faute de normes sectorielles dans les nouveaux marchés libéralisés, la concurrence est répercutée sur les coûts du travail, et les personnels sont exposés à une pression énorme. C'est le cas en particulier pour les activités requérant un personnel nombreux comme la poste, les transports publics de proximité ou les hôpitaux, secteurs où les dépenses de personnel continuent de représenter environ les deux tiers des coûts totaux.

Dans certains de ces secteurs, le phénomène s'amplifie dans la mesure où il ne s'agit pas de marchés en croissance, si bien que la concurrence prend la forme d'une concurrence d'éviction dans laquelle des emplois finissent immanquablement par disparaître. Depuis le début des années 1990, au moins 600000 emplois ont disparu dans les secteurs touchés par la privatisation et la libéralisation (Brandt/Schulten, 2008a, p. 76). Parallèlement, les conditions de travail d'un grand nombre de personnes se sont sensiblement dégradées, avec des salaires plus bas, un allongement des temps de travail et des conditions d'emploi précaires plus fréquentes (Atzmüller/Hermann, 2004b).

\section{Structure des conventions collectives dans les services libéralisés}

La comparaison entre les différentes conventions collectives des secteurs tertiaires libéralisés montre que, selon les branches, soit les entreprises publiques sont encore liées directement aux conventions du service public, soit elles ont conclu des conventions de sous-secteurs ou bien encore, dans le cas des anciens monopoles d'Etat comme la poste ou le rail, leurs salariés bénéficient de conventions d'établissement comparables à celles du service public du point de vue des conditions de travail et de rémunération.

Les nouvelles entreprises privées sont quant à elles très peu liées à des accords de branche ; elles ne disposent le plus souvent que d'accords d'établissement, voire d'aucune couverture conventionnelle (tableau 1). 
Tableau 1 : Structure des conventions collectives et conventions concurrentes dans les secteurs libéralisés et privatisés

\begin{tabular}{|c|c|c|}
\hline Secteur & $\begin{array}{l}\text { Entreprises publiques et } \\
\text { anciens monopoles d'Etat }\end{array}$ & Entreprises privées \\
\hline Télécommunications & $\begin{array}{l}\text { Deutsche Telekom AG : } \\
\text { accords d'établissement } \\
\text { (différents d'une division à } \\
\text { l'autre du groupe) }\end{array}$ & $\begin{array}{l}\text { Accords d'établissement ou } \\
\text { absence de couverture } \\
\text { conventionnelle }\end{array}$ \\
\hline \multirow[t]{2}{*}{$\begin{array}{l}\text { Services postaux / } \\
\text { courrier }\end{array}$} & $\begin{array}{l}\text { Deutsche Post AG : accords } \\
\text { d'établissement (différents } \\
\text { d'une division à l'autre du } \\
\text { groupe) }\end{array}$ & $\begin{array}{l}\text { Absence de couverture } \\
\text { conventionnelle; accords } \\
\text { juridiquement controversés } \\
\text { sur des salaires minimaux } \\
\text { avec le « syndicat des } \\
\text { nouvelles entreprises du } \\
\text { secteur postal » (GNBZ) et la } \\
\text { Confédération chrétienne } \\
\text { (Christlicher } \\
\text { Gewerkschaftsbund) }\end{array}$ \\
\hline & \multicolumn{2}{|c|}{$\begin{array}{l}\text { Accord sur un salaire minimum légal applicable à l'ensemble } \\
\text { du secteur postal en vertu de la loi sur le détachement de } \\
\text { travailleurs }\end{array}$} \\
\hline Rail & $\begin{array}{l}\text { Deutsche Bahn : accords } \\
\text { d'établissement (différents } \\
\text { d'une division à l'autre du } \\
\text { groupe) ; filiale DB } \\
\text { Heidekrautbahn sans } \\
\text { couverture conventionnelle }\end{array}$ & $\begin{array}{l}\text { Accords d'établissement, } \\
\text { accords régionaux } \\
\text { d'associations ou absence de } \\
\text { couverture conventionnelle }\end{array}$ \\
\hline $\begin{array}{l}\text { Transports publics de } \\
\text { proximité }\end{array}$ & $\begin{array}{l}\text { Convention collective du sous- } \\
\text { secteur des transports de } \\
\text { proximité, accords } \\
\text { d'établissement }\end{array}$ & $\begin{array}{l}\text { Accords signés par des } \\
\text { organisations patronales } \\
\text { régionales dans certains cas } \\
\text { (autobus), accords } \\
\text { d'établissement ou absence } \\
\text { de couverture } \\
\text { conventionnelle }\end{array}$ \\
\hline Energie & $\begin{array}{l}\text { Régies municipales : } \\
\text { convention collective du sous- } \\
\text { secteur de } \\
\text { l'approvisionnement, }\end{array}$ & $\begin{array}{l}\text { Conventions collectives de } \\
\text { groupe, conventions } \\
\text { collectives régionales }\end{array}$ \\
\hline
\end{tabular}

${ }^{4}$ Cette loi fédérale (Arbeiterentsendegesetz - AEntG), adoptée en 1996, introduit le principe du pays d'accueil, en vertu duquel les conditions de travail applicables à des salariés d'une entreprise basée à l'étranger sont celles du pays d'accueil, et définit des standards minimaux, pour le secteur du bâtiment notamment, NDT. 


\begin{tabular}{|c|c|c|}
\hline & $\begin{array}{l}\text { conventions collectives } \\
\text { régionales dans certains cas }\end{array}$ & \\
\hline \multirow[t]{2}{*}{$\begin{array}{l}\text { Traitement des } \\
\text { déchets }\end{array}$} & $\begin{array}{l}\text { Convention collective du } \\
\text { service public (TV-ÖD) }\end{array}$ & $\begin{array}{l}\text { Convention collective de } \\
\text { branche pour les opérateurs } \\
\text { privés signée avec la } \\
\text { fédération d'employeurs } \\
\text { BDE } \\
\text { Nombreuses petites } \\
\text { entreprises privées : absence } \\
\text { de couverture } \\
\text { conventionnelle }\end{array}$ \\
\hline & \multicolumn{2}{|c|}{$\begin{array}{l}\text { A partir de mai } 2009 \text { : accord sur un salaire minimal obligatoire } \\
\text { pour tous dans le secteur du traitement des déchets en vertu de } \\
\text { la loi sur le détachement des travailleurs (AEntG) }\end{array}$} \\
\hline Hôpitaux & $\begin{array}{l}\text { TV-ÖD ou convention } \\
\text { collective pour le service } \\
\text { public des Länder (TV-L); } \\
\text { accords d'établissement dans } \\
\text { certains cas }\end{array}$ & $\begin{array}{l}\text { Hôpitaux d'utilité publique : } \\
\text { accords d'établissement ou } \\
\text { absence de couverture } \\
\text { conventionnelle (dispositions } \\
\text { spécifiques dans les } \\
\text { établissements religieux) } \\
\text { Hôpitaux privés : accords } \\
\text { d'établissement ou absence } \\
\text { de couverture } \\
\text { conventionnelle }\end{array}$ \\
\hline
\end{tabular}

Source : Brandt/Schulten (2008c, p. 572, document actualisé).

\section{Télécommunications}

Le secteur des télécommunications présente un paysage conventionnel extrêmement éclaté, constitué pour l'essentiel de conventions d'établissement (Sauerland, 2008). La privatisation des télécommunications, qui relevaient de l'ancienne Deutsche Bundespost, et la création de la Deutsche Telekom AG en 1995 ont entraîné une vaste restructuration de l'entreprise, puis la définition en 2001 d'un nouveau système en remplacement des conventions précédentes, très proches de celles du service public. Parallèlement, la «politique de filialisation» du groupe a également gagné le terrain des conventions collectives, si bien qu'à un ancien champ d'application unique se sont substituées 12 conventions d'établissement distinctes pour les différentes filiales de DT AG (ibid., p. 102). Alors que les personnels déjà en poste conservaient généralement les droits acquis, les nouveaux salariés furent souvent considérablement moins bien lotis. En 2007, Deutsche Telekom a finalement externalisé environ 55000 emplois vers trois sociétés de services. Après 11 semaines de lutte sociale, les revenus existants furent certes garantis, ainsi qu'une sécurité d'emploi de plu- 
sieurs années. Mais, dans le même temps, les salaires d'entrée furent durablement abaissés de $6,5 \%$ et le temps de travail hebdomadaire porté de 34 à 38 heures sans compensation salariale (Schröder, 2007).

Après la libéralisation du secteur en 1998, bon nombre de nouveaux opérateurs de télécommunications se sont pressés sur le marché. Ce sont le plus souvent de petites et moyennes entreprises (PME) au champ d'action local ou régional, n'appliquant pour la plupart d'entre elles aucune convention collective. Les grands opérateurs de téléphonie mobile d'envergure régionale Vodafone, Accor et $\mathrm{O} 2$ disposent d'accords d'établissement, conclus non par ver.di, le syndicat compétent pour le secteur des télécoms, mais par IG Metall, Transnet ou IG BCE. Cette singularité tient à l'histoire de ces entreprises, issues à l'origine de la métallurgie, de la Deutsche Bundesbahn ou de la chimie. L'opérateur E-Plus, présent au niveau national, n'adhère à l'heure actuelle à aucune convention.

\section{Services postaux}

A l'instar de Deutsche Telekom, Deutsche Post AG dispose également d'une série d'accords d'établissement pour les différentes filiales du groupe avec, parfois, des conditions de travail et de rémunération très disparates. Jusqu'à la fin de l'année 2000, les salaires des employés de l'ancienne Bundespost étaient fixés par la convention ad hoc («Tarifvertrag Bundespost»), qui suivait les augmentations de salaire de la convention BAT pour le service public. La décorrélation par rapport aux évolutions de salaire du service public fut définitive après l'entrée en bourse de Deutsche Post AG en 2000 (Brandt/Schulten, 2007, p. 14). La conclusion d'une nouvelle convention cadre pour les ouvriers en 2001 et pour les employés en 2003 signa l'introduction d'une nouvelle structure de rémunération au sein du groupe. Ont été instaurées de fait des rémunérations à « deux vitesses », les personnes recrutées jusqu'en 2001 continuant de bénéficier de la convention «DP AG Besitzstand (DP AG « Droits acquis »), tandis que les nouveaux personnels (soit aujourd'hui environ un quart de l'ensemble des salariés) se voient appliquer la convention DP AG. Les différences de rémunération entre les deux conventions peuvent aller jusqu'à $30 \%$ (Brandt et al., 2007, p. 270).

Avec la libéralisation progressive du marché du courrier depuis 1999, achevée le $1^{\mathrm{er}}$ janvier 2008, une multiplicité de nouvelles entreprises postales privées ont été créées, dont aucune n'est soumise à une convention collective, pas même les deux plus gros concurrents du marché PIN AG Group et TNT, dont le niveau de salaire était en 2006 inférieur de 30 à $60 \%$ à celui de Deutsche Post (ibid.). Le niveau de salaire étant parfois extrêmement bas dans les nouvelles entreprises privées, un salaire minimum légal a été défini à l'automne 2007, à la suite d'une initiative conjointe de ver.di et de la Deutsche Post. En vertu de la loi sur le détachement des travailleurs, ce plancher a été déclaré 
applicable à l'ensemble du secteur postal (Brandt/Schulten, 2008b). L'entreprise néerlandaise TNT en particulier refuse toujours de reconnaître ce salaire minimal et a conclu une convention séparée, d'abord avec le syndicat GNBZ puis avec la confédération chrétienne $\mathrm{CGB}$, avec des salaires minimaux nettement inférieurs. Le tribunal du travail examine actuellement la légalité de ce salaire minimum instauré dans le secteur postal.

\section{Transports ferroviaires}

Jusqu'à la libéralisation formelle de la Deutsche Bundesbahn en 1994, les accords du service public étaient, pour l'essentiel, intégrés aux négociations tarifaires, formellement indépendantes (Keller, 2007). Maintenant, des accords d'établissement autonomes existent au sein de la Deutsche Bahn AG pour les différentes divisions du groupe.

On observe également, dans certains cas, un éclatement du paysage conventionnel entre les catégories professionnelles depuis que le syndicat des conducteurs de locomotives (GDL) a réussi en 2007 à obtenir pour la première fois des dispositions séparées pour les conducteurs de trains (Bispinck/Dribbusch, 2008).

Enfin, avec la DB Heidekraut GmbH, la Deutsche Bahn AG dispose d'une filiale dépourvue de couverture conventionnelle et versant des salaires inférieurs (jusqu'à $20 \%$ ) à ceux généralement pratiqués au sein du groupe. Le syndicat Transnet craint aujourd'hui que la création de nombreuses filiales (jusqu'à 30) dédiées aux transports régionaux, annoncée par la Deutsche Bahn, ne se fasse en dehors de toute convention collective, suivant l'exemple de la Heidekrautbahn.

Dans les transports ferroviaires régionaux précisément, beaucoup de nouvelles entreprises - dont des filiales de groupes de transports présents à l'échelle internationale comme Connex, Keolis ou SBB - se disputent aujourd'hui les marchés publics. Ces dernières disposent généralement d'accords d'établissement ou bien sont couvertes par les conventions collectives signées par les organisations patronales régionales; certaines entreprises ne sont liées à aucune convention collective. Du fait de chevauchements avec les transports de proximité, le syndicat ver.di intervient parfois dans les négociations de ces secteurs, aux côtés des syndicats ferroviaires. Chez les nouveaux opérateurs, le niveau de protection des salariés est toutefois nettement inférieur à celui en vigueur à la Deutsche Bahn AG, ce qui met le groupe sous pression.

Afin de limiter la concurrence et d'endiguer le développement des bas salaires, Transnet propose de fixer, pour l'ensemble du secteur des transports ferroviaires, une grille de salaires minimaux afin de définir, comme dans le secteur du bâtiment, les planchers généralement applicables aux différents niveaux de qualification (Kirchner, 2008, p. 161-162). 


\section{Transports publics de personnes}

Les entreprises de transports publics relèvent traditionnellement du champ d'application des conventions collectives du service public. L'apparition d'entreprises privées de plus en plus nombreuses et la modification des pratiques d'appels d'offres des municipalités ont toutefois fondamentalement changé le paysage conventionnel dans ce secteur (Brandt/Schulten, 2008a, p. 82 sq.). Il existe maintenant dans la plupart des Länder une convention de sous-secteur, la convention des transports de proximité (TV-N), qui suit, surtout dans ses dispositions cadre, celle du service public, tout en étant nettement moins favorable que celle-ci, en particulier pour ce qui est des grilles de rémunération. L'idée initiale était d'établir une nouvelle convention collective pour les transports de proximité intégrant également les domaines nettement moins favorisés des transports privés (autobus p. ex.). Il en est résulté de facto une coexistence entre la convention TV-N (couvrant la grande majorité des entreprises de transports publics), de multiples accords d'établissement et de conventions signées par les organisations régionales d'employeurs, et une absence de couverture conventionnelle dans des entreprises privées du secteur. Les disparités salariales qui en découlent peuvent aller jusqu'à $50 \%$ pour une même activité (ibid.). Les quotas d'appels d'offres, négociés dans les accords d'application du TV-N et limitant la part des prestations de transports qui peuvent être externalisées par les communes, ont un rôle important de stabilisation du système de conventions collectives de ce secteur.

\section{Secteur de l'énergie}

Dans le secteur de l'énergie, le système de conventions collectives est extrêmement complexe. La libéralisation du marché électrique a entraîné la disparition de l'autorité de surveillance de l'électricité et la suppression légale des monopoles de réseaux locaux des grands opérateurs historiques. Les pouvoirs publics ont cédé des participations aux grands groupes électriques. A l'échelon local, de nombreuses régies municipales ont vendu des parts aux grands groupes, désormais au nombre de quatre, depuis 2002 et les vagues de fusion (Brandt/Schulten, 2007). Les employés d'E.ON, RWE et Vattenfall Europe sont rémunérés sur la base de conventions collectives de groupe.

Dans les Länder, des conventions collectives régionales s'appliquent à de nombreux opérateurs régionaux et à quelques régies municipales. En revanche, les régies municipales, le plus souvent détenues par les communes, sont soumises à la convention «Entreprises d'approvisionnement » (TV-V), accord de sous-secteur créé en 2000 en annexe à la convention du service public (BAT/BMTG). Les écarts sont, pour le revenu annuel, de l'ordre de $40 \%$. Ces disparités considérables entraînent un risque de dérapage des salaires, dans une logique de dumping. Une plus grande coordination et une convention collective 
sectorielle, souhaitable à moyen terme, sont selon ver.di le seul moyen d'endiguer la pression sur les salaires et la sortie des conventions qui découlent des externalisations (Bergelin, 2008).

\section{Gestion des déchets}

Le secteur de la gestion des déchets présente un paysage en deux volets (Mendroch, 2008) : les entreprises communales relèvent la plupart du temps de la convention collective du service public (TV-ÖD). Pour le secteur privé, une convention collective sectorielle a été signée entre ver.di et la fédération allemande des entreprises de traitement des déchets (BDE), mais elle couvre à peine un tiers des personnels du secteur, plus des deux tiers des personnes employées dans des entreprises privées de traitement des déchets ne bénéficiant en revanche d'aucune couverture conventionnelle. La concurrence dans ce secteur est surtout attisée par la pratique des appels d'offres, qui expose les entreprises à une énorme pression sur les coûts, celle-ci étant répercutée directement sur les personnels. Pour lutter contre la baisse des salaires, ver.di, la BDE et la fédération des associations d'employeurs communaux (VKA) ont ouvert des négociations sur l'introduction d'un salaire minimum sectoriel, qui doit être déclaré universellement applicable en vertu de la loi sur le détachement des travailleurs (AEntG). Ce principe de salaire minimum se heurte toutefois à l'opposition virulente des PME privées du secteur. Début 2009, le gouvernement fédéral a finalement adopté l'introduction d'un salaire minimum pour le secteur du traitement des déchets, avec effet en mai 2009; ce dispositif établit au moins un plancher afin de limiter le dumping salarial.

\section{Hôpitaux}

La libéralisation touche le secteur hospitalier depuis les années 1990, sous la forme d'une vaste réforme du financement des hôpitaux notamment. Avec l'introduction de budgets plafonnés et de forfaits, les hôpitaux se sont retrouvés face à une pression accrue sur les coûts, et la volonté politique de réduire les capacités hospitalières a entraîné dans certains cas une concurrence d'éviction. Les coûts de personnel étant de plus de $60 \%$ dans le secteur hospitalier, cette concurrence a surtout eu des conséquences pour les salariés. En outre, le cumul de nombreuses années de financement insuffisant a déclenché une vague durable de privatisations des établissements publics qui a entrâné la constitution de groupes hospitaliers privés, obéissant à une logique de profit, comme RhönKlinikum, Helios ou Asklepios.

La structure conventionnelle est très éclatée (Brandt/Schulten, 2008b ; Gröschl-Bahr/Stumpfögger, 2008). Les hôpitaux publics relèvent traditionnellement du champ d'application des conventions du service public mais, pour faire face aux difficultés financières, un certain nombre d'hôpitaux publics ont 
signé des accords de crise leur permettant de déroger provisoirement aux dispositions prévues par ces conventions. Les hôpitaux à but non lucratif, assez nombreux traditionnellement et gérés par des associations religieuses ou caritatives, appliquaient autrefois systématiquement des conditions très proches de celles définies par les conventions collectives du service public. Mais l'exacerbation de la concurrence sur les coûts a fragilisé cette situation (Kühnlein/Wohlfahrt, 2006). Les hôpitaux privés disposent généralement d'accords d'établissement ou bien n'ont même aucune convention collective ; ver.di a récemment réussi à imposer, en particulier pour les grands groupes hospitaliers privés, des accords collectifs qui suivent la convention du service public TV-ÖD. Cependant, les dépenses de personnel sont globalement inférieures de $10 \%$ environ à leur niveau dans les hôpitaux publics (Brandt/Schulten, 2008b).

OUTRE LA DÉCENTRALISATION DE LA POLITIQUE DE DIALOGUE SOCIAL dans les secteurs industriels et la faible couverture conventionnelle dans le tertiaire privé, la libéralisation et la privatisation des services publics constituent un troisième facteur essentiel d'érosion du système allemand de conventions collectives. Le système appliqué au service public, dont le champ d'application était vaste dans le passé, se limite de plus en plus aux activités clés de l'administration publique, tandis que la libéralisation crée de nouveaux marchés qui ne sont pas inscrits dans une régulation globale. Dans le tertiaire privé, les structures de conventions collectives sont au contraire très éclatées, ce qui ne leur permet pas de contenir la concurrence sur les salaires et les coûts du travail. Ces évolutions aboutissent à une «re-marchandisation» des relations économiques et sociales du secteur public (Atzmüller/Hermann, 2004a ; McDonald/Ruiter, 2006).

Dans ce contexte, les syndicats, dont la mission politico-économique fondamentale consiste à juguler la concurrence entre les travailleurs, doivent s'interroger sur les points d'appui politiques possibles afin de parvenir à restaurer des normes (minimales) sectorielles homogènes sur les nouveaux marchés libéralisés. Compte tenu de l'éclatement des structures conventionnelles, il est essentiel que les syndicats créent les conditions d'une politique de négociation coordonnée à l'échelle de la branche. Ceci est tout sauf simple d'un point de vue organisationnel, d'autant que plusieurs syndicats interviennent dans certains secteurs comme les télécommunications, les transports de proximité ou le rail, et qu'une coopération intersyndicale est par conséquent nécessaire.

Autre point d'ancrage possible: la définition de minima de branche, en débat dans certains secteurs et déjà signée dans celui des postes, qui deviendraient applicables à toutes les entreprises allemandes et étrangères en vertu de la loi sur le détachement des travailleurs. Une telle stratégie trouve un appui dans le camp du patronat chez ceux qui souhaitent empêcher une concurrence sauvage et souhaitent à ce titre des conditions de concurrence loyales. Les employeurs disposent toutefois d'une importante force de blocage qui leur permet de refuser à tout moment de signer une convention collective. C'est pourquoi il 
faudrait introduire, à l'appui d'une telle stratégie, un salaire minimum légal, général et interprofessionnel (Bispinck/Schulten, 2008).

Mais le salaire minimum à lui seul ne permet que de limiter partiellement la concurrence sur les salaires et les coûts du travail. C'est pourquoi on peut se demander, pour ouvrir la perspective, comment il est possible de redonner une homogénéité aux structures de conventions collectives disparates dans les secteurs libéralisés et privatisés et de conclure de nouveaux accords sectoriels. Dans les années 1990, cette question a fait l'objet d'un vaste débat au sein des syndicats sur l'établissement de conventions de sous-secteurs dont l'idée fondamentale consistait à préserver la couverture conventionnelle et les négociations dans le service public, tout en intégrant de nouvelles entreprises privées dans des régulations de branche. C'est pourquoi la convention applicable aux transports de proximité ( $T V$-Nahverkehr) définit des rémunérations inférieures à celles du service public mais supérieures à celles prévues dans les accords signés avec les entreprises de transports privées. Les conventions collectives de sous-secteur existantes n'ont cependant pas conduit à l'établissement de nouvelles conventions de branche. A l'origine, la signature d'un accord distinct dans les transports de proximité devait aller de pair avec l'imposition d'une nouvelle régulation, l'Etat s'engageant à n'attribuer de marchés qu'aux entreprises qui appliqueraient l'accord. En pratique, seuls quelques rares Länder ont appliqué de telles régulations, alors qu'une loi fédérale visant à imposer le respect des conventions (Tariftreuegesetz) s'est heurtée à la résistance politique des partis de l'Union (CDU/CSU) (Schulten/Pawicki, 2008).

Dans un arrêt de principe rendu en avril 2008, la Cour de justice de l'Union européenne (CJUE), jugeant les règlements relatifs au respect des conventions contraires à la liberté de prestation des services, les a déclarés irrecevables. On aboutit ainsi à cette situation grotesque que l'Etat, donneur d'ordre obligé de retenir l' «offre la plus économique », se trouve en principe contraint de privilégier les entreprises les moins chères car n'appliquant aucune convention collective. L'Etat participerait ainsi lui-même d'une érosion accrue du système conventionnel. Des dispositions légales en faveur du respect des conventions collectives (Tariftreueregelungen) restent ainsi une condition essentielle à la création de nouvelles conventions de branche dans les secteurs libéralisés, et il faudrait leur donner les garanties nécessaires en droit européen.

En l'absence de dispositions de ce type, les seules possibilités qui restent sont l'applicabilité générale des conventions collectives de branche et l'inscription de l'ensemble de la convention dans la loi sur le détachement des travailleurs afin de contraindre les entreprises étrangères à respecter les mêmes normes de protection lorsque les frontières juridiques et administratives seront supprimées, à compter de 2011 (Bispinck/Schulten, 2009). Or, l'applicabilité générale est un instrument soumis à des règles extrêmement restrictives en Allemagne, ce qui n'est pas le cas dans d'autres pays européens; elle laisse donc aux employeurs une importante force de blocage, conduisant en pratique à la 
réduction continue de la part des conventions collectives généralement applicables depuis les années 1990. Un recours accru à cet instrument supposerait donc préalablement une réforme profonde de simplification et d'allègement des procédures de déclaration d'applicabilité générale (Zachert, 2004).

L'érosion des conventions collectives à la suite de la libéralisation et de la privatisation des services publics fait très nettement apparaitre les responsabilités politiques dans ce processus. Contrairement à la plupart des autres Etats d'Europe de l'Ouest, l'Allemagne ne dispose pas d'institutions pour étayer politiquement le système de protection des salariés (Schulten, 2004). La perte de légitimité de la libéralisation et de la privatisation, très répandue dans l'opinion publique et due notamment à leur impact sur les salariés, pourrait fournir le point de départ d'une réflexion nationale sur la re-stabilisation politique du système de conventions collectives.

Traduction de Marie GRAVEY

\section{Indications bibliographiques}

ARTUS I., Krise des deutschen Tarifsystems. Die Erosion des Flächentarifvertrags in Ost und West, Westdeutscher Verlag, Wiesbaden, 2001

Atzmüller R., Hermann C., « Veränderung öffentlicher Beschäftigung im Prozess der Liberalisierung und Privatisierung », Österreichische Zeitschrift für Soziologie (ÖZS), n 4, 2004a, p. 30-48

Atzmüller R., Hermann C., Liberalisierung öffentlicher Dienstleistungen in der EU und Österreich. Auswirkungen auf Beschäftigung, Arbeitsbedingungen und Arbeitsbeziehungen, Kammer für Arbeiter und Angestellte Wien, Vienne, 2004b (Zur Zukunft öffentlicher Dienstleistungen, 2)

Bergelin S., « Energiewirtschaft », in Brandt T., Schulten T., STERKel G., WiEdemUth J. (eds), Europa im Ausverkauf. Liberalisierung und Privatisierung öffentlicher Dienstleistungen und ihre Folgen für die Tarifpolitik, VSA-Verlag, Hambourg, 2008, p. 121-130

BISPINCK R., « Abschied vom Flächentarifvertrag? Der Umbruch in der deutschen Tariflandschaft », in WSI (ed), WSI-Tarifhandbuch 2006, Francfort s/ Main, 2006a, p. 41-66

BISPINCK R., « Mehr als nur ein Streit um 18 Minuten - Die Tarifauseinandersetzung im öffentlichen Dienst 2006 », WSI-Mitteilungen, $\mathrm{n}^{\circ}$ 7, 2006b, p. 374-388

BISPINCK R. (ed), Wohin treibt das Tarifsystem?, VSA-Verlag, Hambourg, 2007

BISPINCK R., DRIBbUSCH H., « Tarifkonkurrenz der Gewerkschaften zwischen Über- und Unterbietung », Sozialer Fortschritt, ${ }^{\circ}$ 6, 2008, p. 153-163

BISPINCK R.,SCHULTEN T., «Verbetrieblichung der Tarifpolitik? Aktuelle Tendenzen und Einschätzungen aus Sicht von Betriebs- und Personalräten“, WSI Mitteilungen, n 3, 2003, p. 157-166

BISPINCK R., SCHUlten T., « Aktuelle Mindestlohndebatte: Branchenlösungen oder gesetzlicher Mindestlohn?», WSI-Mitteilungen, ${ }^{\circ}$ 3, 2008, p. 151-158

BISPINCK R., SCHUlten T., «Re-Stabilisierung des deutschen Flächentarifvertragsystems », WSI-Mitteilungen, $n^{\circ} 4,2009$, p. 201-209

BORDOGNA L., Industrial relations in the public sector [en ligne], European Foundation for the Improvement of Living and Working Conditions, Dublin, 2007 (Comparative Studies). Disponible sur : http://www.euro found.europa.eu/eiro/studies/tn0611028s/tn0611028s.html [consulté le 21/04/2011] 
BRANDT T., DREWS K., SChUlten T., « Liberalisierung des deutschen Postsektors - Auswirkungen auf Beschäftigung und Tarifpolitik », WSI-Mitteilungen, $n^{\circ}$ 5, 2007, p. 266-273

BRANDT T., SCHULTEN T., Liberalisation and Privatisation of Public Services and the Impact on Labour Relations: A Comparative View from six Countries at the Postal, Hospital, Local Public Transport and Electricity Sector, Report for the EU-Project "Privatisation of Public Services and the Impact on Quality, Employment and Productivity (PIQUE)" [en ligne], Wirtschafts- und sozialwissenschaftliches Institut (WSI), Fondation Hans Böckler, Düsseldorf, 2007. Disponible sur : www.pique.at/reports/pubs/PIQUE_028478_Del8.pdf [consulté le 21/04/2011]

BRAndt T., Schulten T., «Auswirkungen von Privatisierung und Liberalisierung auf die Tarifpolitik in Deutschland. Ein vergleichender Überblick », in BRANDT T., SCHULTEN T., STERKEL G., WIEDEMUTH J. (eds), Europa im Ausverkauf. Liberalisierung und Privatisierung öffentlicher Dienstleistungen und ihre Folgen für die Tarifpolitik, VSA-Verlag, Hambourg, 2008a, p. 68-259

BRANDT T., SCHULTEN T., « Privatisation and Liberalisation of Public Services in Germany: the Example of the Postal and the Hospital Sector », in KeUne M., LeschKe J., WATT A. (eds), Privatisation and Marketisation of Services - Social and Economic Impacts on Employment, Labour Markets and Trade Unions, ETUIREHS, Bruxelles, 2008b, p. [n.c.]

BRANDT T., SCHULTEN T., « Liberalisierung und Privatisierung öffentlicher Dienstleistungen und die Erosion des Flächentarifvertrags », WSI-Mitteilungen [en ligne], n 10, 2008c, p. 570-576. Disponible sur : http://www. boeckler.de/pdf/wsimit_2008_10_brandt.pdf [consulté le 21/04/2011]

BrandT T., SCHUlten T., «The Impact of Liberalisation and Privatisation on Labour Relations », Policy Paper [en ligne], $\mathrm{n}^{\circ}$ 2, 2008d. Disponible sur: http://www.pique.at/reports/pubs/PIQUE_PP2_19_06_2008.pdf [consulté le 21/04/2011]

Brandt T., SChUlten T., Die Folgen von Liberalisierung und Privatisierung für Arbeitsbeziehungen, Kammer für Arbeiter und Angestellte Wien, Vienne, 2009, (Zur Zukunft öffentlicher Dienstleistungen. Die Privatisierung öffentlicher Dienstleistungen und deren Auswirkungen auf Qualität, Beschäftigung und Produktivität,7)

Dribbusch H., Schulten T., « The End of an Era: Structural Changes in German Public Sector Collective Bargaining », in Leisink P., STEIJn B., VeERsma U., Industrial Relations in the New Europe, Edward Elger, Cheltenham, 2007, p. 155-176

EllguTh P., KoHAUT S., « Tarifbindung und betriebliche Interessenvertretung: Aktuelle Ergebnisse aus dem IAB-Betriebspanel $2007 »$, WSI-Mitteilungen, $\mathrm{n}^{\circ}$ 9, 2008, p. 515-519

GröSchl-BAhr G., STUMPfögger N., « Krankenhäuser », in Brandt T., Schulten T., STERKEL G., WiEDEMUTH J. (eds), Europa im Ausverkauf. Liberalisierung und Privatisierung öffentlicher Dienstleistungen und ihre Folgen für die Tarifpolitik, VSA-Verlag, Hambourg, 2008, p. 165-180.

KELLER B., Arbeitspolitik des öffentlichen Sektors, Nomos, Baden-Baden, 1993

Keller B., « Wandel der Arbeitsbeziehungen im öffentlichen Dienst: Entwicklung und Perspektiven », Die Verwaltung, $\mathrm{n}^{\circ} 2$, 2007, p. 173-202

Killian W., Richter P., TrapP J. H., Ausgliederung und Privatisierung in den Kommunen. Empirische Befunde zur Struktur kommunaler Aufgabenwahrnehmung, Sigma, Berlin, 2006

Kirchner A., « Die Bahn », in Brandt T., Schulten T., STERKEl G., Wiedemuth J. (eds), Europa im Ausverkauf. Liberalisierung und Privatisierung öffentlicher Dienstleistungen und ihre Folgen für die Tarifpolitik, VSA-Verlag, Hambourg, 2008, p. 153-164

Kohaut S., SchNabel C., «Zur Erosion des Flächentarifvertrags. Ausmaß, Einflussfaktoren und Gegenmaßnahme », Industrielle Beziehungen, $\mathrm{n}^{\circ}$ 2, 2003, p. 193-219

Kohaut S., Schnabel C., «Tarifliche Öffnungsklauseln. Verbreitung, Inanspruchnahme und Bedeutung », Sozialer Fortschritt, ${ }^{\circ}$ 2, 2007, p. 33-40

KüHNLEIN G., WohlfahrT N., «Soziale Träger auf Niedriglohnkurs? - Zur aktuellen Entwicklung der Arbeits- und Beschäftigungsbedingungen im Sozialsektor », WSI-Mitteilungen, ${ }^{\circ}$ 7, 2006, p. 389-395 
MCDonald D. A., Ruiter G., «Rethinking Privatisation. Towards a Critical Theoretical Perspective », in CHAVez D. (ed), Beyond the Market: The Future of Public Services, Trans National Institute (TNI) Public Services Yearbook 2005/6, 2006, p. 10-20

Mendroch E., « Abfallwirtschaft », in Brandt T., Schulten T., STERKel G., Wiedemuth J. (eds), Europa im Ausverkauf. Liberalisierung und Privatisierung öffentlicher Dienstleistungen und ihre Folgen für die Tarifpolitik, VSA-Verlag, Hambourg, 2008, p. 131-138

OPPHOlzer A., ZACHERT U. (eds), Krise und Zukunft des Flächentarifvertrages, Nomos, Baden-Baden, 2000

REHDER B., Betriebliche Bündnisse für Arbeit in Deutschland. Mitbestimmung und Flächentarif im Wandel, Francfort/New York, 2003 (MPI für Gesellschaftsforschung, 48)

Sauerland F., « Telekommuniktion », in Brandt T., Schulten T., STERKel G., Wiedemuth J. (eds), Europa im Ausverkauf. Liberalisierung und Privatisierung öffentlicher Dienstleistungen und ihre Folgen für die Tarifpolitik, VSA-Verlag, Hambourg, 2008, p. 92-107

SCHNABEL C., «Arbeitnehmervertretungen im öffentlichen Sektor: Bedeutung und (potenzielle) Effizienzwirkungen », Diskussionspapiere des Lehrstuhls für Arbeitsmarkt- und Regionalpolitik an der FriedrichAlexander-Universität in Erlangen-Nürnberg, ${ }^{\circ}$ 52, 2007, p. [n.c.]

SCHRöDER L., « Der Tarifkonflikt bei der Deutschen Telekom AG », WSI-Mitteilungen, n 9, 2007, p. 515518

SCHULTEN T., « (Flächen-)Tarifvertragssysteme in Europa », in WSI (ed), WSI-Handbuch 2004, Francfort s/ Main, 2004, p. 43-64.

Schulten T., PAWICKI M., « Tariftreueregelungen in Deutschland - ein aktueller Überblick », WSI-Mitteilungen, $\mathrm{n}^{\circ} 4,2008$, p. 184-190

Zachert U., « Allgemeinverbindlicherklärung », in Peter G., KemPen O. E., ZaChert U., Die Sicherung tariflicher Mindeststandards, Nomos, Baden-Baden, 2004, p. 11-32. 
- III -

VERS DE NOUVELLES FORMES

D'EMPLOI ET DE MANAGEMENT ? 



\title{
Développement des ressources humaines dans les entreprises françaises de service public
}

\author{
Jean-François AMADIEU
}

Quels facteurs contribuent à l'évolution de la gestion des ressources humaines dans les secteurs libéralisés ou en cours de libéralisation? Le présent article offre des éléments de réponse, et met en lumière l'hétérogénéité des situations, en raison notamment d'un processus d'ouverture à la concurrence plus ou moins avancé selon les branches d'activité ou de la structuration complexe de certains opérateurs sur le marché. Toutefois, apparaissent, dans l'ensemble des secteurs concernés, des phénomènes d'hybridation public/privé.

Pour le compte du Bureau International du Travail, je me suis intéressé il y a quelques années à l'évolution de la gestion des ressources humaines dans l'ensemble des services d'intérêt général, également nommés public utilities. Dans ces secteurs, la France a longtemps évolué dans une situation particulièrement protégée, par ailleurs parfaitement identifiée par l'Union européenne. Cette situation de «surprotection », en comparaison avec d'autres pays, laissait présager de l'impact considérable de l'ouverture des marchés. Dans cette mesure, l'analyse de la situation actuelle se révèle riche d'enseignements. Car si mon propos consistera dans un premier temps à aborder les impacts de la dérégulation sur la gestion des ressources humaines et le management, je préciserai ensuite que ceux-ci sont extrêmement inégaux selon les secteurs, en raison de l'avancée disparate des processus de libéralisation. Dans un troisième temps, qui me paraît le plus important, j'évoquerai le fait que l'évolution considérable du management, de la gestion des ressources humaines et des relations sociales en France dans les entreprises de réseau, les services publics locaux ou le secteur des transports n'est pas uniquement le fruit de la libéralisation, mais s'explique par d'autres facteurs. Cela amène à réfléchir à des processus de fertilisation croisée ou à des évolutions convergentes susceptibles de se produire en France.

\section{Les impacts de la libéralisation en matière de} gestion des ressources humaines, ...

La libéralisation dans les différents secteurs, qu'elle soit achevée, en cours de réalisation ou à venir, exerce une pression notamment sur les salaires, les conditions d'emploi ou les effectifs, conduisant par exemple à des restructurations. Cela est tout à fait caractéristique de La Poste, qui se situe dès à présent en concurrence sur certains de ses marchés (colis, courrier non adressé). Pour les plis en revanche, le processus a été retardé, se déroulant progressivement. L'impact de l'ouverture au marché est colossal en termes d'effectifs. Car bien qu'il soit pos- 
sible d'intégrer des facteurs de protection envers la concurrence, ce qui constitue l'une des particularités du système français, celle-ci peut tout de même s'exercer dans les grandes villes. Cela vaut notamment pour le point névralgique de la clientèle : les entreprises, déterminantes en termes de volume.

Sur les salaires, les conséquences sont liées à l'instauration d'un système de « double échelle » en matière de personnel, qui rassemble employés à statut, ou fonctionnaires, et personnels contractuels, de droit privé. Bien que France Télécom emploie encore une majorité de fonctionnaires, de même que La Poste dans une moindre mesure, il s'agit en réalité de corps en attrition. Au niveau du statut des personnels et de leurs conditions d'emploi, les nouveaux systèmes se révèlent nettement moins favorables que les précédents.

Par ailleurs, les groupes français recourent largement à la filialisation afin de faire évoluer les statuts : nombre d'activités s'exercent sous une forme juridique qui permet d'intégrer des statuts du personnel différents, relevant d'autres conventions collectives. La stratégie de filialisation de ces groupes a pour visée d'exercer à des conditions plus concurrentielles un certain nombre d'activités nouvelles dans d'autres pays européens. Ainsi, via des filiales dévolues, les groupes français sont parfois finalement plus concurrentiels à l'étranger qu'ils ne le seraient sur le territoire national. D'ailleurs, l'une des raisons des levées de fonds au sein de La Poste est de permettre un développement externe ou développement par des activités de filiales, souvent contesté par les syndicats.

Dans le domaine des relations sociales, le fait de prévenir les conflits, de les réguler, comme dans le cas d'Air France en 1998, voire éventuellement de la RATP, correspond à une volonté d'être plus compétitif dans le service au client, dans un contexte plus concurrentiel. Dès lors, la continuité du service, la réduction du niveau de conflictualité, en d'autres termes la «paix sociale », s'inscrivent dans cette démarche.

Il est évident que dans un contexte concurrentiel, l'évaluation de la performance collective et individuelle, et notamment individuelle, revêt une plus grande importance. Au sein de France Télécom, les entretiens d'appréciation ont été mis en place dès le début des années 1990. Toutefois, il y a quelques années encore, la performance de chaque agent n'était pas affichée aux yeux de tous. Dans un mouvement déjà engagé s'est donc instauré un processus de durcissement, également patent sur le sujet de la flexibilité.

\section{... différenciés selon les secteurs}

L'impact de la libéralisation sur les conditions d'emploi de la main-d'œuvre et la gestion des ressources humaines reste toutefois à nuancer, dans un premier temps en raison de la prorogation de situations de monopole, de duopole ou d'oligopole, plaçant les entreprises concernées à l'abri de la concurrence. Par exemple, le secteur de l'eau ou de l'environnement était auparavant aux mains de deux grands opérateurs historiques en France, la Générale des Eaux et Suez. A l'heure ac- 
tuelle, les trois principaux acteurs sur le marché sont Veolia, Suez et La Lyonnaise des Eaux. Dans le secteur des télécoms, on peut se demander si la situation, qui d'ailleurs fait l'objet de contestations, est concurrentielle ou oligopolistique. La question se pose également dans le secteur de l'énergie (gaz, électricité). Et pour ce qui est de la RATP, si l'entreprise se trouve en concurrence sur les marchés de grande banlieue et dans certaines villes de province où elle entreprend des démarches d'implantation, elle est en situation de monopole dans Paris intra muros, qui demeure son cœur de métier. Il est important de rappeler, notamment dans un contexte franco-allemand, que si la situation est telle en France qu'elle a souvent été dénoncée par la Cour des Comptes, cela tient au fait que la concession des services publics locaux par les collectivités territoriales n'obéit pas à certains critères de transparence. Dans un contexte de protection envers la concurrence, les contrats conclus, de longue durée, rendent peu probable l'arrivée d'un concurrent sur le marché concerné.

En outre, lorsque des entreprises se voient privatisées dans le cas français, la forme juridique de l'entreprise change mais le personnel se voit offrir des garanties, faute de quoi ces réformes ne pourraient pas être adoptées. Ces garanties consistent à maintenir le statut en l'état pour les personnels, comme dans le cas récent de La Poste. Certes, cela ne signifie pas que tous les personnels se verront garantir de telles protections, mais elles concernent du moins le «noyau dur» d'agents de ces entreprises. Cela limite d'entrée de jeu la mise en œuvre de diverses mesures de flexibilité ou autres changements.

Ce qui limite aussi l'impact des changements en France est le fait que les entreprises qui interviennent dans ces secteurs ont pour caractéristique d'être des groupes très larges, des consortiums comprenant un nombre extrêmement élevé de filiales. Cette structuration leur permet d'être plus ou moins exposés à la concurrence selon les secteurs dans lesquels ils interviennent. Cela s'applique à des groupes comme Suez, Veolia, mais aussi à Bouygues, Vivendi, etc. Ces groupes présents sur de nombreux secteurs, voire parfois plusieurs secteurs de service d'intérêt général, disposent d'une certaine marge de manœuvre. Il s'agit de multinationales puissantes implantées sur des secteurs parfois extrêmement intéressants et rentables (comme celui des travaux publics), dont la gestion des ressources humaines se révèle hétérogène. Car les groupes Suez et Veolia sont constitués d'une nébuleuse d'entreprises à l'échelle mondiale. La gestion des ressources humaines n'y est pas intégrée: il n'existe en effet pas de politique centrale de gestion des ressources humaines ou de système d'information centralisé que l'on retrouve par exemple chez EDF. La Poste se dirige par ailleurs peu à peu vers cette situation, en ayant des métiers de plus en plus autonomes.

Il est par conséquent difficile de tirer une conclusion générale sur la gestion des ressources humaines au sein des groupes de service d'intérêt général, dont les choix s'avèrent diversifiés : protection du statut pour les uns, évolutions différenciées pour d'autres, ou instauration de filiales en vue de contourner le statut et de proposer des conditions d'emploi moins avantageuses. 


\section{Facteurs d'évolution de la gestion des ressources humaines}

Il apparât légitime de se demander quels facteurs contribuent à l'évolution de la gestion des ressources humaines dans les entreprises de service public. Car la libéralisation ne suffit pas à expliquer la baisse des effectifs. Par exemple, la situation actuelle du fret à la SNCF ne résulte pas uniquement de la mise en concurrence du secteur en France, mais aussi et surtout de la concurrence des modes de transport alternatifs, de sorte que l'évolution du fret dépend aujourd'hui de choix nationaux. Dans le secteur postal, l'opérateur historique se retrouve en grande difficulté pour des raisons à la fois conjoncturelles et structurelles. En effet, la baisse du volume du courrier, corollaire de la concurrence de l'Internet, a un effet direct sur les effectifs. Par conséquent, la baisse de charge ne résulte pas de la présence de la Deutsche Post ou d'autres opérateurs sur le marché.

Plus largement au sein de la fonction publique, il a été décidé de procéder à des baisses d'effectifs. Les plans de restructurations et de réduction d'effectifs menés par la France, certes avec retard par rapport à d'autres pays européens, mais menés effectivement, ne peuvent s'expliquer du fait de la libéralisation mais bien pour des raisons diverses, selon qu'ils concernent la carte militaire, la carte hospitalière, la carte judiciaire ainsi que celle des universités.

Les classifications sont quant à elles désormais établies à partir de cotations de postes et de fonctions, permettant de classer les emplois et de rémunérer le personnel en France. Cela se pratique de plus en plus dans la fonction publique ainsi que dans les entreprises de service d'intérêt général. Au sein de La Poste et de France Télécom, il avait été demandé aux cabinets Hay et Hewitt d'établir des cotations de postes dès le début des années 1990, avant même qu'il ne soit question de libéralisation du secteur postal.

Au regard de l'évolution des classifications en France, il est intéressant de constater que le mouvement qui a mené à l'abandon des logiques de grades de la fonction publique via les grilles Parodi pour s'acheminer vers des logiques de cotation de postes et de fonctions relatives au secteur privé, de type industrielles, a commencé suite à une décision de 1968, pour s'appliquer à partir des années 1974-75. Ainsi, comme pour les baisses d'effectifs, l'évolution des grilles de classifications françaises n'est pas liée au phénomène de libéralisation ou de privatisation des services publics.

Pour ce qui est des relations professionnelles, on assiste à une décentralisation de la régulation qui s'accompagne de la multiplication d'accords locaux conclus à l'échelle de l'entreprise ou d'un établissement, voire d'accords dérogatoires. Ce phénomène, décrit par Jean-Daniel Reynaud dès la fin des années 1970, s'applique non seulement à la France mais aussi à d'autres pays européens. Dans les services d'intérêt général et au sein d'entreprises comme la RATP, la Poste, la décentralisation de la négociation s'est accentuée. Comme dans les autres secteurs, de nombreux accords sont signés chaque année. Ce 
puissant mouvement de régulation ne s'explique pas non plus par une libéralisation du système.

Une autre évolution concerne le service minimum dans le service public. Les travaux de la commission Mandelkern, dont j'ai fait partie, ont donné lieu à la loi de 2007 sur la continuité de service public dans le transport terrestre de voyageurs, portant essentiellement sur les chemins de fer : RATP, SNCF et réseaux de province. La continuité de service fut mise en place non pas pour faire face à la concurrence comme c'était le cas pour Air France en 1998, mais plutôt en raison de la pression de l'opinion, d'ordre politique, et surtout de la nature constitutionnelle de l'exigence de continuité du service. Ainsi, le droit à un service public s'oppose au droit de grève. Il s'agit dès lors de les concilier et d'améliorer le service public.

Dans un contexte franco-allemand, il est intéressant de se demander si le fait de se diriger vers des systèmes de paix sociale prohibant dans une certaine mesure l'exercice de la grève constitue ou non une logique abjecte. Ce n'est toutefois pas le cas en France, où il n'existe pas de réquisition en l'état actuel de la législation : la loi prévoit uniquement de faire une déclaration préalable à la grève. On retrouve par ailleurs un volet de prévention en la matière, qui consiste plutôt à rapprocher la France de ce qui se pratique à l'étranger : c'est-à-dire mieux assumer le service public, la continuité de service dans le transport terrestre.

Dans le domaine des règles et modalités de recrutement du personnel, on observe actuellement des transformations profondes en France, mues par le droit de la discrimination. Celui-ci vient certes d'une impulsion essentiellement européenne qui a obligé la France à créer la Halde et à améliorer son système de lutte contre les discriminations. Dès lors, cela impacte la manière de recruter d'entreprises qui, en France, n'appliquaient pas de politiques appropriées en la matière. Les entreprises du secteur postal, de l'eau, de l'électricité ou du gaz auditent actuellement leurs processus et modifient leur manière de recruter leur personnel. Cette évolution n'a pas de lien avec une libéralisation quelle qu'elle soit, mais découle de l'émergence du droit de la discrimination.

Quant aux déroulements de carrière et salaires, ils sont largement impactés aujourd'hui par l'égalité professionnelle hommes/femmes. L'évolution de la rémunération des femmes à très court terme est désormais conditionnée par la fixation et le renforcement des règles en France en matière d'égalité salariale et de déroulement de carrière. A la demande des pouvoirs publics, les entreprises des secteurs considérés se doivent d'être exemplaires dans leurs politiques. Cela vaut également pour le handicap, les politiques de diversité, la question des seniors, des quartiers difficiles, etc.

Pour finir, la question du risque psychosocial constitue un sujet majeur. Les cas de suicides répertoriés chez France Télécom ne sont pas une nouveauté puisque Renault et EDF avaient été concernés par le passé. Des mesures ont été prises sur les problématiques de harcèlement, qui doivent être complétées. 


\section{Phénomènes d'hybridation public/privé}

Au niveau du recrutement, les entreprises du secteur privé découvrent les vertus des modalités du public. En recourant au CV anonyme, c'est-à-dire en essayant de rendre les modes de recrutement plus impersonnels, les entreprises privées se rapprochent de règles publiques fondées sur les concours. En ce qui concerne les méthodes de rémunération, l'adoption ces quinze dernières années d'une logique de rémunération en fonction des postes occupés par les individus engendre des problèmes de mobilité de la main-d'œuvre. Ainsi, il est tout à fait possible de retrouver des logiques de compétences, ou en d'autres termes des logiques de grades.

Pour ce qui est du traitement des cas individuels, la fonction publique (pas nécessairement les entreprises de réseaux) disposait de commissions administratives paritaires destinées à régler des problèmes individuels. Les entreprises qui appliquent des règles privées découvrent et mettent en place des commissions de ce type. Cela vaut pour la RATP (conformément à son accord de 2001) ou pour La Poste qui met en place des systèmes d'alerte pour l'ensemble du personnel. Des réflexions sont également menées au sein d'EDF. La recherche de systèmes destinés à mieux traiter les litiges individuels constitue un point de convergence, tout comme les enjeux affichés de responsabilité sociale (d'entreprise citoyenne) qui, au-delà de la communication, se rapprochent des préoccupations de service public.

On peut évoquer par ailleurs l'exemple du whistleblowing, en d'autres termes le signalement. L'article $40 \mathrm{du}$ code de procédure pénale peut faire obligation à un fonctionnaire de signaler au procureur les faits dont il serait témoin. La diffusion en France de ces systèmes d'alerte issus de la loi américaine Sarbanes-Oxley suscite des polémiques. Pourtant, il s'agit bien d'un exemple de convergence dans le fait que seront mis en place des mécanismes en interne afin d'empêcher que des faits délictueux, de harcèlement ou de discrimination ne soient perpétrés.

\section{Un taux de couverture conventionnel record en France}

En France, le pourcentage de salariés couverts par un accord ou une convention collective est très élevé. Les entreprises de réseaux notamment s'appuient sur des accords d'entreprises en grand nombre, concernant la garantie voire la création de statuts. On dénombre par ailleurs des accords de branche, notamment dans le secteur des télécommunications. Dans le secteur postal en revanche, le syndicat représentatif a des difficultés à conclure des conventions collectives couvrant l'ensemble du champ. Etant donné que La Poste constitue l'opérateur principal sur son marché, le groupe a tout intérêt à fixer des conventions limitant l'exercice d'une concurrence à bas coûts incarnée par les petits opérateurs. Pour conclure, il est important de noter que la libéralisation ne s'accompagne pas d'un abandon des accords de branche et des accords d'entreprise. Bien au contraire, les accords d'entreprise sont venus s'ajouter aux accords de branche dans les années 1980, de sorte que la régulation sociale connaît aujourd'hui une forte progression. 


\title{
Les entreprises publiques en mutation. Organisation et gestion interne dans les régies municipales
}

\author{
Dieter WAGNER, \\ avec la collaboration rédactionnelle de Magdalena CHMIELEWSKA
}

La recherche menée par Dieter Wagner s'appuie sur l'hypothèse selon laquelle les entreprises publiques posent une limite entre marché et Etat en développant des objectifs à but non lucratif qui leur sont propres. Dans les entreprises publiques, les décisions tireraient leur légitimité de missions déterminées par le politique, la priorité devant être donnée aux " objectifs opérationnels ", définis politiquement, face aux " objectifs organisationnels ", basés sur l'économie.

Mais selon le chercheur, il convient de relativiser cette théorie s'agissant des régies municipales d'électricité, étudiées ici, qui ont été exposées à des dérégulations de diverses natures. Les critères économiques tiennent de plus en plus de place dans leurs objectifs, l'influence politique étant perçue comme un facteur de perturbation. Le modèle est désormais l'entreprise privée, tandis que l'administration publique constitue un repoussoir.

Tout en demeurant le détenteur majoritaire, l'administration s'éloigne de plus en plus de l'entreprise publique.

\section{Grands objectifs des entreprises et priorités guidant leurs décisions internes}

Notre projet de recherche pose la question de l'autonomie du secteur public à côté de la politique et de l'économie. Une telle indépendance devrait se manifester dans des limites de sens (Sinngrenzen) distinguant l'économie publique des autres systèmes sociaux. Les organisations d'un système social font elles aussi la distinction entre nécessités propres et extérieures (cf. Willke, 1995). Ainsi, les administrations basent finalement leurs décisions sur la mise en œuvre de décisions politiques, les entreprises privées, sur les contraintes du marché.

Si l'on suit cette argumentation, la spécificité des entreprises publiques devrait apparaître également dans la singularité des processus de définition et de mise en œuvre de leurs objectifs. Potthoff (1994, p. 311 sq.) souligne que le propre de l'économie publique est le souci de l'intérêt général, avec un sens du service public et des missions à but non lucratif. Abromeit (1985) note que les entreprises publiques doivent définir leurs objectifs non pas à partir des seules forces du marché, mais aussi d'un processus politique. Bien que les «objectifs opérationnels » fixés politiquement aient la priorité sur les « objectifs organisationnels » économiques, leur mise en œuvre peut prendre une forme tout à fait 
« entrepreneuriale » et employer des «moyens conformes au marché » (Eichhorn, 1994b, p. 558).

Cette contribution analyse les principaux objectifs des entreprises publiques en étudiant l'exemple d'entreprises municipales du secteur de l'approvisionnement énergétique ${ }^{1}$. Ce secteur est en train de s'affranchir des anciennes restrictions que lui imposaient les monopoles territoriaux en matière de concurrence. Notre question majeure est la suivante : quels objectifs les régies municipales et les fournisseurs d'énergie communaux que nous avons étudiés poursuivent-ils dans cette période de dérégulation et de soumission accrue aux lois du marché ?

Interrogés sur leurs principes de management, environ les trois quarts des dirigeants de régies municipales et d'entreprises communales du secteur énergétique estiment vouloir servir aussi bien les clients, les collaborateurs et les investisseurs que la société. Leur préférence pour un compromis entre les différents intérêts ne les distingue par de leurs homologues du privé interrogés (cf. graphique 1).

\section{Graphique 1 : Principes de management ${ }^{2}$}

Question: Veuillez vérifier laquelle des thèses suivantes pourrait s'appliquer au mieux à votre entreprise. Merci de ne cocher qu'une seule réponse!

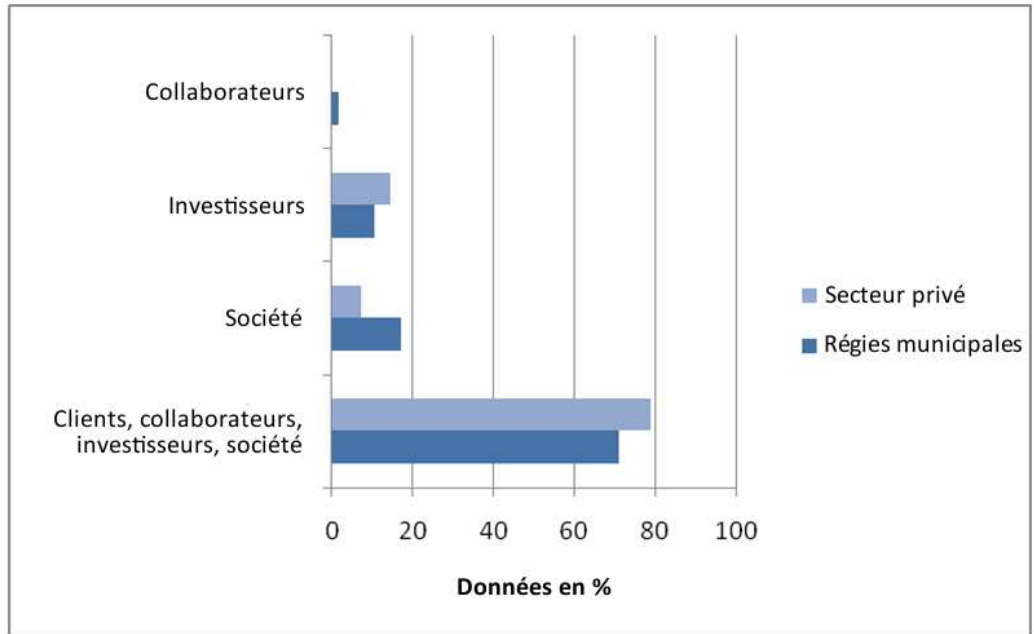

Source : Wagner/Edeling/Stölting, Öffentliche Unternehmen zwischen Privatwirtschaft und öffentlicher Verwaltung, VS Verlag, Wiesbaden, 2004.

\footnotetext{
${ }^{1}$ Nous ne ferons pas ici de distinction entre les fournisseurs d'énergie municipaux et les autres « domaines de régies municipales », quand cela n'est pas indiqué de manière explicite. En règle générale, ces domaines d'entreprises communales étaient interdépendants en raison de la gestion transversale des services publics communaux et, dans les structures d'entreprises, en raison des participations et de l'union personnelle des directions d'entreprises.

${ }^{2}$ Lors d'un colloque sur le management organisé il y a quelques années à Davos, les principes du management ont été soumis à discussion.
} 
Si l'on demande aux dirigeants d'entreprises municipales du secteur énergétique d'apprécier l'influence des objectifs politiques et économiques, on obtient un tableau nuancé (cf. graphique 2). Les objectifs politiques ne sont ni dominants ni complètement dépourvus d'importance. Mais un tiers environ des responsables interrogés indiquent que les objectifs économiques dominent dans leurs entreprises municipales. Environ $40 \%$ d'entre eux déclarent que leur entreprise poursuit des objectifs économiques uniquement dans la mesure où ceux-ci sont acceptables politiquement. On peut en déduire une certaine prédominance des objectifs économiques, sans que l'influence de la politique sur les décisions des entreprises ne soit toutefois complètement niée par les dirigeants des régies municipales.

\section{Graphique 2 : Objectifs globaux de l'entreprise}

Question: Les objectifs globaux peuvent différer d'une entreprise à l'autre. Parmi les déclarations suivantes, laquelle décrit le mieux le rapport entre les objectifs économiques et politiques de votre entreprise? Merci de ne cocher qu'une seule réponse!

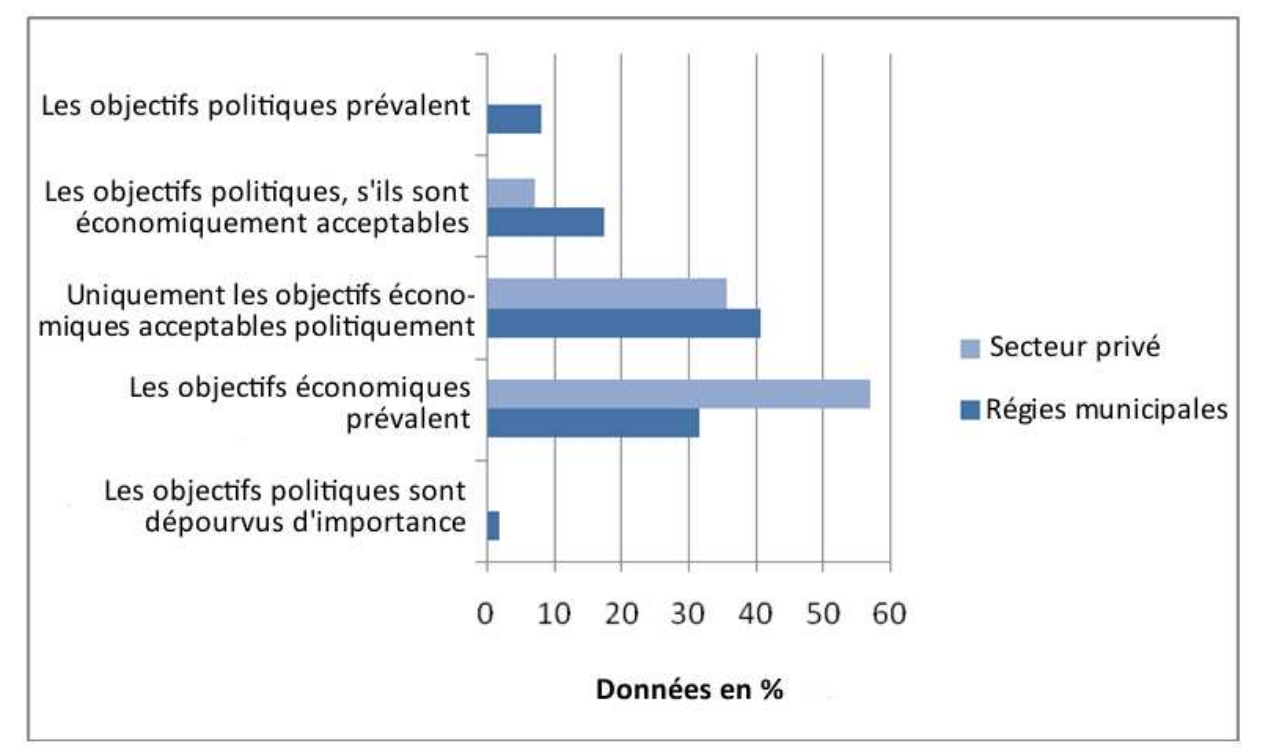

Source : Wagner/Edeling/Stölting, Öffentliche Unternehmen zwischen Privatwirtschaft und öffentlicher Verwaltung, VS Verlag, Wiesbaden, 2004.

Mais cette indécision ne saurait durer : comme celles du secteur privé, les entreprises publiques souhaitent surtout apparaître économiquement performantes, à l'écoute de la clientèle et techniquement innovantes. Les régies municipales semblent moins enclines que les dirigeants d'entreprises équivalentes dans le secteur privé à souhaiter que leur entreprise prenne des orientations favorables 
aux investisseurs de leurs entreprises, au sens de la « valeur pour l'actionnaire » et donc de la valeur de l'entreprise. Par rapport aux responsables des entreprises privées, elles se montrent aussi plutôt moins enclines à prendre des risques. Cette tendance s'explique encore aujourd'hui par la prédominance de capitaux publics dans les régies municipales, qui pour l'instant ne rend pas prioritaire la recherche d'investisseurs extérieurs ou la prise de décisions risquées aux fins de bénéfices maximums ; mais, dans son principe, la différence entre actions «à but non lucratif » et «à but lucratif » est amenée à s'estomper : dans l'idéal managérial des régies municipales, le souci de l'intérêt général des entreprises publiques vient après la performance économique et le souci du client. L'intérêt général ne disparaît pas pour autant des caractéristiques idéales des entreprises municipales, mais son poids est désormais celui d'une caractéristique parmi d'autres : il représente ni plus ni moins que dans les entreprises privées.

Graphique 3 : Caractéristiques idéales de l'entreprise

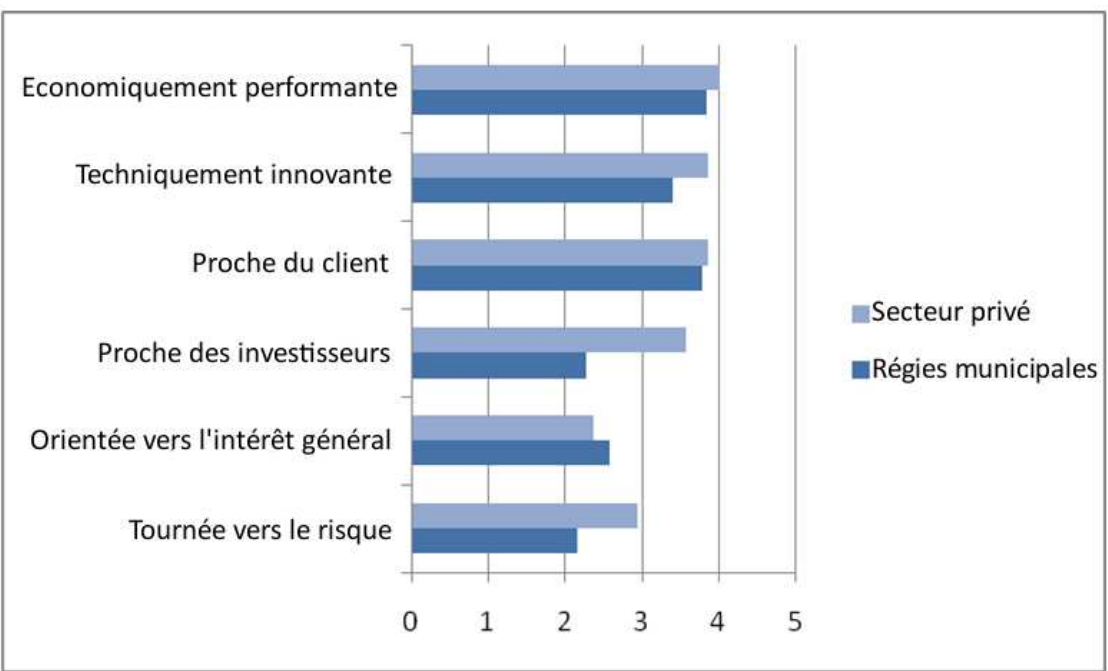

Source : Wagner/Edeling/Stölting, Öffentliche Unternehmen zwischen Privatwirtschaft und öffentlicher Verwaltung, VS Verlag, Wiesbaden, 2004.

Interrogés sur les contraintes qui se posent actuellement dans leurs entreprises en termes de management, les dirigeants des régies municipales soulignent, d'une part, des critères économiques, comme la réussite économique (cf. graphique 4). Mais ils jugent tout aussi important de se baser sur la mission définie par le politique et sur l'intérêt général. Selon les responsables de régies interviewés, c'est en cela que réside, aujourd'hui encore, la différence de gestion entre entreprises publiques et privées. Les notions d'intérêt général et de mission de service public sont, de leur point de vue, moins importantes dans les entreprises privées. Pour les régies ou les opérateurs municipaux, il leur semble 
en revanche nécessaire de garder à l'esprit la réputation de l'entreprise et de trouver un équilibre entre objectifs économiques et préoccupations politiques. Les personnes interrogées jugent également important le soutien de partis et associations. L'on peut déduire des contraintes citées que les objectifs économiques sont actuellement importants pour le management des régies, mais qu'ils sont loin de constituer le seul facteur dont les décisions des entreprises tirent leur légitimité. Mais le fait que l'intérêt général et la mission de service public ne soient plus prioritaires ne signifie pas que ces aspects ne pèsent plus sur les décisions des entreprises.

\section{Graphique 4 : Contraintes en termes de management}

Question:Veuillez énoncer les contraintes imposées aux managers de votre entreprise. Comparez-les à l'idée que vous vous faites des contraintes de l'autre type d'entreprise. (Veuillez énoncer les contraintes imposées aux responsables de votre administration. Comparez-les à l'idée que vous vous faites des contraintes des autres organisations). Barème : 4=très importantes, $1=$ sans importance.

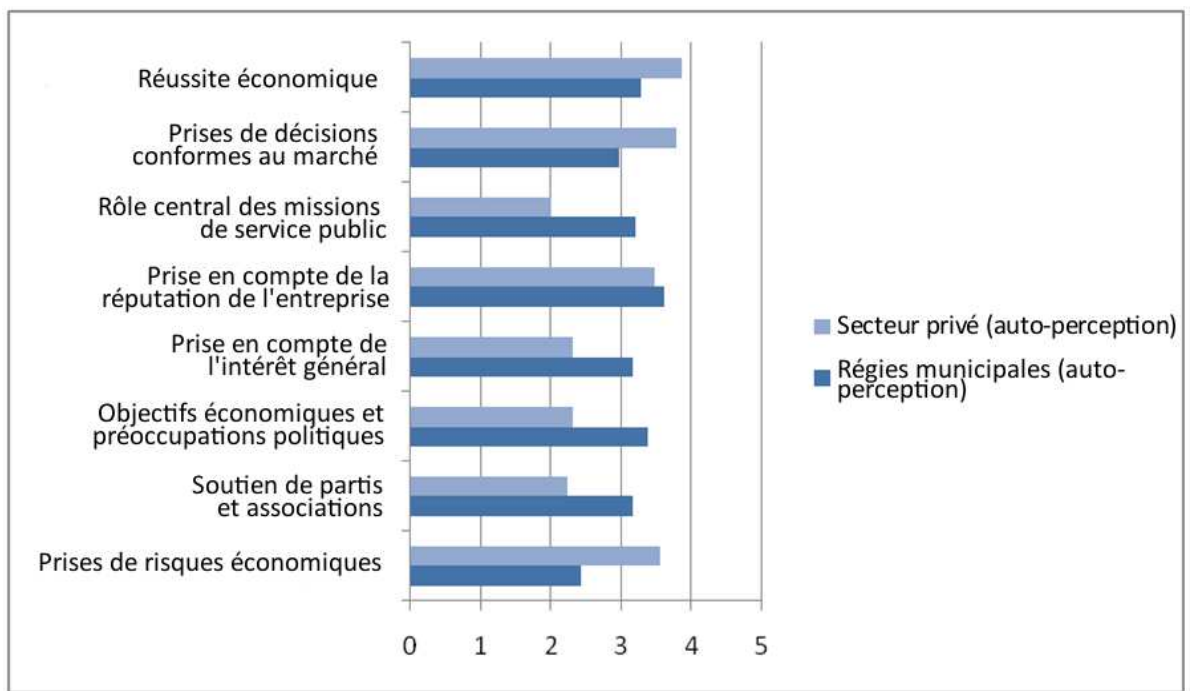

Source : Wagner/Edeling/Stölting, Öffentliche Unternehmen zwischen Privatwirtschaft und öffentlicher Verwaltung, VS Verlag, Wiesbaden, 2004.

Si l'on considère non pas l'ensemble de l'entreprise mais uniquement le domaine de travail immédiat des interviewés, la prédominance des critères économiques se renforce. Interrogés sur les conséquences à prendre en compte dans leur propre domaine de responsabilité directe, les cadres soulignent les critères économiques de leur action, comme la prise en compte de la concurrence, des bénéfices, de la fidélisation de la clientèle et de l'accueil des produits (cf. graphique 5). Ils soulignent également l'importance d'une prestation d'ap- 
provisionnement répondant aux besoins, à la fois en termes de volume et de qualité. L'influence des préoccupations politiques et de l'intérêt général tend à être perçue comme moins importante, y compris pour le domaine de responsabilité direct.

\section{Graphique 5 : Conséquences à prendre en compte}

Question: Quelles conséquences directes ou indirectes de votre action devezvous considérer lors de prises de décisions dans votre domaine de responsabilité ? Barème : $1=$ aucunement, $2=$ =eu, $3=$ prioritairement, $4=$ absolument .

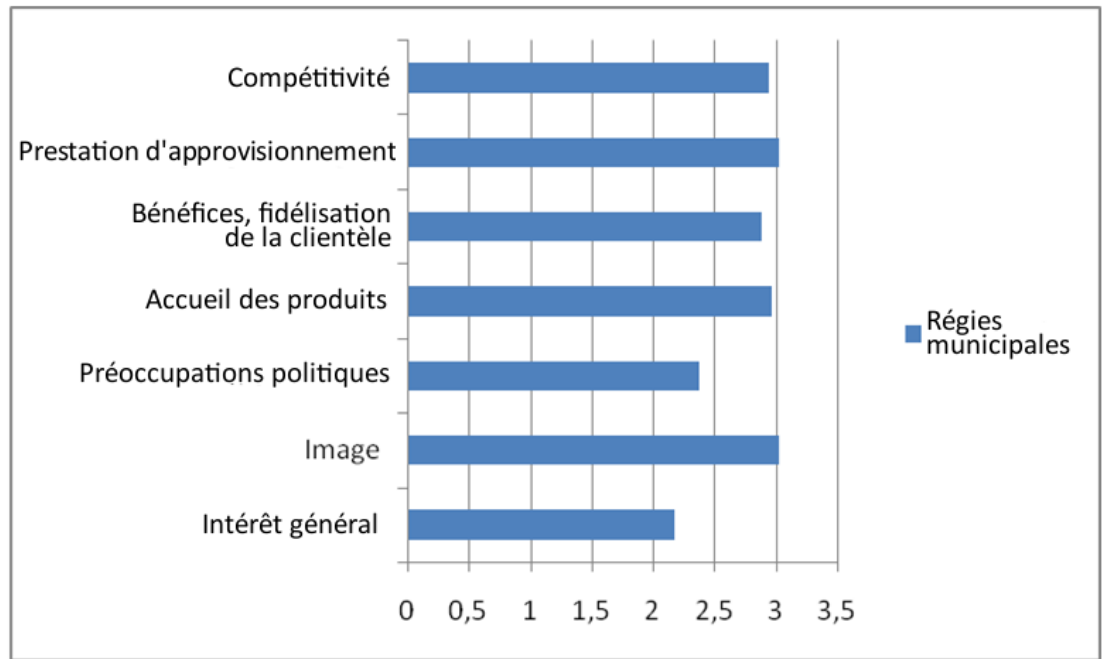

Source : Wagner/Edeling/Stölting, Öffentliche Unternehmen zwischen Privatwirtschaft und öffentlicher Verwaltung, VS Verlag, Wiesbaden, 2004.

Pour leurs prises de décisions, les dirigeants d'entreprises privées doivent - du point de vue des cadres de régies que nous avons interrogés - se baser, plus encore que ceux des entreprises publiques, sur les critères économiques. L'influence de la politique et le poids de l'intérêt général y sont donc faibles. Ces appréciations correspondent tendanciellement à celles des dirigeants des entreprises privées que nous avons interrogés. En cas de cession de participations de leurs entreprises à des investisseurs privés, les personnes interrogées attendent que les nouveaux (co-)détenteurs exercent aussi une influence sur la stratégie commerciale. L'influence de la politique et des autres groupes extérieurs à l'entreprise serait en revanche amenée à diminuer (cf. graphique 6). Les interviewés s'attendent aussi en ce cas à une prise en compte accrue de l'efficacité et de la rationalité technique, un poids plus important de la performance dans la rémunération, un accroissement de la concurrence et une moindre sécurité de l'emploi. 


\section{Graphique 6 : Conséquences de la privatisation}

Question: Au cas où vous travailleriez dans une entreprise publique/privée, quels changements impliquerait selon vous le transfert majoritaire de votre entreprise à des associés privés ? Barème : $1=$ recul, $0=$ stabilité, $1=$ croissance.

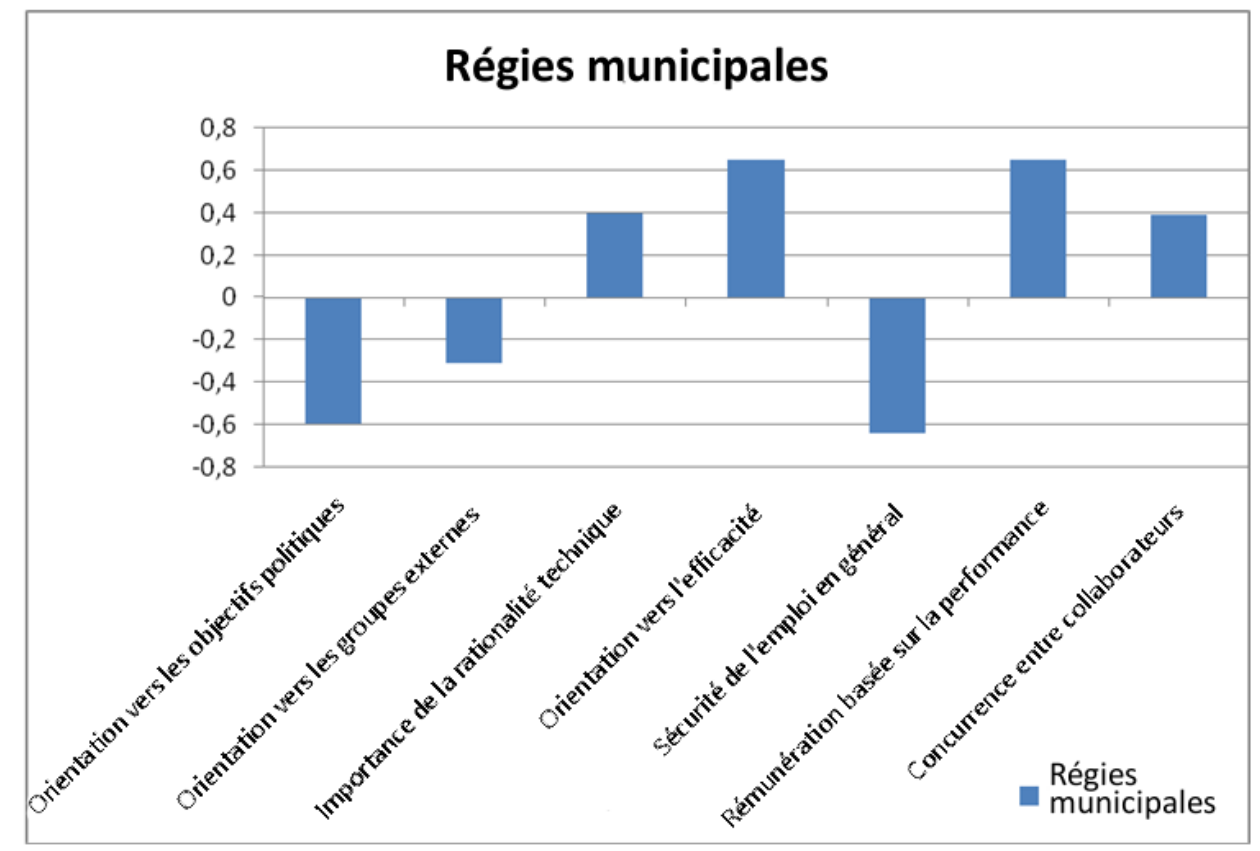

Source : Wagner/Edeling/Stölting, Öffentliche Unternehmen zwischen Privatwirtschaft und öffentlicher Verwaltung, VS Verlag, Wiesbaden, 2004.

Si l'on résume les résultats du questionnaire, les dirigeants de régies soulignent certes un souci des besoins et une influence de la politique municipale sur les décisions de leurs entreprises, ce qu'ils perçoivent comme une différence par rapport aux sociétés privées. Mais, contre toute attente, ces aspects ne sont pas prioritaires sur les objectifs économiques. Ceux-ci, ainsi que les critères qui en découlent et doivent servir de base aux décisions, sont aussi soulignés par les entreprises municipales.

Les cadres de régies considèrent leurs structures d'abord comme des entreprises économiques. Le principal but de l'entreprise cité par les dirigeants comme par les représentants des salariés est par conséquent la rentabilité. Pour tous les fournisseurs publics d'énergie, le modèle est l'entreprise privée, qui réussit à s'affirmer sur le marché. Cette entreprise est l'exemple même d'une structure dont les critères prioritaires sont les coûts et les bénéfices. Pour les responsables de régies interrogés, cette entreprise privée est le modèle que leur propre entreprise doit suivre elle aussi. 
Les critères économiques de la gouvernance d'entreprise ont gagné en importance, sur fond de dérégulation et d'ouverture du marché. Si les entreprises municipales détenaient jadis un monopole territorial qui les mettait à l'abri de la concurrence, celui-ci a volé en éclat dès 1998 s'agissant de la clientèle professionnelle. Et, même sur le marché des particuliers, une concurrence est vite intervenue. Dans ce contexte, ce renforcement du poids des critères économiques dans la gouvernance d'entreprise est tout à fait compréhensible. Pour l'avenir, les dirigeants de régies municipales s'attendent à ce que leurs décisions soient encore plus nettement influencées par les coûts, le marché et les bénéfices. En revanche, d'autres critères comme le financement des infrastructures régionales et la réalisation d'objectifs opérationnels fixés par le politique continueront de perdre du terrain si les régies municipales veulent survivre en tant qu'entreprises.

Les interventions des détenteurs publics ne sont donc pas, contrairement à ce que l'on pourrait attendre, perçues comme un facteur de différenciation par rapport aux entreprises privées, mais interprétées comme des éléments gênants, mettant en péril l'orientation économique des entreprises. L'influence de la politique apparait comme une entrave empêchant les régies municipales de tenir compte encore davantage des coûts et des bénéfices. Ce refus général des interventions politiques est donc partagé aussi par les régies étudiées, qui se distinguent uniquement par les éloges qu'elles font des collectivités locales pour la réserve dont elles font déjà preuve.

Les dirigeants des fournisseurs d'énergie municipaux sont tout à fait conscients des préoccupations politiques des collectivités qui les détiennent, qu'il s'agisse de construire des infrastructures municipales, de protéger des emplois, d'améliorer les infrastructures de la ville, de promouvoir des projets de production d'énergies alternatives, de financer un programme culturel, etc. Mais ils considèrent ce type de projets émanant de la «volonté politique » comme des convoitises entravant la régie municipale dans ses orientations économiques. Du point de vue des dirigeants de régies, la politique introduit des « objectifs secondaires » qui menacent leur objectif principal, à savoir la réussite économique.

Selon eux, si la régie veut s'affirmer face à la concurrence de plus en plus forte sur le marché, il y aura de moins en moins de place pour des prises d'influence politiques affaiblissant l'orientation économique des entreprises.

Alors que l'image de l'entreprise privée, solidement implantée sur le marché, représente la norme en matière d'excellence, l'administration publique en constitue le repoussoir. Sont associés à ce dernier modèle des processus de décision compliqués et beaucoup de formalités, ainsi que l'attitude "souveraine » d'une administration peu en phase avec les besoins des clients. Les dirigeants d'entreprises municipales interrogés se démarquent, eux-mêmes et leurs entreprises, de cet anti-modèle que représente la «boîte de fonctionnaires ». Ils admettent cependant que leurs clients les identifient à cette image négative de l'administration publique. 
L'idéal et le repoussoir constituent un modèle d'interprétation qui évolue au fil du temps. Les régies se démarquent ainsi de leur propre passé de «quasi » entreprises de l'administration publique. Et les dirigeants des structures municipales soulignent qu'ils devront à l'avenir se baser encore plus sur les critères économiques de gouvernance d'entreprise, comme «le privé ».

Le repoussoir que constitue son propre passé d'entreprise monopolistique et celui de l'administration publique sont liés. Les dirigeants des régies se démarquent tout autant de l'entreprise monopolistique protégée des premières années que de l'administration. L'entreprise publique est « sur la voie du secteur privé ». Il faudra à l'avenir, compte tenu notamment de la concurrence européenne croissante, "renoncer aux idéologies, quelles qu'elles soient, et agir uniquement dans une logique d'économie de marché ». "Nous devrons au moins nous adapter dans quelques domaines car il est certain que nous ne sommes pas sortis économiquement de cette logique de monopole du passé [..] et de ce fonctionnement marqué par l'administration ». Tourner la page signifie aussi rompre avec la pratique des solutions optimales d'un point de vue technique, qui avait cours dans le passé.

En somme : l'hypothèse guidant notre recherche considérait que les entreprises publiques tracent une limite entre marché et Etat en développant leurs propres objectifs, à but non lucratif. Dans les entreprises publiques, les décisions devraient tirer leur légitimité de missions définies par le politique, la priorité devant être donnée aux «objectifs opérationnels », fixés politiquement, par rapport aux «objectifs organisationnels », basés sur l'économie. Il convient, pour les fournisseurs d'énergie municipaux analysés, de relativiser cette thèse. Les critères économiques comptent de plus en plus dans les objectifs des régies et autres opérateurs municipaux d'électricité. On constate encore, il est vrai, une influence politique du propriétaire public, mais elle est perçue comme un facteur de perturbation. Les personnes interrogées espèrent pour l'avenir se dégager de plus en plus de cette influence. Sur ce plan, le modèle est d'ores et déjà l'entreprise privée opérant efficacement sur le marché et agissant en fonction de principes économiques; le repoussoir est l'administration publique, qui dépend plus étroitement du politique.

\section{Stratégies de réorganisation et mesures de restructuration formelle}

Les restructurations déjà mises en œuvre ou bien programmées dans les régies municipales sur lesquelles nous avons enquêté suivent le mot d'ordre : « laisser l'économie de marché gagner du terrain dans l'entreprise ». Ces mesures visent ainsi en particulier à améliorer la rentabilité et l'efficacité des entreprises, ainsi qu'à les axer davantage sur le client, le marché et les bénéfices. Les régies municipales interprètent le statut juridique lui-même comme une étape importante de consolidation de leur orientation sur le marché et les bénéfices. Les régies directes (sans personnalité juridique propre) en particulier souhaitent da- 
vantage d'indépendance vis-à-vis de leurs détenteurs municipaux. Elles espèrent qu'une modication de leur forme juridique en une $\mathrm{GmbH}$ ou une AG permettra de réduire l'influence des arguments «extérieurs » sur les décisions de l'entreprise et sa stratégie. Elles escomptent également d'une constitution en $\mathrm{GmbH}$ ou en $\mathrm{AG}$ un raccourcissement des processus de décisions.

Les instances de pilotage dominées par les élus municipaux, comme les comités de travail, entravent en effet la réactivité des régies directes. Or, dans une situation de concurrence, des décisions rapides sont plus que jamais nécessaires pour que l'entreprise ait ses chances dans la course aux clients. Les entreprises déjà constituées en $\mathrm{GmbH}$ ou $\mathrm{AG}$ considèrent ce statut à lui seul comme un signe de gestion de type privé, même lorsque les entreprises sont encore détenues à $100 \%$ par les pouvoirs publics.

Les opérateurs municipaux interrogés souhaitent une interconnexion plus étroite avec d'autres entreprises du même secteur. Ils forment par exemple des groupements d'achat avec d'autres régies afin de bénéficier d'effets d'échelle renforçant leur position sur le marché et donc leur position face à la concurrence. Ils créent aussi des entreprises communes afin d'opérer des synergies.

Certaines entreprises parmi les plus grandes que nous avons interrogées cherchent en outre à échanger des participations croisées avec des opérateurs régionaux ou en détiennent déjà. Ces participations stratégiques ont elles aussi pour objectif de renforcer la position des entreprises concernées face à leurs concurrents potentiels et de les aider à se consolider sur un marché qui s'ouvre.

Toutes les entreprises municipales du secteur ont il est vrai déjà pris conscience de l'importance d'un service commercial et d'un marketing actifs dans ce contexte concurrentiel. Mais, au moment de notre enquête, ces activités n'avaient guère dépassé le stade du projet. Les entreprises ont ainsi commencé à créer des emplois dans le domaine du marketing. Elles se sont aussi mises à réorganiser les services «relations clientèle », l'objectif étant d'être davantage dans une logique de service. L'une des réalisations a par exemple consisté à créer des centres d'appels. Sur le segment des particuliers, les activités se sont généralement limitées à la création ou à l'amélioration d'un service de gestion des réclamations.

Si la concurrence était encore quasi inexistante sur le marché des particuliers au moment de notre enquête, la course aux grands comptes avait commencé dès 1998. Le service commercial a donc fait l'objet de restructurations, s'agissant des clients de l'industrie et de l'artisanat.

Etre présent et aussi à l'écoute de la clientèle que possible est maintenant au centre des nouvelles structures commerciales. Sur le segment des grands comptes, cette préoccupation doit à l'avenir se concrétiser à travers un service clients transversal. Les responsables de grands comptes seront ainsi en charge de toutes les prestations relatives à certains clients clés (Key Account Management). Les visites à la clientèle et les services de contact (Front offices) complètent ce système de suivi. Des conditions attractives, associées à de nouveaux systèmes de suivi de la clien- 
tèle, voilà les moyens à l'aide desquels de nombreuses régies municipales souhaitent désormais fidéliser une partie de leurs clients actuels.

L'ouverture à de nouveaux domaines d'activités était très frileuse au moment de notre enquête. Elle se concentrait principalement sur le secteur des télécommunications. Pour justifier la réserve observée jusque-là, la direction des entreprises invoque surtout les constitutions municipales restrictives, qui encadrent rigoureusement de tels engagements. Les restructurations des opérateurs municipaux se focalisent, outre sur la réorganisation des activités commerciales, sur une meilleure prise en compte des critères de coûts et de bénéfices. Pour améliorer leur santé financière et être en mesure d'affronter la concurrence, ces entreprises sous-traitent les prestations qui peuvent être réalisées à moindre coût à l'extérieur. Sont surtout concernées des prestations qui ne relèvent pas du cœur d'activités, comme le traitement des données, les centres d'appels pour les réclamations de la clientèle ou les travaux allant au-delà de la maintenance des infrastructures de l'entreprise.

Dans certains cas, les régies créent leurs propres filiales pour assurer ces prestations externalisées. Ces filiales sont soumises à d'autres conventions collectives, et peuvent ainsi proposer des prestations à moindre coût sur le marché (exemple : compagnies d'autocars privées).

Ces efforts s'accompagnent de la mise en place dans toute l'entreprise de centres de profits et de coûts, lesquels introduisent le modèle client - fournisseur au sein même de l'organisation. Ce changement vise à rendre transparents les coûts et les prestations des différents services de l'entreprise et à les compenser les uns les autres. Les centres de profits et de coûts doivent aider à déléguer la responsabilité de l'entreprise aux centres de compétence. Les mesures d'amélioration de la gestion de la qualité et les programmes de team building vont également dans le même sens.

Ces mesures, associées à l'organisation de projet et à un aplanissement des hiérarchies, visent à permettre d'activer des potentiels dormants et à rendre les processus plus efficaces et plus axés sur la clientèle. Pour améliorer leur efficacité, certaines entreprises communales ont lancé une réorganisation de leurs processus de fonctionnement. A cette fin, elles ont sélectionné certains processus essentiels dans leur cœur d'activités. Ces processus opérationnels, qui se sont souvent développés historiquement, sont alors optimisés en tenant compte de la rentabilité, de l'efficacité et de l'orientation vers le client.

Du côté du personnel, les efforts de réorganisation réalisés jusque-là se sont accompagnés d'une réduction des effectifs. L'on souligne les efforts de « dégraissage ». Dans la plupart des régies que nous avons étudiées, les postes libérés à la suite de départs en retraite n'ont généralement pas été remplacés ces dernières années. Au lieu de cela, le potentiel d'économies ainsi généré a été consacré aux restructurations.

Si les entreprises tiennent davantage compte du marché et de la concurrence que dans le passé, elles attendent de leurs collaborateurs qu'ils fassent de même. 
C'est pourquoi les réorganisations sont accompagnées de mesures visant à améliorer le souci de la performance et des objectifs chez les collaborateurs comme chez les cadres.

Les changements organisationnels ont pour objectif la « remise en forme » des entreprises, qui opéraient jusque-là sur des monopoles territoriaux protégés, pour qu'elles puissent faire face à la concurrence. Ils visent donc prioritairement l'amélioration de l'approche clientèle et le souci des bénéfices et du rendement. Ces objectifs sont surtout mis en œuvre à travers un système commercial plus soucieux du client et une organisation interne des processus de travail davantage axée sur les coûts et les bénéfices. Si les entreprises municipales que nous avons étudiées ne se démarquent pas dans leurs objectifs, elles se singularisent par le niveau de réalisation et la profondeur des changements visés. Il faut citer les mesures de réorganisation présentées ici - alliances et partenariats stratégiques avec d'autres entreprises, développement de nouveaux domaines d'activités, organisation de la distribution en fonction de segments de clientèle, introduction d'instruments donnant plus de transparence aux performances et aux coûts - qui sont toutefois loin d'avoir été mises en œuvre dans une même mesure par toutes les régies municipales étudiées. On peut constater tendanciellement que les petites structures en particulier se trouvent seulement au début de leurs efforts de réorganisation, tandis que les entreprises plus grandes et plus indépendantes des pouvoirs publics se sont déjà engagées dans cette voie depuis un certain temps.

\section{Graphique 7 : Programmes de management}

Question: On lit tant de choses sur les programmes et instruments modernes de management. Quels programmes sont pratiqués dans votre entreprise et quelle importance leur accordez-vous? (Merci de remplir les deux colonnes!) Barème : $0=$ non pratiqué, $1=$ pratiqué $/ 0=$ sans importance, $1=$ important.

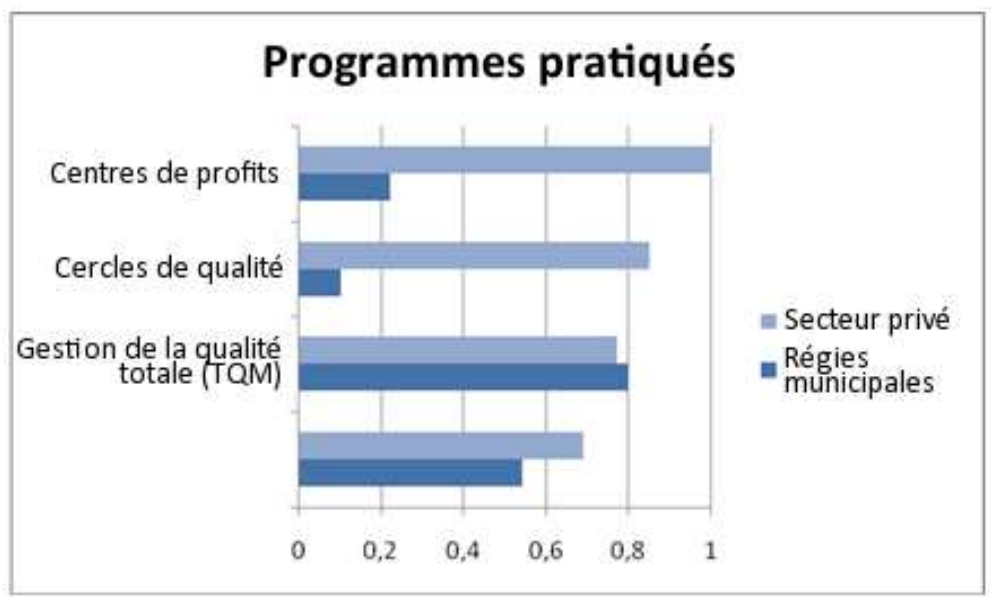



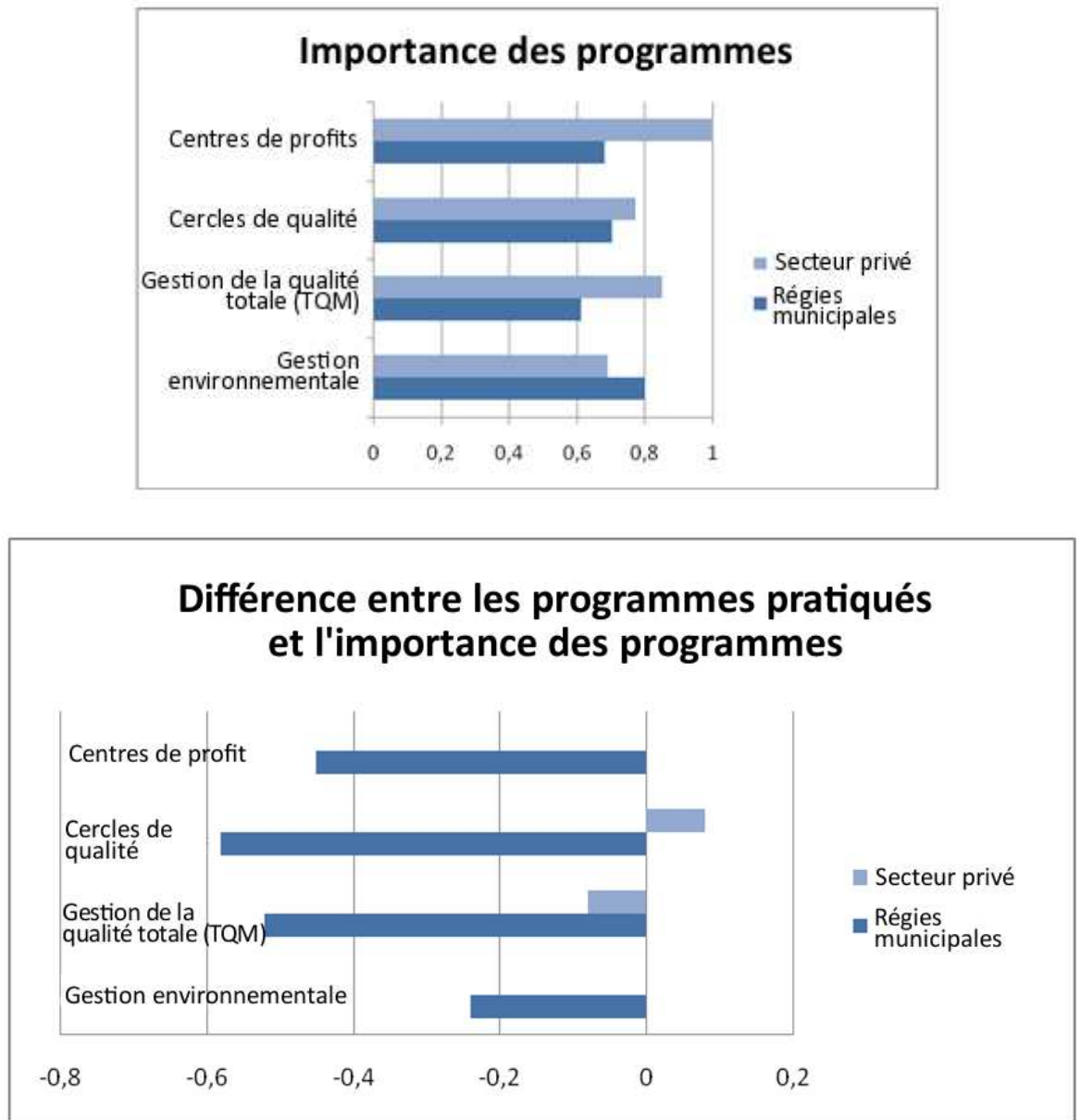

Source : Wagner/Edeling/Stölting, Öffentliche Unternehmen zwischen Privatwirtschaft und öffentlicher Verwaltung, VS Verlag, Wiesbaden, 2004.

Le graphique 7 donne un aperçu de l'état de la réalisation au moment de notre enquête (1998). Si les entreprises pratiquent déjà en majorité une gestion environnementale, les centres de profits et les programmes d'assurance qualité cercles de qualité et gestion de la qualité totale (TQM) par exemple - sont encore très peu appliqués; et ce, bien que le rôle de tels instruments soit jugé très important par les responsables des régies interrogés. Sur l'importance qu'ils accordent aux programmes de management, les cadres de structures publiques ne se démarquent guère de leurs homologues du secteur privé.

Notre thèse selon laquelle un changement de logique de gouvernance des entreprises publiques doit aussi se traduire en stratégies concrètes de réorganisation s'est confirmée. Si les entreprises soulignent d'abord les critères économiques parmi leurs objectifs, comme un souci du marché, des coûts et des béné- 
fices, cette préoccupation trouve aussi sa réalisation concrète dans les stratégies de changement organisationnel. Si les fournisseurs communaux ou les régies municipales prennent pour modèle l'entreprise privée, il n'est pas étonnant que les stratégies et programmes de réorganisation privilégiés issus du secteur privé leur soient également familiers. L'ancrage dans les entreprises publiques que nous avons étudiées d'outils de pilotage basés sur le marché dépasse largement le stade du projet. En effet, les interventions du détenteur communal basées sur des objectifs non économiques sont brutalement rejetées par les entreprises. Les efforts de réorganisation de ces entreprises ne doivent donc pas être interprétés comme le complément partiel d'une politique par ailleurs définie par les directives des municipalités détentrices, mais comme l'expression d'une volonté de suivre le modèle du privé.

\section{Evolution de l'emploi, du recrutement et de la qualification}

L'amendement de la loi sur l'énergie (Energiewirtschaftsgesetz) d'avril 1998 expose les régies municipales et les opérateurs locaux à une concurrence qui leur était inconnue jusqu'alors. Pour être compétitives face à d'autres opérateurs, les régies doivent accroître leur productivité. Dans certains domaines, des emplois disparaissent car certaines prestations ne sont plus réalisées en interne mais externalisées. Nous allons maintenant nous interroger sur les stratégies de réorganisation des ressources humaines poursuivies. Parallèlement aux suppressions de postes, les exigences sont accrues à l'égard des personnels restés dans les entreprises. Des domaines d'activités sont regroupés, dans le service commercial, les centres d'appels du service clientèle ou le marketing par exemple. Les qualifications nécessaires pour ces postes évoluent par conséquent. Nous allons nous demander avec quelles stratégies les régies municipales font face à ces évolutions, en concentrant d'abord notre analyse sur les résultats des entretiens que nous avons eus avec les spécialistes des entreprises. Dans la deuxième partie de cette section, nous analyserons, sur la base de notre enquête quantitative, les outils de planification et de développement des ressources humaines utilisés ou souhaités pour l'avenir, et nous les comparerons à ceux du secteur privé et de l'administration municipale.

Même si c'est à des degrés divers, des emplois ont déjà été supprimés dans toutes les régies que nous avons étudiées. Les cadres et les représentants du personnel soulignent que jusqu'à présent ces suppressions se sont largement déroulées dans des conditions socialement acceptables, à travers le non-remplacement des postes libérés à la suite de départs en retraite ou de préretraites.

Dans le passé déjà, des postes avaient été supprimés dans les régies municipales, souvent au moyen de départs en retraite ou de dispositifs de préretraite. Les restructurations, le regroupement de domaines d'activités, etc. se sont faits avec le personnel restant. Les embauches ont été très limitées, et concernent surtout les domaines comme l'achat d'électricité, le marketing et la distribution, 
pour lesquels les entreprises manquaient de collaborateurs ayant les compétences et expériences spécifiques, importantes d'un point de vue stratégique. Mais les suppressions de postes ne vont pas s'en tenir à la réduction d'effectifs opérée à ce jour, loin de là. Les cadres des régies indiquent que la productivité des collaborateurs doit être encore accrue et les effectifs réduits. A défaut, selon eux, la compétitivité des entreprises est menacée. C'est pourquoi les personnels s'inquiètent de la stabilité des emplois à l'avenir. Car, comparativement aux entreprises privées, les effectifs sont encore importants dans les régies.

Les responsables de ces structures s'attendent encore à des suppressions de postes considérables dans les prochaines années. Les postes maintenus pour les besoins de pointe, que ces entreprises pouvaient encore se permettre dans le passé, seront à l'avenir remplacés par un recours accru aux prestataires externes. Les représentants des salariés espèrent que les suppressions de postes ultérieures seront acceptables socialement, c'est-à-dire qu'elles se feront sans licenciements économiques.

Si tous les dirigeants de régies municipales et même les délégués du personnel ont conscience de la nécessité de continuer à réduire les effectifs, les cadres intermédiaires contestent en revanche l'existence de tels potentiels dans leurs domaines de compétences : s'il faut faire des économies de personnel, cela ne peut être que dans d'autres services, non dans les leurs, estiment-ils.

En plus de la réduction de personnel, les exigences évoluent: le souci du client, du service et des bénéfices fait partie des qualifications requises, à côté des techniques de négociation et de management, pour assurer le management efficace des entreprises dans un contexte de concurrence. Par ailleurs, le découpage des domaines d'attributions a changé, la distribution en étant un exemple : afin d'améliorer le service au client, la séparation jadis usuelle entre gaz, eau et électricité a été supprimée. D'autre part, les tâches d'exécution ont été dissociées des tâches de suivi de la clientèle pures, ce qui signifie que les activités de contact et de «back-office» ont été séparées. Pour ces nouvelles attributions, les entreprises ont eu besoin de collaborateurs ayant d'autres qualifications. Sur le segment des « grands comptes » ou des « clients à contrat spécifique », si important d'un point de vue stratégique, les régies municipales ont décidé d'embaucher du personnel ayant des expériences commerciales acquises dans des domaines de l'économie soumis aux mécanismes du marché.

De même que l'on recherche dans le domaine commercial des collaborateurs sachant négocier et dotés d'une logique entrepreneuriale, les compétences attendues des salariés évoluent aussi dans d'autres domaines.

La stratégie des régies municipales consistant à adapter le profil de qualification du personnel aux nouveaux besoins est donc double : d'une part, elles recrutent du personnel venu de l'extérieur pour les domaines de compétences supplémentaires comme le marketing et le service commercial, la convention collective du service public s'avérant toutefois de plus en plus être une entrave dans ces domaines; d'autre part, les entreprises attendent de leurs personnels qu'ils se qualifient en fonction des nouveaux besoins et structures d'activités. 
Les cadres soulignent que les collaborateurs sont très disposés à s'adapter à de nouvelles activités et à se qualifier pour des missions qui ont évolué. Ils estiment que la situation du marché du travail y est pour beaucoup. Le processus de qualification des collaborateurs n'était pas terminé au moment de notre étude. Ce processus inclut en particulier des formations dans le domaine du contact client, du contrôle et des techniques de management (cf. l'étude PWC) ${ }^{3}$. Si les régies municipales assez importantes utilisent leur propre développement des ressources humaines pour ces mesures, les plus petites font appel aux services de leurs associations professionnelles et à des organismes de formation extérieurs.

Quoique toutes les régies municipales jugent nécessaire une adaptation systématique des qualifications aux nouveaux besoins, toutes n'utilisent pas les outils requis à cet effet en termes de gestion des ressources humaines (RH). Une planification qualitative des RH est certes perçue comme intéressante, mais elle reste encore relativement peu pratiquée (cf. graphique 8$)^{4}$. On peut faire le même constat s'agissant d'un développement des ressources humaines qui encouragerait systématiquement les collaborateurs en fonction de leurs potentiels et de ce que requièrent leurs postes de travail. Là encore, les cadres interrogés ont conscience de l'importance de cet outil pour réussir à maitriser le processus de changement, mais pour l'instant seules quelques rares régies municipales, généralement assez grandes, mettent en place un développement systématique des RH.

Si les grandes régies prévoient même de consolider leur système de développement des RH, une gestion stratégique en ce domaine, c'est-à-dire une planification des qualifications et des compétences du personnel focalisée sur les besoins à venir reste quasi-inexistante dans de nombreuses régies ${ }^{5}$. Et ce, alors que les cadres de ces établissements jugent également cette mesure nécessaire et utile. De même, la pratique d'un marketing actif des ressources humaines, positionnant les régies comme des employeurs attractifs dans les médias publics, est très rare, bien que les cadres interrogés classent cette action comme importante pour attirer des personnels de haut niveau.

Si l'on compare, avec la prudence de rigueur, les régies municipales à notre entreprise de référence du secteur privé, il apparait que les outils de planification, de développement et de recrutement des ressources humaines sont également considérés comme importants dans cette dernière. Mais, à la différence

\footnotetext{
${ }^{3}$ La contribution du personnel à la réussite sur le nouveau marché de l'énergie : une étude réalisée auprès de fournisseurs d'énergie allemands, en comparaison avec des expériences tirées du marché britannique de l'énergie, Francfort-sur-le-Main.

${ }^{4}$ En général, une distinction est faite entre la planification concrète et opérationnelle en termes de ressources humaines et la planification à long terme, tactique et stratégique (cf. Hentze, 1994, p. 86 sq.).

${ }^{5}$ Le concept de gestion stratégique des ressources humaines ou du personnel est à attribuer à Duck. L'intervention stratégique du potentiel intellectuel des collaborateurs est ici mise en avant, en opposition aux méthodes traditionnelles de compression de personnel (cf. aussi Wagner, 1991, p. 67). La motivation, la coopération, la créativité et l'optimisme doivent ainsi permettre d'aboutir, à l'aide des mêmes ressources, à un meilleur résultat.
} 
des entreprises communales, qui en sont encore à devoir introduire de tels outils, ceux-ci sont souvent une pratique déjà courante dans les grandes entreprises privées. Pour l'avenir, les régies prévoient de continuer le "dégraissage » et d'accroître la productivité de leurs salariés. Là encore, leur modèle est le secteur privé, «aguerri à la concurrence ». Sur la base de nos données, un tel modèle peut aussi être déduit des outils privilégiés en matière de recrutement, de planification et de développement des RH. Sur ce point, les entreprises communales ne se démarquent pas de l'administration publique, qui applique également des modèles de planification et de développement des RH ayant déjà cours depuis longtemps dans les grandes entreprises privées.

\section{Graphique 8 : Outils de planification et de développement des RH mis en pratique et souhaités}

Question: On lit tant de choses sur les programmes et instruments modernes de management. Quels programmes sont pratiqués dans votre entreprise (votre administration) et quelle importance leur accordez-vous ? Barème : $0=$ non pratiqué, $1=$ pratiqué $/ 0=$ sans importance, $1=$ important .
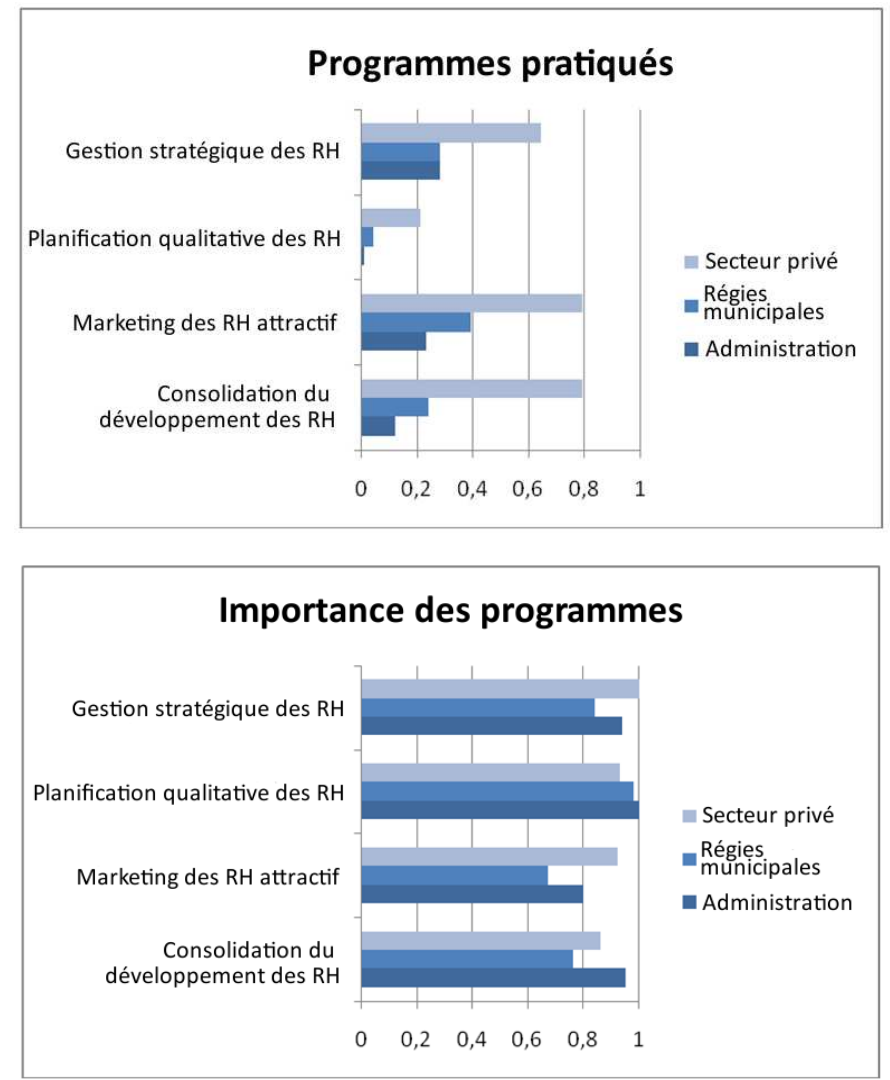


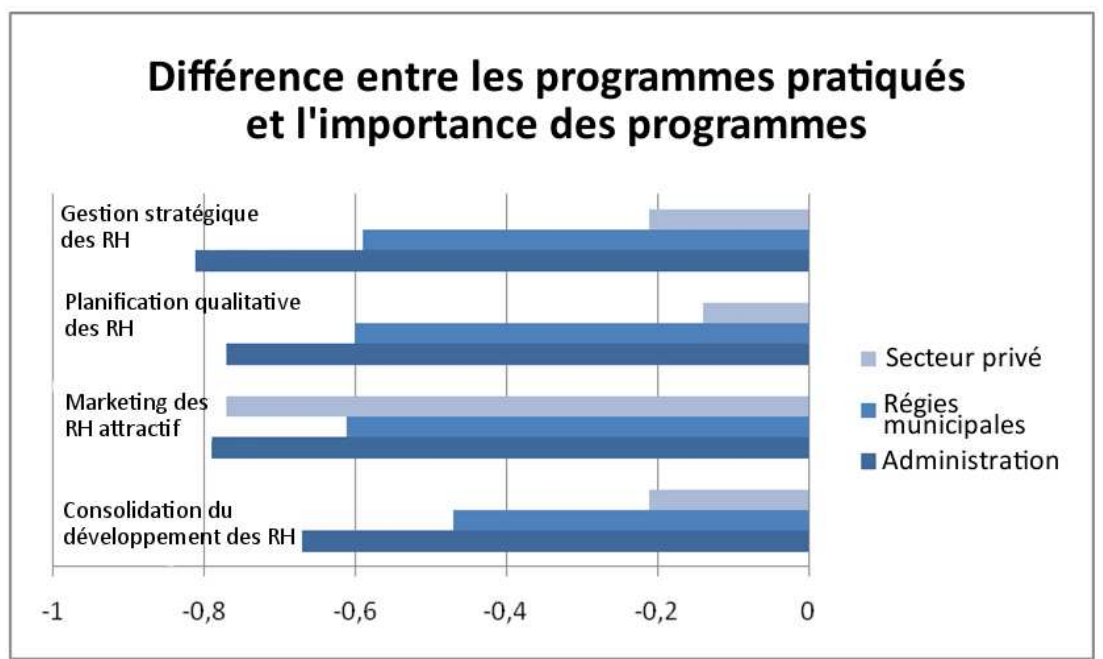

Source : Wagner/Edeling/Stölting, Öffentliche Unternehmen zwischen Privatwirtschaft und öffentlicher Verwaltung, VS Verlag Wiesbaden, 2004.

\section{Rémunération, motivation et management}

Cette partie de notre contribution traite du management des collaborateurs, de leur motivation et des structures de rémunération dans les entreprises communales du secteur énergétique. Comme cela a déjà été montré, les régies municipales souhaitent à l'avenir laisser davantage de place aux objectifs économiques. Nous allons maintenant traiter la question de savoir si une plus forte prise en compte des bénéfices se traduit aussi par l'ajustement en conséquence des outils de management. Nous nous interrogerons également sur le développement de systèmes d'incitation et de motivation des collaborateurs. Il nous faudra aussi nous demander comment il est possible d'apprécier la motivation des salariés dans un contexte de crainte croissante de perte d'emploi. Les résultats de l'enquête normalisée réalisée auprès de cadres de régies municipales sur les outils de management et de rémunération souhaités et pratiqués sont exploités comparativement au secteur privé et à l'administration publique. Ces résultats sont complétés et approfondis à l'aide des informations issues des entretiens semi-directifs réalisés sur le management des régies municipales.

Les cadres de régies interrogés soulignent la nécessité que les collaborateurs s'appuient, plus nettement que dans le passé, sur des indicateurs de performance concrets. Des systèmes de définition des objectifs entre encadrement et salariés doivent y contribuer ${ }^{6}$. Dans la plupart des régies municipales, ces dispositifs

\footnotetext{
${ }^{6}$ Le management par objectifs ou «Management by Objectives » repose sur la définition d'objectifs sur lesquels s'entendent les supérieurs hiérarchiques et les collaborateurs. Cela permet au supérieur de répartir les objectifs de l'entreprise entre les différents collaborateurs. En même temps, ces objectifs individualisés donnent au collaborateur une orientation à l'aide de laquelle il peut adapter son comportement et son travail.
} 
sont considérés comme importants pour résister face à la concurrence, mais seules quelques rares régies les avaient déjà mis en pratique au moment de notre enquête (cf. graphique 9). Ce constat vaut également pour l'évaluation systématique des collaborateurs et des supérieurs hiérarchiques. Quoique perçue comme intéressante, l'évaluation n'a généralement pas encore été introduite dans les régies municipales que nous avons étudiées - alors qu'elle l'a été dans les entreprises privées. Si l'on compare les entreprises publiques et privées, les dispositifs de définition des objectifs et les systèmes d'évaluation sont plus répandus dans ces dernières. De leur côté, les administrations publiques constatent elles aussi le besoin d'outils de ce type ${ }^{7}$.

\section{Graphique 9 : Outils de management et systèmes de rémunération souhaités et pratiqués}

Question: On lit tant de choses sur les programmes et instruments modernes de management. Quels programmes sont pratiqués dans votre entreprise (votre administration) et quelle importance leur accordez-vous ? Barème : $0=$ non pratiqué, $1=$ pratiqué $/ 0=$ sans importance, $1=$ important .

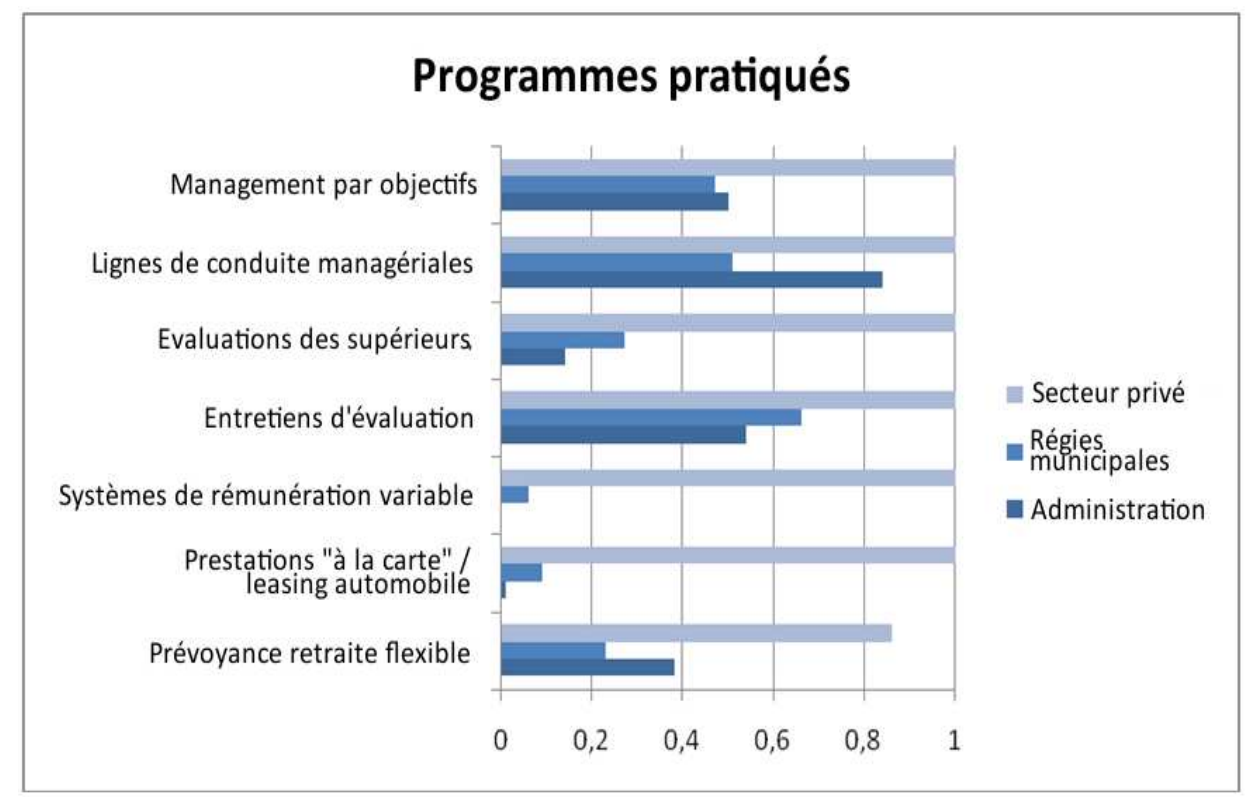

La comparaison régulière entre objectifs et réalité aide ainsi au contrôle et à l'évaluation des performances (cf. aussi Hentze, 1995, p. 219).

7 Sauf exception : les «lignes de conduite managériales » sont plus répandues dans les administrations publiques que nous avons interrogées que dans les entreprises publiques. 

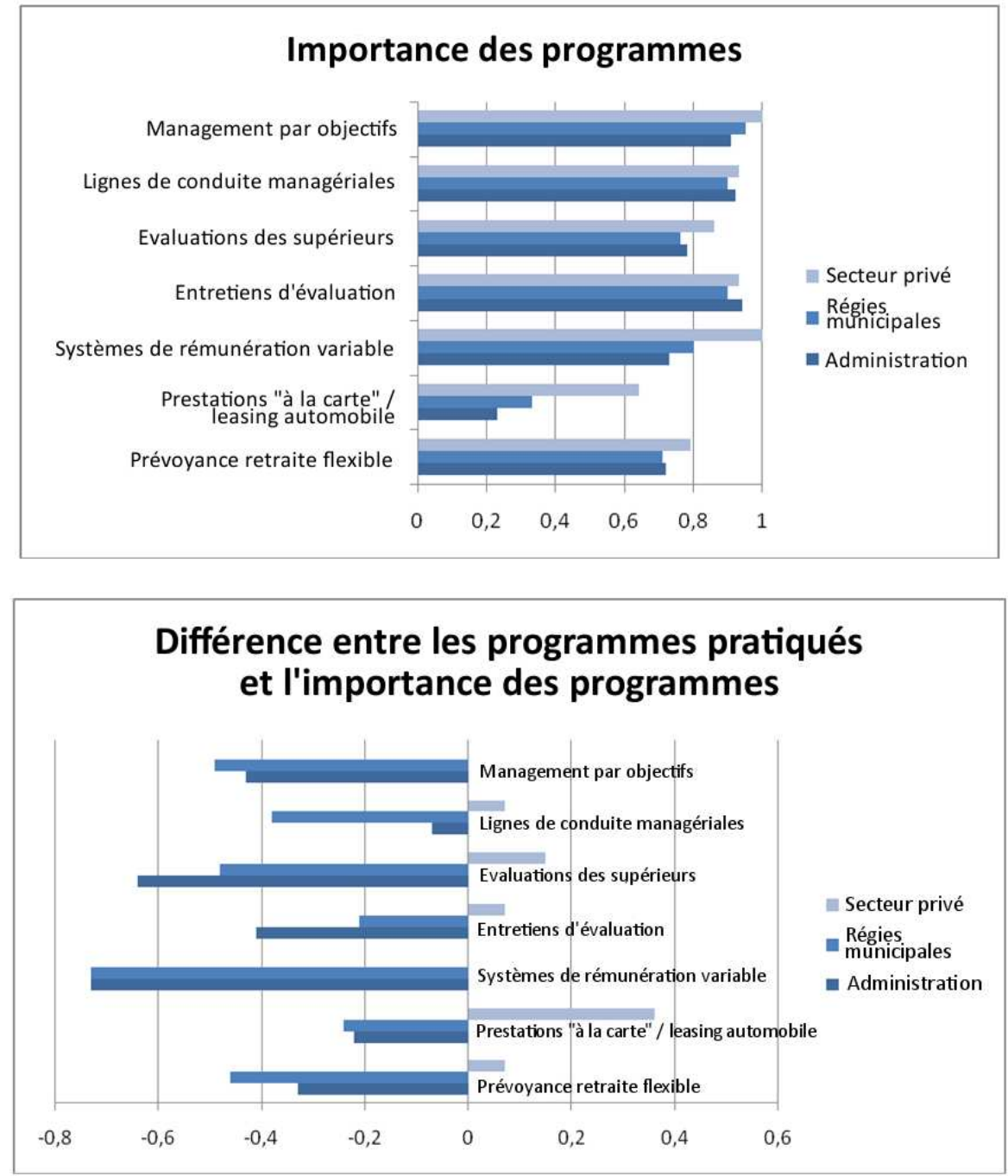

Source : Wagner/Edeling/Stölting, Öffentliche Unternehmen zwischen Privatwirtschaft und öffentlicher Verwaltung, VS Verlag Wiesbaden, 2004.

Nos questions portaient également sur un autre instrument de motivation et de management des collaborateurs : la rémunération liée à la performance. On fait généralement la distinction entre les parts de rémunération fixes et variables (Scholz, 1994, p. 862). La part variable de rémunération peut par exemple être associée à la réalisation d'objectifs opérationnels, personnels ou encore stratégiques dans le périmètre dont le cadre a la responsabilité, ou bien à l'évolution des résultats de l'entreprise. Si les systèmes de définition des objectifs et les dispositifs d'évaluation des collaborateurs permettent de fixer des objectifs concrets et d'évaluer leur réalisation, les systèmes de rémunération variable per- 
mettent de récompenser les performances réalisées. Bien que les programmes et modèles de rémunération plus fortement basée sur les résultats apparaissent comme importants et souhaités, ils sont très rarement mis en pratique. Cela vaut aussi, dans une mesure un peu moindre, pour les dispositifs dits « de cafétéria » ${ }^{8}$ et de prévoyance vieillesse adaptée de manière flexible aux besoins des collaborateurs. En résumé, les régies municipales ne se démarquent pratiquement pas de l'administration publique dans leur souhait de systèmes de rémunération flexibles et basés sur les performances. D'autre part, ce type de systèmes est en pratique plus répandu dans le secteur privé que dans les entreprises et administrations publiques.

C'est précisément sur ce point de la quasi-inexistence de parts variables que s'exerce la principale critique des structures de rémunération pratiquées dans leurs entreprises chez les cadres de régies municipales que nous avons interrogés : ceux-ci considèrent que les systèmes de convention du service public, en particulier la convention des employés du service public (BAT), sont trop rigides et laissent trop peu de place aux performances.

En cela, les cadres que nous avons interrogés sont d'accord avec les délégués du personnel : alors que le système de convention du service public présente de réels avantages pour les salariés les plus âgés et ceux qui ont des enfants, il est trop peu incitatif pour les collaborateurs jeunes et diplômés. Cela rend difficile le recrutement de collaborateurs de haut niveau issus du secteur privé.

Les cadres et les représentants des salariés déplorent la faible attractivité du système de convention du service public, en particulier pour ce type de personnels. Les cadres de régies interrogés voient ici une différence par rapport aux systèmes de rémunération pratiqués dans le privé, plus fortement liés aux résultats.

Ces cadres mettent aussi en exergue un niveau de salaire globalement plus élevé dans le secteur privé que dans le public.

Certaines régies municipales tentent, à l'aide de primes ou de compléments de salaires liés aux résultats, de récompenser les performances de leurs collaborateurs, dans le cadre même des conventions du service public - parfois pour ne pas voir ces salariés rejoindre d'autres entreprises ; dans certains cas, la participation des collaborateurs au succès de l'entreprise est même " honorée » à travers la remise d'actions. Les dispositions concrètes d'intéressement des collaborateurs aux résultats n'en sont toutefois, bien souvent, qu'à leurs balbutiements. Et elles font parfois l'objet de contestations, critiquées comme étant des primes «à la tête » en raison de l'opacité de leur attribution. Lorsque des systèmes de primes d'intéressement sont mis en œuvre, c'est tendanciellement plutôt dans les grandes régies municipales et dans des domaines d'activités stratégiquement importants, comme le service commercial.

Les dirigeants des régies aspirent à faire de leur entreprise, à l'aide de rémunérations plus fortement liées à des critères de performance, un employeur at-

\footnotetext{
${ }^{8}$ Ou système «à la carte », selon lequel le salarié peut choisir entre différentes prestations sociales, dans une limite budgétaire établie par l'entreprise, NDE.
} 
tractif, y compris pour des salariés d'autres domaines de l'économie. Ils espèrent en outre être mieux à même de motiver leurs propres salariés avec une part variable de rémunération.

Comme la critique du système de convention du service public, le souhait d'une rémunération davantage axée sur la performance n'est pas uniquement le fait des cadres des régies, mais émane aussi des représentants du personnel, qui jugent cette évolution importante et nécessaire.

Dans certaines régies, des discussions entre la direction et les représentants des salariés sont en cours sur des accords d'établissement, l'objectif étant pour la direction de définir des structures conventionnelles plus en phase avec le marché. La possibilité d'aboutir à des dispositions au niveau de l'entreprise est toutefois mise en doute, y compris dans certains cas par des cadres des régies estimant que la défense des salariés se mobilise trop pour préserver les acquis.

$\mathrm{Si}$ le secteur privé fait office de modèle en matière de rémunération, les cadres des régies interrogés voient une différence entre leurs entreprises et l'administration publique. Selon eux, celle-ci réside notamment dans la pratique d'avancement et donc, finalement, dans les revenus, plus souples que dans l'administration publique.

$\mathrm{Si}$ les régies municipales s'acheminent globalement vers une rémunération basée sur la performance - notamment afin de motiver davantage les salariés les économies croissantes de personnels créent chez les salariés le souci de la sécurité de leur emploi. Les cadres interrogés citent la sécurité d'emploi supposée comme motivation pour entrer dans le service public et comme compensation pour un salaire moindre. Or, cette motivation va continuer de perdre de l'importance.

Les cadres de régies que nous avons interrogés considèrent que, dans leurs entreprises, les emplois sont directement menacés par la concurrence croissante. Ils soulignent qu'en cela les régies se distinguent de l'administration publique, où les emplois restent plus sûrs.

Certains d'entre eux font le lien entre la motivation et la peur de perdre son emploi. Mais ce point de vue ne fait pas l'unanimité. D'autres managers estiment en effet que la tension qui règne sur le marché de l'emploi peut en soi créer une plus forte motivation pour le personnel.

Pour les salariés des régies municipales, le découpage des activités et les domaines d'attributions changent globalement. Ces évolutions leur demandent d'être très flexibles et prêts à se qualifier en conséquence. Les cadres que nous avons interrogés soulignent que leurs collaborateurs sont motivés à se qualifier et à se réorienter.

Récapitulons les résultats relatifs à cette question : Les régies municipales dans le secteur de l'électricité font de plus en plus dépendre leurs décisions de critères économiques. Si les influences d'un autre ordre, émanant des collectivités locales qui les détiennent, ne sont pour autant pas complètement exclues, elles sont de plus en plus considérées comme des «entraves » qui menacent la 
capacité de survie des entreprises. Pour ce qui est de leurs systèmes de management, de motivation et de rémunération privilégiés, les régies municipales suivent fondamentalement le modèle des entreprises privées : la concurrence entre les entreprises se reflète aussi dans un management de plus en plus basé sur les objectifs et les résultats. Des outils tels que la définition d'objectifs et la rémunération liée aux performances, qui sont depuis longtemps monnaie courante dans les entreprises plus affirmées sur le marché, sont maintenant de plus en plus mis en place dans les régies municipales également. Ce résultat étaie l'hypothèse selon laquelle l'orientation renforcée des régies vers les modèles ayant l'expérience du marché n'est pas seulement un changement au niveau des structures, mais constitue aussi l'indice d'un déplacement de priorité dans ce qui est attendu des collaborateurs et des cadres des entreprises.

\section{Relations sociales et groupes d'influence}

Les régies municipales se trouvent dans une phase de bouleversement. Il leur faut tenir davantage compte du marché pour continuer à exister dans un contexte de concurrence. Ce processus s'accompagne de suppressions d'emplois et de restructurations importantes au plan opérationnel; on peut donc supposer qu'il est particulièrement «explosif » pour les entreprises concernées. Cette partie de notre contribution traite donc des mécanismes de résolution des conflits qui y sont à l'œuvre. Nous posons la question des rapports entre l'encadrement et les représentants des salariés, des groupes les plus influents dans les entreprises et du rôle de la direction dans la médiation des conflits. Les résultats du questionnaire sont complétés et approfondis à l'aide des analyses tirées des entretiens qualitatifs.

Interrogés sur les mécanismes de résolution des conflits de leurs entreprises, les dirigeants des régies fournissent des réponses semblables à celles de leurs homologues des administrations ou du secteur privé (cf. graphique 10) : la discussion des problèmes et la conciliation des intérêts prédominent sur les mécanismes autoritaires de résolution ou d'étouffement des conflits.

Si l'on compare l'influence des différents groupes de l'entreprise dans les régies municipales, les administrations et le secteur privé, on observe la tendance suivante (cf. graphique 11) : dans les régies comme dans les entreprises privées, les décisions sont surtout prises du point de vue des services financiers et commerciaux. Le consensus observé indique que l'observation du marché, des coûts de fonctionnement et des bénéfices, des contraintes liées à la concurrence d'une part, et des rendements escomptés d'autre part, détermine en priorité les décisions dans les entreprises privées comme municipales. Dans les entreprises publiques aussi bien que privées, la prise en compte de la situation financière et des ventes pèse autant sur les décisions stratégiques que l'avis du conseil de surveillance. Dans les entreprises municipales, le conseil de surveillance tend même à avoir davantage de poids que les impératifs économiques, à 
la différence de ce qui se produit dans les entreprises privées, ce qui souligne l'importance des orientations politiques par rapport à une gouvernance qui tiendrait exclusivement compte du marché.

\section{Graphique 10 : Mécanismes de résolution des conflits}

Question: Les conflits font partie du quotidien dans et en dehors des organisations. Dans votre domaine d'activité, comment sont traités les conflits et les problèmes urgents ? Barème: $1=n e$ s'applique pas, $2=n e$ s'applique guère, $3=s^{\prime}$ applique en grande partie, $4=s^{\prime}$ 'applique.

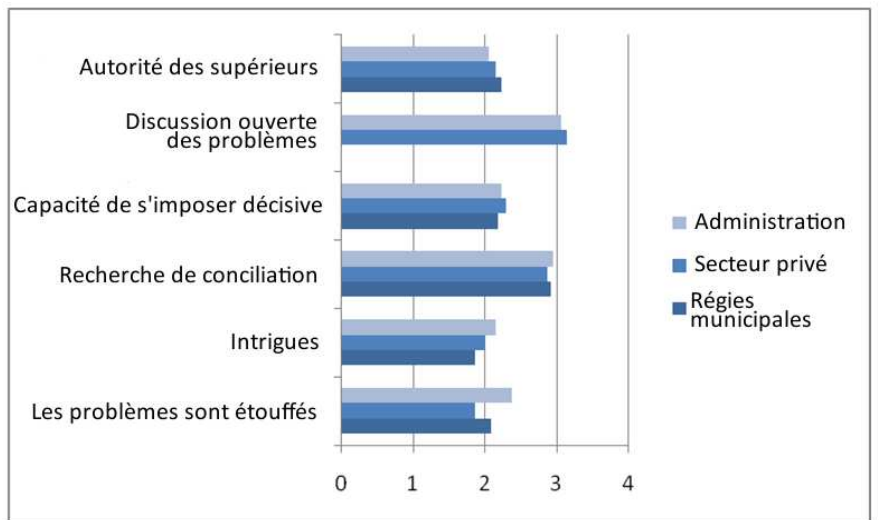

Source : Wagner/Edeling/Stölting, Öffentliche Unternehmen zwischen Privatwirtschaft und öffentlicher Verwaltung, VS Verlag, Wiesbaden, 2004.

\section{Graphique 11 : Influence des groupes de l'entreprise}

Question: Selon votre expérience, quelle influence les secteurs et groupes de l'entreprise (de l'administration) suivants ont-ils sur les décisions de la direction ? Barème : $1=$ aucune, $2=$ faible, $3=$ forte, $4=$ décisive .

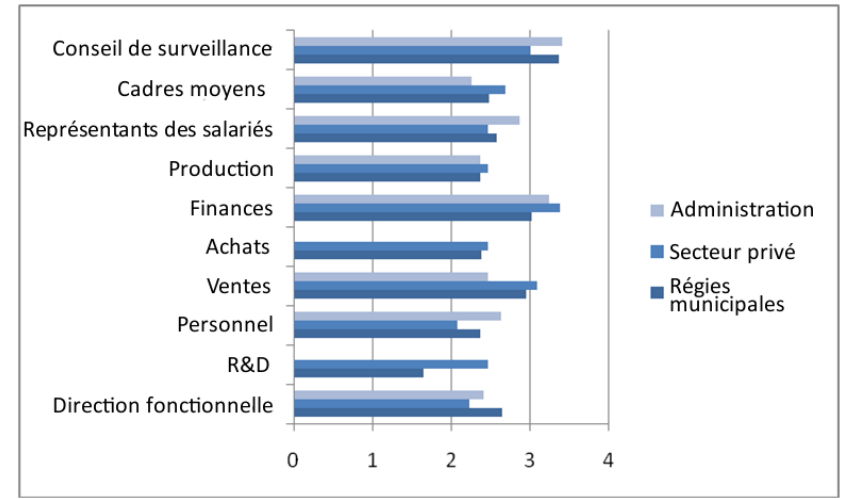

Source : Wagner/Edeling/Stölting, Öffentliche Unternehmen zwischen Privatwirtschaft und öffentlicher Verwaltung, VS Verlag Wiesbaden, 2004. 
Les rapports entre management et représentants du personnel méritent aussi d'être considérés plus en détail. Contrairement à ce que les résultats intermédiaires de l'enquête statistique (graphique 11) pourraient amener à conclure, ces relations pèsent davantage sur les décisions dans les entreprises municipales que dans le secteur privé. Dans presque toutes les régies que nous avons étudiées, les différents acteurs décrivent les rapports entre encadrement et représentants des salariés comme étant coopératifs. Les directeurs disent impliquer les représentants des salariés dans leurs processus de décision, ce que confirment les délégués du personnel. Ceux-ci jugent important d'être informés à temps et associés aux processus de décision.

Si les délégués du personnel confirment l'attitude largement coopérative de la direction de leurs entreprises, les dirigeants considèrent eux aussi les représentants des salariés comme un partenaire important dans le contexte de restructuration (voir aussi, sur le type coopératif de conseil d'entreprise, Kotthoff). Selon la direction des entreprises, cette logique de coopération des délégués du personnel se reflète aussi dans les personnes nommées par les salariés aux conseils de surveillance.

Les directions d'entreprises voient dans les représentants des salariés siégeant dans les organes de surveillance un allié important pour leur politique - y compris face aux détenteurs municipaux. Sur les grandes orientations, les délégués du personnel partagent la même vision que la direction. Les représentants du personnel souhaitent eux aussi assurer la survie de leur entreprise. Or, dans un contexte de concurrence de plus en plus vive, cela ne sera possible qu'en se basant résolument sur les objectifs économiques.

C'est pourquoi les représentants du personnel sont souvent un allié de la direction dans les conseils de surveillance, où il s'agit en effet d'écarter les influences «étrangères » de la politique que tentent de faire peser les représentants municipaux.

L'ATTITUdE COOPÉRATIVE DES DÉLÉGUÉS DU PERSONNEL se traduit aussi par leur disposition à ne pas bloquer la restructuration des régies municipales au fil des besoins du marché, mais, en accord avec la direction, à les rendre socialement acceptables, ce que reconnaît aussi la direction : ainsi, dans le passé, la suppression d'emplois s'est principalement déroulée dans des conditions sociales acceptables, à travers des départs en retraite et des dispositifs de préretraite. Les représentants des salariés espèrent, à l'avenir aussi, éviter des licenciements à l'aide d'accords en ce sens. Dans les régies que nous avons étudiées, les délégués du personnel se montrent tout aussi coopératifs en acceptant d'intégrer aux systèmes de rémunération existants des composantes liées aux résultats.

Traduction de Marie GRAVEY 


\section{Indications bibliographiques}

Aвromeit H., «Öffentlicher Zweck und öffentliche Kontrolle. Ansätze zu einer politischen Theorie der öffentlichen Unternehmung », PVS, vol. 26, n 3, 1985, p. 287-305

Edeling T., Stölting E., WAGner D., Öffentliche Unternehmen zwischen Privatwirtschaft und öffentlicher Verwaltung, VS Verlag, Wiesbaden, 2004

EDEling T. , JANN W., WAGNER D. (eds), Öffentliches und privates Management: Fundamentally alike in all unimportant respects?, Leske + Budrich, Opladen, 1998

Edeling T., Jann W., Wagner, D, Reichard C. (eds), Öffentliche Unternehmen. Entstaatlichung und Privatisierung?, Leske + Budrich, Opladen, 2001

EDELING T. (et al.), Öffentliche Unternehmen zwischen Privatwirtschaft und öffentlicher Verwaltung. Forschungsbericht im DFG-Schwerpunkt: Regulierung und Restrukturierung der Arbeit in den Spannungsfeldern von Globalisierung und Dezentralisierung, Deutsche Forschungsgemeinschaft (DFG) Potsdam, 2001 (DFGForschungsbericht)

EICHHORN P., «Herausforderungen für Führungskräfte in öffentlichen Unternehmen », in EICHHORN P., ENGELHARDT W.W. (eds), Standortbestimmung öffentlicher Unternehmen in der Sozialen Marktwirtschaft, Nomos, Baden-Baden, 1994, p. 227-243

GroseniCK C., Wahl und Gestaltung institutioneller Arrangements in der kommunalen Abfallwirtschaft, wvb, Berlin, 2009

Hentze J., Personalwirtschaftslehre 1: Grundlagen, Personalbedarfsermittlung, -beschaffung, -entwicklung und-einsatz, Haupt, Stuttgart, 1994

LEGEL A., Veränderungen der Steuerungsmechanismen bei der Privatisierung von öffentlichen Unternehmen. Am Beispiel der Deutschen Bahn, wvb, Berlin, 2008

PotтhofF E., "Aufgabenorientierte Überwachung öffentlicher Unternehmen durch Aufsichtsräte », in EICHHORN P, ENGELHARDT W.W. (eds), Standortbestimmung öffentlicher Unternehmen in der Sozialen Marktwirtschaft, Nomos, Baden-Baden, 1994, p. 311-324

Scholz C., Personalmanagement, Vahlen, Munich, 2000

WAGNER D., Organisation, Führung und Personalmanagement, Haufe-Lexware, Fribourg, 1991

WILLKE H., Systemtheorie III: Steuerungstheorie, UTB, Stuttgart, Iéna, 1995. 


\title{
Nouvelles conditions de travail dans les services publics en Allemagne
}

\author{
Achim MEERKAMP
}

Membre du comité directeur national du syndicat ver.di (plus précisément en charge des services publics), Achim Meerkamp se livre ici à une analyse en trois points de la libéralisation des services d'intérêt général outre-Rhin : à l'état des lieux structurel des marchés concernés succède la mesure de l'impact des réformes sur les conventions collectives, puis sur le développement du personnel.

\section{Evolution des marchés libéralisés dans les services publics}

\section{Postes/télécommunications}

A l'été 1989, la Deutsche Bundespost a été scindée en trois domaines d'activités: télécommunications, postes et banque postale. Les anciens représentants du syndicat en charge de la Bundespost ont constaté dans un mémorandum que la privatisation de l'entreprise est à l'origine de la création de ver.di en 2001. Il faut noter à ce propos que les syndicats ne dépendent pas uniquement des organisations autonomes, mais aussi du monde extérieur. Avec un taux de syndicalisation de 85 à $90 \%$ des salariés, le syndicat des postes allemands était particulièrement homogène.

Les trois domaines cités plus haut ont donné naissance à trois entreprises distinctes. Une concurrence forte régnait sur le marché des services express entre des entreprises comme UPS, Hermes, TNT ou DHL, qui avaient toutefois dû, dans le passé, s'en tenir à la clientèle professionnelle. La libéralisation a entraîné une ouverture totale du marché, permettant également aux particuliers de se tourner vers ces entreprises. La nouvelle situation de concurrence qui en a résulté a amené Deutsche Post AG (DPAG) à s'intéresser non plus uniquement à la distribution du courrier, mais également aux services express et logistiques. En rachetant des entreprises d'envergure mondiale, DPAG a réussi à devenir une entreprise internationale, alors que la distribution de courrier correspond à une échelle plutôt nationale.

Au début du processus de privatisation, Deutsche Telekom détenait un monopole absolu sur les télécommunications. Elle s'est retrouvée face à la concurrence, sur le réseau fixe mais aussi pour les opérations de téléphonie mobile. Alors que cette entreprise garde le monopole absolu sur le réseau fixe, les télécommunications sont soumises, dans le monde entier, à une situation concurrentielle nouvelle. L'avenir montrera comment la Commission européenne traite cet aspect de la libéralisation. Il faut noter que, dans le secteur des infrastructures de réseau, Deutsche Telekom est le seul concessionnaire. Cela signifie que tous les autres opérateurs de téléphonie mobile doivent encore faire appel - pour des réparations par exemple - au personnel de cette entreprise. Avec 260000 employés, Deut- 
sche Telekom est devenue un opérateur international : seule une petite moitié du personnel travaille aujourd'hui sur le marché national, et il faut s'attendre à ce que cette part se réduise encore.

Quant à la banque postale Postbank, qui faisait autrefois partie de la Deutsche Bundespost, elle compte encore une clientèle relativement importante aujourd'hui. Avec 14 millions de clients, 21000 salariés et un total de bilan de 237 milliards $€$, il va de soi qu'au même titre que les établissements de droit public (les Sparkassen), cette entreprise n'a pas cessé d'intéresser les banques privées, désireuses de la reprendre. En faisant en 2006 l'acquisition du Beamtenheimstättenwerk (BHW), Postbank s'est étendue au secteur de l'épargne logement. Chose intéressante, le BHW était auparavant détenu à $100 \%$ par les syndicats : la fédération des fonctionnaires (Beamtenbund) et les syndicats à l'origine de ver.di.

\section{Secteur de l'énergie}

Le secteur de l'énergie était autrefois structuré autour de monopoles territoriaux, avec un partage des concessions entre organismes privés et publics. De nouvelles entreprises sont nées du processus de libéralisation en Allemagne, mais d'autres ont disparu, comme la Vereinigte Elektrizitätswerke Westfalen («Union des régies d'électricité de Westphalie »- VEW), qui a fusionné en 2000 avec RWE. Le secteur compte aujourd'hui quatre acteurs mondiaux (E.ON, RWE, le groupe Vattenfall - particulièrement actif dans le nord de l'Allemagne) et l'entreprise EnBW, présente dans le sud du pays, et dont EDF est devenu l'actionnaire majoritaire'. Quelques opérateurs régionaux sont également établis sur le marché, qui opèrent à la fois dans la distribution, la commercialisation et la production. Les numéros cinq et six du secteur électrique en Allemagne sont respectivement les distributeurs régionaux Rheinenergie $\mathrm{AG}$, dont le siège se trouve à Cologne, une entité née des anciennes régies municipales mais dont le champ d'action s'est étendu, et la Mannheimer Verkehrs- und Versorgungsunternehmen (MVV AG). Outre les quatre acteurs mondiaux et les opérateurs régionaux, on dénombre environ 900 régies municipales dans le secteur électrique allemand.

En dépit de cette multiplicité d'opérateurs, E.ON, RWE, Vattenfall et EnBW représentent encore $80 \%$ de la production. Alors qu'un éventuel retour à des régies municipales de droit public fait actuellement l'objet de débats - en particulier dans le sud de l'Allemagne et à Stuttgart -, les quatre principales entreprises du secteur revoient leurs domaines d'activité : E.ON quitte le domaine régulé des activités de réseau pour se concentrer, à l'échelle internationale, sur celui de la production. RWE a quant à elle acquis des entreprises de production dans différents pays. Ces opérateurs, axés sur le profit, sont conscients que la distribution ne leur permet plus de dégager des marges suffisantes et misent donc davantage sur le volet « production».

\footnotetext{
${ }^{1}$ En février 2011, EDF a cédé l'intégralité de ses parts (45\%) au Land de Bade-Wurtemberg, NDE.
} 
Le secteur des régies municipales est quant à lui très hétérogène et regroupe de très petites entreprises, avec un personnel peu nombreux, ainsi que des entités comptant plusieurs milliers de salariés, comme les Stadtwerke de Munich, qui ne se limitent pas à la production et à la distribution, mais ont également leurs propres filiales commerciales. Mais ces monopoles territoriaux sont aujourd'hui complètement exposés à la pression de la concurrence : les grandes entreprises monopolistiques pénètrent maintenant le secteur des régies municipales, et réciproquement. Cette évolution s'accompagne d'interactions significatives, qui ont également des répercussions sur le personnel.

\section{Secteur de l'eau/de l'assainissement}

Les négociations de l'OMC ont permis de constater que l'eau n'est, heureusement, pas encore devenue un bien librement commercialisable à l'échelle mondiale. En Allemagne, les communes doivent donc rester garantes du bon fonctionnement du marché de l'eau et de l'assainissement. La concurrence n'a pas changé grand-chose à cette situation. Malgré la présence de quelques opérateurs privés, ce marché se compose principalement d'entreprises publiques aux formes juridiques diverses, dont, globalement, des régies municipales dans les grandes villes et des organisations autonomes d'exploitation dans l'espace rural, en principe chargées uniquement de la gestion de l'eau. L'Allemagne compte ainsi plusieurs milliers d'entreprises dans le secteur de l'eau, qui ne détiennent parfois qu'un tout petit monopole territorial mais exercent diverses activités : captage, traitement, distribution et commercialisation. Dans le domaine des eaux usées, les communes étant soumises à une pression financière relativement forte, il faut s'attendre à quelques changements. L'Allemagne se caractérise par un haut niveau de développement technologique dans ce secteur qui a connu au cours de la dernière décennie des évolutions positives du point de vue écologique. Or, alors qu'il est urgent de moderniser les réseaux d'assainissement, les communes n'ont actuellement pas les moyens de réaliser les investissements nécessaires, ce qui va bientôt créer une pression financière.

\section{Gestion des déchets}

La gestion des déchets est un marché qui n'est pas vraiment libéralisé alors qu'il est désormais soumis à la concurrence. Ce secteur a connu ces dernières années une segmentation entre les domaines du verre, du papier et, en particulier, du plastique, ce qui a entraîné l'établissement d'une concurrence sur un marché où les concessions font l'objet d'appels d'offres et d'adjudications. Alors que certaines entreprises publiques s'intéressent à l'ensemble du marché, d'autres se concentrent sur les ordures ménagères. Cette activité constitue l'une des missions que doivent garantir les communes en Allemagne et que les grandes municipalités assument généralement elles-mêmes. Dans les grandes villes, la majorité des concurrents relèvent 
encore du secteur public. Dans les districts, on trouve de gros opérateurs privés comme le leader du marché Remondis ou l'entreprise ALBA, surtout présente dans l'ouest et le nord de l'Allemagne. A Berlin, ALBA est en outre l'un des concurrents du service municipal d'enlèvement des ordures (Berliner Stadtreinigung), qui emploie 5500 personnes. Du fait de cette situation concurrentielle, environ $45 \%$ des salariés du secteur de la gestion des déchets ne travaillent plus pour des employeurs publics.

\section{Services d'assistance au sol}

Ce secteur est libéralisé, mais non régulé. Après la privatisation de la Lufthansa, l'Europe s'est intéressée aux services d'assistance au sol et à la sécurité aérienne. Avant la libéralisation, un Land et une commune étaient conjointement en charge d'un aéroport. L'assistance au sol est désormais le fait soit de filiales - les anciennes entreprises d'assistance au sol - soit d'entreprises tierces, c'est-à-dire des entreprises indépendantes, créées par des investisseurs financiers. Par exemple, les deux aéroports qui existent encore à Berlin ne sont plus gérés par des entreprises publiques initiales ou leurs filiales, mais par un sous-traitant.

\section{Deutsche Bahn}

La Deutsche Bahn AG opère à l'échelle nationale et internationale dans les transports de proximité et le trafic grandes lignes ainsi que dans le fret de marchandises. Le gouvernement actuel a pour objectif son démantèlement et la privatisation des infrastructures, du transport de passagers et de marchandises. Bien que ver.di ne soit pas le syndicat compétent pour cette entreprise, il s'intéresse aux conditions de concurrence entre les frets ferroviaire, routier et par voies navigables.

\section{Autonomie contractuelle}

Dans les entreprises, la gestion des ressources humaines doit pour partie s'effectuer au travers de conventions collectives. En effet, la représentation des intérêts est bilatérale en Allemagne, les syndicats n'étant pas seulement des lobbyistes politiques mais également des partenaires sociaux, au niveau fédéral, sectoriel et jusqu'à celui de l'entreprise. En Allemagne, les conseils d'entreprise ou les conseils des délégués du personnel dans le secteur public ne sont pas habilités à signer des conventions collectives. L'usage est de conclure des accords de branche applicables à un territoire : cela signifie que, dans un secteur donné, sont signées des conventions nationales ou régionales, ce qui correspond aussi à la structuration des organisations patronales. La dimension « régionale s signifie qu'il n'y avait par exemple dans l'industrie métallurgique aucun accord de branche applicable à l'ensemble du territoire allemand, même si le syndicat IG Metall mène une politique de négociation à l'échelle du pays entier. Gesamtmetall étant une 
confédération d'employeurs composée de sous-fédérations, IG Metall a toujours traité avec des employeurs représentés par des fédérations et corporations régionales. L'organisation était la même dans le secteur tertiaire.

Ces dernières années, de nouvelles formes de rémunération se sont développées, dans le cadre de l'autonomie contractuelle, de sorte qu' on observe une dispersion des salaires, avec des évolutions vers le bas comme vers le haut. On observe en outre un recul des standards sociaux, l'une des évolutions les plus négatives en Allemagne étant celle de la durée hebdomadaire, mensuelle ou annuelle du travail, qui s'est faite au détriment des salariés.

\section{Désintégration des conventions collectives de branche}

La concurrence et la régulation ont entraîné la création de très nombreuses filiales et, du même coup, la restructuration des appartenances à des associations. La question fut donc de savoir ce que les syndicalistes pouvaient faire pour que le personnel ne se trouve pas sans couverture conventionnelle. Il n'était pas possible de contraindre les entreprises à s'associer à des fédérations sectorielles d'employeurs puisque l'autonomie contractuelle vaut pour les employeurs comme pour les salariés. Les syndicats ont donc tenté de faire signer des conventions collectives, ce qui a entraîné le développement d'une profusion de conventions différentes.

Cette situation concerne la majorité des systèmes conventionnels du service public. Le secteur privé du traitement des déchets entrait dans le champ de compétences de la fédération d'employeurs BDE (Bundesverband der Deutschen Entsorgungswirtschaft e.V.). Mais le BDE ne couvre que 20000 salariés sur les 70000 que compte la branche. Cela veut dire que 50000 salariés travaillent dans des entreprises du secteur qui n'étaient liés à aucune convention. Le syndicat ver.di a tenté de répondre à cette situation par des accords d'établissement, mais cela n'a pas fonctionné globalement. Le débat sur le salaire minimum tient en particulier au fait que, dans certains secteurs, les normes sociales n'ont pas été suffisamment imposées pour préserver les salariés d'une chute libre des salaires. Dans l'est de l'Allemagne par exemple, des salaires particulièrement bas $(3,45 €)$ sont versés aux éboueurs. Ces évolutions ont mis à mal les salariés dans les entreprises dépourvues de conventions collectives, mais également les conventions collectives ellesmêmes, à commencer par celles du privé. Dans le domaine de la gestion des déchets par exemple, les employeurs ont fait pression sur ver.di pour obtenir une couverture conventionnelle. Ver.di a réagi en acceptant l'inclusion de clauses d'ouverture dans les accords collectifs, et en établissant des «accords pour les situations de crise ». Ce principe permet à une entreprise de déroger aux règles prévues par les conventions collectives de branche pour assurer la survie des entreprises et les protéger de la faillite. Dans l'industrie métallurgique par exemple, l'accord de Pforzheim, signé par IG Metall, constitue un accord de ce type, si bien que, fin 2009, un quart des accords signés se trouvait déjà en dessous du niveau de protection sociale de la convention collective de branche. 


\section{Eclatement des grilles de rémunération}

Dans le cadre d'une réforme des conventions collectives de 2005, les salaires d'entrée de l'ensemble du secteur des services publics ont été abaissés de $10 \%$ pour les personnels nouvellement recrutés, ce qui a permis le retour à la compétitivité face aux opérateurs privés. Deuxièmement, de nouveaux planchers ont été créés pour les bas salaires. Troisièmement, on a assisté à un élargissement de l'éventail des salaires dans certaines branches, notamment pour les employés hors conventions collectives. Dans le secteur de l'énergie en particulier, des personnels ont été recrutés avec un niveau de rémunération supérieur à celui de la convention collective. Ver.di a en revanche dû intervenir pour que des salariés recrutés non pas au niveau cadre mais comme employés bénéficient d'une convention collective. Quatrièmement, la concurrence a amené à s'interroger sur les modalités de prise en compte possible de la performance individuelle. Ces dernières années, cette question a suscité en Allemagne un débat politique sur la rémunération de la performance et sur les primes. Dans le secteur public, les primes sont de plus en plus utilisées, qu'il s'agisse de primes d'entreprise concernant l'ensemble du personnel ou de primes réservées à une division en particulier, si bien que l'un des débats très politiques encore d'actualité entre ver.di et les employeurs concerne les rémunérations individualisées de la performance.

Le syndicat mène aussi le débat sur la prévoyance vieillesse d'entreprise - en particulier dans les services publics. En 2003, ver.di a dû procéder, aux côtés des employeurs, à une modification de la prévoyance vieillesse d'entreprise dans le public, liée à la question de la concurrence, mais aussi à celle du financement de l'ancienne prévoyance vieillesse d'entreprise. Cette question va en effet de pair avec le problème des très rapides suppressions de postes. Avec des salariés moins nombreux pour payer la retraite par répartition et un nombre plus important de départs en retraite, il ne sera toutefois plus possible, à un moment ou un autre, de financer la retraite des anciens salariés.

\section{Régression des standards sociaux}

La régression des standards sociaux concerne en premier lieu le maintien du salaire en cas de maladie. Dans le secteur public, le personnel avait droit à un maintien de salaire pendant 18 mois, mais il a été ramené à 6 mois en 2005.

D'autre part, les salariés du public touchaient traditionnellement des primes spéciales pour les vacances, en cas de performances générant des profits, pour les occasions particulières, et en fin d'année - des primes de Noël et de fin d'année, donc. Aujourd'hui, seule subsiste en principe la prime annuelle, dont le niveau a d'ailleurs été abaissé. Dans le cadre d'une redéfinition par l'Etat des motifs donnant lieu à une autorisation d'absence en dehors des congés, ver.di a également dû accepter des restrictions importantes ces dernières années. L'Etat, en tant qu'employeur, a refusé de libérer les personnels qui souhaitaient par exemple faire du 
bénévolat, si bien que ceux qui souhaiteraient faire valoir des engagements bénévoles devront à l'avenir payer pour cela. Cela constituera à court terme une menace en Allemagne, pour les pompiers volontaires par exemple.

Temps de travail

Dans toute l'Allemagne, la durée hebdomadaire moyenne du travail a sensiblement augmenté. Elle est passée d'un peu moins de 38 heures à un peu plus de 40 . Cela tient au fait qu'une partie importante des salariés ne bénéficie pas de conventions collectives, le temps de travail hebdomadaire fixé dans ce type d'accord étant généralement de 35 à 40 heures. Ainsi, les salariés du secteur bancaire travaillent 41 ou 42 heures par semaine. La flexibilisation s'étend, dans l'ensemble du pays : de plus en plus de personnes ont des temps de travail différents. L'homogénéité n'existe plus désormais dans les services publics. Dans les hôpitaux, par exemple, les systèmes classiques de fonctionnement à trois équipes ont été remplacés par une multiplicité de temps de travail différents, du fait de nouvelles exigences. A l'Université de Mayence, environ 258 formes de temps de travail coexistent. Ces exemples montrent le degré de flexibilisation et ses implications d'un point de vue social.

\section{Développement du personnel}

Le développement du personnel regroupe les quatre axes suivants :

- $\quad$ suppressions de postes ;

- réduction des postes à durée indéterminée ;

- segmentation des activités avec une tendance à la professionnalisation ;

- importance nouvelle de la formation initiale, permanente et continue.

Suppressions de postes

Depuis la libéralisation réalisée il y a vingt ans, Deutsche Post a perdu au total 250000 emplois en Allemagne, du fait de restructurations et d'une productivité accrue ; cette évolution s'est faite au détriment des clients, des filiales ayant été fermées. Le secteur de l'énergie a lui aussi procédé à une importante restructuration et supprimé environ un tiers des emplois.

\section{Réduction des emplois permanents}

La concentration sur les activités principales entraîne de nettes réductions des emplois permanents. Les entreprises énergétiques ont externalisé certaines activités. Le secteur des infrastructures de réseau avait déjà été en partie externalisé auparavant. Cela veut dire que la gestion du réseau est déjà partiellement sous-traitée. Outre la réduction des postes permanents, le recours aux emplois temporaires et à 
l'intérim est de plus en plus fréquent, ce qui a constitué ces dernières années l'un des débats entre les partenaires sociaux. Or, la diminution de l'ancrage sur les marchés locaux renforce les fluctuations. Les employeurs publics ont voulu avoir des marchés de l'emploi flexibles dans leurs secteurs, si bien que les fluctuations sont fortes là où il y a pénurie d'emploi. Cela engendre automatiquement des problèmes démographiques. Car, lorsque l'on supprime des postes pendant toute une décennie et que l'on cesse de former ou de recruter suffisamment de personnels, on se trouve confronté, en particulier dans les secteurs de la gestion des déchets, des services aéroportuaires d'assistance au sol et de l'approvisionnement énergétique, à un vieillissement du personnel. Mais cette problématique constitue aussi la mission essentielle des années à venir dans les domaines régaliens classiques du service public.

\section{Segmentation des activités}

La séparation de la production, de la distribution ou des services après-vente entraîne une modification des activités, relevant d'employeurs différents. Cette segmentation mène certes à une professionnalisation, mais aussi à une déqualification dans le secteur à bas salaire qui se développe.

De plus, une pression a été exercée sur la productivité dans le secteur public. A l'échelon fédéral, régional et communal, 1,1 million de postes ont été supprimés ces 15 dernières années sans modification des structures territoriales. Le personnel a dû compenser cette évolution, d'une manière ou d'une autre.

Importance nouvelle de la formation initiale, permanente et continue

La question du recrutement et de l'évolution de l'organisation du travail a pris une importance tout à fait nouvelle. La concurrence et l'évolution des exigences accroissent la pression sur les entreprises et les salariés en termes de qualité. Il a fallu réorganiser les services de développement du personnel car le savoir-faire n'était jusqu'alors pas présent dans les structures des entreprises. La formation continue gagne de l'importance. La représentation des intérêts du personnel conseils d'entreprise, conseils des délégués du personnel dans le secteur public et syndicats - est confrontée à de nouveaux défis.

En Allemagne, La QUeSTion de la gestion des ressources humaines est liée aux structures de protection des salariés. Celles-ci suivent l'évolution des conditions de travail, elles-mêmes liées aux marchés. Si les marchés évoluent, le volet contractuel et la gestion des ressources humaines évoluent également.

Traduction de Marie GRAVEY 


\title{
Ouverture du marché du fret ferroviaire. Du statut du cheminot à son refoulement ?
}

\author{
Marnix DRESSEN
}

Fruit d'une recherche en cours, cet article cherche à comprendre les causes, les enjeux et les moyens de l'ouverture du marché ferroviaire en Europe et plus particulièrement en France avec des aperçus sur l'Allemagne. L'article commence par analyser les causes multifactorielles de la crise du fret, donne des éléments sur les initiatives affichées par la Commission européenne pour le redresser. II analyse également la négociation collective dans le ferroviaire privé et cherche à décrypter le jeu, pour le moins ambigu selon l'auteur, de la direction de l'opérateur historique, la SNCF. L'hypothèse centrale est de considérer que sous couvert de l'ouverture des marchés, il s'agit en fait de réduire les avantages sociaux consentis aux cheminots, incarnation d'un des derniers bastions ouvriers, dans l'espoir d'exacerber la concurrence ainsi que de faire baisser les coûts et réduire les tarifs pour les clients industriels et les voyageurs les plus solvables.

Depuis les années 1980 et 1990, la question de l'ouverture du marché des industries à réseaux (ferroviaire, gaz et électricité, etc.) est régulièrement mise à l'agenda politique par la Commission européenne. Prévaut dans les instances européennes la croyance en la supériorité d'une régulation par le marché sur les régulations étatiques. Il est vrai que dans le cadre de ces «dénationalisations » (privatisation et «européanisation »), les dynamiques de branches, par exemple entre les télécoms et le ferroviaire, sont variables. Les processus, les temporalités et les acteurs mobilisés sont à chaque fois spécifiques (Dressen et Mias, 2009). Ces sociohistoires particulières tiennent à la fois à l'histoire des branches, aux rapports de force sociaux passés et présents dont elles sont le cadre et au degré d'acceptabilité sociale des effets des réformes en cours (Guerre, Lettre de la fondation $n^{\circ} 51$, juin 2010) ${ }^{1}$.

Cet article est plus particulièrement centré sur les transports ferroviaires et plus spécifiquement sur le transport de marchandises. De longue date, il est en crise et l'objectif affiché des restructurations est une « revitalisation » du transport de marchandise. Or, la profondeur temporelle dont on dispose aujourd'hui est suffisante pour montrer que ces orientations ne produisent pas les effets attendus. En effet, dans l'Hexagone, en dépit de ces initiatives, la part du ferroviaire dans le transport de marchandises recule. Mais selon la Commission européenne, jusqu'à la crise financière de 2008, la tendance était inverse au Royaume-Uni et aux Pays-Bas. Il en était de même en Allemagne. Dans ce pays, le fret ferroviaire a crû de $50 \%$ entre 2000 et 2008 alors qu'en France, il

\footnotetext{
${ }^{1}$ Ce qui ouvre à de passionnantes questions de théorie du social : comment d'une branche à l'autre, des acteurs divers dans leurs attentes, intervenant dans des cadres institutionnels hétérogènes peuvent-ils finalement coconstruire des réalités dont les orientations entretiennent pour le moins un air de famille?
} 
chutait assez spectaculairement selon un récent rapport sénatorial ${ }^{2}$ et cela bien avant le ralentissement économique résultant de la crise financière $(-25 \%$ de fret transporté de part et d'autre du Rhin en 2009).

Recul en France, expansion en Allemagne, aux Pays-Bas et au RoyaumeUni, ce constat conduit à se poser la question des variables explicatives à ces situations contrastées.

Sur le plan politique et social, en France, l'ouverture du marché des chemins de fer a entraîné un début d'élaboration d'une convention collective pour les cinq opérateurs privés et celle-ci ouvre une brèche dans le statut des cheminots employés par l'opérateur historique $(\mathrm{OH})$. La diversification des régimes conventionnels couvrant les cheminots du public et du privé contribue aussi à affaiblir un des derniers bastions syndicaux hexagonaux. Il y a de bonnes raisons de penser que si demain cette convention collective est signée par suffisamment de fédérations syndicales et étendue par le ministère du Travail, elle pourrait devenir la «loi de la profession» pour l'ensemble du monde ferroviaire français. C'est ce qu'en 2006, le Premier ministre D. de Villepin appelait un «cadre social harmonisé » (entre cheminots de l'opérateur historique et cheminots du privé).

Mais avant d'approfondir les transactions des acteurs sociaux autour d'une convention collective et d'analyser comment les fédérations syndicales en France ont été associées à la fragilisation du statut du cheminot, voire à son « refoulement ${ }^{3}$ », il convient de brosser un tableau des difficultés du transport ferroviaire de marchandises (depuis le milieu des années 1970) et d'examiner les initiatives de la Commission européenne.

\section{Un recul du fret ferroviaire entamé il y a 35 ans et complexe à analyser}

Le fret ferroviaire français se situe en deuxième position dans l'UE 25, assez loin cependant derrière l'Allemagne nettement leader (en 2004, le ferroviaire français contrôlait $11 \%$ de parts de marché et le ferroviaire allemand $19 \%$ ) alors que la SNCF et la DB sont de taille comparable ${ }^{4}$. Néanmoins, en France, la part du fret dans le transport de marchandises décline 5 . Parti d'une part de marché de $60 \%$ en 1960 , la progression s'observe jusqu'en 1975 pour tomber à $10 \%$ en 2007. En terme de milliards de tonnes.km transportées, l'analyse montre des chutes et des rebonds étonnants, p. ex. entre 1981 et 1998 ou encore 1996 et 2000, 1993 étant en revanche d'un bas niveau historique ${ }^{6}$. Ce recul relatif du

\footnotetext{
${ }^{2}$ http://www.senat.fr/rap/r10-055/r10-055.html (rapport déposé le 20 oct. 2010) [consulté le 18/05/11].

${ }^{3}$ Dans le jargon ferroviaire, « refouler » un train, c'est les faire reculer.

${ }^{4}$ En 2009, le groupe SNCF (toutes filiales comprises) employait 230000 personnes et l'Epic SNCF, 155000. Le groupe DB employait 240000 salariés (170 000 seraient issus de l'ancienne DB publique, «le solde étant logé chez DB Schenker ») [Fried, Les Echos, 14 janv. 2010]. DB Schenker est à la DB ce que Geodis est à la $\mathrm{SNCF}$, une filiale multimodale intégrée.

${ }^{5} \mathrm{http} / / /$ ec.europa.eu/community_law/state_aids/transports-2004/n386-04.pdf [consulté le 12/10/2010].

${ }^{6}$ Pour un chapitre sur nombre de pays européens dont la France, publié en 2001 par l'Observatoire Transport Europe qui se base sur des statistiques du ministère des Transports : http://www.cnt.fr/UserFiles/File/ Commissions_Permanentes/Observatoire/Europe/Dossier_2_Fret-Fer.pdf [consulté en octobre 2010].
} 
ferroviaire s'observait en général dans l'UE. Le recul du transport du fret par chemin de fer s'est poursuivi dans nombre de pays, tout particulièrement en Irlande, en Espagne et dans les nouveaux Etats membres (sauf la Hongrie). Même dans un pays à la culture ferroviaire aussi enracinée que la Suisse, le fret rencontre des difficultés ${ }^{7}$. Globalement, les instances européennes parlent d'une «érosion continue du fret ferroviaire au profit du transport routier ». En effet, alors que le volume transporté par rail a décru de $19 \%$ pendant la même période, en termes absolus, le volume transporté par route a presque doublé en vingt ans (augmentation de $91 \%$ entre 1980 et 2002). «Cette évolution s'est traduite par une augmentation du transport par route de plus de 300000 millions de tonnes kilomètres pour la période 1995-2002, ayant pour conséquences des effets fortement négatifs, notamment en ce qui concerne l'environnement et la congestion routière » notait la Direction générale de l'Energie et des Transports en $2005^{8}$.

Le recul du fret ferroviaire en France a des causes multifactorielles parmi lesquelles les transformations structurelles de l'économie assez spécifiques en France par rapport à l'Allemagne. Dans l'Hexagone, au cours des années 1970, la spectaculaire résorption de la grosse industrie (houille, sidérurgie, etc.) dans le Nord et en Lorraine résulte en partie de l'installation de ce qui en reste à proximité des ports (Dunkerque, Fos-sur-Mer). Comptent aussi la tertiarisation de l'économie et les fragmentations du tissu d'entreprises du secteur secondaire qu'entraînent les intenses politiques de filialisations et d'externalisations de fonctions productives de biens ou de services (Freyssinet, 2006, p. 25). Or, la balkanisation du tissu industriel n'est guère favorable au fer pour diverses raisons. Dans le service que les routiers rendent aux entreprises, le camion se révèle plus souple, permettant le transport de porte à porte du fait d'une meilleure capillarité du réseau routier par rapport au fer $(400000 \mathrm{~km}$ de routes nationales, départementales et d'autoroutes environ contre $30000 \mathrm{~km}$ de voies ferrées) alors que des plans de fermeture de voies ferrées jalonnent l'histoire de la SNCF depuis 1938 au moins. Surtout, un des avantages concurrentiels de la route réside dans sa congruence avec le paradigme productif ohniste (méthode Kanban) auquel adhère un nombre croissant de clients industriels ${ }^{9}$. En vue de réduire les coûts de production et notamment la masse salariale, c'est-à-dire les effectifs salariés, les entreprises se convertissent en effet en nombre à la doctrine du zéro stock, zéro délai (Durand, 2004, chap. 2) et, dans ce domaine, le fer peine à résister au camion et cela alors même que les wagons sont parfois considérés par de gros clients (céréaliers, pétroliers) comme un espace de stockage externalisé

\footnotetext{
${ }^{7}$ http://www.cnt.fr/UserFiles/File/Commissions_Permanentes/Observatoire/Europe/Dossier_2_Fret-Fer.pdf, p. 63 [consulté en octobre 2010].

${ }^{8} \mathrm{http} / / /$ ec.europa.eu/community_law/state_aids/transports-2004/n386-04.pdf [consulté le 12/10/2010].

${ }^{9}$ Ainsi, pour répondre aux exigences croissantes des clients industriels, ECR (Euro Cargo Rail), filiale de DB Schenker, vient d'équiper 30000 wagons de GPS. «A chaque instant, nos clients peuvent savoir où se trouve leurs caisses mobiles ou leur conteneurs et quand ils vont arriver à destination » [Gradt, Les Echos, 14 janv. 2010].
} 
sur la SNCF. On a d'ailleurs pu dire que dans le paradigme ohniste « les stocks voyagent » (Durand, 2004). Sur le plan des coûts, l'avantage comparatif de la route repose sur la flexibilité interne qui pèse sur les camionneurs, surtout lorsqu'ils travaillent sur de longues distances. Il faut ajouter qu'une priorité en investissements est souvent donnée aux transports routiers probablement du fait que les camionneurs exercent de longue date, sur les pouvoirs publics, un efficace lobbying (Neiertz, 1995 ; Chevandier, 2002, p. 265). De 1980 à 1998, les investissements en infrastructures de transport s'élevaient à 297,4 milliards de francs pour le rail (soit 19,4\% du total), contre 1000 milliards de francs pour les routes et les autoroutes (soit 65,4\% de l'ensemble des investissements en infrastructures de transport ${ }^{10}$. Les autoroutes en France ont probablement avant tout été conçues et construites pour les camions (i.e. au détriment du ferroviaire). Au niveau macroéconomique, les entreprises de transports routiers ne payent ni leurs investissements ni leurs externalités négatives (pollution, accidents, encombrements, etc.). De plus, dans l'Hexagone, la taxe sur les carburants constitue une importante source de revenus pour le fisc, raison supplémentaire de la mansuétude des pouvoirs publics à l'égard des camionneurs.

Pour cette longue série de facteurs (souplesse, coût, faiblesse relative des investissements et fiscalité) la route en France bénéficie donc d'une situation structurellement favorable, malgré les atteintes à l'environnement, les heures perdues dans les encombrements routiers et les accidents qu'elle génère. N'estime-t-on pas que la moitié environ des accidents du travail mortels sont désormais des accidents de la route (impliquant et/ou tuant souvent des routiers) ?

On le voit, il n'est nullement nécessaire de partir en chasse des avantages sociaux abusifs dont bénéficieraient les cheminots, c'est plutôt du côté du dumping social qui pèse sur les routiers qu'il faudrait regarder.

En revanche, il est vrai que le fer public est handicapé par l'autofinancement de ses investissements (matériel et exploitation) et par ses frais fixes (80 ou $85 \%$ du prix total d'un transport) alors que sur route, ce coût varie en fonction de l'activité des camions ${ }^{11}$. Ajoutons que l'Etat est bien moins généreux qu'on le dit à l'égard de l'opérateur historique. Ainsi, pour emprunter sur les marchés financiers, la SNCF ne peut que se prévaloir de cautions inférieures à celles que le Premier ministre L. Fabius avait apportées à Eurodisney pour s'installer à Marne-la-Vallée, en $1986^{12}$, alors même que le statut d'Epic (Etablissement Public Industriel et Commercial) est censé lui apporter une garantie financière de tout premier plan qui «exclut la possibilité d'une procédure de faillite ou d'insolvabilité $»^{13}$.

\footnotetext{
${ }^{10} \mathrm{http}: / /$ www.hyperdebat.net/IMG/pdf/doc-47.pdf (Observatoire Transport Europe, 2001, p. 37) [consulté le 9/06/11].

${ }^{11} \mathrm{http} / / /$ ec.europa.eu/community_law/state_aids/transports-2004/n386-04.pdf [consulté le 12/10/2010].

${ }^{12}$ Ainsi l'Etat n'a financé qu'une seule ligne de TGV et à $30 \%$ seulement (la LGV Atlantique) (Mühlstein, 2007).

${ }^{13} \mathrm{http} / / /$ ec.europa.eu/community_law/state_aids/transports-2004/n386-04.pdf [consulté le 12/10/2010].
} 
Depuis la création de l'Epic Réseau Ferré de France (RFF) à côté de l'Epic $\mathrm{SNCF}^{14}, \mathrm{l}^{\prime} \mathrm{OH}$ se voit facturer des « sillons » (droits de passage dans un créneau horaire précis sur une ligne ferroviaire donnée). Les tarifs pratiqués par le propriétaire gestionnaire des infrastructures ferroviaires sont de plus en plus élevés quoique meilleur marché que dans d'autres pays européens où le fret se porte pourtant mieux. Ces coûts sont néanmoins des obstacles pour la SNCF et pour la compétitivité du transport de marchandises. Le montant de ces dépenses résulte probablement de la désintégration de l'ancienne SNCF par la création de RFF. RFF se trouve en effet devant la nécessité d'assurer le financement de la maintenance et surtout de la rénovation d'un réseau qui s'est progressivement dégradé trois décennies durant, faute d'investissements continus à la hauteur des intenses contraintes qu'il subit. Il faut remédier en urgence ou en profondeur aux pannes récurrentes de signalisation et de caténaires, voire de rails cassés. Le réseau était, il y a peu encore, en si mauvais état que les conducteurs de trains étaient contraints à rouler à vitesse réduite sur plusieurs milliers de km (Rapport Rivier \& Putalaz, 2005). Du côté de l'OH, l'âge moyen des «bagnoles » (les wagons) serait de 28 ans alors que ce type de matériel aurait une espérance de vie de 30 ans. La vétusté entraîne des coûts de maintenance considérés deux fois plus élevés et une fréquence des pannes avec leur cortège de conséquences, dont le mécontentement des fameux clients (du fret et du transport voyageurs puisque les deux trafics empruntent généralement les mêmes voies). De manière plus structurelle, le caractère polyvalent des infrastructures se fait au détriment du fret, puisque dans l'ordre des passages, le transport «voyageurs » est prioritaire sur les « patachons » (trains de marchandises).

Enfin, il faut souligner le parti pris de service public qui a longtemps conduit l'opérateur historique à assurer, parallèlement au transport combiné et au train complet, la messagerie ferroviaire dite aussi «lotissement». Cette activité est utile sur le plan économique et environnemental mais peu rentable sur le plan commercial. La messagerie implique en effet la formation de convois à partir de wagons isolés, activité bien plus coûteuse en termes de matériels, d'infrastructures de triage, de personnels nécessaires que la traction de «trains massifs ». La messagerie représentait en 2007 40\% du trafic et $70 \%$ des pertes de la branche fret de la SNCF (Les Echos, 9 sept. 2008). A contrario, les convois complets qui relient un point $\mathrm{A}$ à un point $\mathrm{B}$ sont bien plus lucratifs. C'est d'ailleurs pourquoi les opérateurs privés convoitent cette niche d'activité. Mais la perte de ces trafics par l'Epic l'empêcherait de pratiquer la péréquation entre trafics lucratifs et ceux qui le sont moins. Du coup les difficultés du fret public s'aggravent.

On le voit, les difficultés du fret ferroviaire français ont de multiples origines et il n'est point besoin d'incriminer les cheminots et leurs prétendus avantages indus pour les comprendre. A moins que la stigmatisation des cheminots

\footnotetext{
${ }^{14}$ L'Epic RFF a été créé par la loi du 13 fév. 1997 et l'Epic SNCF par le décret du 18 fév. 1983, consolidé le 30 déc. 2005
} 
ait une autre fonction, celle du démantèlement du service public des transports ferroviaires et de concessions aux entreprises privées ? Pour valider cette hypothèse, il nous faut maintenant examiner la politique européenne en matière d'ouverture des marchés du chemin de fer.

\section{Incitations et injonctions européennes}

«Le développement du fret ferroviaire, est l'une des priorités centrales de la politique communautaire des transports. »

J. Barrot, ancien Commissaire européen au transport (2004)

Depuis 1991, forte des pouvoirs confiés par les Etats-nations (Gobin 2002, Dufresne et alii, 2006), la Commission européenne a multiplié les initiatives de libéralisation, mettant en œuvre des projets dont le Traité de Rome avait envisagé la possibilité et à laquelle, dès les années 1950, les instances dirigeantes de la SNCF se préparent. Des courants d'inspiration néoclassique s'expriment par exemple au niveau du Conseil d'Administration, selon Jean Finez qui prépare une thèse sur le personnel dirigeant des entreprises ferroviaires. Très tôt, les sphères dirigeantes de la SNCF s'inscrivent dans la logique de la Communauté économique européenne qui incite à l'exacerbation de la concurrence. Le rapport annuel de la SNCF pour l'année 1971 (p. 103) est assez clair : «En définitive, nous avons de bonnes raisons de penser qu'avec les structures commerciales ainsi mises en place, la SNCF sera mieux armée qu'autrefois pour affronter la concurrence dans le cadre de l'économie de marché vers laquelle s'oriente la politique des transports en France et dans la C.E.E. Nous sommes en tout cas convaincus qu'elle aura accompli un grand pas vers la complète satisfaction des aspirations de sa clientèle $»^{15}$.

Sur le plan politique, l'ouverture effective du marché ferroviaire ne prend sens que dans le contexte du consensus de Washington (la régulation étatique n'est pas la solution, mais « est le problème ») et de l'implosion du monde soviétique, étant entendu que «les bases juridiques d'une telle politique libérale existent bien depuis $1957 »$ (Mias et Dressen, 2009). Toutefois, les projets des partisans de la fin des monopoles d'Etat ont subi un temps de latence du fait des rapports de forces sociaux relatifs à la triple hostilité des salariés des transports, des usagers et de «l'opinion publique». Puis les rangs de ceux qui pour diverses raisons croient aux vertus de la concurrence se sont étoffés.

Sur le plan politique, juridique et réglementaire, on peut dire que la Commission européenne ne manque pas de constance dans ses orientations relatives à l'ouverture du marché ferroviaire. Adoptant explicitement une politique vantant la concurrence libre et non faussée, la Commission affirme que ses initia-

\footnotetext{
${ }^{15}$ Merci à Jean Finez qui m'a communiqué cet extrait de document et merci aussi pour sa relecture exigeante de l'ensemble de cet article, NDA.
} 
tives permettront de redynamiser le fret et amélioreront le bilan carbone des transports de marchandise tout en en réduisant les coûts.

Les orientations de la Commission ont été matérialisées par trois livres blancs préparatoires à trois «paquets» ferroviaires, contenant au total une dizaine de directives, en comptant la «directive mère » de 1991. Celle-ci crée les conditions de la fin des monopoles historiques en leur faisant injonction de distinguer dans leurs comptes l'infrastructure et l'exploitation (les compagnies exploitantes n'étaient cependant pas tenues de se séparer organiquement de l'infrastructure, la DB par exemple ne l'a pas fait). Il convient de préciser que ces réelles bifurcations ne se sont pas produites sans signes avant-coureurs. D'une part, plusieurs années avant la directive mère de la libéralisation du fret, comme $\mathrm{N}$. Hilal le signale, les interventions des institutions supranationales (Cour de Justice des Communautés européennes et Commission européenne et, dans une moindre mesure, Parlement européen) paraissent non négligeables. Dès le mitan des années 1980, ces instances juridiques sont à l'initiative. Saisie par le Parlement européen, la CJCE rend un arrêt le 22 mai 1985 qui condamne la carence du Conseil des ministres, oblige les Etats à respecter les engagements de 1957 et à ne pas se contenter à l'échelle européenne d'une juxtaposition des réseaux nationaux (Hilal, 2007, p. 18-19). Cela dit, la configuration n'est pas simplement celle d'une commission édictant des oukases en direction des Etats-nations et des $\mathrm{OH}$. Pas plus que les Etats-nations, l'ensemble des organisations syndicales du ferroviaire a toujours été vent debout contre ces orientations, avec lesquelles il a parfois cherché à composer. Comme le dit C. Gobin «adversaires sur certains points, alliés sur d'autres, le «duo » syndicats/commission, oblige, pour comprendre la dynamique, à une maîtrise fine tant des caractéristiques social-historiques nationales de chacun des secteurs envisagés que des rythmes, tensions et légitimité internes propres à l'ensemble institutionnel communautaire » (Soutenance thèse N. Hilal, IEP de Paris, 20 juin 2005). Enfin, il faut aussi considérer que ces réformes ont pris argument d'anciennes difficultés du fret ferroviaire comme on l'a vu supra dans la première partie de cet article. Faute de la fragilisation de l'édifice antérieur, on peut penser que la Commission aurait rencontré plus de difficultés à imposer ses vues.

Quoi qu'il en soit, avec parfois des nuances, des inflexions ou des accélérations, depuis bientôt deux décennies, la Commission européenne suit une ligne intangible consistant à prôner l'introduction de la concurrence comme une panacée dans les services publics ferroviaires et autres services publics à réseaux (gaz, électricité, télécoms). Elle a fait le choix d'ignorer la spécificité du ferroviaire qui ne transporte pas que des électrons ou des fluides mais aussi des voyageurs ${ }^{16}$.

\footnotetext{
${ }^{16}$ Voir Philippe Mühlstein, «sur la libéralisation du transport ferroviaire », 20/9/2007 (l'auteur, ingénieur et cadre à la SNCF, appartient au conseil scientifique d'Attac). Ce texte de 22 p. synthétise clairement quelques traits saillants des évolutions passées et présentes du transport ferroviaire. Accessible sur http://hussonet. free.fr/muhlib.pdf [consulté le 10/02/2010].
} 
Mais d'un point de vue pragmatique, ces orientations, pourtant soigneusement transposées dans la législation et la réglementation ${ }^{17} \mathrm{n}$ 'ont à ce jour pas permis de relancer le transport ferroviaire de marchandises en France ni même, on l'a vu, dans l'UE $25^{18}$. Ces constats factuels ne conduisent pas les autorités européennes à prendre de nouvelles initiatives pragmatiques mais au contraire à persévérer dans leur être et à peser toujours davantage pour une ouverture encore plus nette du marché. Ainsi en juin 2008, A. Tajani, le nouveau Commissaire européen en charge des transports, ouvrait une série de procédures d'infraction adressée à plusieurs Etats-nations contre le manque d'indépendance des sociétés qui gèrent les infrastructures ferroviaires. Ces lettres de mise en demeure mettent notamment en cause les liens persistants entre la SNCF, d'une part, et Réseau Ferré de France (RFF), d'autre part.

Sur le plan économique, on ne peut pourtant ignorer que les nouveaux géants économiques internationaux qui se substituent aux anciens monopoles d'Etat, par ex. dans le fret aérien, organisent parfois des ententes comme la Commission européenne elle-même l'a encore pointé en février 2010 : 18 opérateurs (dont DHL, UPS, Hellmann, Panalpina, DB Schenker, etc.) sont officiellement accusés de mise en place de cartel, une affaire qui évoque le précédent des opérateurs téléphoniques d'ailleurs condamnés pour cela à de significatives amendes (Les Echos, 11 fév. 2010). En somme des monopoles privés cherchent à se construire sur les décombres des monopoles publics. Ajoutons que l'équilibre économique des opérateurs ferroviaires, notamment dans le transport voyageur sur moyenne distance, est menacé par les compagnies aériennes low cost, et il est illusoire de croire que le train pourra être concurrentiel tant que le transport aérien ne sera pas régulé par des considérations environnementales et sociales (à condition de réserver à l'avance, il est aujourd'hui possible de voyager entre Beauvais en Picardie et Milan pour $8 €$ en moyenne...).

Sur le plan idéologique, observons que la Commission européenne raisonne toujours comme si la réduction des coûts était en elle-même souhaitable. On ne perçoit pas grande attention de sa part aux conséquences sociales de cette option. Et même en se plaçant du point de vue d'une critique interne à sa politique, en l'absence d'un réaménagement du territoire et d'une autre division spatiale du travail, il est à craindre que la diminution des frais de transports entraînerait des trajets toujours plus longs pour les marchandises et donc plus d'effets néfastes pour l'environnement. En d'autres termes, la réduction des coûts par principe est grosse d'effets pervers. Néanmoins, pour les marqueteurs, s'il est solvable, la figure du client d'entreprise a progressivement pris la place de l'usager et incarne désormais la loi et les prophètes (une visite dans les «salons grands

\footnotetext{
${ }^{17}$ Le site institutionnel Legifrance fait état de 32 textes transposant des directives européennes concernant totalement ou non le ferroviaire. Le plus ancien date de 1979. Sur les 13 documents qui contiennent le terme «ferroviaire » en titre, le premier date de 1995, le plus récent remonte à 2008 [consultation le 15/10/2009]. Voir http://www.legifrance.gouv.fr/

${ }^{18} \mathrm{http} / / /$ ec.europa.eu/transport/logistics/freight_logistics_action_plan/doc/memo/memo_rail_fr.pdf, 2007 [consulté le 18/05/11], voir le graphique de la p. 4.
} 
voyageurs » des principales gares est à ce titre édifiante). Dans tous les sens du terme, la SNCF est aujourd'hui une société à au moins deux vitesses.

\section{Stratégie industrielle de la SNCF : favoriser les opérateurs ferroviaires de proximité}

Dans le domaine du fret, la direction de l'opérateur historique a mis en œuvre une longue série de mesures et de plans, avec l'objectif affiché de redresser la situation du fret. Ainsi, depuis 1997, Fret SNCF a introduit un système d'information comptable et de contrôle de gestion séparé, qu'il gère de manière autonome pour tout ce qui concerne les constituants du chiffre d'affaires, la comptabilité clients, le crédit clients et l'administration des ventes. Fret SNCF dispose d'un compte de résultats propre, et depuis 2003 d'un bilan, conformément à la directive 91/440 de la Commission européenne, telle que modifiée par la directive $2001 / 12^{19}$. Justifiés par la «relance du fret », les plans d'inspiration le plus souvent malthusienne sont généralement aussi basés sur des réductions d'effectifs. En janvier 2010, la presse financière annonçait près de 2600 suppressions de postes dans le fret public qui s'ajouteront aux 1760 de l'année précédente. «Une décision dictée par les mauvais résultats de cette branche, encore aggravés par la crise et à la volonté affichée par la direction d'abandonner des trafics pas rentables » (La Tribune, 12 janv. 2010). A la demande de la Commission, ces plans s'accompagnent généralement de la sélection de certaines niches et de la fermeture de voies, de dépôts, de triages ${ }^{20}$. Entre autres exemples possibles, la fermeture de la gare de Triage de Somain (Nord), il y a peu encore la première de France, illustre les conséquences de ces plans. Tandis que la CFDT et la FGAAC ont assez facilement accepté certaines de ces restructurations, ces projets se sont heurtés aux résistances de deux organisations syndicales, surtout la CGT et Sud Rail qui y ont vu une stratégie du siège de la SNCF pour « désosser l'entreprise », et finalement la privatiser par appartements. Est aussi inquiétante pour l'avenir du fret public, la présence sur le réseau de deux opérateurs privés mais filiales de l'OH (VFLI et Naviland Cargo) qui l'auto-concurrencent tout en fonctionnant partiellement avec du personnel SNCF. Si ces plans sont jugés inacceptables par une partie des cheminots, c'est aussi qu'ils impliquent de passer au rabot les avantages et les protections dont bénéficient les conducteurs de trains au risque de les conduire à l'échec. Néanmoins, en 2009, certains conducteurs de trains ont accepté de participer à une expérience basée sur le volontariat dans l'espoir par exemple d'un rapprochement avec leur région d'origine. Mais ces plans ont tous échoué. L'absence de volonté politique manifeste de la direction pour sortir de la crise dans laquelle s'enfonce le fret suscite beaucoup de pessimisme sur son avenir.

\footnotetext{
${ }^{19} \mathrm{http}: / /$ ec.europa.eu/community_law/state_aids/transports-2004/n386-04.pdf [consulté le 12/10/2010].

${ }^{20} \mathrm{http}: / /$ ec.europa.eu/community_law/state_aids/transports-2004/n386-04.pdf [consulté le 12/10/2010].
} 
Depuis l'automne 2009, la direction de la SNCF agit autrement. Il s'agit désormais de contourner les résistances et de favoriser des concurrents privés dans le transport de proximité dont elle abandonne clairement l'exploitation, conformément aux conseils de la Commission européenne.

Le 30 septembre 2009, par la bouche de son PDG, la direction de la SNCF a annoncé un engagement national pour le fret ferroviaire, présenté comme «une révolution» «comparable à celle $d u T G V{ }^{21}$. Celle-ci s'articulera sur huit axes dont le développement des opérateurs ferroviaires de proximité (OFP), « une PME ferroviaire qui couvre un territoire ». L'émergence d'une partie au moins des OFP, résulte du désengagement de la SNCF sur ce segment du marché qu'est la courte distance.

Fret SNCF a commencé par être mis sous la tutelle de Geodis, privatisée en 1996 et actuellement filiale à plus de $98 \%$ de la SNCF (depuis juillet 2008). Puis l'opérateur historique s'est engagé à mettre ses propres «ressources » (hommes, savoir-faire, matériels) à la disposition de ses concurrents émergents, les OFP qui eux aussi sont de droit privé. Cela ne se fait pas sans heurts et contradictions entre le gouvernement d'une part et fret SNCF d'autre part puisqu'en juillet 2010, le ministre des Transports Bussereau estimait que la SNCF « sabotait» (sic) la mise en place des OFP (Les Echos, 11 oct. 2010). Après de grandes difficultés ${ }^{22}$, divers OFP ont cependant vu le jour : TPCF (Trains du Pays Cathares et du Fenouillèdes, Ferrovergne) ou sont sur le point de se lancer (dans le Morvan et en Midi-Pyrénées). Ferrovergne par exemple est née cet automne 2010 en Auvergne sur l'initiative des transporteurs et chargeurs de cette région soutenus par la chambre régionale de commerce et d'industrie. Selon un cabinet de consultants, les entreprises partie prenante de cette initiative estimeraient que la SNCF ne répondrait plus à leurs attentes pour six raisons au moins : « ses tarifs, la suppression des sillons, l'insuffisance des fréquences, la rigidité des horaires, le manque de fiabilité des trains, la mixité des lignes entre les voyageurs et les marchandises $»^{23}$.

Ferrovergne aura la mission de regrouper les wagons sur de courtes distances, afin de constituer des convois massifiés notamment dans les ports pour les amener jusqu'à un point d'échange avec la SNCF ou des opérateurs privés. En février 2010, le Directeur général de SNCF Geodis, Pierre Blayau, assurait au nouvel OFP qu'il pourra "compter sur la SNCF en matière technique », l'OH souhaitant s'inscrire dans une logique «partenariale» sur le fret «plus que dans une logique du passé, hégémonique ». Il s'agirait de proposer un plan d'action «pour transformer le transport de marchandises», qui tiendrait

\footnotetext{
${ }^{21}$ Or pour Louis Reybaud, «Une révolution est un prisme dans lequel tout se décompose », 1842, Jérôme Paturot à la recherche d'une position sociale, III, II.

${ }^{22}$ Les OFP paraissent inspirés du rapport commandé par le ministre des Transports et de l'Equipement à Jacques Chauvineau, et intitulé «Transport ferroviaire de fret et développement territorial » (remis en septembre 2006).

${ }^{23}$ http://www.auvergne.cci.fr/IMG/pdf/Dossier_presse_9_.pdf [consulté le 18/05/11].
} 
compte du fait que la SNCF est «plus douée pour le transport massif que pour les dessertes de proximité » (dépêche AFP du 5 février 2010).

En fait, la SNCF semble avoir deux fers au feu : le premier consiste à restructurer son fret autour des trafics rentables matérialisés par les trains complets. Pour ce faire, l'idéal serait une généralisation des autoroutes ferroviaires comme celle qui relie depuis le mois de septembre 2007 le Luxembourg à Perpignan (basée sur le ferroutage, les autoroutes permettent de transporter des camions sur des trains $)^{24}$. Le second fer au feu de la SNCF consisterait à chercher à contrôler les OFP pour les cantonner dans certains créneaux. Cherchant à se défendre des accusations portées par le Secrétaire d'Etat, P. Blayau, le patron du fret du groupe public, répondait en septembre 2010 : « Nous nous sommes positionnés en faveur des OFP. Mais si certains d'entre eux veulent faire concurrence à Fret $S N C F$, nous ne pouvons être d'accord». Le Directeur des affaires publiques et territoriales de SNCF Geodis renchérissait en expliquant que son groupe «soutient ces OFP d'autant plus qu'ils sont complémentaires de Fret $\mathrm{SNCF} »$. Ce à quoi un des fers de lance des OFP répondait : " Compte tenu du poids de la SNCF, il faut une connivence avec elle pour créer un OFP. Mais il ne faut pas que cette connivence devienne prétexte à une mainmise de fait». En somme, les logiques partenariales sont surtout d'apparence et en réalité n'ont guère éliminé les soupçons, les conflits, les rivalités, bref les logiques concurrentielles et surtout la lutte pour les segments du marché les plus lucratifs.

Parfois, fret SNCF semble renoncer face à l'offensive de son grand challenger européen, la Deutsche Bahn. Ainsi, dans le cas de l'OFP du grand port maritime de la Rochelle, le port détient les trois quarts des parts et la DB environ $25 \%$ à travers sa filiale, Euro Cargo Rail ${ }^{25}$. Depuis le 21 janvier 2011, Europorte (relativement puissante filiale fret d'Eurotunnel qui a racheté les activités françaises de Veolia), exploite les lignes ferroviaires du Port de Dunkerque. Cette nouvelle offensive des opérateurs privés a été facilitée par l'ordonnance du 2 août 2005 qui encourage la gestion des voies ferrées portuaires par les établissements portuaires plutôt que par RFF.

Au-delà de ces péripéties, il ne fait guère de doute que l'enjeu des OFP est une privatisation plus ou moins importante du fret ferroviaire, non seulement par l'entrée de nouveaux acteurs (ECR, Europorte, etc.), mais tout autant par le démantèlement de l'opérateur historique, incité en interne et en externe à céder à la concurrence certaines niches du marché. Cela correspond assez largement mais pas complètement à un désengagement de l'OH puisqu'il prend des participations dans certains OFP (15\% par exemple dans Ferrovergne). Mais la tendance lourde est claire : fret SNCF est invité à restreindre son périmètre d'inter-

\footnotetext{
${ }^{24}$ Depuis le mois de septembre 2007 (et inaugurée en mars), cette autoroute ferroviaire relie Bettembourg (Luxembourg) à Le Boulou (Perpignan), sur $1000 \mathrm{~km}$. Le parcours est effectué en $14 \mathrm{~h}$ au lieu de $22 \mathrm{~h}$ par la route. http://www.novethic.fr/novethic/v3/article.jsp?id=112553 [consulté le 13/10/2010].

${ }^{25}$ En sept. 2008, un ancien directeur du port de Dunkerque estimait que $85 \%$ des marchandises acheminées jusqu'aux ports l'étaient par la route. http://www.lavoixdunord.fr/Locales/Dunkerque/actualite/Secteur_ Dunkerque/2008/09/19/article_infrastructures-developper-les-conteneur.shtml [consulté le 18/05/11].
} 
vention. Un tout récent rapport sénatorial préconise des présences ministérielles lors d'inauguration des OFP et ceux-ci devraient être soutenus dans certains cas par les pouvoirs publics : «Il en revient parfois de l'intérêt général de soutenir le wagon isolé, dans une logique d'aménagement du territoire ». Si la situation du fret en général et celui de la SNCF en particulier ne semble pas s'améliorer, la situation du transport voyageurs est en revanche bien meilleure du fait du succès des $1850 \mathrm{~km}$ de LGV (lignes à grande vitesse lancées à partir de 1981) et des TER (Transports Express Régionaux, lancés le $1^{\mathrm{er}}$ janvier 1993). Le nombre de voyageurs transportés par le rail a augmenté de manière très spectaculaire malgré la concurrence de l'avion et la fréquente mauvaise qualité du service offert par le Transilien notamment dans les RER et sur certaines lignes de métro.

Depuis le 10 déc. 2009, le transport voyageurs international est lui aussi sur le papier ouvert à la concurrence et le 13 février 2010, par décret ministériel, Veolia a obtenu sa «licence d'entreprise ferroviaire valable pour effectuer des services de transports voyageurs ». Il y a quelques raisons de penser qu'à partir de 2011 ou de 2012, de nouveaux entrants viendront concurrencer la SNCF sur les segments les plus rentables du marché, notamment les TGV mais peut-être aussi très prochainement les TER dès lors que ce marché sera ouvert à la concurrence, ce que prévoient les textes relatifs aux Obligations de Service Public ${ }^{26}$.

Ayant montré le peu d'efficacité des politiques européennes pour la relance du fret ferroviaire notamment en France, il convient de s'arrêter sur les effets sociaux des initiatives bruxelloises et des réponses apportées par l'OH.

\section{Un cadre social harmonisé pour tous ?}

Les observateurs et les décideurs européens et au niveau des Etats-nations s'attardent rarement sur les conséquences des restructurations sur le contenu du travail et sur les identifications professionnelles des salariés des services publics ${ }^{27}$. A lire les documents officiels, le plus important serait la satisfaction des clients les plus solvables par une politique de baisse des coûts. Or notre angle de vue est différent. Il consiste à montrer que sous couvert d'ouverture des marchés, il s'agit en fait de réduire les avantages sociaux consentis aux cheminots dans l'espoir d'exacerber la concurrence ainsi que de faire baisser les coûts pour réduire les tarifs pour les clients industriels. Nous allons montrer que l'ouverture

\footnotetext{
${ }^{26} \mathrm{http} / / /$ www.dgcl.interieur.gouv.fr/sections/les_collectivites_te/interventions_econom/droit/sieg_et_obligatio ns/services_d_interet_e/view [consulté le 4/11/2010].

${ }^{27}$ Signalons, au titre d'heureuse exception, l'excellent documentaire Les cheminots de Luc Joulé et Sébastien Jousse (2009, 80 min.), qui montre comment dans la région PACA les différents métiers et filières sont déstabilisés par la segmentation de l'entreprise en entités distinctes (fret, grandes lignes, TER, proximité, etc.) incitées à développer des gestions des hommes, des matériels et financière séparées. La fin de l'entreprise intégrée a un coût psycho-social important et nombre de cheminots disent craindre qu'elle nuise à la mise en œuvre de la sécurité.
} 
des marchés entraîne inéluctablement une dégradation de la condition salariale des cheminots qui travaillent pour l'opérateur historique. De plus, à côté des cheminots salariés de l'OH et relevant du «cadre permanent », se développent des entreprises privées dont le personnel travaille sous un autre régime, ce qui est aussi une manière de fragiliser le statut du cheminot.

Voici quelques éléments d'explication sur le contexte et les interactions qui se sont nouées à la faveur de l'ouverture à la concurrence. Une directive européenne sur le temps de travail dans les transports ferroviaires (2005/47) nécessitait en effet une transcription dans les législations nationales avant le 27 juillet 2008. Cette exigence a ouvert la voie à la négociation d'une convention collective pour les 1836 travailleurs du rail salariés du privé dont 390 contractuels de la SNCF (à la date du mois de septembre 2010). C'est qu'à partir de 2006, une demi-douzaine d'opérateurs (Colas Rail, EuRailCo, Euro Cargo2 Rail, Groupe VFLI et surtout Europorte filiale d'Eurotunnel), ont entrepris de disputer à la SNCF ses parts de marché dans le fret ferroviaire ce qui justifiait l'ouverture d'une négociation nationale de branche qui prendrait place à côté du « statut du cheminot » propre à la $\mathrm{SNCF}^{28}$.

Par souci de clarté, on peut périodiser cette négociation collective de la manière suivante.

Etape première (juin-sept. 2006), en vue d'élaborer le décret sur le temps de travail transcrivant la directive européenne, l'Etat voulait se contenter de consulter les OS avant de légiférer. La première réaction des fédérations syndicales a été de demander le statut du cheminot pour tous (cheminots du public ou cheminots privé). Refus de l'État.

Etape 2 (oct.-déc. 2006), la puissance publique accepte que la question du temps de travail soit discutée dans le cadre d'une négociation collective paritaire réunissant autour de la même table, l'organisation patronale Union des Transports Publics (UTP) et des représentants des huit fédérations syndicales représentées à la SNCF. Dans une lettre signée du Premier ministre, les acteurs sociaux étaient invités à travailler à la définition d'un cadre social harmonisé du ferroviaire (CSH). Faute d'accord dans les dix-huit mois, l'Etat annonçait qu'il prendrait unilatéralement ses responsabilités ce qui était une manière de faire peser sur les organisations syndicales le risque d'une décision étatique a minima.

Les deux étapes suivantes se superposent partiellement.

Etape 3 (janv. 2007-juil. 2009), la négociation sur le temps de travail est désormais conçue comme la définition du volet temps de travail de la convention collective nationale $(\mathrm{CCN})$. Des discussions préliminaires s'ouvrent classiquement sur le champ couvert et un accord de méthode figure à l'ordre du jour. Ensuite, s'ouvrent les discussions sur le volet temps de travail. C'est la clé de voûte du système pour les cheminots, surtout pour les roulants, ces derniers as-

\footnotetext{
${ }^{28}$ Dans une interview, G. Pepy, PDG de la SNCF, dénombrait « 13 opérateurs dans le métier du fret en France », sans autre précision. Sans doute intégrait-il les OFP dans ce calcul. Le Figaro, le 4 nov. 2010.
} 
surant leur service $24 \mathrm{~h}$ sur 24, 365 jours par an selon des règles complexes. Les roulants représentent d'ailleurs une proportion importante des salariés des sociétés privées. En octobre 2008, trois fédérations (CFTC, Unsa, CGC) signent cet accord de branche. Deux fédérations (CFDT et FO), d'orientation pourtant modérées, font jouer leur droit d'opposition. Deux autres non signataires (CGT et FGAAC) refusent de leur emboîter le pas. Sud Rail, alors deuxième syndicat de la SNCF, entreprise de référence pour définir la représentativité de la branche, est écarté de manière arbitraire et politique de la négociation, notamment du fait d'une ferme intervention de FO, pourtant peu représentative chez les cheminots de l'opérateur historique. En juillet 2009, après des péripéties, l'accord de branche sur le temps de travail dans le fret privé est pour l'essentiel validé et «étendu » par le ministère du Travail. Il devient donc la norme pour toutes les entreprises du ferroviaire, pour les filiales ferroviaires et peut-être demain pour les milliers de hors statuts directement employés par l'Epic SNCF (en jargon maison les « PS $25 »)^{29}$. L'accord temps de travail est destiné à devenir un des chapitres de la CCN.

Etape 4 (nov. 2008-sept. 2010), la négociation se poursuit sur le contrat de travail, les classifications et les rémunérations. Elle aboutit à un accord soumis à la signature des syndicats le 8 septembre 2010. Il s'agit d'un demi-échec pour l'organisation patronale l'UTP (Union des Transports Publics et Ferroviaires) puisque deux organisations syndicales seulement, acquises d'avance (la CGC et la CFTC) signent cet accord. Faute d'une opposition d'une majorité des fédérations non signataires qui auraient pu faire tomber l'accord, celui-ci a été transmis au ministère du Travail en vue de son « extension » à toutes les entreprises exerçant dans le champ du ferroviaire privé.

Etape 5 (depuis l'automne 2010), ouverture d'une négociation sur la formation professionnelle et la prévoyance.

L'organisation patronale, l'UTP, espère pouvoir aboutir à une convention collective complète en juin 2011. Sans en avoir la certitude, elle escompte la signature de la CFDT qui s'est refusée jusqu'à présent de signer brique après brique, attendant un accord global. A cette date, la négociation aura duré 5 ans.

S'engagera alors une nouvelle négociation pour définir une convention collective du transport voyageur privé. Il n'est pas sûr, là non plus, que Sud Rail, pourtant représentatif à la $\mathrm{SNCF}$, y compris dans le cadre des règles définies par la loi du 20 août 2008 (Andolfatto et Dressen, 2010), sera invité à la table des négociations.

\footnotetext{
${ }^{29}$ Les PS 25 sont des salariés de l'Epic qui ne relèvent pas du «cadre permanent », id est du statut car ils ont été recrutés après l'âge de 30 ans, et/ou n'appartiennent à aucune des 25 nationalités de l'UE et/ou travaillent selon une quotité de moins de $60 \%$ du temps complet. Ces hors statuts qui sont environ 7500 ne bénéficient pas de la même grille de salaires, ne perçoivent pas de treizième mois, ne sont pas couverts par le même régime de médecine du travail et subissent les jours de carence prévus par le Code du travail, à la différence des cheminots relevant du statut. Leur déroulement de carrière est inférieur, comme l'est aussi le niveau de leur pension.
} 
La question de savoir si les salariés des Opérateurs Ferroviaires de Proximité seront concernés par cette convention collective divise l'UTP. Les uns le souhaitent dans un souci de simplification du cadre conventionnel tandis que d'autres se déclarent partisans d'assujettir les OFP à la convention collective des VFIL (Voies Ferrées d'Intérêt Local) ${ }^{30}$. Si cette deuxième option était retenue, on serait alors loin du cadre social harmonisé puisqu'à côté du statut des cheminots, les salariés du privé dépendraient pour les uns de la convention collective VFLI et d'autres de la convention collective négociée par l'UTP. Mais quel que soit le cas de figure, il est à craindre une fragmentation des conditions de travail et d'emploi des cheminots selon l'employeur pour lequel ils travaillent.

\section{Dans les restructurations, les jeux de l'Etat et de la direction de l'opérateur historique}

On l'a vu, la puissance publique n'a pas voulu accéder aux revendications initiales des fédérations syndicales, «statut pour tous » (les cheminots). Puis, sans peut-être que ce soit un projet clairement défini au départ, elle a contribué dans la logique de la transcription d'une directive européenne sur le temps de travail à ce que les acteurs sociaux s'engagent, volet après volet, dans la construction d'une $\mathrm{CCN}$ dont la négociation est toujours en cours.

De son côté, la direction de la SNCF dont l'autonomie dans cette affaire est très relative compte tenu du nombre et du poids de représentants de l'Etat dans son conseil d'administration (7 sièges sur 18), et de la préparation de ses réunions au ministère de l'Economie et des Finances, aurait selon un ancien représentant syndical au Conseil d'Administration, décidé d'apporter sa pierre au dumping social en pesant sur les conditions de travail des salariés des nouveaux entrants. Elle y trouverait ensuite argument pour faire pression sur les acquis consentis aux salariés de l'Epic.

L'ambiguïté de la SNCF par rapport à ses propres salariés est illustrée par son rôle dans la négociation relative aux personnels des opérateurs privés. N'est-elle pas membre de l'UTP, le syndicat patronal négociateur au nom des entreprises privées des accords collectifs susmentionnés ? Elle est même le plus gros contributeur de cette association professionnelle. Son jeu est clairement perçu comme équivoque par les fédérations syndicales du ferroviaire mais aussi par les opérateurs privés. Ces derniers acceptent en effet d'associer la SNCF à la délimitation du mandat de négociation mais ils refusent sa participation à la négociation elle-même.

\footnotetext{
${ }^{30}$ Datant du 26 sept. 1974, elle a été étendue par arrêté du 23 juin 1975 (JORF du 17 juil. 1975) et a fait l'objet d'accords complémentaires ou d'avenants notamment en 1977, 1980 et 1983. Elle couvre divers opérateurs dans le fret et le voyageur notamment sur les lignes secondaires qui sont « en régie » (Ardennes, Drôme Ardèche, Dauphiné, etc.) ou touristiques et qui, depuis 150 ans, déploient leurs activités à côté des grandes Compagnies (jusqu'en 1938), puis de l'OH.
} 
Au vrai, cette stratégie de l'OH n'aurait pas été adoptée d'entrée de jeu mais aurait succédé à deux autres. Dans un premier temps (de 1993 à 1996), soit avant l'ouverture effective du marché en France, la direction de l'OH aurait joué, en convergence avec les organisations syndicales de l'opérateur historique, la carte de la coopération avec quelques partenaires étrangers tout particulièrement la Deutsche Bahn (DB), la Société Nationale des Chemins de fer Belges (SNCB) et la SNC Luxembourgeoise (SNCL). La direction de l'OH aurait tantôt joué la carte de la compétition, tantôt celle de la coopération, tantôt encore celle de la coopétition (coopération et compétition) avec les mêmes partenaires. Cela correspondait aux années 1990 et à la mise en place de l'Eurostar (en 1994), du Thalys (de 1995 à 1999), etc., ces entreprises qu'un responsable CGT décrit comme «biques et boucs » (semi-publiques par le statut de leur personnel et semi-privées par leur partenariat étranger et leur logique lucrative). C'est l'époque de la mise en place de l'autoroute ferroviaire Perpignan/Bettembourg (Luxembourg) dont on a parlé.

Puis, progressivement, la compétition l'aurait emporté sur la coopération. La DB se serait agacée de la stratégie de la SNCF consistant à envoyer sa filiale Keolis chasser sur les terres de la DB dans le transport voyageurs mais qui dénie un droit de réciprocité à la DB et à ses filiales. Les journaux bruissent des polémiques, accusations d'espionnage, plaintes devant les tribunaux ou d'entraves à la concurrence libre et non faussée qui cette dernière décennie opposent les deux leaders européens du chemin de fer, la SNCF et la DB. Dans le domaine du fret, la SNCF semble sur la défensive ou vouloir faire cavalier seul face à son puissant rival d'outre-Rhin ${ }^{31}$.

\section{Un net recul social sur le temps de travail}

Parlons des enjeux. L'élaboration d'un cadre social harmonisé, auquel le Premier ministre invitait les acteurs sociaux dans la phase deux de la négociation collective, n'est pas une catégorie juridique reconnue, contrairement aux notions de convention collective, d'accord de branche (tous deux négociés) ou de statut (en principe « octroyé » et en tout cas validé par l'Etat après une consultation/négociation entre les acteurs sociaux). Mais ce sympathique syntagme « social » et «harmonisé » doit être pris au sérieux, car il pourrait être gros d'un projet d'ampleur : «désanctuariser » le statut du cheminot qui, par le décret 50635 du $1^{\text {er }}$ juin 1950, a succédé à la surprise générale à la convention collective de 1938 inachevée (Le Gall, 1957, p. 148-149).

\footnotetext{
31 «Au plan européen, explique le fondé de pouvoir du groupe Deutsche Bahn, nous participons, avec plusieurs autres compagnies ferroviaires européennes au projet X-Rail, initié avec la Commission européenne pour sauver l'activité de wagon isolé. Il s'agit à la fois de s'entendre sur la définition d'un réseau de points de desserte interconnecté en Europe et surtout, définir quelles garanties offrir aux clients en terme de qualité. Aux dernières nouvelles, la SNCF serait sur le point de sortir de ce projet » [Fried, Les Echos, 14 janv. 2010].
} 
L'objectif serait de procéder à l'alignement de tous les travailleurs du ferroviaire (publics et privés) sur des règles que le patronat du ferroviaire privé, désormais accompagné par l'Etat et de plus en plus souvent par la direction de la SNCF, exigerait devoir être en retrait par rapport à celles qui s'appliquent chez l'opérateur historique.

Ce constat pose deux problèmes : le premier est d'ordre quasi procédural. Une convention collective est-elle nécessairement moins favorable qu'un statut? La question mériterait examen approfondi et la réponse serait sans doute nuancée, d'autant qu'en 1950, la décision qu'a prise la puissance publique d'imposer un statut a soulevé la protestation du syndicat largement hégémonique dans l'entreprise, la CGT. Cette dernière préférait alors travailler à l'actualisation et l'achèvement de la convention collective dont la négociation avait commencé avant guerre, mais n'avait pas pu être achevée du fait de la conjoncture politique de l'époque puis de l'immédiat après-guerre.

La seconde question est d'ordre substantiel et renvoie au contenu de l'accord sur le temps de travail. Avec celui-ci, on peut dire que le loup montre le bout de l'oreille. Signé en octobre 2008, cet accord est très nettement moins protecteur pour les salariés que le statut du cheminot. Par rapport aux normes SNCF (précisées dans le RH0077), l'accord de branche prévoit que la prise de poste peut se faire plus tôt dans la nuit, que le débauchage peut être plus tardif, que l'amplitude de travail est donc plus étendue et que le repos journalier est plus court. Les vrais week-ends (samedi et dimanche) sont bien plus rares et même les deux jours de repos consécutifs sont exceptionnels (en moyenne à peu près un par mois) (Cohen et Dressen, 2010).

La vie de famille et la vie sociale, de toute manière profondément perturbées par l'exercice du métier de conducteur de train par roulements (365 jours/an 24/24) du fait de temporalités irrégulières, est désormais légalement plus pénible encore dans le privé (Cohen et Dressen, 2011).

Ce temps de travail est la clé de voûte du statut des cheminots pour les roulants. Surtout, le RH0077 est en relation étroite avec la valeur sécurité. Il faut être du sérail ou enquêter à la SNCF pour se rendre compte à quel point la sécurité en général et des circulations en particulier sont avec la notion de service public, les deux valeurs cardinales dans l'univers de significations partagées des cheminots.

Partant du constat que «la mesure du temps est ce qui permet de faire de la notion abstraite de travail un objet d'échange » (Supiot, 2004 p. 107), on peut en induire que la valeur d'échange du travail des cheminots privé est bien moindre que celle des cheminots relevant du cadre permanent de la SNCF (« le statut»), puisque les salaires directs dans le privé ne sont pas supérieurs et que les salaires indirects (avantages sociaux, retraites, etc.) sont inférieurs. En somme, l'ouverture des marchés a un coût pour les salariés du rail privés, celui d'une intensification du travail et donc d'une dégradation de leur santé et de leur espérance de vie, le travail de nuit étant le lot commun des conducteurs de train. 
Tableau 1 : Réglementation du travail comparée RH0077 Accord collectif nouveaux entrants

\begin{tabular}{|c|c|c|c|c|c|c|c|c|c|c|}
\hline 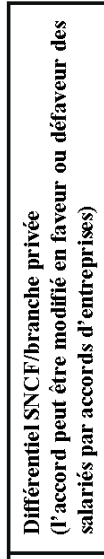 & 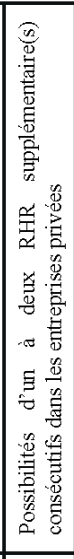 & \multicolumn{2}{|c|}{ 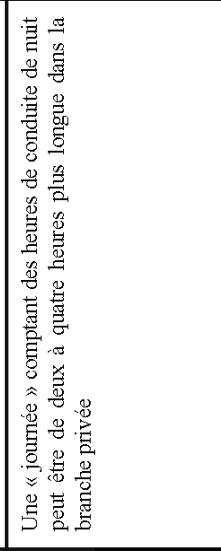 } & 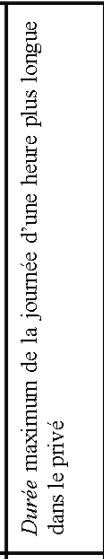 & 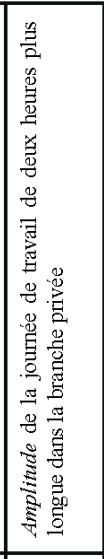 & 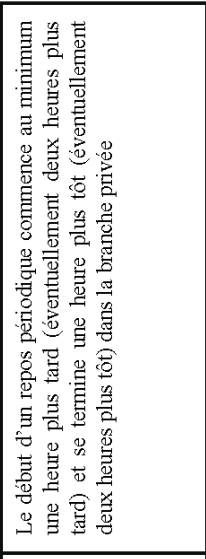 & 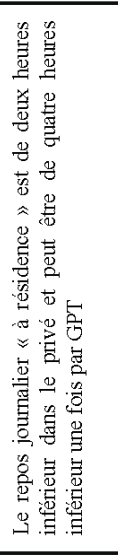 & 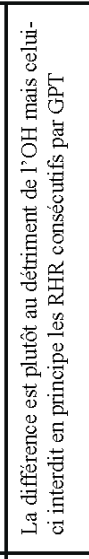 & 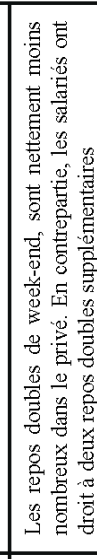 & \\
\hline 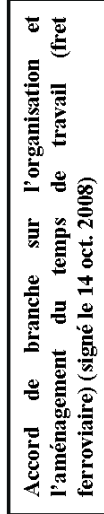 & 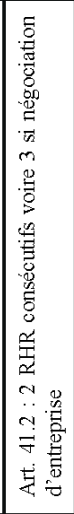 & \multicolumn{2}{|c|}{ 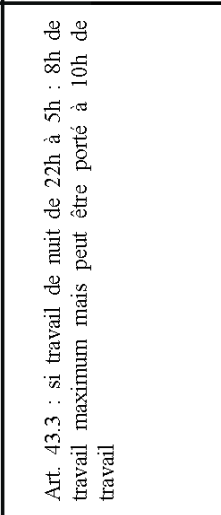 } & 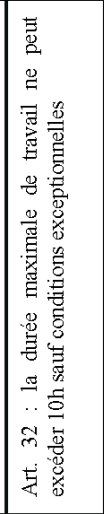 & 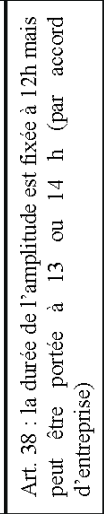 & 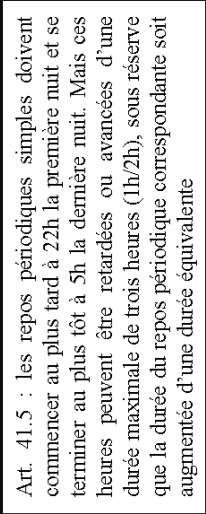 & 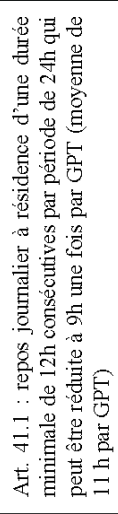 & 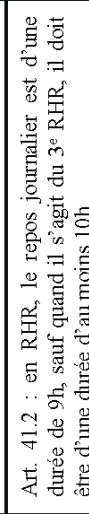 & 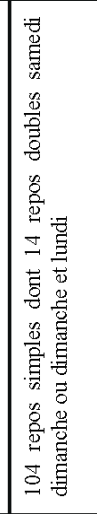 & \\
\hline 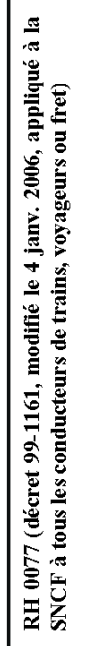 & 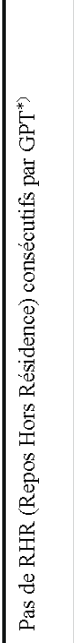 & 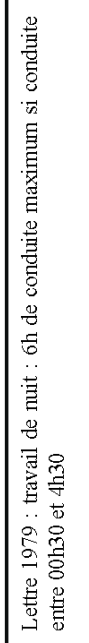 & 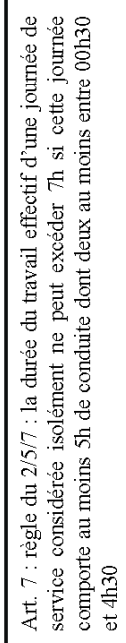 & 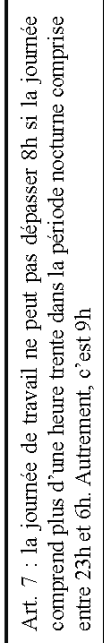 & 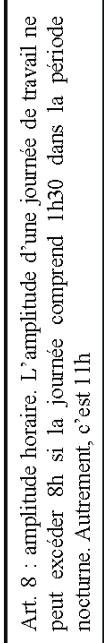 & 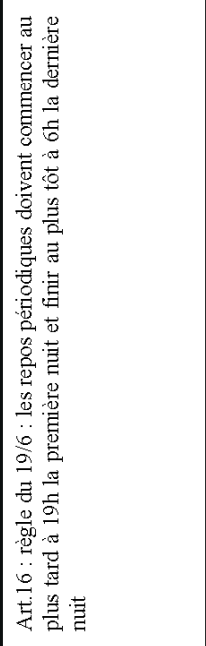 & 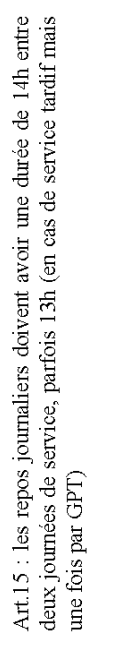 & 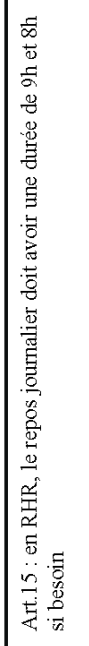 & 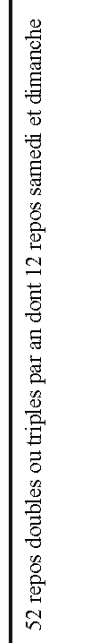 & \\
\hline
\end{tabular}




\section{Un statut des cheminots miné par tous les bouts}

De cette narration et de cette analyse, on peut retirer l'hypothèse que la protection du système est maintenant fissurée du côté des nouveaux entrants et que cette réduction des protections menace désormais les cheminots de l'OH. Les salariés des filiales SNCF (VFLI, Naviland Cargo) qui auto-concurrencent la « maison mère » dans le fret sont en partie concernés par ces nouvelles règles du temps de travail, de la même manière qu'en Allemagne DB Région a contourné les résistances des OS en créant des filiales dans lesquelles les conditions d'emploi sont inférieures à ce qu'elles sont pour les salariés employés en direct par l'opérateur historique. Ainsi en est-il surtout des «PS 25 » dont on a déjà parlé. Au nombre de 7300 (selon le bilan social 2008), en forte progression ces dernières années, leur importante numérique fera que les 60 à $70 \%$ des ayants droit de la nouvelle CCN seront des hors statuts de l'Epic SNCF (mais à ce jour, ce n'est le cas d'aucun conducteur de train de l'opérateur historique, qui sont tous au « cadre permanent »).

En somme, le statut est attaqué de biais. De biais et de toute part, comme le révèle aussi cette statistique : désormais les salariés de l'Epic, membres ou non du cadre permanent, ne représentent plus que $67 \%$ de la force de travail du «groupe SNCF». La dilution progressive de la SNCF dans les filiales des groupes qu'elle contrôle se voit aussi dans ses comptes. "La part de l'Epic dans le chiffres d'affaires total [du groupe SNCF] passe de 72\% à 59\% entre 2009 et 2010 », selon A. Cambi, porte-parole du syndicat Sud Rail (Dépêche AFP, 15 janv. 10). Le centre de gravité de l'activité se déplace de l'entreprise mère vers ses 49 «groupes de sociétés» contrôlant plus de 650 filiales (2006, Rapport anпиel SNCF). Quel que soit l'angle sous lequel on examine le problème, il faut se rendre à l'évidence : «à terme, le statut de cheminot est menacé ». Même les conducteurs de train du fret SNCF sont invités à renoncer à leurs protections et à l'idée du service public au nom de l'impératif de la compétitivité par rapport à la concurrence.

Dans l'ordre symbolique, la logique du volontariat, levier des restructurations dans le fret, vient miner celle des identifications collectives. La stigmatisation des cultures de métiers, suspectées d'archaïsme, relève aussi de cette volonté de réduire le bastion ouvrier qu'incarnaient les cheminots, à commencer par les 15 à 17000 conducteurs de trains. Vont aussi dans le même sens, la mise en veilleuse de la filière apprentissage, voie d'entrée historique dans le métier pour encore tant de cheminots en activité. La recherche d'une réduction de la part des recrutements héréditaires est de même inspiration. En vue de faire reculer la culture corporative, l'enjeu est très officiellement l'introduction de «sang neuf », notamment dans l'encadrement. Les profils de diplômés des grandes écoles de commerce (HEC, ESSEC, etc.), pétris de culture managériale, convaincus de la vertu générale de la mise en concurrence systématique des entreprises mêmes publiques et des salariés qui y travaillent, sont recherchés. 
Le propos n'est pas ici de dire que le statut du cheminot était solide jusqu'à la signature de l'accord d'octobre 2008 relatif aux salariés des entreprises privées. On pense par exemple à la multiplication de longue date des hors statuts. Les «non commissionnés » constituaient et constituent « une figure ancienne et plurielle »(Lada, 2005, p.108).

On peut aussi s'interroger sur la stratégie des organisations syndicales. On ne peut décrire un virage sans précédent. Plusieurs d'entre elles sont majoritairement convaincues du caractère inéluctable voire bénéfique de l'introduction de la concurrence. Hormis Sud-Rail et une partie de la CGT franchement hostile aux évolutions en cours, elles cherchent surtout à freiner ou limiter les effets négatifs pour les salariés des récentes évolutions mais de longue date, elles accompagnent les restructurations par demi-conviction pour les unes ou faute de trouver les moyens de construire de réels rapports de force permettant de s'y opposer pour les autres.

Du fait de la perte de légitimité du conflit redistributif (C. Gobin), les OS parviennent au mieux à «limiter les dégâts », comme on l'a vu à propos de la législation sur le service minimum à l'été 2007 , ou lors du conflit sur les régimes spéciaux de l'automne suivant.

Il est vrai que les syndicats se heurtent à forte partie. Autrefois idéalisés en héros de la bataille du rail (R. Clément, 1946), les cheminots sont progressivement transformés en figure emblématique d'un « corporatisme » archaïque crispé sur son passé, égoïste, symbole d'une corporation « coûteuse pour le contribuable » et incapables de respecter les horaires, plus prompte à se mettre en grève qu'à travailler. Les travailleurs du rail s'attirent une belle collection de stéréotypes. Une lecture de différents blogs sur l'Internet ou des réactions des lecteurs de la presse en ligne à telle ou telle information concernant le fer suscite l'idée que peu d'activités professionnelles s'attirent autant de haines et de préjugés. Des rumeurs malveillantes circulent par exemple sur le fait que les mécaniciens toucheraient une prime de charbon alors que celle-ci, plus précisément appelée «prime d'économie de charbon » a disparu avec les dernières machines à vapeur en $1974 \ldots$ Les salaires de tégévistes en fin de carrière, d'ailleurs souvent exagérés pour les besoins de la démonstration ${ }^{32}$, sont présentés comme étant ceux des agents de la SNCF en général, ce qui ne peut évidemment que blesser les dizaines de milliers de cheminots dont le salaire est inférieur à $1500 €$ par mois.

Quels que soient les auteurs de ces rumeurs (propagées en boucle sur l'Internet, depuis 1995 au moins), cette volonté de déstabiliser les cheminots n'est pas sans rapport avec notre objet. Comment mieux faire accepter à la corporation (en tout cas à ses agents d'exécution et à ses agents de maîtrise) la réduction des avantages acquis qu'en caricaturant leur situation?

\footnotetext{
${ }^{32}$ Ils ne sont pas très loin du salaire moyen à la SNCF, 2709 € par mois en 2008 (selon le bilan social 2008).
} 
Mais ces critiques ne viennent plus seulement de l'extérieur: elles sont désormais souvent exprimées à l'intérieur de l'entreprise. La violence morale est alors à son comble contre tous ceux qui prennent la notion de service public à cœur (Cohen et Dressen, 2011).

DANS CE TROP LONG ARTICLE, nous avons voulu montrer plusieurs choses : premièrement, les causes multifactorielles du déclin du fret pour esquisser la démonstration qu'il n'est nullement besoin de prendre pour cible les avantages sociaux des cheminots pour comprendre la crise du transport de marchandises. Et d'ailleurs, pourquoi les cheminots devraient-ils accepter de perdre des acquis sociaux ? Quel groupe professionnel est prêt à le faire, s'il peut l'éviter ? Nous avons aussi voulu montrer que l'on ne peut comprendre les décompositions et recompositions du monde cheminot qu'en s'intéressant aux politiques de la Commission européenne et à la réponse que chaque Etat-nation et même chaque opérateur historique y apporte. D'ailleurs, les stratégies de la DB, insuffisamment étudiées ici, et celles de la SNCF ne sont pas toujours identiques. Mais elles entretiennent un air de famille en congruence avec l'époque : constituer des géants internationaux multimodaux intégrés, implantés sur plusieurs continents et réduire les avantages sociaux des salariés des opérateurs historiques, encore et toujours au nom de l'impératif de compétitivité. Cela dit, on ne peut accabler exclusivement la Commission européenne car elle agit aussi en fonction des pouvoirs qui lui ont été confiés. On observera cependant qu'elle n'a pas choisi les deux axes qui auraient permis de conforter la place du rail en Europe a) en pesant pour des conditions de travail et d'emploi améliorées chez les routiers afin de réduire l'écart compétitif entre la route et le rail et b) en poussant à la définition d'un statut de tous les cheminots européens qui lui aussi aurait fait obstacle à la concurrence par les coûts, celle-ci se traduisant presque mécaniquement par une réduction des avantages sociaux.

Cette politique s'avère un échec du point de vue de la part de marché du transport de marchandises contrôlée par le rail. L'observateur ne peut que constater un fossé croissant entre les affichages communicationnels et la perte continue des volumes transportés par l'OH au profit des opérateurs privés, qui en France ont conquis environ $10 \%$ de part de marché, au détriment du fret SNCF et non de la route. Le rail privé cannibalise le rail public et la route phagocyte doucement mais sûrement l'ensemble. Ajoutons que depuis la crise financière, la somme de marchandises transportées par voie ferrée (par des entreprises privées ou par l'opérateur historique) accuse encore un recul en valeur absolue $(-25 \%)$ et en valeur relative par rapport à la route. En 2007 encore, dans son langage euphémistique la Commission observait que «la part modale du rail dans le transport de marchandises peine toutefois à progresser ${ }^{33}$. Néanmoins, les partisans des déréglementations/« reréglementations » se refusent à un chan-

\footnotetext{
${ }^{33} \mathrm{http} / / /$ ec.europa.eu/transport/logistics/freight_logistics_action_plan/doc/memo/memo_rail_fr.pdf, 2007 [consulté le 18/05/11].
} 
gement d'orientation, au contraire, ils ne prônent qu'une accentuation des politiques mises en œuvre.

Surtout, nous avons voulu insister sur les conséquences sociales, professionnelles et identitaires des restructurations en cours. Elles se traduisent par un nombre important de suppressions de postes dans le fret ferroviaire public (4 400 sur 2009 et 2010) et aussi par une désorientation importante des cheminots, causée et redoublée par la désarticulation de l'entreprise historique qui était autrefois un univers intégré. Sans tomber dans un naïf organicisme, il est à peine exagéré d'évoquer un univers dont tous les secteurs et les différents organes étaient interdépendants et concouraient nolens volens aux deux grandes valeurs de l'opérateur historique : la sécurité des salariés et des voyageurs et le service public dont il fallait assurer le fonctionnement coûte que coûte 8760 heures par an. Désormais, l'orientation choisie par les pouvoirs publics (largement représentés dans le $\mathrm{CA}$ de la $\mathrm{SNCF}$ ) consiste à expliquer aux cheminots que tout ce qu'ils ont fait jusqu'à présent est dépassé. Sous-traitance et filialisation constituent le nouvel horizon, au risque du dumping social et de situations qui seraient comiques si elles ne révélaient pas des tragédies sociales comme par exemple lorsque G. Pepy, le 9 février 2009, est obligé de reconnaître que les maîtres-chiens des sociétés de gardiennage employés pour surveiller les grandes gares, notamment pour empêcher les sans-papiers d'embarquer clandestinement dans l'Eurostar, sont eux-mêmes dépourvus de titres de séjour.

On observe que privilégier le consommateur sur le producteur n'est pas sans conséquences sociales. On est sans doute ici au cœur des raisons qui expliquent la croissance du nombre de DCI (demandes de concertation immédiate), passage obligé avant le dépôt d'un préavis de grève.

\section{Indications bibliographiques}

ANDOLFATTO D., DRESSEN M., «Transformation de la représentativité syndicale et rénovation de la
négociation collective en France : enjeux et début d'application à la SNCF », in Les systèmes de
représentation au travail : à la mesure des réalités contemporaines ? Employee Representation in the New
World of Work The Dynamics of Rights, Voice, Performance and Power, Colloque international du
CRIMT/47ème congrès de l'ACRI, 16-18 juin 2010, Université Laval, Québec

Chevandier C., Cheminots en grève, ou la construction d'une identité (1848-2001), Maisonneuve et Larose, Paris, 2002

COHEN V., DRESSEN M., « Pénibilité et violence morale chez les conducteurs de trains, Opérateurs privés et opérateur public », in DRESSEN M., DURAND J.-P. (eds), La Violence au travail, Octares, Toulouse, 2011

COUR DES COMPTES (ed), Le réseau ferroviaire Une réforme inachevée, une stratégie incertaine, La Documentation française, Paris, 2008 (Rapport public thématique)

DRESSEN M., «Du statut pour tous au cadre social harmonisé pour chacun ? », in ABALlEA F., MIAS A. (eds), Mondialisation des relations professionnelles, Octares, Toulouse, 2011, p. [n.c.]

DRESSEN M., MiAS A., « Ouverture a la concurrence et négociation collective dans deux secteurs européens des services les télécoms et les transports ferroviaires », in XII journées internationales de Sociologie du Travail, 25-26 juin 2009, Nancy 
Dufresne A., Degryse C., Pochet P. (eds), The Evolution of Sectoral Industrial relations Structures in Europe, The European Sectoral Social Dialogue - Actors, developments and challenges, PIE-Peter Lang, Bruxelles, 2006

DURAND J.-P., La Chaîne invisible, Le Seuil, Paris, 2003

Freyssinet J., Travail et emploi en France, état des lieux et perspectives, La Documentation française, Paris, 2006

GobIN C., «De l'union européenne à... l'européanisation des mouvements sociaux ?», Revue internationale de politique comparée, vol. $9, \mathrm{n}^{\circ} 1,2002$, p. 119-139

Hilal N., L'eurosyndicalisme par l'action. Cheminots et routiers en Europe, L'Harmattan, Paris, 2007 (Logiques politiques)

LADA E., Quand les marges deviennent centre : mise au travail, jeunesses populaires et rapports sociaux de sexe dans le secteur public et associatif, Thèse de sociologie, Université de Paris X- Nanterre, Nanterre, 2005

LE GALl P., Evolution des relations collectives entre les chemins de fer d'intérêt général et leur personnel, Thèse de droit, Université de Paris, Paris, 1957

MüHLSTEIN P., «Sur la libéralisation du transport ferroviaire », Yonne Lautre [en ligne], 27/09/2007, p. [n.c.]. Disponible sur : http://yonne.lautre.net/spip.php?article2539 [consulté le 21/04/2011]

NeIERTZ N., La coordination des transports en France de 1918 à nos jours, Imprimerie nationale/comité pour l'histoire économique et financière de la France, Paris, 1999

Rivier R., PUTAllaz Y., Rapport d'audit sur l'état du réseau ferré national français, sous la direction et la coordination [en ligne], Ecole polytechnique fédérale, Lausanne, 2005. Disponible sur : http://lesrapports.ladocumentationfrancaise.fr/BRP/054000595/0000.pdf [consulté le 21/04/2011]

SuPIOT A., Le Droit du travail, PUF (Que-sais-je ?), Paris, 2004. 



\title{
Gestion des conséquences de la privatisation dans le domaine des ressources humaines: l'exemple de la Deutsche Bahn AG
}

\author{
Claudia PANKE
}

Rédigée par l'ancienne Directrice du personnel de diverses filiales de la Deutsche Bahn $A G$, cette contribution aborde l'aspect juridique de la réforme des chemins de fer allemands et son impact sur le statut des fonctionnaires " privatisés " et des salariés protégés par convention. Conservant une approche relative au droit du travail, Claudia Panke élargit ensuite son propos à d'autres privatisations du secteur public.

Le transfert d'une partie importante des fonctionnaires fédéraux de la sphère de l'administration publique vers celle de l'économie de marché, à la suite de la réforme structurelle des chemins de fer allemands, a eu les conséquences suivantes :

- perte, pour les fonctionnaires, des postes qu'ils détenaient auparavant ;

- risque de perdre leur poste au sens de leur fonction conceptuelle et de leur fonction concrète ;

- risque d'inefficacité de certaines structures fondamentales du droit de la fonction publique ;

- contrainte particulière dans le cadre de la gestion des ressources humaines (GRH) et même, le cas échéant, relativisation de la décision de privatisation du point de vue de l'entreprise privatisée en raison de l'emploi de fonctionnaires.

Pour tous les acteurs de la réforme structurelle des chemins de fer, il s'agit donc de répondre aux questions suivantes :

- quels outils de transfert du personnel sont licites et adaptés ?

- quels principes du droit de la fonction publique sont touchés par la privatisation?

- à quelles conditions est-il possible de mettre en œuvre des mesures de restructuration et d'assainissement financier applicables aux fonctionnaires «privatisés »?

- quelles conséquences tout cela a-t-il en matière de GRH pour l'entreprise privatisée $\mathrm{DB}$ AG ?

A quelques exceptions près, l'exécution de missions publiques sous une forme de droit privé indépendante est licite. Concernant le débat de droit constitutionnel relatif à d'éventuelles contraintes ou limites existant en Allemagne en matière de privatisation, il faut constater qu'il n'y a fondamentalement ni règle de 
privatisation constitutionnelle, en tant qu'expression d'un principe général de subsidiarité pertinent dans le domaine de la politique économique, ni interdiction générale. L'absence de mention d'organismes privés dépositaires de missions administratives aux art. 83 sq. de la Loi fondamentale (Grundgesetz GG) ne permet donc pas de conclure à une interdiction générale de déléguer des compétences administratives à des entités de droit privé.

La thèse selon laquelle les «personnes juridiques de droit privé ne peuvent employer des fonctionnaires » ne tient pas sous cette forme provocante. L'aspect déterminant pour savoir si la législation permet l'emploi de fonctionnaires dans des entreprises du secteur privé est le type de mission ayant vocation à être privatisée ainsi que le type et la portée de la privatisation.

Concernant le transfert des biens ferroviaires à des entités de droit privé, le législateur était conscient des problèmes liés aux conséquences de la privatisation et a eu recours, à l'intérieur même de l'appareil législatif, à une nouvelle réglementation des chemins de fer. A titre de préparation et de sécurisation juridique, il a été procédé, en lien avec la réforme ferroviaire, à d'importantes modifications de la constitution (art. $73, \mathrm{n}^{\circ} 6$; art. $74 \mathrm{n}^{\circ} 23$; art. 80 al. 2 et art. 87 al. 1 phrase $1 \mathrm{GG}$ ) et à l'ajout de nouvelles dispositions ( ${ }^{\circ} 6 \mathrm{a}$ à l'art. $73 \mathrm{GG}$ et les art. 87 e et 143a GG). L'organisation formelle stricte des chemins de fer, avec la création d'une société par actions au sens du nouvel art. 87e GG, qui dégage par conséquent l'entreprise privée de la mission de service public (Daseinsvorsorge), sont les instruments servant à répondre aux objectifs de la réforme ferroviaire.

\section{Transfert des fonctionnaires à la DB AG dans le cadre de la réforme structurelle - Analyse juridique des faits}

En attribuant des prestations de services conformément à la loi, comme cela a été fait dans le modèle ferroviaire, on mettait le pied sur une terra incognita juridique quant aux possibilités de transfert de ressources humaines. Cette manœuvre juridique était nécessaire pour maintenir durablement les activités aux mains d'un personnel compétent puisqu'un transfert ou une fourniture de personnel à une entreprise de droit privé, par la voie d'un détachement ou d'une mutation, n'est pas permise, et que le modèle de la mise à disposition ou de la mise en disponibilité du fonctionnaire pour qu'il exerce ses activités de service dans une entreprise du secteur privé, au sens d'un transfert de personnel rapide et permanent, n'est pas adapté.

Pour mettre en œuvre rapidement le projet de privatisation et à défaut d'autres dispositifs de transfert appropriés, la voie la plus praticable était alors de sécuriser la privatisation de la Deutsche Bundesbahn et de la Deutsche Reichsbahn en modifiant la constitution. Furent invoquées pour justifier l'emploi de grands moyens législatifs l'ampleur et l'importance de la restructuration du géant monopolistique. La teneur de la disposition des art. 143a, 143b GG, 
promulgués en lien avec la privatisation des chemins de fer fédéraux et de la Bundespost, a servi de modèle pour l'ajout de l'art. 123a al. 2 de la loi cadre pour l'uniformisation du droit de la fonction publique (Beamtenrechtsrahmengesetz, BRRG) par la loi de réforme du droit des services publics en 1997. Il en ressort que l'affectation en vertu de l'art. 123a al. 2 BRRG est appropriée pour organiser, en cas de privatisations à venir, le transfert de fonctionnaires du secteur public vers une entreprise de droit privé.

La modification de la constitution, en lien avec la privatisation des postes et des chemins de fer allemands pourrait ainsi être un cas unique dans la pratique de privatisation.

La réforme structurelle des chemins de fer constitue également un pas sur des voies inconnues dans le champ des activités et des services de la fonction publique. On observe un grand changement dans les activités ferroviaires privatisées, en ce qui concerne en particulier l'objectif des services et des prestations. Toute entreprise privée doit faire des bénéfices pour réussir. Un objectif crucial de la réforme ferroviaire consiste à assurer la viabilité financière et l'entrée en bourse, ce qui oriente les entreprises de chemins de fer privatisées vers un souci de réalisation de bénéfices. Or, le principe prédominant de la mission de service public ne doit pas être de maximiser les bénéfices mais de répondre à l'intérêt général. L'une des caractéristiques essentielles d'une administration satisfaisant aux principes du droit public réside dans sa neutralité et son indépendance. L'obligation de prestations désintéressées et impartiales est particulièrement importante pour le droit de la fonction publique et expressément définie dans toutes les lois en la matière. Le caractère altruiste, désintéressé des fonctions officielles, non préoccupé d'avantage personnel, est une base essentielle de la fonction publique professionnelle. Or, ce postulat perd de sa crédibilité s'il est fait appel à des fonctionnaires «privatisés », qui ne sont plus censés défendre l'intérêt général mais les stratégies des entreprises de chemins de fer privées.

Le fonctionnaire accomplissant sa mission sur la base d'un lien de loyauté permanent à son employeur et restant indifférent à tout avantage peut facilement se retrouver pris dans des contradictions inextricables.

L'affectation de fonctionnaires pour des activités au sein de chemins de fer fédéraux organisés selon le droit privé en vertu de l'art. 143a GG associé à l'art. 12 de la loi portant création des chemins de fer fédéraux (Deutsche Bahn Gründungsgesetz, DBGrG) doit être considérée comme une loi spéciale par rapport au détachement possible de fonctionnaires au titre de l'art. 123a BRRG, et se trouve donc dans un rapport de concurrence de droit public avec cette norme. Ce dernier dispositif est donc applicable aux transferts de personnels à des sociétés qui n'entrent pas le cadre d'une entreprise de transports ou d'infrastructures ferroviaires.

La condition absolue pour que l'affectation de fonctionnaires à la Deutsche Bahn AG soit autorisée en vertu de la loi (art. 143a al. 1 phrase 3 GG) est que l'Etat fédéral détienne la majorité des parts de l'entreprise de chemins de fer 
privatisée. Si ce préalable n'est plus réalisé, l'affectation des fonctionnaires prend fin - en conséquence inverse de l'art. 143a al. 1 phrase 3 GG - en vertu de la loi puisqu'il ne s'agit plus d'une compagnie ferroviaire de l'Etat fédéral.

Les dispositions de restructuration des chemins de fer n'ont rien changé au fait que le Bund reste tenu aux obligations émanant du rapport de fonctionnariat de ses agents travaillant pour la Deutsche Bahn AG. Dans le cadre de la supervision légale conformément à l'art. 13 DBGrG, l'Etat fédéral, représenté par le président de la structure de défaisance Bundeseisenbahnvermögen (BEV) ${ }^{1}$, a donc à sa disposition des outils pour assumer sa responsabilité en tant qu'employeur, relativement aux conséquences de la privatisation, vis-à-vis des fonctionnaires «privatisés ». Les possibilités d'intervention de ce contrôle découlent de la qualité d'actionnaire majoritaire du Bund.

\section{Effet de la réforme ferroviaire sur les principes du droit de la fonction publique}

Si la prestation se base sur les objectifs de l'entreprise privée, le risque existe que les obligations de la fonction publique professionnelle, qui découlent de l'art. 33 al. 4 et $5 \mathrm{GG}$, perdent leur principale base de légitimité. On peut douter de la stricte observation des obligations et principes caractéristiques du droit de la fonction publique en cas de prise en compte des conséquences de la privatisation. Un certain nombre d'éléments pourraient au contraire, s'agissant des obligations des fonctionnaires «privatisés » qui n'assument de fait plus de fonctions publiques, plaider pour une prise en compte des principes du droit du travail.

Mais le contenu de l'art. 143a al. 1 GG et l'exigence de conserver le statut qui en découle plaident en sens contraire. Les fonctionnaires "privatisés » sont ainsi assimilés aux fonctionnaires fédéraux encore employés dans le service public. Ces droits vont logiquement de pair avec une série d'obligations de la fonction publique et la poursuite illimitée de la validité des principes traditionnels de la fonction publique professionnelle. En conséquence de la clause de garantie du statut définie à l'art. 143a al. 1 phrase 3 GG, les principes traditionnels de la fonction publique professionnelle, au sens de l'art. 33 al. 5 GG, sont donc fondamentalement applicables, sans restriction, aux fonctionnaires "privatisés » et ont donc effet aussi pour l'employeur de droit privé.

Par conséquent, du fait de la validité notamment du principe de l'emploi à vie et du principe de carrière, il n'est possible de réduire efficacement les effectifs que dans des conditions limitées. De telles pertes de flexibilité en matière de gestion des RH peuvent, dans une perspective entrepreneuriale, entraîner des désavantages concurrentiels si la concurrence n'emploie pas de fonctionnaires. Seul le principe traditionnel d'interdiction des actions de lutte sociale doit être modifié par la réforme structurelle des chemins de fer pour les fonctionnaires

${ }^{1} \mathrm{Cf}$. Gardavaud dans ce volume, NDE. 
mis en disponibilité afin qu'un droit de grève limité leur soit octroyé, à la condition stricte que les actions de lutte se rapportent à des objectifs n'affectant en rien le droit de la fonction publique.

Contrairement aux principes traditionnels de la fonction publique professionnelle au sens de l'art. 33 al. 5 GG, la réforme ferroviaire influence et même modifie le principe du droit à un emploi conforme à sa fonction et les dispositions de durée du travail de la fonction publique.

Le fait que la situation juridique garantie par la constitution reste en vigueur pour les fonctionnaires est déterminant pour l'application du droit disciplinaire aux fonctionnaires affectés et mis en disponibilité. Les devoirs liés à la relation de service et de fidélité découlant de ce droit restent valables. Une sanction disciplinaire pour des manquements commis en dehors de l'exercice des fonctions est donc compatible avec le principe d'égalité au sens de l'art. $3 \mathrm{GG}$, pour les fonctionnaires «privatisés » également.

Le passage des chemins de fer allemands du droit public au droit privé, le détachement des unités opérationnelles de la structure administrative et le transfert des fonctionnaires à la Deutsche Bahn AG ont également eu des répercussions durables, du point de vue opérationnel et juridique, sur la représentation des intérêts des personnels qui y sont employés. Pour accompagner cette mutation au plan des conventions collectives, des dispositions spécifiques ont également été nécessaires au niveau de l'entreprise, lesquelles inaugurent un terrain juridique nouveau.

En conséquence de la fiction juridique définie à l'art. 19 al. 1 DBGrG, les fonctionnaires affectés à la Deutsche Bahn AG en vertu de l'art. 12 al. 2 et 3 DBGrG ou bien à des sociétés de participations en vertu de l'art. 23 DBGrG sont considérés comme des salariés. Cela n'implique toutefois pas que les principes du droit du travail se substituent aux principes traditionnels du droit de la fonction publique. La fiction juridique sert au contraire à protéger les fonctionnaires affectés à des entreprises privées, lesquels se voient notamment confier le droit de vote et d'éligibilité aux conseils des délégués du personnel.

Compte tenu des conditions de participation des personnels définies aux art. 76 al. 1, 78 al. 1 de la loi applicable à la représentation du personnel fédéral (Bundespersonalvertretungsgesetz, BPersVG), les fonctionnaires affectés à des entreprises privées ne sont exclusivement représentés ni par la Personalvertretung propre à la fonction publique ni, conformément aux art. 75 al. 3, 76 al. 2 BPersVG, par les délégués du personnel. En cas de cogestion concurrente au sens de l'art. 76 al. 1 BPersVG et art. 99 de la loi sur l'organisation interne des entreprises, (Betriebsverfassungsgesetz, BetrVG, il existe selon l'interprétation de l'art. 17 al. 2 phrase 1 DBGrG un droit illimité de double participation aux instances de représentation. Le principe catégoriel (Gruppenprinzip) au sens de l'art. 38 al. 1, 2 BPersVG retient l'attention dans la mesure où, dans des affaires spécifiques, liées au droit de leur statut, les fonctionnaires sont représentés exclusivement par le Hauptpersonalrat qu'ils ont élu. 


\section{Effet de la réforme structurelle des chemins de fer sur le personnel couvert par la convention du service public}

S'agissant du transfert du personnel couvert par la convention du service public vers la Deutsche Bahn AG, le législateur a opté pour une transmission à titre particulier à travers un transfert d'entreprise simplifié, fondé à l'art. 14 DBGrG. En tant que loi spéciale, cette disposition a la prépondérance sur celles définies à l'art. 613a du Code civil allemand (Bürgerliches Gesetzbuch, BGB).

Après la réforme des chemins de fer, les personnels transférés ont droit à une prévoyance vieillesse d'entreprise, qui constitue un équivalent au régime de retraite de la fonction publique. Contrairement aux fonctionnaires, les salariés ne peuvent faire valoir après la privatisation la garantie du statut en tant que salarié du service public. Toute analogie avec la garantie du statut des fonctionnaires au sens de l'art. 143a GG est exclue car les fonctions, droits et devoirs de ces catégories respectives sont différents.

\section{Conséquences en termes de gestion du personnel de l'emploi de fonctionnaires " privatisés » ou de personnels aux emplois protégés à la Deutsche Bahn AG}

Si l'on analyse les résultats abstraits constatés à la lumière d'un exemple pratique sur le marché de l'emploi du groupe DB AG, on voit que l'employeur tente de faire le grand écart entre, d'une part, la réalisation des objectifs de la réforme ferroviaire à travers une gestion efficace des ressources humaines et, d'autre part, la préservation des intérêts des personnels concernés et des instances de représentation collective.

Malgré les mesures de rationalisation mises en œuvre pour accroître la productivité et alléger les effectifs, les fonctionnaires et les salariés protégés par convention bénéficient d'une garantie d'emploi du fait de l'organisation du marché du travail au sein du groupe. La sécurité des emplois et la possibilité de réorientation professionnelle ont toutefois leur prix. Les salariés qui signent un contrat de travail avec les sociétés du groupe doivent accepter des préjudices économiques du fait de la réduction de la durée annuelle légale du travail. Leur situation juridique étant garantie par la Loi fondamentale, les fonctionnaires ne sont pas touchés par ces conséquences économiques des mesures de rationalisation.

Bien que les sociétés du groupe ne soient pas des sociétés de participations au sens d'une entreprise de transports ou d'infrastructures ferroviaires au titre de l'art. 3 al. $1 \mathrm{n}^{\circ} 1$ et $2 \mathrm{DBGrG}$ et que la fédération d'employeurs constitue même une institution externe, l'affectation au sens de l'art. 123a BRRG auprès du bureau de placement DB, la mise en disponibilité ou la signature de contrats de mise à disposition constituent des dispositifs suffisants pour un transfert de personnel fonctionnaire. 
On peut retenir dans une perspective critique que le transfert de personnel vers les sociétés du groupe a certes permis de parvenir à réduire le surplus d'effectifs dans les entreprises considérées isolément, mais que cela a pour seul effet un déplacement du problème à l'intérieur du groupe.

En contrepartie de l'accord donné par l'entreprise pour ne pas licencier, les instances de représentation collective du personnel ont donné leur aval à l'organisation d'un marché de l'emploi à l'échelle du groupe. Dans le cadre des négociations sur la conciliation des intérêts ou sur le plan social, ces instances peuvent tenter de compenser ou d'atténuer les préjudices économiques subis par les salariés du fait des mesures de rationalisation.

Il sera à l'avenir plus facile pour les syndicats de mener les négociations collectives puisqu'ils auront en face d'eux un interlocuteur et partenaire unique : la fédération d'employeurs des services de transports et de mobilité (Agv MoVe e.V.). On peut s'attendre à ce que la création de cette fédération débouche notamment sur davantage de transparence et sur une homogénéité accrue des systèmes de conventions collectives. Ce qui est non seulement dans l'intérêt de l'entreprise et des instances de représentation du personnel, mais sert aussi à protéger les salariés.

Avec l'instauration de cette fédération d'employeurs, la compagnie ferroviaire privatisée émet un signal clair quant à son positionnement au sein des instances nationales et internationales dans le domaine de la politique sociale et juridique et quant à son orientation à venir de prestataire de mobilité d'échelle mondiale.

\section{Cadre juridique de l'emploi de fonctionnaires "privatisés " ou de personnels aux emplois protégés dans le cas d'autres privatisations}

Les grandes questions de la gestion des conséquences de la privatisation en matière de ressources humaines montrées dans cette contribution et dans ma thèse sur ce sujet se posent aussi face à d'autres privatisations du secteur public.

Les conclusions en terme d'aménagement du droit des privatisations ne sont néanmoins pas transposables sans réserve à d'autres projets, les conséquences des réformes dépendant très largement de la situation concrète. Pour pouvoir formuler des déclarations générales, valables en toute situation, sur la gestion de la privatisation, il faut d'abord voir si d'autres privatisations d'ampleur comparable à la réforme des chemins de fer peuvent à l'avenir entrer en ligne de compte.

D'une part, il faut sûrement admettre que les privatisations de la Deutsche Bundesbahn et de la Deutsche Bundespost constituaient, de par le transfert de personnel et les conséquences impliquées, des mesures uniques en leur genre, allant jusqu'à justifier des modifications de la Loi fondamentale.

D'autre part, il reste encore au niveau fédéral quelques champs d'action comparables, du point de vue de leur étendue, aux géants monopolistiques res- 
tructurés, et susceptibles de tirer profit, en cas de privatisation, des expériences des réformes passées.

A l'échelon fédéral, on pourrait envisager, au titre de privatisations potentielles, les mesures suivantes :

- la suppression du monopole en matière de placement professionnel et la privatisation de l'Office fédéral de l'emploi ;

- le transfert de l'administration de la Bundeswehr vers une entreprise organisée suivant le droit privé ou

- la privatisation des centres d'entretien routiers pour les autoroutes fédérales.

Les implications juridiques de la privatisation dépendent fondamentalement du modèle choisi, par exemple modèle d'affectation au sens de la réforme ferroviaire ou de la mise à disposition au sens de la réforme des services postaux, des modalités du transfert de personnel et d'une éventuelle garantie du statut de fonctionnaire en droit constitutionnel.

Dans le cas de la réforme ferroviaire comme dans celui de la privatisation de la Deutsche Bundespost, l'art. 143a al. 1 phrase 3, 143b al. 3 GG garantissait constitutionnellement l'affectation des fonctionnaires, et la situation juridique de ceux-ci était également assurée explicitement. Une protection comparable du statut n'est pas fondée pour les fonctionnaires affectés pour des activités dans des entreprises de droit privé en vertu de la norme de loi ordinaire au titre de l'art. 123a BRRG.

Il est donc difficile de proposer une conclusion de portée générale sur la validité des principes traditionnels de la fonction publique professionnelle et sur l'application du droit disciplinaire aux fonctionnaires «privatisés» pour d'autres privatisations à venir; une telle conclusion n'est envisageable qu'en appliquant par analogie les dispositions contenues à l'art. 143a, 143b GG.

De même, il n'y a pas de fiction juridique de salariat pour les fonctionnaires travaillant dans une entreprise du secteur privé sur la base d'une disposition de loi ordinaire. Les dispositions élaborées dans le cadre de la réforme ferroviaire sur l'intégration des fonctionnaires à la cogestion d'entreprise ne contiennent aucune idée juridique générale et ne sont donc pas applicables par analogie aux fonctionnaires affectés au titre de l'art. 123a BRRG. Si des fonctionnaires «privatisés » doivent être intégrés à des dispositions de droit collectif et d'organisation des entreprises à la suite de mesures de privatisation, une loi spéciale est nécessaire pour établir la fiction d'un salariat, par exemple au sens de l'art. 19 DBGrG.

L'art. 15 DBGrG ne pouvant être appliqué par analogie à d'autres projets de privatisation, il faut également créer un dispositif de droit ordinaire comparable dans l'intérêt et pour la protection des personnels, à travers un mandat transitoire du Personalrat, pour éviter qu'un changement d'entreprise n'entraîne un défaut provisoire de représentation du personnel. Les expériences issues de la 
réforme des chemins de fer montrent que, dans le domaine de la gestion et du développement des $\mathrm{RH}$, des normes de loi ordinaire comblant de telles lacunes réglementaires contribuent à la gestion des conséquences de la privatisation.

Le recours à des dispositifs légaux que cela implique ne pourra néanmoins se justifier que dans le cas d'un projet supposant le transfert d'effectifs importants.

Par conséquent, dans le cas de privatisations de faible ampleur, telles que celles qui interviennent au niveau régional et communal, on ne peut faire de constats de portée générale sur la gestion des conséquences de la privatisation que sous réserve, faute de comparabilité. Un cadre juridique pour les fonctionnaires «privatisés » constitue ainsi un préalable à une généralisation de cette gestion.

Si l'on prend en compte un potentiel de privatisation encore considérable au niveau régional ou communal, il est nécessaire d'agir pour clarifier au plan législatif les conséquences de la privatisation pour les fonctionnaires affectés à une entreprise du secteur privé sur la base de l'art. 123a BRRG. A cet égard, les biens fédéraux semblent favorisés par rapport aux biens municipaux, dans la mesure où l'Etat fédéral peut, à travers des législations $a d$ hoc, créer lui-même les conditions pour opérer des privatisations à grande échelle. Les autres entités publiques ne peuvent procéder de la sorte, faute de compétence législative propre.

Les analyses ci-dessus mettent en outre en lumière une nécessité d'agir sur le droit de traitement spécifique applicable aux fonctionnaires affectés à la Deutsche Bahn AG, afin d'harmoniser la structure de traitement et de rémunération pour les fonctionnaires et les salariés. On peut y voir aussi bien un objectif d'organisation plus dynamique, axée sur la performance, du droit régissant les parcours professionnels, qu'une incitation à réformer en profondeur le droit de la fonction publique.

C'est l'Etat fédéral qui détient les compétences décisionnelles et législatives nécessaires pour déterminer les conséquences des privatisations. D'une part, il détient de fait, indépendamment de la priorité de la compétence des Länder liée au système fédéral, la principale compétence en matière de législation; d'autre part, il définit le cadre juridique applicable à la fonction publique professionnelle et donc au développement ultérieur du droit de la fonction publique. La compagnie ferroviaire privatisée ainsi que les instances de représentation des intérêts, autres acteurs de la réforme des chemins de fer, peuvent accompagner ce processus et le soutenir en présentant des valeurs empiriques et des problèmes issus de la pratique.

Posons enfin un pronostic relativement au développement ultérieur de la gestion des conséquences de la privatisation en ce qui concerne les privatisations à venir.

Exiger la suppression générale du statut de fonctionnaire dans le sillage de privatisations est trop audacieux, et irréalisable si l'on prend en compte les aspects à la fois économiques et d'assurance sociale. 
Un aménagement constructif de la gestion des conséquences de la privatisation par le biais d'un droit spécifique de la fonction publique applicable aux fonctionnaires « privatisés » est envisageable.

Les questions de droit de la fonction publique soulevées, dont nous n'avons pu montrer ici qu'un échantillon, sont extraordinairement importantes, compte tenu de l'ampleur du groupe concerné. Pour le seul secteur ferroviaire, ce sont environ 108000 fonctionnaires qui ont été transférés vers des entreprises privatisées à la suite de la réforme des chemins de fer ; pour le secteur postal, pour la seule année 1994, l'effectif a été de 300000 fonctionnaires transférés vers le secteur privé. Le groupe des fonctionnaires «privatisés » est comparable, en effectif et en importance, aux catégories des juges ou des militaires, auxquels s'applique toutefois un droit spécifique.

Bien que les fonctionnaires, au sens d'un modèle d'emploi amené à disparaître, soient désormais employés dans les entreprises du secteur privé, il faut encore s'attendre à une longue période de transition avant que tous les acquis découlant d'un statut de fonctionnaire disparaissent dans la confrontation avec les entreprises privées. Compte tenu du potentiel de privatisation qui subsiste et de la tendance aux privatisations massives que l'on observera vraisemblablement encore dans les années à venir, il pourrait être instructif pour une gestion des conséquences de la privatisation d'étudier scientifiquement la légalité et la pertinence d'un droit de la fonction publique propre aux fonctionnaires «privatisés ».

DES ÉClAIRAGES ET DES CONNAISSANCES SUPPLÉMENTAIRES de portée générale pour une gestion efficace des conséquences de la privatisation pourraient être obtenus en comparant les conséquences de la réforme structurelle des chemins de fer en matière de droit du travail et de GRH aux conséquences de la privatisation des entreprises postales ou en s'appuyant sur une comparaison avec d'autres modèles de privatisation.

Traduction de Marie GRAVEY 
- IV -

MISES EN REGARD SECTORIELLES

FRANCO-ALLEMANDES 



\section{La régulation sociale dans les services postaux}

\section{Dominique BAILLY}

En sa qualité de Directeur du pilotage stratégique RH et Conseiller du Directeur général du groupe La Poste, et en vertu de sa fonction de Président du Comité de dialogue social européen pour le secteur postal, Dominique Bailly dépeint ici les enjeux de la régulation au sein du secteur postal, le positionnement stratégique de La Poste en tant qu'entreprise de services et le modèle social mis en œuvre pour répondre aux défis posés.

\section{Les caractéristiques du secteur postal}

Le marché postal et sa régulation sont déterminés par trois caractéristiques, chacune porteuse de tensions, qui forment un paysage quelque peu complexe.

Comme beaucoup de services d'intérêt général, le secteur postal se situe au carrefour d'enjeux économiques et sociétaux : il fournit un support indispensable à l'activité générale, et subit une pression considérable sur les coûts et la qualité, ce qui est facteur de changement ; il a par ailleurs une forte dimension sociétale en raison de sa présence territoriale, du service universel et de la péréquation tarifaire, et subit une pression de la société en faveur du maintien du cadre traditionnel de son activité.

Par ailleurs, notre modèle de distribution s'inscrit dans un modèle triangulaire fournisseur/client/consommateur. A La Poste, 95\% de nos clients pour le secteur du courrier et du colis sont des entreprises. Ce sont elles qui paient le servie rendu mais ce sont les consommateurs qui reçoivent les bénéfices de la prestation. Il va de soi que les intérêts de nos clients gros émetteurs ne sont pas les mêmes que ceux des consommateurs. Il s'agit là d'un facteur de tension que la régulation doit pouvoir arbitrer de façon correcte. Les opérateurs se retrouvent face à un vrai dilemme stratégique : doivent-ils mettre la priorité sur l'écoute des grands clients et procéder alors à une optimisation industrielle et sociale assez radicale, au risque de perdre leur capital de sympathie auprès des consommateurs ou doivent-ils au contraire être à l'écoute des consommateurs, au risque cependant d'être en difficulté de compétitivité par rapport notamment à des intervenants low cost qui pénètrent le marché ?

La troisième caractéristique réside dans la situation quasi monopolistique, du moins la position ultra dominante des opérateurs postaux, ce qui en droit européen n'est pas quelque chose de condamnable, mais qui explique les pressions exercées par la Commission européenne afin que des brèches s'ouvrent sur ce marché. Néanmoins, le marché est en décroissance, puisqu'il est confronté non seulement à la concurrence électronique et aux phénomènes de substitution, mais aussi à la concurrence d'autres médias publicitaires. Enfin, ce secteur 
représente un employeur important avec 1,7 million de collaborateurs en Europe, ce qui entraîne des défis sociaux extrêmement importants.

\section{Les enjeux de la régulation}

On retrouve par conséquent des enjeux de régulation hautement complexes et sensibles : il s'agit à la fois d'ouvrir le marché, de baisser les prix, d'améliorer la qualité de service tout en défendant les consommateurs, de prendre garde aux effets sociaux et de gérer la problématique du dumping social au niveau national et européen.

A l'échelle européenne, la $3^{\mathrm{e}}$ directive postale fournit le cadre possible de cette régulation, sans l'organiser dans le détail toutefois. Elle rappelle l'obligation d'un service universel 5 jours sur 7 à un prix abordable sur tous les points du territoire (pour mémoire, la France propose un service universel 6 jours sur 7), mais elle renvoie la question du financement de ce service universel à la responsabilité nationale. De ce fait, les conditions de libéralisation du secteur constituent un sujet encore ouvert, d'autant que la dimension sociale de la régulation n'a pas été encore véritablement traitée.

Dans le cadre de ma fonction de Président du Comité sectoriel de dialogue social européen pour le secteur postal, j'ai été assez frappé en effet de voir que, dans l'ensemble des débats sur la régulation du secteur, la question sociale n'était pas abordée. Or, les coûts de personnel de La Poste représentent environ 60 à $70 \%$ des coûts d'exploitation. Les opérateurs historiques peuvent actuellement assez peu jouer sur ces coûts qui représentent une charge fixe. Par conséquent, lorsqu'il existe des écarts de coûts sociaux très importants entre les nouveaux entrants et les opérateurs historiques, on voit apparaître des conditions de distorsion de concurrence, parfois extrêmement déstabilisantes, qu'il est nécessaire de prendre en compte. Des travaux ont été menés dans ce sens : le Comité européen a publié une déclaration en faveur d'une compétition équitable. Le Parlement a proposé une clause sociale, finalement adoptée dans la troisième directive.

La directive doit maintenant être transposée en droit national. En France, la discussion sur la transposition s'est tenue dans le cadre du changement de statut de La Poste, qui est devenue une société anonyme le $1^{\text {er }}$ mars 2010.

\section{Une évolution incertaine du secteur postal}

Au total l'évolution du secteur postal en Europe est assez peu prédictible : on ne sait pas réellement avec quelle rapidité et quelle intensité le marché va s'ouvrir au niveau européen en raison des tentations défensives d'un côté, des pressions exercées par la Commission européenne de l'autre ou encore d'un certain nombre d'aléas en matière de concurrence technologique. Mais, les opérateurs postaux nationaux vont devoir dans tous les cas faire face à la double menace de 
l'érosion du marché du courrier, due à la concurrence d'Internet, et de montée en puissance des nouveaux entrants sur un marché complètement libéralisé.

Par exemple, aux Pays-Bas, où le marché a été ouvert assez tôt sur tout ce qui a trait au marketing direct (qui est une partie très importante et assez rentable de l'activité), TNT a perdu à peu près $15 \%$ de parts de marché en quelques années, ce qui, rapporté au segment ouvert à la concurrence, représente en fait $25 \%$ de perte de parts de marché. Certains économistes estiment l'érosion prévisible du marché du courrier de l'ordre de $3 \%$ par an, hors période de crise. En additionnant cette érosion à la perte due à la libéralisation du secteur postal, le secteur postal pourrait connaître au total en cinq années, dans un scénario extrême, une perte de chiffre d'affaires de l'ordre de $40 \%$, auxquels viennent s'ajouter des prix tirés vers le bas par les opérateurs low cost.

\section{La voie de l'optimisation industrielle et sociale}

Pour rester compétitifs, les opérateurs postaux ont adopté bien souvent une stratégie relativement homogène qui consiste à faire de l'optimisation opérationnelle et sociale assez radicale, en tirant profit des possibilités offertes par les nouvelles technologies. Si la technologie a pour inconvénient de renforcer la concurrence de nouveaux modes de communication, elle a aussi l'avantage, en effet, de faciliter considérablement l'automatisation du traitement du courrier. La plupart des postes européennes sont ainsi engagées dans un grand programme de modernisation pour augmenter la part mécanisée du traitement du courrier. Aujourd'hui, près de $90 \%$ du trafic lettre peut être automatisé. Cette optimisation industrielle de la production courrier a un effet collatéral important, dont l'impact social peut être radical sur le mode de distribution du courrier. Le facteur organise aujourd'hui son temps de travail en deux parties ; au bureau de poste, il trie le courrier tôt le matin dans l'ordre des numéros et des rues pour ensuite partir faire sa tournée. La mécanisation a fait de tels progrès que cette partie de tri préalable a diminué assez considérablement en temps. Les deux activités sont de plus en plus gérées séparément, et le facteur peut ne faire que la distribution physique, ce qui permet d'embaucher des employés à temps partiel ou à statut précaire pour réaliser ce travail.

L'optimisation s'effectue aussi dans les bureaux de poste via la réduction considérable, voire totale, du réseau des bureaux de poste. La poste allemande avait par exemple de l'ordre de 19000 bureaux dans les années 1990, il en reste aujourd'hui environ 12 000, dont 7000 sont sous la bannière Deutsche Post. Les employés de la Deutsche Post travaillent à temps complet dans un nombre réduit de bureaux de poste. Certaines postes européennes ont choisi par ailleurs de supprimer la quasi-totalité de leurs bureaux de poste en n'ayant que des points poste. En France, la politique menée est très différente : nous disposons d'un réseau de 17000 points de contact, dont 12000 constituent des bureaux de plein exercice. 
Cette optimisation opérationnelle conduit à une optimisation sociale pouvant être extrêmement forte. Un des exemples les plus connus est celui de la poste néerlandaise qui emploie pour moitié des facteurs classiques et pour l'autre des livreurs, ou des travailleurs le samedi. Ces livreurs, des employés à temps partiel, sont la plupart du temps des femmes au foyer, des jeunes retraités ou des étudiants, voire des scolaires, car il est possible aux Pays-Bas de travailler à partir de 15 ans dans le cadre des mini-jobs, à des salaires extrêmement faibles. Ainsi, un modèle largement optimisé émerge avec des réseaux de bureaux de poste très allégés, un tri mécanisé et un personnel géré de façon flexible.

Néanmoins, cette optimisation industrielle et sociale a des limites, de sorte que tous les pays n'y recourent pas de manière équivalente. Ces limites sont liées aux conditions de régulation : l'obligation que le régulateur impose à l'opérateur national en matière de service universel, de présence territoriale ou de respect de normes sociales a un effet sur ce degré d'optimisation économique, opérationnel et social. La deuxième limite est liée à l'acceptabilité sociale de ces changements, plus ou moins grande selon les pays. Celle-ci est très forte aux Pays-Bas car il s'agit d'un petit pays où le service universel n'a pas la même dimension qu'en France. La situation est différente de celle d'autres pays comme l'Italie ou l'Allemagne. Enfin les choix stratégiques eux-mêmes peuvent modifier la façon d'aborder l'optimisation industrielle et sociale des activités.

\section{La stratégie équilibrée suivie par La Poste}

En ce qui concerne la France, La Poste a souhaité dès le début associer dans sa stratégie la double dimension économique et sociétale. La stratégie définie sous l'autorité de Jean-Paul Bailly, qui a été appelée «Performance \& Confiance », comprend à la fois un volet économique, sous la forme notamment de grands projets industriels, et un volet sociétal dit de « développement responsable». Cette approche vise à maintenir l'équilibre social interne et l'équilibre sociétal externe. Nous avons résolu le dilemme existant entre le développement de la performance économique et l'exercice de la responsabilité sociétale en choisissant d'être une entreprise de services de proximité, et en exploitant le capital de confiance dont nous disposons auprès des consommateurs et de nos autres partenaires.

Cette orientation nous distingue de la Deutsche Post, qui disposait d'un certain nombre d'atouts, et a fait un choix différent, qui est d'être le leader mondial de la logistique. Grâce notamment à ses revenus issus d'un prix du timbre assez élevé, elle a pu racheter un certain nombre d'entreprises, notamment DHL. Elle est aujourd'hui le premier opérateur mondial en matière de logistique, adoptant une stratégie toujours plus mono-métier puisqu'elle se retire de l'activité bancaire. Sa logique de développement est très industrielle, alors que la nôtre est centrée sur les services.

La Poste française a considéré en effet que le capital de sympathie qu'elle avait vis-à-vis des consommateurs avait une valeur économique : en effet, l'entre- 
prise n'est pas considérée de la même manière que n'importe quel distributeur. En situation de crise, ce capital de sympathie a un effet positif sur l'activité, par exemple pour la Banque Postale, dont l'image est différente de celles des autres opérateurs bancaires. La Poste considère que ce capital de confiance est un actif immatériel qui, dans une économie de services, a une très grande valeur économique et qui devient un critère de diversification. La Poste s'est plus largement diversifiée que la Deutsche Post : elle comprend une activité de courrier, de colis et d'express (mais pas de logistique), ainsi qu'une activité bancaire.

L'orientation vers les services nécessite d'accorder une grande importance à la responsabilité sociale et à l'engagement des salariés. Au sein de la stratégie «Performance et Confiance », le volet sociétal de développement responsable repose sur quatre piliers, qui font partie des engagements les plus fondamentaux du groupe La Poste :

- l'engagement clients grand public (qualité de service, réduction des files d'attente aux guichets) ;

- la présence territoriale et les missions de service public ;

- le développement durable (lutte contre le réchauffement climatique, usage responsable du papier) ;

- un modèle social exceptionnel.

\section{Un modèle social original}

Ce modèle social a été conçu autour de trois engagements. Le premier est celui de la diversité. La Poste veut ressembler à la France. Elle embauche par conséquent des salariés de tous lieux et de toutes origines pour que ses salariés ressemblent à ses consommateurs. En échange de cela, le groupe attend que les postiers embauchés s'engagent dans le service au client. Le deuxième engagement, la qualité de l'emploi, est quant à lui atypique. La Poste a pris l'engagement formel de dé-précariser l'emploi, c'est-à-dire de transformer les CDD en CDI et de transformer en temps complets les temps partiels imposés, de sorte qu'aujourd'hui, la Poste emploie moins de $4 \%$ de ses salariés en CDD, à l'époque où beaucoup d'autres opérateurs font tout le contraire en flexibilisant leur effectif. En échange de cet engagement de la part de l'entreprise, La Poste attend des salariés qu'ils acceptent les changements, et notamment les règles de mobilité interne. Le troisième engagement est celui du développement continu. En contrepartie, l'entreprise va demander aux agents d'accepter être polyvalents et de pouvoir évoluer assez facilement d'un poste à l'autre. L'ensemble converge vers la considération des personnes, c'est-à-dire à la fois des salariés et des consommateurs.

Ces quelques éléments clés qui structurent les politiques sociales constituent un modèle pouvant être qualifié de «flexicurité » interne, alliant la flexibilité et la sécurité, qui permet de tenir un équilibre entre la dimension économique et la dimension sociale interne. 


\section{Une orientation confortée par la crise}

Comme nombre d'acteurs économiques, La Poste est aujourd'hui confrontée à une crise très importante, dont les conséquences se font sentir sur l'activité du courrier en particulier. Bien que la crise appelle de notre part de la vigilance et des plans d'action adaptés, elle conforte le groupe dans ses orientations stratégiques, et notamment le choix d'une diversification permettant de profiter des alternances de cycles selon les secteurs. Il nous semble par ailleurs à La Poste que la crise n'est pas une crise tout à fait comme les autres et qu'elle révèle des attentes sociétales extrêmement fortes. La pression sur les grandes entreprises, et les grandes entreprises de réseau notamment, en matière de responsabilité sociale, va croître dans les années à venir. La Poste y est préparée grâce à sa stratégie à double dimension. Face à la crise, la direction du groupe est certes vigilante, mais aussi confiante quant à ses choix structurants. 


\title{
Historique et évolution du statut. Libéralisation du service public de l'électricité
}

\author{
Bernard CARON
}

Après un rappel historique de l'évolution du secteur électrique et gazier, Bernard Caron, Directeur des Relations Sociales du Groupe EDF, met en évidence l'impact de la loi du 10 février 2000, relative à la modernisation et au développement du service public de l'électricité. A la fois en termes de dialogue social, de conditions d'emploi et de gestion des ressources humaines.

\section{La loi du 8 avril 1946 de nationalisation de l'électricité et du gaz}

La loi du 8 avril 1946 de nationalisation de l'électricité et du gaz a scellé la naissance de deux Etablissements Publics à Caractère Industriel et Commercial (EPIC) : Electricité de France (EDF) et Gaz de France (GDF). Contrairement aux salariés d'entreprises nationalisées comme La Poste et France Télécom, les salariés de ces deux EPIC ne sont pas soumis au statut de la fonction publique mais à leur propre statut. Ainsi dès 1946 existait, sous une forme particulière, une branche des industries électriques et gazières qui comptait au 31 décembre 2008 près de 140 entreprises, dont 117 de moins de 50 salariés. Le secteur dénombre 136000 salariés, dont 95\% sont employés par EDF et GDF SUEZ.

Tel que défini par la loi de 1946, le statut comprend un certain nombre de garanties collectives, reposant sur trois piliers :

- la classification et la rémunération (comme dans toute convention collective) ;

- la protection sociale, avec au sein d'EDF un régime spécial de retraite et un régime spécial maladie, aujourd'hui en pleine évolution ;

- des institutions représentatives du personnel spécifiques que sont le Comité Supérieur Consultatif des Comités Mixtes à la Production (comparable à un comité central d'entreprise) et les Comités Mixtes à la Production (qui étaient l'équivalent des comités d'établissement que nous connaissons aujourd'hui) ainsi que les activités sociales associées, regroupées au sein d'un organisme sui generis de branche, la Caisse Centrale des Activités Sociales (CCAS).

Ce statut, de nature réglementaire, ne peut être modifié que par décret du gouvernement.

La loi de nationalisation de l'électricité et du gaz prévoit de placer le secteur d'activité en dehors du champ de la négociation collective, réservant toutefois, via le ministère chargé de l'Industrie et du Commerce, une possibilité d'extension à l'ensemble des entreprises du secteur des mesures unilatérales de gestion 
des ressources humaines, ou «circulaires internes », prises par les directeurs généraux d'EDF et de GDF en concertation avec les organisations syndicales. Ainsi, lorsque le secteur entre en concurrence en 2000, le statut s'applique non seulement aux deux EPIC, mais aussi à la centaine de petites entreprises locales, souvent répertoriées comme des entreprises non nationalisées. Cela concerne les salaires, la classification, la protection sociale, mais aussi plus globalement toutes les règles de gestion des ressources humaines que peuvent édicter EDF et GDF telles que l'évaluation du personnel.

\section{La loi du 13 novembre 1982}

La loi du 13 novembre 1982 constitue une première modification au sein de ce schéma, accordant la possibilité pour les entreprises à statut de conclure des accords collectifs à leur échelle, sous réserve que ces derniers ne viennent pas modifier le statut.

Ainsi, l'article L. 2233-2 du Code du Travail prévoit que : «... des conventions ou accords d'entreprise peuvent compléter des dispositions statutaires ou en déterminer les modalités d'application dans les limites fixées par le statut ». Bien que cette évolution soit appliquée pour la première fois en 1993 dans les entreprises EDF et GDF, les modalités de fonctionnement de la branche commencent à se rapprocher de celles d'un secteur classique. Car malgré le statut, il existe des domaines ouverts à la négociation collective dans les entreprises. Par ce biais, EDF et GDF ont introduit le passage aux 35 heures, se distinguant ainsi du reste du secteur jusqu'à la loi de 1999 sur la réduction du temps de travail. Une transition s'amorce, du «tout régulé » au niveau de la branche vers un socle régulé à l'échelle du secteur et complété par des accords collectifs d'entreprise.

\section{La loi du 10 février 2000, relative à la modernisation et au développement du service public de l'électricité}

La loi du 10 février 2000 relative à la modernisation et au développement du service public de l'électricité, qui constitue la transposition de la directive européenne, organise l'ouverture des marchés de l'électricité et du gaz à la concurrence. EDF et GDF deviennent des opérateurs parmi d'autres. Ils ne doivent plus pouvoir décider seuls des conditions statutaires dans l'industrie électrique et gazière. La loi de 2000 inscrit le statut du personnel comme étant la «convention collective » de la branche, le statut évoluant comme une convention collective de branche. Elle marque les débuts d'une négociation collective de branche encadrée, s'appliquant à deux domaines d'intervention :

- les terrains nouveaux de négociation. La négociation de branche peut compléter le statut ou en définir les modalités d'application directement via la procédure d'extension (article L. 713-1 du Code du Travail). EDF dispose d'un comité d'extension spécifique, la Commission nationale supérieure du personnel, présidée non pas par un représentant d'EDF ou 
GDF mais par le ministère de tutelle. Néanmoins, les industries électriques et gazières deviennent un secteur d'activité standard au regard de la négociation collective de branche. Par exemple, lors de la négociation de la loi sur la formation au niveau de la branche, le statut a été complété par un accord collectif étendu à toutes les entreprises du secteur ;

- l'adaptation des mesures de branche antérieures à la loi de 2000 (article L. 713.2 du Code du Travail). Dans ce cas, la négociation n'aboutit pas à un accord collectif mais à un relevé de décision transmis aux pouvoirs publics afin de modifier le statut au niveau du secteur. Cela concerne notamment l'évolution du système de retraite ou la révision du système de classification/rémunération en 2010.

A l'heure actuelle, l'ensemble des nouveaux entrants sur le secteur mettent en œuvre le statut du personnel des industries électriques et gazières, dont le champ d'application recouvre les entreprises de distribution, de transport et de production d'électricité et de gaz. Une nuance est à relever toutefois sur le terme de distribution: auparavant, la distribution pouvait être comprise comme le transport depuis la sortie d'un poste très haute tension vers la moyenne tension en électricité ou jusqu'au client final, étant entendu que dans cette hypothèse, la vente au client final faisait partie de la distribution. Aujourd'hui, quelques nouveaux entrants spécialisés dans la commercialisation au client final (tels Energie Directe ou Poweo, bien que Poweo soit entré dans le secteur de la production), n'appliquent pas encore le statut à leur personnel. A cet égard, les groupements d'employeurs de la branche et les organisations syndicales réclament d'une seule voix la modification du premier article du statut.

Au regard de la protection conférée par le statut, il est intéressant de constater que lors de l'entrée de Poweo à l'Union Française de l'Electricité, l'entreprise a réalisé un sondage auprès de son personnel pour savoir s'il préférait conserver son statut actuel ou acquérir celui du personnel des industries électriques et gazières. Pour une large majorité, le personnel s'est prononcé en faveur du statut en vigueur dans l'entreprise. De même, au moment de la restructuration des réseaux de transport du gaz, privatisés dans le sud-ouest et dans une moindre mesure, dans l'ouest de la France, un partage s'est effectué du fait des parts croisées. Les salariés affectés chez Gaz de France, qui jusqu'alors ne bénéficiaient pas du statut des industries électriques et gazières, ne souhaitaient pas changer de statut, considérant que celui qui leur était appliqué était de meilleure qualité que celui des industries électriques et gazières.

\section{La composition de la branche professionnelle des industries électriques et gazières}

La branche professionnelle est composée de deux groupements d'employeurs :

- l'Union Française de l'Electricité (UFE). Existant préalablement à la loi de 2000, elle regroupe les employeurs des entreprises du secteur élec- 
trique, avec pour particularité de couvrir à la fois le domaine social et le domaine industriel. Il s'agit de la principale instance de représentation des employeurs électriciens en Europe ;

- l'Union nationale des employeurs des industries gazières (UNEmIG). L'UNEmIG a fait l'objet d'une création ad hoc en 2000 afin de regrouper les employeurs des entreprises du secteur gazier. Son champ de compétence est exclusivement social, d'autres associations prenant le relais sur le volet industriel.

En matière de représentation du personnel, les fédérations syndicales présentes au niveau des entreprises sont la CGT Energie-Mines, la CFDT EnergieChimie, la CFTC, FO - Fédération Nationale de l'Energie et des Mines et la CFE-CGC. Pour les organisations syndicales, la réorganisation de la branche professionnelle a eu des répercussions aussi importantes que pour les employeurs, initiant de fait un apprentissage collectif. Car auparavant, EDF se concertait avec GDF et édictait ses politiques de ressources humaines après consultation des organisations syndicales. De syndicats d'entreprise au sein d'Electricité et de Gaz de France, les organisations syndicales se sont muées en des fédérations de petites, moyennes et grandes entreprises.

\section{Le fonctionnement de la branche}

Dans le cadre des séminaires des employeurs, ces derniers décident conjointement de leurs positions dans les négociations. Une particularité de la branche réside dans le fait que les employeurs ne peuvent pas signer un accord exclusivement avec l'Union française de l'Électricité et les organisations syndicales, fussent-elles majoritaires. Tout accord nécessite en effet l'approbation conjointe de l'UFE et de l'UNEmIG. Au sein du Comité social de l'UFE et de l'UNEmIG ; les décisions qui permettent d'engager la négociation dans la branche professionnelle sont prises à l'unanimité des représentants des entreprises. Un représentant d'une plus petite entreprise que GDF SUEZ ou EDF peut ainsi bloquer le processus, disposant d'un poids égal dans les décisions en matière de négociation sociale. Toutefois, ces petites entreprises sont souvent regroupées au sein d'associations représentatives.

En second lieu, la Commission paritaire de branche constitue l'instance de négociation entre les employeurs et les organisations syndicales. Elle génère des groupes de travail paritaires pour préparer et faciliter les discussions entre les deux parties.

\section{Les principales évolutions du statut}

Depuis la création de la branche des industries électriques et gazières, le statut évolue régulièrement. Si la grille de rémunération a évolué, les classifications demeurent inchangées à ce jour. Il était prévu d'ouvrir le sujet de la classifi- 
cation/rémunération en 2010 dans la branche des industries électriques et gazières.

En matière de protection sociale, le régime spécial des retraites a été modifié en deux étapes, largement méconnues du grand public. Dans un premier temps, il s'agissait de remanier le financement du régime, jusqu'alors issu du budget d'exploitation des entreprises de la branche, sur la base de la solidarité professionnelle. Dans un secteur désormais ouvert à la concurrence, ce système de financement présentait un risque particulier en cas de faillite de l'entreprise. Moyennant le paiement d'une soulte, le secteur s'est ainsi adossé au régime général (CNAV, ARRCO, AGIRC) qui repose pour sa part sur la solidarité interprofessionnelle. Dans un second temps, par un décret adopté en juillet 2008, le groupe a été soumis à la volonté du gouvernement de modifier, outre le financement, le régime spécial de retraite qui, bien que toujours spécifique sur certains aspects, se rapproche du régime de droit commun français. Depuis lors, le nombre d'annuités de cotisation est passé de 37,5 à 40 , puis 41 . Il existe par ailleurs désormais une caisse nationale des industries électriques et gazières soumise, comme toute caisse de retraite, à la tutelle de la sécurité sociale. Auparavant, la caisse de retraite était gérée par le directeur du personnel et des relations sociales d'EDF-GDF. Le conseil d'administration de la caisse nationale des industries électriques et gazières est paritaire, toutes les décisions étant prises à l'unanimité. En cas d'égalité entre les membres (la situation ne s'étant encore jamais présentée), il appartient au ministre de tutelle de statuer. L'un des derniers chantiers à conduire sur l'évolution des retraites concerne la spécificité des métiers en matière de pénibilité : sous réserve qu'ils aient accompli un certain nombre d'années de service actif, c'est-à-dire d'emploi à forte pénibilité physique, les salariés d'EDF avaient jusqu'à présent la possibilité de partir en retraite de manière anticipée.

La mise en place d'un régime complémentaire maladie est en cours de négociation. Actuellement, le régime spécial maladie couvre comme partout le régime général maladie, mais aussi une petite partie de régime complémentaire, qui ne place pas les industries électriques et gazières à hauteur de la protection sociale proposée par la quasi-totalité des grands groupes. Se met donc actuellement en place une couverture supplémentaire maladie obligatoire instaurée par le biais de la négociation collective de branche, et non par le statut. Par conséquent, une part de la protection sociale maladie dans les industries électriques et gazières sera issue du statut, tandis qu'une autre part résultera de la négociation collective standard.

Elément additionnel d'évolution du statut: la modification en 2007 des instances de représentation du personnel. Le groupe EDF intègre dorénavant les instances classiques en la matière, comprenant un comité central d'entreprise, des comités d'établissement et des délégués du personnel. Outre ces instances, l'entreprise a conservé la Commission secondaire du personnel, où sont examinées un certain nombre de situations individuelles, notamment en ce qui concerne la ré- 
munération et les parcours professionnels. La Commission ne traite cependant pas des cas de discrimination ou de harcèlement, établis à l'aide de processus d'alerte. Ces processus sont suivis par des délégués du personnel, dont le champ de compétences est à peu près identique à celui du droit commun, à l'exception des compétences propres aux commissions secondaires du personnel.

La négociation de branche ne porte pas uniquement sur des éléments obligatoires comme en témoignent les accords suivants :

- l'accord du 16 décembre 2005 relatif à la formation professionnelle continue ;

- l'accord du 5 juillet 2007 sur le travail de nuit ;

- l'accord du 24 avril 2008 sur l'égalité professionnelle entre les hommes et les femmes. Le groupe EDF est précurseur sur ce sujet : l'écart salarial entre les hommes et les femmes y est inférieur à 1\%. EDF a été l'un des premiers groupes à introduire, y compris dans son accord professionnel égalité hommes femmes, la capacité de faire du «rattrapage » sur les écarts salariaux. Pendant quatre à cinq ans, des budgets spéciaux ont été prévus à cette fin ;

- l'accord du 21 février 2008 sur l'accompagnement des parcours professionnels dans la diversité des âges.

Un accord sur les risques psychosociaux a été signé le 12 février 2010 au niveau de la branche. Néanmoins, toutes les entreprises du secteur de plus de 1000 salariés se sont vues dans l'obligation d'en négocier les modalités à leur échelle.

\section{Observations}

Les industries électriques et gazières font actuellement l'apprentissage du dialogue social de branche. Autrefois, EDF et GDF étaient deux EPIC. Il s'agissait de deux entités juridiques séparées, bien que la direction commune EDF-GDF Services, bien connue des consommateurs, pût donner l'impression qu'il s'agissait d'une seule et même entreprise. Mais aujourd'hui, EDF et GDF SUEZ sont deux entreprises concurrentes, au profil différent: en France, GDF SUEZ compte environ 160000 salariés, dont seulement $12 \%$ relèvent de la branche des industries électriques et gazières. Dans le groupe EDF SA en revanche, la quasi-totalité des salariés est au statut des industries électriques et gazières. Ainsi, la politique de ressources humaines, traitée au niveau de la branche puis au niveau de l'entreprise, concerne la quasi-totalité du personnel français d'EDF. La situation est plus complexe au sein de GDF SUEZ, notamment en ce qui concerne les politiques de mobilité menées à l'échelle du groupe, privilégiant la mobilité interne en cas de restructurations industrielles. Un problème se pose dès lors : si un salarié employé dans l'une des filiales de SUEZ Environnement se retrouve redéployé chez GDF SUEZ dans le secteur des industries électriques et gazières, cela viendra achopper sur un principe relatif au statut des in- 
dustries électriques et gazières, selon lequel priorité est accordée au reclassement des salariés à l'intérieur de la branche professionnelle.

En matière de rémunération, les augmentations salariales collectives négociées fin 2009 se sont appliquées à partir de 2010 à l'ensemble des entreprises des industries électriques et gazières. Ce processus inclut également une partie des augmentations individuelles, complétée dans les entreprises par la négociation col-lective d'entreprise. La branche des industries électriques et gazières est en effet le seul secteur d'activité à n'être pas soumis à une négociation annuelle obligatoire au niveau des entreprises, mais où $75 \%$ de celle-ci est réalisée à l'échelle du secteur. Dès lors, toute la complexité réside dans la manière de parvenir à un accord entre employeurs sur le niveau de rémunération à appliquer dans la branche professionnelle sur une année donnée. Les mesures salariales sont ainsi négociées deux fois : dans un premier temps avec les entreprises de la branche, puis avec les organisations syndicales.

La branche présente par ailleurs la particularité de présenter des temps de négociation très longs, du fait de la situation initiale statutaire. Par exemple, la négociation sur les retraites s'est effectuée en deux temps sur l'ensemble de la période 2000-2008. Au sujet de la classification/rémunération, une première tentative a échoué en 2004 : après trois années de négociation, un projet d'accord a fait l'objet d'un droit d'opposition, étant par conséquent réputé non écrit, non valable. Une seconde phase de négociation a été entamée en 2010, d'une durée probable de l'ordre de deux à trois ans. Bien que cela puisse paraitre surprenant, le volet le plus simple dans la négociation relative à la classification/rémunération sera celui de la rémunération. En effet, il n'existe à ce jour pas de réel système de classification de branche. Aboutir à un accord sur la classification, en d'autres termes sur les niveaux de responsabilité des emplois d'une industrie, se révèle autant voire plus complexe que de se mettre d'accord sur les niveaux minimaux de salaire associés à cette classification.

Sous réserve que le jugement (Tribunal d'Instance de Brest, 27 octobre 2009) déclarant non conforme aux lois européennes la loi de 2008 sur la représentativité ne trouve de répercussions dans le droit français, les nouvelles règles en la matière s'appliqueront à l'issue des élections professionnelles de 2010 (dans les entreprises du secteur) et de 2013 (au niveau de la branche). Par conséquent, les interlocuteurs syndicaux changeront. Depuis 1946 et malgré quelques évolutions, le personnel du secteur était représenté par les cinq organisations syndicales le plus souvent présentes en France. Au regard des derniers scores des élections de représentativité de 2010 au niveau d'EDF, une des organisations syndicales n'a pas franchi le seuil des $10 \%$. Elle ne devrait pas franchir non plus en 2013 le seuil des 8\% requis à l'échelle de la branche. En revanche, de nouveaux entrants, comme SUD ou l'UNSA, vont parvenir à s'imposer à l'échelle du groupe EDF. Fin 2009, à EDF SA, SUD était représentative dans 3 établissements, l'UNSA dans un établissement. Toutes deux se sont emparées de la disposition de la loi de 2008 qui permet à des sections syndicales 
non représentatives de désigner des représentants, et sont donc de plus en plus présentes au local. La nouvelle loi aura par conséquent des répercussions sur la manière de conduire les relations sociales. Un seuil de représentativité est par ailleurs requis pour la validation d'un accord, jusqu'à présent réputé valable dans la branche lorsqu'il est signé par trois syndicats. Des changements apparâ̂tront également au niveau des entreprises puisqu'en matière de gestion des ressources humaines, il était jusqu'à présent possible d'établir des avenants, au niveau local, aux accords cadre signés à l'échelle des entreprises, en raison du fait que les cinq organisations syndicales représentatives au niveau de l'entreprise pouvaient être représentatives ou représentées au niveau local.

En définitive, il s'est opéré ces dernières années un grand changement dans le fonctionnement des ressources humaines pour l'ensemble des acteurs. Ce changement s'est accompagné d'un apprentissage de ce qu'est une branche et de ce qu'est une négociation collective ainsi que de la prise en compte d'employeurs de plus petites entreprises de la part d'EDF et GDF SUEZ. L'introduction de la concurrence soulève un certain nombre de questions lors de la négociation d'augmentations collectives à l'intérieur d'un secteur, où un certain nombre d'entreprises locales achètent à un tarif régulé et revendent à un tarif régulé. Par conséquent, leurs marges et leurs capacités de manœuvre sont assez réduites et connues des personnels, qu'ils soient représentants syndicaux ou employeurs.

\section{Facteurs d'évolution de la gestion des ressources humaines au sein d'EDF}

De mon point de vue, l'ouverture des marchés à l'échelle européenne ne constitue pas le vecteur fondamental des changements opérés par le groupe EDF en matière de gestion des ressources humaines. L'émergence d'autres facteurs a joué un rôle dans les processus d'évolution. En effet, dans les années 19801990, on avait l'habitude de dire qu'on « administrait des usagers ». A la fin des années 1980, on a commencé à «servir des clients », en conservant parfois le terme d' « usagers ». Ces dix années de transition, d'une «administration » vers un « service», ont été un vrai choc culturel, tant pour les personnels que pour les politiques de gestion des ressources humaines. L'exigence des clients, exacerbée par d'autres secteurs qui, étant en concurrence, mettaient en évidence des logiques de service différentes, a amené les entreprises à une spécialisation des métiers. Là où hier en monopole, les salariés étaient polyvalents, capables par exemple de traiter le stock du magasin, d'accueillir le client-usager au niveau d'un district local et de faire du conseil facture au téléphone, ils sont aujourd'hui spécialisés afin de répondre aux questions de plus en plus précises des clients, portant notamment sur la domiciliation, la mensualisation. Auparavant, un agent «petites interventions » faisait du conseil sur factures. Aujourd'hui, au sein d'un système régulé, il ne pourra pas se prononcer vis-à-vis du client sur le meilleur opérateur-vendeur d'électricité. Pour autant, et c'est le propre d'entreprises comme EDF qui ont conservé des missions de service public, il peut en- 
core apporter des conseils à un client en situation de précarité afin que celui-ci bénéficie d'un tarif spécifique, applicable à sa situation. ERDF recevra à ce titre une rémunération, au travers de la contribution du service public de l'énergie prélevée sur chacune des factures de l'opérateur-vendeur d'électricité, qu'il s'agisse d'EDF, GDF SUEZ, la SNET ou E.ON. Ainsi, bien que l'on se situe dans un secteur concurrentiel, il est possible de continuer à exercer un certain nombre de missions d'intérêt général faisant l'objet d'un contrat de service public entre l'Etat et l'entreprise.

AINSI, LA GESTION DES RESSOURCES HUMAINES dans les entreprises a davantage évolué du fait des modifications internes à l'entreprise, des nouvelles exigences des clients qu'en raison de la mise en place d'un secteur concurrentiel. Car par exemple, dès les années 1980, EDF disposait d'un système d'évaluation, d'intéressement et d'un système de recrutement non pas sur concours, mais de type classique. Toutefois, l'Europe et l'international jouent un rôle notable dans l'adaptation des politiques de ressources humaines au sein d'un groupe multinational, notamment face à la question de la portabilité et des mobilités intragroupes en Europe ou à l'existence d'un comité d'entreprise européen pour les questions internationales. EDF adopte des politiques de ressources humaines pour l'ensemble du groupe, comme en atteste la signature au niveau mondial du deuxième accord de responsabilité sociale de l'entreprise (RSE), réunissant les organisations syndicales de chaque pays d'implantation et les organisations européennes et mondiales du secteur. Signé pour quatre ans et à l'unanimité, l'accord RSE apporte quelques modifications dans la manière de conduire le dialogue social dans les pays où le groupe EDF est présent. Ainsi, des salariés chinois ont voté pour la première fois de leur vie afin d'être représentés dans le comité annuel dédié au suivi de l'accord à l'échelle mondiale. 



\title{
Régulation sociale et conditions de travail dans le secteur électrique allemand
}

\author{
Heinz-J. BONTRUP
}

En Allemagne, la libéralisation, en cours depuis plus de dix ans dans le secteur électrique, a entraîné des changements divers. Les opérateurs, monopoles naturels avant la libéralisation, ont revu leur stratégie en matière de relations extérieures et adapté leur organisation interne, en modifiant les conditions de travail. La contribution suivante décrit les processus de changement en se concentrant sur les principaux éléments.

\section{Marchés électriques libéralisés à partir de 1998}

Au milieu des années 1990, la directive relative aux services sur le marché intérieur 96/92/CE et la directive dite d'accélération 2003/54/CE ont introduit un changement de paradigme dans le secteur électrique européen. Du fait de la volonté de sécuriser l'approvisionnement, à l'œuvre avant la libéralisation, l'Allemagne dispose d'installations de production dotées de capacités (de réserve) considérables. Dans un premier temps, l'excédent d'électricité a fait nettement baisser les prix - abstraction faite des composantes définies par l'Etat (cf. Bontrup et al., 2008). Les baisses maximales ont été de $41 \%$ pour l'électricité industrielle et de $33 \%$ pour les ménages. Mais elles ont été de courte durée et se sont réduites à mesure que le processus de concentration amorcé dès 1998 avançait du côté des opérateurs. En peu de temps, neuf anciens monopoles précédemment classés comme «naturels » fusionnèrent pour constituer un oligopole de quatre opérateurs dominant le marché (E.ON, RWE, EnBW et Vattenfall Europe). Ces acteurs ont d'abord misé sur des participations ou des acquisitions de fournisseurs régionaux et régies municipales et ont, par ce biais également, rapidement freiné la concurrence escomptée. Si l'on inclut les participations, les quatre grands opérateurs regroupent plus de $80 \%$ des capacités de production en Allemagne (Angenendt et al., 2007). Outre cette stratégie de concentration, E.ON et RWE en particulier poursuivent une stratégie d'internationalisation. Les groupes RWE et Vattenfall Europe, récemment constitués, ont doublé leurs ventes d'électricité depuis la libéralisation, soit en une décennie; EnBW et E.ON ont respectivement triplé et quadruplé leurs ventes.

Dans les régies municipales, gérées par les communes ou par les quatre grands opérateurs, les nouvelles conditions ont également entrainé une réorganisation, en équilibre précaire entre réalisation de la mission de service public, sécurité d'approvisionnement et recherche de profit (Jansen et al., 2007, p. 4). La mort des régies municipales, annoncée par de nombreux observateurs au début de la libéralisation, n'a pas encore eu lieu (cf. sur ce point Wagner et al., 2001, p. 20). Au contraire : même si les fournisseurs communaux d'énergie doivent 
encore affronter des défis importants, la majorité des régies sont bien positionnées (Wübbels, 2007), ce qui tient cependant, il faut l'admettre, à une concurrence qui n'a pas encore véritablement démarré.

Le marché électrique allemand reste très largement concentré. Compte tenu de cette concentration de pouvoir, il n'est pas étonnant que, malgré d'énormes augmentations de productivité entre 1998 et 2007, seules aient perduré des baisses de prix marginales pour le consommateur final, déduction faite des composantes de prix définies par l'Etat ${ }^{1}$. Ainsi, en 2007, les prix étaient inférieurs d'à peine $3 \%$ seulement pour les industriels et d'environ $4,5 \%$ en moyenne pour les ménages à ceux de 1998 - et ce, malgré un accroissement de la productivité du travail (calculée comme production par heure travaillée) de près de $86 \%$ dans le même temps. Face à la volonté politique de stimuler la concurrence, on peut voir dans cette évolution divergente de la productivité et des prix de l'électricité un résultat complètement décevant.

On n'observe pas de résultat satisfaisant non plus sur le terrain des investissements (dans les énergies renouvelables en particulier). Les opérateurs sont restés très réservés après la libéralisation. Cela tient principalement à la mise en œuvre d'un cycle d'investissements initié auparavant et aux nouvelles conditions instaurées par la libéralisation. Au lieu d'investir dans des actifs corporels, les fournisseurs d'électricité ont désinvesti et réduit leurs dépenses pour consolider leur position sur le marché européen. Les entreprises ont par ailleurs utilisé leurs bénéfices pour distribuer des dividendes aux actionnaires, pour se concentrer au plan national afin de générer des effets d'échelle et d'échapper à la pression liée à la concurrence, mais aussi pour prendre des participations internationales afin de diversifier les risques et pour construire des capacités de production à l'étranger.

\section{Suppressions d'emplois}

Outre ces répercussions extérieures, la libéralisation a aussi eu des conséquences massives sur les relations au sein même des entreprises. Les réorientations stratégiques, les fusions et les processus d'internationalisation ont sensiblement modifié les rapports entre capital et travail dans le secteur électrique au cours de la dernière décennie. L'évolution des emplois est un premier indicateur de ces changements structurels.

Dans les entreprises dont l'approvisionnement est l'activité principale, environ 47000 emplois ont été supprimés entre 1998 et 2007, soit pratiquement $19 \%$. Depuis l'ouverture du marché en 1998, l'emploi a donc diminué de

\footnotetext{
${ }^{1}$ Cela inclut les prélèvements résultant des lois sur la cogénération et les énergies renouvelables, ainsi que la taxe sur l'électricité et la TVA, à quoi s'ajoutent les redevances de concession.

${ }^{2}$ Ces vastes suppressions d'emplois sont aussi en partie liées à des fusions d'entreprises, qui ont mené à la cession de pans d'entreprises et au détachement d'établissements et de services ne faisant pas partie du cœur de métier, ceux-ci étant désormais (en partie) intégrés aux statistiques d'autres secteurs d'activité.
} 
2,3 \% par an en moyenne. Avant la libéralisation déjà (de 1992 à 1997), la réorganisation de l'approvisionnement énergétique dans les nouveaux Länder et l'anticipation de la concurrence à laquelle s'attendaient les entreprises du fait de la libéralisation ont entraîné la suppression d'environ 33500 emplois (Bontrup et al., 2008).

Tableau 1 : Evolution des entreprises et des emplois de 1998 à 2007

\begin{tabular}{|c|c|c|c|c|c|c|c|c|c|c|c|c|}
\hline $\begin{array}{l}\text { Répartition } \\
\text { selon } \\
\text { l'effectif }\end{array}$ & & 1998 & 1999 & 2000 & 2001 & 2002 & 2003 & 2004 & 2005 & 2006 & 2007 & $\begin{array}{l}1998 \\
-2007 \\
\text { en \% }\end{array}$ \\
\hline \multirow{2}{*}{$\begin{array}{l}\text { Ensemble des } \\
\text { foumisseurs } \\
\text { d'électricité }\end{array}$} & Nbre d'entreprises & 1229 & 986 & 925 & 919 & 927 & 931 & 960 & 979 & 994 & 1055 & \\
\hline & Effectifs & 251297 & 239777 & 219586 & 205816 & 207419 & 198758 & 209667 & 207654 & 207522 & 204061 & $-18,8$ \\
\hline \multirow{2}{*}{$>500$} & Nbre d'entreprises & 107 & 100 & 88 & 83 & 84 & 81 & 82 & 84 & 83 & 83 & \\
\hline & Effectifs & 194257 & 178888 & 156984 & 146379 & 147000 & 138542 & 147849 & 147148 & 145806 & 141208 & $-27,3$ \\
\hline \multirow{2}{*}{$250-499$} & Nbre d'entreprises & 58 & 62 & 66 & 62 & 67 & 67 & 66 & 60 & 67 & 66 & \\
\hline & Effectifs & 20925 & 22379 & 23620 & 22289 & 23870 & 23626 & 23389 & 21075 & 22865 & 22091 & 5,6 \\
\hline \multirow{2}{*}{$100-249$} & Nbre d'entreprises & 115 & 122 & 126 & 113 & 107 & 109 & 119 & 119 & 120 & 125 & \\
\hline & Effectifs & 17700 & 18662 & 18768 & 16804 & 15963 & 16181 & 17895 & 18343 & 17797 & 18596 & 5,1 \\
\hline \multirow{2}{*}{$50-99$} & Nbre d'entreprises & 148 & 160 & 158 & 170 & 175 & 172 & 173 & 179 & 174 & 185 & \\
\hline & Effectifs & 10453 & 11280 & 11415 & 12102 & 12506 & 12348 & 12413 & 12847 & 12602 & 13336 & 27,6 \\
\hline
\end{tabular}

Source : Office fédéral des statistiques, années diverses, calculs de l'auteur.

C'est surtout le segment des fournisseurs d'électricité de plus de 500 salariés qui a été touché par les suppressions d'emplois. Dans ce groupe d'entreprises, qui réunissait en 2007 environ 140000 emplois, soit environ $70 \%$ de l'ensemble des emplois dans plus de 80 entreprises du secteur électrique, plus d'un emploi sur quatre a disparu depuis 1998. Jusqu'à présent, employeurs et syndicats ont, pour l'essentiel, réussi à opérer cette réduction d'emploi sans licenciements économiques (Bergelin, 2008). Ils ont généralement fait appel à des instruments prévus dans les conventions collectives, parmi lesquels :

- les dispositifs de préretraite et préretraite progressive ;

- la résiliation volontaire des contrats de travail avec versement d'indemnités ;

- la réduction des temps de travail avec ou, dans certains cas, sans compensation salariale ;

- le recours à des sociétés d'emploi et de qualification et

- des accords pour garantir l'emploi en cas de mesures d'externalisation.

Il est intéressant de noter, en matière d'évolution de l'emploi, que les petites et moyennes entreprises d'approvisionnement ont enregistré une hausse de l'emploi de 1998 à 2007. Cette observation vaut en particulier pour les entreprises de 50 à 99 salariés. L'augmentation du nombre d'entreprises, passées de 148 à 185 , est un facteur déterminant. Il s'agit principalement de créations de filiales par les grands fournisseurs d'énergie. 
Dans le secteur électrique allemand, on peut identifier, en plus des évolutions purement quantitatives, trois grandes évolutions qualitatives intervenues en matière d'emploi à la suite de la libéralisation :

- premièrement, chez tous les fournisseurs d'électricité, de nouveaux domaines d'activités et services ont été créés, qui sont caractéristiques des marchés concurrentiels et n'étaient pas nécessaires auparavant du fait des monopoles territoriaux. Parmi ces nouvelles fonctions, citons notamment le marketing, la publicité, la gestion des risques, la gestion des actifs, le négoce d'électricité et de gaz ou trading ainsi que la force de vente et le service commercial (relations clientèle). Il faut également mentionner les activités liées à la gestion de la régulation et à une stratégie d'internationalisation. Les profonds changements de contexte ont eu pour conséquence la création de nouvelles activités et de nouveaux métiers dans le secteur (p. ex le «trader» dans le négoce ou l'agent de centre d'appels au sein du service commercial) ;

- deuxièmement, de nombreux postes ont été externalisés. On peut distinguer deux phases. Dans les années qui ont suivi l'ouverture du marché, les entreprises ont commencé par sous-traiter les activités à bas niveau de qualification (par exemple les emplois liés à la restauration d'entreprise, les services de surveillance ou de logistique). Ces mesures visaient à exploiter les différences de niveau entre les diverses conventions collectives pour en tirer profit en termes de coûts. D'autre part, des postes à haut niveau de spécialisation et de savoir (comme les prestations de services d'information) ont été externalisés, leur caractère généraliste permettant d'envisager la sous-traitance d'activités supplémentaires. Il s'agit souvent, pour ces derniers domaines, d'activités à haut niveau de rémunération. La tendance la plus récente est à la mise en question de services proches du «cœur d'activité », comme les services de réseau, la maintenance des centrales, la facturation ou les services de métrologie et mesure. Ces prestations tendent maintenant à être regroupées dans des « centres de services partagés » (CSP) ; avec, en général, une dégradation des conditions de travail et de rémunération. Est ici à l'œuvre le phénomène ambivalent d' «appréciation de la valeur » professionnelle déjà observé depuis longtemps dans d'autres secteurs de l'économie, en direction des personnels permanents et temporaires ;

- troisièmement, les regroupements d'entreprises (fusions), suivis de restructurations massives des ressources humaines ont contraint de nombreux salariés à la mobilité géographique. Les déménagements importants, impliquant des familles entières, ne sont pas restés limités aux cadres.

La répartition des salariés restés dans les entreprises s'est généralement déplacée des postes techniques vers des postes à niveaux de compétences moyens à élevés, dans les domaines de l'administration, de l'organisation et de la gestion. Ces postes 
sont mieux rémunérés et comportent, en termes de gestion du personnel, des incitations à la motivation. La formation continue et la tentative d'identification à l'entreprise y sont à l'œuvre. Mais, dans le même temps, la gestion des ressources humaines est soumise, dans le secteur électrique comme ailleurs, à la question de l'exclusion des activités moins nécessaires à l'entreprise par le biais de l'externalisation et du déplacement des qualifications moins élevées à la marge de l'entreprise. D'une manière générale, on constate souvent, pour les personnels techniques, une stagnation ou même une détérioration des conditions de rémunération. Globalement, la libéralisation n'a pas entrainé que des destructions d'emploi ou des externalisations avec des conditions d'emploi moins favorables, la répartition des rémunérations en interne s'est également nettement dégradée.

\section{Evolution des rémunérations du travail et répartition de la valeur ajoutée}

Les suppressions de postes ont permis aux fournisseurs d'énergie de réduire leurs dépenses de personnel. De 1998 à 2007, celles-ci ont baissé au total de $3,1 \%$ (soit une moyenne annuelle de $0,3 \%$ ). Malgré un contexte défavorable, la rémunération moyenne des salariés restés dans les entreprises a cependant augmenté, du fait des suppressions de postes («brut employeur», donc cotisations d'assurance sociale et accident incluses), de 19,4\% pendant la période observée (1998-2007), soit une moyenne annuelle de $+2,0 \%$ (cf. tableau 2).

Tableau 2 : Evolution des dépenses de personnel et de la valeur ajoutée nette de 1998 à 2007

\begin{tabular}{|c|c|c|c|c|c|c|c|c|c|c|c|c|}
\hline $\begin{array}{l}\text { Ensemble des fournisseurs } \\
\text { d'électricité }\end{array}$ & 1998 & 1999 & 2000 & 2001 & 2002 & 2003 & 2004 & 2005 & 2006 & 2007 & $\begin{array}{c}1998-2007 \\
\text { en \% }\end{array}$ & $\begin{array}{c}\text { Variation } \\
\text { moyenume an } \\
\text { (en \%) }\end{array}$ \\
\hline Effectifs & 251297 & 239777 & 219586 & 205816 & 207419 & 198758 & 209667 & 207654 & 207480 & 204022 & $-18,8$ & $-2,3$ \\
\hline Heures travallees en milliers & 389726 & 371287 & 343021 & 314633 & 313351 & 303929 & 323137 & 321198 & 318936 & 312630 & $-19,8$ & $-2,4$ \\
\hline $\begin{array}{l}\text { Helres yavalliees } \\
\text { par collab os ateur }\end{array}$ & 1551 & 1548 & 1562 & 1529 & 1511 & 1529 & 1541 & 1547 & 1537 & 1532 & $-1,2$ & $-0,1$ \\
\hline$\ldots$ en millions $€$ & 14136 & 14179 & 14050 & 12329 & 12450 & 12833 & 13264 & 13777 & 15392 & 13699 & $-3,1$ & $-0,3$ \\
\hline par salarié en $€$ & 56251 & 59134 & 63984 & 59903 & 60023 & 64566 & 63262 & 66346 & 74185 & 67145 & 19,4 & 2,0 \\
\hline par heure en $€$ & 36,27 & 38,19 & 40,96 & 39,19 & 39,73 & 42,22 & 41,05 & 42,89 & 48,26 & 43,82 & 20,8 & 2,1 \\
\hline avant impôts en millions $€$ & 25306 & 27536 & 22629 & 21900 & 24442 & 23729 & 29350 & 31388 & 33643 & 37738 & 49,1 & 4,5 \\
\hline par salarie en $€$ & 100703 & 114840 & 103053 & 106406 & 117839 & 119386 & 139984 & 151155 & 162151 & 184970 & 83,7 & 7,0 \\
\hline par heure travaillée en $€$ & 64,93 & 74,16 & 65,97 & 69,60 & 78,00 & 78,07 & 90,83 & 97,72 & 105,49 & 120,71 & 85,9 & 7,1 \\
\hline
\end{tabular}

Source : Office fédéral des statistiques, années diverses, calculs de l'auteur.

Or, la productivité avait quant à elle augmenté de $85,9 \%$ (soit une moyenne annuelle de $+7,1 \%$ ) de 1998 à 2007. La redistribution de la valeur ajoutée de l'entreprise en interne s'est faite de manière très inégale, au détriment du facteur travail. Sur la période étudiée, la valeur ajoutée a augmenté de $49,1 \%$ au total, soit $4,5 \%$ par an en moyenne. Mais, dans le même temps, la part des revenus du travail a diminué de $3,1 \%$ (moyenne annuelle de - 0,35\%), tandis que les revenus après impôts sur les bénéfices ont augmenté de 202,3\% (moyenne annuelle de $+13,1 \%$, voir tableau 3 ). 
Tableau 3 : Répartition de la valeur ajoutée : 1998 - 2007

\begin{tabular}{|c|c|c|c|c|c|c|c|c|c|c|c|c|}
\hline $\begin{array}{l}\text { Ensemble des } \\
\text { fournisseurs d'électricité }\end{array}$ & 1998 & 1999 & 2000 & $\begin{array}{c}2001 \\
\text { en milli }\end{array}$ & $\begin{array}{l}2002 \\
\text { ons } €\end{array}$ & 2003 & 2004 & 2005 & 2006 & 2007 & $\begin{array}{c}1998-2007 \\
\text { en } \%\end{array}$ & \begin{tabular}{|c|} 
Variation \\
moyenmy) \\
(en \%) \\
\end{tabular} \\
\hline Salaires et rémunérations & 14136 & 14179 & 14050 & 12329 & 12450 & 12833 & 13264 & 13777 & 15392 & 13699 & $-3,1$ & $-0,35$ \\
\hline Impôt & 4340 & 4768 & 4181 & 4130 & 4480 & 4549 & 4979 & 5094 & 4986 & 5263 & 21,3 & 2,17 \\
\hline & 1469 & 1636 & 1275 & 1326 & 1238 & 1187 & 1128 & 1137 & 1153 & 1357 & $-7,6$ & $-0,87$ \\
\hline & 4442 & 5986 & 2306 & 2643 & 5138 & 3866 & 7859 & 9092 & 9683 & 13430 & 202,3 & 13,08 \\
\hline Baux et loyers & 920 & 967 & 817 & 1472 & 1136 & 1294 & 2120 & 2288 & 2429 & 3989 & 333,7 & 17,71 \\
\hline Valeur ajoutée totale & 25306 & 27536 & 22629 & 21900 & 24442 & 23729 & 29350 & 31388 & 33643 & 37738 & 49,1 & 4,5 \\
\hline
\end{tabular}

Source : Office fédéral des statistiques, années diverses, calculs de l'auteur.

Contrairement aux salaires et rémunérations des salariés, les revenus du conseil d'administration (CA) et des dirigeants ont eu part aux hausses de la valeur ajoutée. Cela vaut en tout cas pour les quatre grands opérateurs du secteur énergétique en Allemagne. Depuis 2000, ceux-ci ont enregistré dans certains cas des augmentations considérables (voir tableau 4). Cette évolution est surtout liée à l'introduction, à partir de 2003, de systèmes de management et de rémunération axés sur la valeur de l'entreprise, avec un caractère incitatif à long terme. C'est à peu près à partir de cette date que s'opère la dissociation entre les revenus à court terme et l'ensemble des revenus, au moins dans les trois entreprises électriques basées en Allemagne. La rémunération du conseil d'administration de Vattenfall Europe AG, considérée de manière globale comme individuelle, est en revanche faible par rapport à EnBW, qui est une entreprise comparable. Ces différences sont sûrement liées à l'ancrage de Vattenfall en Suède, où prédominent des répartitions de revenus plus égalitaires.

Tableau 4 : Evolution des revenus du conseil d'administration des 4 grands

\begin{tabular}{|c|c|c|c|c|c|c|c|c|}
\hline en milliers $€$ & 2000 & 2001 & 2002 & 2003 & 2004 & 2005 & 2006 & 2007 \\
\hline \multicolumn{9}{|l|}{ E.ON } \\
\hline Somme des revenus à court terme & n.c. & 8800 & 9100 & 17400 & 13459 & 17117 & 16539 & 16058 \\
\hline Ensemble des revenus & 13000 & 8800 & 9800 & 17400 & 13777 & 25076 & 21735 & 20430 \\
\hline Revenus par personne & 2600 & 1760 & 1960 & 2900 & 2296 & 4179 & 3105 & 3405 \\
\hline \multicolumn{9}{|l|}{ RWE } \\
\hline Somme des revenus à court terme & 9310 & 3049 & 9486 & 7651 & 11620 & 10638 & 11371 & 12782 \\
\hline Ensemble des revenus & 9310 & 3049 & 9486 & 13676 & 13846 & 35372 & 35865 & 17376 \\
\hline Revenus par personne & 1862 & 610 & 1581 & 3419 & 2769 & 7074 & 7173 & 3475 \\
\hline \multicolumn{9}{|l|}{ EnBW } \\
\hline Somme des revenus à court terme & 3328 & 4486 & 5553 & 9608 & 9871 & 7927 & 8060 & 9034 \\
\hline Ensemble des revenus & 3328 & 4486 & 5553 & 9608 & 9871 & 10177 & 10311 & 11055 \\
\hline Revenus par personne & 666 & 897 & 925 & 1922 & 1974 & 1696 & 1718 & 1842 \\
\hline \multicolumn{9}{|l|}{ Vattenfall Europe } \\
\hline Somme des revenus à court terme & - & - & 4500 & 3700 & 3841 & 4670 & 5102 & n.c. \\
\hline Ensemble des revenus & - & - & 5200 & 4400 & 4681 & 4690 & 5125 & n.c. \\
\hline Revenus par personne & - & - & 743 & 880 & 780 & 782 & 854 & n.c. \\
\hline
\end{tabular}

Source : chiffres établis par nos soins sur la base des différents rapports d'activité.

\footnotetext{
${ }^{3}$ En raison de l'effet au long cours des systèmes d'incitation, il n'est pas toujours possible de les affecter de manière exacte à l'année correspondante.
} 
Les quatre grands fournisseurs allemands d'énergie appliquent, dans le cadre des revenus à court terme, des parts de rémunération liées aux résultats. Les chiffres correspondants ne variant toutefois pas de plus de $15 \%$ par an, la réalisation des objectifs à court terme semble possible dans un cadre sur lequel les membres du CA peuvent avoir une influence. Les plans d'incitation à long terme font en revanche l'objet de variations plus fortes. Pour certaines entreprises, les incitations à long terme disparaissent presque complètement certaines années. En contrepartie, les revenus des CA sont, avec plus de 7 millions $€$ par an en moyenne, particulièrement élevés les années où les objectifs sont atteints. De 2000 à 2007, les revenus totaux des CA d'E.ON, RWE et EnBW ont pratiquement doublé voire pratiquement triplé.

\section{Organisation syndicale et évolution des conventions collectives}

Les évolutions des revenus du travail et de leur répartition reflètent celles du dialogue social ou des rapports de force entre capital et travail. Le fait qu'une redistribution se soit opérée, comme nous l'avons montré, au détriment des salariés, est lié non seulement au contexte économique général et à celui du secteur électrique en particulier, mais aussi au taux de syndicalisation des personnels. Mais cette évolution est par ailleurs tout aussi conditionnée par les réalités, extrêmement diverses et liées à l'histoire, des relations sociales dans le secteur :

- les salariés sont plus ou moins syndiqués selon leur position dans la chaîne de création de valeur. Le taux de syndicalisation va d'environ 40 à $50 \%$ pour les activités de commerce et distribution à $80-90 \%$ pour la production, en passant par près de $60 \%$ pour les activités de réseau (Brandt/Schulten, 2007, p. 78) ;

- sont applicables dans le secteur des conventions collectives de groupe (pour E.ON, RWE et Vattenfall Europe en particulier), plusieurs conventions régionales (surtout pour les anciens fournisseurs régionaux et les régies municipales) ainsi que les «conventions collectives Entreprises d'approvisionnement Est et Ouest» (TV-V West et TV-V Ost) pour les régies municipales, le plus souvent exploitées par la commune.

Avant la libéralisation du secteur électrique, les régies, majoritairement détenues par les communes, appliquaient pour leurs salariés la convention du service public (BAT/BMTG). Du fait de la concurrence intervenue à court terme après l'ouverture du marché, la convention TV-V avait été définie en octobre 2000, après plus de quatre années de négociations, comme convention catégorielle couvrant spécifiquement le domaine de l'approvisionnement communal de service public, applicable à environ 100000 salariés du secteur de l'approvisionnement énergétique. Pour la première fois, était signée une convention cadre, incluant un accord sur les rémunérations, à la fois pour des ouvriers et des employés du service public. Les conventions TV-V Ouest et Est permettent 
en outre, dans le cadre des « ouvertures » conventionnelles, d'instaurer des parts de rémunération liées aux performances.

Jusqu'en 2007, la TV-V Ouest prévoyait un temps de travail de 38,5 heures pour les anciens Länder (environ 550 régies municipales) et la TV-V Est, une durée hebdomadaire du travail de 40 heures pour les nouveaux Länder (environ 50 régies). Il y avait toutefois des possibilités d'adaptation aux modèles de temps de travail. La convention collective signée en 2008 permet, à compter de juillet 2008, de porter la durée du travail à 39 heures pour les Länder de l'ouest. La grille de rémunération a en général permis d'améliorer les rémunérations et salaires des personnels. Ce succès ne fut toutefois remporté qu'en créant, en contrepartie, une nouvelle catégorie de bas salaires. Celle-ci perçoit environ $20 \%$ de moins que la catégorie de salaire la plus basse auparavant. Pour garantir l'emploi, des clauses d'ouverture peuvent en outre être appliquées, en dérogation aux conventions (Brandt/Schulten, 2007, p. 89).

D'autres organisations d'employeurs comme le groupe AVE Gruppe dans le Land de Hesse, la fédération d'employeurs de Bavière (Tarifgemeinschaft), le groupement Gaz, eau et électricité de la fédération d'employeurs AGWE (Rhénanie du Nord-Westphalie), la fédération des centrales électriques du BadeWurtemberg, l'association Energie Rheinland-Pfalz e.V. ainsi que la fédération des employeurs de l'énergie et de l'approvisionnement (AVEU), présente dans les nouveaux Länder, négocient en outre avec les acteurs syndicaux des conventions collectives spécifiques pour certaines entreprises du secteur de l'approvisionnement énergétique. Ces conventions prévoient généralement elles aussi des clauses d'ouverture afin de préserver l'emploi.

Des conventions de groupe s'appliquent dans les trois grandes entreprises E.ON, RWE et Vattenfall. Elles ont été signées entre les groupes et les syndicats ver.di et IG BCE (mines, chimie, énergie) ainsi que, dans certains cas, IG Metall, qui ont constitué les alliances de négociation ad hoc.

Le plus grand groupe énergétique ayant son siège en Allemagne, E.ON, pratiquait jusqu'en 2007 la semaine de 36 heures. Depuis quelque temps, la direction tente d'imposer une nouvelle disposition allongeant la durée du travail et de suivre la tendance à l'œuvre dans le secteur. Les salariés d'E.ON perçoivent un salaire modulé sur la base de critères extrêmement divers :

- il est d'abord composé d'une rétribution de base, intégrant une composante liée aux qualifications et à l'expérience ;

- s'ajoute ensuite une composante liée à la performance, selon le niveau de réalisation des objectifs correspondant à certaines tâches ;

- les collaborateurs perçoivent en outre une rémunération variable, liée aux résultats, sur la base du ROCE (retour sur capital employé).

Une fois par an, il est par ailleurs proposé aux salariés d'E.ON d'acquérir des actions E.ON à des prix préférentiels. Depuis 2000, près de 2 millions d'actions E.ON ont été cédées aux salariés dans le cadre du paquet d'actions réservées 
aux collaborateurs (Rapport d'activité E.ON 2006, p. 83). Avec la tendance à la hausse de l'action dans les cotations en bourse, les salariés devraient réaliser un revenu supplémentaire non négligeable en cas de cession de leurs actions.

La taille d'un fournisseur d'énergie ne garantit toutefois pas la hausse des revenus et l'amélioration des conditions de travail. Après des négociations difficiles, qui ont duré plusieurs années, RWE s'est récemment entendu avec les syndicats ver.di et IG BCE sur une nouvelle structure de rémunération pour le groupe. Cette convention regroupe d'anciens accords avec d'autres applicables aux sociétés dont RWE a fait l'acquisition récemment. Cet ajustement résulte en un «système de rémunération à deux vitesses » selon que les salariés sont en poste depuis longtemps ou ont été recrutés plus récemment. Les salariés recrutés avant juin 2006 bénéficient en effet d'une disposition sur les droits acquis. De ce fait, les personnels embauchés après juin 2006 touchent environ $30 \%$ de moins que les salariés employés depuis plus longtemps (Brandt/Schulten, 2007, p. 86). Pour porter une appréciation définitive sur la nouvelle convention, il faut toutefois prendre en considération le fait que ce dispositif a pour objectif d'éviter des externalisations supplémentaires et donc des suppressions d'emplois.

Les salariés de RWE peuvent eux aussi acquérir chaque année des actions du personnel à des conditions préférentielles. En 2007, 22062 salariés ont ainsi acheté 457000 actions, soit près de 21 actions par personne. Les salariés de RWE détiennent au total environ $2 \%$ du capital souscrit (rapport d'activité RWE 2007, p. 129).

En 2006, les négociations sur une convention collective de groupe pour Vattenfall Europe ont abouti. Le rapport d'activité 2006 indique à ce propos : «Vattenfall Europe et les syndicats IG BCE, ver.di et IG Metall ont homogénéisé les conditions d'emploi jusque-là très diverses des entreprises clés dans une convention collective commune, réduisant ainsi les disparités liées aux sites » (ibid., p. 37). Des parts de rémunération liées aux performances et aux résultats ont également été introduites chez Vattenfall Europe. L'accord signé exclut les licenciements économiques jusqu'en 2012.

Ces caractérisations rapides des structures conventionnelles montrent déjà l'existence de différences matérielles considérables entre les conventions collectives appliquées dans le secteur. Les écarts sont estimés à environ $40 \%$ du revenu annuel; en 2007, les durées de travail hebdomadaires étaient de 36 à 40 heures. La tendance est clairement à l'allongement du temps de travail, parfois sans compensation financière.

\section{Cogestion : une mission de plus en plus complexe}

Les évolutions décrites plus haut ont aussi fortement marqué et influencé le travail des comités d'entreprise au cours de la dernière décennie. Les attentes étaient fortes à l'égard des codécisionnaires dans ce contexte d'adaptations multiples. Pour faire entendre les intérêts des salariés, sécuriser leurs emplois si possible et 
leur éviter des préjudices économiques, les conseils d'entreprises ont été confrontés à beaucoup de situations et de sollicitations nouvelles. Il leur a fallu développer des initiatives globales sur les projets stratégiques des dirigeants et sur les mesures organisationnelles qui en découlaient. Il a fallu négocier et accepter des compromis. La pression exercée par la direction pour imposer les décisions stratégiques et réaliser des économies le plus vite possible - afin d'empêcher des baisses de bénéfices - a entraîné une augmentation sensible des conflits avec les représentants des salariés. La participation du personnel aux superprofits a été limitée ou empêchée par les équipes dirigeantes. «Alors que, dans le secteur électrique, il était auparavant tout simplement impensable que des comités d'entreprise fassent appel à des organismes de conciliation ou à des tribunaux du travail pour régler des conflits, les cas où il a fallu recourir aux possibilités définies dans la loi sur l'organisation des entreprises (Betriebsverfassungsgesetz) se sont multipliés ces dernières années, y compris dans ce secteur » (Bergelin, 2008, p. 28).

La cogestion, pratiquée depuis toujours dans les entreprises électriques allemandes, n'est pour autant pas encore fondamentalement remise en question ; mais on peut incontestablement voir parmi les nouvelles conditions une complexification des possibilités de cogestion. Parallèlement, les compétences des comités d'entreprise et des salariés en matière de résolution des problèmes sont aujourd'hui plus importantes que jamais. Les directions de nombreuses structures intègrent encore ces ressources productives dans leurs processus quotidiens, afin de préserver ou même d'améliorer la position de leurs entreprises sur le marché. Mais le pouvoir de négociation s'est sensiblement déplacé au profit des employeurs. Les comités d'entreprise (et les syndicats qui les soutiennent) doivent faire des concessions en matière de rémunération et de temps de travail. En raison des politiques de ressources humaines et de considérations sociales, le capital accorde encore aux codécisionnaires l'exclusion des licenciements économiques.

Mais, dans le même temps, sur les questions relatives à l'entreprise, on observe des changements dans les conseils de surveillance en matière de cogestion. La recherche de résultats s'opère, à ce niveau également, de plus en plus souvent sans considération des conséquences à long terme que peuvent avoir les votes, en particulier sur la motivation des salariés. En d'autres termes, avant la libéralisation, le président du conseil de surveillance n'utilisait généralement pas sa voix supplémentaire pour trancher les questions litigieuses lors d'un deuxième vote ; il recourait à la force des arguments face aux alternatives. Dans le secteur électrique allemand, cette époque semble révolue. Du point de vue de nombreux membres de conseils de surveillance, le droit de compter double la voix du président est maintenant tout aussi utilisé que dans d'autres secteurs.

Les changements culturels sont également observables dans les procédures de désignation des « directeurs du travail ${ }^{4} »($ Arbeitsdirektoren) dans le secteur

\footnotetext{
${ }^{4}$ Dans le cadre de la cogestion paritaire des entreprises, l'Arbeitsdirektor est un membre du conseil d'administration de l'entreprise chargé des ressources humaines et des questions sociales, NDT.
} 
de l'énergie (Bergelin, 2008). Dans le passé, ces représentants du personnel et des affaires sociales n'étaient pas nommés contre l'avis des représentants des salariés. Cette procédure débouchait souvent, en pratique, sur le «droit » des salariés non seulement de proposer un Arbeitsdirektor mais aussi de le choisir de manière unilatérale. Les adaptations culturelles ont, de même, largement modifié cette pratique. Aujourd'hui, les codécisionnaires parviennent de plus en plus rarement à imposer leurs propres candidats aux fonctions d'Arbeitsdirektoren au sein des conseils de surveillance.

En somme, les changements de conditions et de stratégies ont des répercussions fondamentales sur les cultures d'entreprises et les relations sociales en leur sein. Le contexte concurrentiel, avec la pression sur les coûts qu'il induit et l'obligation d'optimiser les processus, a mené à recourir davantage aux organismes de conciliation, dont les entreprises n'avaient besoin, avant la libéralisation du marché, que dans des cas absolument exceptionnels.

\section{Synthèse et perspectives}

L'évolution des relations entre capital et travail dans les entreprises du secteur électrique allemand après la libéralisation s'est clairement faite au détriment des salariés. Cela vaut autant pour les ratios de répartition de la valeur ajoutée que pour les conditions de travail, ou encore pour la cogestion. Dans une étude récente, $65 \%$ des conseils d'entreprise interrogés reconnaissaient que les conditions s'étaient dégradées ou fortement dégradées. Seuls $8 \%$ voyaient une amélioration à la suite de la libéralisation (Bontrup/Marquardt, 2010). Il est probable que les tensions continueront de s'accroître et les conditions de se détériorer. Mais de manière sans doute très différente selon les activités de création de valeur ajoutée. La grande question sera de savoir si la convention collective unique pourra être préservée ou si de nouvelles évolutions interviendront en la matière.

Pour les activités de production, on peut s'attendre, dans toutes les entreprises, à un développement des installations de production d'électricité verte ou de cogénération au cours de la prochaine décennie. Ce processus contraindra à revenir dans certains cas à des structures décentralisées, y compris dans les grands groupes. Cette évolution sera également accentuée par la nécessité d'accroître l'efficacité énergétique des prestations, y compris dans le secteur de l'énergie. Ce changement structurel s'accompagne de nouveaux défis en termes de management. Mais il entraîne aussi de nouveaux besoins de qualifications des personnels. Ces évolutions auront des répercussions sur les structures de rémunération, et donc sur la conscience des salariés.

Dans le domaine des activités de réseau, les effets du dispositif de régulation incitative se font parfois déjà sentir, avec une course aux potentiels de rationalisation et de réduction des coûts. Les spécialistes ne considèrent plus les activités de réseau à elles seules comme un modèle économique. Les fournis- 
seurs d'énergie, en particulier les petites et moyennes entreprises, sont par conséquent à l'affût de coopérations. Ces coopérations annoncées visent à générer des potentiels de rationalisation et de synergie en regroupant certains domaines d'activités, en supprimant des emplois et en économisant ainsi sur les dépenses de personnel. C'est la seule stratégie que les dirigeants de nombreuses sociétés et divisions de réseau semblent décidés à employer pour faire face aux nouveaux défis du processus de benchmarking. L'attention des employeurs se porte également sur l'allongement de la durée hebdomadaire du travail, la baisse des grilles de salaires et la réduction voire la suppression des primes spéciales prévues dans les conventions collectives. Ce contexte pourrait favoriser la mise en place d'un processus qui entrainerait l'émergence de structures conventionnelles hétérogènes dans les activités de production et les activités de réseau.

Les rémunérations basées sur les résultats sont habituelles au sein du service commercial et dans la distribution, c'est pourquoi le taux de syndicalisation y est généralement plus bas que dans les autres domaines d'activités. Mais la mise en place et le développement de postes qui n'étaient pas nécessaires dans le passé du fait des monopoles territoriaux, comme le marketing, la gestion des risques, le trading, le conseil en efficacité énergétique, auront aussi des conséquences sur la structure d'emploi et donc sur le pouvoir des syndicats et leurs capacités à s'imposer.

FACE À CES ÉVOLUTIONS et aux importantes disparités matérielles quant aux marges de la création de valeur ajoutée et dans les conventions collectives, les syndicats du secteur électrique restent confrontés à des défis majeurs. Le facteur décisif sera de savoir s'ils parviendront avec les salariés à instaurer une culture de la démocratie participative dans l'entreprise. Il reste à ce jour un immense chemin à parcourir en ce domaine (Bontrup/Marquardt, 2010a).

Traduction de Marie GRAVEY

\section{Indications bibliographiques}

Angenendt N., Müller G., StronziK M., Wissner M., « Stromerzeugung und Stromvertrieb - eine wettbewerbsökonomische Analyse », wik-Diskussionsbeiträge, $\mathrm{n}^{\circ} 297,2007$, p. [n.c.]

BERGElin S., «Energiewirtschaft », in BRANDt T., SCHUlten T., STERKel G., WiEdEMUTH J. (eds), Europa im Ausverkauf - Liberalisierung und Privatisierung öffentlicher Dienstleistungen und ihre Folgen für die Tarifpolitik, VSA-Verlag, Hambourg, 2008, p. 121-130

Bontrup H.-J., MARQUARDT R.-M., VOß W., « Liberalisierung in der Elektrizitätswirtschaft: Zuspitzung der Verteilungskonflikte », WSI-Mitteilungen, ${ }^{\circ}$ 4, 2008, p. 175-183

BontruP H.-J., MARQUARDT R.-M., Kritisches Handbuch der deutschen Elektrizitätswirtschaft, Branchenentwicklung, Unternehmensstrategien, Arbeitsbeziehungen, Edition Sigma, Berlin, 2010

BONTRUP H.-J., MARQUARDT R.-M., « Beschäftigungsbedingungen und Unternehmenskultur in der Elektrizitätswirtschaft », WSI-Mitteilungen, ${ }^{\circ}$ 6, 2010a, p. 291-298 
BOZEM K., Liberalisierung der Energiewirtschaft. Unternehmensstrategien im Umbruch, Energie \& Management, Herrsching, 2007

BRANDT T., SCHUlten T., Pique - Liberalisation and privatisation of public services and the impact on labour relations: a comparative view from six countries in the postal, hospital, local public transport and electricity sectors, Wirtschafts- und Sozialwissenschaftliches Institut (WSI), Fondation Hans Böckler, Düsseldorf, 2007

BUNDESMINISTERIUM FÜR UMWELT (BMU), Erfahrungsbericht 2007 zum Erneuerbaren-Energie-Gesetz gemäß § 20 EEG - BMU-Entwurf-Kurzfassung vom 05.07.2007, BMU, Berlin, 2007

BUNDESREGIERUNG, Verordnung über die Anreizregulierung der Energieversorgungsnetze vom 29. Oktober 2007 (ARegV), (BGB1. I S. 2529), 2007

Held C., THEOBAld C. (eds), Kommunale Wirtschaft im 21. Jahrhundert. Rahmenbedingungen, Strategien und Umsetzungen, VWEW Energieverlag, Francfort s/Main, 2007

JANSEN D., BARNekow S., STOLL U., «Innovationsstrategien von Stadtwerken - lokale Stromversorger zwischen Liberalisierungsdruck und Nachhaltigkeitszielen », FÖV Discussion Papers, n 41, 2007, p. [n.c.]

Parlement européen, Conseil de l'Union européenne, « Directive 96/92/CE du Parlement européen et du Conseil du 19 décembre 1996 concernant des règles communes pour le marché intérieur de l'électricité », Journal Officiel de l'Union européenne, n L 27/20, 31/01/1997

Parlement européen, Conseil de l'Union européenne, « Directive 2003/54/CE du Parlement européen et du Conseil du 26 juin 2003 concernant des règles communes pour le marché intérieur de l'électricité et abrogeant la directive 96/92/CE », Journal Officiel de l'Union européenne, L 176/37, 15/07/2003

Statistisches BundeSAMt, Produzierendes Gewerbe, Fachserie 4, Reihe 4.1: Beschäftigung, Umsatz, Investitionen und Kostenstruktur, Statistisches Bundesamt, Wiesbaden, années diverses

WAgner O., KRISTOF K., « Strategieoptionen kommunaler Energieversorger im Wettbewerb », Wuppertaler Papers, $\mathrm{n}^{\circ} 115,2001$, p. [n.c.]

WÜBBELS M., « Künftige Anforderungen an kommunale Energieversorgungsunternehmen und den VKU », in Held C., TheObald C., Kommunale Wirtschaft im 21. Jahrhundert. Rahmenbedingungen, Strategien und Umsetzungen, VWEW Energieverlag, Francfort s/Main, 2007, p. [n.c.]. 



\section{Le secteur des industries électriques et gazières en France. Evolution des relations sociales de la nationalisation à la privatisation}

\section{Bernard PARMANTIER}

Bernard Parmantier, Directeur des relations sociales au sein du groupe GDF SUEZ, revient dans sa contribution sur le " big bang " du secteur des industries électriques et gazières. La loi du 10 février 2000 a ainsi fait émerger l'idée de branche professionnelle, alors en pleine mutation structurelle (modification des systèmes de retraites, du régime maladie et des relations sociales au sein des entreprises). Au-delà s'impose désormais la notion de groupe, subséquemment à la fusion Gaz de France/Suez.

Le secteur des industries électriques et gazières (IEG) regroupe l'ensemble des entreprises exerçant des activités de production, transport et distribution d'électricité et de gaz. Il comprend environ 144 entreprises, de statuts juridiques divers (Sociétés Anonymes, Sociétés d'Economie Mixte, Régies, Sociétés d'Intérêt Collectif Agricole d'Electricité). Parmi ces entreprises, 117 comptent moins de 50 collaborateurs. Le secteur emploie 139600 salariés, pour 145000 retraités (droits directs et droits dérivés).

\section{D'EDF-GDF à la branche professionnelle}

De 1946 à février 2000

\section{L'histoire de l'après-Seconde Guerre mondiale}

Après la Seconde Guerre mondiale, le Général de Gaulle constitue un gouvernement issu des forces en présence dans la résistance, comprenant notamment un ministre communiste à l'Industrie, dont relevait le secteur du gaz et de l'électricité. Ce dernier fait dès lors l'objet d'une loi de nationalisation en 1946, assortie de l'adoption du statut national des IEG.

\section{Les fondements et éléments constitutifs du statut}

Le statut, élément indissociable de la loi de nationalisation du 8 avril 1946 (art. 47 de la loi), constitue un élément consubstantiel des missions de service public :

- car c'est un outil d'unification du personnel réparti sur tout le territoire qui permet de satisfaire les missions de service public sur l'ensemble du territoire ; 
- car il consacre le paritarisme comme outil de médiation qui permet la recherche de compromis face aux missions de service public confiées aux entreprises du secteur, l'objectif étant de concilier développement industriel et aspects sociaux.

\section{Le statut, ses circulaires, et leurs évolutions}

Au fil des années, le statut du personnel est complété par un corps de règles spécifiques communes au personnel des entreprises relevant des IEG : plus de 1000 circulaires d'application précisent les dispositions du statut et recouvrent tous ses champs. Cette variété de textes faisant force de loi a rendu ces derniers difficilement lisibles.

\section{De 1946 à février 2000 : synthèse}

Pendant plus de soixante ans, le dialogue social est centralisé au niveau des directions générales des sociétés EDF et Gaz de France. Durant cette période, les accords de branche n'existent pas. Les décisions sont prises par les deux grands établissements publics EDF et Gaz de France. Le ministère de l'Industrie procède par la suite à l'extension de tout ou partie des textes aux Entreprises Non Nationalisées (ENN), certains textes ne s'appliquant pas aux ENN. Le statut du personnel des IEG reste stable.

Par ailleurs, les pouvoirs publics demeurent très présents dans le champ social. Dans le cadre du paritarisme, la CGT occupe une place centrale et les Directions ou les pouvoirs publics prennent peu de décisions sociales sans son approbation implicite. Toutefois, les relations sociales pouvent paraître très conflictuelles vues de l'extérieur. Ainsi tout se joue sur deux niveaux, avec au sommet une concertation avec les appareils syndicaux, appareils politiques et responsables d'entreprises et, sur le terrain, un contexte plus conflictuel.

$$
\text { Le « big bang » de la branche }
$$

\section{Le « big bang » de la branche}

La loi du 10 février 2000, relative à la modernisation et au développement du service public de l'électricité, va poser les conditions de l'ouverture du marché. De même qu'en 1946, cette loi, qui a d'abord une portée industrielle d'organisation de la concurrence, revêt une dimension sociale à l'article 45 , qui va bouleverser les relations sociales. L'article précise que «des accords professionnels peuvent compléter, [...] les dispositions statutaires ou en déterminer les modalités d'application dans les limites fixées au statut», appelant pour la première fois à la création d' « organisations d'employeurs les plus représentatives dans la branche». 


\section{Les effets du « big bang »}

Deux organismes patronaux sont mis en place : l'Union Française de l'Electricité (UFE) et l'Union Nationale des Employeurs des Industries Gazières (UNEmIG). Les accords collectifs sont signés non plus uniquement au niveau d'EDF, mais par les représentants des employeurs de la branche. Dès lors, les accords de branche sont appliqués automatiquement aux entreprises adhérentes de l'UFE et de l'UNEmIG et étendus aux autres entreprises après un passage en Commission Supérieure Nationale du Personnel.

Au niveau de la branche professionnelle, les employeurs définissent dans un premier temps des positions de négociation. Sont alors réunis des responsables issus d'EDF, Gaz de France, Suez (qui possédait quelques entités dans le secteur de l'électricité et du gaz), ainsi que des petites et moyennes entreprises. Le dialogue inclut par la suite les organisations syndicales au niveau de la branche dans le cadre de commissions paritaires de branche, qui se tiennent une à deux fois par mois. Ainsi, aussi bien au niveau patronal que syndical, les petites entreprises peuvent exprimer leurs points de vue et elles se sont d'ailleurs regroupées au sein d'organismes patronaux distincts.

Suite à l'adoption de la loi de 2000, le statut du personnel des IEG évolue (réforme des retraites, réforme du régime complémentaire maladie, négociation salariale...). Les pouvoirs publics se désengagent progressivement du dialogue social. Les relations sociales se sont déplacées au niveau de la branche professionnelle. On assiste dès lors à un vrai dialogue social, avec la signature d'un bon nombre d'accords.

\section{Les principales évolutions structurelles}

Des évolutions de fond sont venues bouleverser le mode de relations et de dialogue avec les organisations syndicales: elles concernent les systèmes de retraite, le régime maladie et les relations sociales au sein des entreprises.

\section{Evolution du régime de retraite}

Quelques chiffres permettent d'appréhender la problématique: les IEG comptent 142520 actifs cotisants dans 138 entreprises pour 145341 pensionnés (dont pensions de réversion). L'âge moyen (réel) de départ à la retraite est situé à 56 ans. Le montant de la pension s'élève à près de $70 \%$ du dernier salaire hors primes, pour un total de 3,3 milliards $€$ de retraites versées.

Un problème démographique se pose : en 1983, on comptait 2,17 actifs pour 1 inactif, en 2006, 1,37 actif pour 1 inactif, pour arriver en 2015 à un ratio de $1: 1$. Un second problème découle de la modification de la structure des entreprises : en passant du statut d'établissements publics à celui de sociétés anonymes, EDF et Gaz de France doivent désormais appliquer les normes comp- 
tables internationales (IFRS). Celles-ci imposent de provisionner les engagements des entreprises pour le futur, notamment les avantages consentis aux inactifs par l'entreprise. Dans ce système de retraites «maison », les provisions se montent à 35 milliards $€$. Il était par conséquent impossible, notamment pour EDF qui représente plus de $80 \%$ de la branche professionnelle, d'intégrer cette somme. Les normes internationales de comptabilité ont donc incité les entreprises à modifier la structure de financement des retraites, avec un adossement sur les régimes de droit commun. Auparavant, le système appliqué aux IEG était isolé par rapport au régime de base de la sécurité sociale et aux régimes complémentaires français. On va passer à un système à trois échelons : le régime de base de la sécurité sociale, un régime assis sur la partie complémentaire et un troisième niveau financé par les entreprises de la branche.

Avant la loi de 2000, la gestion du régime était le fait d'un service d'EDF. En raison de l'évolution du secteur des IEG, il n'était pas possible de poursuivre ce mode de gestion en interne. Une Caisse Nationale des IEG (CNIEG) a été mise en place pour la gestion des pensions. Il s'agit d'un organisme de sécurité sociale à gestion paritaire, destiné à maintenir les modalités du régime spécial et à sécuriser son financement. Ce régime est adossé au régime général de base (CNAV) et au régime de retraite complémentaire (ARRCO-AGIRC), avec versement d'une soulte de 7,6 milliards $€$. La Contribution Tarifaire d'Acheminement (CTA), créé par ailleurs pour le financement des retraites du secteur régulé, n'induit pas de surcoût pour les clients.

\section{Evolution du régime spécial maladie}

Dans la branche des IEG, tous les remboursements maladie étaient gérés par les caisses mutuelles d'action sociale (CMCAS) sous la responsabilité des organisations syndicales. Or dans les années 2000, on s'aperçoit que l'équilibre financier de ce système est précaire, le déficit cumulé se montant à plusieurs dizaines de millions $€$. Les frais de gestion sont trop élevés du fait d'une gouvernance ne permettant pas un pilotage efficace : la gestion est répartie entre 107 caisses locales et un organisme national dont les pouvoirs sont limités. Les systèmes d'information se révèlent défaillants à brève échéance, du fait des évolutions récentes du régime général.

La problématique liée aux normes comptables apparaît également car ce système fonctionne à la fois pour les actifs et les inactifs. Bien que le système soit géré par les partenaires sociaux, les entreprises de la branche auraient dû provisionner les avantages liés aux inactifs. Un organisme de sécurité sociale de droit privé est mis en place à l'échelle nationale, la CAMIEG. Centralisé, il est chargé uniquement de la gestion du régime spécial d'assurance maladie-maternité des IEG (actifs et inactifs). Il est séparé juridiquement des Caisses locales, en charge des œuvres sociales (qui restent gérées par les organisations syndicales). Son conseil d'administration est composé de représentants des ouvrants 
droit. La CAMIEG est par ailleurs dotée d'un directeur et d'un agent comptable, nommés par arrêté. Compte tenu du fait que cette caisse gère à la fois les actifs et les inactifs, les employeurs ne sont pas présents dans cet organisme de sécurité sociale. Ils le sont néanmoins à travers une commission nationale de suivi, instance consultative et paritaire externe, qui permet de préparer les décisions.

La structure de financement du régime est modifiée, via notamment l'arrêt de la cotisation des employeurs vers la section des inactifs. Il existe dès lors une séparation forte entre le régime spécial maladie couvrant les actifs, financé par les entreprises et les salariés, et celui couvrant les inactifs, financé par ces derniers, et dans une moindre mesure, par une cotisation de solidarité payée par les salariés actifs.

Pour les organisations syndicales, la réforme du régime spécial maladie constitue une évolution plus forte que celle du régime des retraites : la maîtrise du système de santé qu'elles géraient jusqu'alors diminue fortement et elles ne l'exercent plus qu'à travers leur présence au conseil d'administration.

\section{Evolution des Institutions Représentatives du Personnel (IRP)}

Avant la réforme, les élections de représentativité organisées tous les trois ans jouaient un rôle central en matière de relations professionnelles dans les IEG. Elles déterminaient le poids de chaque organisation syndicale, ainsi que le nombre de sièges dans les instances au niveau local.

Contrairement au droit commun, la désignation syndicale primait sur l'élection. En cas de désaccord avec un de ses représentants dans une instance, l'organisation syndicale avait la possibilité de le destituer du jour au lendemain. Dans le droit commun, un membre d'un comité d'entreprise sur une liste syndicale peut conserver ce mandat, même s'il choisit de changer de syndicat après son élection. Il ne peut pas être destitué par l'organisation syndicale.

Par ailleurs, les instances de représentation du personnel étaient non conformes aux dispositions du Code du travail. Il n'existait pas de comité d'entreprise mais un Comité Mixte à la Production (CMP) et un Comité Supérieur Consultatif des CMP. La mission des délégués du personnel était réalisée par une Commission Supérieure du Personnel (CNSP), chargée notamment de gérer les réclamations des cadres (traitées au niveau national) et, au niveau local, par une Commission Secondaire du Personnel pour les personnels «non cadre ».

En 2007, après un an et demi de concertation au niveau de la branche professionnelle, le gouvernement français a adopté deux décrets destinés à modifier le système de représentation du personnel dans la branche. Le premier décret stipule que les entreprises de la branche professionnelle doivent appliquer les dispositions du Code du travail relatives aux délégués du personnel, aux comités d'entreprise, aux CHSCT, notamment celles concernant leurs élections et leurs attributions. Les premières élections ont eu lieu en octobre 2007, ces nouvelles instances de représentation du personnel devant être installées au plus 
tard au 31 décembre 2007. Les secondes élections se sont tenues fin 2010. Ainsi ont été créés :

- des comités d'entreprise (en lieu et place des Comités Mixtes à la Production) ;

- des délégués du personnel.

Quelques spécificités demeurent : en plus de ces structures classiques, les commissions secondaires continuent d'exister, mais de manière plus décentralisée : les réclamations pour les personnels «cadre » et «non cadre » sont désormais gérées sans distinction au niveau des entreprises. Un deuxième décret en Conseil d'Etat conserve une dérogation par rapport au Code du travail : les œuvres sociales ne sont pas gérées par les comités d'entreprises, mais par une Caisse Centrale des Activités Sociales (CCAS) en dehors des entreprises, financée par 1\% des recettes du gaz et de l'électricité. L'évolution des instances représentatives du personnel est très importante dans le mode de fonctionnement des organisations syndicales. Les organisations syndicales doivent se concentrer davantage sur les enjeux locaux et moins sur les problématiques politiques.

\section{De la branche professionnelle à la notion de groupe}

\section{La fusion entre Gaz de France et Suez}

L'évolution des relations sociales due à l'ouverture du marché est assez considérable. Elle va se poursuivre avec la fusion entre Gaz de France et Suez, initiée deux ans avant sa réalisation effective. Le projet de fusion a fait l'objet d'une première annonce en février 2006 par Dominique de Villepin. Y ont fait suite un certain nombre de conflits et de procès, de sorte que le projet s'est retrouvé bloqué à la fin de l'année 2006. Le processus a été relancé après les élections présidentielles, lors du deuxième semestre 2007, pour aboutir le 22 juillet 2008 avec la création de GDF SUEZ. GDF SUEZ est présent sur l'ensemble de la chaîne de l'énergie, en électricité et en gaz naturel, de l'amont à l'aval :

- production, achat et commercialisation de gaz naturel et d'électricité ;

- transport, distribution, gestion et développement de grandes infrastructures de gaz naturel et d'électricité (réseaux de transport et de distribution, sites de stockage, terminaux méthaniers) ;

- conception et commercialisation des services énergétiques et de services liés à l'environnement (Lyonnaise des Eaux, Sita...).

\section{Chiffre d'affaires, effectifs et capacités par région}

Le groupe a réalisé en 2009 un chiffre d'affaires mondial de 79,9 milliards $€$. Il employait 200650 collaborateurs au 31 décembre 2009. En Europe, GDF SUEZ emploie 180000 salariés pour un chiffre d'affaires de 68,6 milliards $€$. 


\section{De la notion de branche à la notion de groupe}

La fusion entre Gaz de France et Suez va avoir un autre impact sur les relations sociales. Le groupe GDF SUEZ en France compte 106000 salariés, dont 29000 seulement travaillent dans le secteur de l'énergie. Le secteur des services à l'énergie se classe en première position avec un peu moins de 41000 salariés, suivi par l'environnement avec 33000 salariés. Par conséquent, les sociétés du groupe GDF SUEZ, très éclaté en structures juridiques et diversifié en termes d'activités, appliquent plusieurs conventions collectives. Néanmoins, si les salariés des IEG sont minoritaires, les leaders syndicaux sont pour la plupart issus de la branche des IEG, en raison du poids important des moyens des organisations syndicales dans cette branche.

Ce n'était pas le cas du groupe Suez auparavant, qui n'avait pas la même formalisation des moyens aux organisations syndicales. Dès lors, quand vont se constituer des équipes de négociation pour discuter à l'échelle du groupe, le poids des leaders syndicaux issus de Gaz de France sera le plus important en nombre et en compétences notamment car ils s'étaient spécialisés sur des sujets tels que la retraite, la maladie ou encore l'épargne salariale.

Un aspect nouveau pour les syndicats issus de Gaz de France va jouer dans les relations sociales : la notion de groupe par rapport à la notion de branche. Jusqu'à présent, les syndicats de Gaz de France appartenaient à la branche des IEG. Malgré une décentralisation progressive vers le niveau des entreprises, il existait une cohérence au niveau de la branche professionnelle. Après la fusion vont être abordés des sujets transversaux à l'échelle du groupe, concernant les entreprises du groupe, qu'elles appartiennent aux IEG ou aux autres secteurs d'activité. Les représentants syndicaux «historiques » de la branche des IEG vont avoir des difficultés à appréhender :

- des discussions par entreprise et non plus par branche ;

- des discussions groupe avec une diversité de situations et de métiers ;

- des sujets abordés au niveau du groupe qui sont aussi abordés au niveau de la branche professionnelle (seniors, risques psycho-sociaux, pénibilité...).

Le sujet de la mobilité illustre parfaitement cette problématique : logiquement dans un même groupe, la mobilité est prônée au sein des entreprises du groupe. Mais le secteur des IEG a aussi organisé sa mobilité au sein des entreprises de la branche.

Cette continuité est désormais souhaitée au sein de l'ensemble du groupe GDF SUEZ, ce qui se «télescope » avec la mobilité au sein de la branche des IEG.

Ces changements viennent renforcer la tendance à la décentralisation de la négociation au niveau des entités juridiques de plus en plus nombreuses dans la branche des IEG. 
L'ÉVOLUTION PROFONDE DE LA BRANCHE DES IEG s'est produite dans un laps de temps relativement restreint (moins de 10 ans) alors qu'il y avait eu une grande stabilité entre 1946 et 2000. Des changements ont été introduits dans le contexte concurrentiel, législatif (instances de représentation du personnel), normatif (IFRS) et juridique. Les mutations en termes de relations sociales et de conditions de travail se sont traduites par un nombre (relativement) réduit de conflits sociaux, en raison de la qualité du dialogue social au niveau de la branche professionnelle. La tradition de concertation au sommet avec les organisations syndicales a perduré et a permis que ces évolutions se fassent avec peu de conflits sociaux, au regard de l'évolution réelle des relations sociales du secteur des IEG. 


\title{
Relations sociales et gestion des ressources humaines à la Deutsche Bahn AG dans le contexte de l'ouverture à la concurrence
}

\author{
Christophe GARDAVAUD
}

\begin{abstract}
Responsable de la stratégie réglementaire Europe à la Deutsche Bahn AG, Christophe Gardavaud explique les modalités de la réforme ferroviaire allemande de 1993 pour en dresser ensuite le bilan, au regard des principaux objectifs visés (allègement de la dette publique et revitalisation du transport ferroviaire). II décrit enfin le processus d'adaptation de la gestion du personnel à un contexte concurrentiel pour en souligner l'efficacité, notamment dans le secteur du transport ferroviaire régional.
\end{abstract}

Si la dimension historique de l'unification allemande en 1990 et l'accélération du processus d'élargissement de l'Union européenne qu'elle a permise ont largement marqué les esprits, moins connues sont les gageures quasi sans précédent que cet événement a emporté pour la conduite des affaires publiques. La réunion de deux appareils d'Etat nécessitait en effet d'harmoniser, d'homogénéiser mais aussi de rationaliser les structures.

Parmi elles, l'armée et les chemins de fer constituaient deux défis majeurs pour la nouvelle République fédérale d'Allemagne. Il est d'usage, à ce propos, de citer l'ancien chancelier Helmut Schmidt qui considérait, qu'en l'état, l'Allemagne ne pouvait continuer à financer qu'un seul des deux ${ }^{1}$.

A cette époque, la situation des chemins de fer est effectivement précaire. Deux compagnies ferroviaires coexistent encore: à l'ouest la Deutsche Bundesbahn, créée le 7 septembre 1949, et à l'est, la Deutsche Reichsbahn, héritière de la compagnie nationale. Leurs dettes cumulées s'élèvent à quelque 66 milliards de marks (soit 33 milliards $€$ environ) et, en l'absence de réforme structurelle majeure, sont appelées à augmenter continuellement.

En 1990, les deux entreprises emploient à elles deux environ 489000 personnes : la Reichsbahn, 253 000, et la Bundesbahn 236 000. Ces derniers se partagent entre personnels ayant un statut de fonctionnaire et salariés de droit commun.

Les deux entreprises ont un mode de fonctionnement inefficient et bureaucratique. Il faut ainsi plus d'employés côté est-allemand pour faire circuler moitié moins de trains sur un réseau deux fois plus petit qu'en Allemagne de l'ouest. Côté ouest-allemand, malgré un plan de relance de la compétitivité lancé dans les années 1980 et baptisé DB $90^{2}$, la Bundesbahn n'atteindra jamais les objectifs de hausse de

\footnotetext{
${ }^{1}$ «Deutschland könne sich entweder die Bundeswehr oder die Bundesbahn leisten ».

${ }^{2}$ La faible productivité et compétitivité de l'entreprise ferroviaire tenait à sa vocation administrative loin des préoccupations d'une gestion économique et entrepreneuriale dynamique. Certes l'article $28 \S 1$ de la loi sur les chemins de fer prévoyait que la Bundesbahn devait être gérée comme toute autre entreprise, mais le même article insistait particulièrement sur les sujétions de service public pesant sur elle. La loi aménageait en outre
} 
la productivité de $40 \%$ et de réduction des coûts - notamment de personnel - de $25 \%$ à $30 \%$ qu'elle s'était fixée.

Avant même la réunification, la République fédérale d'Allemagne savait qu'une restructuration s'imposait si l'on voulait éviter la faillite du système ferroviaire. La prise de conscience des enjeux environnementaux et le besoin de se doter d'infrastructures de transports en commun performants pour desservir les nombreuses agglomérations jouent aussi en faveur du changement. Comme partout à cette époque, les décideurs politiques pensent trouver la solution dans une transformation du système combinant dérégulation et modification législative permettant à l'entreprise ferroviaire d'adopter des règles de gestion basées sur les coûts et les résultats économiques.

La réunification va rendre cette restructuration inéluctable. Le 2 décembre 1993, une révision de la Loi fondamentale et un paquet de lois ouvrant la voie à une grande réforme ferroviaire sont adoptés par une écrasante majorité (559 voix pour, 12 contre, 4 abstentions).

\section{La réforme ferroviaire (décembre 1993)}

Les bases de la réforme sont posées par une révision de la Loi fondamentale, d'une part, et le vote de six lois distinctes regroupées dans un document unique remodelant le paysage ferroviaire ${ }^{3}$. Citons-en les éléments les plus importants :

- la Deutsche Bahn est créée, constituée en société par actions ${ }^{4}$ et regroupant les deux anciennes compagnies nationales en son sein ;

- la nouvelle entreprise adopte des règles de droit privé et gère ses activités selon des considérations de marché. La Deutsche Bahn est ainsi formellement libérée de ses obligations de service public et l'objectif de quête du profit est clairement affirmé. D'emblée, la possibilité d'une cession par l'Etat de tout ou partie du capital est envisagée ; les activités de gestion et d'exploitation de l'infrastructure sont néanmoins exclues de ce projet ;

- l'Etat demeure seul garant du bien public en matière de chemins de fer. Ceci doit se matérialiser par le financement régulier des investissements dans le réseau ferroviaire ;

- une structure de défaisance, le «Bundeseisenbahnvermögen » (BEV) est créée. Elle reprend la totalité des dettes des deux entreprises (environ 66 milliards de marks) et perçoit en contrepartie les biens immobiliers

une forte capacité d'influence au pouvoir politique sur les décisions prises par l'entreprise. Dans ce contexte, la Bundesbahn ne pouvait pas faire jeu égal avec ses concurrents privés.

${ }^{3}$ La loi dite Eisenbahnneuordnungsgesetz (ENeuOG) regroupe plusieurs lois présentées chacune dans un article particulier de l'ENeuOG dont notammenat à l'art.1, loi d'unification et de restructuration des deux entreprises ferroviaires ; art. 2, loi portant création d'une société par actions, la Deutsche Bahn ; art. 3, loi relative à l'administration des chemins de fer par l'Etat fédéral ; art. 4, loi de régionalisation des transports en commun de proximité ; art. 5, loi ferroviaire générale.

${ }^{4}$ Deutsche Bahn Gründungsgesetz, 27 décembre 1993 (BGB1. I S. 2378, 2386; 1994 I S. 2439), dernièrement modifié par l'article 307 de la directive du 31 octobre 2006 (BGB1. I S. 2407). 
non indispensables à l'exploitation ferroviaire ${ }^{5}$ (en particulier de nombreux bâtiments de représentation en ex-Allemagne de l'Est). Le BEV dépend du ministère des Transports et siège à Bonn ;

- les transports de proximité sont régionalisés : les Länder deviennent des autorités organisatrices de transport, et obtiennent, chaque année, de l'Etat fédéral une dotation de financement pour le lancement de programmes d'investissement et la conclusion de nouveaux contrats. La mise en œuvre de la régionalisation prend effet le $1^{\mathrm{er}}$ janvier 1996 ;

- le réseau ferré national est enfin entièrement ouvert à la concurrence, que ce soit pour effectuer du transport ferroviaire de marchandises que pour du transport ferroviaire de voyageurs. Les concurrents apparaissent rapidement soit après avoir remporté un appel d'offres - c'est le cas du transport régional (cf. infra) où la concurrence se fait «pour » un marché donné, administré par une autorité organisatrice de transport - soit par volonté d'exploiter un nouveau marché - c'est le cas du transport de marchandises ou des dessertes voyageurs grandes lignes où la concurrence se fait «sur » le marché.

Le grand mérite de cette réforme est de clarifier la répartition des rôles entre la force publique et l'entreprise ferroviaire. Si la première exécute les tâches administratives -gestion de la dette et des personnels fonctionnaires, contrôle du respect du droit applicable et de la sécurité des transports - il incombe à l'entreprise ferroviaire, d'une part, et à l'entreprise gestionnaire du réseau ferroviaire, d'autre part, respectivement d'opérer les dessertes ferroviaires et d'exploiter l'infrastructure. La réforme connaît cinq ans plus tard, en 1999, une deuxième étape qui conduit à une modification de la structure de la DB AG. Celle-ci est constituée en holding et cinq filiales - DB Reise \& Touristik $\mathrm{AG}^{6}$, DB Regio AG, DB Cargo $\mathrm{AG}^{7}, \mathrm{DB}$ Netz AG, DB Station \& Service $\mathrm{GmbH}$. Les actifs sont à cette occasion répartis entre les différentes filiales.

\section{Bilan de la réforme : un succès partagé}

Il est important de mesurer le succès de la réforme à l'aune des objectifs initiaux.

L'objectif d'allègement de la charge publique tout d'abord est pleinement atteint. Avant l'adoption de la réforme, plusieurs scénarios avaient été envisagés. D'après ces estimations, une prorogation de l'état existant aurait conduit à alourdir la charge budgétaire consacrée au transport ferroviaire de $41 \%$ en sept ans, jusqu'à atteindre 32,3 milliards $€$ en 2001 . En votant la réforme, le législateur espérait maintenir le niveau des dépenses à niveau constant (hors effets de l'inflation) et ainsi limiter la dépense publique à 22,2 milliards $€$ en 2001 (soit $31 \%$ de moins que dans le premier scénario).

\footnotetext{
${ }^{5}$ „die nicht für den Eisenbahnbetrieb notwendigen Immobilien“.

${ }^{6}$ Aujourd'hui DB Fernverkehr AG.

${ }^{7}$ Aujourd'hui DB Schenker Rail AG.
} 
Les résultats enregistrés par la réforme dépassent les meilleurs pronostics : en 2001, le niveau de la dépense publique allouée au transport ferroviaire est abaissé à 16,1 milliards $€$, divisant de moitié la charge publique qui aurait été consacrée au ferroviaire sans intervention du législateur. Cette tendance s'est depuis poursuivie : en 2008, quelque 15,3 milliards $€$ ont été consacrés au transport ferroviaire.

Par ailleurs, le second objectif de la réforme que constituait la revitalisation du transport ferroviaire est lui aussi atteint. L'activité a fortement augmenté tant dans le domaine du transport de marchandises ( $+52 \%$ en 15 ans) que dans celui du transport de voyageurs $(+25 \%$ sur la même période). La part de marché intermodale du transport ferroviaire s'est elle aussi renforcée et consolidée $(+1$ point entre 1994 et 2008 dans le transport de marchandises, $+2,5$ points dans le transport voyageurs). Le nombre de concurrents n'a cessé de croître jusqu'à atteindre 323 entreprises ferroviaires actives sur le réseau ferré allemand en 2010. Enfin, la productivité de la compagnie nationale croît de $280 \%$ entre 1994 et 2008, dépassant les espérances des concepteurs du plan DB 90 d'à l'époque.

Le transport régional de voyageurs constitue sans doute la meilleure synthèse de ce succès. Les autorités organisatrices de transport observent ainsi une baisse de $20 \%$ à $30 \%$ de leurs coûts pour un taux de satisfaction et une qualité bien supérieurs (cf. infra).

Enfin, le BEV, bien qu'héritant de la dette colossale des deux anciennes compagnies, a pu céder la grande partie des biens immobiliers qui lui avaient été transférés et réaliser au passage quelques plus-values. De plus, le nombre de personnels fonctionnaires décroissant, les charges de personnel pesant sur le BEV diminuent régulièrement, tout comme la dette qui s'amortit elle aussi progressivement.

\section{Les répercussions de la réforme ferroviaire en matière de gestion des ressources humaines}

A la fusion des deux anciennes entreprises, en 1994, 220000 salariés de la Deutsche Bundesbahn et 160000 salariés de la Deutsche Reichsbahn rejoignent la Deutsche Bahn AG. La réforme prévoit de reprendre l'ensemble de ces salariés dans un premier temps et de continuer à les employer à des conditions financières identiques. Ceci constituait une véritable gageure tant les systèmes sociaux regroupés étaient différents. Deux éléments en particulier peuvent alors constituer les écueils mettant en péril cette fusion : la reprise des personnels fonctionnaires de la Deutsche Bundesbahn tout d'abord, et la nécessaire restructuration de l'entreprise ensuite.

\section{Les règles de reprise des personnels fonctionnaires de la Deutsche Bundesbahn}

Trois catégories d'employés coexistent au moment de la réunification, la difficulté étant de les intégrer harmonieusement au sein de la Deutsche Bahn AG, une entreprise de droit privé : 
- les employés de la Deutsche Reichsbahn, anciens employés d'une entreprise publique du bloc communiste. Ceux-ci ne peuvent pas être considérés comme des fonctionnaires à proprement parler et ne répondent pas entièrement à la définition d'un salarié de droit commun selon des standards occidentaux. Pour autant, ils sont plus proches de cette seconde catégorie ;

- les employés de droit commun de la Deutsche Bundesbahn ;

- les employés de la Deutsche Bundesbahn, possédant le statut de fonctionnaire.

La réforme ferroviaire n'autorise plus que la conclusion de contrat de travail de droit privé entre l'entreprise et ses salariés. Les anciens contrats sont toutefois repris par la Deutsche Bahn. Ceci a pour effet de réduire à deux les catégories de personnel dans l'entreprise : les fonctionnaires issus de la Bundesbahn et les autres.

La réforme introduit cependant une subtilité complémentaire en souhaitant que la Deutsche Bahn ne gère que des salariés de droit commun. La solution à l'équation est une nouvelle fois apportée par le Bundeseisenbahnvermögen (BEV).

Dans la nouvelle organisation, les personnels au statut sont officiellement rattachés au BEV, leur autorité de tutelle. Ce dernier les met à la disposition de la Deutsche Bahn qui constitue leur autorité fonctionnelle. Celle-ci rémunère tous ses salariés selon les mêmes grilles de rémunération, valables pour tout son personnel, en fonction d'un poste et d'une classe donnés. La particularité des personnels au statut est qu'ils continuent de toucher un traitement que le BEV et non la Deutsche Bahn leur verse à la fin du mois. Conformément aux dispositions du droit public, ce traitement est fonction du grade et de l'échelon de l'employé. L'écart qui peut exister entre la rémunération et le traitement (positif ou négatif) est absorbé par le $\mathrm{BEV}^{8}$.

Le même système s'applique aux règles de couverture sociale et de prévoyance. En 1994 quelque 430000 personnes (salariées ou retraitées) sont concernées par ces mesures. La loi portant création de la Deutsche Bahn AG (Deutsche Bahn Gründungsgesetz - DBGrG) expose à l'article 14 alinéa $2^{9}$ complété par l'article 21 alinéa 4 les modalités de poursuite des mécanismes de prévoyance. Comme pour les dispositifs liés à la rémunération, le mécanisme retenu confère au BEV le financement de l'écart entre le montant des cotisations versées par la Deutsche Bahn AG et celui des cotisations applicables aux fonctionnaires.

Par ce mécanisme, le statut des salariés est fictivement harmonisé. L'État prend à son compte la charge résultant du maintien des contrats à statut. Le tout est isolé dans le budget du BEV ce qui présente l'avantage de pouvoir identifier la masse

\footnotetext{
${ }^{8}$ C'est pourquoi les allemands surnomment ce système « indemnisation des coûts comme si » (Als-ObKosten-Erstattung), c'est-à-dire « comme si » tous les salariés étaient de droit commun.

9 «(...) Die im Zeitpunkt der Eintragung der Gesellschaft in das Handelsregister bestehenden Pflichtversicherungen bei der Bahnversicherungsanstalt Abteilung B, ab 1. Oktober 2005 Deutsche Rentenversicherung Knappschaft-Bahn-See, werden durch das Bundeseisenbahnvermögen fortgeführt; die durch eine Schließung des Versichertenbestandes entstehenden Aufwendungen aus der Pflichtversicherung trägt das Bundeseisenbahnvermögen $(\ldots) »$.
} 
salariale bénéficiant du statut de fonctionnaire et d'en suivre l'évolution - en l'occurrence l'érosion régulière.

\section{L'accompagnement social de la restructuration de l'entreprise} et de la rationalisation de son fonctionnement

Le deuxième défi imposé à la Deutsche Bahn AG en échange de son désendettement était de renouer avec la rentabilité et les profits. Une drastique restructuration accompagnée d'une rationalisation du fonctionnement de l'entreprise étaient donc nécessaires. Et ceci passait par l'exigence d'une réduction de la masse salariale.

Pour préserver un bon climat social, la Deutsche Bahn AG fait d'emblée le choix d'opérer cette réduction d'effectifs sans licenciement. Elle fixe avec les représentants des salariés les modalités de gestion des effectifs dans un accord d'entreprise, le Beschäftigungsbündnis Bahn, signé en 1996. Elargi en 2005 et prolongé jusqu'à la fin de l'année 2010, cet accord se compose, d'une part, d'un accord collectif sur la garantie de l'emploi - Beschäftigungssicherungstarifvertrag et, d'autre part, d'un accord sur le marché interne du travail - Konzernbetriebsvereinbarung Konzernarbeitsmarkt.

Le premier accord vise à prévenir la perte d'emploi du fait de la suppression d'une activité et fixe même une règle d'exclusion du licenciement économique pour tout salarié employé depuis plus de cinq ans par la DB AG. En cas de cessation d'une activité (perte d'un marché public par exemple), les salariés peuvent être affectés à d'autres tâches le temps de leur retrouver un nouvel emploi au sein de l'entreprise. En contrepartie, l'accord sur le marché interne du travail entérine une triple flexibilité à laquelle les salariés s'engagent :

- flexibilité géographique, d'une part : le reclassement peut être proposé en n'importe quel lieu du territoire fédéral ;

- flexibilité salariale, d'autre part: la rémunération liée au nouveau poste peut éventuellement être abaissée à $80 \%$ du salaire précédent. Notons au passage que ceci ne concerne que les salariés disposant d'un contrat de droit commun puisque les salariés à statut demeurent rétribués selon les grilles de la fonction publique ;

- flexibilité fonctionnelle, enfin : le nouveau poste peut nécessiter une formation complémentaire voire signifier une totale réorientation de carrière.

Grâce à cette flexibilité négociée, l'emploi peut être garanti aux salariés. Mais surtout, la Deutsche Bahn AG n'est pas entravée dans son développement face à la concurrence.

Par ailleurs, pour optimiser le fonctionnement de ce système, la Deutsche Bahn AG crée une filiale, aujourd'hui dénommée DB Job Service $\mathrm{GmbH}^{10}$, fonctionnant sur le modèle d'une agence pour l'emploi.

\footnotetext{
${ }^{10}$ Jusqu'au 31 mars 1999, la structure n'était juridiquement pas distincte de l'entreprise et s'appelait Dienstleistungszentrum Arbeit.
} 
Tout événement entraînant une réduction d'effectifs au sein d'une entité du groupe DB (restructuration, cessation d'activité, perte de marché, etc.) entraîne le transfert des salariés vers DB Job Service. Après un bilan de compétences et un entretien servant à recueillir les vœux du salarié, DB Job Service est chargé de son reclassement à l'intérieur (ou le cas échéant à l'extérieur) de l'entreprise. Souvent, le salarié se voit en outre proposer une formation complémentaire lui permettant de réorienter sa carrière.

Au total, depuis la mise en place de DB Job Service, quelque 44000 salariés ont été pris en charge par cette entité et reclassés avec succès.

Si l'on veut brosser un tableau complet des raisons du succès de la restructuration de l'entreprise ferroviaire historique, un autre facteur structurel doit néanmoins être évoqué : la pyramide des âges au sein de la Deutsche Bahn. Les nombreux départs à la retraite ou en préretraite non renouvelés, auxquels se sont ajoutés quelques départs négociés, ont ainsi permis de réduire les effectifs de 100000 personnes entre 1994 et 2001, année du rachat du logisticien Schenker. Cette tendance s'est poursuivie même après puisque, à périmètre constant, c'est-à-dire sans l'effet des acquisitions, les effectifs en Allemagne ont continué de décroître, atteignant 179000 salariés aujourd'hui.

\section{Récapitulatif des principaux accords d'entreprises}

Avant la réforme, les deux anciennes compagnies nationales disposaient chacune de leurs propres grilles de rémunération et convention collective.

Avec la première étape de la réforme ferroviaire en 1994, plusieurs accords collectifs sont négociés avec les syndicats, les plus importants étant une nouvelle convention collective unique, une nouvelle grille de rémunération et un accord de protection de l'emploi. Le premier objectif était avant tout de garantir les niveaux de protection sociale existants.

La deuxième étape de la réforme, en 1999, a consisté du point de vue de la gestion des ressources humaines à accompagner la transformation juridique de l'entreprise, désormais composée d'une structure holding chapeautant plusieurs filiales prenant la forme d'une société par actions ou d'une SARL. Les accords signés avec les syndicats ont ainsi nécessité une déclinaison pour chacune des filiales.

En 2004, des critères de flexibilité sont introduits en contrepartie de la sécurité de l'emploi offerte par l'entreprise à ses salariés. En échange de la renonciation par la DB AG de procéder jusqu'à fin 2010 à des licenciements économiques, les représentants des salariés acceptent d'augmenter la durée du travail hebdomadaire à 40 heures par semaine et de renoncer à un jour de congé payé par an.

2007 marque l'achèvement de ce processus : la convention collective est simplifiée, unifiée et surtout, signée par les trois syndicats de l'entreprise - Transnet, $\mathrm{GBDA}^{11}$ et $\mathrm{GDL}^{12}$.

\footnotetext{
${ }^{11}$ GBDA: Gewerkschaft Deutscher Bundesbahnbeamten und Anwärter. Avec Transnet, de loin le premier syndicat, le GBDA représente l'ensemble des salariés des chemins de fer.
} 


\section{Le transport ferroviaire régional, paradigme du succès de la gestion du personnel à la Deutsche Bahn AG}

La situation existant dans le transport ferroviaire régional a mis la robustesse du mécanisme de gestion du personnel que nous venons de décrire particulièrement à l'épreuve.

Les autorités organisatrices de transport chargées de gérer la délégation de service public de transport ont en effet rapidement mesuré l'avantage qu'elles pouvaient tirer de la concurrence : trafic accru, matériel roulant moderne, innovation et qualité en hausse, le tout pour des prix de $20 \%$ à $30 \%$ inférieurs ${ }^{13}$. La pratique des appels d'offres, ou pour le moins de consultations auprès de plusieurs entreprises ferroviaires, s'est ainsi développée. Ce sont aujourd'hui quelque $30 \%$ des marchés publics de transport régional qui ont été attribués par voie d'appel d'offres. De plus en plus, la Deutsche Bahn est concurrencée sur les marchés qu'elle gérait jusqu'alors en situation de monopole.

Quelques chiffres en attestent : en 2003, les concurrents ne possèdent encore que $9,9 \%$ des parts de marché. Six ans plus tard, ce chiffre atteint $20,3 \%{ }^{14}$. Il est en outre déjà possible d'affirmer que cette tendance va se poursuivre en observant le taux de réussite des concurrents aux appels d'offres passés. Ces derniers précèdent d'une à deux années l'entrée en service si bien qu'il est possible d'anticiper avec certitude la structure du paysage concurrentiel avec quelques années d'avance.

Si entre 2005 et 2007, la Deutsche Bahn a été en mesure de remporter $70 \%$ des marchés renouvelés en moyenne, en 2008 seuls $28 \%$ lui sont revenus. Ceci devrait donc se répercuter durablement dans les parts de marché de la concurrence en 2011 (les marchés étant attribués pour une durée oscillant entre 10 et 15 ans).

GRÂCE AU BESCHÄFTIGUNGSBÜNDNIS BAHN garantissant aux salariés de la Deutsche Bahn le maintien de leur emploi en échange de leur flexibilité (cf. supra), l'entreprise a pu négocier le tournant de la concurrence sans traumatisme. En cas de perte de marché, les salariés qui acceptent la proposition de reclassement continuent de travailler au sein de la Deutsche Bahn AG et celle-ci ne subit pas de double sanction - perte du marché, sureffectifs.

Elle peut combler des besoins existant dans d'autres de ses activités ou réemployer ces personnes à des postes identiques ailleurs en Allemagne. Le savoirfaire de ces salariés n'est ainsi pas perdu pour la Deutsche Bahn AG et la culture d'entreprise est renforcée.

\footnotetext{
${ }^{12}$ GDL: Gewerkschaft Deutscher Lokomotivführer. Ce syndicat représente les intérêts spécifiques de quelque 34000 conducteurs de locomotives.

${ }^{13}$ Source : BAG-SPNV, Association des autorités organisatrices de transport en Allemagne.

${ }^{14}$ Parts de marché calculées en millions de trains.km.
} 


\title{
Les évolutions de la régulation sociale dans le contexte de la libéralisation des transports ferroviaires en France
}

\author{
Dominique AUBRY
}

L'article suivant présente le point de vue du syndicat CFDT sur les nouveaux modes de dialogue social induits par la libéralisation du fret ferroviaire en France. II revient sur les accords conclus ces dernières années dans la branche et met en lumière l'impact de ces derniers sur la négociation sociale et les conditions de travail des salariés, aussi bien au sein de l'opérateur historique que des nouveaux entrants sur le marché.

Mon intervention consistera à montrer une chronologie d'évènements qui ont modifié le climat et le paysage du dialogue social dans le système ferroviaire français en quelques années.

\section{Libéralisation du fret ferroviaire et politique de réduction des coûts}

En France, l'ouverture à la concurrence du marché du fret ferroviaire international est effective depuis le 31 mars 2003, avec neuf mois d'avance sur le calendrier européen. Cette anticipation a été décidée par le gouvernement français en échange d'une autorisation de Bruxelles de recapitaliser l'activité fret SNCF, en vue de moderniser le parc de locomotives notamment.

Dès lors, la direction de l'entreprise a mis en œuvre un plan de réduction des coûts de production du transport de fret, afin de les rapprocher de ceux des nouveaux opérateurs ferroviaires privés. Cette recherche effrénée de gains de productivité s'est faite au détriment des conditions sociales des cheminots (atteintes à la réglementation et au temps de travail, à l'organisation, etc....).

La CFDT, avec d'autres organisations syndicales, a dénoncé le risque grave de détérioration des conditions de vie personnelle et de travail des salariés, au détriment de la santé des agents et de la sécurité de l'exploitation ferroviaire. Le climat social, déjà fragile dans l'entreprise, n'a alors cessé de se dégrader depuis.

\section{Accord sur l'amélioration du dialogue social}

Cependant, l'accord sur l'amélioration du dialogue social et la prévention des conflits conclu en 2006 a permis de diminuer significativement la conflictualité à la SNCF. Le nombre de préavis de grève et celui des grèves réelles ont fortement chuté.

La CFDT s'est engagée dans cet accord pour redynamiser la politique contractuelle à la SNCF et dépasser le rituel classique «demande d'audience - dépôt 
d'un préavis de grève - grève - négociation grâce à la grève - résultats - reprise du travail ». Cet accord permettait aussi d'éviter que le Parlement ne légifère sur le sujet en instaurant des dispositions encore plus restrictives sur le droit de grève dans les transports, le rendant difficile d'utilisation et inopérant.

La CFDT a toujours indiqué que le droit de grève ne doit s'exercer que lorsque tous les moyens de discussion et de négociation ont échoué pour obtenir un accord satisfaisant entre les parties.

\section{Elaboration du cadre social harmonisé}

Puis la construction d'un «cadre social harmonisé » dans le secteur ferroviaire ouvert à la concurrence, initié par le Premier ministre Dominique de Villepin, a conduit début 2007 à la négociation d'une convention collective nationale de la branche ferroviaire française applicable aux «nouveaux opérateurs ferroviaires privés ».

La partie temps de travail de cette convention a fait l'objet fin 2008 d'un accord signé par les syndicats CFTC (chrétien), UNSA (autonome) et CFE-CGC (cadres).

Les syndicats CFDT et FO ont quant à eux engagé la procédure d'opposition légale à ce texte.

Les syndicats CGT et FGAAC (conducteurs) n'ont pas souhaité approuver ce texte. Le syndicat SUD-Rail ne participait pas à la négociation.

Nous constatons une dispersion syndicale sur un sujet majeur et un accord particulièrement minoritaire puisque signé par trois organisations syndicales ayant ensemble une représentativité d'à peine $20 \%$ des salariés.

Mais en l'absence de dénonciation par une ou plusieurs organisations syndicales représentant 50\% des salariés du secteur, cet accord est validé, malgré les 120 observations très critiques du ministère du Travail effectuées en 2009.

La CFDT a donc dénoncé cet accord car les dispositions relatives au temps de travail sont très nettement inférieures aux règles de référence applicables actuellement dans l'entreprise publique ferroviaire nationale, mais aussi parce qu'il se situe bien en deçà de l'accord conclu le 27 janvier 2004 pour le transport transfrontalier de fret ferroviaire entre le patronat ferroviaire européen (C.E.R.) et la Fédération Européenne des Transports (E.T.F.) pour la partie salariés.

Pour la CFDT, avec une durée journalière de service nettement augmentée, notamment de nuit, une amplitude de travail démesurée et la possibilité de travailler avec trois Repos Hors Résidence consécutifs pour les conducteurs, cet accord entérine des reculs sociaux inacceptables.

\section{Le programme « Destination 2012 » de la SCNF}

Lors de la Convention Cadres à la SNCF les 18 et 19 juin 2008, Guillaume Pépy, président de la SNCF, a présenté à 3000 cadres son programme «Destination 2012 » qu'il a traduit par la formule «Simplifier et Rassembler». « Simplifier » car selon le dirigeant de l'entreprise publique, la SNCF doit modifier 
considérablement son organisation structurelle et managériale pour affronter la réalité économique imposée par l'ouverture des transports ferroviaires à la concurrence en Europe.

« Rassembler » fut le mot d'ordre rajouté à l'automne 2008 parce que les propositions non définitives formulées par le Président Pépy ont provoqué les inquiétudes les plus vives chez tous les cadres de l'entreprise, de tous les métiers, activités et niveaux hiérarchiques. C'est certainement la première fois depuis la grande réorganisation géographique et structurelle de 1972 à la SNCF que l'encadrement n'est pas en capacité de se projeter dans son avenir immédiat.

D'ailleurs, l'encadrement n'est plus associé à la définition de la stratégie de l'entreprise, il est mis à l'écart et subit les évènements au même titre que les autres salariés, d'où la quête de sens au travail des cadres aujourd'hui, qui ne peuvent plus être le relais naturel de la stratégie d'entreprise entre la direction et la base. Cela rend difficile le rôle du management de proximité dans la régulation sociale. L'entreprise sclérose elle-même un outil essentiel de la régulation sociale.

\section{La loi sur le « service minimum dans les transports »}

La loi sur le «service minimum dans les transports » du 21 août 2007 est venue impacter l'accord sur l'amélioration du dialogue social et la prévention des conflits que j'ai évoqué précédemment.

L'introduction de la déclaration individuelle d'intention de faire grève 48 heures avant le début de celle-ci a reporté la responsabilité collective de la grève sur le salarié, le plaçant en situation délicate face à son encadrement de proximité, ce dernier étant lui-même soumis à des pressions hiérarchiques supérieures.

De même, l'instauration d'un délai maximum de 8 jours à partir de la fin de la négociation pour déposer un préavis de grève, ainsi que le vote à bulletin secret sur la poursuite ou non de la grève après 8 jours de conflit sont de mauvais coups portés à l'action syndicale par essence collective.

Un point positif est à noter avec l'obligation pour l'employeur de recevoir les organisations syndicales dans un délai de trois jours. Précisons que dans le cadre des dispositions statutaires SNCF, une demande d'audience officielle effectuée par un syndicat représentatif ne connaissait pas de date limite minimale ou maximale pour la rencontre.

Dans la vie sociale «courante », et selon la force électorale du syndicat demandeur, certaines audiences ont été accordées avec un délai long de plusieurs semaines voire plusieurs mois! Ce qui n'est pas synonyme d'un dialogue social dynamique et constant.

\section{Conséquences de la réforme de la représentativité syndicale}

La loi du 20 août 2008 dite de «modernisation sociale», relative notamment à la représentativité des syndicats, impose désormais aux organisations syndicales 
de salariés d'obtenir $10 \%$ des suffrages exprimés au $1^{\mathrm{er}}$ tour des élections aux comités d'entreprise pour être représentatives au niveau national et ainsi négocier des accords collectifs à ce niveau.

Ce texte législatif prévoit aussi qu'un accord collectif n'est valable que s'il a été signé par une ou plusieurs organisations syndicales représentant au moins $30 \%$ des suffrages exprimés à cette même élection. La dénonciation d'un accord peut s'opérer par des syndicats représentant au moins $50 \%$ des salariés qui se sont exprimés.

A l'issue des élections professionnelles du 26 mars 2009 à la SNCF, seules quatre organisations syndicales sur huit ont gagné leur représentativité nationale. CFDT, CGT, SUD et UNSA sont désormais placées devant leurs responsabilités de négociateurs.

Le pôle progressiste constitué de la CFDT et de l'UNSA n'atteint cependant pas la barre fatidique des $30 \%$ nécessaire pour la validation d'un accord.

Il y a fort à parier qu'il se passera de longs mois avant qu'un accord de progrès social ne soit à nouveau signé à la SNCF.

Dans le contexte de crise économique et financière, de salaires et de pouvoir d'achat insuffisants que connaissent les salariés, la perspective de ne pas aboutir prochainement à un accord salarial à la SNCF risque d'accroître la tension du climat social dans l'entreprise.

Nous constatons qu'en peu de temps, deux lois viennent rendre plus difficile le dialogue social dans les entreprises de transport public en France.

La CFDT entend cependant confirmer toute sa place dans le champ de la politique contractuelle basée sur les revendications des cheminots et la mise en place des moyens économiques et des nouveaux droits sociaux pour assurer un développement harmonieux du secteur du transport ferroviaire.

\section{Détérioration du dialogue social chez un opérateur privé...}

La CFDT est aujourd'hui l'organisation syndicale majoritaire dans une nouvelle entreprise de transport ferroviaire privée opérant sur le marché français. Cette société entend développer tous les moyens et pratiques managériales pour conquérir des parts de marché. En interne, la direction entend stimuler sa productivité par une mise en concurrence de ses différentes unités de production entre elles. A cela, s'ajoute, la mise en concurrence des salariés entre eux par l'octroi de primes diverses et de conditions horaires souvent détériorées.

Aux plus jeunes de ses salariés d'accepter les horaires difficiles, les trajets effectués en toute illégalité par rapport au socle minimaliste du Code du travail français, et gare à ceux qui oseraient se plaindre! Les menaces non voilées sur l'emploi sont aussi facilement utilisées. Voilà ce qu'il en est des relations professionnelles au quotidien.

En ce qui concerne les relations sociales, un climat de défiance a été instauré par la direction par rapport aux syndicats et aux représentants du personnel. 
Ceux-ci sont clairement désignés comme des freins au développement de l'entreprise par leur souci de ne pas travailler plus de sept jours d'affilée par exemple ou de réaliser une journée d'amplitude de travail de 14 heures voire plus !

Pour éviter tout problème juridique, les «délits d'entrave » aux responsabilités de délégués du personnel et plus largement des Institutions Représentatives du Personnel sont courants. Le harcèlement est communément admis quand il s'agit de faire pression sur un délégué qui est contrôlé sur l'utilisation de son temps de représentation, voire mis « sous tutelle » lorsqu'il s'agit de limiter les revendications collectives et individuelles.

Cette « mise sous tutelle » s'exerce par une opération dilatoire consistant à surveiller avec plus d'acuité les faits et propos professionnels du salarié délégué du personnel sur un semestre, par exemple.

Dans cette entreprise, la politique contractuelle est inexistante, le débat se résume souvent à un monologue patronal justifiant les efforts de tous et le nonrespect des droits des salariés.

La CFDT s'est donc positionnée pour défendre les salariés et leurs revendications sur la base des textes juridiques existants (Code du Travail pour l'essentiel et jurisprudences).

... et contournement des organisations syndicales chez l'opérateur historique

Certains tentent de mettre en œuvre de nouveaux outils de régulation sociale, contournant les organisations syndicales. A la SNCF, il s'agit par exemple de consultation directe des salariés par sondages, de stigmatisation des salariés des entreprises ferroviaires dans l'opinion publique. Ceci par de nouvelles pratiques managériales utilisant les médias internes ou externes à l'entreprise (journaux, Intranet, Internet, blogs...).

Ainsi, depuis le conflit des retraites en 2007, la direction de la SNCF n'hésite pas à mettre en porte-à-faux l'ensemble des cheminots.

En témoignent les tracts et informations aux usagers qui indiquent l'absentéisme des cheminots.

Un exemple d'information sonore le 31 décembre à la gare de Paris Nord: « en raison du nombre élevé d'agents s'étant déclarés malades, un service allégé sera mis en place...».

C'est ainsi que l'usager peut penser qu'il est facile de "se déclarer malade » et qu'il s'agit du choix du cheminot, un choix qui relève du confort en cette période de fêtes.

D'aucuns voudraient faire croire que les cheminots en maladie durant cette période sont des malhonnêtes et des délinquants, qu'ils ne s'y prendraient pas mieux !

Nul doute que ce type d'intervention publique qui jette l'opprobre sur tous les cheminots quels qu'ils soient, est un très mauvais coup porté au dialogue social dans l'entreprise publique. Cette forme de violence verbale est particulièrement contre-productive. 
TOUTES CES PRATIQUES ne vont pas dans le sens d'une plus grande responsabilisation des acteurs sociaux.

La CFDT prône quant à elle un retour à de bonnes pratiques, dans le cadre du respect et de la reconnaissance mutuels des partenaires sociaux, pour une meilleure régulation sociale dans les entreprises.

Réconcilier l'économique et le social est une impérieuse nécessité, le premier ne réussira pas sans le second, il faudra bien trouver le point d'équilibre. Le développement durable de l'être humain doit constituer le socle des politiques de développement durable. 
$-\mathrm{V}$ -

ÉCLAIRAGES D'AUTRES PAYS MEMBRES DE L'UE 



\title{
De la nationalisation à la privatisation : l'exemple de la Grande-Bretagne
}

\author{
Richard POND
}

Cette contribution présente le cas d'un pays précurseur en matière de libéralisation des services publics : la Grande-Bretagne. La privatisation des industries nationalisées, l'ouverture à la concurrence des services aux organismes publics et l'introduction des partenariats public-privé (PPP) en sont diverses modalités, dont les conséquences en termes d'emploi et de régulation sociale sont évoquées ci-après.

Dans les années d'immédiat après-guerre, la Grande-Bretagne connut un vaste programme de nationalisation sous le gouvernement travailliste, de 1945 à 1951. L'une des impulsions majeures à l'œuvre derrière ces mesures fut l'idée qu'il fallait réorganiser les industries significatives et essentielles comme l'électricité et le charbon afin de répondre à une demande d'énergie plus importante. Ces industries étaient fragmentées, le secteur charbonnier privé présentant un bilan très insatisfaisant en matière de sécurité alors que le secteur électrique était dominé par un grand nombre de fournisseurs détenus par les municipalités.

La création d'un régime public de soins (National Health Service) marqua également une évolution majeure dans le secteur public britannique. Dans ce secteur aussi, l'idée qui présidait aux changements était qu'un système organisé et financé par l'Etat pourrait bénéficier d'une meilleure répartition des ressources que la myriade de services locaux fournis par une diversité d'organisations publiques, privées et caritatives.

L'accent était mis sur l'augmentation de la production et sur l'amélioration des conditions d'emploi et de travail.

Pendant les années 1950 et, plus encore, les années 1960 et 1970, l'emploi public se développa, à mesure de l'expansion des services publics aussi bien locaux que nationaux.

Les syndicats, dont beaucoup étaient affiliés au parti travailliste qui forma encore des gouvernements en 1964-1970 puis entre 1974 et 1979, étaient très favorables à la création et au développement ultérieur des industries nationalisées et des services publics. Ils purent bénéficier de relations bien meilleures avec leurs nouveaux employeurs, négocier de meilleures conditions d'emploi et de travail, y compris de vastes conventions collectives sectorielles, et augmenter leur nombre d'adhérents.

Les perspectives commencèrent à changer pour le secteur public dans les années 1970, lorsque la crise économique mondiale frappa et que les dépenses publiques se retrouvèrent sous pression. A la fin de cette même décennie, le gouvernement travailliste décida de vendre une tranche d'actions de la compa- 
gnie pétrolière nationalisée, British Petroleum (BP), afin d'accroître les recettes. Ce fut de fait la première grande mesure de privatisation, quoique ce fût plus une opération ponctuelle que le début véritable d'une stratégie de privatisation.

La pression sur les dépenses publiques et les tentatives de contrôler l'inflation en limitant les augmentations de salaires furent au cœur de ce que l'on appela «l'hiver du mécontentement », considéré comme le début de la fin du gouvernement travailliste et l'une des principales raisons du soutien croissant au parti conservateur et à ses idées visant à réduire le rôle et l'ampleur du secteur public. Ces idées faisaient certes partie du programme du parti pour les élections générales de 1979, mais les propositions spécifiques de privatisations n'en étaient pas une composante majeure.

On peut retenir du premier gouvernement conservateur des années 1980 (1979-1983) deux orientations en matière de privatisation et de libéralisation. D'une part, une stratégie commençait à se dessiner autour de la vente d'entreprises nationalisées, tandis que, de l'autre, l'objectif du gouvernement était d'introduire la concurrence dans les services fournis par les organismes locaux et autres entités publiques comme les institutions sanitaires et les ministères.

\section{Dénationalisation}

La vente d'industries nationalisées démarra lentement avec une autre vente d'actions de BP. Il fut également procédé à une vente d'actions de British Aerospace, l'entreprise d'armement et aéronautique, ainsi qu'à la privatisation totale d'Amersham International, une société de biochimie. Il fallut attendre le second gouvernement Thatcher (1983-1987) pour que certaines des grandes privatisations fussent entreprises avec British Telecom (BT) en 1983, marquant une nouvelle étape importante dans la stratégie des conservateurs.

Cette évolution est remarquable car elle impliquait de résister à une campagne menée par le mouvement syndical en général et au sein de l'entreprise elle-même où le taux de syndicalisation était élevé. La stratégie du parti conservateur intégrait non seulement une campagne à destination directe des salariés, avec des offres d'actions gratuites et bon marché, mais également une campagne nationale pour inciter les particuliers à prendre des actions BT.

Il faut noter en toile de fond qu'une autre stratégie essentielle des Conservateurs résidait dans de grands changements en matière de législation du travail, introduisant des restrictions en matière de grève et proscrivant efficacement toute grève contre la privatisation car ces actions seraient considérées comme des grèves politiques et, partant, comme illégales. Les syndicats se seraient alors retrouvés en situation de défier la loi et d'encourir des amendes potentiellement considérables.

Une autre tactique dans la cession d'industries nationalisées, incluant la vente de $\mathrm{BT}$, consista à sous-évaluer les actions. Cette pratique visait à assurer le succès de la vente. Dans le cas de BT et d'autres privatisations ultérieures, 
des particuliers purent acquérir des actions bon marché en sachant qu'ils pourraient les revendre presque immédiatement avec des bénéfices importants. Les ventes d'actions étaient encadrées, de sorte qu'un quota d'actions était réservé aux particuliers et que le nombre d'actions disponibles pour les investisseurs institutionnels était limité. Ceux-ci, fonds de pension et compagnies d'assurance par exemple, tentèrent généralement de maintenir l'équilibre de leurs portefeuilles d'action, ce qui signifie qu'ils voulaient renforcer leurs participations dans les grandes entreprises privatisées. Il en résulta, après la plupart des privatisations, une hausse des prix des actions, lorsque des particuliers cédèrent leurs actions à des investisseurs institutionnels.

L'autre caractéristique importante de la vente de BT, également présente dans la vente de British Gas en 1986, fut le maintien de la position monopolistique des entreprises. Prenant conscience qu'aucune des entreprises ne serait confrontée à une concurrence significative dans un avenir proche, le gouvernement introduisit un système de régulateurs chargés de surveiller les sociétés et d'établir un ensemble d'indicateurs de performance, notamment des limites de hausse des prix.

Dans le cas des privatisations ultérieures, l'approche du gouvernement fut différente, avec des systèmes centralisés comme dans le secteur électrique ou les chemins de fer, scindés en un grand nombre d'entreprises différentes opérant au niveau régional ou national. Ce changement déboucha sur un éclatement des systèmes nationaux de conventions collectives sectorielles. Ce fut le cas dans le secteur ferroviaire et celui des transports en autocars, ainsi que dans ceux de l'électricité et de l'eau.

Les changements affectèrent aussi les systèmes de rémunération, avec l'introduction de contrats personnels pour les cadres dans le secteur de l'énergie et l'expansion de rémunérations liées à la performance. Une grande réforme fut entreprise en la matière dans le secteur des chemins de fer, où la plupart des nouvelles sociétés adoptèrent de nouvelles structures de rémunération, avec des rétributions composées de salaires de base plus élevés, de préférence au bas niveau de salaire de base combiné à de nombreux versements et indemnités supplémentaires qui prévalait à l'époque de British Rail.

Globalement, l'impact de la dénationalisation ne fut pas si dramatique pour les salariés qui conservaient des emplois permanents. Avec un taux de syndicalisation restant relativement élevé, comparé au reste du secteur privé, les syndicats réussirent à préserver leurs exigences en matière de conventions et donc à protéger le niveau de rémunération et des conditions de travail dans la plupart des entreprises privatisées. Dans le secteur électrique, par exemple, le taux de syndicalisation est tombé de $67 \%$ à la veille de la privatisation à $49 \%$ aujourd'hui, mais c'est encore près de trois fois plus que dans le secteur privé en général $(17 \%)$.

Cela n'empêcha pas les suppressions d'emplois massives avant et après la privatisation dans beaucoup de ces entreprises, souvent facilitées par le recours 
aux fonds de pension pour financer de bonnes prestations de préretraite. Ainsi, entre 1990 et 1997, le nombre d'emplois dans le secteur électrique a diminué de $50 \%$. Certains de ces programmes draconiens de réduction d'effectifs eurent pour conséquence le recours accru aux emplois à durée déterminée et à l'intérim, en tout cas dans le secteur de l'énergie et chez BT. Quoique les syndicats aient pu, là encore, faire face ; chez BT, le syndicat des travailleurs en communication $(\mathrm{CWU})$ en particulier, parvint à fédérer des intérimaires, à négocier des conventions couvrant leurs conditions d'emploi et de travail et à assurer certaines garanties relatives à la conversion des postes de certains intérimaires en postes permanents.

Dans le secteur ferroviaire, les syndicats ont réussi à coordonner leurs négociations dans les différentes sociétés de chemin de fer et à préserver leur structuration. Le principal syndicat, le RMT, continue de se battre pour un retour aux négociations sectorielles.

Alors que les syndicats ont pu préserver les conditions de rémunération et de travail de leurs membres dans bon nombre d'entreprises privatisées, les personnels qui ont le plus bénéficié des changements sont les cadres supérieurs, dont beaucoup étaient déjà à la tête des structures avant la privatisation. En plus de voir leurs rémunérations augmenter rapidement, ils ont généralement pu bénéficier de participations substantielles dans les entreprises privatisées et des programmes de stock-options courants dans la plupart des entreprises cotées en bourse. Ces dispositifs permettent aux hauts dirigeants d'acquérir des actions à un certain prix pendant une période donnée, avec la perspective de pouvoir profiter d'une hausse du cours de l'action, mais aussi de la liberté de ne pas exercer leurs options si les cours de l'action baissent.

\section{Concurrence en matière de prestation de services}

Deux principaux développements contribuèrent à la campagne d'introduction de la concurrence dans la prestation d'un certain nombre de services aux organismes publics. Le premier fut la suppression par le gouvernement de la clause sur les salaires équitables dans les organismes publics locaux. Cette disposition obligeait les sous-traitants à verser des salaires comparables à ceux pratiqués pour les personnes employées directement par les instances locales. A une époque où il n'existait pas de salaire minimum légal, sa suppression signifiait que les entreprises étaient libres de réduire les salaires et de se positionner de manière avantageuse face à la concurrence.

Dans l'intervalle, un certain nombre d'organismes locaux contrôlés par les Conservateurs avaient sous-traité toute une série de services afin de réduire les coûts.

Les principales prestations visées étaient notamment l'enlèvement des ordures, le nettoyage des rues, le nettoyage de bâtiments et la restauration, des secteurs où les entreprises profitaient des conditions de rémunération et de tra- 
vail moins favorables proposées à leurs salariés pour faire des offres à bas prix afin de remporter les marchés.

Malgré des services manifestement de mauvaise qualité et des manquements aux contrats de la part d'entreprises plus préoccupées par la réduction des coûts que par la fourniture de services de qualité à l'aide d'une maind'œuvre correctement payée, la sous-traitance se répandit, avec l'entrée sur le marché d'un grand nombre de grandes entreprises et de multinationales. En 1983, le gouvernement publia une circulaire à destination de toutes les organisations du service national de santé, les appelant à soumettre leurs services à une mise en concurrence systématique (market testing). Cette procédure mena encore à sous-traiter des services comme le gardiennage, la sécurité, le nettoyage et la restauration.

Cependant, le gouvernement était déçu que l'idée de soumission à la concurrence n'ait pas été adoptée plus largement dans les organismes publics locaux, de nombreuses structures locales dirigées par les travaillistes refusant d'ouvrir leurs services à la concurrence. Par conséquent, le gouvernement opta pour la contrainte et introduisit en 1987 une législation de soumission obligatoire à la concurrence qui obligeait les pouvoirs publics locaux à soumettre un certain nombre de services à des appels d'offres, notamment le nettoyage de bâtiments et des rues, l'enlèvement des ordures, la restauration et la gestion des parcs automobiles. D'autres services comme la gestion du logement vinrent s'ajouter par la suite.

Là encore, la facilité consistait pour les entreprises à faire des offres moins chères que les offres internes tout simplement en ayant moins de salariés pour assurer ce service et en proposant des conditions de rémunération et de travail plus mauvaises. Cela incluait, outre les salaires, les retraites, les congés maladie, les droits aux congés annuels et d'autres conditions. Cette évolution eut un impact important sur la syndicalisation, impliquant le transfert d'un grand nombre de salariés hors de la convention sectorielle applicable à la fonction publique locale. Si les syndicats réussirent à conserver une partie de leurs membres employés dans les entreprises privées de sous-traitance, il leur fallut souvent accepter des conventions collectives moins favorables voire, parfois, l'absence de convention. Un certain nombre de sous-traitants remportant de nombreux marchés et devenant des acteurs nationaux importants, les syndicats parvinrent à négocier des conventions cadre, mais celles-ci ne garantissaient pas des niveaux de salaires à l'échelon local.

Pour les syndicats, le défi était de taille. Jusqu'aux années 1980, ceux qui étaient présents dans les structures locales (les précurseurs de Unison, Unite et du GMB) avaient des sections importantes, couvrant des centaines, sinon des milliers voire, dans quelques cas, des dizaines de milliers de membres. A la fin des années 1990, l'ampleur de la sous-traitance était telle qu'une seule section Unison ne devait plus faire face à un employeur local, mais à des dizaines, sinon des centaines d'employeurs du secteur privé. 
Les salariés transférés vers des entreprises privées étaient souvent confrontés à des conditions de rémunération et de travail plus défavorables, malgré la protection légale temporaire que leur apportait la disposition relative au transfert de salariés (en application de la directive relative aux droits acquis). Ils découvrirent aussi qu'ils avaient des conditions de travail et de rémunération différentes de celles des autres salariés de l'entreprise ayant des fonctions similaires. Les sous-traitants avaient des niveaux de salaires différents selon le marché remporté et des niveaux de rémunération différents pour les nouveaux salariés intégrés pour travailler sur ces marchés. Ce processus a entraîné des traitements à deux vitesses voire plus dans les entreprises de sous-traitance, avec des personnes ayant des conditions de travail et de rémunération différentes.

\section{7-2010 : les travaillistes au pouvoir}

Dans la période qui précéda les élections générales de 1997, le parti travailliste prit un certain nombre d'engagements envers les syndicats, dont certains faisaient partie des plus gros affiliés au parti. L'introduction d'un salaire minimum national en 1999 était l'un de ces engagements, particulièrement salué par les syndicats même s'ils jugèrent décevant le niveau de départ. Les syndicats du secteur public furent particulièrement satisfaits que le nouveau gouvernement travailliste supprime d'emblée le système de soumission obligatoire à la concurrence (CCT). Il fut toutefois remplacé par un nouveau système, celui de l'offre la plus avantageuse (Best Value), qui obligeait les organismes locaux à mettre leurs services en concurrence, mais en s'intéressant à la valeur globale du contrat et non en se focalisant étroitement sur le prix le plus bas. Telle était en tout cas l'intention. Selon l'expérience des syndicats, la manière dont ce système fut mis en œuvre dans de nombreux organismes revenait à instaurer un régime qui n'était pas si différent du système CCT.

Les syndicats connurent une grande déception, avec la tendance du Labour à considérer le secteur privé comme étant le plus à même de proposer des prestations de services publics plus efficaces. Même si le gouvernement ne le déclara pas aussi nettement, ce présupposé apparaissait dans ses actions, en particulier dans la promotion de l'initiative de financement privé (PFI) ou ce que l'on connaît davantage aujourd'hui sous le nom de partenariats public-privé adoptés dans toute l'Europe.

La politique des contrats de PFI fut développée par les gouvernements conservateurs sous John Major (1990-1997) afin de donner au secteur privé une place bien plus importante dans des projets de construction relevant du public, comme la construction d'hôpitaux. Au lieu de financements publics, des consortiums d'entreprises privées devaient porter la responsabilité du financement, de la conception, de la construction et de l'exploitation d'équipements publics. Les pouvoirs publics devaient ensuite utiliser ces équipements sur la base d'un ac- 
cord à long terme (20 ans ou plus) dans le cadre duquel ils verseraient au consortium un droit annuel fixe avec un ajustement autorisé pour l'inflation.

Ce dispositif ne se répandit pas très vite pendant les gouvernements conservateurs, mais il fut repris avec enthousiasme par le gouvernement travailliste. L'un de ses principaux attraits résidait dans le fait que de nombreux grands projets de construction pouvaient être lancés sans augmenter les emprunts publics. Les syndicats du secteur public étaient généralement opposés à ce système car ils pensaient que les organismes publics se retrouveraient liés à des contrats à très long terme avec une marge limitée de renégociation. Ils s'inquiétaient aussi de l'inclusion dans beaucoup des projets initiaux de contrats concernant l'exploitation d'équipements comme des hôpitaux et des écoles, ce qui signifiait la privatisation d'un éventail de services comme le nettoyage, la maintenance, la sécurité, etc.

Les syndicats se préoccupaient aussi de l'inégalité des conditions s'agissant de choisir entre financement public et contrat de PFI. Ils firent valoir que les hypothèses relatives au risque et au taux de rendement étaient construites en faveur des PFI, de sorte que les projets de nouveaux hôpitaux ou écoles allaient opter pour des PFI. L'un des marchés les plus importants et le plus controversé porta sur la maintenance du métro londonien, dont la sous-traitance fut imposée à l'organisme Transport for London par le gouvernement travailliste. Cette expérience se solda par un échec au bout de quelques années seulement. Le consortium privé en charge de ce service échoua à plusieurs reprises à atteindre ses objectifs de performance et la maintenance dut être reprise par Transport for London.

Le gouvernement travailliste fit quelques concessions sur les PFI et la privatisation, généralement en négociant des accords protégeant les conditions de rémunération et de travail de certains salariés. Des dispositions spécifiques existaient dans le secteur sanitaire pour couvrir les salariés transférés vers des entreprises privées sous contrat de PFI, ainsi qu'un accord national impliquant les organisations patronales du secteur privé dans la gestion locale, visant à juguler le développement de conditions de travail à deux vitesses. Les syndicats furent sans aucun doute satisfaits de cette disposition, même s'il ne s'avéra pas si facile d'assurer l'application de l'accord localement.

\section{Situation actuelle}

Le taux de syndicalisation dans le secteur public est encore d'environ $60 \%$, soit un niveau beaucoup plus élevé que dans le privé où il est de $17 \%$. Les grandes conventions sectorielles continuent de définir les principales conditions de rémunération et de travail de centaines de milliers de salariés dans les organismes publics locaux et les services de santé. Malgré une décentralisation significative de la négociation dans la fonction publique et les secteurs privatisés comme le rail et l'électricité, la structure syndicale a bien résisté et les conventions collec- 
tives ont été maintenues. Les retraites ont également été préservées alors que c'est maintenant le point sur lequel la réforme se focalise. La grande majorité des régimes de retraite du secteur privé est passée de prestations définies à des cotisations définies, tandis que la plupart des salariés du secteur public restent couverts par des régimes à prestations définies. La privatisation et l'externalisation sont courantes et l'on s'attend à ce qu'elles se répandent au fil des coupes faites dans le secteur public pour répondre à la crise.

Traduction de Marie GRAVEY 


\title{
Libéralisation des services publics, stratégies d'entreprises et conséquences pour l'emploi : le secteur postal et électrique en Autriche
}

\author{
Christoph HERMANN, Dominik LINDNER
}

Les services publics organisés et gérés par l'Etat ont longtemps fait partie intégrante du modèle social européen (Frangakis et al., 2009). L'Autriche ne fait pas exception en la matière. L'adhésion à l'Union européenne en 1995 a conduit non seulement à une libéralisation de l'économie du pays, mais aussi, par la suite, à la suppression des monopoles dans les services d'intérêt général. L'introduction de la concurrence a eu un impact important non seulement sur les stratégies des entreprises mais également sur l'emploi dans les services publics qui constituaient, avec les autres domaines du secteur public, un pilier du modèle d'emploi autrichien (Hermann/Flecker, 2009). Cette contribution met en lumière les changements survenus dans les services postaux et le secteur électrique. Afin de mieux saisir le lien entre la restructuration des entreprises et la transformation des relations sociales, l'analyse s'effectue surtout à l'échelle de l'entreprise. Les résultats présentés ci-dessous ont été recueillis dans le cadre d'un projet de recherche européen sur la libéralisation des services publics'.

Cette contribution commence par un aperçu des processus de libéralisation et de privatisation dans les services postaux et le secteur électrique. Elle présente ensuite les résultats issus de deux études de cas dans ces secteurs. Elle décrit enfin les conséquences en termes de relations sociales et présente les conclusions qui peuvent être tirées de ces résultats.

Le processus de libéralisation et de privatisation

La libéralisation du marché postal autrichien s'est faite pour l'essentiel en suivant les directives européennes, sous forme d'ouverture progressive du marché. A partir de 1998, les colis de 350 grammes et plus ou les courriers coûtant plus de cinq fois le tarif d'affranchissement standard ont été exclus du monopole postal. Dans le même temps, la Post- und Telekom AG, séparée de l'administration d'Etat, fut déclarée prestataire de services universel, et un certain nombre de critères furent définis afin de garantir un service de base. La loi relative aux postes, adoptée en 1997, fut modifiée en conséquence. Depuis 2006, le domaine réservé, dont la poste autrichienne autrefois publique détient encore le monopole, est limité aux courriers de moins de 50 grammes (Hermann, 2009, p. 238 ; Hofbauer, 2006a). A l'origine, la libéralisation complète au sein de

1 Le projet s'intitule « Privatisation of Public Services and the Impact on Quality, Employment and Productivity » (PIQUE). Il a bénéficié d'un financement de l'Union européenne dans le cadre du $6{ }^{\mathrm{e}}$ PCRD (projet $\mathrm{n}^{\circ}$ CIT-2006-028478). Pour plus d'informations : www.pique.at. Le Centre de recherches et de conseil sur le monde du travail (FORBA) à Vienne était en charge de la coordination et de la partie autrichienne du projet. 
l'UE était prévue pour 2009. A la suite des protestations de certains Etats membres, elle a été différée à 2011 (et même 2013 pour certains pays).

Une nouvelle loi relative au marché postal a été adoptée en 2009 pour répondre à cette ambition de libéralisation complète. Cette loi a instauré pour la première fois un régime de concession pour les nouveaux entrants sur le marché du courrier adressé (qui n'avaient jusque-là qu'à déclarer leur activité). La loi prévoit désormais un minimum de 1650 relais postaux, ceux-ci pouvant toutefois offrir moins de services que les bureaux de poste traditionnels et pouvant être gérés par un partenaire privé de la Poste (mais l'entreprise n'est en droit de fermer un bureau de poste que si elle trouve un «partenaire postal»). Les concurrents privés ayant un chiffre d'affaires annuel de plus d'un million $€$ doivent dorénavant contribuer à un fonds de péréquation afin de participer au financement du service universel. L'obligation faite aux concessionnaires d'offrir à leurs salariés « des conditions de travail adaptées, en vigueur en Autriche, y compris en termes de rémunération », fut un point particulièrement controversé de la réforme. Sont considérées comme adaptées au sens de la nouvelle loi les conditions de travail « définies dans la convention collective de travail ». Mais les partenaires sociaux débattent toujours d'une convention collective de branche pour le secteur postal.

L'ancien monopole, l'Österreichische Post AG, est détenu à $51 \%$ par l'Etat, les autres parts ayant été vendues en bourse en 2006 à des investisseurs privés (une petite part des actions fut aussi cédée à des conditions préférentielles aux personnels de l'entreprise). Jusqu'à présent, l'entreprise a fait obstacle à toutes les tentatives de ses concurrents de s'établir durablement sur le marché. Le transporteur allemand de colis Hermes a pendant quelque temps essayé de prendre pied dans certaines agglomérations. Malgré des contrats lucratifs avec de grandes sociétés de vente par correspondance, l'opérateur privé a fini par se retirer du marché autrichien. La distribution du courrier a connu des phénomènes semblables : Redmail, concurrent renommé, se trouvait sur ce marché, mais a dû se retirer, provisoirement au moins, de la distribution du courrier adressé. Cette entreprise, basée sur une joint-venture entre une maison d'édition autrichienne et la Poste néerlandaise, est encore présente dans la distribution de courrier non adressé et de publicité (outre la distribution de journaux). Mais, dans ce domaine, l'ancienne entreprise monopolistique s'est judicieusement préparée à affronter la concurrence en rachetant le plus gros concurrent du marché, la société Feibra. Une concurrence forte règne sur le marché de la livraison express. Très tôt, l'opérateur historique autrichien a cédé ce marché aux entreprises privées; il ne tente que depuis quelques années de conquérir des parts de marché avec ses propres services express.

En réponse à la première directive européenne de réforme du secteur électrique de 1996, l'Autriche a commencé à libéraliser, à la fin des années 1990, le marché de l'électricité. La transposition de la directive s'est faite dans le cadre de la loi d'organisation du marché électrique, adoptée en 1998 et modifiée en 2000 (Lindner, 2008, p. 28 sq). Suivant les obligations définies par la directive européenne, les activités 
de transport et de distribution ont été séparées de la production et des activités commerciales. La première étape a été une séparation comptable des différentes activités au sein de la même entreprise ; puis, des entreprises formellement indépendantes ont été créées (Hofbauer, 2006b). L'ouverture du marché électrique se fait progressive-ment depuis 1999. Dans un premier temps, seuls les usagers ayant une consommation annuelle de plus de $40 \mathrm{GWh}$ ont pu choisir leur fournisseur. Puis, cette liberté de choix a été progressivement étendue aux clients ayant une consommation annuelle supérieure à 20 et $9 \mathrm{GWh}$. Depuis 2001, tous les consommateurs peuvent choisir leur fournisseur. L'Autriche est ainsi allée au-delà des exigences minimales de l'UE, qui prévoyaient une libéralisation complète pour 2007 (ibid.). Alors que les grandes entreprises ont, pour certaines, fait usage de cette liberté de choix, à ce jour, seul un très petit nombre de ménages a changé de fournisseur d'électricité. Cela s'explique par la notoriété importante des anciens opérateurs et par l'identification des consommateurs aux entreprises régionales et aux fournisseurs municipaux, qui restent prédominants.

La structure du marché électrique n'a pratiquement pas changé. Avant la libéralisation, ce marché était dominé par un opérateur national (Verbundgesellschaft), neuf entreprises régionales, correspondant aux neuf Länder autrichiens, et cinq entreprises municipales situées dans les grandes villes. Malgré la libéralisation, la compagnie nationale et les neuf entreprises régionales continuent de produire $95 \%$ de l'électricité du pays. Mais, ce qui a changé, c'est que des entreprises qui coopéraient jadis - la compagnie nationale assurait la production électrique et le transport, les entreprises régionales la distribution et la vente - sont maintenant manifestement concurrentes (mais, dans le même temps, certaines entreprises régionales détiennent des participations dans l'opérateur national). L'une des évolutions les plus importantes est par conséquent que la compagnie nationale opère également depuis peu dans la vente d'électricité, et vise la même clientèle que les entreprises régionales et les compagnies municipales de distribution. Le deuxième changement essentiel réside dans le fait que des entreprises allemandes et françaises du secteur électrique ont acquis des actions dans certaines entreprises régionales, dans le cadre de privatisations partielles. Malgré l'entrée d'investisseurs étrangers sur le marché, les entreprises électriques autrichiennes sont restées majoritairement publiques. Autre spécificité autrichienne : la politique a contraint les entreprises de s'unir contre la concurrence étrangère, dans le cadre de la « solution électrique autrichienne ». De ce dispositif est né le groupe Energie Allianz, qui comprend uniquement, après le retrait du fournisseur de Haute-Autriche, les entreprises régionales de Vienne, de Basse-Autriche et du Burgenland (Lindner, 2008, p. 32-33).

\section{Exemples de réactions d'entreprises}

Nous présentons dans cette section les résultats de deux études de cas d'entreprises : l'une, consacrée aux services postaux ; l'autre, au secteur électrique. Ces 
études de cas se basent sur respectivement huit et onze entretiens avec des dirigeants (ou des anciens dirigeants dans le cas des postes), des délégués du personnel et des collaborateurs. Le regard d'interlocuteurs différents nous permet de reconstituer le « cas d'entreprise ».

\section{Services postaux}

L'étude de cas consacrée au secteur postal traite l'opérateur qui détenait jadis le monopole. Avant même la libéralisation du marché, l'administration des postes et des télégraphes fut séparée en 1996 de l'administration publique et transférée à une société par actions. Au début, les différents domaines d'activité relevaient encore de l'entreprise Post und Telekom AG. Mais, à partir de 1999, de plus en plus de domaines en furent détachés, à commencer par les télécommunications, puis la société de transports Postbus et enfin la Postsparkasse. Depuis 2002, Post AG regroupe essentiellement les activités de l'ancienne Poste (jaune). Les premières années, l'entreprise démantelée tenta surtout de faire du lobbying contre la libéralisation. Elle lança dans le même temps son premier programme de réduction des coûts qui se concentrait, d'une part, sur la réduction des emplois au travers d'un programme de préretraite et de primes de départ volontaire. D'autre part, un programme de rationalisation fut mis en place, dont l'axe majeur était l'instauration de centres de tri entièrement automatisés. La politique de réforme s'accéléra à compter de l'année 2000. Selon les propos d'un ancien dirigeant, la direction de l'entreprise s'est alors rendu compte que la libéralisation ne pouvait être arrêtée et que l'entreprise devait revoir son positionnement sur le marché pour pouvoir survivre après la fin du monopole. Selon le point de vue d'un autre ancien manager, la pression réformatrice émanait moins de la concurrence que du gouvernement qui ne voulait plus continuer à soutenir les pertes de l'entreprise. Le délégué du personnel souligne quant à lui que la libéralisation de l'entreprise a été utilisée comme une menace vis-à-vis des salariés.

Ces évolutions ont débouché sur une profonde réforme structurelle visant à réduire les coûts. Cette réforme comportait plusieurs volets. Premièrement, une réforme organisationnelle fut lancée, à l'issue de laquelle l'entreprise fut divisée en trois domaines d'activités : les secteurs du courrier, des colis et de la logistique, et le réseau de filiales. Le secteur du courrier (domaine réservé inclus) représente environ $60 \%$ du chiffre d'affaires; les colis et la logistique, un peu plus de $30 \%$; et le réseau de filiales, à peine $10 \%$. Ce changement eut pour conséquence la distribution séparée du courrier et des colis ainsi que, dans de nombreux cas, la gestion sur des sites distincts d'opérations autrefois effectuées dans les bureaux de poste. Deuxièmement, le réseau de filiales fut considérablement réduit et fit l'objet d'une concentration importante (voir ci-dessous). Par ailleurs, l'entreprise prit des mesures supplémentaires pour défendre sa position de leader sur le marché postal. Elle reprit en particulier son plus gros concurrent dans le secteur de la distribution de supports publicitaires. En 2001, la Poste autri- 
chienne acquit $74,9 \%$ de la Feibra. Depuis 2005, ce distributeur de publicité est une filiale détenue à $100 \%$ par l'opérateur historique autrichien. Comme le souligne un ancien dirigeant, cette opération fut un « coup de génie ». Grâce à l'acquisition de la Feibra, l'entreprise est en mesure de tenir à distance la concurrence low-cost, et peut par ailleurs exercer une pression sur ses propres salariés.

Le rachat de Feibra a en revanche épuisée les possibilités concrètes de croissance sur le marché postal. Post AG compte il est vrai sur une progression dans le secteur des services express, mais elle peut s'attendre à subir des pertes dans la distribution de courrier à partir de 2011. Afin de pouvoir poursuivre sa croissance malgré tout, et de réinvestir au moins une partie de ses énormes bénéfices, l'entreprise s'est engagée, d'une part, dans d'autres secteurs, et d'autre part, à l'étranger. Parmi les acquisitions en Autriche, on peut citer le rachat d'entreprises de logistique et d'un journal gratuit à Vienne. Les engagements à l'étranger ont connu une forte progression surtout après l'entrée en bourse en 2006. Alors que la part du chiffre d'affaires générée à l'étranger était encore inférieure à $3 \%$ en 2006, elle était déjà de $27 \%$ en 2007 ; elle représentait alors même plus de $70 \%$ pour les livraisons de colis (Post 2007, p. 40-41).

La stratégie de diversification ne s'est pas limitée aux acquisitions. L'offre de produits a également été étendue, et le suivi de la clientèle a été davantage axé sur différents groupes-cibles. Dans le premier cas, l'offre de produits a été élargie dans les filiales. Depuis peu, la direction recommence à miser davantage sur les services financiers, après la cession de la Postsparkasse en 2002 (Post 2007, p. 12). Dans le second cas, des structures ont été créées pour le suivi des grands comptes («Key-Account-Management»). Les clients stratégiques de la Poste autrichienne - dont la vente par correspondance, des prestataires de services financiers, des opérateurs de téléphonie mobile, mais aussi des administrations - constituent l'essentiel du chiffre d'affaires, tandis que les particuliers en représentent à peine plus de $5 \%$. Pour citer un ancien dirigeant de l'entreprise : «Le particulier, [...] sincèrement, n'a plus aucune importance pour la Poste [...], il joue un rôle politiquement [...] et les politiques le flattent et parlent de lui, mais de fait, ce n'est pas lui qui rapporte de l'argent. » Dans le secteur des services colis, les tarifs font déjà l'objet de négociations de gré à gré avec les gros clients. Alors que ces clients négocient les prix, la Poste a considérablement augmenté les tarifs standards en 2003. A côté des économies réalisées sur les coûts, l'augmentation des tarifs a contribué pour une part non négligeable à l'augmentation des bénéfices de l'entreprise.

Comme nous l'avons mentionné plus haut, Post AG a massivement investi dans la modernisation de son réseau de distribution (c'est-à-dire dans le retrait, le tri et la distribution du courrier). Entre 1999 et 2005, avant la privatisation partielle, donc, plus de 300 millions $€$ ont été dépensés pour la logistique et la modernisation des centres de distribution (Post 2005, p. 34). A partir de 2002, la modernisation s'est surtout faite sous la pression de la mise en œuvre des livraisons $\mathrm{J}+1$, un cadre qui impose la livraison de $95 \%$ des envois postaux dès le 
jour suivant. Outre le renforcement des capacités techniques, l'entreprise a procédé à une vaste concentration de son réseau de distributeurs et à une réduction draconienne de son réseau de filiales. Avant le début du processus de restructuration, on comptait 39 centres de distribution en Autriche. Il n'en reste plus que six aujourd'hui, et tous sont très largement automatisés (Post 2007, p. 46). Le réseau de distribution a été soumis à un processus similaire : il comptait encore 1880 points de distribution en 2001, dont seuls 320 subsistent aujourd'hui pour l'ensemble du territoire autrichien (ibid.). Cette concentration a été favorisée notamment par un changement de système de transport: au lieu du rail, Post AG fait aujourd'hui exclusivement appel aux transporteurs routiers.

Du point de vue de la direction, cette concentration permet la mobilisation plus efficace du personnel. La réduction des points de distribution a été assortie d'un agrandissement des zones. Selon le rapport d'activité 2003, l'entreprise a pu, en établissant des équipes de distribution plus étoffées et en réduisant parallèlement les effectifs, réaliser des économies massives et accroître sa productivité (Post 2003, p. 32). Mais, comme cela apparaît plus en détail ci-dessous, l'augmentation de la productivité s'appuyait aussi sur une intensification considérable du travail. Ce processus n'est nullement terminé. Les points de distribution sont en permanence soumis à des contrôles de capacités. Une valeur-temps est attribuée à chaque étape de travail, ce qui permet de calculer, sur la base du volume de courrier moyen, les effectifs nécessaires. Sauf exceptions, les résultats de ces études, utilisés en interne pour élaborer des modèles systématiques, débouchent sur des réductions d'effectifs. L'une des personnes interviewées décrit la procédure ainsi :

"Par exemple, quand Mobilkom a été retiré du $2^{e}$ arrondissement, ils sont tout de suite venus et nous ont dit: vous avez tant d'envois en moins. Ça fait un demi-poste... Ils sont assis là avec leur notebook et font le calcul devant nous. »

Outre la concentration des centres de tri et de distribution, il y a eu une réduction draconienne du réseau de filiales. Entre 2002 et 2007, 951 filiales (les anciens bureaux de poste) ont été fermées, en deux étapes (2002-2003 et 2004-2005) (Tamme, 2007). Aujourd'hui, on compte 1334 filiales, soit seulement $57 \%$ du réseau de 1998. Dans le même temps, le réseau de boîtes aux lettres de la Poste a été réduit lui aussi. De 2004 à 2006 seulement, 2800 boîtes aux lettres ont été démontées (ibid.). Des solutions de remplacement ont été trouvées dans certains cas, en proposant une partie des services des filiales à des partenaires locaux. C'est une forme d'externalisation. Le transport est un autre domaine dans lequel des externalisations ont été réalisées. Depuis le passage au transport routier, une partie des chargements est sous-traitée à des transporteurs privés. On compte également quelques distributeurs indépendants, rémunérés en fonction du nombre d'envois postaux délivrés.

\section{Emploi}

Comme le montre le graphique, le nombre de salariés hors entreprises rachetées a été réduit de 13467 personnes entre 1998 et 2007, soit une baisse de $37 \%$. 
Ce chiffre correspond à plus de 1300 départs annuels en moyenne. Depuis la libéralisation, l'emploi à temps partiel a connu une augmentation continue. Ce constat vaut également pour la Poste. Le rapport d'activité 2003 relève à ce propos une intensification du travail à temps partiel afin de pouvoir réagir avec flexibilité aux fluctuations de volume (Post 2003, p. 53). Mais sur ce point, l'ancien détenteur du monopole autrichien semble en retard par rapport aux évolutions internationales. La Poste néerlandaise emploie déjà la moitié de son personnel à temps partiel, et son homologue allemande un tiers (Hermann/Brandt/Schulten, 2008).

\section{Graphique 1: Evolution de l'emploi, étude de cas Post AG}

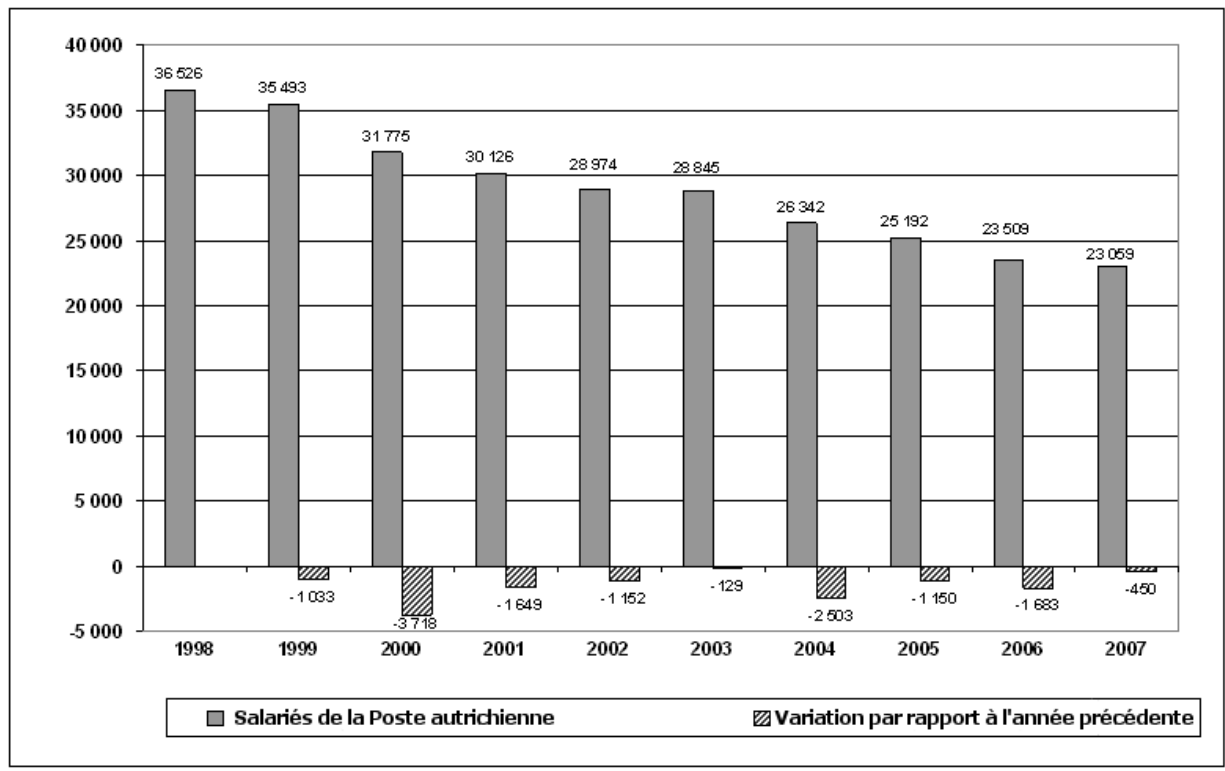

Source : rapports d'entreprise.

Au cours de la phase initiale (1997-1998), la direction de l'entreprise a instauré des programmes spécifiques afin de supprimer des emplois. Ces programmes comportaient, d'une part, des dispositions de départ en préretraite pour les fonctionnaires, d'autre part, des indemnités pour les salariés qui quittaient volontairement l'entreprise (dites «poignées de main dorées »). Ce dernier dispositif a surtout été utilisé par de jeunes collaborateurs dynamiques qui croyaient à une carrière possible dans le privé (Blum et al., 1999, p. 36 sq.). Dans le secteur des activités postales, 1946 personnes ont fait valoir leurs droits à la préretraite, et 307 ont accepté le départ volontaire avant 1998 (Post 1998, p. 13). Depuis, la réduction des effectifs consiste surtout dans le faible taux de remplacement des salariés qui quittent l'entreprise. Les personnels en surnombre, pour lesquels il 
n'y a plus d'emploi, sont regroupés dans une division séparée. Anciennement dénommée «Jobline», cette division s'appelle depuis 2005 «Karriere- und Entwicklungscenter» («centre de carrière et d'évolution»). Selon le rapport d'activité 2005, la Poste soutient ainsi la «nouvelle évolution professionnelle des collaborateurs touchés par les mesures de restructuration [...]. L'objectif prioritaire est de les réintégrer le plus tôt possible dans le processus opérationnel de l'entreprise » (Post 2005, p. 43). Selon les délégués du personnel, ce «centre de carrière et d'évolution » s'avère en réalité plutôt une impasse, d'où les collaborateurs ne sortent plus, à quelques exceptions près. "Personne n'y $a$ encore fait carrière ou poursuivi son évolution [...]. En vérité, [...] les personnels y perdent toute perspective. » La direction a annoncé à l'été 2010 son intention de fermer d'ici la fin de l'année ce centre, où plus de 500 personnes étaient récemment parquées (Standard, 11 août 2010).

\section{Organisation et conditions de travail}

La réorganisation de l'entreprise et les investissements dans des technologies nouvelles et économes en travail ont sans aucun doute contribué à accroître la productivité. Mais l'augmentation de la charge de travail des salariés a été au moins aussi importante pour la hausse de productivité. Avec la réorganisation des zones de distribution, basée sur un mélange de mesure taylorienne des temps de travail et l'introduction du travail en équipe à la japonaise, un facteur moyen se voit attribuer deux fois plus de points de dépôt aujourd'hui qu'il y a dix ans (soit 800 à 1000 contre 400 à 500). Alors que les facteurs particulièrement rapides effectuaient autrefois leur tournée en six heures, les délégués du personnel assurent que personne n'a fini en moins de huit heures aujourd'hui. Il n'est donc plus possible désormais de reprendre la zone d'un collègue absent, maintenant répartie au sein d'une équipe. Beaucoup de salariés comprennent tout à fait que l'efficacité ait été renforcée. Mais le problème est que ce processus semble sans fin. "La gestion des tâches était autrefois conçue de telle manière qu'on [...] y arrivait sur le temps de service. Maintenant, malheureusement, il faut vraiment te battre [...] pour t'en sortir pendant ton temps de service. »Un ancien responsable atteste lui aussi que «dans beaucoup de domaines, on a atteint les limites. » De nombreuses personnes, dont certaines ne sont certes plus toutes jeunes, disent être au maximum de leurs capacités physiques. "C'est quand même fatiguant [...] de porter ces paquets : j'y ai participé deux, trois fois. Le soir, je n'en pouvais plus.»

Aux guichets également, la charge de travail a augmenté. Avec le passage au guichet universel (il y avait autrefois des guichets spécifiques pour les différents services comme le retrait du courrier et des colis ; aujourd'hui, la plupart des opérations sont proposées au même guichet) et les économies permanentes de personnel, les salariés qui sont encore là ont parfois du mal à prendre les pauses auxquelles ils ont droit. "Quand les clients arrivent régulièrement, [...] 
tu restes sans arrêt derrière le guichet. [...] Si le flux de clients s'interrompt, tu peux aller boire un café, mais sinon, si c'est un bureau de Poste où viennent tous les chauffeurs et tout, tu restes mobilisé jusqu'à ta pause, ou bien souvent on n'a pas de pause, jusqu'à la fin du service. »Selon les salariés, la réorganisation et la détérioration des conditions de travail ont aussi des répercussions sur la qualité des services. "Le courrier arrive sûrement plus vite. Mais le contact des clients [...] avec le facteur, il est de moins en moins là, car le facteur n'a plus le temps, en fait [...]. La seule chose qui ait augmenté, c'est la vitesse d'acheminement. Mais la qualité en elle-même, le service au client, vraiment trop peu. »

\section{Electricité}

L'entreprise étudiée est un fournisseur municipal d'électricité, détenu à $100 \%$ par la commune, donc par la puissance publique. Cette compagnie a toutefois changé de forme juridique et opère maintenant comme entreprise de droit privé. Après avoir perdu le monopole du territoire, elle a dû faire face à la concurrence. L'entreprise présentée dans cette étude de cas a réagi à cette situation nouvelle notamment en renforçant la coopération avec d'autres fournisseurs d'électricité autrichiens. Une société créée conjointement est chargée de la distribution aux gros clients et du négoce d'électricité au niveau européen. Parallèlement, l'entreprise s'engage davantage dans la construction de centrales à l'étranger. D'une manière générale, le champ d'action de cet opérateur s'est fortement élargi du fait de la libéralisation. Autrefois, sa mission consistait à approvisionner en toute sécurité les particuliers et les entreprises de l'ensemble de la ville. La libéralisation l'a conduite à devenir une entreprise opérant à l'échelle internationale et obligée, sur le marché libéralisé, de s'éloigner de plus en plus de sa mission principale : l'approvisionnement électrique de la ville.

L'un des changements majeurs dus à l'ouverture du marché a concerné sa politique tarifaire. La compagnie distingue principalement trois segments de clientèle, différenciés par les volumes consommés. Ces segments sont les grands comptes, les entreprises et le marché de masse (les ménages). C'est le secteur des grands comptes qui a été le plus affecté par la libéralisation. Auparavant, des tentatives ont été faites pour fidéliser davantage cette clientèle, afin de résister dans un contexte de concurrence d'éviction. Les premières négociations tarifaires ont été menées dès 1999 sur ce segment. Après la libéralisation en 2001, l'entreprise se trouvait, pour ses plus gros consommateurs, face à une concurrence de cinq à six autres opérateurs, ce qui a entraîné une forte baisse de prix $^{2}$. L'étape suivante a consisté en baisses de prix pour les clients des secteurs de l'industrie et de l'agriculture. Le marché de masse étant le moins touché par

\footnotetext{
${ }^{2}$ Le prix est ainsi tombé à 17 groschen $(1,23$ cent) par KWh. Il oscille actuellement entre 6 et 7 cents pour les grands comptes (en fonction de la taille du client), ce qui selon les responsables de l'entreprise garantit la couverture des frais et ne laisse aucune marge.
} 
la concurrence, c'est sur ce segment que les baisses de prix furent les moins importantes. L'entreprise étudiée a pu jouer le jeu des baisses de prix car le rendement, et donc la productivité de ses centrales, est supérieur à celui de la plupart de ses concurrents, du fait d'une utilisation extensive de la cogénération.

Mais sa stratégie consiste globalement à se positionner comme opérateur de qualité. Si ses prix sont supérieurs à ceux des fournisseurs low-cost, elle offre en contrepartie de meilleurs services. La différence avec cette concurrence lowcost réside surtout dans le fait que l'opérateur étudié dispose de son propre centre d'appels, avec des collaborateurs formés spécifiquement. Une partie de ses concurrents recourt au contraire à des centres d'appels externes. La qualité d'information et la communication avec le reste de l'entreprise en pâtissent. Mais, même dans l'entreprise étudiée, on s'efforce de standardiser la communication avec la masse de la clientèle et de traiter autant que possible les contacts clients non pas via des centres de SAV locaux mais par téléphone ou par courriel. D'autre part, sur le plan du réseau, les contraintes tarifaires strictes imposées par le régulateur ont pour conséquence des investissements insuffisants dans l'entretien des infrastructures. La direction redoute à long terme des pertes de qualité susceptibles d'avoir des répercussions négatives sur l'approvisionnement électrique.

\section{Emploi}

Comme cela apparaît sur le graphique, de 1994 à 2007, le personnel de l'entreprise a diminué de plus de 1000 personnes, soit $25 \%$ de l'ensemble des effectifs.

Graphique 2 : Evolution de l'emploi, étude de cas électricité

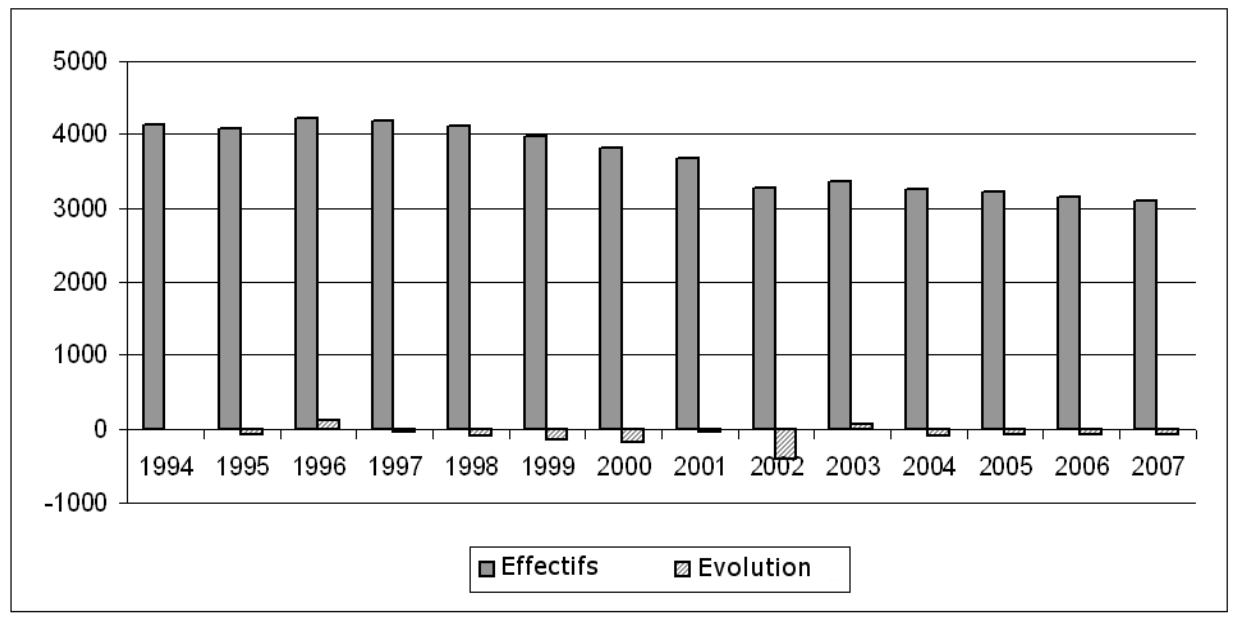

Source : rapports d'entreprise. 
En valeur absolue, le nombre de salariés est passé de 4150 à 3088 soit, pour la période observée, une suppression moyenne de plus de 80 postes par an. La réduction particulièrement élevée des effectifs en 2002 correspond à une vague de départs en préretraite dite «Plan 55 ». Ces destructions d'emploi massives peuvent s'interpréter comme une réponse immédiate à la libéralisation et à la pression sur les coûts qu'elle a entraînée. L'entreprise a dû, une fois son monopole perdu, accroître sa rentabilité et réaliser des économies dans tous les domaines. Pour renforcer sa compétitivité, elle a réduit de manière draconienne le facteur de coût que constitue le personnel, sans aller toutefois jusqu'à des vagues de licenciement. Les suppressions de postes ont pris exclusivement la forme de «départs naturels ». Cela signifie que cette réduction s'est déroulée principalement au travers de départs en retraite normale, de préretraites et de départs volontaires. Actuellement, sur ces départs, seul un poste sur trois est remplacé. La réduction du personnel à l'aide de départs naturels a été favorisée par la structure des âges du personnel actif. Avant la libéralisation, environ un collaborateur sur quatre avait plus de 50 ans.

\section{Organisation et conditions de travail}

L'organisation du travail a surtout été modifiée au travers d'une transformation structurelle des services existants et de la création de nouvelles divisions dans des domaines d'activités devenus plus importants du fait de la libéralisation et de la concurrence. Tous les domaines n'ont pas été touchés de la même manière par les changements. Dans celui, bien «implanté » des centrales, les changements se sont limités au regroupement de services afin de bénéficier de synergies. Mais cela n'a pas entraîné de modifications sévères en termes d'organisation ou de processus de travail. La création de nouvelles unités, comme la gestion des réclamations, a en revanche généré davantage de changements. Les nouvelles unités ont pour l'essentiel été constituées à partir des ressources humaines de l'entreprise. Cela signifie que les collaborateurs d'autres services ont été affectés à de nouvelles divisions, et donc à des activités complètement nouvelles, avec les formations correspondantes et un changement considérable au quotidien. Le service de gestion des réclamations compte par exemple des personnes qui travaillaient avant la libéralisation dans des domaines aussi divers que la facturation, la comptabilité, la technique ou le service des contrats.

L'organisation du travail a connu des modifications très importantes à la suite de la création par l'entreprise de sa propre société de réseau. Celle-ci ne compte que 37 collaborateurs, responsables de la planification stratégique dans le domaine des infrastructures de réseau (il a été question récemment d'affecter davantage de salariés à ce secteur, ce que refuse une partie du personnel en y voyant une scission de fait de l'entreprise). Pour toutes les autres activités, il faut emprunter du personnel à l'entreprise affiliée. Ce système entrâne une augmentation considérable des tâches administratives, toutes les activités devant 
être comptabilisées et faire l'objet de contrats de prestations. Cette évolution n'est pas très cohérente avec la prétention de devenir une entreprise de services moderne, une structure moins lourde. Les diverses contraintes propres à un secteur régulé augmentent en outre la bureaucratie. Mais la marge de décision et la flexibilité au sein des services ont été réduites par le transfert des compétences essentielles de planification à la société de réseau, ce qui représente aussi un changement crucial au quotidien.

Alors que les exigences à l'égard des salariés et la complexité des processus de travail se sont considérablement accrues, les effectifs ont été réduits de près d'un quart en quelques années. La charge de travail a donc énormément augmenté. Cette charge accrue est observable dans tous les secteurs de l'entreprise, et tous les interlocteurs, depuis la direction jusqu'aux simples collaborateurs, en passant par les délégués du personnel, l'ont jugée très élevée comparativement à ce qu'elle était auparavant.

Graphique 3 : Evolution de l'emploi dans les services postaux et le secteur électrique

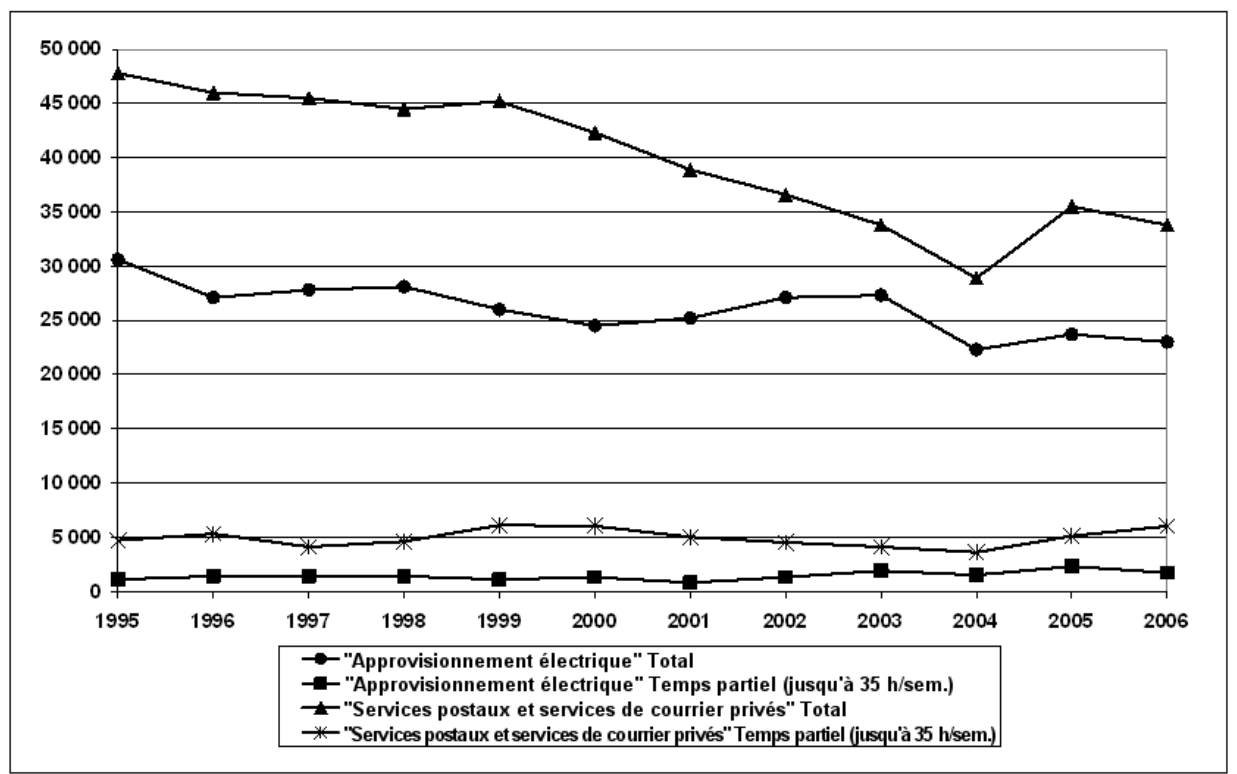

Source : Statistik Austria Mikrozensus, dépouillement pour le projet PIQUE.

Les pertes d'emplois évidentes relevées dans les études de cas des services postaux et du secteur électrique ne sont pas spécifiques à ces deux entreprises. On les retrouve plus largement au niveau des secteurs. Comme le montre le graphique, entre 1995 et 2006, l'emploi a diminué de $29 \%$ dans le secteur des services postaux et des services de courrier privés, et de $25 \%$ dans le secteur élec- 
trique. Ces chiffres démontrent que les emplois créés par les nouveaux opérateurs sont loin de compenser les pertes d'emplois survenues dans les anciens monopoles. Les données montrent aussi, dans les deux secteurs et pour la même période, une augmentation fulgurante du travail à temps partiel, en croissance de $28 \%$ dans le cas des services postaux et de $55 \%$ dans celui du secteur électrique; et encore, la part de l'emploi à temps partiel est encore relativement faible dans le secteur électrique comparativement à d'autres secteurs.

\section{Relations sociales}

Les relations sociales dans le service public présentaient dans le passé un ensemble de spécificités (Hermann, 2008, p. 213-214). Le service public faisait notamment exception à la loi constitutionnelle sur le travail (Arbeitsverfassungsgesetz ${ }^{3}$ ) et n'avait pas de droit des conventions collectives. Les salaires et temps de travail n'étaient pas définis dans des conventions, mais fixés, dans le cadre de la législation, par des règlements de service. Les augmentations de salaires annuelles étaient négociées au cours de discussions informelles entre les responsables syndicaux et les représentants du gouvernement. Dans certains secteurs, les salariés bénéficiaient de droits de cogestion plus vastes que ceux du secteur privé, permettant aux délégués ou aux représentants du personnel d'exercer une influence sur la direction des entreprises publiques. La protection en matière de mutation constituait un argument fort dans les négociations de ce type, les fonctionnaires ne pouvant se voir affectés à un autre poste contre leur volonté. Les syndicats du service public opéraient souvent au niveau de l'entreprise plutôt que de la branche, les opérateurs monopolistiques publics comme les chemins de fer autrichiens et l'administration des postes et télégraphes couvrant l'ensemble de leurs secteurs respectifs. De fait, les syndicats du service public avaient une forte influence sur les conditions d'emploi, notamment en raison du taux de syndicalisation élevé - plus de $80 \%$ dans le public, contre moins de $40 \%$ dans le privé - et de leur présence importante au niveau des entreprises.

L'ancienne administration publique des postes et télégraphes est un exemple révélateur des relations sociales dans le service public. Les salariés étaient fonctionnaires ou avaient un statut équivalent. Les conditions d'emploi étaient définies dans le cadre de règlements de service. Les augmentations de salaire étaient négociées, conjointement avec les autres syndicats du service public, directement avec le gouvernement. Avec la transformation de la Poste autrichienne en entreprise de droit privé, l'ancienne Post und Telekom AG s'est vu accorder en 1996 le droit de négocier des conventions collectives. Depuis, la direction négocie une convention collective d'établissement avec le syndicat des services postaux et des télécommunications. Cet accord s'applique aux colla-

\footnotetext{
${ }^{3}$ Equivalent autrichien du Code du travail, NDT.
} 
borateurs ayant rejoint l'entreprise après 1996 et recrutés sous statut d'employé. Un règlement de service reste en vigueur pour les fonctionnaires, mais les augmentations de salaires qui les concernent ne sont plus négociées entre le syndicat et les représentants du gouvernement, mais entre le syndicat et la direction de l'entreprise (ibid., p. 224-225). Les collaborateurs recrutés depuis juillet 2009 pâtissent d'un autre désavantage : ils gagnent jusqu'à $25 \%$ de moins que leurs collègues en service depuis plus longtemps. L'écart est moindre en cas d'heures supplémentaires car l'indemnisation des heures supplémentaires a été revalorisée. Le syndicat des postes a accepté les réductions de salaire, la direction de l'entreprise ayant menacé d'externaliser la distribution en la confiant à sa filiale Feibra (Hermann, 2009, p. 248).

Outre une fragmentation accrue chez l'opérateur historique, la libéralisation a entraîné l'instauration de conditions de travail et d'emploi extrêmement disparates au sein du secteur. Dans le domaine des services postaux (courrier, colis, services express et distribution de prospectus), au moins cinq conventions collectives différentes coexistent en Autriche, avec des dispositions différentes en matière de temps de travail et de rémunération (Haidinger/Hermann, 2008). Cette fragmentation est encore renforcée par le fait que $90 \%$ des distributeurs travaillant pour des nouveaux opérateurs de distribution du courrier et de publicité sont employés comme indépendants. Ils n'ont donc aucune protection sociale. Ils sont payés aux pièces et gagnent en moyenne environ la moitié du salaire d'entrée d'un facteur à la Poste autrichienne (Hermann/Brandt/Schulten, 2008). Du fait de cette faible rémunération, ces distributeurs sont à Vienne soit des frontaliers venant de Slovaquie ou de République tchèque soit des demandeurs d'asile en attente de régularisation. Il faut souligner que la Poste autrichienne pratique elle-même des conditions d'emploi de ce type par l'intermédiaire de sa filiale Feibra. Dans le secteur de la distribution de colis et des services express, les personnes ne sont en revanche généralement pas indépendantes. La gestion d'une grande partie des activités de distribution et de retrait par des petites entreprises de sous-traitance entraîne cependant régulièrement des violations du droit du travail, comme le non-respect du principe d'indemnisation correcte du travail supplémentaire et des heures supplémentaires.

Dans le secteur électrique, le système de relations sociales était dans une situation privilégiée, l'opérateur national et les neuf entreprises régionales ayant toujours fait exception aux négociations avec le service public. Mais ces entreprises publiques ne relevaient pas non plus de la chambre de commerce, qui défend les intérêts des employeurs du privé. Au lieu de cela, elles constituaient un groupe d'intérêts distinct, l'Union des entreprises électriques d'Autriche (Hermann, 2008, p. 220 sq.). Bien qu'il s'agisse d'entreprises publiques, leurs ouvriers sont par ailleurs représentés par le syndicat Métal, textile, alimentation (GMTN) et les employés par le syndicat des employés du privé Imprimerie, papier, journalisme (GPA-DJP). Les deux organisations syndicales négocient avec l'Union des entreprises électriques d'Autriche sur la convention collective de 
branche applicable aux fournisseurs d'électricité. Du point de vue des salariés, cette convention est l'une des plus favorables en Autriche, avec des salaires nettement supérieurs à la moyenne nationale (Adam, 2005). De ce fait, les caractéristiques du système conventionnel du public présentées plus haut s'appliquent aussi au secteur électrique, même si les acteurs concernés ne font pas partie du service public. Le taux de syndicalisation, de $80 \%$, est lui aussi d'un niveau semblable à celui du public. La convention collective des fournisseurs d'électricité s'applique aux deux tiers environ des 24000 salariés que compte approximativement le secteur au total (ibid.).

Outre l'opérateur national et les neuf sociétés régionales, il y a aussi des fournisseurs d'électricité municipaux. Que la fourniture d'électricité relève de l'entreprise municipale d'approvisionnement ou soit gérée par une entreprise indépendante, de droit privé, les salariés étaient défendus par le syndicat des employés municipaux. Contrairement à ceux des autres fournisseurs, les employés des entreprises municipales, pour autant que leurs activités ne fussent pas externalisées, relevaient autrefois du système conventionnel du public. Le syndicat des employés municipaux et le syndicat Service public (Gewerkschaft Öffentlicher Dienst) négociaient avec des représentants du gouvernement fédéral, des Länder et des communes sur les augmentations annuelles de salaires. En matière de salaires et de temps de travail, cela n'était pas source d'avantages vis-àvis des employés de la compagnie nationale et des sociétés régionales. A l'époque des critères de Maastricht et de réductions budgétaires, il était plutôt plus difficile d'imposer des augmentations de salaires pour les employés municipaux que pour leurs collègues de la compagnie nationale, formellement indépendante, et des sociétés régionales. Mais ils bénéficiaient du statut de fonctionnaire ou de conditions d'emploi équivalentes. Si les changements sont restés limités, comparativement aux bouleversements survenus dans les services postaux, de nouvelles dispositions ont aussi affecté le système conventionnel du secteur électrique.

Dans tous les domaines, des services ont été externalisés vers des prestataires de services privés. Les employés de ces entreprises privées, comme les sociétés de nettoyage, travaillent, à de rares exceptions près, dans des conditions nettement plus mauvaises que les employés du secteur électrique. Les fournisseurs d'électricité recourent plus souvent à l'intérim qu'auparavant. Les personnels permanents des entreprises non municipales ont subi un préjudice, avec la suppression de prestations sociales volontaires supplémentaires. Pour répondre à la séparation entre distribution et vente imposée par les directives européennes, la plupart des opérateurs ont en outre créé des sociétés propres pour le commerce d'électricité. Certains dirigeants estiment que ces nouvelles entreprises ne relèveraient donc plus de la convention collective du secteur électrique mais de la convention générale du commerce, nettement moins favorable du point de vue des salariés. Les syndicats sont parvenus à empêcher ce transfert. Ils ont toutefois dû accepter des concessions en matière de 
flexibilité du temps de travail et de rémunération sur objectifs pour les personnels employés par ces sociétés commerciales (ibid.) ${ }^{4}$.

La libéralisation a entraîné des changements plus importants pour les opérateurs municipaux. Après la libéralisation, certains ont adhéré à l'Association des entreprises électriques autrichiennes et ont adopté la convention collective du secteur électrique. D'autres, faisant partie des régies municipales, ont conservé leurs propres conventions. Les nouveaux salariés des services municipaux ne sont par conséquent plus recrutés sur des postes de fonctionnaires mais d'employés, ce qui constitue non seulement une dégradation en termes de sécurité d'emploi, mais aussi, dans certains cas, de rémunération. En ce qui concerne l'entreprise que nous avons étudiée, les personnels embauchés depuis juillet 2001 et rémunérés suivant une convention d'entreprise gagnent environ $13 \%$ de moins que leurs homologues travaillant depuis plus longtemps dans la même société. On observe aussi une dégradation sur le plan de l'avancement. L'avancement d'échelon n'intervient plus que tous les quatre ans pour les «nouveaux » collaborateurs, alors que pour ceux qui bénéficient de l'ancien statut de la fonction publique, cet avancement s'effectue tous les deux ans. Les nouveaux employés sont par ailleurs désavantagés dans la mesure où, les périodes de service précédant le recrutement n'étant plus prises en compte, ils commencent toujours au premier échelon.

LA LIBÉRALISATION DES SERVICES PUBLICS EN AUTRICHE a de larges conséquences pour l'emploi, les conditions de travail et les relations sociales. Depuis le début du processus de libéralisation, les opérateurs historiques des postes et de l'électricité ont clairement supprimé des emplois, au travers notamment de départs en retraite anticipés et d'incitations financières au départ volontaire. Dans le cas de la Poste, les salariés « en trop » sont regroupés dans une division distincte où ils sont condamnés à ne rien faire. Dans certains cas, des économies d'emplois ont été réalisées en introduisant de nouvelles technologies, mais aussi, dans les services postaux au moins, en réduisant et en allégeant le réseau postal. Malgré l'introduction de nouvelles technologies, l'intensité du travail s'est sensiblement accrue depuis la libéralisation. Outre des charges de travail plus élevées, les personnels se débattent avec de plus fortes exigences de flexibilité de la part des entreprises.

La suppression de postes et l'intensification du travail participent d'une stratégie globale visant à économiser des coûts, une autre stratégie consistant à verser des salaires plus bas. Dans les deux études de cas présentées, les entreprises ont réduit les traitements des «nouveaux » collaborateurs, c'est-à-dire des personnels

\footnotetext{
${ }^{4}$ Pour les employés de ces entreprises indépendantes du secteur électrique de commerce et de distribution, la convention collective inclut des dispositions complémentaires en matière de temps de travail et de rémunération. Ces employés peuvent ainsi, en cas d'horaires flexibles, travailler jusqu'à 10 heures par jour tandis que le calcul du salaire minimum de base prend en compte la performance ainsi que les provisions sur les ventes et sur le négoce. Dans les deux cas, la signature d'une convention d'entreprise est nécessaire.
} 
embauchés après une certaine date. Une autre option consiste à recourir à des formes d'emploi atypiques et à externaliser certaines prestations. Les deux secteurs considérés recourent fortement au travail à temps partiel, celui-ci restant toutefois dans le secteur électrique nettement inférieur à la moyenne nationale. Dans ce secteur, l'intérim est très répandu, alors que les services postaux emploient de plus en plus souvent des facteurs indépendants. On observe aussi, en lien avec le recours à l'emploi atypique, des fluctuations plus importantes, le vieux modèle de l'emploi à vie dans le service public diminuant à vue d'œil.

Ces changements débouchent sur une fracture croissante entre les personnels : «anciens » et «nouveaux » salariés, temps plein et temps partiel, personnel permanent et temporaire, etc. Ces divisions de plus en plus marquées se traduisent aussi dans le domaine des relations sociales. Alors qu'en Autriche le système de relations sociales se caractérise généralement par un niveau élevé de couverture et de concentration, les situations des services publics libéralisés présentent précisément une fragmentation croissante des conditions d'emploi. Les deux entités étudiées ont par exemple leur propre convention collective d'entreprise. La coexistence de conditions d'emplois hétérogènes entraîne une «spirale négative », les entreprises détériorant progressivement la situation.

Ce qui est intéressant dans le cas autrichien, c'est que ces changements se produisent alors que l'intensité de la concurrence consécutive à la libéralisation reste pour l'instant modérée. Alors que les entreprises partiellement privatisées ont demandé des concessions à leurs salariés, elles tentent de couper court à la concurrence. Et, alors qu'elles font des économies sur les dépenses de personnel et, parfois, sur l'offre et la qualité, elles investissent à l'étranger et dans d'autres activités lucratives. En définitive, on se demande quel rôle les services publics jouent encore après la libéralisation. En tout cas, ils ne contribuent plus à davantage d'emploi et à de l'emploi de qualité.

\section{Traduction de Marie GRAVEY}

\section{Indications bibliographiques}

ADAM G., Questionnaire for EIRO comparative study on industrial relations in the public sector, focusing on the public utilities - The case of Austria [en ligne], European Foundation for the Improvement of Living and Working Conditions, Dublin, 2005. Disponible sur : http://www.eurofound.europa.eu/eiro/2005/02/word/at 0411203s.doc [consulté le 17/05/2011]

Blum M., Flecker J., Fischer L., Hermann C., Collective Bargaining on Employment and Competitiveness in Austria. FORBA Forschungsbericht [en ligne], FORBA, Vienne, 1999. Disponible sur : http://www. forba.at/data/downloads/file/28-FORBA_FB_99-3.pdf [consulté le 20/04/2011]

Frangakis M., Hermann C., Huffschmid J., Lorant K., Privatisation Against the European Social Model. A Critique of European Policies and Proposals for Alternatives, Palgrave Macmillan, Houndsmills, 1999

Haidinger B., Hermann C., Arbeitsbedingungen bei den neuen Postdienstleistern. FORBA-Forschungsbericht im Auftrag der Kammer für Arbeiter und Angestellte [en ligne], FORBA, Vienne, 2008. Disponible sur : http://www.forba.at/data/downloads/file/298-FB\%2004-2008.pdf [consulté le 20/04/2011] 
Hermann C., « Die Privatisierung der Gesundheit in Europa », Sozialpolitik in Diskussion, n 5, 2007, p. 5-21

HermanN C., «Durch Privatisierung zum Ausnahmefall: Fragmentierung der Arbeitsbeziehungen in öffentlichen Dienstleistungen in Österreich », in BRANDT T., SCHUlten T., STERKEL G., WiedeMUTH J. (eds), Europa im Ausverkauf. Liberalisierung und Privatisierung öffentlicher Dienstleistungen und Folgen für die Tarifpolitik, VSA-Verlag, Hambourg, 2008, p. 212-232

Hermann C., «Die Liberalisierung des österreichischen Postmarktes, neue Unternehmensstrategien und die Folgen für Beschäftigung und Arbeitsbedingungen », Arbeit \& Wirtschaft, vol. 35, n 2, 2009, p. 237-255

Hermann C., Brandt T., SChulten T., «Commodification, casualisation and intensification of work in liberalised European postal markets », The new gold rush: the new multinationals and the commodification of public sector work, vol. $2, \mathrm{n}^{\circ} 2,2008$, p. 40-55

Hermann C., Flecker J., «Das "Modell" Österreich im Wandel », in Hermann C., AtzmüLler R. (eds), Die Dynamik des „österreichischen Modells“. Brüche und Kontinuitäten im Beschäftigungs- und Sozialsystem, Sigma, Berlin, 2009, p. 17-44

Hofbauer I., Liberalisation, Privatisation and Regulation in the Austrian Postal Service Sector, Rapport d'étude PIQUE, Vienne, 2006a [en ligne] : http://www.pique.at/reports/pubs/PIQUE_CountryReports_Post_ Austria_November2006.pdf [consulté le 20/04/2011]

HOFBAUER I., Liberalisation, privatisation and regulation in the Austrian electricity sector [en ligne], Country report on liberalisation and privatisation processes and forms of regulation, FORBA, Vienne, $2006 \mathrm{~b}$. Disponible sur: http://www.pique.at/reports/pubs/PIQUE_CountryReports_Electricity_Austria_November 2006.pdf [consulté le 20/04/2011]

LINDNER D., Die Liberalisierung der österreichischen Elektrizitätswirtschaft und ihre Auswirkungen auf die Beschäftigungssituation und Versorgungssicherheit, Diplomarbeit, Université de Vienne, Vienne, 2008

ÖSterreichische Post AG, Geschäftsberichte [rapports d'activité], Österreichische Post AG, Vienne, années diverses

TAMme O., Sind die Postdienste auf dem Land noch flächendeckend? Postversorgung auf dem Land nach der Schließungswelle, Bundesanstalt für Bergbauernfragen, Vienne, 2007. 


\title{
Conséquences sociales des processus de « marchéisation » dans le secteur électrique et les services postaux suédois
}

\author{
Monica ANDERSSON BÄCK, Jesper HAMARK, Christer THÖRNQVIST
}

Dans cet article, les auteurs évoquent les processus de marchéisation en Suède et leurs conséquences sociales en s'appuyant sur des exemples issus des services postaux et du secteur électrique suédois. Cette recherche est le résultat de travaux collectifs : Christer Thörnqvist, Jesper Hamark et Monica Andersson Bäck (qui travaillent à l'Université de Göteborg) ont constitué l'équipe suédoise du projet de recherche européen PIQUE.

Le projet PIQUE couvre quatre secteurs - l'électricité, les services postaux, les transports publics locaux et les services de santé/les hôpitaux - et six pays européens : Allemagne, Autriche, Belgique, Grande-Bretagne, Pologne et Suède. Une partie importante de cette présentation est issue du chapitre Marketisation in Swedish electricity and postal services de Christer Thörnqvist, paru dans l'ouvrage Privatisation and liberalisation of public services in Europe édité par Keune, Leschke \& Watt en 2008.

\section{Création des services électriques et postaux : avant la libéralisation.}

Le « modèle suédois » de relations sociales est renommé bien au-delà des frontières du pays (Thörnqvist, 2008, p. 125), et nous y reviendrons plus tard lorsque nous évoquerons les différences entre la Suède et les autres pays observés dans le cadre de ce projet de recherche. On sait moins en revanche qu'il existait il y a près d'un siècle un modèle suédois d'infrastructure économique (cette notion a été développée pour la première fois par Arne Kaijser en 1994) basé sur des facteurs prioritairement sociaux et non techniques.

Ce modèle d'infrastructure commence à se constituer à la fin du XIX ${ }^{\mathrm{e}}$ siècle, avec le développement des centrales hydroélectriques en Suède ; il couvrait par conséquent les secteurs de l'électricité, le système ferroviaire, les téléphones et télégraphes ainsi que des entreprises privées (Thörnqvist, 2008, p. 126). La société avait désespérément besoin d'une alimentation continue en énergie «nouvelle » : l'électricité (Kaijser, 1994, p. 166-80 ; Jakobsson, 1996, p. 74-76). La singularité de ce modèle consistait, selon Kaijser, dans le partage de responsabilité entre organes gouvernementaux, acteurs municipaux et privés.

Ainsi, au sein du marché électrique, il existait un système d'infrastructure informel résultant de la coopération entre l'Etat suédois et d'autres parties intéressées. Les organes gouvernementaux étaient responsables de l'échelon national, alors que les autorités locales étaient chargées de l'échelon régional. Les contacts étaient souvent personnels, et il n'y avait pas d'autorité publique de supervision (Thörnqvist, 2008, p. 126). 
De même, la coopération entre l'Etat suédois et les entreprises privées se traduisait par un généreux soutien du gouvernement aux «champions nationaux ». Les compétences techniques étaient très demandées, et la coopération prospérait, avec l'objectif d'inventions techniques et de solutions innovantes. L'Etat suédois remplissait par conséquent les carnets de commandes d'un certain nombre de grandes entreprises du pays pour leur donner la possibilité de développer des produits à leur propre rythme, à l'abri de la concurrence du marché. La coopération entre l'Etat et ASEA, le prédécesseur d'ABB, constitue l'exemple le plus important de cette relation de symbiose (Thörnqvist, 2008, p. 126).

Le groupe ABB est aujourd'hui leader dans les technologies électriques et d'automatisation ; il est présent dans une centaine de pays et emploie environ 117000 personnes (www.abb.com [consulté le 18/05/11]).

Le système postal constituait toutefois une exception de majeure ; très formaliste, il fut financé, organisé et administré comme une entité strictement publique depuis le milieu du XVII ${ }^{\mathrm{e}}$ siècle jusqu'à la libéralisation dans les années 1990 (Thörnqvist, 2008, p. 126).

La comparaison entre ces deux secteurs de l'électricité et des services postaux est un défi pédagogique et intéressant que nous allons essayer de relever. Ces deux exemples peuvent être considérés comme les deux pôles opposés de part et d'autre d'un continuum d'activités publiques marchéisées.

\section{Le système postal suédois}

Le système postal est l'une des structures publiques les plus anciennes en Suède. Ses origines remontent à la Guerre de Trente ans et à des besoins de renseignement militaire fiable. Les premiers statuts de l'Office postal suédois portent la date du 20 février 1636. Les services initiaux s'appuyaient sur des paysans et des personnes spécialement nommées à cet effet dans les villes, qui pouvaient délivrer les lettres et autres envois postaux. Toutefois, l'introduction d'un système ferroviaire ainsi que la curiosité de l'opinion suscitée par les tendances internationales amenèrent à développer des services postaux modernes avec des boîtes aux lettres, des timbres et un tarif d'affranchissement commun. A cette époque, vers 1860, les services postaux étaient considérés à la fois comme un service commun et comme un outil et un atout pour l'administration publique. Les bureaux de poste se mirent par conséquent à transmettre des paiements, à gérer des mandats-poste et un système de dépôt et livraison. Quelques années plus tard, la structure se vit aussi accorder le statut de caisse d'épargne et, à partir de 1888, on considère qu'elle détenait le monopole des services postaux suédois (Thörnqvist, 2008, p. 131).

\section{Le secteur électrique suédois}

Le secteur électrique suédois a été créé au début des années 1900, s'appuyant sur un besoin d'électricité, un consensus idéologique entre les entreprises et le 
gouvernement, des acteurs résolus à investir, mais aussi sur des conflits avec les droits des riverains (par exemple le droit de chasser le tétras) et de fermiers locaux qui risquaient d'être sérieusement enfreints en cas d'expansion du système hydro-électrique (Thörnqvist, 2008, p. 127).

Mais les obstacles furent facilement levés grâce au consensus entre l'Etat et les capitalistes industriels qui siégeaient au tribunal de l'eau pour définir une nouvelle législation. A l'époque, les détenteurs de droits de riveraineté n'avaient même pas le droit de vote du fait du seuil de revenus/de fortune requis. La levée des obstacles a également eu un impact sur l'emploi. La suppression des droits de riveraineté entraîna le déclin rapide de ce groupe, mais une augmentation du nombre d'ouvriers dans l'industrie avec des emplois comparativement sûrs dans le secteur électrique (Jakobsson, 1996).

Tableau 1 : Les services postaux et le secteur électrique suédois avant la libéralisation

\begin{tabular}{|l|l|l|}
\hline & SERVICES POSTAUX & MARCHE ELECTRIQUE \\
\hline Développement & $\begin{array}{l}1636: \text { création } \\
1850: \text { boite aux lettres, timbres, etc. } \\
1880: \text { caisse d'épargne postale } \\
1888: \text { monopole sur les services postaux }\end{array}$ & $\begin{array}{l}\text { Milieu des années 1800: développement } \\
\text { del'énergie hydro-électrique } \\
\text { Rentable avec système triphasé }\end{array}$ \\
\hline Besoins & $\begin{array}{l}\text { Service de renseignement militaire fiable } \\
\text { Tendances internes et besoin de } \\
\text { communiquer dans une société industrielle }\end{array}$ & $\begin{array}{l}\text { Approvisionnement continu des usines } \\
\text { et des ménages en électricité }\end{array}$ \\
\hline Relation & $\begin{array}{l}\text { Formelle } \\
\text { Structure }\end{array}$ & $\begin{array}{l}\text { Administration formelle et entité } \\
\text { strictement publique }\end{array}$ \\
\hline Public et salariés & $\begin{array}{l}\text { Paysans et personnes nommées à cet effet } \\
\text { Facteurs et guichetiers des bureaux de poste } \\
\text { employés }\end{array}$ & $\begin{array}{l}\text { Déclin des droits de riveraineté } \\
\text { Augmentation des ouvriers dans l'industrie }\end{array}$ \\
\hline
\end{tabular}

Source : Thörnqvist, 2008.

Ce socle historique a bien sûr eu des répercussions importantes sur le développement ultérieur, et donc sur la situation actuelle.

\section{Le point de départ du processus de marchéisation : les arguments en faveur de changements}

Les lecteurs les plus observateurs auront déjà remarqué que nous parlons de libéralisation et de privatisation en termes de «marchéisation » (marketization). C'est un choix délibéré car nous considérons que la notion de "marchéisation » rend bien compte des évolutions en Suède. Cette notion est liée au concept de 
New Public Management [nouvelle gestion publique - NGP], qui englobe la rhétorique et la pratique de la gestion issues des entreprises privées et transposées dans le secteur public. La NGP renvoie à une gouvernance visant la concurrence, le contrôle et la passation de marchés (Almqvist, 2006). Ce concept a été introduit par le chercheur britannique Christopher Hood, de la London School of Economics. Selon Hood, la Suède fait partie des pays qui ont fait le choix d'une mise en œuvre précoce de la NPG, à la suite des exemples américains et britanniques, en même temps que le Canada, la Nouvelle-Zélande et l'Australie (Hood, 1995). La France se trouvait dans la moyenne, tandis que l'Allemagne a procédé à une mise en œuvre tardive de la NPG (Hood, 1995).

Le développement du secteur public et les investissements qui y ont été réalisés après la Seconde Guerre mondiale sont des facteurs importants de l'histoire de la Suède. On peut considérer que l'ouverture de marchés et les dérégulations ont démarré avec la crise financière et la réduction des dépenses au milieu des années 1970 et ont été progressivement réalisées jusqu' aux années 1990.

Au milieu des années 1970, la longue période de croissance économique subit un coup d'arrêt avec la crise pétrolière de 1973, qui représente le tournant ayant ouvert la voie d'une approche néolibérale dans les années 1980 (Lindvall, 2004).

Dans les années 1980, alors que l'économie suédoise souffrait de toute évidence d'une baisse de productivité, la restructuration du secteur public fut remise à l'ordre du jour. En 1989, le gouvernement social-démocrate lança ce qui fut appelé une «enquête publique » sur la faible productivité, qui prit fin deux années plus tard. Les conclusions étaient basées sur la théorie microéconomique néoclassique et niaient toute différence fondamentale entre industrie et services (Thörnqvist, 2007, p. 19-21). Afin de remettre la productivité sur les rails, le groupe d'économistes, de plus en plus influent dans les services publics suédois, recommandait de réduire les coûts et d'exposer à la concurrence les activités qui en étaient protégées (SOU, 1991, n 82, p. 335-42).

Sur fond de difficultés répétées (déflation, chômage, perte de confiance) causées par la grande récession du début des années 1990, un âpre débat politique eut lieu sur la pression nécessaire en faveur de réformes. Le débat portait alors sur deux problèmes principaux, auxquels était apportée une solution commune basée sur les marchés et la gestion.

L'Union européenne et ses critères de convergence furent d'autres facteurs essentiels, qui ont fortement influencé l'économie suédoise ainsi que les niveaux de dépenses publiques (Andersson Bäck, 2008).

Le passage d'un gouvernement social-démocrate à une majorité de droite (1991-1994) entraîna un grand changement d'idéologie. Cependant, la libéralisation fut le résultat de vingt années de débats (Antman, 1994, p. 19).

Le processus fut marqué par de nombreux petits changements qui transformaient peu à peu les marchés d'infrastructures (Thörnqvist, 2008, p. 129). Ainsi, aucun projet de loi ou décision parlementaire ne marque le début de la libéralisation (SOU, 2005, $\mathrm{n}^{\circ}$ 4, p. 150-52). Les réformes se sont développées pendant 
la plus grande crise économique de l'après-guerre en Suède, et ce contexte a été mis en avant comme étant une force majeure à l'œuvre derrière les réformes, plus déterminante que l'idéologie (SOU, 2005, $\mathrm{n}^{\circ} 4$, p. 152-54). Cela signifie que les raisons de la réforme étaient annoncées comme pragmatiques, mais que ses conséquences ont incontestablement eu une portée idéologique.

Un consensus s'établit entre les socio-démocrates et les partis conservateurs sur la nécessité d'ouvrir les marchés protégés, mais selon le baromètre annuel, l'opinion ne soutenait guère ce type de mesures. Depuis 1993, les avis favorables et hostiles à la privatisation des entreprises publiques étaient à peu près à égalité, ou penchaient légèrement contre de telles mesures. Comme un vaste débat public sur la question avait eu lieu au milieu des années 1980 lors des projets de vente de la compagnie suédoise de téléphonie Telia, la plupart des gens avaient déjà un avis sur la privatisation au tournant des années 1990. En outre, les sondages montrent clairement que la position de l'opinion à l'égard de la privatisation n'était pas liée à la satisfaction ou l'insatisfaction vis-à-vis des services publics existants, mais qu'elle était plutôt de nature idéologique (Nilsson, 2001).

Les services postaux et l'électricité furent dérégulés et exposés à la concurrence en même temps. Au-delà des considérations politiques, il est en fait surprenant que ces deux secteurs aient été privatisés alors que l'un et l'autre fonctionnaient bien et avaient une bonne réputation (Högselius et Kaijser, 2007).

\section{Restructuration - forme juridique, parts de marché et nombre d'acteurs en concurrence}

Changements dans le système postal

C'est donc à une histoire de plus de 350 ans d'administration publique qu'il fut mis un terme en mars 1994 lorsque la Poste suédoise fut démantelée et transformée en la société Posten AB. En Suède, le «marché postal » recouvre la distribution de tous les envois postaux. Seule la distribution d'envois postaux de moins de deux kilos fut libéralisée en 1994. Les envois plus lourds ainsi que les journaux etc. ont toujours été ouverts aux entrepreneurs privés. L'une des lignes de partage importantes entre le courrier couvert par le monopole et celui qui était ouvert aux entreprises privées consistait à savoir si l'envoi était «écrit »ou « imprimé », et s'il était adressé à un destinataire unique ou faisait l'objet d'un envoi en nombre (Thörnqvist, 2008, p. 131).

Lorsque l'entreprise Posten AB fut créée en 1994, il lui fallut reprendre la plupart des obligations de la Poste suédoise - contrairement à ses concurrents « libres ». Posten AB doit fournir des services quotidiens à l'ensemble de la population suédoise.

Cela signifie que l'entreprise doit être en mesure de distribuer 20 millions d'envois postaux par jour, expédiés et reçus par 4,5 millions de ménages et 900000 sociétés, cinq jours par semaine. Les obligations de Posten AB, mais 
aussi du gouvernement suédois sont réglementées par cinq lois et décrets différents (Thörnqvist, 2008, p. 133).

Peu de grandes sociétés privées ont été tentées de prendre des parts du marché du courrier en Suède. En fait, le seul concurrent important, CityMail, est détenu par la Poste norvégienne. Grâce à son monopole en Norvège, la Poste norvégienne peut utiliser son excédent pour se développer sur le marché concurrentiel suédois du courrier. Il est vrai que CityMail ne détient qu'une part de 5 à $7 \%$ du marché. L'entreprise n'a jamais eu l'intention de concurrencer Posten $\mathrm{AB}$ dans tous les domaines, mais uniquement sur les segments les plus rentables (Hamark et Thörnqvist, 2007).

Tableau 2 : Restructuration des services postaux et du secteur électrique (1990-2003)

\begin{tabular}{|c|c|}
\hline SERVICES POSTAUX & MARCHE DE L'ELECTRICITE \\
\hline 1998 Directive (67/97/CE) & $\begin{array}{l}1990 \text { Les sociaux-démocrates lancent une } \\
\text { réforme de transformation } \\
\text { des organismes publics }\end{array}$ \\
\hline $\begin{array}{l}1993 \text { Projet de loi suédois de dérégulation } \\
\text { entrant en vigueur en } 1994 \text { - les envois } \\
\text { postaux de moins de deux kilos sont } \\
\text { libéralisés ; envois écrits et non imprimés }\end{array}$ & $\begin{array}{l}1996 \text { Une loi ouvre la production et la } \\
\text { fourniture à la libre concurrence. } \\
\text { Le transport d'électricité n'est pas soumis à } \\
\text { la «marchéisation» }\end{array}$ \\
\hline $\begin{array}{l}1994 \text { Les Postes suédoises sont démantelées et } \\
\text { transformées en société Posten AB }\end{array}$ & $\begin{array}{l}1992 \text { Vattenfall est démantelé et transformé } \\
\text { en la société par actions détenue par le } \\
\text { gouvernement Vattenfall AB }\end{array}$ \\
\hline $\begin{array}{l}\text { Obligations réglementées par cinq lois et } \\
\text { décrets. Services quotidiens à destination } \\
\text { de toute la population suédoise : } \\
20 \text { millions d'envois postaux / jour } \\
4,5 \text { millions de foyers et } 900000 \text { entreprises, } \\
\text { cinq jours par semaine }\end{array}$ & $\begin{array}{l}\text { Réglementation juridique plus stricte. } \\
\text { L'autorité publique de régulation, } \\
\text { Energimyndigheten, } \\
\text { reçoit ses instructions sous forme } \\
\text { de « lettres de régulation » annuelles (lois } \\
\text { européennes et décrets inclus) }\end{array}$ \\
\hline $\begin{array}{l}\text { Concurrent CityMail, sur le segment } \\
\text { des services postaux aux entreprises. } \\
\text { Envois en nombre }\end{array}$ & Voir tableau 3 \\
\hline $\begin{array}{l}\text { Parts de marché en } \mathbf{2 0 0 3} \\
\text { Posten } \mathrm{AB} 92,9 \% \text { du marché } \\
\text { CityMail } 6,6 \% \\
\text { Autres concurrents } 0,5 \%\end{array}$ & \\
\hline
\end{tabular}

Source : Thörnqvist, 2008.

La société CityMail a été créée juste avant la libéralisation, en 1991, en s'appuyant sur une innovation de son fondateur, un expert dans le domaine de la logistique. Cette innovation est issue du développement des TIC, qui a rendu possible un tri plus efficient des destinataires et des envois, permettant ainsi de ré- 
duire les prix. Cette innovation a eu pour résultat la création de services postaux industrialisés. Ce segment lucratif couvrait les envois de masse émanant d'entreprises ayant de nombreux clients ; il se gérait donc sur d'importants volumes faciles à gérer. C'est-à-dire que Posten $\mathrm{AB}$ a des obligations spécifiques et que la concurrence ne s'est présentée que sur le segment particulier des services postaux aux entreprises. Cette concurrence avec Posten $A B$ fut très favorable à CityMail. L'entreprise est également en charge de la loterie nationale, basée sur le code postal des personnes, une activité très populaire et très lucrative (Hamark et Thörnqvist, 2007).

La commission nationale chargée de la libéralisation suédoise fait valoir que la concurrence a eu un effet global positif sur le marché du courrier en Suède. Les preuves empiriques à l'appui de cette conclusion sont cependant assez minces. Posten $\mathrm{AB}$ n'a qu'un concurrent significatif sur ce marché, à savoir CityMail, qui se concentre en fait sur un segment de clientèle. En 2003, dix ans après la libéralisation, Posten $\mathrm{AB}$ détenait toujours $92,9 \%$ du marché ; CityMail en avait 6,6\% et d'autres concurrents se partageaient les $0,5 \%$ restants.

Afin de devenir plus compétitif sur le marché nordique, Posten $A B$ et son homologue danois ont annoncé en 2008 leur intention de fusionner en une société qu'ils détiendraient conjointement. L'information a été rendue officielle le $1^{\text {er }}$ avril 2008, jour des poissons d'avril ; ce fut une nouvelle difficile à croire pour les Suédois qui regardaient la télévision ce jour-là, car les Suédois et les Danois sont des voisins qui aiment faire de mauvaises blagues l'un sur l'autre. Comme si la France et la Belgique s'engageaient dans une entreprise conjointe de services postaux.

\section{Changements dans le secteur électrique}

Le 26 octobre 1990, le gouvernement social-démocrate lance une dynamique de réforme pour faire face à la crise monétaire et rendre plus efficiente la gestion des biens publics; l'une des mesures consista à transformer Vattenfall en une société par actions aux mains de la puissance publique (Thörnqvist, 2008, p. 134).

Après un certain nombre de préparatifs, fusions et autres changements organisationnels, la nouvelle société Vattenfall, dont les activités sont scindées, est créée le $1^{\text {er }}$ janvier 1992.

Le $1^{\text {er }}$ janvier 1996, une nouvelle loi est adoptée qui ouvre à la fois la production et la distribution à la libre concurrence. Le transport, qui se fait à grande échelle, n'est pas marchéisé. Les trois niveaux de transport existants, national, régional et local, forment ensemble un monopole «naturel », et il y a peu à gagner de la privatisation ou de la concurrence. Par conséquent, le marché de l'électricité est désormais composé de trois segments : production, transport et distribution à destination des différents clients, ménages compris (Högselius et Kaijser, 2007, p. 138-39 ; Andersson et Thörnqvist, 2007a). 
Après la dérégulation, le secteur fut soumis à des réglementations juridiques plus strictes. L'autorité publique de régulation, Energimyndigheten, reçoit ses instructions sous la forme de «lettres de régulation» annuelles. Ces consignes sont très détaillées et réglementent non seulement les questions de concurrence et de sécurité mais aussi la recherche et l'introduction/diffusion de sources d'énergie alternatives, en particulier les centrales éoliennes. Toutes les lois et tous les décrets européens ont été transposés en droit suédois et leur contenu est donc traité par les lettres de régulation (Andersson et Thörnqvist, 2007a).

Berg (1999) indique que la libéralisation du marché électrique suédois avait trois motivations principales :

- le souhait de s'adapter aux politiques de libre marché de l'AELE et de l'UE ;

- l'ouverture d'autres marchés de pays nordiques - en particulier la libéralisation norvégienne en 1991 et la création du marché commun nordique de l'électricité, Nord Pool; et

- une «forte volonté politique » de libéraliser et de privatiser les biens publics. Ce dernier facteur fut sans doute, comme nous l'avons suggéré, le plus important.

Le tableau ci-dessous résume et compare les situations d'avant 1996 et de 2004, en s'intéressant au «monopole » et aux principaux nouveaux acteurs du marché.

Tableau 3 : Le marché électrique suédois avant le 1er janvier 1996 et en 2004

\begin{tabular}{|c|c|c|}
\hline $\begin{array}{l}\text { Secteur du } \\
\text { marché }\end{array}$ & Avant le $1^{\text {er janvier } 1996}$ & 2004 \\
\hline Production & $\begin{array}{l}\text { - VATTENFALL (puissance } \\
\text { publique) } \\
\text { «clubs» détenus par des } \\
\text { actionnaires privés ou } \\
\text { par les municipalités, } \\
\text { sous } 1 \text { 'influence des } \\
\text { pouvoirs publics }\end{array}$ & 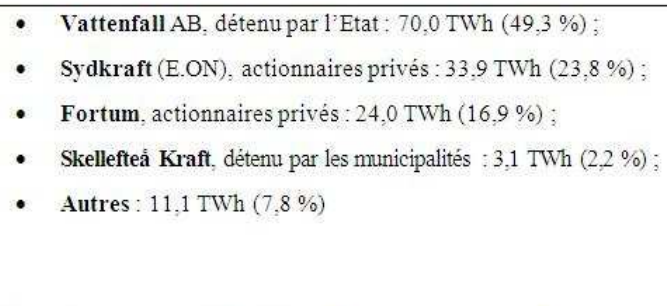 \\
\hline Transport & $\begin{array}{l}\text { Stamnätsklubben (un « club» } \\
\text { administré par les pouvoirs } \\
\text { publics; membres privés) }\end{array}$ & $\begin{array}{l}\text { Svenska Kraftnät (entreprise publique ayant des filiales } \\
\text { détenues par des actionnaires privés et des municipalités, } \\
\text { dont les plus importantes sont Vattenfall, Sydkraft et Fortum) }\end{array}$ \\
\hline $\begin{array}{l}\text { Distribution } \\
\text { vente }\end{array}$ & Vattenfallsverket + les clubs & $\begin{array}{l}\text { - Vattenfall AB, détenu par 1'Etat }(22,7 \%) \text {; } \\
\text { - E.ON (Sydkraft), actionnaires privés }(17,3 \%) \text {; } \\
\text { - Fortum, actionnaires privés }(17,3 \%) \\
\text { - } \\
\text { Autres }(42,7 \%)\end{array}$ \\
\hline
\end{tabular}

Source : Energimyndigheten [en ligne]. Disponible sur http://www.stem.se (production); Energimarknadsinspektionen [en ligne]. Disponible sur: http://www.energimarknadsinspektionen.se (distribution/vente) [consultés en janvier 2008]. 
Cette comparaison montre que les résultats diffèrent fortement à certains égards : les services postaux restent reconnaissables par rapport à leur forme précédente, un opérateur, Posten $\mathrm{AB}$, détenant encore plus de $90 \%$ du marché (malgré la présence sur certains segments importants du seul concurrent sérieux, CityMail).

Le secteur électrique, quant à lui, s'est trouvé réparti entre différents acteurs présentant une grande variété de formes juridiques. Il est difficile d'établir un tableau complet de ce secteur, en particulier sur le marché de détail (Thörnqvist, 2008, p. 125).

Le marché des transports reste fortement monopolistique. Les monopoles sont régionaux, cependant, et couvrent différentes parties de la Suède. Paradoxalement, cela signifie que Vattenfall, Fortum et Sydkraft - autrement dit, les mêmes concurrents que sur les segments de la production et de la distribution d'électricité - détiennent chacun leur propre monopole régional et ne sont donc pas en concurrence. Outre ces trois acteurs, on compte quelques municipalités et entreprises disposant de leur propre réseau régional de transport (Svenska Kraftnät [en ligne]. Disponible sur : http://www.svk.se [consulté en janvier 2008]).

\section{Conséquences en termes de qualité de service et de productivité}

On pourrait trouver des exemples de forte productivité et d'amélioration de la disponibilité du service sur certains segments de clientèle, et une qualité globalement moindre sur d'autres.

Pour l'usager suédois moyen, il apparaît qu'il ne s'est pas passé grandchose ; le seul changement qui ait directement affecté la vie quotidienne des gens est la fermeture de bureaux de poste - les petites « agences postales » destinées aux opérations financières sont supprimées, et la vente de timbres ainsi que l'expédition et la réception de colis trop encombrants pour une boîte aux lettres sont maintenant gérées par des supermarchés et autres magasins. Les citoyens suédois ont en outre assisté à une diminution des services postaux, à la fermeture de bureaux de poste et à la disparition de boîtes aux lettres.

Dans le secteur électrique, la libéralisation a entraîné une hausse des prix à la consommation. Or, les prérequis pour la transformation d'électricité n'ont pas changé depuis une centaine d'années, c'est toujours la même technologie (Thörnqvist, 2008, p. 133).

En 2005, une «commission publique » évaluait les effets du processus de marchéisation. Elle a été appelée «commission des règlements » (Regelutredningen). Même si l'intention annoncée était de créer une commission impartiale, les commissions sont rarement aussi «neutres » qu'elles le prétendent, et celle-ci ne fit pas exception. La commission des règlements avait pour mission d'examiner les effets du processus de libéralisation sur les prix à la consommation, et il était entendu que le résultat devait être positif. Mais la commission ne parvenant pas à trouver de tels résultats, elle se contenta de s'excuser de leur absence (Thörnqvist, 2008, p. 129). 
La commission a donc avancé un certain nombre d'arguments pour expliquer pourquoi la libéralisation n'avait pas produit les effets escomptés, par exemple le fait que le processus de marchéisation n'avait pas été mis en œuvre correctement. Cependant les analyses furent menées sans remettre en cause la cohérence de la théorie néoclassique selon laquelle un «marché en concurrence parfaite » est préférable pour le consommateur à un monopole (cf. Keen, 2004, p. 97-107). Pas un mot ne mentionne le fait que la marchéisation puisse avoir ses inconvénients et entraîner d'autres problèmes, peut-être aussi graves. La commission a annoncé cependant d'emblée qu'elle avait d'importants problèmes méthodologiques en raison d'un manque d'enquêtes et d'informations pertinentes (Thörnqvist, 2008, p. 131).

Tableau 4 : Développements dans six grands secteurs après la libéralisation, Suède

\begin{tabular}{|c|c|c|c|c|c|c|}
\hline & Postes & Taxis & $\begin{array}{l}\text { Chemins de } \\
\text { fer }\end{array}$ & $\begin{array}{l}\text { Aviation } \\
\text { nationale }\end{array}$ & Electricité & Télécoms \\
\hline $\begin{array}{l}\text { 1. Prix comparés à la } \\
\text { tendance générale des } \\
\text { prix }\end{array}$ & Hausse & Hausse & Hausse & Hausse & Hausse & Baisse \\
\hline 2. Volume de production & Baisse & - & Hausse & Baisse & Inchangé & Hausse \\
\hline 3. Rentabilité & Inchangée & Hausse & Inchangée & Baisse & - & Inchangée \\
\hline 4. Emploi & Baisse & Hausse & Baisse & Baisse & Baisse & Baisse \\
\hline $\begin{array}{l}\text { 5. Revenus parrapport } \\
\text { au commerce et à } \\
1 \text { 'industrie en général }\end{array}$ & Hausse & Baisse & Baisse & Baisse & Baisse & Hausse \\
\hline 6. Productivité & Hausse & - & Hausse & Hausse & - & Hausse \\
\hline $\begin{array}{l}\text { 7. Concentration du } \\
\text { marché }\end{array}$ & Baisse & Baisse & Baisse & Baisse & Baisse & Baisse \\
\hline 8. Accessibilité & - & Hausse & Hausse & - & - & Hausse \\
\hline
\end{tabular}

Source : Regelutredningen, SOU, 2005, n 4, p. 48.

La commission a analysé l'évolution après la libéralisation en Suède dans six secteurs importants. L'évaluation a pris en compte huit aspects de la libéralisation, sans que soient sérieusement discutés d'autres paramètres que l'efficience (productivité) et les prix à la consommation.

1. Le prix par rapport à la tendance générale des prix a augmenté à la fois dans le secteur postal et celui de l'électricité, comme dans tous les secteurs comparés sauf les télécoms, où l'on a observé une baisse des tarifs.

Le rapport faisait par ailleurs valoir que, même si la marchéisation n'a pas eu de bénéfices visibles pour les consommateurs d'électricité ou pour la société dans son ensemble, la situation aurait été bien pire en l'absence de dérégulation (Damsgaard et Green, 2005). Cette conclusion s'appuie toutefois uniquement, dans une démarche hypothético-déductive, sur l'analyse de ce qui aurait pu arriver si la régulation de marché avait été maintenue telle qu'elle était avant 1996. De plus, la recherche a été menée sous l'influence d'un «groupe de réfé- 
rence » composé de représentants de plus ou moins toutes les entreprises et structures publiques (Damsgaard et Green, 2005, p. 10) susceptibles de bénéficier d'une manière ou d'une autre d'un résultat « positif » montrant que la marchéisation était et reste la seule voie à suivre. Aucune voix d'opposition n'était intégrée au groupe de référence et tous les modèles testés étaient inspirés de l'économie néoclassique, ce qui ne laissa aucune place à d'autres solutions que l'économie de marché d'influence néolibérale.

2. Le volume de production a diminué dans les services postaux et est resté inchangé dans le secteur électrique.

3. La rentabilité est restée inchangée dans les services postaux et il est difficile de trouver des informations pour l'ensemble des acteurs du secteur électrique, mais certains sont gagnants, d'autres perdants.

4. L'emploi est en baisse dans le secteur postal comme dans celui de l'électricité, de même que dans tous les autres secteurs étudiés, à l'exception des taxis.

5. Les revenus par rapport au commerce et à l'industrie en général augmentent pour les services postaux et sont en baisse dans le secteur électrique

6. La productivité a augmenté dans les services postaux, et il est difficile de présenter des chiffres pour l'ensemble du secteur électrique.

7. La concentration du marché a été réduite dans les six secteurs qui ont été libéralisés.

8. Il est difficile d'affirmer quoi que ce soit concernant l'accessibilité.

\section{Conséquences en termes d'emploi et de conditions de travail}

Bien souvent, les salariés restés dans les secteurs évoqués doivent s'accommoder des changements. L'une des principales conclusions, c'est que la marchéisation, menée au nom de la concurrence et, en outre, en tant qu'effet d'une concurrence accrue, a eu pour conséquence une pression élevée de réduction des coûts du travail et des réductions d'effectifs. La rationalisation et l'externalisation font partie des effets de la libéralisation, de même que l'intensification du travail, le stress et la détérioration des conditions de travail.

Les personnes interviewées dans le cadre du projet Pique

Les syndicalistes de Posten $\mathrm{AB}$ interviewés sont tous les trois membres de la principale confédération d'ouvriers de la fonction publique, SEKO.

Un grand nombre de salariés de Vattenfall étant des employés, le troisième et dernier partenaire de l'entreprise interviewé est issu de la plus importante confédération de salariés, Unionen. Comme nous le verrons, les visions divergent considérablement sur plusieurs points entre les syndicats d'ouvriers et d'employés (Thörnqvist, 2008, p. 138). 


\section{Posten $A B$}

Les évolutions et la concurrence accrue dans les services postaux ont eu notamment pour conséquence une baisse constante du nombre de salariés depuis le milieu des années 1990. Le personnel de Posten AB est passé de 35250 salariés en 1990 à environ 15000 employés des postes à plein temps fin 2007 (Thörnqvist, 2008, p. 139- 140).

Les tâches sont fondamentalement les mêmes qu'avant la restructuration. Environ $80 \%$ des facteurs sont employés sur des contrats à plein temps et la répartition hommes/femmes est à peu près équilibrée. Les heures de travail ont été généralement réduites à la fin des années 1990, avec le passage de 40 à 38 heures hebdomadaires. Les emplois du temps sont cependant définis sur une base mensuelle afin de faire face aux fluctuations de volume. Les interviewés font valoir que les salariés ont déjà "payé » pour l'accord sur la semaine de 38 heures avec des augmentations de salaires très faibles depuis plusieurs années. En outre, la rémunération totale a de facto diminué depuis la restructuration dans la mesure où les possibilités d'heures supplémentaires ont disparu ou se sont au moins considérablement réduites (Thörnqvist, 2008, p. 140).

Au départ, CityMail a adopté une attitude plutôt hostile à l'égard des syndicats mais, depuis lors, l'entreprise s'y est montrée plus favorable. Le taux de syndicalisation plus faible chez CityMail tient surtout à la différence de politique d'emploi par rapport à Posten $\mathrm{AB}$. Il a toujours été entendu entre Posten $\mathrm{AB}$ et le syndicat qu'être facteur constitue une véritable profession ; c'est un métier qu'il faut apprendre et perfectionner en le pratiquant, et c'est un métier que les gens sont susceptibles de poursuivre jusqu'à leur départ en retraite. Pour CityMail, en revanche, la distribution de courrier est un emploi que les gens peuvent prendre pour une courte durée, généralement quand ils sont jeunes et en attente d'autres opportunités de carrière.

Le turnover annuel est actuellement d'environ $30 \%$ chez CityMail, selon SEKO. Les jeunes salariés ne voient donc pas vraiment la nécessité que les syndicats interviennent pour sécuriser les conventions collectives, les assurances, etc. D'un autre côté, les syndicalistes que nous avons interviewés indiquent que CityMail est parfois plus favorable aux salariés que Posten $\mathrm{AB}$, particulièrement en ce qui concerne le recrutement. L'entreprise propose au départ un contrat temporaire et, à échéance de ce contrat, le salarié peut choisir de partir ou de prendre un poste permanent.

Posten $\mathrm{AB}$ a en revanche « une capacité de tout fiche en l'air !» La société propose trop de formes différentes de contrats temporaires et peu de postes à durée indéterminée (Thörnqvist, 2008, p. 140-141).

Vattenfall

Programmes de réduction des effectifs et de rationalisation - ratios

La pression renforcée sur les coûts et sur la rentabilité chez Vattenfall AB a entraîné des programmes de rationalisation qui ont eu pour conséquence de 
lourdes réductions d'emplois. Ce sont en particulier les emplois techniques dans le domaine du support, de la maintenance et de l'investissement qui ont été touchés par la rationalisation. Les ventes et le marketing, à l'inverse, se sont développés. Les chiffres communiqués par le syndicat SEKO montrent qu'environ un tiers des installateurs ont perdu leur emploi. Le ratio entre personnels administratifs et «ouvriers de terrain » était de 30/70 avant la marchéisation et est approximativement passé à 70/30. Cela montre sans doute la préférence générale de l'entreprise pour les ventes, au détriment de la production et de la maintenance « traditionnelles » (Thörnqvist, 2008, p 141-142).

Tableau 5 : Restructuration de l'emploi dans les services postaux et le secteur électrique (1980-2010)

\begin{tabular}{|c|c|c|}
\hline Vattenfall & $\begin{array}{l}\text { Ouvriers sur des postes techniques } \\
\text { support, maintenance et investissement } \\
\text { (par exemple installateurs) } \\
\text { représentés by SEKO }\end{array}$ & $\begin{array}{l}\text { Emplois administratifs dans les } \\
\text { ventes et le marketing - } \\
\text { Représentés par UNIONEN }\end{array}$ \\
\hline $\begin{array}{l}\text { Années } 1980 \\
\text { Entreprise publique regroupan } \\
9 \text { à } 10000 \text { salariés en Suède }\end{array}$ & & \\
\hline Avant la marchéisation & $70 \%$ & $30 \%$ \\
\hline $\begin{array}{l}2010 \\
\text { Groupe nord-européen comptant } \\
32300 \text { salariés au total, } \\
\text { dont } 8400 \text { travaillent en Suède }\end{array}$ & & \\
\hline Après la marchéisation & $30 \%$ & $70 \%$ \\
\hline
\end{tabular}

Source : Thörnqvist, 2008.

Cette évolution résulte en une préparation insuffisante aux coupures d'électricité. Les interruptions à petite échelle sont courantes depuis toujours, mais on a vu ces cinq dernières années environ des tempêtes assez grosses pour mettre à mal l'ensemble du système électrique, privant les consommateurs de lumière, d'appareils ménagers, de réfrigérateurs, télévision, etc. Ni les personnes que nous avons interrogées ni les documents officiels de SEKO n'incriminent explicitement les nouvelles formes d'organisation, mais il est clair que le problème tient, au moins en partie, au manque de personnel compétent du fait de l' «allégement » de l'organisation.

En dépit de ce qu'avance SEKO, il est difficile de dire si la restructuration a entraîné une réduction d'effectifs ou non. Vattenfall était une entreprise publique comptant 9 à 10000 salariés en Suède à la fin des années 1980 ; c'est aujourd'hui un groupe nord-européen employant 32300 salariés au total, dont 8400 travaillent en Suède (Thörnqvist, 2008, p. 141-142). 
Le représentant d'Unionen était quant à lui moins hostile aux programmes de rationalisation. Il estime que Vattenfall devait réagir pour faire face à la concurrence nouvelle et que l'entreprise a agi en bon employeur, en expérimentant différents modèles et méthodes douces pour réduire les effectifs, permettant aux salariés licenciés de trouver de nouvelles opportunités (Sabel, 2001).

\section{Gestion des ressources humaines pour les ouvriers}

Les représentants du syndicat SEKO interviewés soulignent fortement le fait que la restructuration a entraîné une perte de qualification. Ils déplorent un manque de ressources pour la formation après la réorganisation.

De plus, l'âge moyen des salariés sur des emplois techniques est élevé et le recrutement «quasi inexistant » ce qui, ajouté au manque de développement des compétences, aggrave le manque de préparation de la main-d'œuvre à affronter l'avenir. Du fait de licenciements, préretraites forcées et du manque de recrutement, la plupart des monteurs électriciens ont aujourd'hui plus de 50 ans. L'âge officiel de départ en retraite pour la plupart des emplois est de 65 ans en Suède, mais en raison de la pénibilité physique, rares sont les monteurs qui gardent leur poste aussi tard.

Le nombre d'adhérents de SEKO ayant des contrats à durée indéterminée a diminué peu à peu car les entreprises principales - c'est-à-dire pas seulement Vattenfall - ont préféré acheter les services de sociétés plus petites, spécialisées, répondant davantage à la volonté d'alléger l'organisation. La nouvelle politique de gestion des ressources humaines (GRH) après la restructuration - c'est-à-dire l'externalisation de certaines tâches de maintenance et d'autres services - a entrâné une division du personnel entre une « catégorie A et B » (cf. Atkinson et Meager, 1986).

L'environnement de travail s'est déjà considérablement dégradé du fait des licenciements, qui ont accru la charge de travail et le stress des ouvriers. L'un des enjeux cruciaux sera par conséquent la préservation du contenu des conventions collectives pour assurer que les ouvriers "périphériques » et intérimaires aient eux aussi des standards et des salaires corrects (Thörnqvist, 2008, p. 141-142).

\section{Gestion des ressources humaines pour les employés}

Aucun des problèmes soulevés par les représentants de SEKO ne semblait constituer une menace pour le membre d'Unionen.

En réponse à une question directe, notre interlocuteur a déclaré ne voir aucune espèce de conséquence négative du fait de la restructuration. Au contraire, le propriétaire - c'est-à-dire l'Etat - ne s'immisce plus dans des détails comme il le faisait auparavant.

Mais le répondant montrait une certaine déception quant au déclin de l'attention accordée à la perspective à long terme : Vattenfall est devenu plus tributaire de résultats trimestriels positifs (Thörnqvist, 2008, p. 141-142). 


\section{Similarités et différences de " marchéisation » entre la Suède et les autres pays étudiés. Eléments d'explication}

\section{Le modèle suédois de relations sociales}

Beaucoup de salariés du secteur électrique et des services postaux ont subi une dégradation de leur situation, ce que les salariés suédois ont en commun avec leurs homologues des autres pays étudiés dans le cadre du projet PIQUE. Dans une certaine mesure, les syndicats ont perdu de l'influence, les conditions d'emploi et de travail se sont détériorées à mesure que les entreprises privées se concentraient uniquement sur les aspects financiers.

L'on pourrait dire que ce qui est gagné du côté du consommateur est perdu du côté du producteur.

Il semble toutefois que les exemples suédois diffèrent à certains égards des autres pays du projet de recherche PIQUE. Le système suédois de conventions collectives, très solide, n'a pas été particulièrement affecté par le processus de libéralisation (Thörnqvist, 2008, p. 140).

Les caractéristiques suivantes décrivent les relations sociales suédoises ${ }^{1}$. Voir aussi Andersson et Thörnqvist (2007b)

- Des positions fortes des syndicats (malgré un affaiblissement avec l'actuel gouvernement de droite) ;

- une structure conventionnelle fortement centralisée (quoique les salaires négociés individuellement soient fréquents);

- formation centralisée des salaires, malgré une part croissante de dispersion salariale ;

- la stabilité à long terme des relations d'emploi, autrefois importante, est affaiblie ;

- instrument important pour les politiques nationales d'emploi.

Comme le souligne Kjellberg (2000, 2006), le taux de syndicalisation et la force des syndicats dépendent largement d'une combinaison d'organisation centrale et de structures locales. Le pouvoir de négociation centralisée empêche la couverture conventionnelle de se fragmenter et facilite les «politiques salariales solidaires », alors que des réseaux extensifs de sections syndicales locales, bien intégrées aux syndicats nationaux, rapprochent les syndicats de leur base.

La Suède a des syndicats forts en raison de ce qu'on appelle le "système Ghent », c'est-à-dire des régimes d'assurance chômage gérés par des syndicats, financés à l'aide de fonds publics, ce qui les met en contact avec les personnes en recherche d'emploi ou bénéficiaires de prestations. Or, pour être efficace, ce

\footnotetext{
${ }^{1}$ Cela inclut les prélèvements résultant des lois sur la cogénération et les énergies renouvelables, ainsi que la taxe sur l'électricité et la TVA, à quoi s'ajoutent les redevances de concession.
} 
système doit rester associé à des organisations syndicales bien structurées en confédérations au niveau des secteurs et présentes dans les entreprises (Kjellberg, 2000).

La Suède se démarque par ailleurs d'autres pays «centralisés » dans la mesure où elle s'appuie davantage sur la négociation collective que sur la législation. Il faut par exemple noter que la législation suédoise ne définit pas de salaires minimums, une problématique couverte par les conventions collectives.

Les conventions collectives couvrent près de $90 \%$ des salariés suédois et tous les secteurs du marché de l'emploi. En outre, les syndicats suédois négocient les salaires pour les ouvriers dans les cadres définis par les accords sectoriels. La Suède n'a pas de comités d'entreprise ; au lieu de cela, toutes les négociations, à tous les niveaux de l'entreprise, sur les salaires et les conditions de travail, sont traitées par une section syndicale locale.

Il en coûte cependant relativement peu aux employeurs suédois, quelle que soit la taille de l'entreprise, de licencier des salariés. Si une société ne peut plus se permettre de garder sa main-d'œuvre, elle peut licencier ses salariés avec seulement six mois de préavis.

Il existe un filet de sécurité fort pour les personnes sans emploi, même si ce dispositif est en érosion constante depuis les années 1990, une tendance qui à ce jour (en 2007) ne s'est toujours pas inversée.

Paradoxalement, Le pays qui constate aujourd'hui le moins de disparités, la Suède, pourrait dans un avenir proche être celui où les syndicats auront le plus de difficultés à défendre le système de rémunération. Le système suédois, dépourvu de salaire minimum légal, pourrait facilement être la proie du dumping social du fait d'entreprises étrangères amenant avec elle des salariés venus du pays où se trouve le siège de l'entreprise.

Traduction de Marie GRAVEY

\section{Indications bibliographiques}

ABB ASEa Brown Boveri LTD, ABB Power and Productivity for a better world [en ligne]. Disponible sur : http://www.abb.com [consulté le 17/05/11]

ALMQVist R., New Public Management - om konkurrensutsättning, kontrakt och kontroll., Liber, Malmö, 2006

ANDERSSON M., THöRNQVIST C., Liberalisation, privatisation and regulation in the Swedish electricity sector. Country report on liberalisation and privatisation processes and forms of regulation [en ligne], Université de Göteborg, Göteborg, 2007a. Disponible sur : http://www.pique.at/reports/reports.html [consulté le $11 / 05 / 11]$

ANDERSSON M., Thornqvist C., «Determining wage in Europe's SMEs: how relevant are the trade unions? », Transfer, vol. $13, \mathrm{n}^{\circ} 1,2007 \mathrm{~b}$, p. 55-74

ANDERSSON BäCK M., Conceptions, conflicts and contradictions at the introduction of a Swedish Health Call Centre, Thèse de sciences du travail, Université de Göteborg, Göteborg, 2008 
ANTMAN P., « Vägen till systemskifte - den offentliga sektorn i politiken 1970-1992 », in GUSTAFSSON R. A. (ed), Köp och sälj, var god svälj? - vårdens nya ekonomistyrningssystem $i$ ett arbetsmiljöperspektiv, Arbetsmiljöfonden, Stockholm, 1994, p. 19-66

AtKinson J., Meager N., Changing working patterns: how companies achieve flexibility to meet new needs, National Economic Development Office, Londres, 1986

BERG A., Staten som kapitalist: Marknadsanpassningen av de affärsdrivande verken 1976-1994, Acta Universitatis Upsaliensis, Uppsala, 1999

ENERGIMYNDIGHETEN, Energimyndigheten [en ligne]. Disponible sur : http://www.stem.se [consulté en janvier 2008]

ENERGIMARKNADSINSPEKTIONEN, Energimarknadsinspektionen [en ligne]. Disponible sur: www.energi marknadsinspektionen.se [consulté en janvier 2008]

Hamark J., ThörnQvist C., 'Post Modernism or Post Mortem?' A Case Study of the Swedish Post for the PIQUE Meeting in Düsseldorf, 18-19. October 2007, [s.e.], [s.1.], 2007

HögSEliUS P., KAIJSER A., När folkhemselen blev internationell: Elavregleringen i historiskt perspektiv, SNS, Stockholm, 2007

Hood C., « The "New Public Management" in the 1980s: variation on a theme », Accounting, Organizations and Society, vol. 20, n 2-3/1995, p. 93-109

JАKOBSSON E., Industrialisering av älvar: Studier kring svensk vattenkraftsutbyggnad 1900-1918, Avhandlingar från Historiska institutionen i Göteborg 13, Thèse, Université de Göteborg, Göteborg, 1996

KAIJSER A., I fädrens spår: Den svenska infrastrukturens historiska utveckling och framtida utmaningar, Carlssons, Stockholm, 1994

KeEn S., Debunking Economics: The Naked Emperor of the Social Sciences, Zed Books, Londres \& New York, 2004

KJEllberg A., "The Multitude of Challenges Facing Swedish Trade Unions », in WadDINGTON J., HofFMANN R (eds), Trade Unions in Europe, ETUI, Bruxelles, 2000, p. 529-573

KJELLBERG A., « The Swedish unemployment insurance: will the Ghent system survive? », Transfer, vol. 12, $\mathrm{n}^{\circ} 1,2006$, p. 87-98

Lindvall J., The Politics of Purpose: Swedish Macroeconomic Policy after the Golden Age, Thèse de Science Politique, Université de Göteborg, Göteborg, 2004

NiLSSON L., «Förnyad polarisering i välfärdspolitiken », in HolMBERG S., WEIBULL L. (eds), Land du välsignade? SOM-undersökningen 2000, rapport $\mathrm{n}^{\circ}$ 26, Université de Göteborg, Institut SOM, Göteborg, 2001, p. 79-91

SABEL O., « Personalminskning och kompetensväxling inom Vattenfall - Uppföljning av ett omställningsprojekt », in GRI-rapport, Institut de Recherches de Göteborg, Ecole d'Economie et de Droit commercial, Université de Göteborg, Göteborg, 2001, p. [n.c.]

SOU, Drivkrafter för produktivitet och välstånd: Produktivitetsdelegationens betänkande, Allmänna Förlaget, Stockholm, 1991, $\mathrm{n}^{\circ} 82$

SOU, Liberalisering, regler och marknader: Betänkande av Regelutredningen, Statens offentliga utredningar, [s.e.], Stockholm, 2005, $\mathrm{n}^{\circ} 4$

SvensKa Kraftnät, Svenska Kraftnät [en ligne]. Disponible sur :http://www.svk.se [consulté en janvier 2008]

Thörnqvist C., «Marketisation in Swedish electricity and postal services », in KEUNE M., LESCHKE J., WATT A. (eds), Privatisation and liberalisation of public services in Europe, ETUI-REHS, Bruxelles, 2008, p. [n.c.]

Thörnqvist C., «Changing Industrial Relations in the Swedish Public Sector: New Tensions within the Old Framework of Corporatism », International Journal of Public Sector Management, vol. 20, n 12007, p. 16-33. 



\section{LES AUTEURS}

\section{Jean-François AMADIEU}

Directeur du Centre d'Etude et de Recherche sur les Organisations

et la Gestion des Relations Sociales (CERGORS), Université Paris I

\section{Monika ANDERSSON BÄCK}

Chargée de recherches au Département des sciences du travail, Université de Göteborg

\section{Dominique AUBRY}

Secrétaire général adjoint, CFDT Cheminots

\section{Dominique BAILLY}

Directeur du pilotage stratégique RH, Groupe La Poste

Président du Comité de dialogue social européen pour le secteur postal

\section{Jean-Pierre BASILIEN}

Directeur de projets, Entreprise\&Personnel, Paris

\section{Pierre BAUBY}

Conseiller du Secrétariat général du Centre européen des entreprises à participation publique et des entreprises d'intérêt économique général (CEEP) sur les « Services d'intérêt général »

\section{Heinz-J. BONTRUP}

Professeur à la Fachhochschule de Gelsenkirchen, Département de droit économique

\section{Jean-Claude BOUAL}

Animateur du Comité européen de liaison sur les services d'intérêt général (Celsig)

\section{Torsten BRANDT}

Chercheur à l'Institut des Sciences Economiques et Sociales (Wirtschafts- und Sozialwissen schaftliches Institut, WSI) de la Fondation Hans Böckler, Düsseldorf (jusqu'en 2009) Actuellement Chercheur à l'Institut de Sociologie de l'Université de Duisburg-Essen

\section{Bernard CARON}

Directeur des Relations Sociales du Groupe EDF (jusqu'en 2010)

Actuellement Directeur de la Direction des Services Partagés du Groupe EDF

\section{Magdalena CHMIELEWSKA}

Collaboratrice scientifique à la Chaire Organisation et Personnel, Université de Potsdam

\section{Corinne DEQUECKER}

Consultante et Responsable de la Société A.3.C

(Atelier, Compréhension et Conduite du Changement) 


\section{Marnix DRESSEN}

Chercheur au Centre Lillois d'Etudes et de Recherches

Sociologiques et Economiques (CLERSE), Université de Lille I

\section{Christophe GARDAVAUD}

Responsable de la stratégie réglementaire Europe, Deutsche Bahn AG

\section{Samuel GREEF}

Chercheur au Département «Système politique de la République Fédérale d'Allemagne », Université de Kassel

\section{Jesper HAMARK}

Doctorant au Département d'histoire économique, Université de Göteborg

\section{Solène HAZOUARD}

Ingénieur d'études au CIRAC, Cergy-Pontoise

\section{Christoph HERMANN}

Chargé de recherches, Centre de recherche sur le marché du travail (FORBA), Vienne

\section{Viktoria KALASS}

Doctorante au Département «Système politique de la République Fédérale d'Allemagne », Université de Kassel

\section{Jean LAPEYRE}

Expert national détaché, Secrétariat du Secrétaire général,

Comité économique et social Européen (CESE/EESC), Bruxelles (jusqu'en 2009)

Actuellement Chargé de mission Europe, Bureau de représentation de Syndex à Bruxelles

\section{René LASSERRE}

Directeur du CIRAC, Professeur à l'Université de Cergy-Pontoise

\section{Dominik LINDNER}

A coopéré en 2008 au projet « Privatisation of Public Services and the Impact on Quality, Employment and Productivity » (PIQUE)

Travaille actuellement dans le secteur électrique

\section{Achim MEERKAMP}

Membre du Comité directeur de ver.di

\section{Claudia PANKE}

Maire de la ville de Wülfrath, Ancienne Directrice du personnel de DB European Railservice GmbH, DB AutoZug GmbH et CityNightLine AG CNL 


\section{Bernard PARMANTIER}

Directeur des relations sociales, GDF SUEZ

\section{Richard POND}

Membre de la Fédération Syndicale Européenne des Services Publics (FSESP)

\section{Wolfgang SCHROEDER}

Professeur des Universités en sciences politiques, Département «Système politique de la République Fédérale d'Allemagne et Innovation », Université de Kassel

Depuis novembre 2009, Secrétaire d'Etat au ministère du Travail, des Affaires sociales, des

Femmes et de la Famille du Land de Brandebourg

\section{Thorsten SCHULTEN}

Chef du service « Politique de l'emploi et politique tarifaire européennes », Institut des Sciences Economiques et Sociales (Wirtschafts- und Sozialwissenschaftliches Institut, WSI) de la Fondation Hans Böckler, Düsseldorf

\section{Christer THÖRNQVIST}

Professeur associé au Département des sciences du travail, Université de Göteborg

\section{Pierre-Eric TIXIER}

Professeur des Universités, Institut d'Etudes Politiques de Paris, Chercheur au Centre de Sociologie des Organisations (CSO)

\section{Henrik UTERWEDDE}

Directeur adjoint de l'Institut Franco-Allemand de Ludwigsburg (DFI)

\section{Dieter WAGNER}

Titulaire de la Chaire Organisation et Personnel, Université de Potsdam 
Composition : CIRAC
www.cirac.u-cergy.fr

CIRAC, c/o Université de Cergy-Pontoise

33 boulevard du Port - 95011 CERGY-PONTOISE CEDEX

Impression : Dupli-print

2 rue Descartes - Z.I. Sezac - 95330 DOMONT

Dépôt légal : août 2011

Imprimé en France 


\section{Une comparaison France-Allem agne}

Sous la direction de Solène HAZ OUARD, René LASS ERRE, Herrik UT'ERWJEDDE

Dans le cortexte de la promotion et de l'intégration du marché intérieur européen initíes pax liscte unique un processus de dérégutation dars les services publics d'intérêt général a été engagó à l’aube des années 1900 a u riveau corrmuna utaire en vie de favoriser l'émergence fun grand marché des servicer à l'échelle euxopéenne Ce processur de dérégulation avait poux objet dounir à h concurrence les services d'intérêt général des pays membre qui, jusqu ưalos, étaiert généralement onganisér en monopoles publics et cors tituaient poux airs i dire des enchwes na tionales échappart à h foi aux règles cormmunes de $\mathrm{h}$ concurrenoe et, de surcroît, au droit cormum du travail dans les pays membres. De ce fait mềne, bs directives successines adoptéer a u niveau cormmuna utaire, puis transp os ées en droit national, ort non seulement mo difié les modes de régulation économique, mais plus profondément encore les systèmes de régulation socio-profers ionnelle et de ger tion des ress ouxcer humainer de oe secteux, remettant ainsi en question les particularités sociales du service public qui s'étaient affirmées dero h plupart des Etat membre depuis h fin de h Seconde Guerre mondile

Tel est Pobjet du présent oumage qui étudie et compare, principalement à travers $\mathbf{~} \mathbf{b}$ cas de h France et de l'allemagne qui sont au certre de Panalyse, mais aursi ì partir fautres exemples européers, tels que cehi de l'Autriche, de la Grande-Bretagne et de la S uz̀de, les incidences de oette politique de déréguhtion sux les relations collectives de tavail et le raragement socill des enteprises dart le secteur, désigrié désormais sous le terme de servioes dintérêt économiyu général (SIEG). Rlt deli des singularités des expériences nationa les qui ont marqué la structuxa tion initile, pui la transformation des anciens servioes publics, il ressort de Panalyse der effet que h dérégulation a induits dans le charpq social un tableau d'ersemble trèr differencié, darr lequel coexistent des évohutiors régressines en termes d'acquis sociaux statutaires, mais également de nouvelles formes of orgarisation des rapport de travall Les unes et les autres concourent à h moderrisation éconorrique et socinle de oe vaste ersemble qui, inchuant der secteurs aussi essentiek que oux de l'énergie,

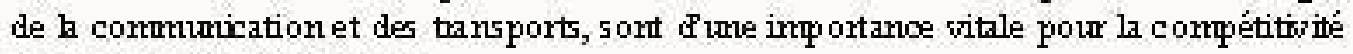
der économies européermer.

Ounage publié avec le concoux du CIERA et du CICC (Uriversité de Cergy-Pontoise)

$$
\begin{gathered}
25 € \text { T'TC } \\
\text { ISBN : } 978-2-905518-40-8
\end{gathered}
$$
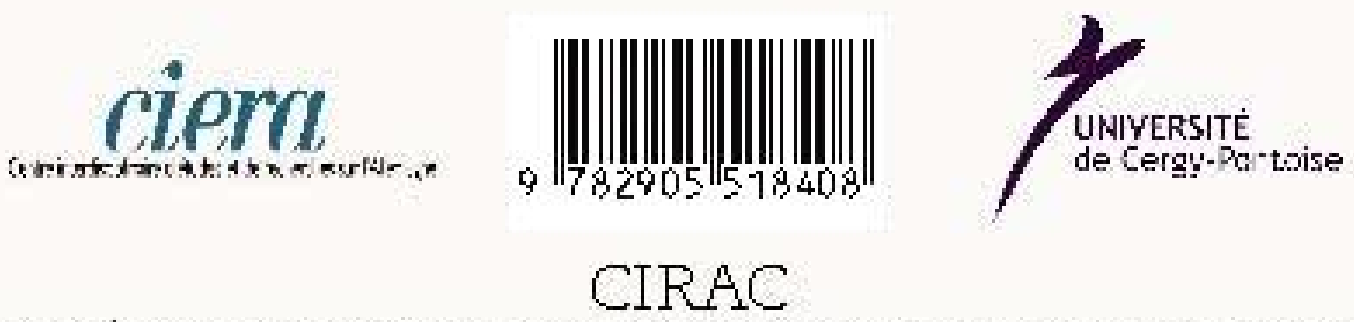